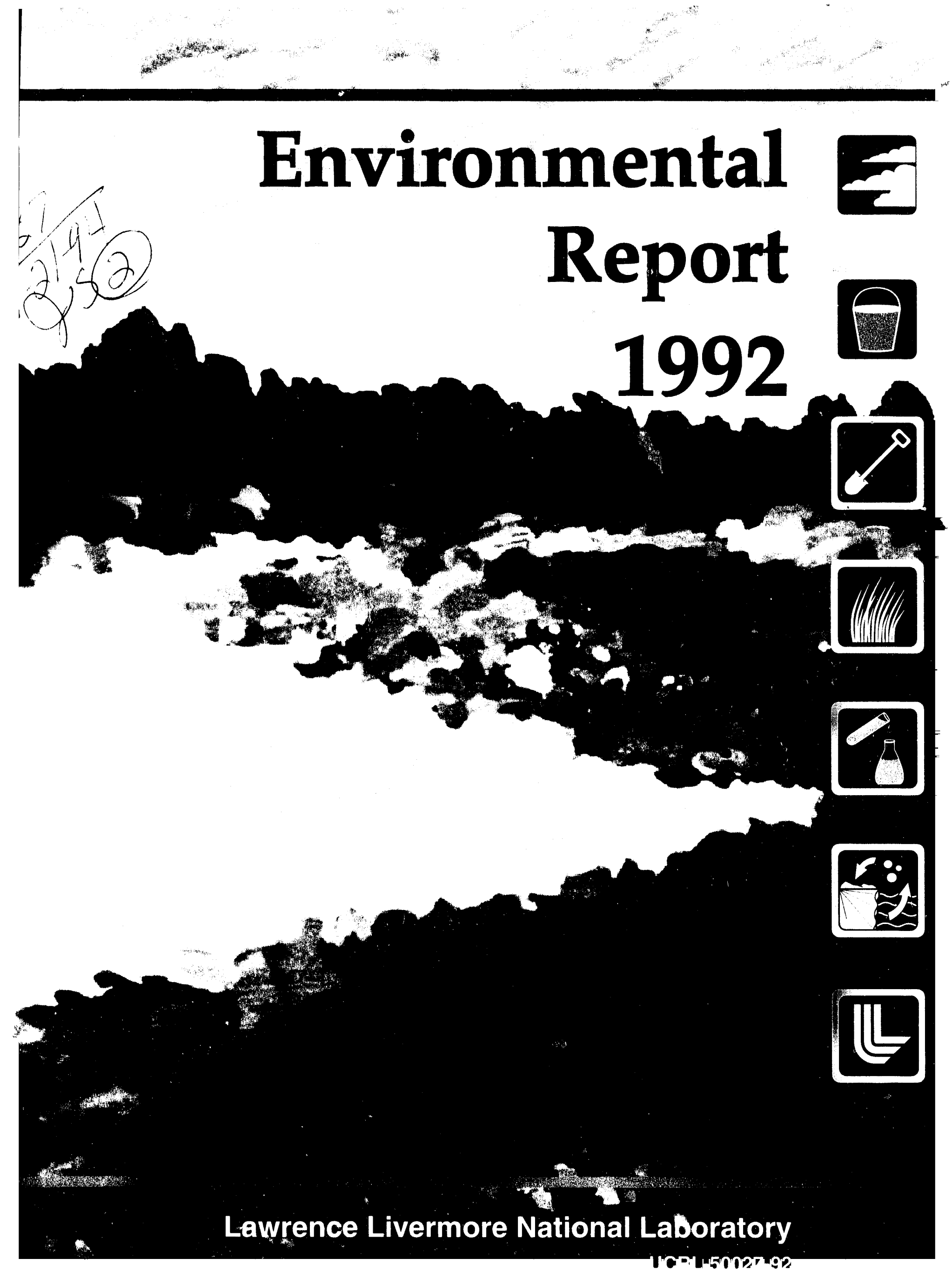




\section{Lead Compositor}

Beverly L. Chamberlain

\section{Art and Design}

Laura M.H. Rose

\section{Composition}

Sherry A. Emmons

Jill S. Sprinkle

\section{Publication Services}

Candy K. Justin
The cover for this year's publication was painted electronically by Laura M.H. Rose of LLNL's Technical Information Department. Laura studied art and design at the University of Arizona, and was lead artist and designer for the Environmental Reports for 1991 and 1992. Starting with a scanned photograph, she used Fractal Design's Painter and a CalComp drawing tablet to create the painted image of the Central Drainage Basin at the Livermore site. She then used Adobe Photoshop to convert it to monochrome and stretch it. The final cover was composited in Quark XPress.

This report was created using electronic publishing. The word processing and layout were performed using Microsoft Word on the Macintosh, and the art was created using Adobe Illustrator.

For additional information about this report, please contact:

Bert Heffner, Area Relations Manager, LLNL Public Affairs Department

P.O. Box 808, L-404

Livermore, CA 94550

(510) 424-4026

\section{DISCLAIMER}

This document was prepared as an account of work sponsored by an agency of the United States Government. Neither the United States Government nor the University of California nor any of their employees, makes any warranty, express or implied, or assumes any legal liability or responsibility for the accuracy, completeness, or usefulness of any information, apparatus, product, or process disclosed, or represents that its use would not infringe on privately owned rights. Reterences herein to any specific commercial products, process or service by trade name, trademark, manufacturer, or otherwise, does not necessarily constitute or imply its endorsement, recommendation, or favoring by the United States Government or the University of California. The views and opinions of authors expressed herein do not necessarily reflect those of the United States Government or the University of California, and shall not be used for advertising or product endorsement purposes. 


\title{
Environmental Report 1992
}

\author{
Authors \\ Gretchen M. Gallegos \\ Saundra M. Wander \\ Brian K. Balke \\ Eric Christofferson \\ Paula J. Tate \\ Kris A. Surano \\ Robert J. Harrach \\ Lucinda M. Garcia \\ Barbara C. Fields \\ William G. Hoppes \\ Rebecca A. Failor
}
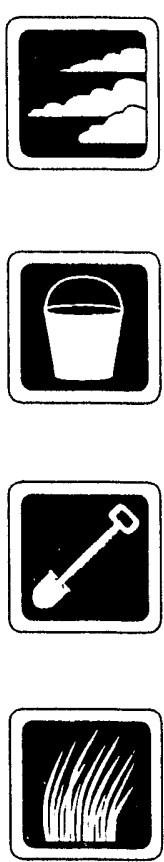

Contributing Authors

Albert L. Lamarre

Richard G. Blake

Duane W. Rueppel

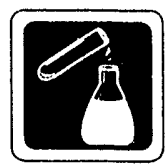

William F. Isherwood Donald H. MacQueen Robert L. Berger

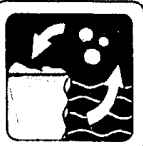

Editor

Gloria C. Wilt

\section{MASTER}

Lawrence Livermore National Laboratory 
This report is prepared for the U.S. Department of Energy (DOE) by the Environmental Protection Department (EPD) at the Lawrence Livermore National Laboratory (LLNL). This publication presents the results of LLNL's environmental monitoring and compliance effort and provides an assessment of the impact of LLNL operations on the environment.

As in the 1991 report, data are presented in Système International (SI) units. This is consistent with the federal law stated in the Metric Conversion Action of 1975 (15 United States Code 205a et seq.); Presidential Executive Order 12770, Metric Usage in Federal Government programs (July 25, 1991); and DOE's policy on the use of the metric system (DOE Order 5900.2), which states in part that "All Departmental Elements should take steps, as directed by Executive Order 12770, to use the Metric System (SI) in procurements, grants, publications, and other business-related activities." Radiological results are presented in becquerels, and summary statistics are repeated in curies $\left(1 \mathrm{~Bq}=2.7 \times 10^{-11} \mathrm{Ci}\right)$. Similarly, dose is presented in millisieverts and summary statistics are repeated in millirem $(1 \mathrm{mSv}$ $=100 \mathrm{mrem}$ ).

Results for both the Livermore site and Site 300 are presented in each chapter, and trends in monitoring data are shown graphically.

This document is the responsibility of the Environmental Monitoring and Analysis Division of the EPD. Within this division, personnel from the Environmental Monitoring Section (EMS) sampled the environment, interpreted the results, performed the impact analysis of radiological emissions from LLNL, and compiled the information presented here.

Monitoring data were obtained through the combined efforts of the EMS, the Nuclear Chemistry Division, the Radiological Analytical Sciences laboratory, and the Hazards Control Department of LLNL. Special recognition is deserved for the dedication and professionalism of these environmental monitoring technicians-Paris E. Althouse, Paul C. Dickinson, David L. Graves, Renee Needens, ${ }^{*}$ Donald G. Ramsey, Rebecca J. Ward, and Rhonda L. Welsh-and to these data management personnel-Nina Hankla,* Jennifer Clark, ${ }^{*}$ Colleen R. Frost, and Connie Wells.* 
In addition, the following people made significant contributions to this report:

\section{Other EPD Contributors}

David H. Armstrong

Shari L. Brigdon

Bruce E. Campbell

Tina M. Carlsen

Sabre J. Coleman

Michelle L. Corcoran

Barbara L. Danielson

T. David Epley

Robert P. Felicitas

Robert P. Fischer

Karen J. Folks

Stephanie S. Goodwin

Charlene $\mathrm{H}$. Grandfield

Allen R. Grayson

Amelia Hagen

Howard L. Hall

Marion G. Heaton

Richard K. Henry

Joy M. Hirabayashi

Daniel B. Knight

Richard K. Landgraf

Jennifer Larson*

\author{
David V. Martini \\ William A. McConachie \\ Bruce K. McDowell \\ Joseph R. McIntyre \\ Michael P. Meltzer \\ Richard L. Michalik \\ Holly $\mathrm{H}$. Miller \\ Harold E. Pfeifer \\ Ruth A. Presley \\ David W. Rice \\ Maureen N. Ridley \\ William W. Schwartz \\ David J. Shoemaker \\ John M. Sims \\ Michael J. Taffet \\ Stanley H. Terusaki \\ Janet L. Tweed \\ Charlotte VanWarmerdam \\ Jill Watz \\ Robin A. Wendt \\ Kenneth C. Zahn \\ John P. Ziagos
}

\section{Contributors from Other Departments}

Karen L. Anderson, Assurances Office James Merrigan, Nuclear Chemistry

Ruth M. Anderson, Nuclear Chemist. J Richard J. Nagle, Nuclear Chemistry

Arthur H. Biermann, Hazards Control

V. Janet Brown, Nuclear Chemistry

Ruth M. Nuckolls, Nuclear Chemistry

Neil B. Crow, Health \& Ecological

Ann M. Ruth, ${ }^{*}$ Nuclear Chemistry

Assessment

Gary L. Seibel, Hazards Control

Rohit K. Shah, Hazards Control

Janet C. Garrison, Nuclear Chemistry

Frank J. Gouveia, Health \& Ecological

Assessment

Duane P. Straub, Hazards Control

Gerry Stuart, ${ }^{*}$ Nuclear Chemistry

David G. Trombino, Hazards Control

G. Bryant Hudson, Nuclear Chemistry

Joane M. Westermark, Hazards Control

Terry Jokela, Health \& Ecological

Assessment

Kai M. Wong, Health \& Ecological

Assessment

Ann Lee, ${ }^{*}$ Nuclear Chemistry

Mary A. Lee, Hazards Control

Susan C. MacLean, Nuclear Chemistry $\quad{ }^{*}$ Contract employee 
List of Figures...................................................................................................................... x. xiii

List of Tables.......................................................................................................................... xvii

Executive Summary …........................................................................................ EX-1

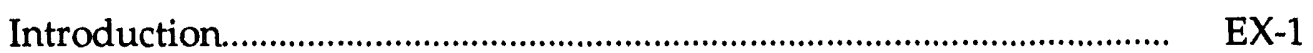

Environmental Monitoring Results....................................................... EX-1

Radiological Impact Assessment.......................................................... EX-5

Environmental Compliance Activities ………………………............... EX-6

Conclusion .............................................................................. EX-8

1. Site Overview................................................................................... 1-1

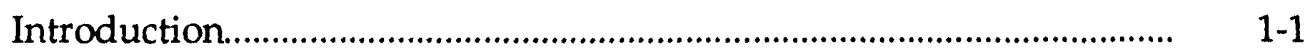

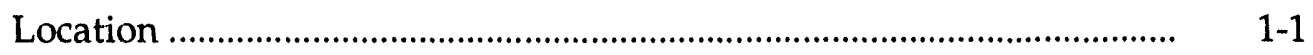

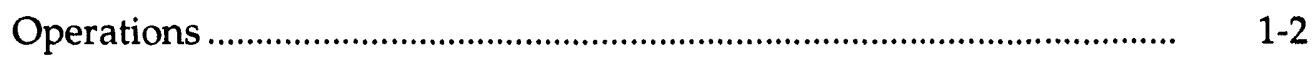

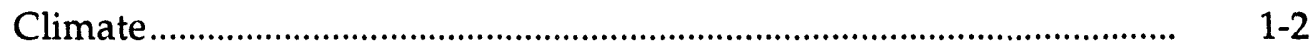

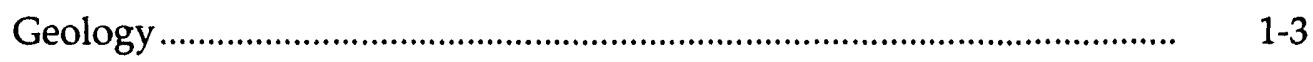

Topography ....................................................................

Hydrogeology .....................................................................

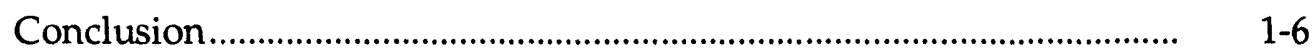

2. Compliance Summary ………………..........................................................

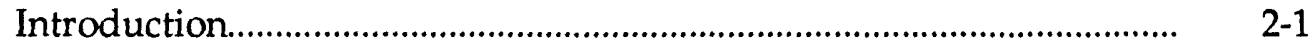

Department of Energy Tiger Team ....................................................... 2-1

CERCLA/SARA-Title I.................................................................. 2-2

Livermore-Site Ground Water Project......................................... 2-2

Site 300 Environmental Restoration Project ............................... 2-4

SARA-Title III ............................................................................

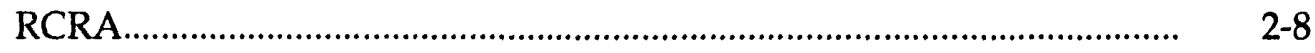

Hazardous Waste Management Activities .................................. 2-. 2-8

DOE Hazardous Waste Disposal Moratorium .......................... 2- 2-8

Hazardous Waste Report for 1992 ............................................... $\quad 2-8$ 
Hazardous Waste Permits............................................................. 2-9

Extremely Hazardous Waste Permit ........................................... 2-. 2-9

Hazardous Waste Transport Registration ................................. 2 2-10

Medical Waste Permit............................................................... 2-. 2-10

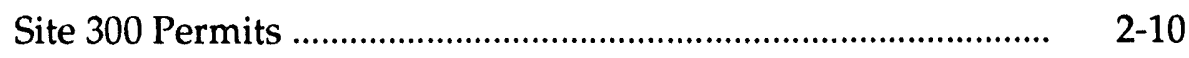

Inspections ......................................................................... 2-.

Waste Accumulation Areas ..................................................... 2-. 2-12

Underground Storage Tank Management............................................. 2-13

Underground Tank Permits....................................................... 2-13

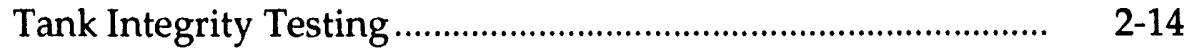

Closure and Leak Documentation .............................................. 2-14

Tank Upgrade Project ............................................................... 2-15

Remedial Activities ………………………………............... 2-15

Inspections .......................................................................... 2-16

National Environmental Policy Act (NEPA) and California

Environmental Quality Act (CEQA)................................................... 2-17

NEPA ............................................................................. 2-17

Environmental Assessments Submitted to DOE ....................... 2-18

Environmental Impact Statement/Environmental

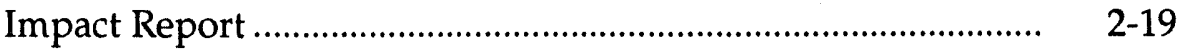

CEQA …

Endangered Species Act .................................................................... 2-20

National Historic Preservation Act......................................................... 2-20

Clean Water Act-NPDES/Waste Discharge Requirements ................. 2- 2-21

Ground Water and Surface Water Discharge Permits.............. 2 2-21

Wastewater Permits................................................................. 2-2 2-2

Inspections ........................................................................... 2-23

Streambed Alteration Agreements ............................................ 2-23

Clean Air Act/Air Quality Management Activities............................... 2- 2-24

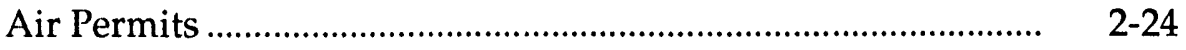

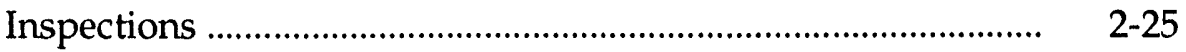

National Emission Standards for Hazardous Air

Pollutants (NESHAPs) ............................................................ 2-25

Inspections ......................................................................... 2-26

Toxic Substances Control Act (TSCA).................................................. 2-27

Current Issues and Actions................................................................ 2-27 
3. Environmental Program Information............................................................. 3-1

Introduction.................................................................................. 3-1

Environmental Protection Department............................................... 3-1

Operations and Regulatory Affairs Division............................... 3-2

Hazardous Waste Management Division ................................... 3-3

Environmental Restoration Division ........................................ 3-3

Environmental Monitoring and Analysis Division.................... 3-4

Self-Monitoring Programs....................................................................... 3-5

Treatment Facility A …......................................................

Treatment Facility B ........................................................... 3-5

Treatment Facility F .......................................................... 3-5

General Construction Activity................................................... 3-6

General Industrial Activity .................................................... 3-6

Central Drainage Basin .................................................................... 3-6

Quarterly Minor Spill Report .................................................. 3-6

Cooling Tower ................................................................................ 3-7

Waste Minimization and Pollution Abatement ....................................... 3-7

Waste Minimization and Pollution Prevention

Awareness Plan .......................................................................... 3-7

Waste Minimization .................................................................. 3-9

Nonhazardous-Materials Recycling ......................................... 3-11

Compliance Activities.................................................................... $\quad 3-12$

Building Inspections ................................................................... 3-12

Waste Accumulation Area Inspections ...................................... 3-13

Spill Response ......................................................................... 3-13

Site Evaluations Prior to Construction......................................... 3-13

Environmental Training ........................................................... $3-14$

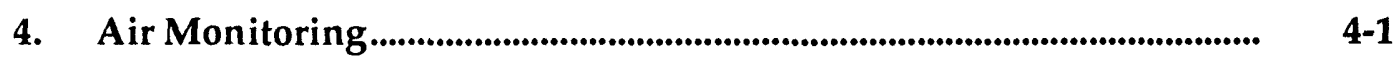

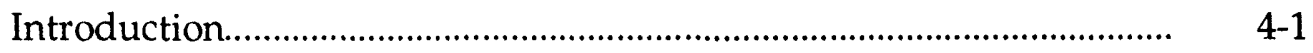

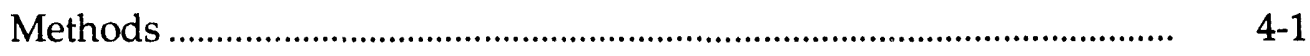

Results ....................................................................................... 4-5

Livermore Site................................................................ $4-5$

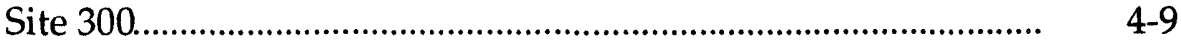

Environmental Impact ......................................................................... 4-10

Radioactive Effluents .......................................................... $\quad 4-10$

Nonradioactive Effluents ....................................................... $\quad 4-10$ 
5. Sewage Monitoring.......................................................................................... 5-1

Introduction ...............................................................................

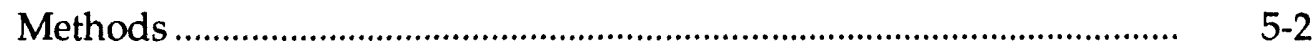

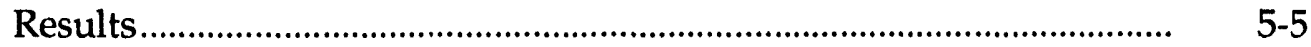

Radioactivity in Sewage ............................................................ 5-5

Nonradioactive Pollutants in Sewage ...................................... 5-7

Environmental Impact ......................................................................... 5- 5

Radioactivity in Sewage .......................................................

Nonradioactive Liquid Effluents ............................................. $\quad 5-10$

6. Surveillance Water Monitoring................................................................... 6-1

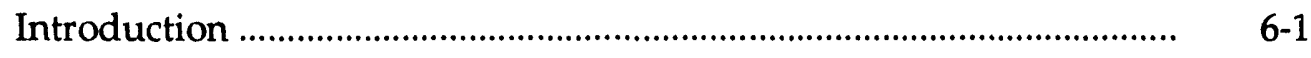

Methods ........................................................................................

Livermore Water Reclamation Plant ......................................... 6-2

Livermore Valley ....................................................................

Livermore Site..................................................................

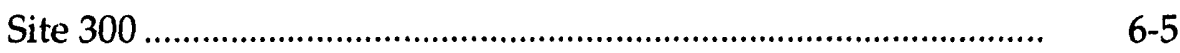

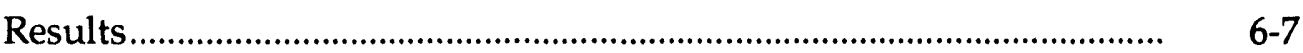

Livermore Site/Livermore Valley, Radioactivity in

Water.

Livermore Site/Livermore Valley, Nonradioactive

Pollutants in Water.....................................................................

Site 300, Radioactivity in Surface Water ..................................... 6-.

Site 300, Radioactivity in Ground Water ...................................

Site 300, Nonradioactive Pollutants in Water .............................. 6- 6-9

Environmental Impact ............................................................................. 6-11

Livermore Site ........................................................................ 6-11

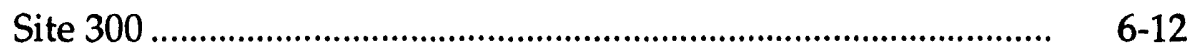

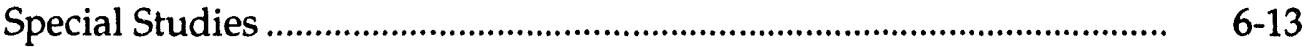

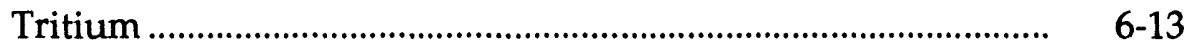

Central Drainage Basin ............................................................... 6... 6-15

7. Ground Water Protection..........................................................................

Introduction ................................................................................

Livermore Site............................................................................

Ground Water Remediation ....................................................

Ground Water Protection........................................................... 
Site 300 . 7-10

Ground Water Remediation.................................................... 7-10

Ground Water Protection Monitoring......................................... $\quad$ 7-13

Environmental Impact ....................................................................... 7-20

Livermore Site........................................................................... $\quad 7-20$

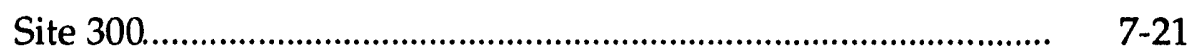

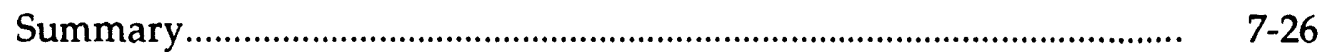

8. Soil and Sediment Monitoring ............................................................ 8-1

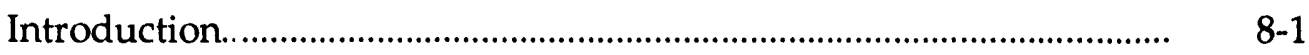

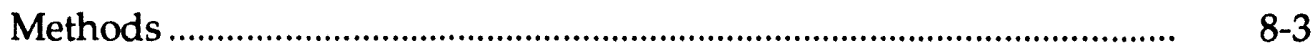

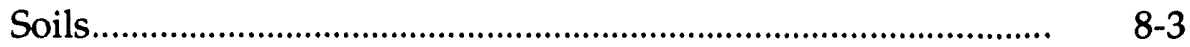

Stream Sediments ................................................................ 8-6

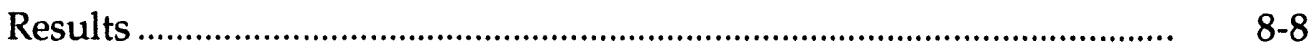

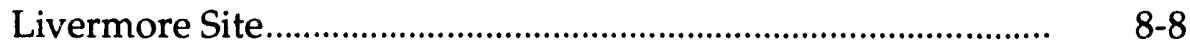

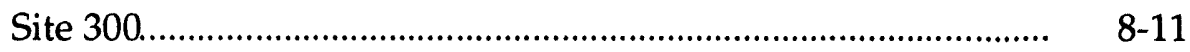

Environmental Impact ................................................................... $\quad 8-12$

Livermore Site........................................................................ $8-12$

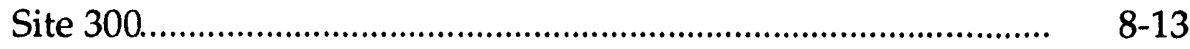

Special Projects: Shallow Sediment Sampling ..................................... 8-13

9. Vegetation and Foodstuff Monitoring .................................................... 9-1

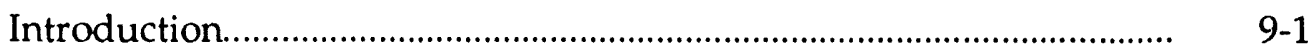

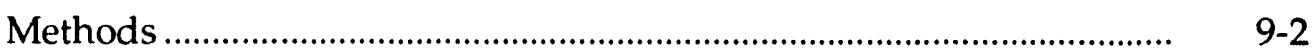

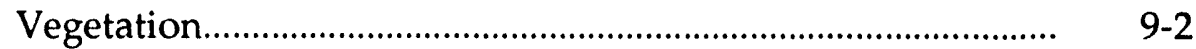

Milk ........................................................................................... $9-5$

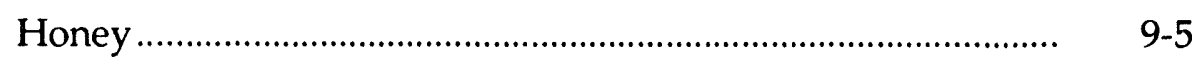

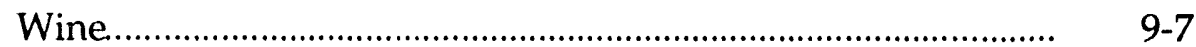

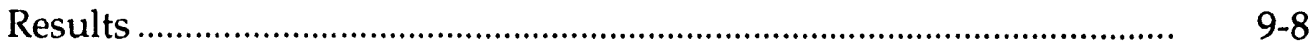

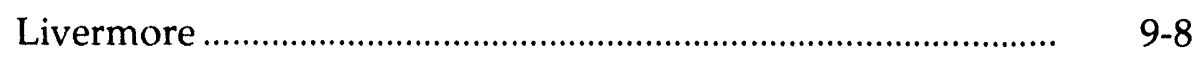

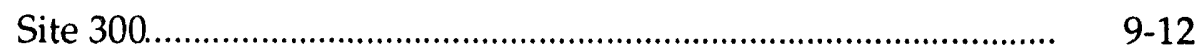

Environmental Impact ....................................................................... $9-13$

Livermore Site...................................................................... $9-13$

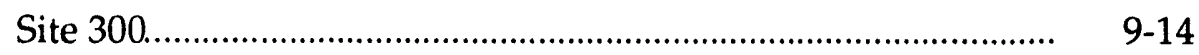

10. Environmental Radiation Monitoring ................................................... 10-1

Introduction.................................................................................. 10-1 
Methods

$10-1$

Results 10-3

Livermore Site.

$10-3$

Site 300 .

$10-4$

Environmental Impact ............................................................. $\quad 10-5$

Livermore Site................................................................ $10-5$

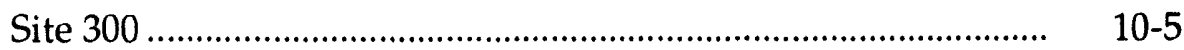

Special Study ................................................................................ $\quad 10-5$

11. Radiological Dose Assessment .............................................................. 11-1

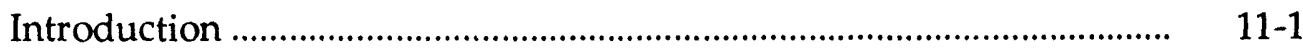

Natural and Man-Made Radiation ............................................. 11-1

Radioactivity ......................................................................... 11-2

Measurement of Radioactivity and Dose................................... 11-3

Sources of Natural Radioactivity ............................................. 11-4

Radiation Sources, Control Measures, and Standards......................... 11-5

Radionuclides at LLNL........................................................ 11-5

LLNL's Radiation Control Program ......................................... 11-5

Radiation Protection Standards................................................ 11-6

Radiological Doses from Air Emissions ............................................... 11-7

Monitored Releases of Radioactive Materials ........................... 11-8

Air Emissions Data................................................................... 11-9

LLNL Buildings with Radionuclide Release Potential .............. 11-9

Calculations of Effective Dose Equivalents ........................................ 11-10

Description of Dose Model, Input Parameters, and

Defined Terms ........................................................................ 11-10

Calculated Results Summary-Livermore Site and

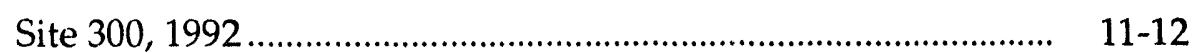

Summary and Conclusion.......................................................... 11-14

12. Non radiological Impact .................................................................... 12-1

Introduction .............................................................................. 12-1

Air Monitoring .............................................................................. $12-1$

Sewage Monitoring ......................................................................... 12-2

Water Monitoring ....................................................................... $12-4$

Soil and Sediment Monitoring........................................................ 12-5

Noise Monitoring ….......................................................................... $12-7$ 
Environmental Occurrences.

Superfund Amendments and Reauthorization Act (SARA)

Title III Reporting Requirements.

13. Quality Assurance

Introduction.

Deviations and Changes to the Sampling Program

13-2

Sampling Location Designators

13-2

Air Sampling Program.

13-2

Sewage Monitoring Program.

13-2

Routine Water Sampling Program

13-3

Vegetation and Foodstuff Sampling Program

13-3

Environmental Radiation Monitoring Program.

13-4

Meteorology Monitoring Program

13-4

Soils/Sediments Monitoring Program....

$13-4$

Compliance Ground Water Sampling Program.

13-5

Routine Ground Water Monitoring

13-6

Quality Assurance Activities.....

13-6

Participation in Laboratory Intercomparison Studies............... 13-6

Duplicate Analyses .............................................................. 13-8

Statistical Methods.......................................................................... 13-8

Radiological Data ................................................................ 13-8

Nonradiological Data........................................................... 13-9

Statistical Comparisons......................................................... 13-9

Summary Statistics................................................................ 13-9

Radiation Units ................................................................ $\quad 13-10$

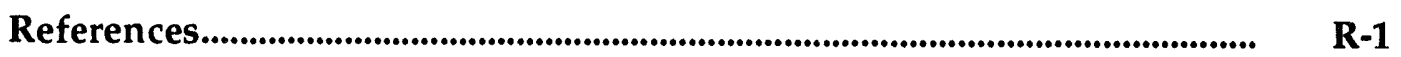

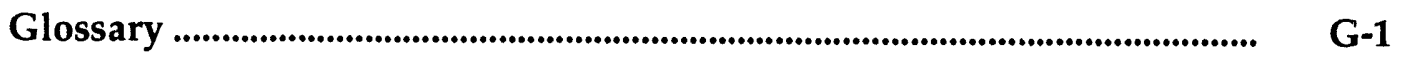

Appendices

Appendix A. 1992 EPD Publications ….................................................. A- A-1

Appendix B. Methods of Dose Calculations ......................................... B-1

Appendix C. Ground Water Protection Tables..................................... C-1

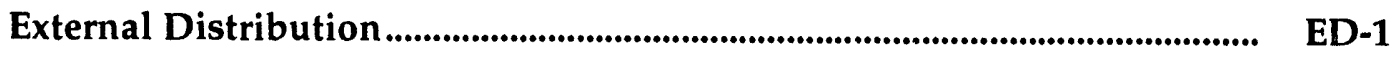


1-1. Wind rose showing the average annual wind speed, frequency of occurrence, and direction at the Livermore site, 1992

1-2. Wind rose showing the average annual wind speed, frequency of occurrence, and direction at Site 300, 1992

1-3. Approximate ground water and surface elevation contours, Livermore site and vicinity

1-4. Ground water and surface elevations at Site 300, 1992

4-1. Air particulate and tritium sampling locations,

Livermore site, 1992

4-2. Air particulate and tritium sampling locations,

Livermore Valley, 1992

4-3. Air particulate sampling locations, Site 300, 1992

4-4. Mean plutonium concentrations on air monitors at two

locations, 1982-1992.

4-5. Mean concentration of beryllium on air filters,

Livermore-site perimeter, 1974-1992.

5-1. Sewer monitoring network, 1992

5-2. LLNL tritium concentration in sewage effluent, 1992

5-3. LLNL monthly average plutonium and cesium

concentrations in sewage

6-1. Ground water well sampling locations, Livermore

Valley, 1992

6-2. Surface and drinking water sampling locations,

Livermore Valley, 1992 
6-3. Rain sampling locations, Livermore site and Livermore Valley, 1992

6-4. Storm water runoff sampling locations, Livermore site and vicinity, 1992.

6-5 Water sampling locations and access roads, Site 300 and vicinity, 1992

6-6. Tritium activity in the Livermore Valley, 1989-1992

7-2 Ground water monitoring locations with tritium concentrations exceeding $37 \mathrm{~Bq} / \mathrm{L}(1000 \mathrm{pCi} / \mathrm{L})$ at LLNL, January 1992

7-3 Several environmental restoration activities are in progress at Site 300 .

7-4 Landfills (active and inactive) and associated monitoring wells, Site 300, 1992

7-5 Tritium activities $(\mathrm{Bq} / \mathrm{L})$ in ground water in the Pit 7 Complex, Building 850/Doall Ravine, and EFA subareas, EFA/WFA study area, 4th Quarter 1991

Freon-113 in wells K1-05, K1-08, and K1-09, 1992

7-8 Trichloroethane in wells W-817-03, W-817-03A, and

Tritium in well K1-02B, 1989-1992

8-1. Soil sampling locations, Livermore Valley, 1992

8-2. Site 300 soil sampling locations, 1992 ..

8-3. Arroyo and drainage basin sediment sampling locations, 1992

8-4. Plutonium concentration in surface soils, 1980-1992.

8-5. Uranium concentration in surface soils, 1980-1992

9-1. Livermore Valley vegetation sampling locations, 1992 


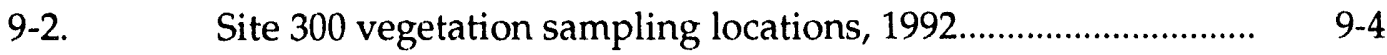

9-3. Goat and cow milk sampling locations, 1992.......................... 9-6

9-4. Mean tritium in retail wines, 1977-1992, plotted by sampling year (error bars are \pm 1 standard error)

9-5. Mean tritium in retail wines, 1980-1991, decay-corrected and plotted by vintage year (error bars are \pm 1 standard error) ....... $\quad 9-10$

9-6. Tritium concentration in honey (error bars are \pm 1 standard error)

10-1. Gamma and neutron dosimeter locations, Livermoresite, 1992

10-2. Gamma dosimeter locations, Livermore Valley, 1992 $10-3$

10-3. Gamma dosimeter locations, Site 300, 1992.

$10-4$

10-4. Gamma dosimeter locations, San Francisco Bay Area, 1992.... 10 10-6

10-5. Off-site gamma radiation background dose frequency distribution, Livermore, 1992

11-1. Typical annual radiation doses from various sources (NCRP 1987)

12-1. Monthly mean concentrations of beryllium on air filters, Livermore site and Site 300, 1992

12-2. Summary of monthly average results for nonradiological substances in the sanitary sewer

12-3. Comparison of maximum values of nonradiological water pollutants at Site 300 locations to their regulatory limits.

12-4. Average concentration of selected analytes in sediments and soils as a percent of regulatory limits for disposal

12-5. Noise monitoring locations in Tracy and surrounding area, 1992. 
1-1. Average annual percent frequency of wind direction at $10 \mathrm{~m}$ above ground level, versus wind speed $(\mathrm{m} / \mathrm{s})$ for the Livermore site and Site 300, 1992 (one-hour averages).............. 1-8

2-1. Summary of Permits as of March 15, 1993 ............................... 2-28

3-1. TFA WDR self-monitoring sampling and analysis schedule.

4-1. Gamma activity on air filters, Livermore-site perimeter, 1992.

4-2. Plutonium-239 activity on air filters (in $10^{-13} \mathrm{~Bq} / \mathrm{mL}$ ),

Livermore Valley, 1992

4-3. Plutonium and uranium activity on air filters,

Livermore-site perimeter, 1992

4-4. Plutonium-239 activity on air filters (in $10^{-13} \mathrm{~Bq} / \mathrm{mL}$ ), $1992 \ldots$

4-5. Tritium in air (in $10^{-7} \mathrm{~Bq} / \mathrm{mL}$ ), Livermore-site perimeter, 1992.

4-6. Tritium in air (in $10^{-7} \mathrm{~Bq} / \mathrm{mL}$ ), special study, 1992 ................. 4-22

4-7. Tritium in $\operatorname{air}$ (in $10^{-7} \mathrm{~Bq} / \mathrm{mL}$ ), Livermore Valley, 1992........... 4-23

4-8. Beryllium on air filters (in $\mathrm{pg} / \mathrm{m}^{3}$ ), Livermore-site perimeter, 1992.

4-9. Gamma activity on air filters, Site 300, 1992.

4-10. Plutonium and uranium activity on air filters,

Site 300, 1992

4-11. Beryllium on air filters (in $\mathrm{pg} / \mathrm{m}^{3}$ ), Site 300, 1992

4-12. Radioactive airborne effluent releases from the

Livermore site, 1981 through 1992. 
4-13. Nonradioactive air emissions, Livermore site and

Site 300, 1992

5-1. Estimated total radioactivity in sanitary sewer effluent,

LLNL, 1992

5-2. Sewer discharge release limits for tritium $\left({ }^{3} \mathrm{H}\right),{ }^{137} \mathrm{Cs}$, and ${ }^{239} \mathrm{Pu}$

5-3. Various radionuclides in sanitary sewer effluents (in $10^{-6} \mathrm{~Bq} / \mathrm{mL}$ ), LLNL and LWRP, 1992

5-4. Metals discharged to sanitary sewer system (in $\mathrm{mg} / \mathrm{L}$ ), 1992 summary

5-5. Positively detected parameters in LLNL sanitary sewer effluent, 1992

5-6. Radioactive liquid effluent releases from the Livermore site, 1983-1992

5-7. Summary of contaminant releases detected by the sewer monitoring system, 1992

6-1. $\quad$ EPA standard methods for water sampling. 6-17

6-2. Radioactivity in storm water runoff (in Bq/L), 1992 6-18

6-3. Radioactivity in water, Livermore Valley (in Bq/L), 1992 ........

6-4. Tritium in rain, Livermore site and Livermore Valley (in Bq/L), 1992

6-5. Tritium activity in wells, Livermore Valley (in Bq/L), 1992.....

6-6. LLNL storm water runoff, nonradioactive parameters, 1992

6-7. Quarterly radioactivity in surface water (in $\mathrm{Bq} / \mathrm{L}$ ),

Site 300, 1992 .

6-8. Analyses of water from supply wells near Site 300, 1992.........

6-9. Quarterly analyses of water from surveillance monitoring wells, Site 300, 1992

6-10. Analyses of water from supply wells, Site 300, 1992 
6-11. Central Drainage Basin management results

(in $\mathrm{mg} / \mathrm{L}$ ), 1992

6-12. Central Drainage Basin discharge results (in $\mu \mathrm{g} / \mathrm{L}), 1992$........ 6-50

7-1. Water quality parameters and detection limits for monitoring wells, Site 300, 1992

7-2. Parameters sampled in Pits 1 and 2 Barcad samplers, Site 300, 1992

8-1. Positively detected radionuclides and beryllium in soil sampled at depths from 0 to $50 \mathrm{~mm}$, Livermore Valley, 1992 ..

8-2. Positively detected radionuclides in arroyo sediments near the Livermore site, 1992

8-3. Positively detected metals (in $\mathrm{mg} / \mathrm{L}$ ) in arroyo sedir ents near the Livermore site, 1992.

8-4. Positively detected organic compounds (in $\mathrm{mg} / \mathrm{kg}$ ) in arroyo sediments at the Livermore site, 1992.

8-5. Positively detected radionuclides and beryllium in soil sampled at depths from 0 to $50 \mathrm{~mm}$, Site 300, 1992.

8-6. Positively detected radionuclides in arroyo sediments ( 0 to $50 \mathrm{~mm}$ ) near the Livermore site, 1992.

8-7. Positively detected metals (in $\mathrm{mg} / \mathrm{L}$ ) in arroyo sediments

( 0 to $50 \mathrm{~mm}$ depth) near the Livermore site, 1992

9-1. Tritium in vegetation (in $\mathrm{Bq} / \mathrm{L}$ of moisture content), 1992 ....... 9-15

9-2. Tritium in retail wine (in Bq/L), 1992 .................................... 9-17

9-3. Tritium in honey (in Bq/L in recovered water), 1992 ............... 9-17

9-4. Tritium in goat's milk (in Bq/L), 1992 _..................................... 9-18

9-5. Potassium-40 in milk (in Bq/L), 1992 ..................................... 9-20

9-6. Cesium-137 in milk (in Bq/L), 1992 ........................................ 9-22

9-7. Tritium in vegetation (in $\mathrm{Bq} / \mathrm{L}$ of moisture content), Site 300,1992 
10-1. TLD environmental radiation measurements (in $\mathrm{mSv}$ ),

Livermore-site perimeter, 1992.................................................. 10-9

10-2. TI,D environmental radiation measurements (in $\mathrm{nSV}$ ),

Livermore Valley, 1992 .............................................................. 10-10

10-3. Neutron environmental radiation measurements (in $\mathrm{mSv}$ ),

Livermore-site perimeter, 1992.

$10-12$

10-4. TLD environmental radiation measurements (in mSv),

Site 300 perimeter and off-site locations, 1992

10-5. TLD environmental radiation measurements (in $\mathrm{mSv}$ ), special study, Site 300 vicinity, 1992

10-6. TLD environmental radiation measurements (in mSv), special study, San Francisco Bay Area, 1992-1993

11-1. LLNL buildings containing RMMAs

11-2. Annual radiation dose via airborne emissions, 1992

11-3. Comparison of radiation doses, LLNL, 1992

13-1. LLNL/Livermore Valley sampling location designators

13-2. Site 300 sampling location designators

13-3. Results from the EPA Environmental Monitoring Systems Laboratory (EMSL) Intercomparison Program, 1992

13-4. Results from the California Department of Health Services Environmental Laboratory Accreditation Program (ELAP) Water Pollution Studies.

13-5. Results from the DOE Environmental Measurements Laboratory (EML) Quality Assurance Program, 1992

13-6. Quality assurance duplicate sampling, selected parameters, 1992 


\section{Introduction}

Lawrence Livermore National Laboratory (LLNL), a U.S. Department of Energy facility operated by the University of California, serves as a national resource of scientific, technical, and engineering capability with a special focus on national security. Over the years, the Laboratory's mission has been broadened to encompass such areas as strategic defense, energy, the environment, biomedicine, the economy, and education. The Laboratory carries out this mission in compliance with local, state, and federal environmental regulatory requirements and takes measures to ensure that its operations do not adversely affect the environment or public health. It does so with the support of the Environmental Protection Department, which is responsible for environmental monitoring, environmental compliance, environmental restoration, and hazardous waste management. The first activity, environmental monitoring, is the focus of this report.

LLNL comprises two sites: the Livermore site and Site 300. The Livermore site occupies an area of 3.28 square kilometers on the eastern edge of Livermore, California. Site 300, LLNL's experimental testing site, is located 24 kilometers to the east in the Altamont Hills, and occupies an area of 30.3 square kilometers. Environmental monitoring activities are conducted at both sites as well as in surrounding areas.

During 1992, the Envirc inmental Protection Department conducted sampling of air, sewage effluent, ground water, surface water, soil, vegetation and foodstuffs, and took measurements of environmental radiation. It performed more than 150,000 analyses of environmental samples. The analytical results are summarized in the chapters that follow, along with evaluations of the impact of radioactive and nonradioactive materials, a discussion of the effects of LLNL operations on the environment, and a summary of the activities undertaken to comply with local, state, and federal environmental laws.

\section{Environmental Monitoring Results}

LLNL's sampling networks undergo constant evaluation; changes to them are made, as necessary, to ensure adequate monitoring of all media potentially affected by LLNL operations. Once samples are collected, they are analyzed for radioactive and nonradioactive substances using standard methods such as EPA- 
approved analytical procedures, special systems such as the continuous monitoring system for Livermore site sewage, or special analytical techniques designed to measure very low levels of radionuclides. Environmental radiation is also measured directly using dosimeters.

The primary ongoing pollutant of concern continues to be tritium, the radioactive isotope of hydrogen. The emissions of tritium at the Livermore site were significantly lower in 1992 than in past years. The primary source of tritium emissions, the Tritium Facility, has ceased operations except for inventory reduction activities. The lower tritium emissions are reflected in the tritium measured in surface water, rain water, and runoff. Small decreases have also been seen in measured values for tritium in air and vegetation.

Particulate matter in air is monitored for beryllium and for radionuclides, including plutonium and uranium. Most of the radioactivity detected is from naturally occurring radionuclides and global fallout from historical nuclear weapons testing. Plutonium is found at low levels around the perimeter of the Livermore site and at even lower levels at Site 300. The highest average plutonium value was measured at a location on the Livermore site near Building 531. The average concentration there was less than $2.7 \times 10^{-13}$ bequerels per milliliter $\left(\mathrm{Bq} / \mathrm{mL}\right.$ ) of air (less than $7 \times 10^{-18} \mu \mathrm{Ci} / \mathrm{mL}$ ), a small fraction of the regulatory exposure guideline of $7.4 \times 10^{-10} \mathrm{~Bq} / \mathrm{mL}$ of air $\left(2 \times 10^{-14} \mu \mathrm{Ci} / \mathrm{mL}\right)$.

To determine whether operations at Site 300 are affecting the amounts of uranium, which occurs naturally, environmental analysts measure the relative amounts of two isotopes of uranium, uranium-238 and uranium-235. Natural uranium is primarily uranium-238, with $0.7 \%$ uranium- 235 . Most LLNL uranium operations use uranium-238, a slightly radioactive isotope. The observed ratio of the two isotopes, therefore, can reveal whether LLNL operations have added uranium-238 to the environment at Site 300 . The ratios of airborne particulate uranium in January and October 1992 deviate from the natural ratio, indicating the presence of airborne uranium-238 from Site 300 operations. The measured concentrations of uranium-238, however, are only a small fraction $(24 / 100,000)$ of the regulatory exposure guideline of $0.03 \mu \mathrm{g} / \mathrm{m}^{3}$.

Discharges of radioactive and hazardous materials to the combined sanitary and industrial sewer at the Livermore site are controlled by limiting the use of those materials, implementing engineering controls, and routing discharged material to retention tanks for later characterization and treatment. Flow proportional samples of discharged wastewater are regularly collected and analyzed to assure that LLNL's sewage effluent meets the requirements of the permit granted by the City of Livermore. In addition, effluent is monitored continuously for $\mathrm{pH}$, selected metals, and radioactivity. Should concentrations be detected above warning levels, LLNL's Sewer Diversion System would be automatically activated. The diversion system will capture all but the first few 
minutes of wastewater flow that causes an alarm, thereby protecting the Livermore Water Reclamation Plant (LWRP) and minimizing any required cleanup. Eight minor metal releases were detected in 1992. In every case, a more detailed analysis of the diverted sewage showed that the slight contamination did not represent a threat to LWRP operations. With the exception of elevated levels of oil and grease in the first half of 1992, no regulatory violations were noted. The results of the effluent monitoring program demonstrate the success of LLNL's discharge control programs.

As in 1991, monitoring results for sewage show concentrations of plutonium and cesium slightly higher than the lowest values seen historically. These results are related to sewer cleaning with new, more effective equipment. However, the measured plutonium activities were further elevated during the months of January through March 1992. For those three months, the data increase by a factor of ten above values measured during 1991 and the other months of 1992. Possible causes of the elevated levels were investigated, but no definite determination of the cause could be made. Nonetheless, the plutonium levels observed in LLNL effluent are 20 times below the drinking water standard for alpha radiation, and the highest activities observed in LWRP sludge are more than 16 times below the proposed EPA guideline for the unrestricted use of soil.

Water sampling and analysis are a large part of the LLNL surveillance monitoring effort. The waters monitored include lakes, streams, rainfall, tap water, storm water runoff, drinking water supply wells, and ground water monitoring wells. The samples are analyzed for gross alpha and beta radiation, tritium, and nonradioactive pollutants including solvents, metals, and pesticides. No radiological or nonradiological pollutant was observed in storm water above regulatory limits at the Livermore site. All tritium concentrations in drinking water supply wells at Site 300 were less than 2/1000 of the California drinking water standard for tritium. Trichloroethene was the major nonradiological constituent of concern in Site $\mathbf{3 0 0}$ ground water monitoring. It was detected in a backup water supply well, but its concentration in the samples from this well never exceeded one-fourth of the drinking water standard. Nevertheless, an investigation of the source of the trichloroethene is continuing.

Ground water protection activities at LLNL are performed as part of the investigation, study, monitoring and remediation of contamination caused by past practices. Ground water monitoring at the Livermore site investigates contamination at the site according to the Federal Facility Agreement for the Comprehensive Emergency Response, Compensation, and Liability Act (CERCLA). LLNL conducts ground water monitoring at Site 300 under two programs, one to meet regulatory commitments established for site-specific CERCLA investigations, and a second consisting of routine compliance monitoring around landfill and wastewater surface impoundment units. 
During 1992, the major remediation effort for the Livermore site focused on submittal of the Record of Decision in the CERCLA process and bringing one additional ground water treatment unit on-line. At Site 300, monitoring efforts continued to support ongoing remediation efforts and the preparation of a sitewide remedial investigation report. Other major efforts included closure plans of Pits 1 and 7, and conducting "pump and treat" operations in the General Services Area. Although compliance monitoring documented several areas at Site 300 where contaminants in ground water exceeded the State Maximum Contaminant Levels (MCLs) for drinking water, they were all in areas of known contamination and at levels similar to previous years, or following predicted trends. In no case did humans or animals drink or use the water.

Vegetation and foodstuffs are monitored for their tritium content; milk is further analyzed to determine the amounts of cesium-137 (a fallout radionuclide) and potassium-40 (a naturally occurring radionuclide). Generally, the milk samples taken are of goat's milk, but in 1992 one of the participants in the monitoring program acquired a cow, and this milk was analyzed as well. As in past years, the levels of radiation in milk were minimal and for the most part could not be distinguished from background radiation levels. The tritium concentrations in vegetation samples taken near the Livermore site were greater than those taken from more distant locations. The nearby vegetation tritium concentrations were about one-half those reported in 1991, while the concentrations in samples taken at greater distances were essentially unchanged from 1991. As in the past. the tritium concentrations in Livermore Valley wines are slightly above those for wines tested from Europe and other locations in California; however, even the highest detected value, $8.8 \mathrm{~Bq} / \mathrm{L}(240 \mathrm{pCi} / \mathrm{L})$, is just over $1 \%$ of the amount California allows in drinking water.

Soils are sampled at the Livermore site and Site 300 and are analyzed for radionuclides and beryllium; arroyo sediments from storm water drainage channels are sampled at the Livermore site and analyzed for radionuclides, metals, organic compounds, and pesticides. All measured values for 1992 were consistent with historic data and were well below regulatory limits. A special study of shallower sediment samples that were analyzed for metals and radionuclides showed beryllium-7, zinc, and copper at higher concentrations in the shallow samples. In fact, beryllium-7 was only detected in shallow samples, but because it is generated from cosmic rays and has a short half-life (53.4 days), there is little chance for it to migrate to deeper levels before decaying. The higher concentrations of zinc and copper could be attributed to the normal operation of motor vehicles. Copper is found in brake pads and diesel fuel, and zinc is present in tires; both are regarded as potential sources of storm water pollution, and motor vehicles provide a constant source of such pollutants. 
LLNL also maintains an extensive network of direct radiation monitors, using thermoluminescent dosimeters (TLDs) for gamma radiation and neutron monitors developed at LLNL for neutron radiation. TLD measurements at the Livermore-site perimeter averaged $0.65 \mathrm{mSv}(65 \mathrm{mrem})$ and at the Site 300 perimeter averaged $0.78 \mathrm{mSv}(78 \mathrm{mrem})$. Neutron measurements at the Livermore-site perimeter averaged $0.057 \mathrm{mSv}(57 \mathrm{mrem})$. All of these measurements are equivalent to natural background radiation levels.

\section{Radiological Impact Assessment}

The DOE environmental radiation protection standards are provided in DOE Order 5400.5, Radiation Protection of the Public and the Environment. The primary DOE radiation standards for protection of the public are $1 \mathrm{mSv} / \mathrm{y}(100 \mathrm{mrem} / \mathrm{y})$ effective dose equivalent for prolonged exposure, and $5 \mathrm{mSv} / \mathrm{y}(500 \mathrm{mrem} / \mathrm{y})$ effective dose equivalent for occasional exposure. DOE and LLNL also comply with the EPA's standard for radiation protection, which applies to air emissions. It limits the whole-body effective dose equivalent to $0.1 \mathrm{mSv} / \mathrm{y}(10 \mathrm{mrem} / \mathrm{y})$. Both limits are based on the maximum exposure that any individual member of the public could receive. Compliance with the limits is determined by computer modeling, which includes conservative assumptions and results in calculated exposures larger than exposures actually received by members of the public.

Routine LLNL operations during 1992 released a total of $6.55 \times 10^{12} \mathrm{~Bq}$ $(177 \mathrm{Ci})$ of tritium to the atmosphere; of that, $3.7 \times 10^{12} \mathrm{~Bq}(100 \mathrm{Ci})$ was in the form of tritiated water. An additional $9.77 \times 10^{12} \mathrm{~Bq}(264 \mathrm{Ci})$ was released by Sandia National Laboratories, California (SNL/CA), with approximately $4.81 \times 10^{12} \mathrm{~Bq}(130 \mathrm{Ci})$ in the form of tritiated water. The amount of radioactivity released from LLNL during 1992 was less than that released during 1991, and was below the range of previous years. There were no accidental radionuclide releases to the atmosphere at either the Livermore site or Site 300 during 1992.

The total dose calculated for point source emissions, for a theoretical person having the greatest possible exposure at the Livermore site, is $0.69 \mu \mathrm{Sv}$ $(0.069 \mathrm{mrem})$ and from diffuse emissions is $0.1 \mu \mathrm{Sv}(0.010 \mathrm{mrem})$, yielding a total dose of $0.79 \mu \mathrm{Sv}(0.079 \mathrm{mrem})$ for the Livermore site in 1992. This dose represents less than $1 \%$ of the EPA limit of $0.1 \mathrm{mSv} / \mathrm{y}(10 \mathrm{mrem} / \mathrm{y})$ for airborne emissions. This value is much lower than those for previous years: dose values reported for 1991 and 1990 were $2.34 \mu \mathrm{Sv}(0.234 \mathrm{mrem})$ and $2.40 \mu \mathrm{Sv}$ (0.240 mrem). Reduced emissions of tritium from the Tritium Facility account for much of this decline.

The calculated dose to a person having the greatest possible exposure at Site 300 in 1992 was calculated to be $0.21 \mu \mathrm{Sv}(0.021 \mathrm{mrem})$ effective dose equivalent. Virtually all of this resulted from Building 801 firing table emissions that used depleted uranium during open-air high-explosives tests. The dose 
contributed by firing table operations at Site 300 has steadily declined: in 1991 it was $0.44 \mu \mathrm{Sv}(0.044 \mathrm{mrem})$, and in 1990 it was $0.57 \mu \mathrm{Sv}(0.057 \mathrm{mrem})$.

The principal radionuclides contributing to these doses are tritium in the tritiated water form-which accounts for about $49 \%$ of the dose at the Livermore site-and the isotopes of uranium with atomic numbers 238,234, and 235, which account for about $32 \%$ of the dose at the Livermore site and all of the Site 300 dose.

The collective effective dose equivalent for LLNL 1992 operations was 0.015 person-Sv (1.5 person-rem) from the Livermore site and 0.071 person-Sv (7.1 person-rem) from Site 300, including all pathways (ingestion, air immersion, surface exposure, and inhalation pathways). These collective effective dose equivalents from the two LLNL sites are quite small compared to that from natural sources of radiation, which was estimated to be 19,000 person-Sv $(1,900,000$ person-rem).

\section{Environmental Compliance Activities}

Both the Livermore site and Site 300 are Superfund sites under the Comprehensive Environmental Response, Compensation, and Liability Act (CERCLA) as amended by the Superfund Amendments and Reauthorization Act (SARA). The proposed technique for cleaning up ground water at the Livermore site consists of managed ground water extraction at 18 locations and surface treatment. Contaminated sediments in the unsaturated zone will be treated by extracting fuel hydrocarbons or volatile organic compounds by vacuum-induced venting and treatment of the vapors. The U.S. Environmental Protection Agency and state and local agencies have approved this plan. In 1992, ground water was treated at three pilot treatment facilities: Treatment Facility A treated more than 71 million liters of ground water, removing and destroying about 10 kilograms of volatile organic compounds; Treatment Facility B treated about 9.4 million liters of ground water, removing and destroying about 1.5 kilograms of volatile organic compounds; and during four 10-hour tests, Treatment Facility F recovered about 76 liters of gasoline. The Superfund activities at Site 300 are at an earlier stage; LLNL is currently completing a site-wide remedial investigation report for the site.

In January 1992, LLNL submitted a revised Part B Permit application under the Resource Conservation and Recovery Act (RCRA). This document was again revised in April 1993. Because RCRA program authorization has been delegated to the State of California, LLNL now works with the California Department of Toxic Substances Control in seeking hazardous waste permits.

Another major document completed during 1992 was the Final Environmental Impact Statement and Environmental Impact Report (EIS/EIR) for the Continued Operation of Lawrence Livermore National Laboratory and Sandia National 
Laboratories, Livermore. This document was prepared to comply with the National Environmental Policy Act and the California Environmental Quality Act. The EIS/EIR assessed the environmental impacts of the Laboratories' operations on air and water quality, geological and ecological systems, occupational and public health, prehistoric and historic resources, endangered species, floodplains and wetlands, socioeconomic resources, hazardous waste management, site contamination, and other environmental issues. After a public hearing, written comments were incorporated and the Final EIS/EIR was published, then certified by the Regents of the University of California and accepted by the Department of Energy.

LLNL continues to perform all activities necessary to comply with clean air and clean water requirements. In 1992, the Bay Area Air Quality Management District issued 111 permits for the Livermore site. The San Joaquin Valley Unified Air Pollution Control District issued 25 permits for Site 300 covering the operation of equipment that has emissions to the air. LLNL has permits for discharge of treated ground water, for discharges of industrial and sanitary sewage, and for discharges of storm water. Site 300 has four additional permits for inactive landfills; cooling tower discharges; operation of the sewer lagoon, septic tanks, and leach fields; and discharge of treated ground water. LLNL complies with all requirements for self-monitoring and inspections associated with these permits.

Four quarterly reports were prepared in 1992 to demonstrate compliance with National Emissions Standards for Hazardous Air Pollutants (NESHAPs). As of March 31, 1993, all 56 of the Livermore site and Site 300 facilities having the potential to release radionuclides to the atmosphere had been evaluated. Annual doses resulting from total actual emissions (after controls) were found to be well below the NESHAPs standard of $100 \mu \mathrm{Sv} / \mathrm{y}(10 \mathrm{mrem} / \mathrm{y})$. Estimates of effective dose equivalent for all evaluated facilities, assuming normal operations but no pollution control devices, have been calculated. Three facilities at the Livermore site have been identified as having potential unmitigated effective dose equivalent estimates greater than $1 \mu \mathrm{Sv} / \mathrm{y}(0.1 \mathrm{mrem} / \mathrm{y})$, and these facilities have appropriate continuous radionuclide sampling systems in place, as required by NESHAPs. Several other Livermore-site facilities also have existing sampling systems; however, potential unmitigated effective dose equivalent estimates were less than $1 \mu \mathrm{Sv} / \mathrm{y}(0.1 \mathrm{mrem} / \mathrm{y})$, and they do not require continuous monitoring under NESHAPs regulations. No other facilities, including all those at Site 300, were found to require continuous monitoring systems.

LLNL has one endangered species, the Amsinckia grandiflora (large-flowered fiddleneck), which is found at Site 300. Representatives of the California Department of Fish and Game and the U.S. Wildlife Service visited Site 300 on 
March 26, 1992, and counted a record number (546 plants) of these flowers located at Building 858 Drop Tower.

\section{Conclusion}

Like many other organizations and individuals who are conserned about the environment, LLNL is committed to protecting the environment and ensuring that operations are conducted in accordance with applicable laws and regulations that have been enacted to protect the environment. The current techniques used at LLNL for environmental monitoring are very sensitive, allowing observation of extremely low levels of constituents. The monitoring results for 1992 generally show that these low concentrations are but a very small fraction of regulatory standards. Because the analytical results and evaluations generally show a decrease in contaminant levels, they reflect the responsiveness of the Laboratory in controlling pollutants. In summary, the results of the 1992 environmental monitoring program demonstrate that the environmental impacts of LLNL are minimal and pose no threat to the public or the environment. 


\section{Introduction}

Just as rain, wind, and geography affect our personal lives every day, climate and geography also play primary roles in how the environment is affected by human actions. Dispersal of particles in the air, for example, is influenced by wind patterns and rainfall, which in turn are influenced by geographical characteristics. Similarly, the dispersal of ground water is constrained by the particular geology of the site. Thus data on wind, rainfall, and geological and geographical characteristics are used to calculate the effects that operations at LLNL might have on the surrounding environment. Some history and a description of these data help us understand the relationship of the Laboratory to its setting.

\section{Location}

Forty years ago, when LLNL was founded to conduct weapons research and development, the Livermore site was relatively isolated, approximately 1.6 kilometers from the Livermore city limits. Over the years, Livermore evolved from a small town of less than 7000 people to its present population of nearly 55,000 . The economy diversified from being primarily agricultural to including light industry and business parks. Within the last few years, low-density, singlefamily residential development has filled the formerly vacant fields, bringing the city limits of Livermore to LLNL's western boundary.

LLNL's Livermore site occupies an area of 3.28 square kilometers, including the land that serves as a buffer zone around the site. Immediately to the south is Sandia National Laboratories, California (SNL/CA), operated by AT\&T Technologies, Inc., under DOE contract. SNL/CA provides research and development associated with nuclear weapons systems engineering, as well as related national security tasks. Although their primary missions are similar, LLNL and SNL/CA are separate facilities, each with its own management and each reporting to a different DOE operations office.

Other property to the south includes agricultural areas devoted to grazing, orchards, and vineyards; some low-density residential areas; and a business park. Farther south, property is primarily open space or rural ranchettes, with some agricultural use. A very small amount of low-density residential development lies to the east of the Livermore site, and agricultural land extends 
to the foothills of the intercoastal range that defines the eastern margin of the Livermore Valley.

A business park is located north of the Livermore site, and a 199.6-hectare parcel of open space to the northeast has been rezoned to allow development of a center for heavy industry.

Site 300, LLNL's experimental testing site, is located 24 kilometers east of the Livermore site in the Altamont Hills of the Diablo Range and occupies an area of 30.3 square kilometers. It is in close proximity to two other testing facilities; Physics International operates a testing facility that is adjacent and to the east of Site 300, and SRI International operates the other facility, which is located approximately 1 kilometer south of Site 300. The Carnegie State Vehicular Recreation Area is located south of the western portion of Site 300, and wind turbine generators line the hills northwest of the site. The remainder of the surrounding area is in agricultural use, primarily grazing land for cattle and sheep. The nearest residential area is the town of Tracy (population 25,000), located 10 kilometers to the northeast.

\section{Operations}

LLNL is a research laboratory with the infrastructure-including security, fire, and medical departments-necessary to support its operations and personnel. Cafeteria, banking, and limited shopping services are available on site, and electrical, sewerage, engineering, maintenance, and waste management activities support LLNL's operations.

LLNL's mission is to serve as a national resource of scientific, technical, and engineering capability with a special focus on national security. Over the years, this mission has evolved to include a wide variety of activities including inertial confinement fusion, laser isotope separation, magnetic fusion energy, biomedical and environmental research, energy and resources, environmental restoration and waste management, and scientific and institutional support.

\section{Climate}

Mild, rainy winters and warm, dry summers characterize the climate of the Livermore Valley. The mean annual temperature is $12.5^{\circ} \mathrm{C}$; the normal seasonal temperature range is defined by nighttime winter lows in the vicinity of $0^{\circ} \mathrm{C}$ and summer daytime highs around $38^{\circ} \mathrm{C}$. Annual wind data for the Livermore site are given in Figure 1-1 and in Table 1-1. For approximately $45 \%$ of the year, prevailing winds are from the west and southwest. During the remainder of the year, it is calm (approximately $33 \%$ of the year) or wind directions are variable. 
Both rainfall and wind exhibit a strong seasonal pattern. Most of the precipitation occurs between October and April, with very little rainfall during the warmer months. The highest and lowest annual rainfalls on record are 782 millimeters and 138 millimeters. In 1992, the Livermore site received 299 millimeters of rain.

The climate at Site 300, while generally similar to the Livermore site, is modified by higher elevation and more pronounced relief. The temperature range is somewhat more extreme than the Livermore site, and topography significantly influences surface wind patterns. Annual wind data are presented in Figure 1-2 and Table 1-1. Rainfall for 1992 was 305 millimeters.

\section{Geology}

\section{Topography}

Livermore Site. $\mathrm{Th} \mathrm{e}$ Livermore site is located in the southeastern portion of the Livermore Valley, a topographic and structural depression oriented east-west within the Diablo Range of the California Coast Range Province. The Livermore Valley forms an irregularly shaped lowland area approximately 26 kilometers long and an average of 11 kilometers wide. The floor of the valley slopes from an elevation of approximately 200 meters at the eastern end to approximately 90 meters at its drainage outlet, which is located near the southwestern corner.

Site 300. The topography of Site 300 is much more irregular than that of the Livermore site; a series of steep hills and ridges are oriented along a generally northwest/southeast trend and are separated by intervening ravines. The elevation ranges from approximately 150 meters

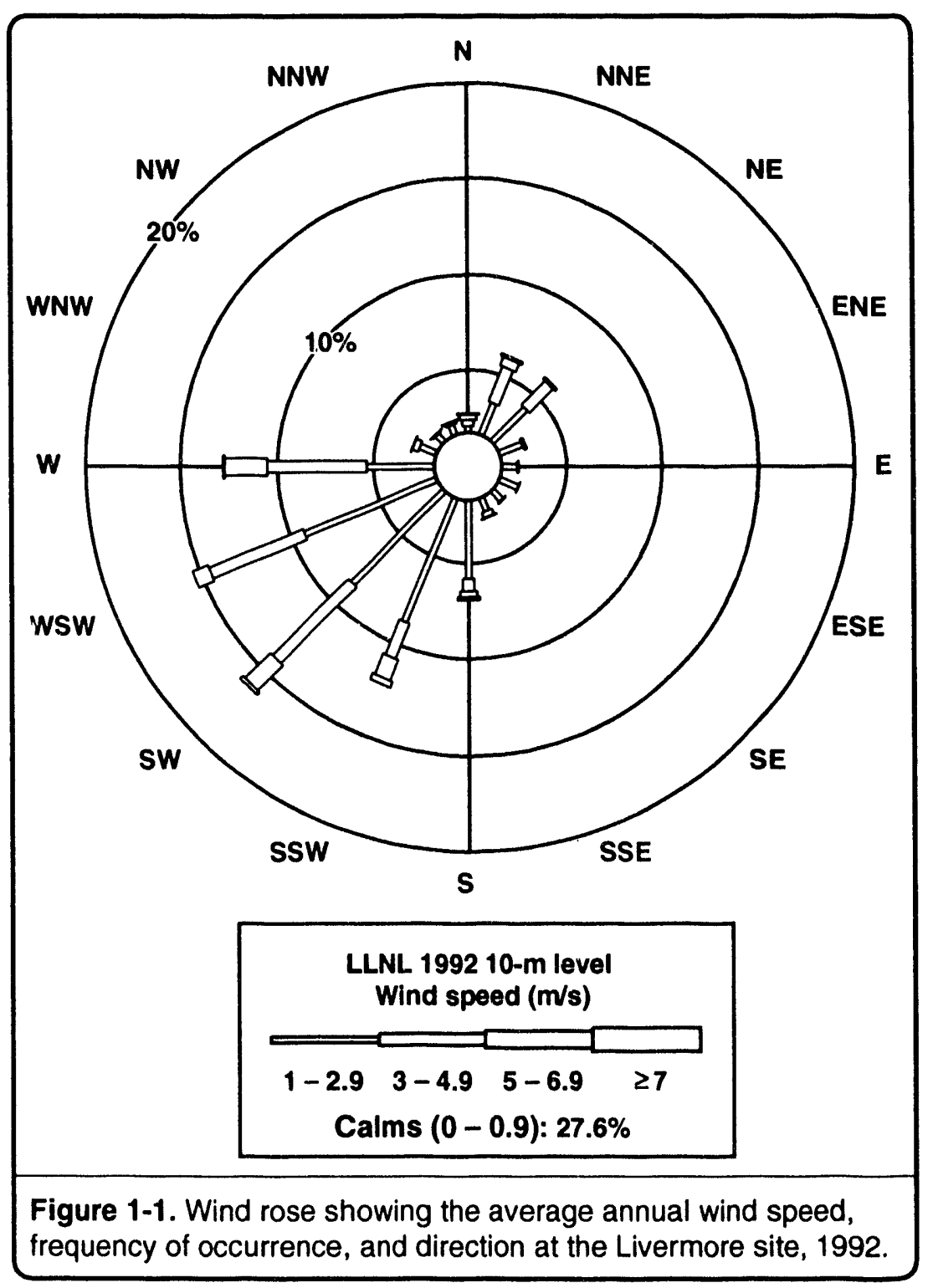


at the southeast corner of the site to approximately 538 meters in the northwestern portion.

\section{Hydrogeology}

Livermore Site. The hydrogeology and movement of ground water in the vicinity of the Livermore site have been the subjects of several recent and continuing investigations. Detailed discussions of these investigations can be found in Stone and Ruggieri (1983); Carpenter et al. (1984); Webster-Scholten and Hall (1988); Dresen et al. (1989a-f, 1990); and Thorpe et al. (1990). This section has been summarized from the reports of these investigations and from data supplied by Alameda County Flood Control and Water Conservation District,

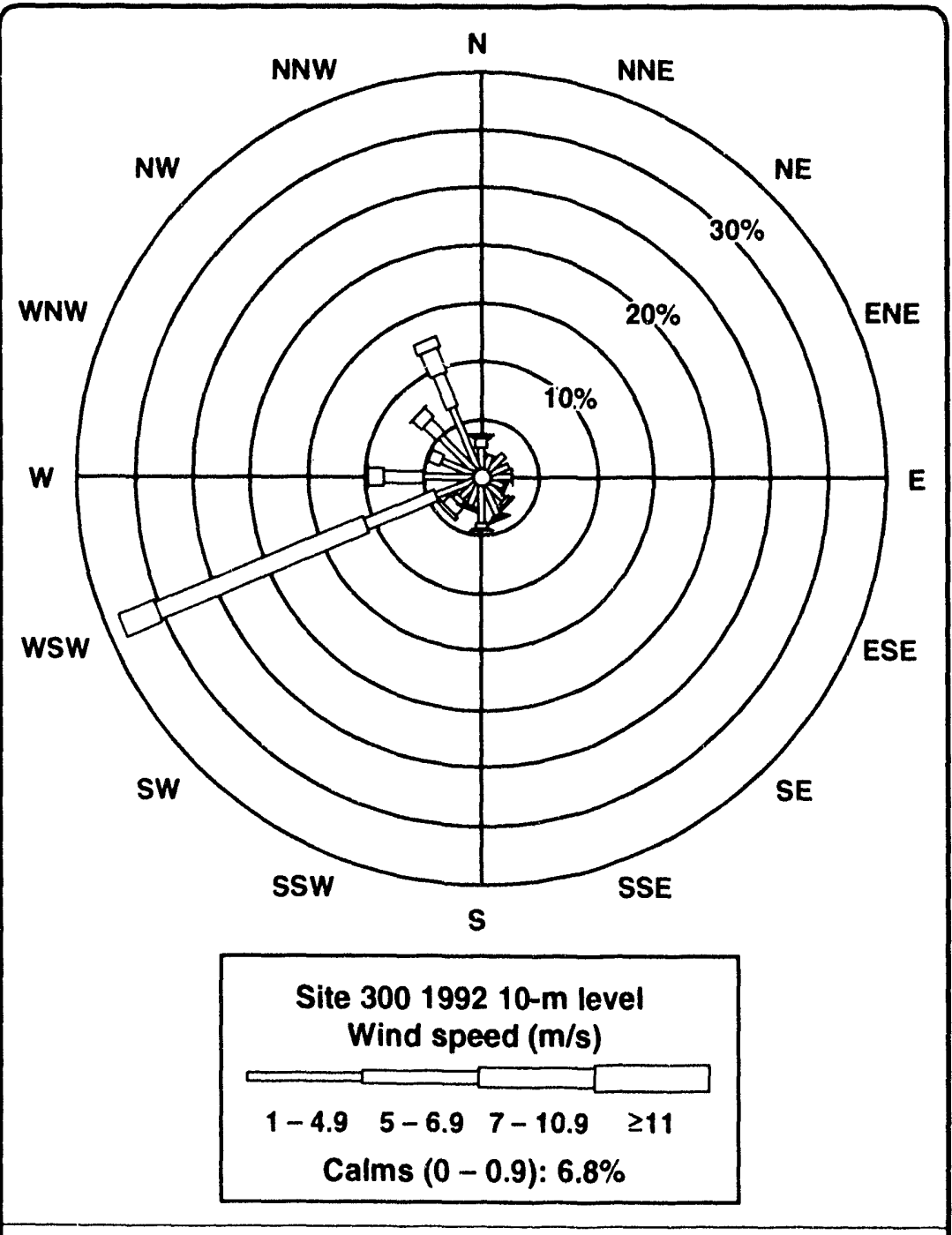

Figure 1-2. Wind rose showing the average annual wind speed, frequency of occurrence, and direction at Site 300, 1992.
Zone 7 , the agency responsible for ground water management in the Livermore Valley basin.

The Livermore Formation (and overlying alluvial deposits) contains the aquifers of the Livermore Valley ground water basin, an important waterbearing formation. Natural recharge occurs primarily along the fringes of the basin and through the arroyos during periods of winter flow. Artificial recharge, if needed to maintain ground water levels, is accomplished by releasing water from Lake Del Valle or from the South Bay Aqueduct into arroyo channels in the east. Ground water flow in the valley generally moves toward the central eastwest axis of the valley and then westward through the central basin. Ground water flow in the basin is generally assumed to be primarily horizontal, although a significant vertical component probably exists in fringe areas and under localized sources of recharge. 
Beneath the Livermore site, the depth to the water table varies from about 8 to 43 meters. Figure 1-3 shows a contour map of water-table elevations (feet above mean sea level) for the Livermore-site area. Although water-table elevations vary slightly with seasonal and year-to-year variations in both natural and artificial recharge, the qualitative patterns shown in Figure 1-3 are generally maintained. At the eastern edge of the Livermore site, ground water gradients (change in elevation per unit of horizontal distance) are relatively steep, but under most of the site and farther to the west, the contours flatten to a gradient of approximately 0.003 . Ground water flow under the northern and western portions of the site is generally westward. Aquifer tests on monitoring wells in the vicinity of the Livermore site indicate that the hydraulic conductivity of the permeable sediments ranges from 1 to 16 meters per day (Isherwood et al. 1991).

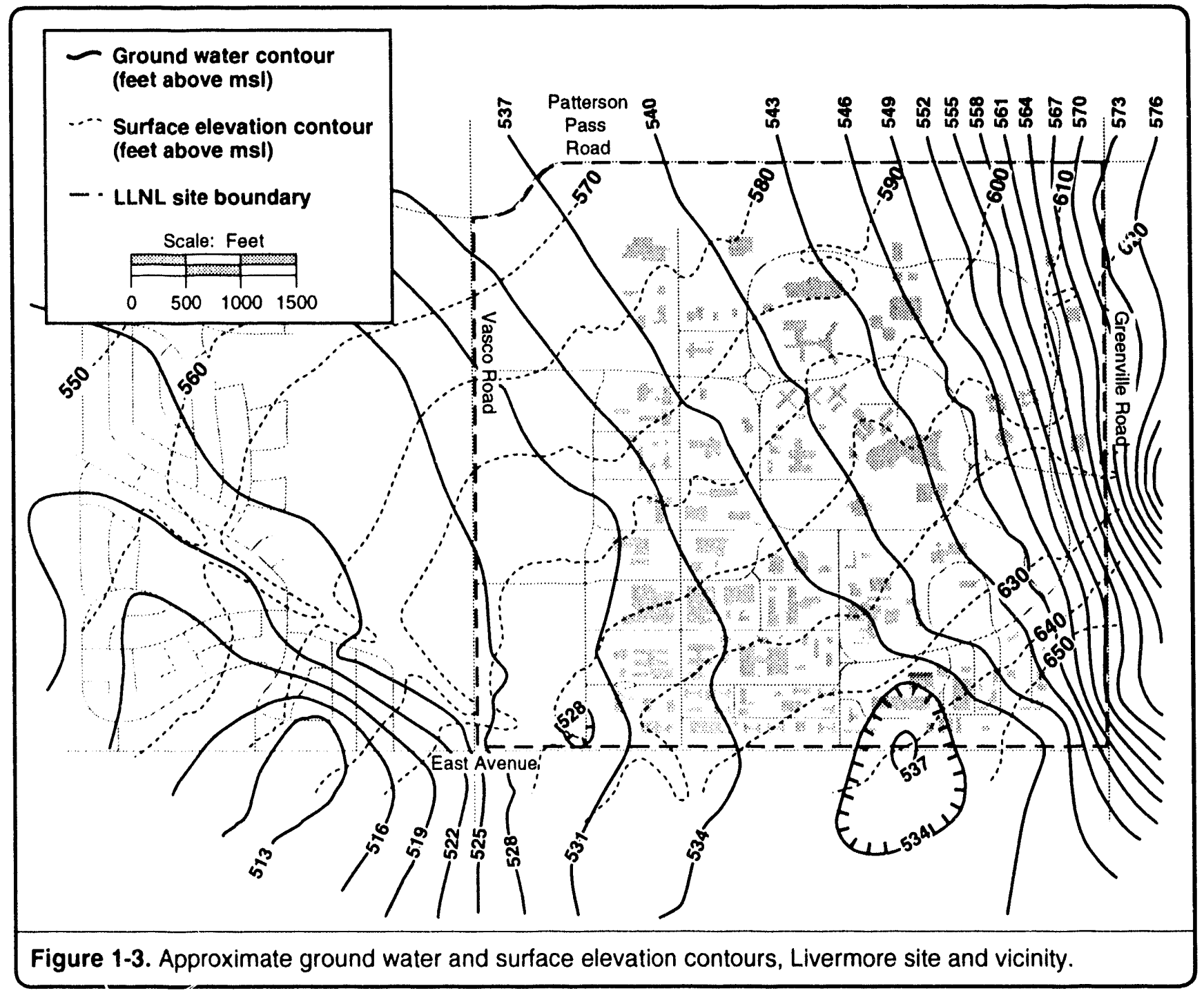


This, in combination with the observed water-table gradients, yields an average ground water velocity estimate of 20 meters per year (Thorpe et al. 1990). The range in these values reflects the heterogeneity typical of the more permeable of the alluvial sediments that underlie the area.

Site 300. At Site 300, local perched aquifers containing small amounts of water occur in some of the channel deposits in the nonmarine Tertiary sequence. Perched aquifers contain unconfined water that is separated from an underlying main body of water by impermeable layers; normally they are small and highly localized. Because water quality is poor and yields are low, these perched waterbearing zones do not meet the State of California criteria for aquifers that are potential water supplies.

The thick Neroly sandstone, stratigraphically near the base of the formation, contains confined water. Wells located in the western part of the General Services Area are completed in this aquifer, and are used to supply drinking and process water.

Figure 1-4 shows the elevation contours for water in the regional aquifer at Site 300 . The map of the piezometric surface (the level to which water rises in a well that penetrates a confined or unconfined aquifer) is based primarily on water levels in the Neroly lower blue sandstone aquifer.

\section{Conclusion}

LLNL appreciates the importance of our environment and recognizes that our setting provides a habitat for a number of species of native flora and fauna. Like many other organizations concerned about the environment, LLNL is committed to protecting it and ensuring that operations at the Laboratory are conducted in accordance with applicable laws and regulations that have been enacted to protect the environment. 


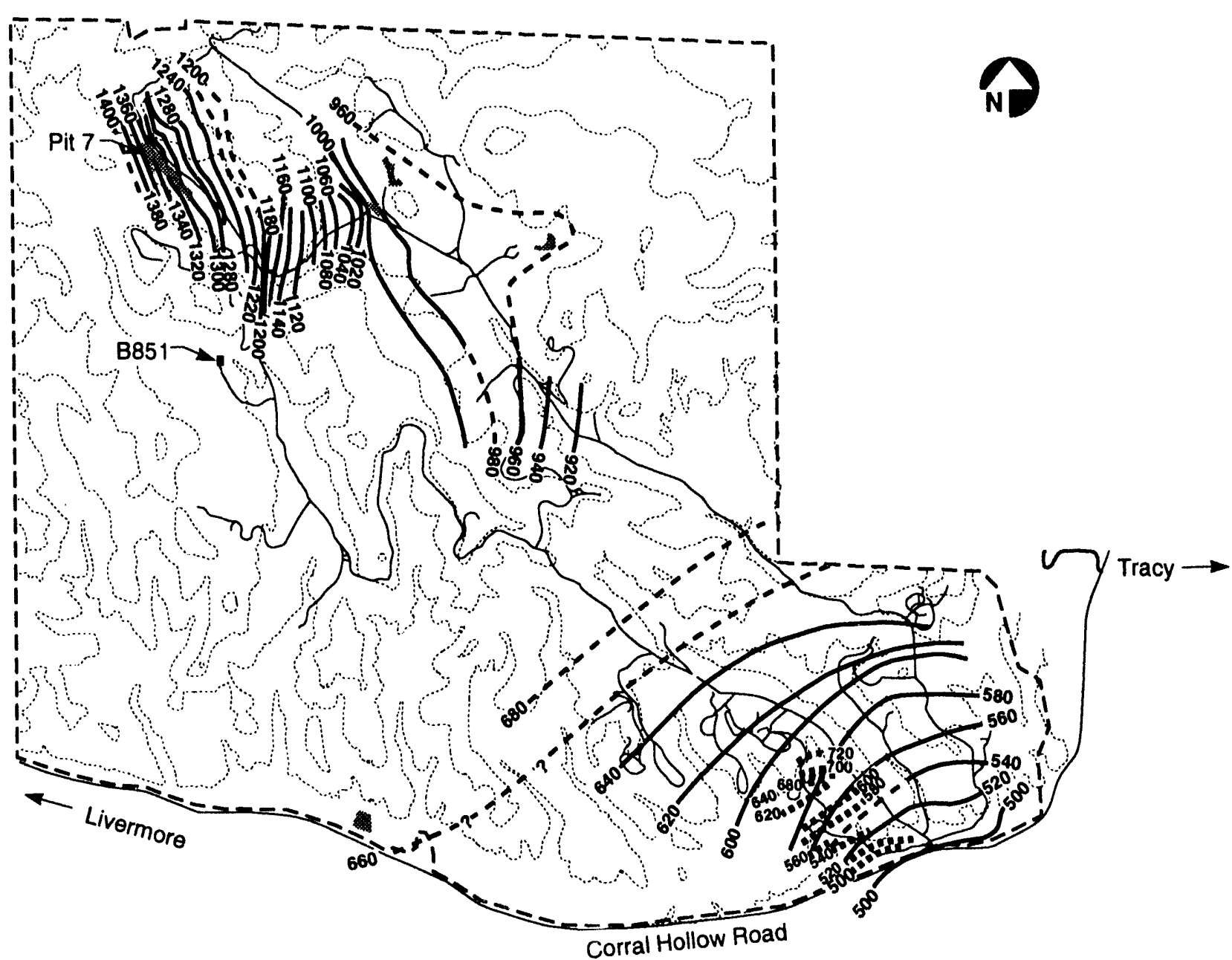

- - Site 300 perimeter

Surface elevation contour (feet above msl)

.... HE Process Area ground water elevation contour

\section{- Ground water elevation contour (feet above msl) \\ - dashed were approximate, \\ -?. queried where uncertain

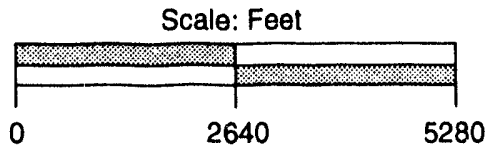

Figure 1-4. Ground water and surface elevations at Site 300, 1992. 
Table 1-1. Average annual percent frequency of wind direction at $10 \mathrm{~m}$ above ground level, versus wind speed $(\mathrm{m} / \mathrm{s})$ for the Livermore site and Site 300, 1992 (one-hour averages).

\begin{tabular}{|c|c|c|c|c|c|c|}
\hline \multirow[b]{2}{*}{ Direction } & \multicolumn{6}{|c|}{ Llvermore site } \\
\hline & $\begin{array}{c}\text { Calm } \\
0.0-0.9\end{array}$ & $1.0-2.9$ & $3.0-4.9$ & $5.0-6.9$ & $>7.0$ & Total \\
\hline $\mathbf{N}$ & 1.7 & 0.4 & 0.3 & 0.3 & 0.1 & 2.8 \\
\hline NNE & 1.7 & 1.8 & 2.0 & 0.5 & 0.1 & 6.1 \\
\hline NE & 1.7 & 2.5 & 1.7 & 0.1 & 0.0 & 6.0 \\
\hline ENE & 1.7 & 1.3 & 0.2 & 0.0 & 0.0 & 3.2 \\
\hline $\mathbf{E}$ & 1.7 & 0.9 & 0.1 & 0.0 & 0.0 & 2.7 \\
\hline ESE & 1.7 & 1.1 & 0.0 & 0.0 & 0.0 & 2.8 \\
\hline SE & 1.7 & 0.7 & 0.1 & 0.0 & 0.0 & 2.5 \\
\hline SSE & 1.7 & 0.8 & 0.3 & 0.0 & 0.0 & 2.8 \\
\hline $\mathbf{s}$ & 1.7 & 4.1 & 0.7 & 0.3 & 0.2 & 7.0 \\
\hline SSW & 1.7 & 7.0 & 2.0 & 1.2 & 0.3 & 12.2 \\
\hline sw & 1.7 & 6.8 & 5.6 & 1.8 & 0.2 & 16.1 \\
\hline Wsw & 1.7 & 7.4 & 5.3 & 0.9 & 0.0 & 15.3 \\
\hline$w$ & 1.7 & 3.6 & 5.1 & 2.2 & 0.1 & 12.7 \\
\hline WNW & 1.7 & 0.9 & 0.3 & 0.0 & 0.0 & 2.9 \\
\hline NW & 1.7 & 0.6 & 0.1 & 0.0 & 0.0 & 2.4 \\
\hline NNW & 1.7 & 0.6 & 0.1 & 0.1 & 0.0 & 2.5 \\
\hline \multirow[t]{3}{*}{ Total } & 27.2 & 40.4 & 23.9 & 7.4 & 1.0 & 100.0 \\
\hline & \multicolumn{6}{|c|}{ Site 300} \\
\hline & $\begin{array}{c}\text { Calm } \\
0.0-0.9\end{array}$ & $1.0-4.9$ & $5.0-6.9$ & $7.0-10.9$ & $>11.0$ & Total \\
\hline $\mathbf{N}$ & 0.4 & 2.0 & 0.8 & 0.2 & 0.1 & 3.5 \\
\hline NNE & 0.4 & 1.4 & 0.0 & 0.0 & 0.0 & 1.8 \\
\hline NE & 0.4 & 2.0 & 0.0 & 0.0 & 0.0 & 2.4 \\
\hline ENE & 0.4 & 1.9 & 0.0 & 0.0 & 0.0 & 2.3 \\
\hline $\mathbf{E}$ & 0.4 & 1.9 & 0.1 & 0.0 & 0.0 & 2.4 \\
\hline ESE & 0.4 & 1.4 & 0.2 & 0.1 & 0.0 & 2.1 \\
\hline SE & 0.4 & 2.1 & 0.3 & 0.2 & 0.0 & 3.0 \\
\hline SSE & 0.4 & 2.9 & 0.2 & 0.2 & 0.0 & 3.7 \\
\hline $\mathbf{s}$ & 0.4 & 3.3 & 0.5 & 0.2 & 0.0 & 4.4 \\
\hline ssw & 0.4 & 2.0 & 0.2 & 0.1 & 0.0 & 2.7 \\
\hline sw & 0.4 & 1.8 & 0.5 & 0.9 & 0.3 & 4.0 \\
\hline Wsw & 0.4 & 3.6 & 6.6 & 19.5 & 3.3 & 33.4 \\
\hline$w$ & 0.4 & 4.3 & 3.4 & 1.4 & 0.0 & 9.5 \\
\hline WNW & 0.4 & 3.0 & 1.1 & 0.1 & 0.0 & 4.6 \\
\hline NW & 0.4 & 4.3 & 1.6 & 1.1 & 0.0 & 7.4 \\
\hline NNW & 0.4 & 5.8 & 3.0 & 2.2 & 1.1 & 12.5 \\
\hline Total & 6.4 & 43.7 & 18.5 & 26.2 & 4.8 & 100.0 \\
\hline
\end{tabular}




\section{Introduction}

In 1992, LLNL conducted numerous environmental activities to comply with legal and internal requirements. These activities, performed at both the Livermore site and Site 300, encompassed environmental safety and health, hazardous waste management, wastewater, air quality, and general environmental issues. Documents that provide detailed information on these activities can be found at the LLNL Visitors Center and the City of Livermore Public Library. A summary of the environmental permits issued to or held by LLNL during the year is presented at the end of this chapter (Table 2-1). In addition, a list of 1992 Environmental Protection Department (EPD) publications is presented in Appendix A. This chapter includes background information to place the 1992 activities in context, as well as information about activities in the first quarter of 1993.

\section{Department of Energy Tiger Team}

The Department of Energy (DOE) Tiger Team Assessment of LLNL Environmental, Safety, and Health (ES\&H) Programs in 1990 concluded that curtailment or cessation of any LLNL operations was not warranted and that compliance issues identified by the team are known to local, state, and federal regulatory agencies. Specific findings are presented in more detail in the Tiger Team Assessment (U.S. Department of Energy 1990).

In response to the Tiger Team Assessment, LLNL developed an Action Plan, which was approved by the DOE in early October of 1990, in which LLNL committed to correct identified deficiencies. The Action Plan describes changes in organizational structure to strengthen line management responsibility and accountability for ES\&H/quality assurance issues; self-assessment activities to identify, resolve, and prevent future noncompliance with regulations; and commitment of significant funding for corrective actions.

The Action Plan also includes descriptions of the actions to be taken a: the site to respond to the findings, along with schedules and estimated costs for each action. A progress report issued on a semiannual basis contains a brief status of the activity, the individual responsible for the action, and any revisions in the scheduled completion date. The Tiger Team Action Plan has been extended from a five-year to a seven-year plan (FY91-FY97). 
LLNL continues to undertake those activities identified in its Action Plan, and significant progress has been made towards the 581 subtasks identified in it. As of December 31, 1992, approximately $66 \%$ of these subtasks have been completed, $3 \%$ are on schedule, $1 \%$ are behind schedule, and $27 \%$ are considered late, with 20 low priority subtasks (the remaining 3\%) not funded. The majority of those subtasks behind schedule or late are a result of funding limitations in fiscal year 1993 or the inability in fiscal year 1991 to acquire qualified staff as fast as originally anticipated.

\section{CERCLA/SARA-Title I}

Also known as the "Superfund" Act, the Comprehensive Environmental Response, Compensation, and Liability Act (CERCLA) is a federal statute that gives the federal government the authority to identify and clean up sites when hazardous chemicals and substances have been released to the environment. The U.S. Environmental Protection Agency (EPA) is responsible for managing CERCLA.

The Superfund Amendments and Reauthorization Act (SARA) amended various provisions of CERCLA, including the establishment of a process for federal facility participation in CERCLA regulation.

\section{Livermore-Site Ground Water Project}

The Ground Water Project continues to address CERCLA compliance issues at the Livermore site. Because some of the ground water at LLNL contains volatile organic compounds (VOCs), primarily trichloroethene (TCE) and tetrachloroethene (PCE), the site is on the EPA's National Priorities List (NPL). The Ground Water Project complies with provisions specified in a Federal Facility Agreement (FFA) made by the EPA, DOE, the California EPA Department of Toxic Substances Control (DTSC), and the Regional Water Quality Control Board (RWQCB), San Francisco Bay Region. As required by the FFA, the project addresses compliance issues through investigations of potential source areas (such as old release sites, solvent handling areas, and leaking underground tank systems), continued monitoring of ground water, and remediation.

Source investigations are used to evaluate all possible contamination areas. A computerized source investigation tracking system has been implemented to aid the investigation of all potential sources at LLNL. If any contamination above regulatory standards is detected, the area is evaluated further to determine the extent of the contamination and to assess its potential for migration.

Based on compliance monitoring and sampling of ground water, surface water, soil, sediments, and air for toxic materials, the primary medium of concern appears to be ground water and the principal compounds of potential concern appear to be VOCs and fuel hydrocarbons. This was fully confirmed 
by the CERCLA Remedial Investigation Report for the LLNL Livermore-Site (Thorpe et al. 1990).

The Record of Decision (ROD) and Responsiveness Summary were signed by the U.S. EPA on August 5, 1992. On January 6, 1993, LLNL completed the Remedial Action Implementation Plan (RAIP) for cleaning up ground water and soil at the Livermore site. The RAIP is the first step in the Remedial Design/Remedial Action (RD/RA) process under CERCLA. Its proposed technique for cleaning up ground water consists of managed ground water extraction at 18 locations and surface treatment. Contaminated sediments in the unsaturated zone will be treated by extracting fuel hydrocarbons or VOCs through vacuum-induced venting and oxidizing the vapors at the surface. The EPA, DTSC, and RWQCB have approved the RAIP.

The scope of these assessment and restoration efforts encompasses a set of diverse activities designed to investigate, evaluate, and eventually remediate potentially hazardous compounds that may have affected soil and ground water. These activities include the following: investigation of the hydrology, geology, soil properties, and hydrogeology of the site; investigation (including searches of historical records) of known and suspected sources of contamination; installation of piezometers and monitoring and extraction wells; sampling and analysis of soil and ground water; aquifer testing; development of computer models and a conceptual site model to aid ground water study; assessment of potential risks to public health from contaminants in soil and ground water; provision of project information to the public and coordination of community involvement; evaluation of numerous studies designed to aid site remediation; preparation of reports documenting the investigation, evaluation, and remediation; and testing and implementation of appropriate technologies to accomplish environmentally responsive cleanup.

On March 12, 1993, the first of five Remedial Design (RD) Reports for LLNL was completed. As described in the RAIP, the five RD Reports will be prepared over a three-year period to be consistent with projected funding levels and to optimize the cleanup. The first RD Report is for Treatment Facilities A and B and their respective extraction wells and piezometer networks. Both of these facilities were constructed and tested under an EPA-approved pilot study for the Southwest Corner/Offsite Area. Treatment Facility A (TFA) has been operating since September 1989, and Treatment Facility B (TFB) has operated intermittently since October 1990. In addition, work continues on various computer models and algorithms to support and optimize remediation design and planning activities. Effective remediation will require the use of our predictive capabilities during both the phased-in construction of extraction and treatment facilities and in fullscale operations. 
TFA treated more than 71 million liters of ground water during 1992, removing and destroying approximately 10 kilograms $(6.3 \mathrm{~L})$ of VOCs. About 200 million liters have been treated since TFA began operating. TFB treated about 9.4 million liters of water in 1992, removing and destroying approximately 1.5 kilograms $(0.9 \mathrm{~L})$ of VOCs from ground water. More than 15 million liters have been treated since TFB began operating.

Construction activities for Treatment Facility F (TFF), a steam injection/ ground water and vapor extraction and treatment system, continued at the Gasoline Spill Area throughout 1992. TFF supports the DOE-sponsored Dynamic Stripping research project as well as unsaturated zone and ground water remediation. Remediation operations at TFF during 1992 were limited to four 10-hour soil vapor extraction tests conducted between August and December. Vapor extraction occurred for a total of about 40 hours at a combined vapor extraction rate of about 50 standard cubic feet per minute $(\mathrm{scfm})$. Total fuel hydrocarbon concentrations measured in extracted air ranged from about 4000 to 6000 parts per million by volume (ppmv), resulting in a fuel hydrocarbon removal in 1992 of about 76 liters of gasoline. Full operation of TFF began in February 1993.

Previous remedial activities from 1988 through 1991 at TFF resulted in a total removal of about 7500 liters of gasoline. Therefore, through 1992 a total of about 7600 liters of gasoline have been removed. Results for 1993 TFF performance are currently being evaluated and will be recorded in future reports.

The LLNL Ground Water Project continued its community relations program by holding four Community Work Group meetings in 1992, and by publishing quarterly Ground Water Update information to keep interested parties informed. The draft revised Community Relations Plan (CRP) was completed on January 29, 1993, and submitted to regulatory agencies as well as the Community Work Group. Copies of all LLNL ground water restoration documents, including monthly progress reports and annual project reports, which provide detailed information about the LLNL Ground Water Project's activities, are available at the LLNL Visitors Center and the Livermore Public Library.

\section{Site 300 Environmental Restoration Project}

At Site 300, ongoing remedial investigations, feasibility studies, and remedial actions are being performed as a part of the Environmental Restoration Project. Site 300 investigations and remedial actions are conducted under the joint oversight of the EPA, Central Valley RWQCB, and DTSC under the authority of a separate FFA. The Site 300 FFA was negotiated to encompass the environmental restoration requirements of RCRA as well as CERCLA. The FFA negotiations began in the fall of 1990 and the FFA was executed in June 1992. 
The issues and areas of investigation at Site 300 include (1) TCE in soil, sediment, rock, and/or ground water at the Building 834 Complex, High Explosives (HE) Process Area, General Services Area (GSA), Pits 5, 6, and 8, and Building 833 Area; (2) HE compounds in the HE Process Area; and (3) tritium in the East and West Firing Areas. Highlights of these activities are presented below; the area-specific individual draft Remedial Investigation (RI) and Feasibility Study (FS) reports should be consulted for additional details.

As discussed in the Environmental Report for 1991, a number of draft RI and FS reports were completed for Site 300 during 1991. These reports present the results of investigations in a given study area; they were submitted to regulatory agencies for consideration of appropriate choices for remediation. The draft RI reports include detailed discussions of the environment, geology and hydrogeology, environmental risk of any toxic chemicals encountered, and assessment of the risk to public health and safety. The FS reports include proposals for remedial action alternatives with cost estimates under several conditions, from no action to full remediation.

In mid-1991, the regulatory agencies requested that LLNL prepare a site-wide remedial investigation report to replace the previously submitted individual draft RI reports. Compilation of the draft site-wide remedial investigation (SWRI) report began in 1991 and is scheduled to be completed in August of 1993. The SWRI is organized by study areas that roughly correspond to the areas covered by the individual RI reports. It contains a detailed risk assessment for the entire site. Final FS reports for the individual study areas will be prepared after submittal of the SWRI.

General Services Area (GSA) Study Area. According to the draft RI (McIlvride et al. 1990) and draft FS (Ferry et al. 1990) reports, VOCs have been identified in the shallow ground water beneath the GSA in three localities. One plume is in the western GSA, three small plumes are in the central GSA, and one plume is in the eastern GSA. Additional investigation revealed that the plume located in the western part of the study area originates from the HE Process Area. Therefore, this plume is included in the HE Process Area study area in the SWRI. The eastern GSA plume extends about 1.2 kilometers off site in the gravels of Corral Hollow Creek. A ground water treatment unit to remove VOCs from the eastern GSA was designed, put into operation in June 1991 as a CERCLA Removal Action, and operated throughout 1992. Total treated water at the GSA through February 1993 was about 42 million liters. The average TCE concentration (TCE is the primary VOC in the ground water) in the water treated at the eastern GSA treatment facility between June 1991 and February 1993 was $38 \mu \mathrm{g} / \mathrm{L}$; thus, approximately 160 grams of TCE have been removed. Design work began on a ground water treatment and vapor extraction unit to remove VOCs from the central GSA. 
Building 834 Complex Study Area. An isolated, perched aquifer that contains TCE has been defined and reported in the draft RI/FS report (Bryn et al. 1990). Techniques for removal of TCE vapor from the vadose zone above the water table and from the shallow perched water have been evaluated and pilot-tested. From January to March 1992, under funding from the EPA Emerging Technologies Program, LLNL in conjunction with Purus, Inc., evaluated a treatment technology fur TCE. An ultraviolet flashlamp system was used to destroy TCE in air from the vacuum extraction system. Although $99 \%$ of the ingoing TCE was destroyed, by-product analyses showed that oxidation was not complete.

HE Process Area Study Area. The ground water beneath a small part of the HE Process Area near Buildings 815 and 817 contains low concentrations of the HE compound RDX (Crow and Lamarre 1990). Trace concentrations of the HE compound HMX, in water from one well, were found very near the detection limit. The area where $\mathrm{HE}$ compounds are present in the ground water has been successfully defined.

TCE has been detected at levels above maximum contaminant levels (MCLs) in the first two aquifers beneath an area near and southeast of Building 815 . The data collected from several monitoring wells in the central and southern parts of the HE Process Area identified the TCE plume to extend into the western GSA.

East Firing Area and West Firing Area Study Area. The draft RI of the Building 850/East Firing Area (Taffet et al. 1992) and the draft FS (Taffet and Oberdorfer 1991) discuss the distribution of tritium in the ground water near the firing table at Building 850, in the alluvium in Doall Ravine to the southeast, and in the adjacent part of the East Firing Area to the southeast along Elk Ravine. The draft FS report includes various remediation alternatives. The RI/FS of the Pit 7 Complex (Taffet et al. 1989b) discusses the distribution of tritium and TCE in the ground water in the vicinity of the Pit 7 Complex, which is located in the West Firing Area, as well as various remediation alternatives.

Pit 6 Area Study Area. The draft RI of the Pit 6 Landfill Area (Taffet 1990) discusses the small plume of TCE in ground water, which discharges to the surface at small springs at the southeastern edge of the Pit 6 Area. The draft FS (Taffet et al. 1991) discusses options for its remediation.

As discussed in the Environmental Report for 1991 (Gallegos et al. 1992a), during geologic mapping in the vicinity of Pit 6, a fault showing evidence of Holocene displacement was observed in a cut slope on State of California property (the State Vehicle Recreation Area Manager's residence), about 300 meters southeast of the landfill. As a result of subsequent detailed geologic investigations, project staff have determined that the fault represents a potentially active strand of the Corral Hollow-Carnegie Fault Zone, which is located generally south and west of the Site 300 property. Details of the 
investigation are described in Investigation of Holocene Faulting Near Closed Landfill Pit 6, Lawrence Livermore National Laboratory Site 300 (Carpenter et al. 1991).

Building 833 Area Study Area. Low concentrations of TCE and associated VOCs have been detected in shallow soils and sediments (to a depth of 15 meters) beneath the Building 833 Area. During the remedial investigation of the Building 833 Area, concentrations of up to $1,800 \mu \mathrm{g} / \mathrm{L}(\mathrm{ppb})$ were detected in ground water in two boreholes. Results of the investigation were published in Webster-Scholten et al. (1991).

Closure of Landfill Pits 1 and 7. During December of 1992, LLNL completed the capping of landfill Pits 1 and 7, located at the northern part of Site 300. This work was done under an LLNL RCRA closure plan previously approved by the EPA, DTSC, and the Central Valley RWQCB. The primary components of the closure design consist of a closure cover system, surface water control system, and subsurface water control system. The Construction Quality Assurance Closure Report, Lawrence Livermore National Laboratory Site 300, Pits 1 and 7 (Golder Associates, Inc. 1993) documents the construction quality assurance (CQA) activities for the final closure of Pits 1 and 7. This documentation includes construction and testing of the test fill pad and the sealed double-ring infiltrometer, construction and CQA verification of the closure cover cap system, construction of the subsurface interceptor drainage trench (Pit 7), and construction and CQA testing of the concrete surface water control channels.

\section{SARA-Title III}

The Superfund Amendments and Reauthorization Act (SARA) Title III also is known as the Emergency Planning and Community Right-to-Know Act. It requires owners/operators of facilities that have certain hazardous chemicals on site to provide information on the manufacture, use, and storage of those chemicals to organizations responsible for emergency-response planning. In California, this information must be provided to the California Office of Emergency Services, the county, and the local fire department.

A physical inventory of all chemicals subject to reporting under SARA Title III was conducted during the months of November and December 1988. The inventory included the chemical name, location, weight, type of container, and physical state of the substance. The inventory included the Livermore site, Site 300, and leased space off site. In early 1989, the data were entered into a computerized database system and reports were generated for local emergency planning agencies. As in previous years, in 1992 EPD continued updates of the inventory to reflect changes in chemicals handled on site. 


\section{RCRA}

\section{Hazardous Waste Management Activities}

The Resource Conservation and Recovery Act (RCRA) provides the framework at the federal level for regulating the generation and management of solid wastes, including wastes designated as hazardous. Similarly, the California Hazardous Waste Control Act (HWCA) sets requirements for the management of hazardous wastes in California. RCRA and HWCA regulate treatment, storage, and disposal facilities, including permit requirements. In July 1992, California was authorized to administer the federal RCRA program and DTSC became the primary agency administering these activities. The permit process requires DTSC to review and approve Part A and Part B permit applications, which provide information on the facility and the wastes managed therein.

As discussed in last year's report, LLNL is continuing discussions with DTSC regarding LLNL's 52 waste accumulation areas (WAAs). In December 1992, DTSC added the WAAs to the LLNL Interim Status Document (ISD CA 2890012584). LLNL is awaiting the final decision from DTSC as to whether the WAAs will need to be included in the Part B permit application and subsequently permitted (see further discussion in "Current Issues and Actions" at the end of this chapter).

\section{DOE Hazardous Waste Disposal Moratorium}

In May 1991, DOE Headquarters imposed a moratorium on the shipment of hazardous waste from all DOE facilities to off-site treatment and disposal facilities. The moratorium was in response to concerns that a DOE contractor on the East Coast (not associated with LLNL) had inadvertently shipped radioactive hazardous waste to a hazardous waste treatment facility.

The lifting of this moratorium was contingent upon developing criteria and procedures to satisfy DOE Headquarters that shipments of hazardous waste would have no added radioactivity from DOE activities. After a series of meetings to resolve comments on LLNL's draft criteria and procedures, DOE Headquarters lifted the moratorium for LLNL on December 20, 1991. DOE's San Francisco Operations Office lifted the final restrictions on January 20, 1992.

\section{Hazardous Waste Report for 1992}

The Hazardous Waste Management Division completed the 1992 Hazardous Waste Report, which is required by DTSC under 22 CCR 66264.75, by the April 1, 1993, deadline.

The report, which is maintained on file at the request of DTSC, comprises four forms. The identification and certification (IC) form provides general facility information, including addresses, contacts, and general waste minimization information. The waste generation and management (GM) form represents 
"cradle-to-grave" tracking of each waste stream category. The waste received from off site (WR) form includes descriptions and quantities of wastes that were received from the off-site facilities (Site 300 and the Livermore Airport), and the process system (PS) form includes waste quantities treated by each waste management unit on site.

\section{Hazardous Waste Permits}

The Livermore-site hazardous waste storage and treatment management units continue to operate under interim status provisions (ISD CA2890012584). Waste management units include container storage, tank storage, and various treatment processes (e.g., wastewater filtration, blending, and size reduction).

With RCRA program authorization being given to the State of California, LLNL is now working solely with DTSC in maintaining hazardous waste permits. Since the submittal of a revised Part B permit application on January 17, 1992, LLNL has deemed it necessary to make further additions and revisions. Accordingly, a new Part B revision was submitted to DTSC on April 30, 1993.

An update to the waste management units mentioned in last year's report follows:

- Building 419 will be closed under interim status. Present plans call for closure to commence in 1994.

- The Building 624 incinerator has been closed, and the incinerator itself has been dismantled. LLNL has received acknowledgment of closure from DTSC.

- Closure of the Area 612-3 Container Storage Unit began in November 1992. Operation of the Building 693 Container Storage Unit began that same month.

- The Building 233 Container Storage Unit will be upgraded to meet current seismic standards and has been added to thr new Part B permit application.

- Five mixed waste treatment units have been added to the Building 514-1 Complex.

\section{Extremely Hazardous Waste Permit}

Permit Number 2-13640 is required, pursuant to 22 CCR 67430.1, to transport extremely hazardous waste to an off-site hazardous waste disposal facility. As a condition of the permit, LLNL must prepare a list of the extremely hazardous wastes (including concentration, quantity, packaging, proposed hauler, disposal facility, and proposed method of disposal) and submit it to DTSC two weeks prior to the shipment of any such waste. This permit must be renewed annually; the application for renewal was submitted in August 1992. 


\section{Hazardous Waste Transport Registration}

This registration is required, pursuant to $22 \mathrm{CCR} 66263.10$, to transport hazardous wastes over public roads (e.g., from one LLNL site to another). Conditions for registration include annual inspections of transport vehicles and trailers by the California Highway Patrol, special training and annual physical examinations for drivers, and annual submission of lists of transport vehicles and trailers to DTSC. The registration renewal application was sent to DTSC in October 1992.

\section{Medical Waste Permit}

LLNL generates several types of medical wastes (previously identified as infectious wastes). In July 1991, LLNL submitted an application for a medical waste permit to the Alameda County Environmental Health Services (ACEHS). The application contained detailed information concerning the management and treatment of medical wastes generated by LLNL's biomedical research and health services facilities (including Site 300 medical waste generated by the Center for Chemical Forensics). The permit, which is valid for five years with annual renewal of registration, was issued in August 1991.

\section{Site 300 Permits}

Landfill Pits 1 and 7 were operated at Site 300 under provisions of ISD CA2890090002 until November 1988, when their use ceased. The landfills had been used for disposing of firing-table gravel and debris. The closures of these landfills wcre completed in 1992 in accordance with the closure plan approved by EPA, DTSC, and the Central Valley RWQCB.

The Site 300 Building 883 Hazardous Waste Container Storage Facility continued to operate through 1992 under provisions of the Part B permits (Part B CA2890090002) issued by EPA and DTSC in November 1989. These permits allow the facility to store specific wastes in containers, provided that certain administrative and operational conditions are met.

A High Explosives (HE) burn facility operated at Site 300 under interim status standards until November 1992. Work has begun on the design and permitting of a new HE open burn/open detonation facility to replace this unit. The RCRA Part B permit application for a Site 300 Explosive Waste Storage Facility was submitted to DTSC on July 20, 1992. This facility will allow for longer-than-90-day storage of explosive wastes prior to treatment. The application is still in the initial review process.

\section{Inspections}

The DTSC performed its annual inspection of the Livermore site on June 30; on July 1, 2, 7, and 8; and on August 3, 1992. The inspection included the review of the retention tank system on the east side of Building 141, which includes two 
aboveground tanks (141-R2O3 and 141-R5O1) used to collect hazardous rinse waters. These tanks had been operated as less-than-90-day hazardous waste collection tanks but were removed from service in 1991. The DTSC inspector informed LLNL that closure plans would be required for closure of these tanks but that the regulatory agencies would not have to review or approve them because their purpose is simply to document how the tank is closed. Closure plans for the two tanks were completed in February 1993, and the tanks will be closed in 1993.

Following its inspection of the Livermore site, DTSC issued a Report of Violation (ROV) that LLNL received on August 18, 1992. Eleven violations were noted, and LLNL took the following actions in response to the ROV:

- Revised the tank certification procedures.

- Segregated waste in the Building 614 East Cells Container Storage Unit.

- Expanded the EP0006 training course to cover chemical compatibility, to reinforce 90-day regulatory storage limitations at the WAA, to include Contingency Plan implementation, and to reinforce one-year storage limitation at the point of generation.

- Developed an improved system of labeling to address the storage of incompatible waste.

- Repaired paint cracks in the secondary containment of the Area 514 Storage Tank 514-R5O1 Unit.

- Trained hazardous waste management employees in proper inspection techniques of secondary containment.

- Developed and installed a computerized WAA inventory tracking system.

- Established a Continuous Quality Improvement group to identify and resolve 90-day storage issues at the WAA.

- Implemented a continuous operating record at the WAA.

- Corrected all labels that were incomplete or inaccurate.

- Updated the Battery Shop practices for the management of lead/tin solder dross.

- Relocated the eyewash/safety shower unit at the Building 361 WAA.

On November 20, 1992, DTSC and Department of Health Services (DHS) visited the Livermore site to review the proposed Mixed Waste Management Facility technologies. The visit was for information only; no Notices of Violation (NOVs) or Notices of Deficiencies (NODs) resulted from this visit. 
DTSC conducted an inspection of the Site 300 hazardous waste facilities in January 1992. Following its inspection of Site 300, DTSC issued an ROV received at LLNL on April 8, 1992. Thirteen violations were noted in the ROV; LLNL took the following actions in response:

- Expedited the analysis, handling, and tracking of hazardous waste in the WAAs.

- Corrected labels that were incomplete or inaccurate.

- Revised RCRA landfill cover inspection procedures.

- Replaced eyewash units at Building 827.

- Corrected the 1989 Annual Report on quantity of explosive wastes treated at Site 300, and revised record-keeping procedures.

- Implemented new DTSC requirements to keep hazardous waste containers of oily rags shut tight.

- Closed the Building 836 WAA.

- Modified WAA inspection frequency to every 7 days or less.

- Modified the Building 829 Waste Acceptance Plan to include peroxides.

- Removed all waste not included in the Building 883 permit.

- Negotiated with the State to resolve waste code issues in Building 883 hazardous waste storage.

- Used the U.S. EPA number of Livermore site as the transporter identification number on Hazardous Waste Manifests.

Pits 1 and 7 at Site 300 were inspected by DTSC, Central Valley RWQCB, and EPA on December 15, 1992. No NOVs or NODs resulted from this inspection.

On January 14, 1993, DTSC visited Site 300 to review current and future Explosive Waste Treatment and Storage Facilities. The visit was for information only and no NOVs or NODs were issued.

\section{Waste Accumulation Areas}

During 1992, there were 52 WAAs in operation at LLNL: 51 WAAs at the Livermore site and 1 WAA at the Livermore Airport. Environmental Protection Department (EPD) personnel performed 1040 biweekly WAA walk-throughs at the Livermore site WAAs and 21 biweekly WAA walk-throughs at the Airport WAA during 1992. During the first quarter of 1993, EPD personnel performed 306 biweekly WAA walk-throughs at the Livermore site and 6 biweekly WAA walk-throughs at the Airport WAA. The walk-throughs are informal checks of 
items such as capacity, labeling, and secondary containment; formal inspections of these items are conducted by program personnel.

There were 14 WAAs being operated at Site 300 during 1992. The Building 836 WAA was taken out of service in October 1992, leaving a total of 13 active WAAs at Site 300. EPD personnel performed 330 biweekly WAA walkthroughs at Site 300 during 1992. As of the first quarter of 1993, 13 WAAs remain in operation at Site 300 and during this quarter, 65 biweekly WAA walkthroughs were performed.

\section{Underground Storage Tank Management}

Federal and state laws provide comprehensive regulation of the design, operation, monitoring, and closing of tank systems that contain hazardous products or hazardous waste. The California Underground Tank Regulations, found in 23 CCR Chapter 16, regulate underground storage tank (UST) systems used for containment of hazardous products or hazardous waste. The state underground tank regulations are administered at the Livermore site by the Alameda County Environmental Health Services (ACEHS) and at Site 300 by the San Joaquin County Public Health Services (SJCPHS). Federal standards for design, construction, operation, maintenance, and closure of these USTs are found in 40 CFR 280. Additional federal standards for petroleum tanks are given in 40 CFR 112. Standards for design, construction, operation, maintenance, and closure of USTs that contain hazardous wastes (both aboveground and underground) are the subject of RCRA regulations in 40 CFR 265.

The Livermore site has 46 aboveground and 49 underground tanks in service, which may contain either hazardous products or hazardous waste. Of these, 36 tanks contain wastewater, and the other 59 tanks contain hazardous products.

Underground tanks at Site 300 contain diesel fuel, gasoline, and contaminated wastewater; aboveground tanks contain diesel fuel, insulating oil, trichloroethene, and contaminated wastewater. Some of the wastewater systems are a combination of underground and aboveground tanks. Site 300 currently has 12 aboveground and 16 underground tanks in service, of which 21 contain hazardous products and 7 contain hazardous wastewater.

\section{Underground Tank Permits}

In 1992, LLNL paid annual fees to the ACEHS for 49 hazardous waste, mixed waste, and hazardous product underground storage tank permits for the Livermore site. The number of underground tanks that require permits remained the same as in 1991. Three underground storage tanks were closed, one underground storage tank was reclassified as nonhazardous because of changes in use, and four mixed waste underground storage tanks were added to the list. 
In 1992, LLNL paid fees to SJCPHS for 16 petroleum underground storage tanks at Site 300; all of these tanks remain in service.

\section{Tank Integrity Testing}

Under the tank leak-tightmess testing program, underground tank systems are tested to determine structural integrity, in accordance with requirements established in state and federal regulations. Single-walled, underground wastewater systems are tested, including those not presently regulated. The underground portions of tank systems are tested (as a whole or by component parts) using methods that may include precision tests, dye tests, helium-injection detection, arid hydrostatic tests. All leak-tightness test results for regulated systems are provided to ACEHS or SJCPHS. Twenty-three underground diesel fuel and gasoline systems at the Livermore site and 10 systems at Site 300 were tested in 1992. Testing of petroleum tanks in 1992 revealed two system failures at the Livermore site. One system was repaired, retested, and returned to service; the second system was removed from service. For the repaired system, the contaminated soil was removed at the time of repair. For the other system, most of the contaminated soil was excavated when the tank system was removed from service; the corrective action plan will specify any additional soil removed and any further sampling, if appropriate.

In addition, 15 (11 at the Livermore site and 4 at Site 300) underground wastewater retention systems were tested in 1992. These systems receive and retain wastewater from processes of potential concern (i.e., metal plating, printed-circuit boards, semiconductors, and oily water from floor cleanup and steam-cleaning operations). Water is retained by these systems until the appropriate mode of disposal has been determined, based on the results of tank content analyses. Testing in 1992 identified one wastewater system at Site 300 that had a leak in its secondary system. This system was removed from service and is awaiting repair. The leak was minor and did not result in an adverse impact on the environment.

\section{Closure and Leak Documentation}

Closure plans, closure reports, and leak reports were completed in 1992 for tank systems (or portions of systems) that were taken out of service, previously removed (but not officially closed), or expected to be removed from service. Closure requirements for hazardous USTs include a closure plan prior to commencing work to close the system, quarterly reports if a leak has been identified, and a closure report upon completion of closure activities. The closure plans require a detailed review of the uses of the tank, a sampling plan, a site plan, and other information to verify that no environmental contamination has occurredor, if it has occurred, to ensure its cleanup. Thirty-five closure plans and reports for hazardous waste tank systems and six leak reports for hazardous and 
nonhazardous waste tank systems were submitted for regulatory review in 1992. Although not required for submittal to regulatory agencies, three closure plans and reports were prepared for nonhazardous waste tank systems as a part of LLNL's best management practices.

\section{Tank Upgrade Project}

In fiscal year 1992, LLNL received line item funding for four years to upgrade or close approximately 126 tanks in accordance with existing local, state, and federal tank regulations or to decrease the potential for contamination of the environment as the result of a release from a tank or its appurtenances. These tanks include wastewater retention tanks (nonhazardous, hazardous, mixed, and radioactive) and product retention tanks (including petroleum products). In fiscal year 1993, additional funding was granted to provide overflow and spill protection to aboveground oil-filled electrical equipment (e.g., transformers) and additional aboveground petroleum tanks, giving a revised total of 214 tanks or transformers being closed or upgraded.

As of the first quarter of 1993, construction was completed for one tank and that tank is in use; construction is in progress for 15 tanks; design is in progress for 52 tanks; and another 58 tanks are in the preliminary stage of the upgrade process. Overflow and spill protection design and construction have not yet begun for the remaining tanks and transformers. Engineering assessments to certify the integrity of all single-walled hazardous waste systems and all newly installed systems are in progress.

\section{Remedial Activities}

At the Building 292 area, a leak of tritiated rinse water from an underground storage tank was discovered in July 1989. The tank was immediately taken out of service, but not disconnected from the influent piping. On December 23, 1990, freezing weather caused a water pipe in Building 292 to break, allowing clean water to flow into floor drains and discharge into the tank. Subsequently, the floor drains leading from the building to the tank were sealed.

The tritium distribution and ground water flow conditions around the Building 292 area have been characterized using chemical and hydraulic data from 18 boreholes and wells, tritium ground surface flux measurements, and tritium transpiration measurements from selected vegetation in the area. Since the fall of 1990, the maximum detected ground water tritium activity was $280 \mathrm{~Bq} / \mathrm{L}(7,700 \mathrm{pCi} / \mathrm{L})$ from piezometer UP-292-001 measured on November 30, 1990. In 1992, an additional piezometer, UP-292-020, was installed approximately 6 meters west of Building 292. Preliminary data for this piezometer indicate that no tritium is present in saturated and unsaturated sediment samples from this borehole. Also, no tritium activity was detected in the ground water collected from this piezometer. There are currently eight piezometers in wells in the 
Building 292 area that are being monitored quarterly. In 1993, tritium in the area will continue to be monitored.

In December 1992, the underground diesel fuel tank at Building 298 showed a leak in the fuel supply line, which was reported to ACEHS. The tank has been removed from service and is scheduled for removal and closure in 1993. Soil samples from beneath the tank and its piping will be collected at that time. Contaminated soil, if found during closure activities, will be removed in accordance with applicable regulations.

Soil samples collected from the base of an excavation during closure of a diesel fuel tank at Site 300's Building 877 showed diesel fuel contamination. The presence of diesel fuel in the soil is believed to have resulted from occasional overfilling. A Corrective Action Plan, which specifies excavation to remove the contaminated materials and additional sampling to confirm that the contamination has been removed, has been prepared for review and approval by SJCPHS.

In the Site 300 Building 879 automotive fleet facility, mineral oil was released through an underground casing for the ram of the hydraulic hoist system. Contaminated soil is planned to be removed in 1994 according to the procedure established with the Central Valley RWQCB.

\section{Inspections}

On February 19 and March 27, 1992, an inspector from ACEHS inspected the progress of the installation of new underground portions of the Building 161 wastewater retention tank system.

On March 10, 1992, an inspector from ACEHS witnessed the removal of a 1100-liter underground diesel fuel tank at Building 424. The tank (424-D1U1) formerly supplied fuel to the Building 424 emergency generator.

On April 2 and 3, 1992, an inspector from ACEHS witnessed closure activities (tank removal and soil sampling) for the closure of the 231-R3U1 and 231-R3U2 tank systems. The two 57,000-liter tanks and their associated piping were located at the southwest corner of Building 231 and had been used to supply fuel oil for two boilers located inside Building 231.

On May 20, 1992, an inspector from ACEHS witnessed closure activities (inplace closure and soil sampling) for the closure of the 325-I1U1 tank system. The closed 12,800-liter tank is located on the east side of Building 325 and had been used to retain wastewater from the mixed-bed ion exchangers located inside Building 325.

On October 14, 1992, an inspector from ACEHS inspected the sources of wastewater discharge into the Building 298 wastewater retention tank system. This inspection was for information only. 
On May 4 and 5, 1992, an inspector from SJCPHS inspected the closure and closure sampling of the underground diesel fuel tank systems at Buildings 875 and 877.

On June 1, 5, 10,11, July 23, and August 17, 1992, an inspector from SJCPHS inspected the construction and testing of the newly installed underground diesel fuel system at Building 875.

No NOVs or NODs were received as a result of any of these inspections.

\section{National Environmental Policy Act (NEPA) and California Environmental Quality Act (CEQA)}

\section{NEPA}

NEPA (42 U.S.C. 4371 et seq.) establishes federal policy for protection of environmental quality. The major method of achieving established NEPA goals is the requirement for federal agencies to assess the potential environmental impacts of their proposed actions through preparation of an Environmental Impact Statement (EIS) for any major federal or federally funded project that may have significant impact on the quality of the human environment. If the need for an EIS is not clear, an Environmental Assessment (EA) must be prepared, which leads to a definitive conclusion concerning whether to prepare an EIS or whether a Finding of No Significant Impact (FONSI) can be issued. In the latter case, an EIS is not required.

Certain groups of actions that do not have a significant effect on the environment, either individually or cumulatively, can be categorically excluded from further NEPA review. Subpart D of the April 1992 DOE NEPA Implementing Procedures identifies those actions that do not require the preparation of either an EA or an EIS.

Within DOE, an Action Description Memorandum (ADM) often is prepared when the proposed action does not clearly fit one of the categories of actions outlined in DOE NEPA Implementing Procedures as specifically requiring preparation of an EA or an EIS. This document provides DOE with sufficient information to determine which type of assessment document may be needed.

In accordance with the DOE Secretary of Energy Notice 15-90 and supplemental procedural guidance for its implementation, proposed actions that could be categorically excluded under the proposed revision to the DOE NEPA Guidelines (55 Federal Register 213) would require the preparation of a Brief EA until such time as these guidelines were approved. Because of the extremely limited potential for significant environmental impacts, Brief EAs were to be relatively short ( 3 to 10 pages). A number of these Brief EAs were prepared; then, after the revised DOE NEPA Implementing Procedures were finalized (April 24, 1992), draft Brief EAs still being reviewed by DOE were either processed as EAs 
or, if appropriate, were converted to Categorical Exclusions (CXs) in accordance with the April 1992 DOE NEPA Implementing Procedures.

In 1992 and first quarter 1993, LLNL prepared 117 documents in accordance with policies and procedures for DOE review and determination as to compliance with the NEPA. These included 2 ADMs, 111 CXs, 3 EAs, and 1 EIS (described below). DOE issued the following 11 FONSIs during that period for previously submitted EAs or Brief EAs:

- Microwave Tokamak Experiment/Experimental Test Accelerator II (MTX/ETA II)

- Super High Altitude Research Project (SHARP), formerly Cheap Access to Orbit (CATO) Experimental Tests

- Brilliant Pebbles Tether Test at the Nevada Test Site

- Pilot Tritium Evaporator at the LLNL Site 300 Well 8 Spring

- Vacuum Process Laboratory Relocation

- Electric Utility System Distribution, Replacements, and Upgrades

- Advanced Materials Configuration Laboratory

- Security Administration Building

- Diamond-Anvil High Pressure Cell

- Laser Guide Star Experiment

- Multipass Beamlet Testbed in Building 381

\section{Environmental Assessments Submitted to DOE}

Brief descriptions of the three proposed actions for which NEPA EAs were prepared during 1992 and first quarter 1993 are presented below.

Environmental Assessment for the Verification, International Security, and Technology Analysis (VISTA) Center addressed the potential environmental impacts associated with the proposed construction and use of a two-story building, which would be adjacent to and become part of Building 261, to be used for offices and bench-scale laboratories at the Livermore site. This proposed project is discussed in the Environmental Impact Statement and Environmental Impact Report for Continued Operation of Lawrence Livermore National Laboratory and Sandia National Laboratories, Livermore (EIS/EIR) for the entire site (U.S. DOE and UC 1992b). At this time, DOE is still reviewing the draft EA as well as the information contained within the EIS.

Environmental Assessment for the Site 300 Explosive Waste Storage Facility addressed the potential environmental impacts associated with the construction and operation of the storage facility for explosive wastes at Site 300. This 
proposed project is also discussed in the EIS/EIR for the entire site (U.S. DOE and UC 1992b). At this time, DOE is still reviewing the draft EA as well as the information contained within the EIS.

Environmental Assessment for the Building 197 Physics Laboratory addressed the potential environmental inpacts associated with current and planned operations in Building 197 at the Livermore site. The proposed action includes development of advanced techniques and technology for production of extremely high-speed integrated semiconductor circuits for high-performance supercomputers, optics for the wide-field-of-view telescope and the x-ray microscope, and feasibility of laser-initiated propulsion for launching moderate masses into orbit. At this time, DOE is still reviewing this draft EA.

\section{Environmental Impact Statement/Environmental Impact Report}

The Draft Environmental Impact Statement and Environmental Impact Report for Continued Operation of Lawrence Livermore National Laboratory and Sandia National Laboratories, Livermore (EIS/EIR; U.S. DOE and UC 1992a) was issued for public comment in March 1992. The EIS/EIR analyzed the potential environmental impact of continued operation, including near-term (within 5 to 10 years) proposed projects, of LLNL and SNL/CA. The EIS/EIR also analyzed alternatives that involved continuing operation at fiscal year 1992 funding levels without further growth, modifying the continued operations alternative that reduced adverse environmental impacts of operation or facilities, and shutting down and decommissioning. To meet CEQA requirements, the EIS/EIR also examined the alternative of discontinuing the University of California's management of LLNL after September 1992. (In the fall of 1992, UC was awarded a 5-year contract to continue management of LLNL.) The EIS/EIR assessed the environmental impacts of the laboratories' operations on air and water quality, geological and ecological systems, occupational and public health, prehistoric and historic resources, endangered species, floodplains and wetlands, socioeconomic resources, hazardous waste management, site contamination, and other environmental issues. A public hearing on the Draft EIS/EIR was held on April 30, 1992. Written comments were accepted for 90 days, from March 11 until June 11, 1992. The Final EIS/EIR, with comments incorporated, was published in August 1992 (U.S. DOE and UC 1992b). The EIS/EIR was certified on November 25,1992 , by the Board of Regents, concluding the CEQA evaluation process. The NEPA Record of Decision (ROD) was signed by the Secretary of Energy on January 1, 1993. When DOE publishes the appropriate Mitigation Action Plan (MAP), the DOE NEPA process will be complete.

\section{CEQA}

CEQA (California Public Resources Code Sections 21000 et seq.) establishes state policy for protection of environmental quality. The goals of CEQA are 
achieved by requiring local and state agencies to assess the potential environmental impacts of proposed actions for which they may have a decisionmaking role. This is done through the preparation of an Initial Study (IS), which leads to issuance of a Negative Declaration or a requirement to prepare an Environmental Impact Report (EIR). An EIR may also be prepared directly for projects that may have significant environmental impacts.

One EIR was prepared under the UC CEQA procedures and submitted to the UC Regents in August 1992. This document, the Final Environmental Impact Statement and Environmental Impact Report for Continued Operation of Lawrence Livermore National Laboratory and Sandia National Laboratories, Livermore (U.S. DOE and UC 1992b), also served as a DOE EIS and has been previously discussed under the information on NEPA implementation.

One IS for LLNL's proposed Explosive Waste Storage Facility (EWSF) at Site 300 was submitted to DTSC to assist that agency in meeting its CEQA responsibilities. At this time, DTSC is still reviewing the IS.

\section{Endangered Species Act}

Under Section 7 of the U.S. Endangered Species Act, a list is prepared of endangered or threatened species and other species of concern that may exist or are known to exist at the LLNL and SNL/CA sites. LLNL conducted numerous field surveys during 1991, and these were reported in the 1992 EIS/EIR.

In November 1992, LLNL surveyed the northern Livermore-site boundary when conditions in Arroyo Las Positas provided an opportunity to inventory animal tracks. Six mammal species, two bird species, and miscellaneous insect species were observed during the survey. No signs of sensitive species were observed along the arroyo, and species signs observed were a subset of the species identified in the 1992 EIS/EIR for the Livermore site.

In 1992, LLNL also conducted (for a Site 300 proposed project) a survey of potential dens of the San Joaquin kit fox (Vulpes microtis mutica) and known burrows of two other sensitive species [American Badger (Taxidea taxus) and burrowing owl (Athene cunicularia)]. No potential kit fox dens or known burrows for the other two of these species were noted during this survey. Representatives of the California Department of Fish and Game and the U.S. Fish and Wildlife Service visited Site 300 on March 26, 1992, and counted a record number (546 plants) of mature large-flowered fiddleneck (Amsinckia grandiflora), an endangered species, located at the Building 858 Drop Tower.

\section{National Historic Preservation Act}

Under Section 106 of the National Historic Preservation Act, a cultural resource assessment of the Livermore site and of a single historic archeological 
property within Site 300 was conducted in 1992 by William Self Associates (William Self Associates 1992). The purpose of the assessment was to determine National Register of Historic Places (NRHP) eligibility potential of all or part of the Livermore site and Site 300 historic property. Twenty-three buildings at the Livermore site were examined (some cursorily) pursuant to NRHP criteria. LLNL is studying the preliminary conclusions in the report.

\section{Clean Water Act-NPDES/Waste Discharge Requirements}

Clean water is the subject of local, state, and federal regulations. The National Pollutant Discharge Elimination System (NPDES), under the Federal Clean Water Act, establishes permit requirements for discharges into surface waterways. In addition, the State of California requires permits, known as Waste Discharge Requirement (WDR) Orders, for any discharges that could adversely affect the beneficial uses of waters of the state. The Regional Water Quality Control Boards (RWQCBs) are responsible for issuance and enforcement of both permit types. Finally, the Livermore Water Reclamation Plant (LWRP) requires permits for discharges to the city sanitary sewer system.

\section{Ground Water and Surface Water Discharge Permits}

WDR Order No. 88-075, issued by the San Francisco Bay RWQCB, pertains to activities undertaken to investigate and remediate contaminants in ground water at the Livermore site. The order allows treated ground water that meets specified standards to be discharged to specified areas on DOE property. The NPDES permit (CA0029289, also designated as WDR Order No. 91-091) applies to treated ground water discharged to the ground from monitoring wells used in the ground water investigation and to other treated ground water discharged to ground and surface waters. The self-monitoring programs required by these permits are described in Chapter 3, Environmental Program Information.

Two Notices of Intent (NOI) were submitted to the State Water Resources Control Board to cover the Livermore site and Site 300 under the California Industrial Activity Storm Water NPDES permit during the firsi quarter of 1992. This permit allows LLNL storm water discharges under the State General Permit, which became effective October 1, 1992. In addition, three NOIs were submitted to the State Water Resources Control Board to cover construction projects greater than 5 acres under the General Construction Activity Storm Water NPDES permit. The NOIs were submitted for the Livermore-site Building 132 and for the Site 300 Roadway Improvement Project and closure of landfill Pits 1 and 7. Both the Livermore site and Site 300 are implementing draft Storm Water Pollution Prevention Plans. The storm water monitoring programs were implemented by January 1, 1993, as required by the State General Discharge Permit. 
Site 300 has four additional permits issued by the Central Valley RWQCB: WDR Order No. 80-184 pertains to inactive landfills; WDR Order No. 82-105 applies to the cooling-tower discharges (an NPDES permit-CA0081396-is also required for the cooling-tower discharges because discharges may enter storm water drains); WDR Order No. $85-188$ is a permit for operation of the sewer lagoon, septic tanks, leachfields, and the Class II surface impoundment for HE rinse water; and WDR Order No. 91-052 (NPDES Permit No. CA0082651) is a permit for discharge of treated ground water from the eastern General Services Area ground water treatment facility to Corral Hollow Creek. The selfmonitoring programs for these permits are also discussed in Chapter 3, Environmental Program Information.

LLNL obtained permission from the San Francisco Bay RWQCB to temporarily discharge treated ground water from Treatment Facility A (TFA) into the on-site Central Drainage Basin to maintain the basin's water level and to prevent deterioration of its water quality due to evaporative losses. LLNL began discharging TFA water in October 1992 and discontinued discharging in December 1992 because of increased rainfall. The self-monitoring agreement submitted to the San Francisco Bay RWQCB for discharges from the Central Drainage Basin is detailed in Chapter 3, Environmental Program Information.

\section{Wastewater Permits}

A Wastewater Discharge Permit from the Livermore Water Reclamation Plant (LWRP) provides for the continued discharge of LLNL sanitary and industrial effluent to the city sewer system. Permit No. 1250(91-92) was in effect from September 1991 through September 1992, and renewal Permit No. 1250(9293) is effective from September 1992 to September 1993. Under the provisions of this permit, LLNL is required to conduct a monitoring program at its outfall into the LWRP sewer system. Daily and monthly effluent sampling are performed to satisfy permit compliance requirements. The daily samples are composited to represent (and are reported as) weekly values. The monitoring results of the total LLNL effluent are reported monthly to the LWRP. In accordance with federal pretreatment regulations, additional quarterly and semiannual sampling is required of all identified categorical processes. These additional monitoring results are reported semiannually to LWRP.

Processes that are regulated under 40 CFR 403 (categorical processes) include metal-finishing and certain electrical and electronic processes. As stated in Permit No. 1250(92-93), there are 22 individual processes. Some processes discharge to the sanitary sewer and are monitored; some processes do not discharge to the sanitary sewer and are not monitored; and some processes discharge to one of five tank systems, which are monitored. All of these 
discharges are reported to the LWRP in the Semiannual Wastewater Reports (see e.g., Grayson and Brigdon 1992, Grayson 1992).

LWRP personnel spent five days on site during 1992 (in February, April, July, October, and November), inspecting and sampling discharges. LLNL was granted three Discharge Permits by the LWRP for discharge to the sanitary sewer during 1992: additional ground water discharge Permit No. 1508G for discharge of sewerable waste from Treatment Facility F, ground water discharge Permit No. 1510G for an Environmental Restoration Division treatability study, and wastewater discharge Permit No. 1250(92-93) for site-wide discharge of wastewater.

The LWRP issued two NOVs to LLNL in 1992: one for violation of the permitted effluent limitation of oil and grease, and one for violation of the permitted effluent limitation of cupper. Neither of these violations resulted in any fines. LLNL also received a letter of concern regarding elevated levels of oil and grease in the LLNL effluent. None of these actions resulted in any adverse impact on the operation of the LWRP.

\section{Inspections}

The San Francisco Bay RWQCB spent one-half day on September 1, 1992, meeting with LLNL and inspecting areas of recent minor spills. Areas visited were Building 341, where a recent release of low conductivity water had occurred, and the Area 612 Facility yard, where rinse water from a tanker that had contained sewerable water had been released. These incidents are discussed in more detail in Chapter 12 under the Environmental Occurrences subsection.

California Department of Fish and Game personnel inspected the Livermore site on February 5, 1993, to determine the need for a streambed alteration agreement for proposed work to reinforce the Arroyo Seco bank where it crosses the southwestern corner of the Livermore site. Fish and Game personnel inspected Site 300 twice during 1992 to determine conditions for the Main Roadway Improvement Project Streambed Alteration Agreement. The first inspection occurred on June 1, 1992, and the second inspection was on August 3, 1992.

\section{Streambed Alteration Agreements}

Two streambed alteration agreements were issued by the California Department of Fish and Game for construction projects impacting the natural drainage at Site 300. The projects were the Super High Altitude Research Project (SHARP), which was formerly CATO (Cheap Access to Orbit), and the Main Roadway Improvement Project. 


\section{Clean Air Act/Air Quality Management Activities}

The Clean Air Act is the federal law that authorizes the EPA to set air quality standards and to assist state and local governments in developing and executing air pollution prevention and control programs. The Bay Area Air Quality Management District (BAAQMD) is the agency responsible for enforcing these standards (as well as the state and local standards) for air emissions from stationary sources in the San Francisco Bay Area (including the Livermore site), and the San Joaquin Valley Unified Air Pollution Control District (SJVUAPCD) is the agency responsible for enforcing these standards in San Joaquin County (including Site 300).

After reviewing LLNL's air toxics emission inventory that was submitted in November 1989, as required by the Air Toxics "Hot Spots" Information and Assessment Act of 1987 (AB 2588, California Health and Safety Code Section 44360 et seq.), the BAAQMD requested that LLNL assess the impact of toxic air emissions on the surrounding area. A screening assessment was completed and risk was found to be below the threshold values that are used to determine need for further evaluation. Results of this work were submitted to the BAAQMD in fall of 1990.

After reviewing LLNL's AB 2588 inventory for Site 300, which was submitted in June 1990, the SJVUAPCD requested that LLNL provide additional emissions data for a small burner used for disposal of explosives waste. Test results were sent to SJVUAPCD in March 1993.

These air toxics emission inventories are required every two years, and both updated inventories (Livermore site and Site 300) were submitted during the summer of 1992.

\section{Air Permits}

Air permits are obtained from the BAAQMD for the Livermore site and from the SJVUAPCD for Site 300. In 1992, the BAAQMD issued 111 permits for the Livermore site. These permits authorize 19 operations that involve the use of solvent cleaners, 16 degreasers, 9 ovens, 8 furnaces, 6 printing presses, 5 paint spray booths, 4 explosive operations, 3 machine tool lathes, 3 plating operations, 3 silk-screen operations, 3 semiconductor fabrication operations, 3 etchers, 2 storage tanks, 2 polishing operations, 1 rock crusher, 1 roof mixer, 1 drum crusher, 1 transfer tank, 1 extraction/treatment system, 1 oil-shale combuster, 1 metal-finishing operation, $1 \mathrm{kiln}, 1$ shale hopper and preheater, 1 fire test cell, 1 optic coating operation, 1 paper pulverizer system, 1 vacuum chamber, 1 milling machine, 1 dry-film developer, 1 welding operation, 1 filter test, 1 service station, and 7 miscellaneous uses. 
In 1992, the SJVUAPCD issued 25 permits covering the operation of 9 internal combustion engines/emergency power generators, 7 drying ovens (one permit), 5 boilers, 2 gasoline tanks with vapor recovery (one permit), 2 dispensing systems with vapor recovery, 1 machining equipment with dust filter, 1 evaporative tower, 1 carbon absorption system, 1 portable air compressor, 1 sawdust collector, 1 paint spray booth, and 1 crane.

\section{Inspections}

BAAQMD personnel conducted 14 days of inspections at the Livermore site during 1992. On January 8, 1992, BAAQMD issued an NOV citing excessive pressure in the spray wand of a degreaser. The equipment was immediately brought into compliance.

The SJVUAPCD conducted one inspection at Site 300 during 1992, and no NOVs were issued.

During the first quarter of 1993, BAAQMD conducted five days of inspections at the Livermore site. As a result of these inspections, LLNL received one NOV, issued for Building 511 for use of adhesive without a permit. LLNL has applied to the BAAQMD for permits authorizing the use of adhesives.

\section{National Emission Standards for Hazardous Air Pollutants (NESHAPs)}

in October 1989, the EPA issued new NESHAPs standards concerning radionuclide emissions from DOE facilities. DOE initiated discussions with the EPA to develop a Memorandum of Understanding on how the DOE facilities would achieve compliance with these standards. Pending resolution of the DOE/EPA discussions, LLNL drafted a work plan to satisfy the new requirements and began to assemble information needed to meet the new standards. This effort included reviewing all operations that employ radionuclides and gathering data to model each source.

After reviewing the initial data, the EPA found that LLNL could not demonstrate compliance with the new standards. On April 19, 1991, a Finding of Violation and an order to come into compliance was issued by the EPA to UC and DOE. The law requires that every release-point from an operation using radionuclides must be evaluated using an EPA-approved computer model or be continuously monitored. Under NESHAPs requirements, the decision to install stack monitoring or to perform periodic monitoring depends on the calculated, potential, unabated off-site dose from stack emissions, and whether the resultant dose exceeds or is less than one percent of the NESHAPs standard ( $100 \mu \mathrm{Sv} / \mathrm{y}$ or $10 \mathrm{mrem} / \mathrm{y}$ ). EPA acknowledged in the Finding of Violation document that it appeared that LLNL had monitored and modeled the major contributors to its emissions. 
LLNL completed its evaluations of emissions in the first quarter of 1993, as in a proposed schedule agreed upon by EPA, DOE, and LLNL as part of negotiations for establishing a Federal Facilitities Compliance Agreement (FFCA).

In the NESHAPs Action Plan dated May 23, 1991, which was submitted by LLNL and DOE to the EPA, LLNL made a commitment to provide quarterly progress reports until all buildings in which radionuclides are used, or activation products occur, are assessed for compliance with NESHAPs regulations (40 CFR 61). Thereafter, annual summary reports will be submitted.

Four quarterly reports were prepared in 1992 (Lamson 1992; Biermann and Lamson 1992a, b; Biermann et al. 1992) and one in 1993 (Biermann et al. 1993). As of March 31, 1993, all 56 of the Livermore site and Site 300 facilities having the potential to release radionuclides to the atmosphere have been evaluated for compliance with the NESHAPs regulations. These include facilities designated as high-priority, medium-priority, and low-priority. Operations involving nonnuclear explosive testing at Site 300 were also evaluated. Additionally, eight continuously monitored facilities have been evaluated.

Annual doses resulting from total actual emissions (after controls) were not found to exceed the NESHAPs standard of $100 \mu \mathrm{Sv} / \mathrm{y}(10 \mathrm{mrem} / \mathrm{y})$. Estimates of effective dose equivalent (EDE) for all evaluated facilities, assuming normal operations and no control devices, have been calculated. Three facilities at the Livermore site (Buildings 331 and 332 and the hardened portion of Building 251) have been identified as having potential unmitigated EDE estimates greater than $1 \mu \mathrm{Sv} / \mathrm{y}(0.1 \mathrm{mrem} / \mathrm{y})$, and these facilities have appropriate radionuclide monitoring systems in place, as required by NESHAPs. Several other Livermore-site facilities (Buildings 175, 231, 419,490, and 491, and the unhardened portion of Building 251) also have monitoring systems; however, their potential unmitigated EDE estimates were less than $1 \mu \mathrm{Sv} / \mathrm{y}(0.1 \mathrm{mrem} / \mathrm{y})$ and do not require continuous monitoring under NESHAPs regulations. No other facilities, including all those at Site 300, were found to require continuous monitoring systems. Descriptions of, and testing conducted on, the existing sampling systems indicate that representative sampling is being performed. Therefore, LLNL has demonstrated compliance with the 40 CFR 61.93 NESHAPs regulations.

\section{Inspections}

On December 14 and 15, 1992, EPA personnel performed an inspection to evaluate LLNL progress toward compliance with NESHAPs regulations. The inspector stated that all deficiencies had been identified by LLNL in the quarterly reports and should continue to be addressed as actions are taken under the existing compliance schedule. 


\section{Toxic Substances Control Act (TSCA)}

TSCA regulations affecting the Livermore site are those that regulate the storage and disposal of polychlorinated biphenyls (PCBs) and asbestos wastes. The PCB annual report, required under 40 CFR 761.180, is a record of PCBcontaining equipment in service, taken out of service, or disposed of during the year. At LLNL, equipment containing PCBs is used in a totally enclosed manner until the equipment is taken out of service, at which time it is removed to Hazardous Waste Management for disposal at an approved site. Statistics for PCBs were compiled in 1992 and are kept on file, available for EPA inspection. Asbestos wastes are reported in the Hazardous Waste Report, which is required by DTSC under 22 CCR 66264.75 .

\section{Current Issues and Actions}

Uncertainty remains over the applicability of Assembly Bill 107 (Section 25123 of the California Health and Safety Code) to LLNL's Waste Accumulation Areas (WAAs). DTSC headquarters and Region 2 have not agreed on the interpretation of the new definition of a storage facility and whether or not LLNL must submit permit applications for all its WAAs.

In response to an NOD from EPA on the LLNL 1989 RCRA Part B permit application, LLNL substantially revised the permit application. A 38-volume Part B permit application was submitted on January 17, 1992, fully describing each of the 24 waste management units to be permitted. The revised permit application fully addressed EPA's comments and concerns and better reflected operations and management of the LLNL Hazardous Waste Management Facilities. In addition to the revised Part B, the Part A permit application was also revised to reflect current operations. The revised Part A was submitted on December 13, 1991.

Both parts have since been submitted again-Part A on December 18, 1992, and Part B on April 30, 1993. These newest revisions include addition of the Building 233 Facility (container storage unit for hazardous and mixed wastes), several mixed waste treatment facilities in the Building 514-1 Complex, and a revised Waste Analysis Plan.

Closure plans for the Building 419 Size Reduction Unit and the Building 419 Solidification Unit were submitted to DTSC on February 7, 1992. Size reduction operations were transferred to the Building 612 Size Reduction Unit, and solidification was transferred to the existing solidification operations at the Building 513 Solidification Unit. This change is described in the revised Part B permit application. Additionally, closure has begun on the Area 612-3 Container Storage Unit and is in progress on the Building 169 Temporary WAA. 
In 1991, LLNL was concerned about the need to offset newly identified air emissions. On February 14, 1992, LLNL appealed BAAQMD's requirement to offset eight precursor organic compound (POC) emission sources. BAAQMD granted LLNL's appeal, with the result that LLNL has more POC emission credits available for future projects.

Table 2-1. Summary of Permits as of March 15, 1993.

\begin{tabular}{|c|c|c|}
\hline $\begin{array}{l}\text { Type of } \\
\text { Permit }\end{array}$ & Uvermore Site & Site 300 \\
\hline Air & 111 (Various equipment) & 25 (Various equipment) \\
\hline \multirow[t]{6}{*}{ Water } & WDR Order No. 88-075 & WDR Order No. 80-184 \\
\hline & $\begin{array}{l}\text { WDR Order No. 91-091, } \\
\text { NPDES Permit No. CA0029289 }\end{array}$ & $\begin{array}{l}\text { WDR Order No. 82-105, } \\
\text { NPDES Permit No. CA0081396 }\end{array}$ \\
\hline & & WDR Order No. 85-188 \\
\hline & & $\begin{array}{l}\text { WDR Order No. 91-052, } \\
\text { NPDES Permit No. CA0082651 }\end{array}$ \\
\hline & $\begin{array}{l}\text { WDR Order No. 91-13-DWQ (as } \\
\text { amended by Order No. 92-12-DWQ) } \\
\text { NPDES General Permit } \\
\text { No. CAS000n01 }\end{array}$ & $\begin{array}{l}\text { WDR Order No. 91-13-DWQ (As } \\
\text { amended hy Order No. 92-12-DWQ) } \\
\text { NPDES General Fermit } \\
\text { No. CAS000001 }\end{array}$ \\
\hline & $\begin{array}{l}\text { WDR Order No. 92-08-DWQ } \\
\text { NPDES General Permit } \\
\text { No. CASO00002 } \\
\text { Site ld. No. Bldg. } 1322015300881\end{array}$ & $\begin{array}{l}\text { WDR Order No. 92-08-DWQ } \\
\text { NPDES General Permit } \\
\text { No. CAS000002 } \\
\text { Site Id. Nos. 5B395300882 } \\
\text { Main Gate Road Improvement } \\
\text { Pits } 1 \text { and } 7 \text { Closure 5B395300882 }\end{array}$ \\
\hline \multirow{3}{*}{$\begin{array}{l}\text { Hazardous } \\
\text { waste }\end{array}$} & ISD CA2890012584 & Part B CA2890090002 \\
\hline & $\begin{array}{l}\text { DTSC Permit No. 2-13640 for disposal of } \\
\text { extremely hazardous waste }\end{array}$ & ISD CA2890090002 \\
\hline & $\begin{array}{l}\text { Hazardous waste transport registration } \\
1351\end{array}$ & \\
\hline Sewer & $\begin{array}{l}\text { Discharge Permit Nos. 1250(92-93), } \\
\text { 1508G, and } 1510 \mathrm{G}\end{array}$ & \\
\hline Tanks & Fees paid for $\mathbf{4 9}$ tanks & Fees paid for 16 tanks \\
\hline Other & $\begin{array}{l}\text { FFA, ground water investigation/ } \\
\text { remediation; ACEHS medical waste } \\
\text { permits for treatment and storage }\end{array}$ & \\
\hline
\end{tabular}


Saundra M. Wander

Rebecca A. Failor

\section{Introduction}

LLNL has a substantial commitment to environmental compliance and accountability. Each year, the Environmental Protection Department (EPD) monitors the environment surrounding the Livermore site and Site 300. In 1992, over 150,000 analyses of environmental samples were performed. This effort, which is conducted in accordance with DOE Orders 5400.1, 5400.5, and 5484.1, has three purposes: (1) to assess the effectiveness of pollution control programs, (2) to assess compliance with applicable environmental laws and regulations, and (3) to evaluate the impact of operations on the environment. Data are produced from sampling of air, sewage effluent, ground water, surface water, soil, vegetation, foodstuff, and environmental radiation; the type of samples collected at a specific location depends on the site and the potential pollutants to be monitored. Details regarding individual monitoring activities can be found in specific chapters herein for each environmental medium. These chapters also document a special study of tritium in rain and storm u'ater runoff, discussed in Chapter 6 on Surveillance Water Monitoring. Summary information on monitoring activities can be found in Chapter 11 on Radiological Dose Assessment and Chapter 12 on Nonradiological Impact.

Although its monitoring activities are quite comprehensive, EPD's main mission is to interact with LLNL programs to ensure that operations are conducted in a manner that limits environmental impacts to levels consistent with regulatory guidelines. The activities that are required by environmental statutes are described in the preceding chapter. In addition to giving a brief description of each division of EPD, this chapter describes the activities, carried out by EPD, that were mandated by DOE or implemented independently by LLNL.

\section{Environmental Protection Department}

LLNL management has designated EPD as the lead environmental support organization, with responsibility for providing the guidance and expertise to ensure that LLNL meets the environmental requirements of all relevant local, state, and federal regulations and DOE Orders. [The primary responsibility for formal, active implementation of the Laboratory's Environmental, Safety, and Health (ES\&H) issues rests with the Associate Directors of the Laboratory, who 
form the ES\&H Council. The ES\&H Working Group, which includes each Associate Director's Assurance Officer, heads of ES\&H departments, and environmental legal counsel, assists the ES\&H Council in implementing ES\&H policies and actions (LLNL 1991).]

Principal functions of EPD operations include preparing and maintaining LLNL environmental plans and guidelines, informing management about pending changes in environmental regulations impacting LLNL, representing LLNL in day-to-day interactions with regulatory agencies, assessing the effectiveness of pollution control programs, and monitoring the environment to determine the impact of LLNL operations. EPD also helps LLNL programs manage and minimize hazardous, radioactive, and mixed wastes; determines the concentrations of environmental contaminants remaining from past activities; cleans up environmental contamination to acceptable standards; and responds to emergencies so as to minimize any impact on the environment.

Training is also a principal function of EPD. Major efforts are ongoing to provide LLNL employees with training on environmentally related topics and improve their ability to comply with environmental regulations. Training tasks address both specialized training for environmental professionals in EPD and training in a variety of environmental topics for employees at all levels throughout LLNL.

EPD is divided into four operating divisions: Operations and Regulatory Affairs Division (ORAD), Hazardous Waste Management Division (HWMD), Environmental Restoration Division (ERD), and Environmental Monitoring and Analysis Division (EMAD).

\section{Operations and Regulatory Affairs Division}

ORAD helps LLNL programs operate in a manner that meets all environmental compliance requirements. The ORAD staff consults with program personnel on regulatory compliance, permitting issues, waste minimization, pollution abatement, inspections, and monitoring to help identify and mitigate potential environmental contamination and compliance concerns in existing and planned projects.

The ORAD staff works with LLNL programs in all facets of environmental permitting activities. They interpret pertinent environmental regulations and requirements; they analyze existing operations and evaluate new facilities and operations during planning stages to identify environmental concerns and permitting needs; and they obtain the necessary permits. After a permit has been obtained, ORAD staff assists programs in evaluating environmental protection controls and procedures so that these controls and procedures are properly implemented. 
Other permitting activities include guiding LLNL programs through the regulatory process to help ensure that they meet regulatory requirements; meeting and conferring with regulators regarding permitting and compliance issues; and coordinating regulatory requirements for tank systems. ORAD staff also responds to emergencies and other urgent concerns to advise on environmental cleanup, sampling, and regulatory reporting.

ORAD prepares and maintains many types of documents for LLNL programs. ORAD staff issues guidance documents that describe how environmental laws, regulations, and DOE Orders are implemented at LLNL; issues templates for operational plans for tank systems and provides guidance and assistance to the programs in preparation of these plans; develops and maintains chemicaltracking databases; and prepares permits and regulatory documents, including documentation for compliance with the National Environmental Policy Act (NEPA) and the California Environmental Quality Act (CEQA). In addition, ORAD staff works with a Training and Development Group to prepare and present in-house training programs for LLNL employees on environmental issues, sampling methodologies, and waste-handling protocols.

\section{Hazardous Waste Management Division}

HWMD manages all hazardous, radioactive, and mixed wastes generated at all LLNL facilities. Operationally, HWMD staff processes these wastes for temporary storage, treatment, and transportation for either recycling or off-site disposal. Principally, the HWMD staff processes, stores, packages, solidifies, treats, or prepares waste for shipment and disposal, recycling, or discharge to the sanitary sewer.

HWMD tracks and documents the movement of hazardous, mixed, and radioactive wastes from waste accumulation areas to final disposition; decontaminates LLNL equipment; ensures that containers for shipment of waste meet specifications of the U.S. Department of Transportation and other regulatory agencies; responds to emergencies; and participates in the cleanup of hazardous and radioactive spills at LLNL facilities. HWMD staff prepares annual and biennial hazardous waste reports, which are required by the state and federal environmental protection agencies.

Additionally, HWMD is actively involved in locating and evaluating facilities that may accept mixed waste for storage or disposal, and subsequently ensuring that shipments from LLNL meet the acceptance criteria of any selected site.

\section{Environmental Restoration Division}

ERD was established to evaluate and remediate problems resulting from past hazardous materials handling and disposal practices for hazardous materials and from leaks and spills that have occurred at the Livermore site and Site 300, both prior to and during LLNL operations. These past practices and spills have 
resulted in concentrations of environmental contaminants that are unacceptable by current standards. In addressing Comprehensive Environmental Response, Compensation, and Liability Act (CERCLA) compliance issues, ERD staff plans, directs, and conducts assessments to determine both the impact of such releases on the environment and the restoration activities needed to reduce contamination concentrations to protect human health and the environment.

The ERD staff investigates field sites to determine the existence and extent of contamination and its impact on human health and the environment. ERD evaluates various remediation technologies, including state-of-the-art technologies, and recommends and implements actions for site restoration. Finally, ERD is responsible for managing remediation activities, such as soil removal and ground water and surface water treatment, and for assisting in closing inactive facilities in a manner designed to prevent environmental contamination.

\section{Environmental Monitoring and Analysis Division}

EMAD functions include effluent and surveillance monitoring, wastewater regulatory guidance, sample analysis, risk assessment, and reporting. EMAD provides guidance on how to implement laws and regulations and DOE Orders relating to wastewater. EMAD is responsible for monitoring the environmental effects, both radiological and nonradiological, of effluent streams (air, sewage, and storm water runoff) and for sampling federally regulated point-source discharges for compliance with federal regulations. The division also conducts surveillance monitoring of direct radiation as well as radiological and nonradiological surveillance monitoring of air, soil, water, ground water, vegetation, and foodstuff. Wastewater effluent monitoring and regulatory guidance is the responsibility of EMAD, and its staff is working with the programs to prepare storm water pollution prevention plans and eliminate illicit wastewater discharges. EMAD staff supports LLNL site waste generators, as well as the HWMD, in performing chemical and radiological analysis to identify, characterize, and certify waste for proper disposal. EMAD staff also conducts risk assessment and impact modeling and analysis.

EMAD is responsible for producing this annual LLNL Environmental Report, as well as for radionuclide effluent reporting under the National Emission Standards for Hazardous Air Pollutants (NESHAPs) of the Clean Air Act; the Quarterly Ground Water Report for the California Regional Water Quality Control Board, Central Valley Region; the Semiannual Wastewater Report; and a number of other documents including those dealing with wastewater management for regulatory compliance, including permit applications, monitoring reports, and compliance plans. 


\section{Self-Monitoring Programs}

Self-monitoring programs are required by the following permits issued by the San Francisco Bay Regional Water Quality Control Board: Waste Discharge Requirements (WDR) Order No. 88-075 for discharge of treated ground water to a percolation pond or for on-site use; and National Pollutant Discharge Elimination System (NPDES), Permit No. CA0029289, WDR Order No. 91-091 for discharge of treated ground water to the surface drainage system (this WDR Order replaced WDR Order No. 90-106 on June 19, 1991).

Monitoring conducted to fulfill the requirements of WDR Orders 80-114 (1980) and 85-188 for Pit 1 Area, Pit 7 Complex, Building 817 HE Process Area, and Pit 6 Area at Site 300 is discussed in detail in Chapter 7, Ground Water Protection.

\section{Treatment Facility $A$}

During the past year, LLNL continued a self-monitoring program, as required by the WDR Order No. 88-075, to verify the performance and effectiveness of Treatment Facility A (TFA) and the LLNL recharge basin, located south of East Avenue. In 1992, TFA successfully treated over 71 million liters of ground water containing volatile organic compounds (VOCs). Analytic results of TFA effluent samples indicate that WDR Order No. 88-075 discharge limits were not exceeded during 1992. The TFA total VOC discharge limit is $5 \mathrm{ppb}$. As an example, the self-monitoring sample collection schedule for TFA is presented in Table 3-1.

\section{Treatment Facility $B$}

LLNL also continued the self-monitoring program, as required by WDR Order No. 91-091, to verify the performance and effectiveness of Treatment Facility B (TFB). In 1992, TFB successfully treated more than 9.4 million liters of ground water.

Analytic results of TFB effluent samples indicate that the WDR Order No. 91-091 VOC discharge limit, which is 5 ppb, was not exceeded during 1992. The discharge limit for hexavalent chromium, $11 \mathrm{ppb}$, is very close to the detection limit of $10 \mathrm{ppb}$. Although this limit was exceeded in 1992, LLNL has taken measures to reduce the levels of hexavalent chromium, and the levels are being controlled.

\section{Treatment Facility $F$}

Treatment Facility F (TFF) is used in support of the DOE-sponsored Dynamic Stripping research project as well as soil and ground water remediation. During 1992, its operations were limited to four 10-hour soil vapor extraction tests conducted between August and December at the Gasoline Spill Area. The extracted air had fuel hydrocarbon concentrations ranging from 4000 to 6000 parts per 
million by volume, resulting in removal of about 76 liters of gasoline. When added to the 7500 liters of gasoline removed in remedial activities from 1988 to 1991, TFF has removed close to 7600 liters of gasoline through 1992.

\section{General Construction Activity}

Visual observance monitoring required by the California General Construction Activity Permit for three construction projects began in December 1992. Moriitoring for the General Construction Activity Storm Water NPDES Permit includes visual observations of construction sites before anticipated storms and after each actual storm causing runoff from the construction site. This monitoring is used to determine that best management practices are in place and are adequate, and to identify areas contributing to a storm water discharge.

\section{General Industrial Activity}

Self-monitoring required by the General Industrial Activity Storm Water NPDES Permit consists of sampling two storms per year, one which must be during the rainy season. (This sampling occurred in January 1993.) In addition, during the rainy season, monthly observations were performed of the discharge outfalls from our one storm water runoff event. During the dry season, monitoring is required twice to identify any dry weather flows. Finally, the permit requires an annual facility inspection to ensure that best management practices to prevent storm water pollution are adequate and in place.

The self-monitoring sample collection procedure and schedule for both Livermore site and Site 300 are presented in the storm water monitoring procedures. An annual certification of compliance with the provisions of the General Industrial Activity Storm Water Permit and the Storm Water Pollution Prevention Plans will be submitted on July 1 of each year in the annual monitoring report to the Regional Water Quality Control Boards (RWQCBs).

\section{Central Drainage Basin}

Monitoring of discharges from the Central Drainage Basin under NPDES Permit No. CA0029289 began in December 1992 and continued through the first quarter of 1993. Monitoring data for the drainage basin are presented in Chapter 6, Surveillance Water Monitoring.

LLNL submitted to the RWQCB a proposed monitoring plan, which includes monitoring releases occurring after the first storm of the season and monthly thereafter, through the end of the 1992/1993 rainy season. Future discharge monitoring includes the first release storm and two additional storm water releases performed in conjunction with NPDES storm water monitoring.

Quarterly Minor Spill Report

The Minor Spill Report is based on an agreement with the San Francisco Bay RWQCB and provisions in NPDES Permit No. CA0029289 and WDR Order 
No. 88-075 that require a report of all releases to the ground or surface waters that are not specifically allowed in the permit. The permit does not specify a de minimis level. The only discharge allowed by these permits is treated ground water. This report is used for minor spills that are below reportable quantities and are well contained and characterized. Should a spill of a reportable quantity of material or one that is not contained occur, the appropriate agencies are contacted immediately.

\section{Cooling Tower}

The Cooling Tower Report is submitted to the Central Valley RWQCB according to the provisions in NPDES Permit No. CA0081396 (WDR Order No. 82-105). LLNL submits quarterly data on temperature, $\mathrm{pH}$, flow, and total dissolved solids (TDS). Because cooling tower $\mathrm{pH}$ values exceed the permit limitations approximately $95 \%$ of the time, LLNL has submitted a time schedule to the Central Valley RWQCB identifying actions to eliminate surface water discharges from 13 of the 16 cooling towers that could discharge to surface drainage ways. LLNL proposes to continue discharging the three remaining cooling towers as before but has requested the Central Valley RWQCB to expand the allowable $\mathrm{pH}$ discharge range.

\section{Waste Minimization and Pollution Abatement}

\section{Waste Minimization and Pollution Prevention Awareness Plan}

The combined Waste Minimization and Pollution Prevention Awareness Plan was prepared in accordance with DOE Order 5400.1 and is dated May 31, 1992. This plan was originally issued on May 31, 1991, but since then there have been significant changes in regulatory requirements that have affected Laboratory operations.

Some changes involved new laws: California passed a law requiring reporting of recycled non-RCRA wastes; the California Hazardous Waste Management and Source Reduction Review Act led LLNL to identify its largest hazardous waste streams and select waste minimization options for them, along with a commitment for their implementation; the Pollution Prevention Act of 1990 required significant modifications to the existing LLNL hazardous waste data reporting procedures; and the Land Ban placed new restrictions on the generation of mixed wastes.

Other changes resulted from new definitions, requirements, and restrictions. These include the reduction by DOE of the level of radioactivity that defines waste as radioactive or mixed; new offset requirements for local air emissions that have increased the difficulty of obtaining air permits; an Executive Order issued in September 1991 that requires new emphasis on the use of recycled materials by all federal facilities; California's increased restrictions on the 
quantities of nonhazardous wastes that may be disposed of in landfills; and the directives by the DOE Secretary to participate in the EPA 33/50 program to reduce emissions of 17 priority chemicals and to submit progress reports on the program, as well as to phase out Class I ozone-depleting chemicals (ODCs) by the end of 1995 and to submit progress reports on this phase-out.

With these changes in mind, the strategies proposed in the Waste Minimization and Pollution Prevention Awareness Plan include the following actions. The first is to develop specific ways for the programs to conserve resources, minimize waste generation, and prevent pollution. This includes creating incentives for pollution prevention; developing specific goals and schedules for waste minimization activities; promoting the use of nonhazardous materials; substituting, reformulating, modifying, managing, and/or recycling waste materials to achieve minimal adverse effects; targeting policies, procedures, or practices that may be barriers to waste minimization; and integrating and coordinating waste generators and waste managers on waste minimization issues.

The second action is to enhance communication of waste minimization goals and ideas. This involves developing and implementing employee pollution prevention awareness and occupational training programs; collecting and exchanging waste minimization information through technology transfer, outreach, and educational networks; and developing mechanisms for fully disseminating current technical information.

The third action involves identifying and implementing methods and technologies for waste minimization, for example, characterizing waste streams and developing a baseline of waste generation data.

In 1992 LLNL conducted a number of activities in support of this plan. LLNL continued to communicate management's commitment to curtail pollution, and publicized the goals of pollution prevention through posters and articles in Newsline (the I.LNL biweekly newspaper). LLNL conducted formal training on pollution prevention and on the responsibilities of waste generators. Pollution prevention displays and handouts were presented during Earth Week, at two Earth Day Fairs (April 1992 and April 1993), and at an on-site Energy Fair (October 1992). Environmental Alerts (one-page flyers) were published and distributed to all LLNL employees, conveying information on environmental problems and possible solutions, on recommended practices, and on pertinent environmental regulatory issues. Changes and additions to regulatory requirements, new technologies, and management changes related to environmental issues continued to be conveyed in 1992 by the environmental analysts assigned to specific site areas. Finally, the LLNL-wide safety and health awards program was expanded in 1992 to include environmental ideas. 


\section{Waste Minimization}

The Waste Minimization Project (WMP), assisted by program representatives, prepared the Annual Waste Reduction Report for the Department of Energy on both the Livermore site and Site 300. WMP also updated the 1991 Livermore site SB14 report and submitted a report to Alameda County concerning recycling of non-RCRA hazardous wastes.

The reduction of waste has been a major effort at LLNL. A significant part of this effort has concentrated on both liquid solvents, which are disposed of as liquid hazardous waste, and volatile solvents, which evaporate into the air. Several LLNL programs are collaborating in a project to evaluate the substitution of less hazardous solvents or cleaners for chemicals currently in use. Preliminary results are very encouraging and have led to the elimination of some cleaning operations that used halogenated solvents.

For example, Chemistry and Materials Science has reduced by about $70 \%$ the amount of hazardous solvents used in radioactive materials management areas, representing a significant reduction in mixed wastes. This reduction is a direct result of employee education and of switching to nonhazardous solvents. Chemistry and Materials Science has also implemented a procedure to remove the chlorofluorocarbon (CFC) solvent from a degreaser whenever the machine is not in use, thereby reducing CFCs lost through evaporation by approximately 250 kilograms per year.

The Lasers Directorate reduced evaporative losses of CFCs by switching to a formulation that has a lower vapor pressure and by installing a CFC recovery system. The Lasers program also implemented a system to reuse optical cleaning solutions in Building 490, thereby reducing generation of metal-contaminated wastewater by 2636 kilograms per year, and installed oil-free vacuum pumps to reduce both lubricating oil wastes and system maintenance costs.

The volume of hazardous wastewater produced at LLNL has also been reduced through use of Transportable Treatment Units (TTU) to process hazardous wastewater from retention tanks. Since mid-1992, TTUs have treated more than 84,000 kilograms of metal-contaminated wastewater from Building 141. Treated wastewater was discharged to the sewer system instead of being hauled off site for disposal, resulting in a cost savings of more than $\$ 66,000$. LLNL has initiated procurement actions to extend this service to all other qualified waste streams.

The Engineering Directorate converted the rinsing operation for the manufacture of printed wiring boards to series flow, which reduced (by about $90 \%$ ) the quantity of its largest hazardous waste stream-contaminated rinse water. The Machine Shop installed a recycling system in Building 321 that has reduced the generation of waste machine-tool coolant by about $90 \%$ and eliminated more than 9100 kilograms of waste per year. During its first full year 
of operation, the Plating Shop rinse water recycling system processed 265 million liters of water and generated only 1100 liters of metal-bearing aqueous waste, a reduction of $99.7 \%$. The Lasers Directorate reduced alcohol solvent use by about 800 liters per year by switching to a nonhazardous alternative cleaner.

Other changes in operations, as well as reuse and recycling of materials, have led to substantial reductions in wastes. Personnel from Nuclear Design and from Materials Fabrication at Site 300 continue to recycle and reuse high explosives pieces for shot tank evaluations, microbarograph testing, and employee training exercises. Employees at Site 300 continue to segregate packaging to ensure that only those wrappers that contact high explosives are disposed of as hazardous waste.

Energy Research used to store processed oil shale in 55-gallon drums that were used only once, then disposed of as hazardous waste. The program now uses recyclable transportainers to store processed shale that is awaiting disposal. This eliminated more than 550 kilograms per year of hazardous waste. Magnetic Fusion Energy now returns spent insulating oil to the manufacturer, where it is purified for reuse; this reduced oily waste by about 2000 kilograms per year. Plant Operations also ceased regenerating its ion exchanger resin beds on site in 1991. The resin vendor now regenerates these beds off site, eliminating a large wastewater stream contaminated with metals.

Automotive Fleet Division of Business Services has substituted reusable stainless steel containers that use pressurized air as propellant in place of aerosol cans at Site 300 . This provides an $80 \%$ reduction in the amount of waste generated and a savings of $\$ 500$ per year. Plant Operations now changes compressor and generator lubricating oils only when analyses show high metal content; previously, oil changes followed a time schedule. The Motor Pool started using recapped tires instead of buying new ones for certain vehicles. The Hazardous Waste Management Division sent between 135 and 180 kilograms of metallic lead bricks, hammers, and shot to LLNL salvage operations for off-site recycling.

LLNL initiated an alternative solvent and coolants research project to minimize the use of ozone-depleting chemicals, halogenated hydrocarbons, and material with volatile organic compounds. The WMP solicits LLNL programs for parts that are currently cleaned with toxic solvents. After cleaning these parts with alternative solvents, the WMP returns them to the programs with suggestions for alternative solvent use. Substituting less toxic cleaning materials minimizes environmental management and waste management costs, minimizes employee health risk exposures, reduces potential impact to the environment, and correlates well with guidance and objectives from the U.S. EPA.

LLNL evaluated the effectiveness of more than 40 alternative solvents for cleaning grease, oil, and dirt from commonly used substrates such as optical 
glass, aluminum, other machined metals, printed wiring boards, and plastic. Advanced spectrometry methods are used to quantify cleanliness achieved with the various solvent cleaners. In addition, aquatic toxicity tests are conducted on materials that provide promising cleaning results. Several alternative solvents have proved to be not only less hazardous but also better cleaners than the more toxic material currently used.

Some of the solvent substitutions to date include: the main bay machine shop substituted a nonhazardous alternative cleaner (815GD) for 1,1,2-trichloroethane (TCA); the Plating Shop replaced its potassium ferrocyanide chromate conversion coating process with a less hazardous noncyanide process and substituted oxalic acid for sulfuric acid in its aluminum anodizing process; the Engineering Records Center replaced alcohol with vinegar and water for daily cleaning of the camera bed and is using diluted sodium hypochlorite instead of standard sulfonic acid and dichromate tray cleaner for cleaning processor racks; the Building 141 machine shop substituted nonhazardous cleaner SD1291 for TCA; Plant Engineering switched to 1990GD for spray washing in Building 511; and the Technical Information Department replaced a high-speed printing press with a dry-toner copier (eliminating 102 liters per year of hazardous chemicals).

TSCA hazardous waste decreased about 6400 kilograms; RCRA hazardous waste increased about 152,800 kilograms; and non-RCRA hazardous waste decreased about 173,400 kilograms. Overall, hazardous waste generation declined nearly 28,110 kilograms or $2.8 \%$ during 1992 , and low-level radioactive waste generation decreased about 10,000 kilograms or $4.5 \%$. Mixed waste generation increased about 43,000 kilograms or $42 \%$; most of this increase has been traced to one building's retention tank, which has since been replaced, and the increase can thus be considered as nonrecurring. Transuranic waste increased because of intentional inventory reduction in the plutonium facility and because some of the processing equipment was shut down during part of 1991, resulting in lower generation for that year.

\section{Nonhazardous Materials Recycling}

In April 1991, LLNL implemented a site-wide white paper recycling program that has collected more than 200 tons of paper in its first year.

The WMP completed the first of a series of nonhazardous waste assessments. Paper waste constituted approximately $50 \%$ (by weight) of the total material sampled. The paper waste was sorted into subcategories, including newsprint, colored paper, coated paper, computer (green bar) paper, and paper towels. Only minor quantities of white paper were observed during the assessment, indicating success with the white paper recycling program. Cardboard comprised the second largest waste stream category. Plastics, primarily polystyrene from food service wastes, comprised the third. Glass and aluminum cans represented a very 
small portion of the waste stream, indicating that individual employee efforts to collect and recycle these materials are successful.

Because cardboard comprises the second largest waste stream, LLNL has implemented a pilot program for cardboard recycling with Biology and Biotechnology, Business Operations, Engineering, Shipping, and Salvage. Also, LLNL has implemented a pilot program within the Environmental Protection Department to substitute rechargeable batteries for alkaline batteries in pagers and other small electronic equipment. The WMP is studying the feasibility of recycling polystyrene at LLNL's three cafeterias.

LLNL has established a Buy Recycle Committee in response to Executive Order 12780, which mandates federal facilities to increase use of recycled materials. LLNL Stores now carries a white ledger paper with $50 \%$ recycled fiber content, refillable bottles that replace aerosol cans, aqueous-base correction fluid, low-alkalinity dishwashing compound, and refurbished laser printer toner cartridges. The Technical Information Department demonstrated that recycled paper could be used in most copiers and laser printers, stimulating acceptance by many other departments.

\section{Compliance Activities}

The Environmental Operations Group within ORAD is responsible for working closely with LLNL programs and departments to implement environmental requirements so that planned and current operations and experiments comply with local, state, and federal environmental regulations. The group consists of environmental analysts assigned to specific LLNL programs and departments to assist in day-to-day environmental activities. In addition to advising LLNL staff, analysts review and verify that proper procedures are followed for the management of hazardous wastes and for the implementation of appropriate pollution abatement controls. Compliance status is maintained and verified by the analysts who keep abreast of existing and planned activities, review construction documents, inspect facilities, and assess waste management procedures.

\section{Building Inspections}

Formal, detailed building inspections for each LLNL facility are conducted based on a schedule established by the facility manager with the Hazards Control Health and Safety Team. These inspections scrutinize proper handling and management of hazardous and radioactive wastes and waste streams, management and maintenance of waste accumulation areas (WAAs), potential release pathways to the environment (e.g., storm and sanitary sewer drains), hazardous product storage areas, wastewater retention systems, operating equipment (e.g., vacuum pumps, transformers, capacitors, and baghouses), and 
laboratory and machine shop areas. An inspection report is prepared for the program or department, and follow-up checks are conducted to ensure implementation of recommendations or corrections. Walk-through inspections are conducted on an as-needed basis. During 1992, 190 formal building inspections were conducted and 9 audits of Building 883 at Site 300 were completed. In addition, nine Hazardous Waste Management Facility audits were conducted.

For the first quarter of 1993, 49 formal building inspections were conducted and 3 audits of Building 883 at Site 300 were completed. In addition, three Hazardous Waste Management Facility audits were conducted.

\section{Waste Accumulation Area Inspections}

Inspections are conducted at least weekly at all WAAs by program representatives to help ensure that WAAs are operated in compliance with regulatory requirements. An inspection checklist is completed and corrective actions are implemented. In addition, EPD staff conducts biweekly routine checks at all WAAs to help ensure that programs manage their WAAs and wastes in compliance with federal and state requirements. During 1992, 1040 biweekly WAA routine checks were conducted on the Livermore site, 21 biweekly checks at the Livermore Airport, and 330 biweekly checks at Site 300 . Additional information is provided in Chapter 2 , under the subsection titled Waste Accumulation Areas.

\section{Spill Response}

Investigation, sampling, and evaluation of all spills and leaks that are potentially hazardous to the environment are conducted when necessary. The spill response process includes identifying the spill or leak, shutting off the source (if safe to do so), eliminating ignition sources, contacting appropriate emergency personnel, cordoning off the area, containing the spill or leak, absorbing and neutralizing the spill or leak, assisting in clean up of the spill or leak, determining if a spill or leak must be reported to regulatory agencies, and verifying that cleanup (including decontaminating and replenishing spill equipment) is complete. Environmental analysts also provide guidance to the programs on preventing spill recurrence.

\section{Site Evaluations Prior to Construction}

Sampling and analysis for potential contaminants are conducted at all proposed construction sites to ensure that any soil and debris generated is disposed of appropriately. During 1992, environmental analysts conducted preconstruction site evaluations for 79 construction projects. During the first quarter of 1993, 22 additional preconstruction site evaluations were conducted. 


\section{Environmental Training}

In 1992, the Environmental Protection Department (EPD) sponsored training, including RCRA and SARA/OSHA programs, for 4490 people for a total of 19,084 contact hours. For the first quarter 1993, EPD trained 614 people for a total of 3269 contact hours. These Training Program statistics do not include the number of hours devoted to on-the-job training, which is handled separately by each division. Additionally, EPD routinely participates in biweekly briefings to orient all new employees concerning approved environmental practices and procedures, including persons to contact during emergencies.

Table 3-1. TFA WDR self-monitoring sampling and analysis schedule.

\begin{tabular}{|c|c|c|c|c|}
\hline Frequency & TFA-1001 & TFA-PP3 & $\begin{array}{l}\text { TFA-C1E/C1W, } \\
\text { TFA-C2E/C2W }\end{array}$ & $\begin{array}{c}\text { Land } \\
\text { observation }\end{array}$ \\
\hline Daily & Flow rate & Flow rate & - & - \\
\hline Weekly & - & EPA 601 & - & $\begin{array}{l}\text { Perform } \\
\text { observation }\end{array}$ \\
\hline Monthly & $\begin{array}{l}\text { EPA } 301 \\
\text { pH }\end{array}$ & $\begin{array}{l}\text { Temperature } \\
\mathrm{pH}\end{array}$ & & Complete report \\
\hline $\begin{array}{l}\text { Quarterly } \\
\text { (Mar., June, } \\
\text { Sept., Dec.) }\end{array}$ & Chlorides & $\begin{array}{l}\text { Chlorides } \\
\text { Fish toxicitya } \\
\text { Turbidity }^{\mathbf{b}}\end{array}$ & $\begin{array}{l}\text { Chlorides } \\
\text { EPA601 }\end{array}$ & - \\
\hline $\begin{array}{l}\text { Semiannually } \\
\text { (Apr., Oct.) }\end{array}$ & Metals ${ }^{c}$ & Metals $^{c}$ & $\begin{array}{l}\text { Metals }^{\mathrm{c}} \\
\text { Gross alpha and } \\
\text { beta particles, } \\
\text { tritium } \\
\text { Fish toxicity }\end{array}$ & \\
\hline $\begin{array}{l}\text { Annually } \\
\text { (Apr.) }\end{array}$ & $\begin{array}{l}\text { EPA } 624^{d} \\
\text { EPA } 625\end{array}$ & $\begin{array}{l}\text { EPA } 624^{\mathrm{d}} \\
\text { EPA } 625 \\
\text { Gross alpha and } \\
\text { beta particles, } \\
\text { tritium }\end{array}$ & $\begin{array}{l}\text { EPA } 602 \\
\text { EPA } 625\end{array}$ & \\
\hline
\end{tabular}

Specific requirements:

- Sampling of TFA-C1E/C1W and TFA-C2E/C2W should be coincident with TFA-1001 and TFAPP3 sampling.

- If any instantaneous maximum limit is exceeded, then sampling frequency shall be increased to daily, until two samples collected on consecutive days show compliance with instantaneous maximum.

a Fish toxicity, 96 hours survival in undiluted waste.

b Jackson turbidity units.

c Priority pollutant metals: antimony, chromium (total), mercury, silver, arsenic, copper, nickel, thallium, beryllium, lead, selenium, zinc, cadmium. Samples also are analyzed for boron, chromium $(+6)$, iron, manganese, and cyanide.

d When schedule calls for coincident EPA 601 and 624 analyses, perform only EPA 624. 


\section{Introduction}

Air surveillance monitoring is performed to evaluate compliance with local, state, and federal regulations, which ensure that human health and the environment are protected from hazardous emissions into the air. LLNL complies with all appropriate local, state, and federal environmental air quality laws and U.S. Department of Energy (DOE) regulations including: 22 CCR 67264.700 and 66265.710, Environmental and Compliance Monitoring; 40 CFR 61, the National Emissions Standards for Hazardous Air Pollutants (NESHAPs) section of the Clean Air Act; Environmental Regulatory Guide for Radiological Effluent Monitoring and Environmental Surveillance (U.S. Department of Energy 1991); and DOE Orders 5400.1, General Environmental Protection Program, and 5400.5, Radiation Protection of the Public and the Environment. In general, LLNL analyzes for most constituents at levels that are far below regulatory standards to determine any environmental impact.

LLNL monitors ambient air to determine if airborne radionuclides or hazardous materials are being released by Laboratory operations, what the concentrations are, and what the trends are in the LLNL environs. Air monitoring involves measurement of particles collected on filters, or of vapor chemically trapped on a collection medium. Concentrations of various airborne radionuclides (including particulates and tritium water vapor), beryllium, and lead are measured at the Livermore site, Site 300, at off-site locations throughout the Livermore Valley, and at an off-site location in Tracy.

\section{Methods}

LLNL maintains eight (including two used for a special study) continuously operating, high-volume, air particulate samplers on the Livermore site (Figure 4-1), eleven in the Livermore Valley (Figure 4-2), eight at Site 300, and one in Tracy (Figure 4-3). The samplers are positioned to ensure reasonable probability that any significant concentration of particulate effluents from LLNL operations will be detected. Geographical details of particulate sampling locations are outlined in a written procedure, which is included in Appendix B of the Environmental Monitoring Plan (Gallegos et al. 1992b).

Each air particulate sampler pulls air continuously at a constant rate of 400 liters per minute through a $20.3 \mathrm{~cm} \times 25.4 \mathrm{~cm}$ Whatman-41 cellulose filter. The flow is maintained at better than the DOE requirement of $\pm 20 \%$ of the nominal flow by using a mass flow controller that adjusts motor speed. These flow rates are verified at regular intervals with a portable field-calibration unit. If a sampler fails, it is repaired and then calibrated with a 


\section{Air Monitoring}

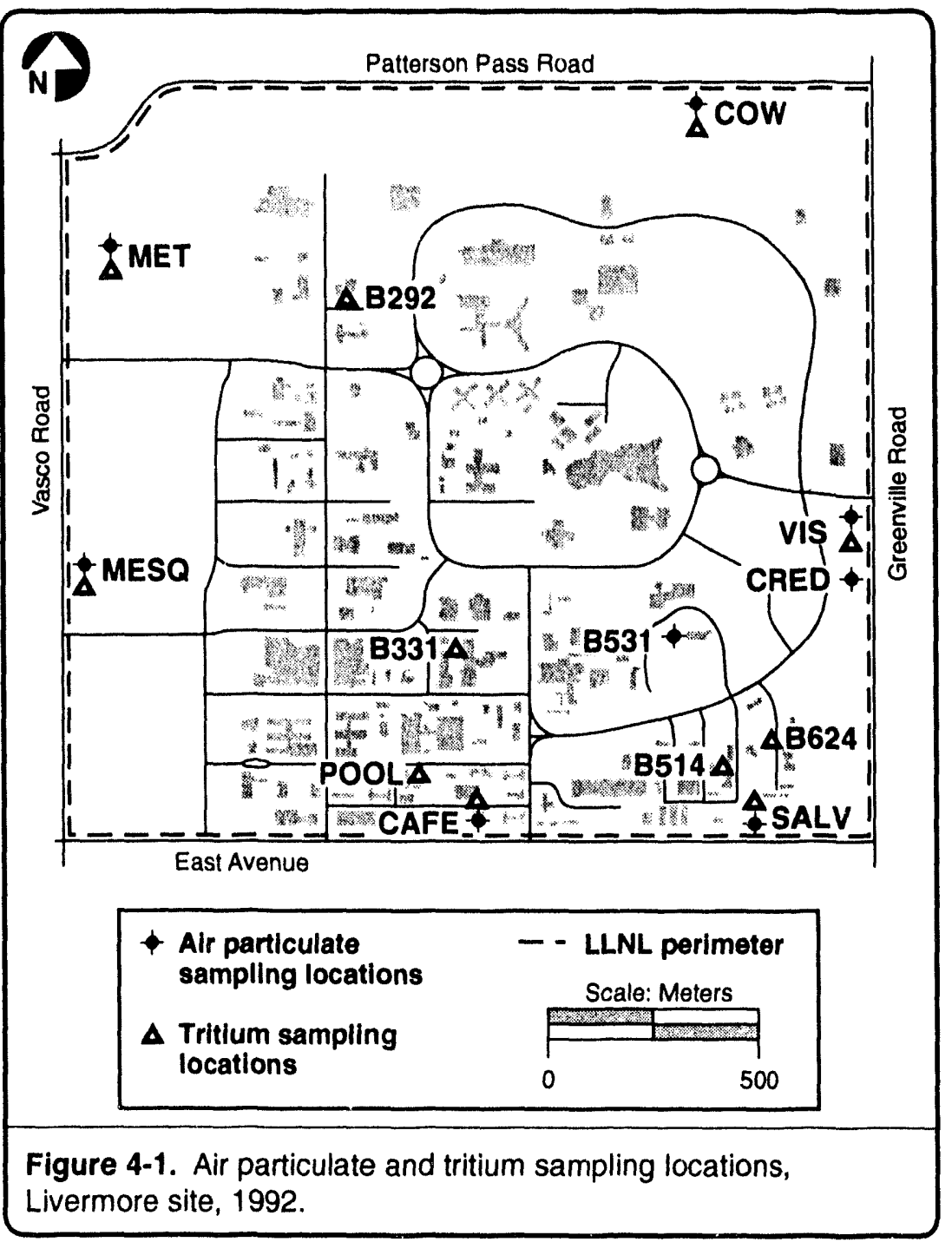

spirometer that itself was calibrated using a unit traceable to National Institute for Standards and Technology (NIST).

An easily dissolvable filter and a low trace-metal background are required for airborne-beryllium analyses. Whatman-41 filters provide a balance between such requirements and particulatecollection efficiency (Lindeken et al. 1963).

Particulate filters are changed each week at all locations. After each particulate filter is removed from a sampler, it is identified by location, date on, date off, elapsed time, and flow rate, and is given a sample identifier (a four-field code) that accompanies it throughout the analysis. Filters are then placed in glassine envelopes and transported to the Livermore site, where all recorded information is transferred to $\log$ sheets. After a four-day delay for decay of the radon-thoron daughters, gross alpha and beta activities on the filters are determined with a gas-flow proportional counter.

Separate ${ }^{239} \mathrm{Pu}$ and ${ }^{90} \mathrm{Sr}$ calibration sources determine alpha and beta counting efficiencies, respectively. These standards are traceable to NIST or equivalent certification. Counting-efficiency measurements are made with each set of counted filters. A background count is taken at the beginning of each run and between each set of ten samples. Records are kept of background and counting-efficiency variations that occur in the counting equipment

Monthly composites of filters from each of the Livermore-site perimeter locations (SALV, MESQ, CAFE, MET, VIS, and COW) are placed into individual plastic bags. The six bags are then combined and sealed in a $214-\mathrm{cm}^{3}$ aluminum can and are counted for gamma-emitting radionuclides using low-background $\mathrm{Ge}(\mathrm{Li})$ detectors. The Site 300 filters are sealed and counted in a similar manner. Following gamma counting, the composited filters from each Livermore-site perimeter location are analyzed for the presence of ${ }^{239} \mathrm{Pu},{ }^{235} \mathrm{U}$, and ${ }^{238} \mathrm{U}$. The off-site samples from the Livermore Valley are analyzed for ${ }^{239} \mathrm{Pu}$, and all of the Site 300 samples are composited and analyzed for ${ }^{23}>\mathrm{Pu}$, ${ }^{235} \mathrm{U}$, and ${ }^{238} \mathrm{U}$. The filters are ashed and then dissolved in a mixture of nitric acid 
$\left(\mathrm{HNO}_{3}\right)$ and hydrochloric acid $(\mathrm{HCl})$ and/or hydrofluoric acid $(\mathrm{HF})$. Plutonium and uranium are separated by an ion-exchange process. Each separated element is purified further by ion exchange. Then any plutonium is electroplated onto a stainless steel disk and submitted for alpha spectrometry, while uranium is submitted for analysis by mass spectrometry.

Replicate samples are processed to confirm the results obtained from the samplers. In addition, a duplicate QA/QC sampler is operated for two months in parallel with the permanent one at a given site. The $\mathrm{QA} / \mathrm{QC}$ fitters also are exchanged weekly, and both filter sets are submitted for analysis in the usual manner. After two months, the $\mathrm{QA} / \mathrm{QC}$ sampler is rotated to another location.

A total volume of approximately 4 million liters of air is sampled at each location per week. LLNL's Radiation Analytical Sciences laboratory analyzes the filters for radionuclides, including mixed fission products, plutonium, and uranium. The details of air particulate sampling and sample change-out are described in Appendix B of the Environmental Monitoring Plan (Gallegos et al. 1992b). Details of high-volume sampler flow calibration are also discussed in an Environmental Monitoring Section procedure, and details of air sample analysis procedures are outlined in Garrison et al. (1985).

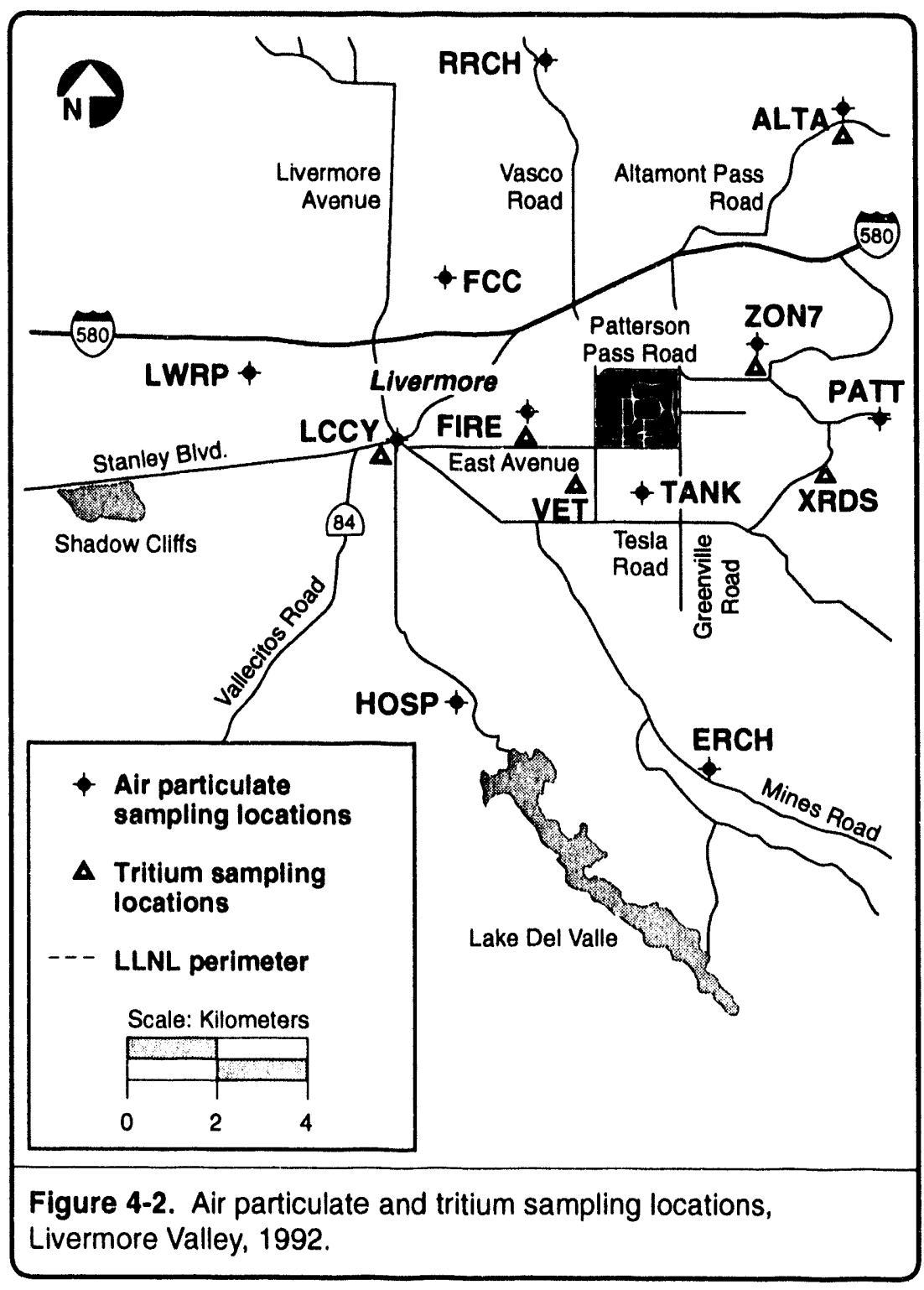

As outlined in U.S. Department of Energy (1991), gross alpha and gross beta air filter results are used only as trend indicators; specific radionuclide analvsis is done for plutonium, uranium, and all gamma emitters. All analytical results are reported as a measured concentration, or at the minimum detection limit (MDL) when no activity is detected. In all cases, the MDL is more than adequate for demonstrating compliance with the pertinent regulatory requirements for radionuclides that are present or may be present in the air sample. 
Particle size distributions are not determined, because the estimated effective dose equivalent to the maximally exposed individual is well below the $0.01 \mathrm{mSv}(1 \mathrm{mrem}$ ) allowable limit.

In addition to radionuclide analyses, beryllium measurements are performed on Livermore-site perimeter filters. Beryllium and lead measurements are performed on Site 300 samples. Each month, half of each of the weekly air filters are composited by sample location. The filters are wet-ashed with nitric acid and quantification is done by atomic absorption spectroscopy. These analyses are performed by an outside contract laboratory.

Beryllium measurements from half of each of the weekly air filters from the Livermore-site perimeter and Site 300 samplers are composited by sampling location every inonth. Two paper filters at a time are wet-ashed with a mixture of $80 \% \mathrm{HNO}_{3}$ and $20 \%$ perchloric acid $\left(\mathrm{HClO}_{4}\right)$. The solutions then are evaporated to a few milliliters (care is taken to prevent the samples from baking dry). The samples are diluted

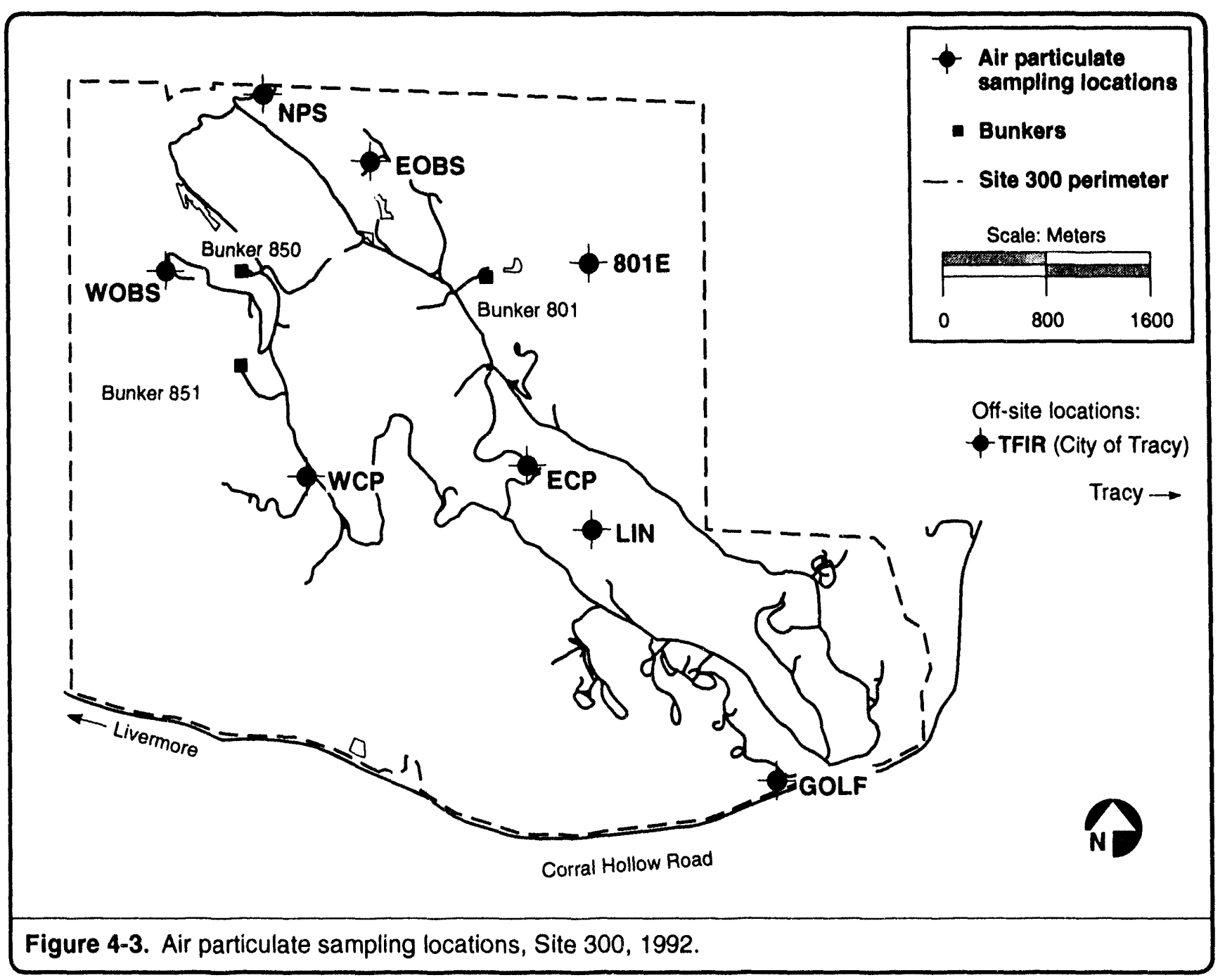


with water and filtered. Any residues are discarded, and the filtrates are diluted to $100 \mathrm{~mL}$ with deionized water. Quantification is accomplished by atomic absorption spectrophotometry with electrothermal atomization.

LLNL also maintains 11 continuously operating airborne tritium samplers on the Livermore site (Figure 4-1) and 6 samplers in the Livermore Valley (Figure 4-2). Four of the Livermore site locations (B331, B292, B514, and B624) are part of a special study. The tritium sample locations are detailed in Appendix $B$ of the Environmental Monitoring Plan (Gallegos et al. 1992b). The tritium samplers, operating at a flow rate of $700 \mathrm{~mL} / \mathrm{min}$, use silica-gel in flasks to trap water vapor. These flasks are changed every two weeks, and the samples are identified by location, date on, date off, elapsed sampling time, and flow rate. The flow rate is the average of the initial and final flow rates, which are measured biweekly with a rotometer that is calibrated once a year. Each sample is given a sample identifier that accompanies it through analysis. Two additional samplers are rotated among the locations at two-month intervals to provide a duplicate QA/QC sample. Details of the actual tritium sampling and a description of tritium sampler calibration can be found in Appendix B of the Environmental Monitoring Plan (Gallegos et al. 1992b).

The tritium concentration in the collected water vapor is determined by liquidscintillation counting. Airborne tritium sample analysis is done by LLNL's Radiation Analytical Sciences laboratory. Details of the analytical procedure are described in Garrison et al. (1985).

\section{Results}

\section{Livermore Site}

Airborne Radioactivity. The values reported for gross alpha and gross beta activities are similar to those observed in previous years and show no significant differences between samples taken at the Livermore-site perimeter and Livermore Valley. Most of the gross alpha determinations were at or near the detection limit of the method. Typical gross alpha activity is less than $1.3 \times 10^{-10} \mathrm{~Bq} / \mathrm{mL}\left(<3.4 \times 10^{-15} \mu \mathrm{Ci} / \mathrm{mL}\right)$, and typical beta activity is $5.4 \times 10^{-10} \mathrm{~Bq} / \mathrm{mL}\left(1.5 \times 10^{-14} \mu \mathrm{Ci} / \mathrm{mL}\right)$. The primary sources of observed alpha and beta activity are the naturally occurring radioisotopes of uranium and thorium and their decay products.

Table 4-1 lists the activities of gamma-emitting radionuclides that contribute to the activity in the Livermore-site perimeter samples. Of the nuclides tabulated, ${ }^{7} \mathrm{Be},{ }^{40} \mathrm{~K},{ }^{226} \mathrm{Ra},{ }^{228} \mathrm{Ra}$, and ${ }^{228} \mathrm{Th}$ occur naturally. The primary source of ${ }^{137} \mathrm{Cs}$ is long-term global fallout and fallout resuspension. An investigation to determine the source of ${ }^{22} \mathrm{Na}$ has begun.

In addition to providing baseline data on global fallout, analysis of these fission products enables LLNL to monitor the containment of the small inventories of mixed fission products and radiochemical tracers used at LLNL. The Derived Concentration Guides (DCGs) for these radionuclides are also shown in Table 4-1. For air, DCGs specify the concentrations of radionuclides that could be inhaled continuously 365 days a year without exceeding the DOE primary 
radiation protection standard to the public, which is $1 \mathrm{mSv} / \mathrm{y}(100 \mathrm{mrem} / \mathrm{y})$ effective dose equivalent (DOE Order 5400.5). (Chapter 11 on Radiological Dose Assessment provides an explanation of this and other units of dose.) Finally, the percentage of the DCGs is presented. These values demonstrate the low levels of gamma activity present at the Livermore-site perimeter.

Table 4-2 shows the concentration of plutonium on air filter sa collected in the Livermore Valley. The highest off-site concentration c occurred in June at Location LWRP (at the Livermore Water Reclamation a The LWRP contains some contamination principally resulting from slu $-\varepsilon^{r e-}$ spreading operations following an estimated $32 \mathrm{mCi}$ of plutonium released to the sewer in 1967 (see Chapter 8 on Soil and Sediment Monitoring). In June of 1992, the LWRP began construction of a new clarifier tank located approximately 25 meters upwind of the air sampling station. These construction activities caused large amounts of resuspension that could be responsible for the elevated ${ }^{239} \mathrm{Pu}$ results in June. Table 4-3 shows the concentrations of airborne ${ }^{239} \mathrm{Pu},{ }^{235} \mathrm{U}$, and ${ }^{238} \mathrm{U}$ (depleted uranium) on air filter samples from the Livermore-site perimeter. For these data, the DCG is based on the typical environmental oxide chemical form. Because of problems with the analysis of the April samples for Locations SALV, MESQ, CAFE, MET, VIS, and COW, some 5.1-cm-diameter sections of these samples were retrieved from another analytical lab and used for reanalysis. Although the results are higher than normal for these locations, they represent the limit of detection for filter" "s of this size. The April sample for Location SALV, which is on the : registered the highest concentrations : onium in air of all perimeter sampling locations. The concentration reported for this sample, $2.11 \times$ $10^{-13} \mathrm{~Bq} / \mathrm{mL}\left(5.70 \times 10^{-18} \mu \mathrm{Ci} / \mathrm{mL}\right)$, represents 0.00029 of the DCG. No other statistically significant differences between locations or samples were noted, and the overall ${ }^{239} \mathrm{Pu}$ levels essentially were the same as those reported in 1991 . The maximum measured concentrations of $238 \mathrm{U}$ are less than 0.0006 of the DCG (DOE Order 5400.5). Except for the April results, when analytical problems occurred, all ${ }^{235} U /{ }^{238} U$ ratios are as expected for naturally occurring uranium.

Figure 4-4 shows the annual mean concentrations of ${ }^{239} \mathrm{Pu}$ for Locations SALV (on site) and FCC (off site) from 1982 to 1992. Location FCC represents a typical background location and SALV represents the perimeter location having the highest annual average over this 11 -year period. The higher values at SALV may be attributed to historical activities at LLNL.

In June 1991, two air-particulate sampling locations (B531 and CRED) were added as part of a special study to investigate elevated levels of plutonium in air and surface soil in the southeast quadrant of the Livermore site [see the 1991 Soil and Sediment Monitoring chapter for general background on this study (Gallegos et al. 1992a)]. Table 4-4 shows the monthly concentrations of airborne ${ }^{239} \mathrm{Pu}$ at these locations. The average concentration of less than 
$2.74 \times 10^{-13} \mathrm{~Bq} / \mathrm{mL}\left(<7.4 \times 10^{-18} \mu \mathrm{Ci} / \mathrm{mL}\right)$ at Location $\mathrm{B} 531$ is higher than the average concentration for any of the other air particulate sampling locations but are still far below the DCG. These sampling locations will become part of our routine monitoring network and will provide data for diffuse source dose assessments.

Table 4-5 shows the average biweekly concentrations of tritiated water-vapor that were observed at the Livermore-site perimeter sampling locations. The highest annual mean concentration was observed at Location POOL. The mean concentration at POOL was $3.3 \times 10^{-7} \mathrm{~Bq} / \mathrm{mL}\left(8.9 \times 10^{-12} \mu \mathrm{Ci} / \mathrm{mL}\right)$, or 0.00009 of the DCG.

Four additional air tritium sampling locations (B331, B292, B514, and B624) were installed in July 1991 to evaluate diffuse sources of tritium on the Livermore site. Table 4-6 shows the average biweekly concentrations of tritiated water-vapor for the new sampling locations. The highest average concentrations were observed at Locations B292 and B624. These concentrations were $5.0 \times$ $10^{-6} \mathrm{~Bq} / \mathrm{mL}\left(1.4 \times 10^{-10} \mu \mathrm{Ci} / \mathrm{mL}\right)$ and $1.7 \times 10^{-6} \mathrm{~Bq} / \mathrm{mL}\left(4.5 \times 10^{-11} \mu \mathrm{Ci} / \mathrm{mL}\right)$, respectively, and represent 0.0014 and 0.0005 of the DCG. The 1992 average concentrations at B292 and B624 are lower than the average concentrations in 1991. The highest biweekly tritium concentrations of $2.5 \times 10^{-5} \mathrm{~Bq} / \mathrm{mL}(6.8 \times$ $\left.10^{-10} \mu \mathrm{Ci} / \mathrm{mL}\right)$ in May and $1.2 \times 10^{-5} \mathrm{~Bq} / \mathrm{mL}\left(3.3 \times 10^{-10} \mu \mathrm{Ci} / \mathrm{mL}\right)$ in August

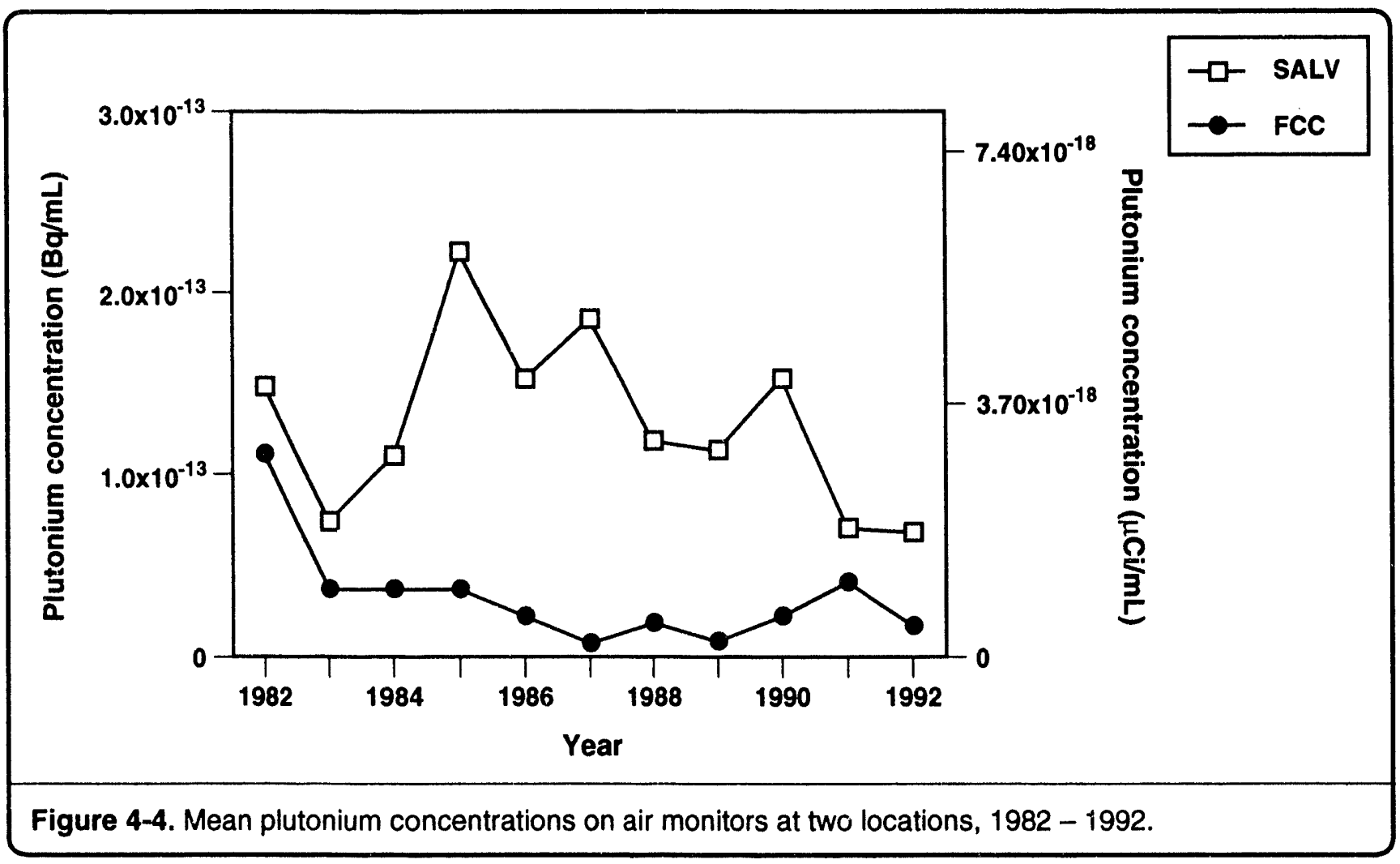


were observed at Location B292. These concentrations represent 0.0068 and 0.0032 of the DCG, respectively. The B292 area is adjacent to an underground retention tank that had previously leaked (see the Underground Storage Tank Management section of Chapter 2, Compliance Summary, for information regarding the B292 area). Another noteworthy biweekly tritium concentration of $1.0 \times 10^{-5} \mathrm{~Bq} / \mathrm{mL}\left(2.7 \times 10^{-10} \mu \mathrm{Ci} / \mathrm{mL}\right)$ was observed at Location B331 in July. Although this concentration is much higher than any biweekly concentrations in 1991, the 1992 average concentration at B331 is the same as 1991. As with the two new air particulate sampling locations, the new tritium sampling locations will be continued to provide diffuse emission source data.

Table 4-7 shows the average biweekly concentrations of tritiated water-vapor for the Livermore Valley sampling locations. The highest annual average concentrations were observed at Locations ZON7 and VET. This concentration of approximately $9.5 \times 10^{-8} \mathrm{~Bq} / \mathrm{mL}\left(2.6 \times 10^{-12} \mu \mathrm{Ci} / \mathrm{mL}\right)$ represents 0.00002 of the DCG. The highest biweekly concentration was observed in February at Location VET. This concentration, $2.6 \times 10^{-7} \mathrm{~Bq} / \mathrm{mL}\left(7.0 \times 10^{-12} \mu \mathrm{Ci} / \mathrm{mL}\right)$, is 0.00007 of the DCG. These 1992 tritium values generally are lower than those reported last year.

Beryllium in Air. The average monthly concentrations of airborne beryllium for the Livermore-site perimeter sampling locations are shown in

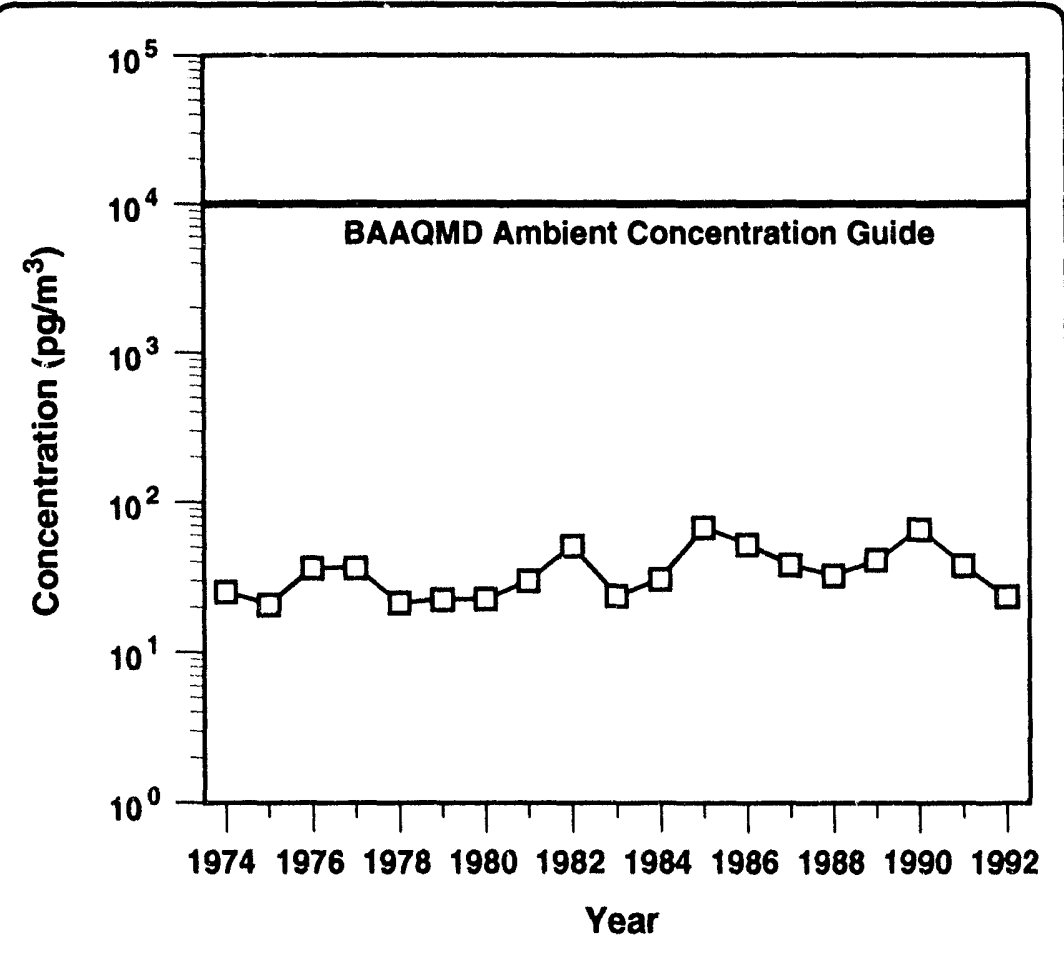

Figure 4-5. Mean concentration of beryllium on air filters, Livermore-site perimeter, 1974 - 1992. (Median used in 1981, 1989, and 1990.)
Table 4-8. The concentrations are less than 0.007 of the monthly ambient concentration limit of $10,000 \mathrm{pg} / \mathrm{m}^{3}$ established by the Bay Area Air Quality Management District (BAAQMD).

Figure 4-5 is a plot of the average beryllium concentration at the LLNL-site perimeter from 1974 through 1992 (the medians of 1981, 1989, and 1990 values are shown because some of the varying analytical techniques have high detection limit:). The average concentration was calculated to be 0.004 of the ambient concentration guide. Unless there is a change in LLNL's operations, it is expected that the beryllium levels will remain unchanged. 
Site 300

Airborne Radioactivity. Most gross alpha determinations were at, or near, the analytical limit of detection for the method. Typical gross alpha activity is $<1.2 \times 10^{-10} \mathrm{~Bq} / \mathrm{mL}\left(<3.2 \times 10^{-15} \mu \mathrm{Ci} / \mathrm{mL}\right)$. Typical beta activity is $5.0 \times 10^{-10} \mathrm{~Bq} / \mathrm{mL}\left(1.4 \times 10^{-14} \mu \mathrm{Ci} / \mathrm{mL}\right)$. The primary sources of observed gross alpha and beta activity are naturally occurring radioisotopes of uranium and thorium and their decay products.

Table 4-9 lists the activities, as well as the DCGs, of gamma-emitting radionuclides in samples from Site 300 and Tracy. All these radionuclides were measured at concentrations significantly below the DCGs. Of the nuclides tabulated, ${ }^{7} \mathrm{Be},{ }^{40} \mathrm{~K},{ }^{226} \mathrm{Ra},{ }^{228} \mathrm{Ra}$, and ${ }^{228} \mathrm{Th}$ are naturally occurring. The primary source of ${ }^{137} \mathrm{Cs}$ normally is long-term global fallout and fallout resuspension. An investigation to determine the source of ${ }^{22} \mathrm{Na}$ has begun.

Table 4-10 shows the concentration of ${ }^{239} \mathrm{Pu},{ }^{238} \mathrm{U}$, and ${ }^{235} \mathrm{U}$ on air filter samples collected from Site 300 . The highest concentrations of ${ }^{239} \mathrm{Pu}$ were observed in the April and May composites at a level of approximately $3.1 \times$ $10^{-14} \mathrm{~Bq} / \mathrm{mL}\left(8.2 \times 10^{-19} \mu \mathrm{Ci} / \mathrm{mL}\right.$, or 0.00004 of the DCG). The highest concentration of $238 \mathrm{U}$ was observed in the April composite at a level of $2.5 \times$ $10^{-4} \mu \mathrm{g} / \mathrm{m}^{3}\left(0.0008\right.$ of the DCG). The highest concentration of ${ }^{235} \mathrm{U}$ was also observed in the April composite at a level of $1.7 \times 10^{-6} \mu \mathrm{g} / \mathrm{m}^{3}(0.00004$ of the DCG). No other significant differences between locations or samples were noted. The overall levels were essentially the same as those reported in previous years.

The ratio of ${ }^{235} \mathrm{U}$ to ${ }^{238} \mathrm{U}$ can be used as an indicator of the source of the uranium. Both $235 \mathrm{U}$ and $238 \mathrm{U}$ occur naturally in the area, but only $0.7 \%$ of the naturally occurring uranium is ${ }^{235} \mathrm{U}$, and the remainder is ${ }^{238} \mathrm{U}$. Because LLNL uses ${ }^{238} \mathrm{U}$ at Site 300 , but does not use ${ }^{235} \mathrm{U}$, it follows that if the ratio remains constant and near $0.7 \%$ (within the limit of sampling and analytical error), then the ${ }^{238} \mathrm{U}$ measured is from natural sources. The ${ }^{235} \mathrm{U} /{ }^{238} \mathrm{U}$ ratio for January and October show statistically significant deviations from the natural ratio, indicating the presence of airborne depleted uranium from Site 300 operations. The measured concentrations of ${ }^{238} \mathrm{U}$, however, are only 0.00024 of the DCG (DOE Order 5400.5).

Beryllium in Air. The average monthly concentrations of airborne beryllium for the Site 300 sampling locations are shown in Table 4-11. The highest beryllium concentration of $304.9 \mathrm{pg} / \mathrm{m}^{3}$ occurred at Location WOBS in July. We will continue to investigate beryllium concentrations at this location. The concentrations average 0.003 or less (with the highest value being 0.03 ) of the federal standard, which is $10,000 \mathrm{pg} / \mathrm{m}^{3}$. These data are well below the standards and do not indicate the presence of a threat to the environment or public health. 


\section{Environmental Impact}

\section{Radioactive Effluents}

In 1992, a total of $6.55 \times 10^{12} \mathrm{~Bq}(177 \mathrm{Ci})$ of tritium was released to the atmosphere from operations at LLNL. Of this, approximately $3.70 \times 10^{12} \mathrm{~Bq}$ $(100 \mathrm{Ci})$ were released as tritiated water (HTO). The remaining tritium was elemental tritium gas (HT). Once released to the environment, the potential dose from elemental tritium gas is approximately 25,000 times lower than a dose from a comparable release of tritiated water. Therefore, the tritiated hydrogen gas did not contribute significantly in calculations of the overall dose. Most of the tritium discharged to the atmosphere by LLNL in 1992 came from the Tritium Facility (Building 331). The highest single weekly emission from the facility was $4.81 \times 10^{11} \mathrm{~Bq}(13 \mathrm{Ci})$, of which $3.70 \times 10^{11} \mathrm{~Bq}(10 \mathrm{Ci})$ was elemental tritium gas. This emission was measured between August 6 and August 25, 1992. The diffuse tritium sources at B292, B331, B514, and B624 have a localized effect; however, no high tritium concentrations were detected at the site perimeter or off site.

The electron-positron linear accelerator at B194 was not activated in 1992. Therefore, no releases of ${ }^{13} \mathrm{~N}$ and ${ }^{15} \mathrm{O}$ occurred. Comparative releases of radioactive effluents at LLNL during the 12-year period from 1981 through 1992 are shown in Table 4-12. The radioactive atmospheric emissions from LLNL operations during 1992 were much lower than previous years.

The concentrations of beryllium and radionuclides measured around Site 300 and in the City of Tracy were well below all standards and, except for uranium isotopes, reflect background or naturally occurring levels of these chemicals. Two of the ${ }^{235} \mathrm{U} /{ }^{238} \mathrm{U}$ ratios shown in Table 4-11 are less than the ratio of naturally occurring concentrations of these isotopes, and this suggests the presence of LLNL-induced depleted uranium in air samples from Site 300, although the actual concentrations are not statistically elevated over background.

\section{Nonradioactive Effluents}

The concentrations of beryllium at both sites can be attributed to resuspension of surface soil containing naturally occurring beryllium. Local soils contain approximately $1 \mathrm{ppm}$ of beryllium, and the Livermore area and Central Valley air typically contains 10 to $100 \mu \mathrm{g}$ of particulates per cubic meter. Using a value of $50 \mu \mathrm{g} / \mathrm{m}^{3}$ for an average dust load and $1 \mathrm{ppm}$ for beryllium content of dust, an airborne beryllium concentration of $50 \mathrm{pg} / \mathrm{m}^{3}$ can be calculated. In view of the range of natural variation expected in these background values, this value is in excellent agreement with the data in Tables 4-8 and 4-9.

The estimated releases from exempt and permitted sources of air pollutants at the Livermore site can be compared to the most recent estimated 1992 daily release of air pollutants for the entire Bay Area. For example, the total emissions of oxides of nitrogen (NOx) released in the Bay Area is 500 metric tons per day compared to LLNL's 0.079 metric tons per day (0.0002 of total Bay Area 
emissions). The Bay Area Air Quality Management District estimate for reactive organic emissions (ROG) is at 743 metric tons/day, versus Livermore site's 0.013 metric tons/day (0.00002 of total Bay Area emissions) in 1992.

Table 4-13 lists the estimated LLNL 1992 total releases for organic precursor and nonprecursor compouiuls, chlorofluorocarbons (an organic nonprecursor), and other LLNL airborne emissions.

Certain equipment operations at Site 300 require permits from San Joaquin County. Total estimated emissions are reported to the San Joaquin Valley Unified Air Pollution Control District. The total estimated air emissions during 1992 from equipment operations at Site 300 are given in Table 4-13. 
Table 4-1. Gamma activity on air filters, Livermore-site perimeter, 1992. a

\begin{tabular}{|c|c|c|c|c|}
\hline \multirow[b]{2}{*}{ Month } & \multirow{2}{*}{$\frac{\left[10^{-9} \mathrm{~Bq} / \mathrm{mL}\right]}{{ }^{7} \mathrm{Be}}$} & \multicolumn{3}{|c|}{$\left[10^{-12} \mathrm{~Bq} / \mathrm{mL}\right]$} \\
\hline & & ${ }^{40} K$ & ${ }^{137} \mathrm{Cs}$ & ${ }^{22} \mathrm{Na}$ \\
\hline Jan. & $2.69 \pm 0.04$ & $<4.18$ & $<0.17$ & $<0.19$ \\
\hline Feb. & $3.91 \pm 0.06$ & $<5.81$ & $<0.20$ & $<0.23$ \\
\hline Mar. & $3.87 \pm 0.08$ & $<6.92$ & $<0.26$ & $<0.28$ \\
\hline Apr. & $5.34 \pm 0.09$ & $<4.54$ & $<0.17$ & $0.76 \pm 0.41$ \\
\hline May & $5.82 \pm 0.09$ & $<5.89$ & $<0.21$ & $0.70 \pm 0.49$ \\
\hline June & $3.17 \pm 0.05$ & $<6.80$ & $<0.23$ & $<0.26$ \\
\hline July & $4.10 \pm 0.10$ & $23.41 \pm 13.91$ & $<0.16$ & $<0.19$ \\
\hline Aug. & $5.91 \pm 0.09$ & $23.69 \pm 11.18$ & $0.62 \pm 0.44$ & $0.73 \pm 0.48$ \\
\hline Sept. & $5.45 \pm 0.09$ & $35.83 \pm 14.69$ & $1.05 \pm 0.48$ & $<0.22$ \\
\hline Oct. & $6.10 \pm 0.10$ & $15.82 \pm 10.79$ & $0.53 \pm 0.31$ & $<0.19$ \\
\hline Nov. & $5.75 \pm 0.15$ & $<6.50$ & $<0.21$ & $0.81 \pm 0.54$ \\
\hline Dec. & $4.00 \pm 0.09$ & $<4.21$ & $<0.23$ & $<0.24$ \\
\hline Median & $4.68^{b}$ & $<6.65$ & $<0.22$ & $<0.25$ \\
\hline MAD & $1.18^{c}$ & $-^{d}$ & $-d$ & $-d$ \\
\hline DCG & $1.5 \times 10^{-3}$ & $3.3 \times 10^{-5}$ & $1.5 \times 10^{-5}$ & $3.7 \times 10^{-5}$ \\
\hline \multirow[t]{2}{*}{$\begin{array}{l}\text { Fraction } \\
\text { of DCG }\end{array}$} & $3.1 \times 10^{-6}$ & $<2.0 \times 10^{-7}$ & $<1.5 \times 10^{-8}$ & $<6.8 \times 10^{-9}$ \\
\hline & \multicolumn{4}{|c|}{$[\mu \mathrm{Ci} / \mathrm{mL}]$} \\
\hline Median & $1.3 \times 10^{-13 b}$ & $<1.8 \times 10^{-16}$ & $<6.0 \times 10^{-18}$ & $<6.8 \times 10^{-18}$ \\
\hline MAD & $3.2 \times 10^{-14 c}$ & $-^{d}$ & $-^{d}$ & $-^{d}$ \\
\hline DCG & $4 \times 10^{-8}$ & $9 \times 10^{-10}$ & $4 \times 10^{-10}$ & $1 \times 10^{-9}$ \\
\hline
\end{tabular}


Table 4-1. Gamma activity on air filters, Livermore-site perimeter, $1992^{a}$ (concluded).

\begin{tabular}{|c|c|c|c|}
\hline \multirow[b]{2}{*}{ Month } & \multicolumn{3}{|c|}{$\left[10^{-12} \mathrm{~Bq} / \mathrm{mL}\right]$} \\
\hline & ${ }^{226} \mathbf{R a}$ & ${ }^{228} \mathbf{R a}$ & ${ }^{228} \mathrm{Th}$ \\
\hline Jan. & $<0.37$ & $<0.70$ & $<0.38$ \\
\hline Feb. & $<0.43$ & $<1.60$ & $<0.42$ \\
\hline Mar. & $<0.53$ & $<1.17$ & $<0.61$ \\
\hline Apr. & $0.90 \pm 0.51$ & $1.97 \pm 1.51$ & $1.12 \pm 0.64$ \\
\hline May & $<0.98$ & $<1.22$ & $<0.53$ \\
\hline June & $<1.03$ & $<1.09$ & $<0.53$ \\
\hline July & $<0.70$ & $2.16 \pm 1.48$ & $0.92 \pm 0.65$ \\
\hline Aug. & $1.70 \pm 1.10$ & $3.14 \pm 1.36$ & $2.06 \pm 1.23$ \\
\hline Sept. & $2.38 \pm 0.82$ & $3.10 \pm 1.53$ & $2.11 \pm 0.98$ \\
\hline Oct. & $1.51 \pm 0.64$ & $2.39 \pm 1.29$ & $1.57 \pm 0.85$ \\
\hline Nov. & $<0.48$ & $<0.97$ & $<0.54$ \\
\hline Dec. & $<0.50$ & $<0.88$ & $<0.60$ \\
\hline Median & $<0.80$ & $<1.41$ & $<0.61$ \\
\hline MAD & -d & $-d$ & - d $^{d}$ \\
\hline DCG & $3.7 \times 10^{-8}$ & $1.1 \times 10^{-7}$ & $1.5 \times 10^{-9}$ \\
\hline \multirow{2}{*}{$\begin{array}{l}\text { Fraction } \\
\text { of DCG }\end{array}$} & $<2.2 \times 10^{-5}$ & $<1.3 \times 10^{-5}$ & $<4.0 \times 10^{-4}$ \\
\hline & & {$[\mu \mathrm{Ci} / \mathrm{mL}]$} & \\
\hline Median & $<2.2 \times 10^{-17}$ & $<3.8 \times 10^{-17}$ & $<1.6 \times 10^{-17}$ \\
\hline MAD & -d & $-d$ & $-^{d}$ \\
\hline DCG & $1 \times 10^{-12}$ & $3 \times 10^{-12}$ & $4 \times 10^{-14}$ \\
\hline
\end{tabular}

Note: Radionuclide results are reported $\pm 2 \sigma$; see Quality Assurance chapter.

a All Livermore-site perimeter samples composited.

b Mean value reported because of QA requirements; see Quality Assurance chapter.

c Standard deviation reported because of QA requirements; see Quality Assurance chapter.

d No measure of dispersion calculated; see Quality Assurance chapter. 
Table 4-2. Plutonium-239 activity on air filters (in 10-13 Bq/mL), Livermore Valley, 1992.

\begin{tabular}{|c|c|c|c|c|c|c|}
\hline \multirow[b]{2}{*}{ Month } & \multicolumn{6}{|c|}{ Sampling location ${ }^{a}$} \\
\hline & TANK & ZON7 & FCC & HOSP & LWRP & FIRE \\
\hline Jan. & $0.56 \pm 0.18$ & $<0.08$ & $0.24 \pm 0.13$ & $0.50 \pm 0.20$ & $0.51 \pm 0.20$ & $0.23 \pm 0.15$ \\
\hline Feb. & $<0.07$ & $0.34 \pm 0.16$ & $0.13 \pm 0.10$ & $-b$ & $0.48 \pm 0.23$ & $0.20 \pm 0.16$ \\
\hline Mar. & $0.09 \pm 0.09$ & $0.31 \pm 0.16$ & $0.16 \pm 0.10$ & $<0.09$ & $0.35 \pm 0.15$ & $0.10 \pm 0.09$ \\
\hline Apr. & $0.19 \pm 0.10$ & $<0.03$ & $<0.06$ & $0.10 \pm 0.07$ & $0.75 \pm 0.20$ & $0.19 \pm 0.11$ \\
\hline May & $<0.05$ & $<0.02$ & $<0.05$ & $0.08 \pm 0.08$ & $0.28 \pm 0.20$ & $<0.06$ \\
\hline June & $<0.03$ & $<0.07$ & $<0.08$ & $<0.21$ & $2.23 \pm 0.45$ & $<0.12$ \\
\hline July & $<0.02$ & $0.10 \pm 0.07$ & $<0.03$ & $<0.03$ & $0.36 \pm 0.25$ & $<0.22$ \\
\hline Aug. & $<0.07$ & $0.09 \pm 0.09$ & $<0.38$ & $<0.08$ & $0.57 \pm 0.29$ & $0.10 \pm 0.08$ \\
\hline Sept. & $0.63 \pm 0.56$ & $0.31 \pm 0.20$ & $0.13 \pm 0.11$ & $0.15 \pm 0.13$ & $0.42 \pm 0.23$ & $<1.25$ \\
\hline Oct. & $<0.02$ & $0.13 \pm 0.11$ & $<0.46$ & $<0.23$ & $0.71 \pm 0.20$ & $<0.01$ \\
\hline Nov. & $<0.23$ & $<0.05$ & $<0.29$ & $<0.06$ & $0.31 \pm 0.14$ & $<0.04$ \\
\hline Dec. & $<0.06$ & $<0.04$ & $<0.02$ & $<0.02$ & $<0.34$ & $<0.01$ \\
\hline Median & $<0.07$ & $<0.09$ & $<0.13$ & $<0.09$ & $<0.61^{c}$ & $<0.11$ \\
\hline MAD & -0 & -0 & - & -0 & $>0.53^{d}$ & - \\
\hline \multirow{2}{*}{$\begin{array}{l}\text { Fraction } \\
\text { of DCG }\end{array}$} & $<9.3 \times 10^{-6}$ & $<1.2 \times 10^{-5}$ & $<1.8 \times 10^{-5}$ & $<1.2 \times 10^{-5}$ & $<8.2 \times 10^{-5}$ & $<1.5 \times 10^{-5}$ \\
\hline & \multicolumn{6}{|c|}{$[\mu \mathrm{Ci} / \mathrm{mL}]$} \\
\hline Median & $<1.9 \times 10^{-19}$ & $<2.3 \times 10^{-19}$ & $<3.5 \times 10^{-19}$ & $<2.5 \times 10^{-19}$ & $<1.6 \times 10^{-1 R C}$ & $<3.0 \times 10^{-19}$ \\
\hline MAD & - & - & - & - & $>1.4 \times 10^{-18 d}$ & -0 \\
\hline
\end{tabular}


Table 4-2. Plutonium-239 activity on air filters (in $10^{-13} \mathrm{~Bq} / \mathrm{mL}$ ), Livermore Valley, 1992 (concluded).

\begin{tabular}{|c|c|c|c|c|c|c|}
\hline \multirow[b]{2}{*}{ Month } & \multicolumn{6}{|c|}{ Sampling locationa } \\
\hline & TFIR & ALTA & ERCH & LCCY & RRCH & PATT \\
\hline Jan. & $<0.10$ & $0.16 \pm 0.10$ & $0.08 \pm 0.07$ & $0.25 \pm 0.15$ & $0.74 \pm 0.23$ & $0.09 \pm 0.07$ \\
\hline Feb. & $0.19 \pm 0.14$ & $0.19 \pm 0.13$ & $0.56 \pm 0.29$ & $0.32 \pm 0.18$ & $0.16 \pm 0.12$ & $0.61 \pm 0.25$ \\
\hline Mar. & $0.20 \pm 0.12$ & $0.22 \pm 0.12$ & $0.19 \pm 0.11$ & $0.16 \pm 0.11$ & $0.20 \pm 0.11$ & $0.24 \pm 0.13$ \\
\hline Apr. & $0.13 \pm 0.09$ & $0.16 \pm 0.10$ & $0.13 \pm 0.09$ & $0.20 \pm 0.11$ & $0.12 \pm 0.08$ & $0.10 \pm 0.05$ \\
\hline May & $<0.04$ & $<0.04$ & $0.28 \pm 0.14$ & $<0.02$ & $<0.05$ & $<0.04$ \\
\hline June & $0.12 \pm 0.09$ & $<0.13$ & $0.42 \pm 0.26$ & $0.23 \pm 0.21$ & $<0.04$ & $<0.22$ \\
\hline July & $<0.05$ & $<0.06$ & $0.14 \pm 0.13$ & $<0.05$ & $<0.37$ & $<0.05$ \\
\hline Aug. & $0.68 \pm 0.36$ & $0.20 \pm 0.14$ & $0.27 \pm 0.15$ & $<0.42$ & $<0.001$ & $<0.002$ \\
\hline Sept. & $0.21 \pm 0.17$ & $<1.22$ & $<0.04$ & $0.19 \pm 0.14$ & $0.15 \pm 0.11$ & $<0.92$ \\
\hline Oct. & $<0.33$ & $<0.21$ & $<0.08$ & $<0.09$ & $0.14 \pm 0.11$ & $0.15 \pm 0.11$ \\
\hline Nov. & $<0.004$ & $<0.20$ & $<0.17$ & $0.10 \pm 0.08$ & $0.07 \pm 0.07$ & $<0.18$ \\
\hline Dec. & $<0.15$ & $<0.33$ & $<0.33$ & $0.21 \pm 0.17$ & $<0.09$ & $<0.24$ \\
\hline Median & $<0.14$ & $<0.20$ & $<0.22^{c}$ & $<0.19^{c}$ & $<0.13$ & $<0.17$ \\
\hline MAD & - & -o & -0 & -0 & - & -0 \\
\hline \multirow{2}{*}{$\begin{array}{l}\text { Fraction } \\
\text { of DCG }\end{array}$} & $<1.9 \times 10^{-5}$ & $<2.7 \times 10^{-5}$ & $<3.0 \times 10^{-5}$ & $<2.5 \times 10^{-5}$ & $<1.8 \times 10^{-5}$ & $<2.2 \times 10^{-5}$ \\
\hline & \multicolumn{6}{|c|}{$[\mu \mathrm{Ci} / \mathrm{mL}]$} \\
\hline Median & $<3.8 \times 10^{-19}$ & $<5.3 \times 10^{-19}$ & $<6.1 \times 10^{-19^{C}}$ & $<5.1 \times 10^{-19^{c}}$ & $<3.5 \times 10^{-19}$ & $<4.5 \times 10^{-19}$ \\
\hline MAD & -0 & -0 & - & - & - & - \\
\hline
\end{tabular}

Note: Radionuclide results are reported $\pm 2 \sigma ;$ see Quality Assurance chapter.

a See Fig. 4-2 for sample locations. Location TFIR is in the city of Tracy.

b No data; see Quality Assurance chapter.

c Mean value reported because of QA requirements; see Quality Assurance chapter.

d Standard deviation reported because of QA requirements; see Quality Assurance chapter.

- No measure of dispersion calculated; see Quality Assurance chapter.

$1 D C G=7.4 \times 10^{-10} \mathrm{~Bq} / \mathrm{mL}$ for ${ }^{239} \mathrm{Pu}$ activity in air $\left(2 \times 10^{-14} \mu \mathrm{Ci} / \mathrm{mL}\right)$. 
Table 4-3. Plutonium and uranium activity on air filters, Livermore-site perimeter, 1992.

\begin{tabular}{|c|c|c|c|c|c|}
\hline & & {$\left[10^{-13} \mathrm{~Bq} / \mathrm{mL}\right]$} & {$\left[10^{-5} \mu \mathrm{g} / \mathrm{m}^{3}\right]$} & {$\left[10^{-7} \mu \mathrm{g} / \mathrm{m}^{3}\right]$} & {$\left[10^{-3}\right]$} \\
\hline Location & Month & ${ }^{239} \mathrm{Pu}$ & ${ }^{235} U$ & ${ }^{238} \mathrm{U}$ & ${ }^{235} U /{ }^{238} U$ \\
\hline \multirow[t]{12}{*}{ SALV } & Jan. & $0.23 \pm 0.14$ & 2.06 & 1.50 & 7.28 \\
\hline & Feb. & $0.50 \pm 0.26$ & 2.37 & 1.68 & 7.09 \\
\hline & Mar. & $0.23 \pm 0.12$ & 2.74 & 1.92 & 7.00 \\
\hline & Apr.' & $2.11 \pm 1.01$ & 2.41 & 1.38 & 5.72 \\
\hline & May & $0.91 \pm 0.24$ & 11.40 & 8.10 & 7.11 \\
\hline & June & $0.47 \pm 0.19$ & 10.50 & 7.54 & 7.21 \\
\hline & July & $1.72 \pm 0.74$ & 8.35 & 6.13 & 7.35 \\
\hline & Aug. & $0.47 \pm 0.20$ & 11.90 & 8.59 & 7.23 \\
\hline & Sept. & $0.69 \pm 0.25$ & 18.30 & 13.30 & 7.29 \\
\hline & Oct. & $0.25 \pm 0.24$ & 7.89 & 5.66 & 7.18 \\
\hline & Nov. & $0.17 \pm 0.14$ & 7.13 & 5.11 & 7.17 \\
\hline & Dec. & $0.35 \pm 0.22$ & 2.24 & 1.60 & 7.16 \\
\hline \multirow[t]{12}{*}{ MESQ } & Jan. & $0.15 \pm 0.10$ & 2.79 & 1.98 & 7.10 \\
\hline & Feb. & $0.12 \pm 0.11$ & 3.32 & 2.40 & 7.21 \\
\hline & Mar. & $0.19 \pm 0.13$ & 3.54 & 2.52 & 7.11 \\
\hline & Apr. ${ }^{b}$ & $0.98 \pm 0.70$ & 2.90 & 1.65 & 5.68 \\
\hline & May & $0.30 \pm 0.14$ & 5.79 & 4.16 & 7.18 \\
\hline & June & $0.14 \pm 0.10$ & 8.68 & 5.70 & 6.56 \\
\hline & July & $<0.28$ & 6.04 & 4.40 & 7.29 \\
\hline & Aug. & $0.40 \pm 0.17$ & 7.60 & 5.56 & 7.32 \\
\hline & Sept. & $0.44 \pm 0.24$ & 15.10 & 11.00 & 7.26 \\
\hline & Oct. & $0.14 \pm 0.12$ & 8.16 & 5.79 & 7.10 \\
\hline & Nov. & $0.2^{7} \pm 0.13$ & 6.83 & 4.85 & 7.09 \\
\hline & Dec. & $0.27 \pm 0.18$ & 2.54 & 1.87 & 7.37 \\
\hline \multirow[t]{12}{*}{ CAFE } & Jan. & $<0.10$ & 5.08 & 3.58 & 7.04 \\
\hline & Feb. & $0.30 \pm 0.15$ & 5.46 & 3.94 & 7.21 \\
\hline & Mar. & $0.41 \pm 0.20$ & 6.66 & 4.68 & 7.02 \\
\hline & Apr.' & $0.94 \pm 0.85$ & 3.90 & 3.62 & 9.28 \\
\hline & May & $0.41 \pm 0.20$ & 9.89 & 7.11 & 7.18 \\
\hline & June & $0.24 \pm 0.18$ & 8.56 & 6.10 & 7.13 \\
\hline & July & $0.34 \pm 0.13$ & 9.11 & 6.82 & 7.49 \\
\hline & Aug. & $0.40 \pm 0.21$ & 14.20 & 10.20 & 7.20 \\
\hline & Sept. & $0.51 \pm 0.23$ & 16.00 & 11.80 & 7.33 \\
\hline & Oct. & $0.21 \pm 0.13$ & 10.90 & 7.89 & 7.24 \\
\hline & Nov. & $0.26 \pm 0.19$ & 7.07 & 5.15 & 7.28 \\
\hline & Dec. & $0.34 \pm 0.20$ & 4.23 & 3.10 & 7.31 \\
\hline
\end{tabular}


Table 4-3. Plutonium and uranium activity on air filters, Livermore-site perimeter, 1992 (continued).

\begin{tabular}{|c|c|c|c|c|c|}
\hline & & [10-13 Bq/mL] & {$\left[10^{-5} \mu g / \mathrm{m}^{3}\right]$} & {$\left[10^{-7} \mu \mathrm{g} / \mathrm{m}^{3}\right]$} & {$\left[10^{-3}\right]$} \\
\hline Locationa & Month & ${ }^{239} \mathrm{Pu}$ & $235 U$ & $238 \mathrm{U}$ & ${ }^{235} U /{ }^{238} U$ \\
\hline \multirow[t]{12}{*}{ MET } & Jan. & $0.22 \pm 0.13$ & 2.06 & 1.48 & 7.20 \\
\hline & Feb. & $0.16 \pm 0.12$ & 2.75 & 1.99 & 7.23 \\
\hline & Mar. & $0.14 \pm 0.10$ & 2.76 & 1.98 & 7.17 \\
\hline & Apr.b & $0.67 \pm 0.53$ & 2.84 & 1.87 & 6.61 \\
\hline & May & $0.28 \pm 0.13$ & 5.10 & 3.68 & 7.22 \\
\hline & June & $0.98 \pm 0.41$ & 5.33 & 3.56 & 6.69 \\
\hline & July & $<0.09$ & 4.34 & 3.16 & 7.27 \\
\hline & Aug. & $0.34 \pm 0.19$ & 8.27 & 6.21 & 7.51 \\
\hline & Sept. & $0.35 \pm 0.15$ & 9.63 & 6.98 & 7.25 \\
\hline & Oct. & $0.23 \pm 0.17$ & 7.57 & 5.49 & 7.25 \\
\hline & Nov. & $0.24 \pm 0.14$ & 6.11 & 4.54 & 7.43 \\
\hline & Dec. & $<0.07$ & 2.27 & 1.67 & 7.35 \\
\hline \multirow[t]{12}{*}{ VIS } & Jan. & $0.28 \pm 0.15$ & 2.46 & 1.74 & 7.05 \\
\hline & Feb. & $0.23 \pm 0.14$ & 2.92 & 2.08 & 7.13 \\
\hline & Mar. & $0.31 \pm 0.16$ & 2.77 & 1.97 & 7.11 \\
\hline & Apr..$^{b}$ & $0.86 \pm 0.65$ & $? .30$ & 1.66 & 6.12 \\
\hline & May & $0.37 \pm 0.17$ & 3.97 & 2.86 & 7.20 \\
\hline & June & $0.28 \pm 0.13$ & 5.01 & 3.29 & 6.57 \\
\hline & July & $0.34 \pm 0.13$ & 4.41 & 3.30 & 7.49 \\
\hline & Aug. & $0.51 \pm 0.24$ & 6.72 & 4.97 & 7.40 \\
\hline & Sept. & $0.62 \pm 0.24$ & 9.67 & 6.96 & 7.20 \\
\hline & Oct. & $0.33 \pm 0.25$ & 6.36 & 4.6 .4 & 7.29 \\
\hline & Nov. & $0.30 \pm 0.18$ & 5.57 & 4.08 & 7.32 \\
\hline & Dec. & $0.16 \pm 0.15$ & 2.06 & 1.47 & 7.13 \\
\hline \multirow[t]{12}{*}{ cow } & Jan. & $0.30 \pm 0.16$ & 3.40 & 2.42 & 7.11 \\
\hline & Feb. & $0.30 \pm 0.16$ & 3.92 & 2.78 & 7.11 \\
\hline & Mar. & $0.45 \pm 0.23$ & 5.38 & 3.79 & 7.05 \\
\hline & Apr.b & $1.03 \pm 0.71$ & 4.97 & 3.33 & 6.70 \\
\hline & May & $0.17 \pm 0.12$ & 10.70 & 7.73 & 7.21 \\
\hline & June & $0.51 \pm 0.26$ & 8.76 & 6.23 & 7.11 \\
\hline & July & $0.30 \pm 0.22$ & 7.42 & 5.45 & 7.35 \\
\hline & Aug. & $0.31 \pm 0.17$ & 10.10 & 7.23 & 7.13 \\
\hline & Sept. & $0.26 \pm 0.15$ & 12.90 & 8.89 & 6.89 \\
\hline & Oct. & $<0.20$ & 9.03 & 6.64 & 7.14 \\
\hline & Nov. & $0.17 \pm 0.13$ & 6.97 & 4.83 & 6.93 \\
\hline & Dec. & $0.25 \pm 0.16$ & 2.45 & 1.81 & 7.36 \\
\hline
\end{tabular}


Table 4-3. Plutonium and uranium activity on air filters, Livermore-site perimeter, 1992 (concluded).

\begin{tabular}{|c|c|c|c|c|c|c|}
\hline Sampling location & SALV & MESQ & CAFE & MET & VIS & cow \\
\hline \multicolumn{7}{|l|}{${ }^{239} \mathrm{Pu}$} \\
\hline Mean $\left(1 \sigma^{-13} \mathrm{~Bq} / \mathrm{mL}\right)$ & 0.68 & $<0.31$ & $<0.37$ & $<0.31$ & 0.38 & $<0.35$ \\
\hline Std $\operatorname{dev}\left(10^{-13} \mathrm{~Bq} / \mathrm{mL}\right)$ & 0.62 & $>0.23$ & $>0.21$ & $>0.26$ & 0.19 & $>0.24$ \\
\hline Fraction of $D^{-C G}$ & $9.13 \times 10^{-5}$ & $<4.13 \times 10^{-5}$ & $<5.03 \times 10^{-5}$ & $<4.23 \times 10^{-5}$ & $5.17 \times 10^{-5}$ & $<4.79 \times 10^{-5}$ \\
\hline Mean $\left(1 \sigma^{-18} \mu \mathrm{Cl} / \mathrm{mL}\right)$ & 1.83 & $<0.83$ & $<1.01$ & $<0.85$ & 1.03 & $<0.96$ \\
\hline Std dev $\left(10^{-18} \mu \mathrm{Cl} / \mathrm{mL}\right)$ & 1.68 & $>0.63$ & $>0.57$ & $>0.71$ & 0.53 & $>0.64$ \\
\hline \multicolumn{7}{|l|}{$238 \mathrm{U}$} \\
\hline $\operatorname{Mean}\left(1 \sigma^{5} \mu g / m^{3}\right)$ & 7.27 & 6.11 & 8.42 & 4.92 & 4.52 & 7.17 \\
\hline Std $\operatorname{dev}\left(10^{-5} \mu g / \mathrm{m}^{3}\right)$ & 5.16 & 3.60 & 3.84 & 2.55 & 2.28 & 3.23 \\
\hline Fraction of DCGd & $2.42 \times 10^{-4}$ & $2.04 \times 10^{-4}$ & $2.81 \times 10^{-4}$ & $1.64 \times 10^{-4}$ & $1.51 \times 10^{-4}$ & $2.39 \times 10^{-4}$ \\
\hline \multicolumn{7}{|l|}{$235 U$} \\
\hline $\operatorname{Mean}\left(10^{-7} \mu \mathrm{g} / \mathrm{m}^{3}\right)$ & 5.21 & 4.32 & 6.17 & 3.55 & 3.25 & 5.09 \\
\hline Std dev $\left(10^{-7} \mu g / \mathrm{m}^{3}\right)$ & 3.77 & 2.63 & 2.75 & 1.89 & 1.66 & 2.29 \\
\hline Fraction of DCG & $1.11 \times 10^{-5}$ & $9.20 \times 10^{-6}$ & $1.31 \times 10^{-5}$ & $7.55 \times 10^{-6}$ & $6.92 \times 10^{-6}$ & $1.08 \times 10^{-5}$ \\
\hline
\end{tabular}

Note: Radionuclide results are reported $\pm 2 \alpha$; see Quality Assurance chapter.

a See Fig. 4-1 for sample locations.

b Problems with the analysis of these samples occurred (see text).

c $D C G=7.4 \times 10^{-10} \mathrm{~Bq} / \mathrm{mL}$ for ${ }^{239} \mathrm{Pu}$ activity in air $\left(2 \times 10^{-14} \mu \mathrm{Ci} / \mathrm{mL}\right)$.

d $D C G=0.3 \mu \mathrm{g} / \mathrm{m}^{3}$ for ${ }^{238} \mathrm{U}$ activity in air.

- $D C G=0.047 \mu \mathrm{g} / \mathrm{m}^{3}$ for ${ }^{235} \mathrm{U}$ activity in air. 
Table 4-4. Plutonium-239 activity on air filters (in $10^{-13} \mathrm{~Bq} / \mathrm{mL}$ ), 1992.

\begin{tabular}{|c|c|c|}
\hline \multirow[b]{2}{*}{ Month } & \multicolumn{2}{|c|}{ Sampling location } \\
\hline & B531 & CRED \\
\hline Jan. & $0.98 \pm 0.30$ & $0.26 \pm 0.13$ \\
\hline Fob. & $0.77 \pm 0.26$ & $0.21 \pm 0.19$ \\
\hline Mar. & $0.14 \pm 0.03$ & $0.35 \pm 0.17$ \\
\hline Apr. & $1.99 \pm 0.61$ & $0.18 \pm 0.11$ \\
\hline May & $3.92 \pm 0.54$ & $0.26 \pm 0.16$ \\
\hline June & $4.44 \pm 0.84$ & $0.32 \pm 0.16$ \\
\hline July & $2.82 \pm 0.60$ & $0.46 \pm 0.23$ \\
\hline Aug. & $3.16 \pm 0.46$ & $0.40 \pm 0.18$ \\
\hline Sept. & $9.99 \pm 0.80$ & $0.47 \pm 0.22$ \\
\hline Oct. & $3.51 \pm 0.61$ & $<0.41$ \\
\hline Nov. & $1.12 \pm 0.25$ & $<0.01$ \\
\hline Dec. & $<0.02$ & $<0.01$ \\
\hline Mean & $<2.74$ & $<0.28$ \\
\hline Std dev & $>2.73$ & -b \\
\hline \multirow[t]{2}{*}{ Fraction of DCGC } & $<3.70 \times 10^{-4}$ & $<3.76 \times 10^{-5}$ \\
\hline & \multicolumn{2}{|c|}{$[\mu \mathrm{C} / / \mathrm{mL}]$} \\
\hline Mean $(\mu \mathrm{C} / \mathrm{mL})$ & $<7.40 \times 10^{-18}$ & $<7.52 \times 10^{-19}$ \\
\hline Std $\operatorname{dev}(\mu \mathrm{Ci} / \mathrm{mL})$ & $>7.38 \times 10^{-18}$ & -b $^{\mathrm{b}}$ \\
\hline
\end{tabular}

Note: Radionuclide results are reported $\pm 2 \sigma ;$ see Quality Assurance chapter.

a See Fig. 4-1 for sample locations.

b No measure of dispersion calculated; see Quality Assurance chapter.

c $D C G=7.4 \times 10^{-10} \mathrm{~Bq} / \mathrm{mL}$ for ${ }^{239} \mathrm{Pu}$ activity in air $\left(2 \times 10^{-14} \mu \mathrm{Ci} / \mathrm{mL}\right)$. 
Table 4-5. Tritium in air (in 10-7 Bq/mL), Livermore-site perimeter, 1992.

\begin{tabular}{|c|c|c|c|c|}
\hline \multirow[b]{2}{*}{ Month } & \multicolumn{4}{|c|}{ Sampling location } \\
\hline & SALV & MESQ & CAFE & MET \\
\hline \multirow[t]{2}{*}{ Jan. } & $1.31 \pm 0.11$ & $0.11 \pm 0.08$ & $2.09 \pm 0.14$ & $1.01 \pm 0.11$ \\
\hline & $0.70 \pm 0.11$ & $0.08 \pm 0.08$ & $0.18 \pm 0.09$ & $1.93 \pm 0.14$ \\
\hline \multirow[t]{2}{*}{ Feb. } & $1.92 \pm 0.18$ & $2.58 \pm 0.19$ & $3.32 \pm 0.21$ & $1.98 \pm 0.19$ \\
\hline & $1.35 \pm 0.12$ & $1.77 \pm 0.18$ & $3.17 \pm 0.20$ & $0.94 \pm 0.12$ \\
\hline \multirow[t]{2}{*}{ Mar. } & $2.86 \pm 0.18$ & $2.15 \pm 0.21$ & $2.98 \pm 0.19$ & $1.46 \pm 0.14$ \\
\hline & $1.99 \pm 0.19$ & $1.80 \pm 0.19$ & $2.29 \pm 0.19$ & $0.99 \pm 0.16$ \\
\hline \multirow[t]{2}{*}{ Apr. } & $3.85 \pm 0.22$ & $1.17 \pm 0.16$ & $1.70 \pm 0.16$ & $0.63 \pm 0.20$ \\
\hline & $1.67 \pm 0.18$ & $-\mathbf{b}$ & $1.74 \pm 0.18$ & $0.43 \pm 0.14$ \\
\hline \multirow[t]{2}{*}{ May } & $2.83 \pm 0.20$ & $1.07 \pm 0.15$ & $1.98 \pm 0.17$ & $0.85 \pm 0.14$ \\
\hline & $1.72 \pm 0.17$ & $0.67 \pm 0.14$ & $1.58 \pm 0.17$ & $0.53 \pm 0.13$ \\
\hline \multirow[t]{2}{*}{ June } & $2.28 \pm 0.16$ & $0.20 \pm 0.10$ & $0.54 \pm 0.13$ & $0.29 \pm 0.26$ \\
\hline & $2.26 \pm 0.20$ & $0.15 \pm 0.12$ & $0.42 \pm 0.13$ & $0.14 \pm 0.12$ \\
\hline \multirow[t]{3}{*}{ July } & $2.11 \pm 0.24$ & $0.24 \pm 0.18$ & $0.43 \pm 0.17$ & $<0.17$ \\
\hline & $2.14 \pm 0.22$ & $0.61 \pm 0.18$ & $1.13 \pm 0.19$ & $0.33 \pm 0.16$ \\
\hline & $3.43 \pm 0.31$ & $<0.19$ & $0.50 \pm 0.19$ & $<0.16$ \\
\hline \multirow[t]{2}{*}{ Aug. } & $3.61 \pm 0.25$ & $0.75 \pm 0.18$ & $1.40 \pm 0.19$ & $0.74 \pm 0.17$ \\
\hline & $3.34 \pm 0.23$ & $1.85 \pm 0.20$ & $3.20 \pm 0.24$ & $-^{b}$ \\
\hline \multirow[t]{2}{*}{ Sept. } & $2.73 \pm 0.25$ & $1.04 \pm 0.19$ & $1.05 \pm 0.19$ & $0.43 \pm 0.17$ \\
\hline & $2.37 \pm 0.15$ & $1.11 \pm 0.20$ & $1.64 \pm 0.21$ & $0.70 \pm 0.13$ \\
\hline \multirow[t]{2}{*}{ Oct. } & $1.47 \pm 0.19$ & $4.03 \pm 0.26$ & $3.04 \pm 0.23$ & $1.47 \pm 0.12$ \\
\hline & $2.05 \pm 0.22$ & $1.91 \pm 0.19$ & $2.67 \pm 0.32$ & $1.12 \pm 0.17$ \\
\hline \multirow[t]{2}{*}{ Nov. } & - $^{b}$ & $0.95 \pm 0.18$ & $2.63 \pm 0.25$ & $1.90 \pm 0.32$ \\
\hline & $2.05 \pm 0.16$ & $9.69 \pm 0.28$ & $5.29 \pm 0.22$ & $3.01 \pm 0.19$ \\
\hline \multirow[t]{3}{*}{ Dec. } & $1.30 \pm 0.14$ & $1.61 \pm 0.15$ & $2.14 \pm 0.14$ & $1.24 \pm 0.13$ \\
\hline & $1.46 \pm 0.15$ & $0.64 \pm 0.16$ & $2.23 \pm 0.20$ & $0.73 \pm 0.17$ \\
\hline & $1.53 \pm 0.19$ & $0.83 \pm 0.15$ & $1.77 \pm 0.17$ & $0.95 \pm 0.17$ \\
\hline Meanc & 2.17 & $<1.49$ & 1.97 & $<0.97$ \\
\hline Std dev & 0.80 & $>1.94$ & 1.16 & $>0.69$ \\
\hline $\begin{array}{l}\text { Fraction } \\
\text { of DCG }\end{array}$ & $5.88 \times 10^{-5}$ & $<4.02 \times 10^{-5}$ & $5.31 \times 10^{-5}$ & $<2.61 \times 10^{-5}$ \\
\hline \multirow[t]{2}{*}{ Dose $(m S v)^{9}$} & $4.7 \times 10^{-5}$ & $<3.2 \times 10^{-5}$ & $4.2 \times 10^{-5}$ & $<2.1 \times 10^{-5}$ \\
\hline & \multicolumn{4}{|c|}{$[\mu \mathrm{Ci} / \mathrm{mL}]$} \\
\hline Mean & $5.88 \times 10^{-12}$ & $<4.02 \times 10^{-12}$ & $5.31 \times 10^{-12}$ & $<2.61 \times 10^{-12}$ \\
\hline Std dev & $2.17 \times 10^{-12}$ & $>5.25 \times 10^{-12}$ & $3.14 \times 10^{-12}$ & $>1.87 \times 10^{-12}$ \\
\hline Dose (mrem) ${ }^{\ominus}$ & $4.7 \times 10^{-3}$ & $<3.2 \times 10^{-3}$ & $4.2 \times 10^{-3}$ & $<2.1 \times 10^{-3}$ \\
\hline
\end{tabular}

...continued 
Table 4-5. Tritium in air (in 10-7 Bq/mL), Livermore-site perimeter, 1992 (concluded).

\begin{tabular}{|c|c|c|c|}
\hline \multirow[b]{2}{*}{ Month } & \multicolumn{3}{|c|}{ Sampling location ${ }^{\mathrm{a}}$} \\
\hline & VIS & cow & POOL \\
\hline \multirow[t]{2}{*}{ Jan. } & $1.61 \pm 0.15$ & $1.27 \pm 0.13$ & $3.89 \pm 0.21$ \\
\hline & $0.31 \pm 0.10$ & $0.32 \pm 0.09$ & $0.10 \pm 0.09$ \\
\hline \multirow[t]{2}{*}{ Feb. } & $2.11 \pm 0.18$ & $1.00 \pm 0.08$ & $5.11 \pm 0.27$ \\
\hline & $1.67 \pm 0.19$ & $0.96 \pm 0.17$ & $3.74 \pm 0.23$ \\
\hline \multirow[t]{2}{*}{ Mar. } & $1.95 \pm 0.14$ & $2.53 \pm 0.18$ & $5.66 \pm 0.30$ \\
\hline & $1.68 \pm 0.20$ & $1.37 \pm 0.19$ & $5.00 \pm 0.27$ \\
\hline \multirow[t]{2}{*}{ Apr. } & $1.32 \pm 0.17$ & $0.95 \pm 0.13$ & $4.07 \pm 0.26$ \\
\hline & $1.49 \pm 0.19$ & $0.88 \pm 0.14$ & $1.76 \pm 0.17$ \\
\hline \multirow[t]{2}{*}{ May } & $1.44 \pm 0.16$ & $0.93 \pm 0.14$ & $2.28 \pm 0.21$ \\
\hline & $1.61 \pm 0.18$ & $0.91 \pm 0.14$ & $2.00 \pm 0.19$ \\
\hline \multirow[t]{2}{*}{ June } & $1.90 \pm 0.15$ & $0.87 \pm 0.10$ & $1.30 \pm 0.12$ \\
\hline & $1.41 \pm 0.18$ & $0.51 \pm 0.12$ & $1.12 \pm 0.15$ \\
\hline \multirow[t]{3}{*}{ July } & $1.96 \pm 0.25$ & $1.01 \pm 0.19$ & $1.38 \pm 0.30$ \\
\hline & $1.95 \pm 0.22$ & $1.04 \pm 0.17$ & $2.57 \pm 0.27$ \\
\hline & $1.64 \pm 0.22$ & $0.75 \pm 0.17$ & $1.47 \pm 0.30$ \\
\hline \multirow[t]{2}{*}{ Aug. } & $2.18 \pm 0.21$ & $1.05 \pm 0.16$ & $2.38 \pm 0.21$ \\
\hline & $2.60 \pm 0.22$ & $1.42 \pm 0.17$ & $3.41 \pm 0.24$ \\
\hline \multirow[t]{2}{*}{ Sept. } & $1.73 \pm 0.21$ & $0.57 \pm 0.15$ & $3.89 \pm 0.38$ \\
\hline & $1.54 \pm 0.13$ & $1.18 \pm 0.10$ & $2.49 \pm 0.23$ \\
\hline \multirow[t]{2}{*}{ Oct. } & $0.80 \pm 0.19$ & $0.61 \pm 0.10$ & $6.33 \pm 0.32$ \\
\hline & $1.74 \pm 0.22$ & $1.07 \pm 0.17$ & $4.29 \pm 0.27$ \\
\hline \multirow[t]{2}{*}{ Nov. } & $2.24 \pm 0.26$ & $1.55 \pm 0.29$ & $4.59 \pm 0.33$ \\
\hline & $1.39 \pm 0.14$ & $-b$ & $9.55 \pm 0.34$ \\
\hline \multirow[t]{3}{*}{ Dec. } & $0.74 \pm 0.11$ & $0.68 \pm 0.11$ & $3.14 \pm 0.17$ \\
\hline & $1.62 \pm 0.19$ & $0.92 \pm 0.19$ & $2.14 \pm 0.18$ \\
\hline & $1.11 \pm 0.15$ & $1.06 \pm 0.16$ & $1.91 \pm 0.16$ \\
\hline Meanc & 1.61 & 1.02 & 3.29 \\
\hline Std dev & 0.49 & 0.43 & 2.01 \\
\hline $\begin{array}{l}\text { Fraction } \\
\text { of DCGd }\end{array}$ & $4.34 \times 10^{-5}$ & $2.75 \times 10^{-5}$ & $8.89 \times 10^{-5}$ \\
\hline \multirow[t]{2}{*}{ Dose (mSv) } & $3.4 \times 10^{-5}$ & $2.2 \times 10^{-5}$ & $7.1 \times 10^{-5}$ \\
\hline & & {$[\mu \mathrm{Ci} / \mathrm{mL}]$} & \\
\hline Mean & $4.34 \times 10^{-12}$ & $2.75 \times 10^{-12}$ & $8.89 \times 10^{-12}$ \\
\hline Std dev & $1.32 \times 10^{-12}$ & $1.15 \times 10^{-12}$ & $5.42 \times 10^{-12}$ \\
\hline Dose (mrem) ${ }^{\bullet}$ & $3.4 \times 10^{-3}$ & $2.2 \times 10^{-3}$ & $7.1 \times 10^{-3}$ \\
\hline
\end{tabular}

Note: Radionuclide results are reported $\pm 2 \sigma$; see Quality Assurance chapter.

a See Fig. 4-1 for sampling locations.

b No data; see Quality Assurance chapter.

c Livermore-site perimeter overall average $=1.8 \times 10^{-7} \mathrm{~Bq} / \mathrm{mL}$.

d $\mathrm{DCG}=3.7 \times 10^{-3} \mathrm{~Bq} / \mathrm{mL}\left(1 \times 10^{-7} \mu \mathrm{Ci} / \mathrm{mL}\right)$.

- This dose is the effective dose equivalent. 
Table 4-6. Tritium in air (in 10-7 Bq/mL), special study, 1992.

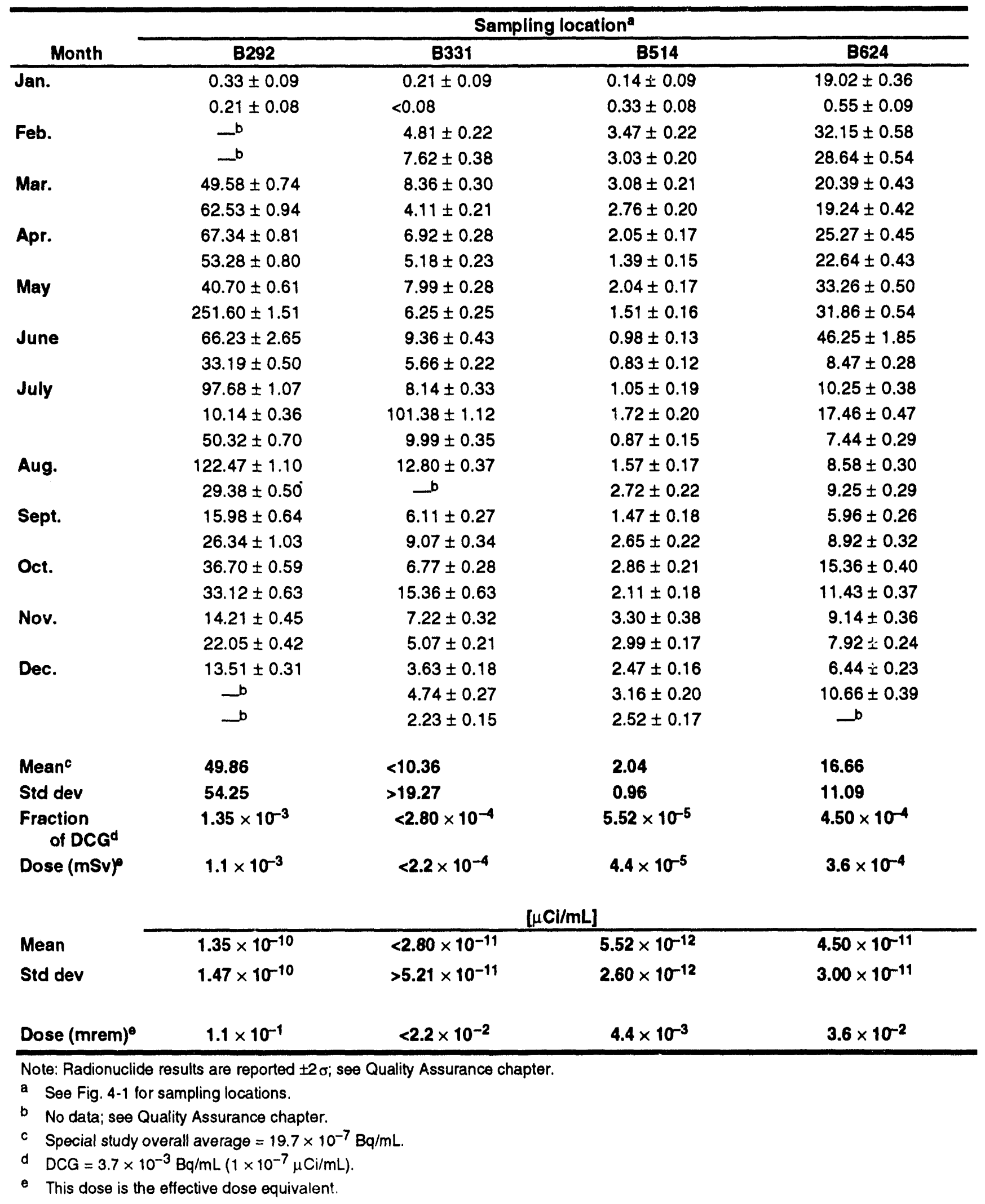


Table 4-7. Tritium in air (in 10-7 Bq/mL), Livermore Valley, 1992.

\begin{tabular}{|c|c|c|c|}
\hline \multirow[b]{2}{*}{ Month } & \multicolumn{3}{|c|}{ Sampling location } \\
\hline & ZON7 & ALTA & LCCY \\
\hline \multirow[t]{2}{*}{ Jan. } & $0.67 \pm 0.12$ & $0.25 \pm 0.12$ & $0.37 \pm 0.10$ \\
\hline & $1.17 \pm 0.13$ & $0.38 \pm 0.11$ & $0.14 \pm 0.09$ \\
\hline \multirow[t]{2}{*}{ Feb. } & $1.26 \pm 0.16$ & $0.42 \pm 0.18$ & $1.12 \pm 0.25$ \\
\hline & $1.16 \pm 0.13$ & $0.44 \pm 0.14$ & $1.11 \pm 0.23$ \\
\hline \multirow[t]{2}{*}{ Mar. } & $1.36 \pm 0.19$ & $0.81 \pm 0.19$ & $0.72 \pm 0.18$ \\
\hline & $0.91 \pm 0.16$ & $0.68 \pm 0.17$ & $0.61 \pm 0.18$ \\
\hline \multirow[t]{2}{*}{ Apr. } & $0.93 \pm 0.15$ & $0.67 \pm 0.15$ & $0.15 \pm 0.13$ \\
\hline & $0.68 \pm 0.15$ & $0.51 \pm 0.17$ & $0.19 \pm 0.16$ \\
\hline \multirow[t]{2}{*}{ May } & $0.98 \pm 0.14$ & $0.67 \pm 0.15$ & $0.28 \pm 0.12$ \\
\hline & $0.68 \pm 0.13$ & $0.33 \pm 0.13$ & $0.15 \pm 0.15$ \\
\hline \multirow[t]{2}{*}{ June } & $1.23 \pm 0.12$ & $0.25 \pm 0.09$ & $<0.67$ \\
\hline & $0.88 \pm 0.16$ & $0.28 \pm 0.15$ & $0.15 \pm 0.15$ \\
\hline \multirow[t]{3}{*}{ July } & $1.07 \pm 0.20$ & $0.39 \pm 0.19$ & $0.23 \pm 0.16$ \\
\hline & $1.08 \pm 0.19$ & $0.48 \pm 0.18$ & $<0.15$ \\
\hline & $1.24 \pm 0.21$ & $<0.19$ & $<0.18$ \\
\hline \multirow[t]{2}{*}{ Aug. } & $1.18 \pm 0.20$ & $<0.17$ & $<0.18$ \\
\hline & $1.40 \pm 0.17$ & $0.61 \pm 0.15$ & $0.26 \pm 0.17$ \\
\hline \multirow[t]{2}{*}{ Sept. } & $0.82 \pm 0.10$ & $0.26 \pm 0.11$ & $0.38 \pm 0.29$ \\
\hline & $1.02 \pm 0.13$ & $0.39 \pm 0.12$ & $<0.14$ \\
\hline \multirow[t]{2}{*}{ Oct. } & $0.55 \pm 0.15$ & $0.64 \pm 0.20$ & $0.55 \pm 0.16$ \\
\hline & $1.02 \pm 0.21$ & $0.42 \pm 0.19$ & $0.60 \pm 0.17$ \\
\hline \multirow[t]{2}{*}{ Nov. } & $1.30 \pm 0.24$ & $0.62 \pm 0.30$ & $0.48 \pm 0.20$ \\
\hline & $0.65 \pm 0.11$ & $0.50 \pm 0.11$ & $1.01 \pm 0.12$ \\
\hline \multirow[t]{3}{*}{ Dec. } & $0.38 \pm 0.12$ & $-b$ & $0.39 \pm 0.12$ \\
\hline & $0.67 \pm 0.14$ & $0.28 \pm 0.21$ & $0.15 \pm 0.12$ \\
\hline & $0.78 \pm 0.18$ & $0.24 \pm 0.16$ & $0.24 \pm 0.10$ \\
\hline Meanc & 0.97 & $<0.43$ & $<0.41$ \\
\hline Std dev & 0.27 & $>0.18$ & -d \\
\hline Fraction of DCG & $2.61 \times 10^{-5}$ & $<1.17 \times 10^{-5}$ & $<1.10 \times 10^{-5}$ \\
\hline \multirow[t]{2}{*}{ Dose $(m S v)^{t}$} & $2.1 \times 10^{-5}$ & $<9.3 \times 10^{-6}$ & $<8.8 \times 10^{-6}$ \\
\hline & & {$[\mu \mathrm{Ci} / \mathrm{mL}]$} & \\
\hline Mean & $2.61 \times 10^{-12}$ & $<1.17 \times 10^{-12}$ & $<1.10 \times 10^{-12}$ \\
\hline Std dev & $7.38 \times 10^{-13}$ & $>4.83 \times 10^{-13}$ & -d \\
\hline Dose (mrem) ${ }^{\prime}$ & $2.1 \times 10^{-3}$ & $<9.3 \times 10^{-4}$ & $<8.8 \times 10^{-4}$ \\
\hline
\end{tabular}


Table 4-7. Tritium in air (in 10-7 Bq/mL), Livermore Valley, 1992 (concluded).

\begin{tabular}{|c|c|c|c|}
\hline \multirow[b]{2}{*}{ Month } & \multicolumn{3}{|c|}{ Sampling location } \\
\hline & FIRE & XRDS & VET \\
\hline \multirow[t]{2}{*}{ Jan. } & $0.67 \pm 0.12$ & $0.81 \pm 0.14$ & $1.10 \pm 0.13$ \\
\hline & $0.58 \pm 0.10$ & $1.62 \pm 0.15$ & $0.67 \pm 0.11$ \\
\hline \multirow[t]{2}{*}{ Feb. } & $0.95 \pm 0.15$ & $1.45 \pm 0.22$ & $2.59 \pm 0.22$ \\
\hline & $0.77 \pm 0.15$ & $0.79 \pm 0.19$ & $1.53 \pm 0.18$ \\
\hline \multirow[t]{2}{*}{ Mar. } & $0.97 \pm 0.18$ & $1.31 \pm 0.21$ & $1.61 \pm 0.20$ \\
\hline & $0.57 \pm 0.15$ & $0.73 \pm 0.18$ & $1.44 \pm 0.19$ \\
\hline \multirow[t]{2}{*}{ Apr. } & $0.20 \pm 0.12$ & $0.50 \pm 0.16$ & $0.65 \pm 0.15$ \\
\hline & $0.22 \pm 0.14$ & $0.45 \pm 0.18$ & $0.35 \pm 0.15$ \\
\hline \multirow[t]{2}{*}{ May } & $0.45 \pm 0.15$ & $0.87 \pm 0.15$ & $0.94 \pm 0.15$ \\
\hline & $<0.13$ & $0.34 \pm 0.14$ & $0.53 \pm 0.14$ \\
\hline \multirow[t]{2}{*}{ June } & $0.24 \pm 0.13$ & $0.63 \pm 0.13$ & $0.28 \pm 0.13$ \\
\hline & $0.21 \pm 0.13$ & $0.47 \pm 0.15$ & $<0.13$ \\
\hline \multirow[t]{3}{*}{ July } & $<0.17$ & $0.61 \pm 0.20$ & $<0.18$ \\
\hline & $0.27 \pm 0.17$ & $0.60 \pm 0.18$ & $0.27 \pm 0.16$ \\
\hline & $<0.16$ & $0.37 \pm 0.17$ & $<0.54$ \\
\hline \multirow[t]{2}{*}{ Aug. } & $0.27 \pm 0.15$ & $0.51 \pm 0.17$ & $0.51 \pm 0.16$ \\
\hline & $0.59 \pm 0.15$ & $1.05 \pm 0.16$ & $1.27 \pm 0.17$ \\
\hline \multirow[t]{2}{*}{ Sept. } & $0.31 \pm 0.08$ & $0.32 \pm 0.08$ & $0.45 \pm 0.09$ \\
\hline & $0.19 \pm 0.11$ & $0.58 \pm 0.12$ & $0.37 \pm 0.12$ \\
\hline \multirow[t]{2}{*}{ Oct. } & $0.93 \pm 0.16$ & $0.36 \pm 0.14$ & $1.56 \pm 0.18$ \\
\hline & $0.94 \pm 0.19$ & $0.67 \pm 0.19$ & $1.39 \pm 0.29$ \\
\hline \multirow[t]{2}{*}{ Nov. } & $0.87 \pm 0.23$ & $0.71 \pm 0.24$ & $0.97 \pm 0.23$ \\
\hline & $1.62 \pm 0.14$ & $0.45 \pm 0.10$ & $2.06 \pm 0.16$ \\
\hline \multirow[t]{3}{*}{ Dec. } & $-^{b}$ & $0.27 \pm 0.10$ & $0.96 \pm 0.13$ \\
\hline & $0.25 \pm 0.17$ & $0.20 \pm 0.16$ & $0.68 \pm 0.15$ \\
\hline & $0.53 \pm 0.13$ & $0.50 \pm 0.16$ & $1.13 \pm 0.14$ \\
\hline Meanc & $<0.52$ & 0.66 & $<0.93$ \\
\hline Std dev & $>0.37$ & 0.36 & $>0.62$ \\
\hline Fraction of $\mathrm{DCG}^{\circ}$ & $<1.41 \times 10^{-5}$ & $1.78 \times 10^{-5}$ & $<2.51 \times 10^{-5}$ \\
\hline \multirow[t]{2}{*}{ Dose (mSv) } & $<1.1 \times 10^{-5}$ & $1.4 \times 10^{-5}$ & $<2.0 \times 10^{-5}$ \\
\hline & \multicolumn{3}{|c|}{$[\mu \mathrm{Ci} / \mathrm{mL}]$} \\
\hline Mean & $<1.41 \times 10^{-12}$ & $1.78 \times 10^{-12}$ & $<2.51 \times 10^{-12}$ \\
\hline Std dev & $>1.00 \times 10^{-12}$ & $9.66 \times 10^{-13}$ & $>1.67 \times 10^{-12}$ \\
\hline Dose (mrem) & $<1.1 \times 10^{-3}$ & $1.4 \times 10^{-3}$ & $<2.0 \times 10^{-3}$ \\
\hline
\end{tabular}

Note: Radionuclide results are reported $\pm 2 \sigma$; see Quality Assurance chapter.

a See Fig. 4-2 for sampling locations.

b No data; see Quality Assurance chapter.

c Livermore Valley overall average $=6.6 \times 10^{-8} \mathrm{~Bq} / \mathrm{mL}$.

d No measure of dispersion calculated; see Quality Assurance chapter.

- $D C G=3.7 \times 10^{-3} \mathrm{~Bq} / \mathrm{mL}\left(1 \times 10^{-7} \mu \mathrm{Ci} / \mathrm{mL}\right)$.

1 This dose is the effective dose equivalent. 
Table 4-8. Beryllium on air filters (in pg/m³), Livermore-site perimeter, 1992.

\begin{tabular}{lrrrrrr}
\hline & \multicolumn{6}{c}{ Sampling location } \\
\cline { 2 - 7 } \multicolumn{1}{c}{ Month } & SALV & MESQ & CAFE & MET & \multicolumn{1}{c}{ VIS } & COW \\
\hline Jan. & 16.8 & 22.5 & 42.5 & 14.3 & 15.0 & 18.2 \\
Feb. & 15.2 & 17.5 & 31.0 & 15.7 & 12.3 & 20.5 \\
Mar. & 10.9 & 10.6 & 20.2 & 10.0 & 9.5 & 15.5 \\
Apr. & 30.8 & 24.7 & 27.9 & 11.8 & 12.9 & 19.4 \\
May & 37.5 & 28.8 & 35.4 & 23.3 & 22.0 & 39.7 \\
June & 8.1 & $<5.4$ & $<5.4$ & $<5.8$ & $<5.4$ & $<7.2$ \\
July & 42.9 & 24.6 & 28.1 & 20.5 & 18.5 & 27.0 \\
Aug. & 33.1 & 34.7 & 43.7 & 32.6 & 27.3 & 39.4 \\
Sept. & 57.5 & 56.9 & 45.8 & 36.1 & 30.8 & 65.9 \\
Oct. & 23.7 & 22.5 & 25.6 & 21.9 & 17.4 & 21.9 \\
Nov. & 41.8 & 23.3 & 24.2 & 17.2 & 31.2 & 18.4 \\
Dec. & 8.3 & 9.9 & 13.6 & 6.3 & 13.2 & 13.7 \\
& & & & & & \\
Meany ${ }^{b}$ & 27.2 & $<23.5$ & $<28.6$ & $<18.0$ & $<18.0$ & $<25.6$ \\
Std dev & 15.9 & $>13.4$ & $>12.2$ & $>9.5$ & $>8.3$ & $>15.9$ \\
\hline
\end{tabular}

Note: The monthly ambient concentration guide (ACG) set by the BAAQMD is $10,000 \mathrm{pg} / \mathrm{m}^{3}$. To determine the fraction each value is of the monthly standard, divide the reported value for the month by 10,$000 ; \theta .9 ., 35.0+10,000=0.000350$.

a See Fig 4-1 for sampling locations.

b Livermore-site perimeter overall annual average is $23.5 \mathrm{pg} / \mathrm{m}^{3}$. 
Table 4-9. Gamma activity on air filters, Site 300, 1992. a

\begin{tabular}{|c|c|c|c|c|}
\hline \multirow[b]{2}{*}{ Month } & \multirow{2}{*}{$\frac{\left[10^{-9} \mathrm{~Bq} / \mathrm{mL}\right]}{{ }^{7} \mathrm{Be}}$} & \multicolumn{3}{|c|}{$\left[10^{-12} \mathrm{Bg} / \mathrm{mL}\right]$} \\
\hline & & ${ }^{40} K$ & ${ }^{137} \mathrm{Cs}$ & ${ }^{22} \mathrm{Na}$ \\
\hline Jan. & $1.79 \pm 0.03$ & $<3.97$ & $<0.13$ & $<0.14$ \\
\hline Feb. & $3.33 \pm 0.06$ & $<5.03$ & $<0.16$ & $0.45 \pm 0.38$ \\
\hline Mar. & $3.73 \pm 0.07$ & $<3.98$ & $<0.15$ & $0.58 \pm 0.39$ \\
\hline Apr. & $5.50 \pm 0.12$ & $<6.69$ & $<0.16$ & $0.70 \pm 0.35$ \\
\hline May & $7.27 \pm 0.17$ & $22.51 \pm 11.53$ & $<0.20$ & $0.98 \pm 0.58$ \\
\hline June & $4.06 \pm 0.07$ & $<4.13$ & $<0.16$ & $0.64 \pm 0.37$ \\
\hline July & $5.48 \pm 0.09$ & $<3.27$ & $<0.12$ & $0.55 \pm 0.29$ \\
\hline Aug. & $7.64 \pm 0.17$ & $19.04 \pm 10.39$ & $<0.16$ & $0.63 \pm 0.34$ \\
\hline Sept. & $5.47 \pm 0.11$ & $27.33 \pm 13.88$ & $0.79 \pm 0.61$ & $<0.26$ \\
\hline Oct. & $5.57 \pm 0.09$ & $15.55 \pm 9.64$ & $0.39 \pm 0.29$ & $0.38 \pm 0.30$ \\
\hline Nov. & $5.30 \pm 0.08$ & $<5.20$ & $<0.18$ & $<0.21$ \\
\hline Dec. & $3.57 \pm 0.07$ & $<5.11$ & $<0.19$ & $<0.21$ \\
\hline Median & $4.89^{b}$ & $<5.15$ & $<0.16$ & $<0.48$ \\
\hline MAD & $1.67^{c}$ & -d & -d & $>0.25$ \\
\hline DCG & $1.5 \times 10^{-3}$ & $3.3 \times 10^{-5}$ & $1.5 \times 10^{-5}$ & $3.7 \times 10^{-5 b}$ \\
\hline \multirow{2}{*}{$\begin{array}{l}\text { Fraction } \\
\text { of DCG }\end{array}$} & $3.3 \times 10^{-6}$ & $<1.6 \times 10^{-7}$ & $<1.1 \times 10^{-8}$ & $<1.3 \times 10^{-8^{c}}$ \\
\hline & \multicolumn{4}{|c|}{$[\mu \mathrm{Ci} / \mathrm{mL}]$} \\
\hline Median & $1.3 \times 10^{-13 b}$ & $<1.4 \times 10^{-16}$ & $<4.4 \times 10^{-18}$ & $<1.3 \times 10^{-17 b}$ \\
\hline MAD & $4.5 \times 10^{-14 c}$ & -d & $-d$ & $>6.7 \times 10^{-18 c}$ \\
\hline DCG & $4 \times 10^{-8}$ & $9 \times 10^{-10}$ & $4 \times 10^{-9}$ & $1 \times 10^{-9}$ \\
\hline
\end{tabular}


Table 4-9. Gamma activity on air filters, Site 300, 1992. ${ }^{a}$ (concluded).

\begin{tabular}{|c|c|c|c|}
\hline \multirow[b]{2}{*}{ Month } & \multicolumn{3}{|c|}{$\left[10^{-12} \mathrm{~Bq} / \mathrm{mL}\right]$} \\
\hline & ${ }^{226} R a$ & ${ }^{228} \mathrm{Ra}$ & ${ }^{228} \mathrm{Th}$ \\
\hline Jan. & $<0.29$ & $<0.65$ & $<0.31$ \\
\hline Feb. & $<0.35$ & $<0.75$ & $<0.41$ \\
\hline Mar. & $<0.30$ & $1.85 \pm 1.56$ & $<0.36$ \\
\hline Apr. & $1.04 \pm 0.69$ & $<0.70$ & $<0.45$ \\
\hline May & $<1.22$ & $<0.85$ & $<0.57$ \\
\hline June & $1.00 \pm 0.59$ & $<0.64$ & $0.83 \pm 0.53$ \\
\hline July & $0.92 \pm 0.54$ & $1.32 \pm 0.83$ & $0.93 \pm 0.58$ \\
\hline Aug. & $0.89 \pm 0.64$ & $2.22 \pm 1.21$ & $<0.47$ \\
\hline Sept. & $2.01 \pm 0.80$ & $<1.04$ & $1.39 \pm 0.96$ \\
\hline Oct. & $1.14 \pm 0.56$ & $<0.69$ & $<0.41$ \\
\hline Nov. & $<0.40$ & $<0.84$ & $<0.48$ \\
\hline Dec. & $<0.37$ & $<0.84$ & $<0.47$ \\
\hline Median & $<0.90$ & $<0.84$ & $<0.47$ \\
\hline MAD & $-^{d}$ & $-^{d}$ & 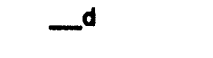 \\
\hline DCG & $3.7 \times 10^{-8}$ & $1.1 \times 10^{-7}$ & $1.5 \times 10^{-9}$ \\
\hline \multirow{2}{*}{$\begin{array}{l}\text { Fraction } \\
\text { of DCG }\end{array}$} & $<2.4 \times 10^{-5}$ & $<7.7 \times 10^{-6}$ & $<3.1 \times 10^{-4}$ \\
\hline & \multicolumn{3}{|c|}{$[\mu \mathrm{Ci} / \mathrm{mL}]$} \\
\hline Median & $<2.4 \times 10^{-17}$ & $<2.3 \times 10^{-17}$ & $<1.3 \times 10^{-17}$ \\
\hline MAD & $-d$ & $-d$ & -d \\
\hline DCG & $1 \times 10^{-12}$ & $3 \times 10^{-12}$ & $4 \times 10^{-14}$ \\
\hline
\end{tabular}

Note: Radionuclide results are reported $\pm 2 \sigma$; see Quality Assurance chapter.

a All Site 300 perimeter samples composited.

b Mean value reported because of QA requirements; see Quality Assurance chapter.

c Standard deviation reported because of QA requirements; see Quality Assurance chapter.

d No measure of dispersion calculated; see Quality Assurance chapter. 
Table 4-10. Plutonium and uranium activity on air filters, Site $300,1992 .^{a}$

\begin{tabular}{|c|c|c|c|c|}
\hline & {$\left[10^{-13} \mathrm{~Bq} / \mathrm{mL}\right]$} & {$\left[10^{-5} \mu \mathrm{g} / \mathrm{m}^{3}\right]$} & {$\left[10^{-7} \mu g / \mathrm{m}^{3}\right]$} & {$\left[10^{-3}\right]$} \\
\hline Month & ${ }^{239} \mathrm{Pu}$ & ${ }^{238} \mathrm{U}$ & $235 \mathrm{U}$ & $235 / 238 \mathrm{U}$ \\
\hline Jan. & $0.02 \pm 0.01$ & 4.36 & 2.03 & 4.7 \\
\hline Feb. & $0.04 \pm 0.03$ & 2.04 & 1.33 & 6.5 \\
\hline Mar. & $0.06 \pm 0.05$ & 1.98 & 1.37 & 6.9 \\
\hline Apr. & $0.30 \pm 0.19$ & 24.80 & 17.00 & 6.9 \\
\hline May & $0.31 \pm 0.05$ & 7.36 & 5.25 & 7.1 \\
\hline June & $0.05 \pm 0.03$ & 5.20 & 3.53 & 6.8 \\
\hline July & $0.05 \pm 0.02$ & 5.04 & 3.33 & 6.6 \\
\hline Aug. & $0.06 \pm 0.03$ & 7.00 & 4.76 & 6.8 \\
\hline Sept. & $0.14 \pm 0.07$ & 10.60 & 7.37 & 6.9 \\
\hline Oct. & $0.09 \pm 0.03$ & 11.10 & 6.47 & 5.8 \\
\hline Nov. & $0.03 \pm 0.02$ & 5.77 & 4.07 & 7.1 \\
\hline Dec. & $0.06 \pm 0.03$ & 1.92 & 1.32 & 6.9 \\
\hline Mean & 0.10 & 7.26 & 4.82 & \\
\hline Std dev & 0.10 & 6.30 & 4.33 & \\
\hline \multirow[t]{2}{*}{ Fraction of DCG } & $1.4 \times 10^{-5 b}$ & $2.4 \times 10^{-4^{c}}$ & $1.0 \times 10^{-5^{d}}$ & \\
\hline & {$[\mu \mathrm{Ci} / \mathrm{mL}]$} & & & \\
\hline Mean & $2.7 \times 10^{-19}$ & & & \\
\hline Std dev & $2.7 \times 10^{-19}$ & & & \\
\hline
\end{tabular}

Note: Radionuclide results are reported $\pm 2 \sigma ; s e \theta$ Quality Assurance chapter.

a Composite of all Site 300 samples. See Fig. 4-3 for sample locations.

b $D C G=7.4 \times 10^{-10} \mathrm{~Bq} / \mathrm{mL}$ for ${ }^{239} \mathrm{Pu}$ activity in air $\left(2 \times 10^{-14} \mu \mathrm{Ci} / \mathrm{mL}\right)$.

c $D C G=0.3 \mu \mathrm{g} / \mathrm{m}^{3}$ for ${ }^{238} \mathrm{U}$ activity in air.

d $D C G=0.047 \mu \mathrm{g} / \mathrm{m}^{3}$ for $235 \mathrm{U}$ activity in air. 
Table 4-i1. Beryllium on air filters (in pg/m³), Site 300, 1992.

\begin{tabular}{lrrrrrrrrrr}
\hline & \multicolumn{10}{c}{ Sampling locationa } \\
\cline { 2 - 11 } Month & EOBS & ECP & WCP & \multicolumn{1}{c}{ LIN } & GOLF & TFIR & NPS & WOBS & $\mathbf{8 0 1 E}$ \\
\hline Jan. & 6.6 & $<4.4$ & $<4.4$ & $<4.4$ & $<4.3$ & 14.1 & $<4.3$ & $<4.4$ & $<4.4$ \\
Feb. & 13.1 & 11.7 & 18.7 & 9.2 & 8.5 & 22.1 & 9.1 & 9.2 & 5.2 \\
Mar. & 10.9 & 16.3 & 13.5 & 9.5 & 17.6 & 17.4 & 11.2 & 9.4 & 8.1 \\
Apr. & 20.5 & 26.1 & 30.4 & 22.9 & 23.8 & 29.6 & 17.4 & 16.9 & 14.1 \\
May & 43.3 & 40.4 & 38.0 & 44.2 & 24.1 & 41.7 & 29.4 & 27.9 & 12.1 \\
June & 37.1 & 18.9 & 33.9 & 8.1 & $<5.4$ & $<5.4$ & 18.9 & 18.8 & 6.8 \\
July & 34.6 & 41.0 & 35.2 & 28.2 & 44.4 & 35.3 & 29.6 & 304.9 & 30.6 \\
Aug. & 28.9 & 20.8 & 33.5 & 31.6 & 108.4 & 25.2 & 31.7 & 32.2 & 20.9 \\
Sept. & 48.0 & 38.9 & 60.7 & 40.2 & 90.7 & 73.8 & 47.2 & 43.3 & 43.9 \\
Oct. & 29.3 & 18.6 & 21.7 & 20.8 & 38.8 & 30.9 & 23.7 & 25.5 & 29.2 \\
Nov. & 16.2 & 13.6 & 16.2 & 18.4 & 36.6 & 38.7 & 11.8 & 16.8 & 15.0 \\
Dec. & 13.5 & 9.8 & 6.2 & 5.0 & 12.4 & 24.8 & 6.2 & 5.2 & 6.2 \\
& & & & & & & & $<2.2$ \\
Meanc & 25.2 & $<21.7$ & $<26.0$ & $<20.2$ & $<34.6$ & $<29.9$ & $<20.0$ & $<42.9$ & $<16.4$ \\
Std dev & 13.6 & $>12.4$ & $>15.8$ & $>13.6$ & $>33.3$ & $>17.3$ & $>12.7$ & $>83.3$ & $>12.4$ \\
\hline
\end{tabular}

Note: The monthly ambient concentration guide (ACG) set by the BAAQMD is $10,000 \mathrm{pg} / \mathrm{m}^{3}$. To determine the fraction each value is of the monthly standard, divide the reported value for the month by 10,000 ;

e.g., $14.1+10,000=0.00141$.

a See Fig. 4-3 for sampling locations.

b Location TFIR is in the city of Tracy.

c Site 300 overall annual average is $26.3 \mathrm{pg} / \mathrm{m}^{3}$. 


\section{Air Monitoring}

Table 4-12. Radioactive airborne effluent releases from the Livermore site, 1981 through 1992.

\begin{tabular}{lrrrr}
\hline & \multicolumn{5}{c}{ Airborne effluents } \\
\cline { 2 - 5 } Year & ${ }^{3} \mathbf{H}(\mathbf{G B q})$ & ${ }^{3} \mathrm{H}(\mathrm{Ci})$ & ${ }^{3} \mathrm{~N}$ and ${ }^{15} \mathrm{O}(\mathrm{GBq})$ & ${ }^{3} \mathrm{~N}$ and ${ }^{15} \mathrm{O}(\mathrm{Ci})$ \\
\hline 1981 & 96,900 & 2,619 & 12,700 & 344 \\
1982 & 74,520 & 2,014 & 21,600 & 584 \\
1983 & 120,100 & 3,245 & 31,600 & 855 \\
1984 & 272,100 & 7,354 & 3,000 & 81 \\
1985 & 81,550 & 2,204 & 19,200 & 520 \\
1986 & 46,400 & 1,254 & 4,180 & 113 \\
1987 & 101,800 & 2,751 & 2,300 & 62 \\
1988 & 147,400 & 3,983 & 1,100 & 30 \\
1989 & 109,200 & 2,952 & 1,600 & 42 \\
1990 & 47,430 & 1,282 & 1,800 & 48 \\
1991 & 41,140 & 1,112 & 440 & 12 \\
1992 & 6,550 & 177 & 0 & 0 \\
\hline
\end{tabular}

Table 4-13. Nonradioactive air emissions, Livermore site and Site 300, 1992.

\begin{tabular}{lcc}
\hline & \multicolumn{2}{c}{ Estimated releases [metric tons/day] } \\
\cline { 2 - 3 } \multicolumn{1}{c}{ Pollutant } & Livermore site & Site 300 \\
\hline Carbon monoxide & 0.01632 & 0.00288 \\
Chlorofluorocarbons & 0.02886 & 0.00212 \\
Organic compounds & 0.01284 & 0.00126 \\
Oxides of nitrogen & 0.07905 & 0.00653 \\
Oxides of sulfur & 0.00219 & 0.00035 \\
Particulates & 0.00336 & 0.00054 \\
\hline
\end{tabular}




\section{Introduction}

The Livermore site discharges approximately 1.1 million liters per day of wastewater to the City of Livermore sewer system, an amount that constitutes less than $7 \%$ of the total flow to the system. This volume includes wastewater generated by Sandia National Laboratories, California (SNL/CA), which is discharged to the LLNL collection system, and combines with LLNL sewage before it is released at a single point to the municipal collection system. The wastewater contains sanitary sewage and industrial effluent and is discharged in accordance with permit requirements and the City of Livermore Municipal Code.

The effluent is processed at the Livermore Water Reclamation Plant (LWRP). As part of the Livermore-Amador Valley Wastewater Management Program, the treated sanitary wastewater is transported out of the valley through a pipeline and discharged into San Francisco Bay. A small portion of the treated effluent is used for summer irrigation of the adjacent municipal golf course. Sludge from the treatment process is disposed of in landfills.

LLNL receives water from two suppliers. During the summer months, June through August, deliveries are taken primarily from the Alameda County Flood Control and Water Quality Conservation District Zone 7. This water is a mixture of ground water and water from the South Bay Aqueduct of the State Water Project. For the remainder of the year, LLNL's water usually is supplied from the Hetch-Hetchy Aqueduct. Water quality parameters for these sources are obtained from the suppliers and are used to evaluate compliance with the discharge permit conditions that limit changes in water quality between receipt and discharge.

Administrative and engineering controls at the Livermore site prevent potentially contaminated wastewater from being discharged directly to the sanitary sewer. Waste generators receive training on proper waste handling. Environmental Protection Department personnel review facility procedures and inspect processes for inappropriate discharges. Retention tanks are used to collect wastewater from processes that might release large quantities of contaminants (to validate the integrity of this equipment, LLNL undertook an extensive evaluation, completed in 1993, of drain connections from buildings to the sanitary and storm sewer systems). Finally, to verify that training and control equipment function properly, wastewaters are sampled and analyzed both at the point of generation and at the point of discharge to the municipal sewer system. 
For facilities with installed retention tank systems, collected wastewater is discharged to the sanitary sewer only if laboratory results show that pollutant levels are within allowable limits (Grandfield 1989). LLNL has developed internal discharge guidelines for specific sources and operations to ensure that sewer effluent for the entire site complies with LLNL's waste discharge permit. If pollutant levels exceed permissible concentrations, the wastewater is treated to reduce pollutants to the lowest levels practical and below LLNL guidelines, or the wastewater is shipped to an off-site treatment or disposal facility. Liquids containing radioactivity are treated on site, using processes that reduce the activity to levels well below DOE Order 5400.5 and LWRP discharge limits.

The discharge permit requires continuous monitoring of the effluent flow rate and $\mathrm{pH}$. A flow-proportional composite sampler at the outfall collects samples that are analyzed for metals, radioactivity, toxic chemicals and water quality parameters. In addition, the outflow to the municipal collection system is sampled continuously and analyzed for conditions that may upset the LWRP treatment process or otherwise impact the public welfare. The effluent is continuously analyzed for $\mathrm{pH}$ (as mentioned above), selected metals, and radioactivity. If concentrations above warning levels are detected, an alarm is registered at the LLNL Fire Dispatcher's Station, which is attended 24 hours a day. The monitoring system provides a continuous check on sewage control and, since July of 1990, automatically notifies the LWRP in the event that contaminants are detected.

In 1991, LLNL completed construction of a diversion system that is automatically activated when unacceptable conditions are detected by the monitoring system. The diversion system ensures that all but the first few minutes of the affected wastewater is retained at LLNL, thereby protecting the LWRP and minimizing any required cleanup. Up to 775,000 liters of potentially contaminated sewage can be held pending analysis to determine the appropriate handling method. The diverted effluent may be returned to the sanitary sewer, shipped for off-site disposal, or treated at LLNL's Hazardous Waste Management Facility.

LLNL has also implemented a system of satellite monitoring stations that operates in conjunction with the sewer monitoring system (Figure 5-1). The satellite monitoring stations are positioned at strategic locations within the main sewer system to help pinpoint the area on site from which a spill has originated. Each station consists of an automatic sampler that collects samples on a timeproportional basis. In the event of a spill, these samples are analyzed.

\section{Methods}

A 24-hr composite of Livermore-site sewage effluent is collected daily by a peristaltic pump that functions for $4 \mathrm{~s}$ for every $3875 \mathrm{~L}$ of effluent. Aliquots of this composite are 
transferred to plastic bottles and submitted for analysis. Treated effluent from LWRP is collected daily by LWRP employees. Aliquots are placed in $500-\mathrm{mL}$ polyethylene bottles, which are retained for weekly collection by LLNL. Composite samples from the LWRP digesters are collected monthly. The composites consist of aliquots taken from the circulating sludge three times a week.

Standard quality control and quality assurance procedures are maintained. When each sewage field sample is collected, it is labeled with the sampling location and date of sampling. In the laboratory, each sample is assigned a number that accompanies that sample during analysis.

The daily composite samples are analyzed for gross alpha, gross beta, and tritium activity. A monthly composite of the Livermore-site and LWRP effluents is analyzed for ${ }^{137} \mathrm{Cs}$ and ${ }^{239} \mathrm{Pu}$. Weekly composites of LLNL effluent are analyzed for metals. In addition, composite samples from the LWRP digesters are analyzed monthly for gross radioactivity and metals; composites of the monthly samples are analyzed quarterly for plutonium and cesium content and for gamma-emitting radionuclides.

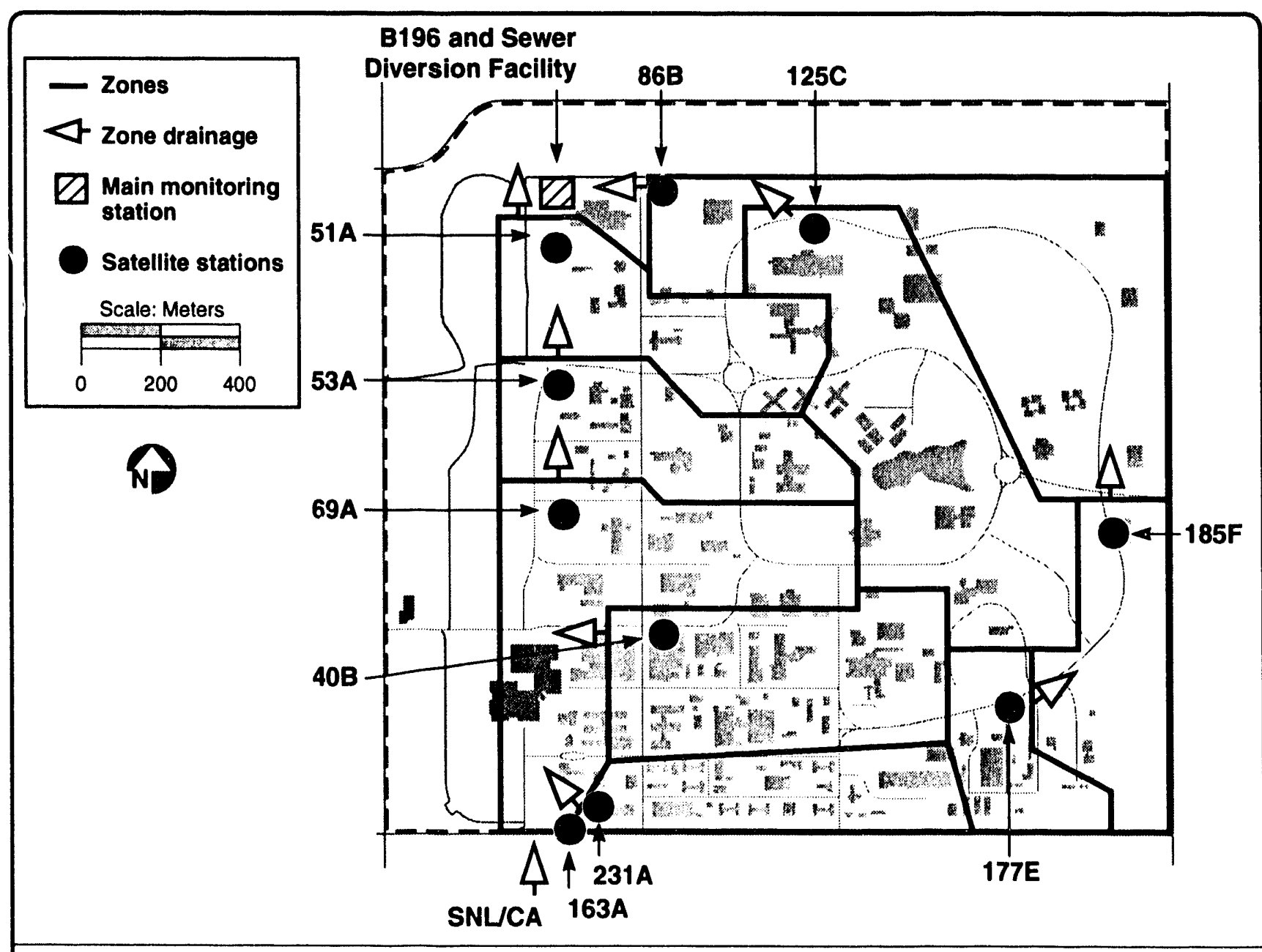

Figure 5-1. Sewer monitoring network, 1992. 
Water quality parameters and organic compounds are also monitored. Once each month, a 24-hr composite sample and an instantaneous grab sample of the LLNL sewage effluent are subjected to an extensive set of analyses. These analyses include parameters specified on LWRP's National Pollutant Discharge Elimination System permit, including metals, nutrients, pesticides, and priority pollutants. The federal priority pollutants are measured using EPA Methods 624 and 625 to establish baseline information for these parameters.

Quarterly samples also are collected at the point of discharge of specified metal finishing and electrical (and electronic) component processes to assure compliance with EPA discharge limits for those processes. LLNL reports the results of these analyses semiannually to the LWRP, the agency delegated by EPA to implement those regulations. The results are not reported here.

For those samples analyzed for gross alpha and beta activity, about $25 \mathrm{~mL}$ of an $80 \% / 20 \%$ mixture of $\mathrm{HNO}_{3}$ and $\mathrm{HClO}_{4}$ is added to an aliquot $(350 \mathrm{~mL}$ if the sample is from Livermore site, $100 \mathrm{~mL}$ if from LWRP), and the liquid is evaporated from the mixture. The residue is taken up in $10 \mathrm{~mL}$ of $\mathrm{HNO}_{3}$ and $5 \mathrm{~mL}$ of $5 \% \mathrm{H}_{2} \mathrm{O}_{2}$. The volume is reduced to 3 to $5 \mathrm{~mL}$ on a hotplate, transferred to 5 -cm-diameter planchets, and counted. The alpha yield is calibrated with ${ }^{239} \mathrm{Pu}$, and the beta yield is calibrated with ${ }^{90} \mathrm{Sr}$.

For tritium analyses, a 5-mL aliquot of distilled sewage sample is added to $15 \mathrm{~mL}$ of Packard Instagel in a 25-mL, screw-cap polyethylene bottle. Another $15 \mathrm{~mL}$ of Instagel is used as a control blank. Both the sample and the blank are placed in the refrigerated detection chamber of the scintillation counter for a 1-hr temperature equilibration prior to counting. Each sample is counted for $100 \mathrm{~min}$.

Plutonium is separated by coprecipitation with manganese dioxide $\left(\mathrm{MnO}_{2}\right)$. The $\mathrm{MnO}_{2}$ then is ashed and dissolved in $\mathrm{HNO}_{3}$ and $\mathrm{H}_{2} \mathrm{O}_{2}$. Plutonium is purified by ion exchange, electroplated onto a stainless steel disk, and submitted for alpha spectroscopy.

Cesium is separated by ion exchange with ammonium molybdophosphate (AMP). The AMP crystals are transferred to a counting vial with concentrated $\mathrm{HCl}$, which then is submitted for gamma spectroscopy.

Weekly composites of Livermore-site sewage effluent are analyzed for arsenic, cadmium, chromium, copper, iron, lead, nickel, silver, aluminum, beryllium, and zinc. The weekly composite sample is prepared by combining $500-\mathrm{mL}$ daily samples. A solution of $\mathrm{HNO}_{3}(80 \%)$ and $\mathrm{HClO}_{4}(20 \%)$ is added to the first 500 -mL daily sample of the month, and the mixture is reduced in volume to about $100 \mathrm{~mL}$. Each subsequent day, the daily sample is added and the total volume is reduced to about $100 \mathrm{~mL}$. After the $500-\mathrm{mL}$ aliquot from the last day has been added, the combined volume is reduced to about $50 \mathrm{~mL}$. When the solution is cool, about $50 \mathrm{~mL}$ of deionized water is added, and the liquid is filtered through Whatman- 40 paper. The paper is placed in a platinum crucible and digested with about $5 \mathrm{~mL}$ of $\mathrm{HF}, 16 \mathrm{~mL}$ of $\mathrm{HNO}_{3}$, and $4 \mathrm{~mL}$ of $\mathrm{HClO}_{4}$ to a residue of about 4 to $5 \mathrm{~mL}$. Water is added and the liquid is refiltered; the residue is discarded. The filtrates are combined, transferred to a $100-\mathrm{mL}$ volumetric flask, and brought to volume with deionized water. The metal content is determined by means of inductively coupled plasma (ICP) mass spectrometry. The required calibration standards are prepared by dilution of a 1000-ppm, NIST-traceable stock solution. 
For mercury analysis, $25 \mathrm{~mL}$ is taken from the daily sample and transferred to a glass bottle with a ground-glass stopper. Prior to analysis, $100 \mathrm{~mL}$ of the solution is reduced with $5 \mathrm{~mL}$ of $\mathrm{H}_{2} \mathrm{SO}_{4}$ and $5 \mathrm{~mL}$ of $\mathrm{HNO}_{3}(80 \%)$. Potassium permanganate then is added to eliminate sulfides and chlorides. The sample is heated in a water bath for $2 \mathrm{hr}$ at $95^{\circ} \mathrm{C}$. After cooling, $6 \mathrm{~mL}$ of sodium chloride-hydroxylamine sulfate reduces the excess permanganate, after which $5 \mathrm{~mL}$ of stannous sulfate is added. The resulting solution is analyzed by cold-vapor atomic absorption. The analysis is performed twice a month, to avoid exceeding EPA-prescribed holding times on the weekly samples. Each time the analysis is performed, standards are run to calibrate the method, as described above.

\section{Results}

\section{Radioactivity in Sewage}

Determination of the total radioactivity released as tritium, alpha emitters, and beta emitters is based either on the measured radioactivity in the effluent or on the limit of sensitivity, whichever is higher (see Table 5-1). The combined releases of tritium, alpha, and beta radiation $-8.0 \mathrm{GBq}\left(\mathrm{GBq}=10^{9} \mathrm{~Bq}\right)$, or $0.22 \mathrm{Ci}$-were $22 \%$ of the California Title 17 limit, which is $37 \mathrm{GBq}$ (Table $\mathbf{5 - 2}$ summarizes regulatory limits applied to the discharge of radionuclides in LLNL sewage). The total is based on the results shown in Table 5-1, reduced by reported SNL/CA tritium releases of $2.2 \mathrm{GBq}(0.06 \mathrm{Ci})$. Figure 5-2 shows the daily concentration of tritium for 1992 . The concentrations of ${ }^{239} \mathrm{Pu},{ }^{137} \mathrm{Cs}$, and tritium measured in the sanitary sewer effluent from LLNL and LWRP are presented in Table 5-3. During 1992, there were no inadvertent releases that exceeded any discharge limits for release of radioactive materials to the sanitary sewer system.

The annual average concentration of tritium in LLNL sanitary sewer effluent was $0.019 \mathrm{~Bq} / \mathrm{mL}(0.7 \mathrm{pCi} / \mathrm{mL}$ ), about $0.0037 \%$ (that is, 0.000037$)$ of the DOE Derived Concentration Guide (DCG) to a public sewer system that, if exceeded, requires the application of best available technology, or BAT (facilities that exceed the DOE DCG over the course of a month must evaluate the need to implement additional control measures to reduce effluent concentrations). The daily concentration presented in Figure 5-2 was multiplied by the volume of sewage released on that day and then summed to determine the total radiation released in the form of tritium, as given in Table 5-1. The annual average concentration of ${ }^{137} \mathrm{Cs}$ was $5.4 \mu \mathrm{Bq} / \mathrm{mL}\left(1.4 \times 10^{-4} \mathrm{pCi} / \mathrm{mL}\right)$, only 0.0000045 of the DOE DCG. The annual average ${ }^{239} \mathrm{Pu}$ concentration was $5.2 \mu \mathrm{Bq} / \mathrm{mL}$ $\left(1.4 \times 10^{-4} \mathrm{pCi} / \mathrm{mL}\right)$, which was 0.000014 of the DOE DCG. Figure 5-3 shows the average monthly plutonium and cesium concentrations in sewage since 1973. 


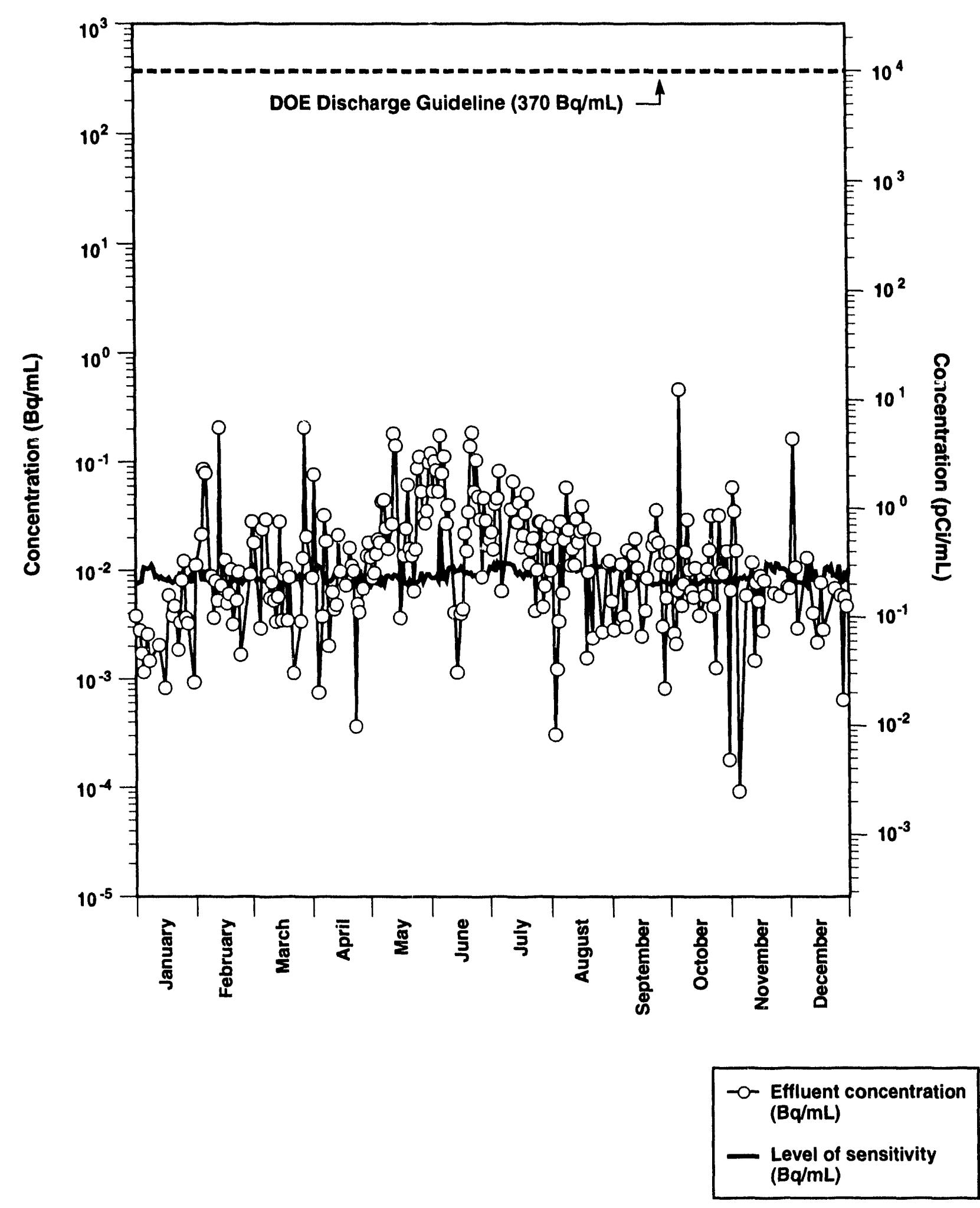

Figure 5-2. LLNL tritium concentration in sewage effluent, 1992. Values below the level of sensitivity are possibly due to analytical uncertainty rather than the presence of tritium. 


\section{Nonradioactive Pollutants in Sewage}

Table 5-4 presents monthly average metal concentrations in LLNL's sanitary sewer effluent. At the bottom of the table, the annual average concentration for each metal is compared to the discharge limit. The metals that approached closest to the discharge limits were mercury, lead and copper at $14 \%, 13 \%$, and $8 \%$, respectively, of the discharge concentrations. Results of monthly monitoring for metals and other physical and chemical characteristics of the sanitary sewer effluent are provided in Table 5-5. With the exception of the oil and grease results, the results are all well below the pollutant limitations stipulated in the discharge permit. The oil and grease results are discussed in the following section.

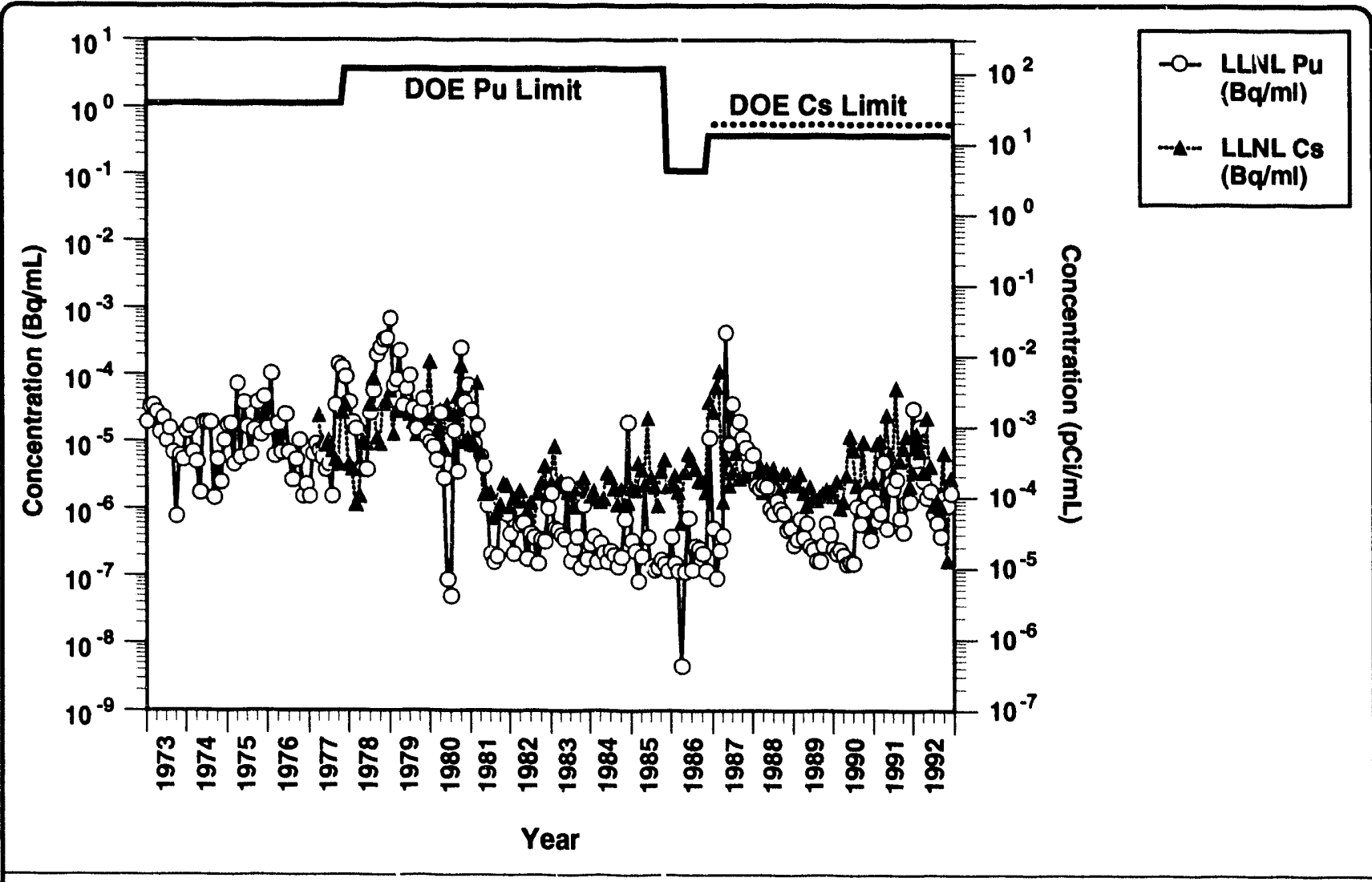

Figure 5-3. LLNL monthly average plutonium and cє sium concentrations in sewage. 


\section{Environmental Impact}

\section{Radioactivity in Sewage}

To prevent discharges of radioactive materials to the sanitary sewer, LLNL limits the use of those materials and implements engineering controls to prevent the release of radioactivity to the environment. When a process does noi lend itself to such controls, or when an extra measure of security is warranted, liquid effluents with potential for containing radioactive materials are not discharged directly into the sanitary sewer but are routed to retention tanks. The retention tanks collect potentially contaminated liquids until a thorough characterization can be performed. When a retention tank becomes full, a sample of the liquid is analyzed to determine pollutant concentrations. If necessary, the wastewater can be treated at LLNL's Hazardous Waste Management Facility to filter out radioactivity to the lowest levels reasonably achievable, and well below discharge limits. Once treated and below accepiable limits (verified by resampling and analysis), the liquid waste is discharged to the City of Livermore sanitary sewer system.

DOE requires that radiological releases to the sanitary sewer comply with local and state regulations. The most stringent of these limits is applied by 17 CCR. Furthermore, DOE has established criteria for the application of BAT to protect public health adequately and minimize degradation of the environment.

Table 5-6 summarizes the radioactivity in liquid effluent released over the past nine years. During 1992, a total of $8.0 \mathrm{GBq}(0.22 \mathrm{Ci})$ of tritium was discharged to the sanitary sewer. This is the combined release from the Livermore site and from SNL/CA, whose records account for $2.2 \mathrm{GBq}(0.06 \mathrm{Ci})$ of this amount; LLNL therefore released $5.8 \mathrm{GBq}(0.16 \mathrm{Ci})$, an amount that is well within environmental protection standards established by DOE and is less than the range reported in the past.

In 1992, the total LLNL releases, in the form of alpha and beta emitters (including tritium), were $16 \%$ of the $17 \mathrm{CCR}$ limit, which is $37 \mathrm{GBq}$. For specific isotopes, the average tritium concentration was 0.0000037 of the DOE DCG, while the average ${ }^{239} \mathrm{Pu}$ and ${ }^{137} \mathrm{Cs}$ discharges were 0.000014 and 0.0000045 of the DOE DCG.

Figure 5-3 summarizes the ${ }^{239} \mathrm{Pu}$ monitoring data since 1973 . The historical levels observed since 1981 are approximately $0.37 \mathrm{~Bq} / \mathrm{mL}\left(1 \times 10^{-5} \mathrm{pCi} / \mathrm{mL}\right)$, with the exception of a peak in 1987. Even this peak is well below the applicable DOE DCG. Historically, levels generally are one-millionth $(0.000001)$ of that limit. The greatest part of the plutonium discharged in LLNL effluent is ultimately concentrated in LWRP sludge, which is dried and disposed of at a landfill. The plutonium concentration observed in 1992 sludge, $15.2 \mathrm{mBq} / \mathrm{dry} \mathrm{g}$ 
$(0.56 \mathrm{pCi} / \mathrm{dry} \mathrm{g})$, is 30 times lower than the proposed EPA guideline for unrestricted use of soil ( $480 \mathrm{mBq} /$ dry g).

As first discussed in the Environmental Report for 1991 (Gallegos et al. 1992a), concentrations of plutonium and cesium remain slightly elevated over the lowest values seen historically. As was established in 1991, the overall upward trend is related to sewer cleaning with new, more effective equipment. Cleaning activity around the site was extensive during 1992, as utilities personnel worked to complete their assessment of the condition of the sewer system.

Along with this observation, however, the measured plutonium activities were further elevated during the months of January through March. For those three months, the data show an increase of a factor of 10 above values seen during 1991 and the other months of 1992. The effects of this discharge are visible in the activities measured in LWRP process streams (effluent and sludge). The highest activities observed in LWRP sludge are more than 10 times below the proposed EPA guideline for the unrestricted use of soil, and the levels observed in LLNL effluent are 20 times below the most applicable drinking water standard (for alpha radiation). Because of the longer retention times in the LWRP sludge digesters (roughly 30 days), slighily higher activities were seen in LWRP sludge through the second quarter of the year.

LLNL has investigated possible causes for the elevated levels, including inadvertent releases from LLNL operations, releases from retention tanks and sewer cleaning operations. No definite determination of the cause could be made. Retention tank releases could account for, at most, $10 \%$ of the plutonium discharged. Sewer cleaning was scattered across the LLNL site during this period, but only one location was cleaned during all three months. The cleaning at that location was necessitated by pipe degradation, and that section of the sewerage was replaced in April 1992. The higher levels could also be related to elevated levels of alpha radioactivity detected in LLNL's effluent on several days during the first quarter of 1992.

Small releases of alpha radiation are observed in LLNL sewage several times each year, but do not usually correlate with any increase in plutonium. However, in order to better understand occasional discharges of alpha-emitting radioactivity above routine levels, a protocol has been developed for further analysis when higher levels are seen. The information obtained-the specific radioisotopes detected in a release-will be used to achieve a greater understanding of these discharges. Should any specific radioisotope be commonly observed in alpha radiation releases, LLNL personnel will be able to focus their efforts to decrease future discharges of that type. The program of extended evaluation of alpha radiation releases was initiated in 1993, but L.LNL will analyze archived samples held from the first quarter of 1992. 


\section{Nonradioactive Liquid Effluents}

For the year as a whole, the monitoring data reflect the success of LLNL's discharge control program in preventing any significant impact on the operations of the City's treatment plant. LLNL was issued a single Notice of Violation during 1992, for elevated concentrations of oil and grease seen in the monthly grab sample taken in May. Examination of the historical record indicates that oil and grease concentrations in sanitary sewage began to rise in the summer of 1990. This correlates with improvements to the sewer maintenance program, which led to the liberation of historical residue in the sewers. In addition, evaluations by discharge control personnel have identified deficiencies in the design of grease abatement measures in use at LLNL cafeterias. Improvements have been designed and scheduled for implementation.

As a result of this incident, the LWRP suggested changes to LLNL's sampling procedure for oil and grease monitoring. The LWRP was concerned that a single grab sample would not adequately characterize the average concentration of oil and grease in LLNL effluent. Because the LWRP is primarily concerned with controlling the total daily inflow to the plant, they requested that LLNL institute a more comprehensive sampling protocol for oil and grease. In response, LLNL now collects four oil and grease samples at 4-hour intervals, beginning at 6 a.m. and ending at 6 p.m. The sampling times are distributed so that periods of peak and low flow are represented proportionately in the total volume of wastewater discharged during each period. The results for the four samples can therefore be averaged to produce a measure of the average daily oil and grease discharge.

Table 5-7 summarizes the incidents during 1992 when LLNL's continuous monitoring system identified instantaneous excursions of pollutant concentrations above alarm limits. A total of ten incidents was reported to the LWRP and DOE during 1992. For incidents that lasted more than 5 minutes, the Sewer Diversion Facility was activated to contain the remainder of the release. In all cases, later analysis of the diverted effluent showed that the average concentration of the contaninant was sufficiently low to allow release of the wastewater back to the sanitary sewer. In no case did an incident represent a threat to the integrity of operations at the LWRP.

Examination of the list of incidents reveals a marked difference between the first and second halves of the year. During the first six months of 1992, the recorded incidents were quite similar to the kinds of releases that dominated the list of events in 1991. During April, it was determined that the bulk of the zinc discharges was probably caused by use of a common household cleaner that contained zinc in high concentrations as an artifact of the manufacturing process. The second most frequent type of alarm-brief releases of silver-ceased recurring in June. Continued reviews of silver processing operations by environmental operations analysts, identification of potential sources during a 
comprehensive inventory and evaluation of sanitary sewer connections, a poster campaign targeted to reduce silver discharges, and a general laboratory information campaign successfully sensitized personnel as to the importance of controlling silver discharges.

In addition to showing success in controlling zinc and silver discharges, the list in Table 5-7 indicates that the magnitude and seriousness of the releases decreased in the second half of the year. The instantaneous concentrations observed were only marginally higher than the discharge guidelines. More than the absolute numerical reduction in the number of events (down from 14 observed during 1991), the decrease in the significance of the incidents indicates the responsiveriess of LLNL programs and personnel to the need to control the discharge of pollutants to the sanitary sewer. 
Table 5-1. Estimated total radioactivity in sanitary sewer effluent, LLNL, 1992.

\begin{tabular}{lcc}
\hline Radioactive emitter & $\begin{array}{c}\text { Estimate based on effluent } \\
\text { concentration [GBq] }\end{array}$ & $\begin{array}{c}\text { Limit of sensitivity } \\
{[\mathrm{GBq}]^{\mathrm{a}}}\end{array}$ \\
\hline Tritium & $7.9^{\mathrm{b}, \mathrm{c}}$ & 3.7 \\
Alpha radiation & 0.026 & $0.041^{\mathrm{b}}$ \\
Beta radiation & $0.26^{\mathrm{b}}$ & 0.06 \\
\hline
\end{tabular}

a $\mathrm{GBq}=10^{9} \mathrm{~Bq}$, or $0.028 \mathrm{pCi}$.

b Used for determination of the ha al radiation release because it is the more conservative value.

c $7.9 \mathrm{GBq}$ includes $5.7 \mathrm{GBq}$ from LLNL plus $2.2 \mathrm{GBq}$ from SNUCA.

Table 5-2. Sewer discharge release limits for tritium $\left({ }^{3} \mathrm{H}\right),{ }^{137} \mathrm{Cs}$, and $239 \mathrm{Pu}$.

\begin{tabular}{|c|c|c|c|c|}
\hline & & $3 \mathbf{H}$ & ${ }^{137} \mathrm{Cs}$ & ${ }^{239} \mathrm{Pu}$ \\
\hline \multicolumn{2}{|c|}{$\begin{array}{l}\text { CA Title } 17 \text { concentrations used } \\
\text { to establish release limits }\end{array}$} & $3.7 \times 10^{3} \mathrm{~Bq} / \mathrm{mL}$ & $15 \mathrm{~Bq} / \mathrm{mL}$ & $30 \mathrm{~Bq} / \mathrm{mL}$ \\
\hline \multirow[t]{3}{*}{ CA Title 17} & daily & $37 \mathrm{GBq}^{\mathrm{a}}$ & $16 \mathrm{GBq}^{\mathrm{a}}$ & $32 \mathrm{GBq}^{\mathrm{a}}$ \\
\hline & monthly & $37 \mathrm{GBq}^{\mathrm{b}}$ & $37 \mathrm{GBq}^{\mathrm{b}}$ & $37 \mathrm{GBq}^{\mathrm{b}}$ \\
\hline & yearly & $37 \mathrm{GBq}^{\mathrm{a}}$ & $37 \mathrm{GBq}^{\mathrm{a}}$ & $37 \mathrm{GBq}^{\mathrm{a}}$ \\
\hline \multicolumn{2}{|c|}{$\begin{array}{l}\text { DOE annualized discharge limit } \\
\text { for application of BATC }\end{array}$} & $370 \mathrm{~Bq} / \mathrm{mL}$ & $0.55 \mathrm{~Bq} / \mathrm{mL}$ & $0.37 \mathrm{~Bq} / \mathrm{mL}$ \\
\hline
\end{tabular}

a CA Title 17 regulations impose a 37-GBq combined limit on the total of all radiation released, i.e., the total release of all isotopes must not exceed $37 \mathrm{GBq}$. If a total of $37 \mathrm{GBq}$ of a particular isotope was released during the year, this would require that no other isotopes be released.

b Limits similar to those applied to daily releases except total releases for the month must not exceed these quantities.

c The DOE annualized discharge limit for application of Best Available Technology is five times the DCG (ingested water) for each radionuclide released. 
Table 5-3. Various radionuclides in sanitary sewer effluents (in $10^{-6} \mathrm{~Bq} / \mathrm{mL}$ ), LLNL and LWRP, 1992.

\begin{tabular}{|c|c|c|c|c|c|c|c|}
\hline \multirow[b]{2}{*}{ Month } & \multicolumn{2}{|c|}{$\begin{array}{c}{ }^{3} \mathrm{H} \\
(\mathrm{mBg} / \mathrm{mL})\end{array}$} & \multicolumn{2}{|c|}{$\begin{array}{c}137 \mathrm{Cs} \\
(\mu \mathrm{Bg} / \mathrm{mL})\end{array}$} & \multicolumn{2}{|c|}{$\begin{array}{c}{ }^{239} \mathrm{Pu} \\
(\mu \mathrm{Bg} / \mathrm{mL})\end{array}$} & \multirow{2}{*}{ 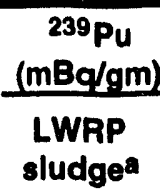 } \\
\hline & LLNL & LWRP & LLNL. & LWRP & LLNL & LWRP & \\
\hline Jan. & $<8.7$ & $<9.3$ & $3.3 \pm 0.7$ & $0.4 \pm 0.1$ & $30 \pm 1$ & $0.087 \pm 0.023$ & \\
\hline Feb. & $21 \pm 3$ & $<7.9$ & $12.6 \pm 0.9$ & $1.2 \pm 0.5$ & $5.2 \pm 0.2$ & $0.25 \pm 0.04$ & \\
\hline Mar. & $14 \pm 2$ & $<8.5$ & $6.9 \pm 0.8$ & $0.5 \pm 0.2$ & $16 \pm 1$ & $0.062 \pm 0.019$ & $29.7 \pm 1.3$ \\
\hline Apr. & $10 \pm 2$ & $<8.6$ & $3.4 \pm 0.6$ & $<0.4$ & $2.5 \pm 0.1$ & $0.021 \pm 0.009$ & \\
\hline May & $38 \pm 3$ & $<9.2$ & $22 \pm 3$ & $<1.1$ & $1.4 \pm 0.2$ & $0.013 \pm 0.013$ & \\
\hline June & $47 \pm 4$ & $<9.6$ & $4.2 \pm 0.6$ & $<3.9$ & $1.8 \pm 0.2$ & $<0.037$ & $20.9 \pm 0.8$ \\
\hline July & $20 \pm 3$ & $<10.1$ & $1.1 \pm 0.3$ & $<0.2$ & $0.8 \pm 0.1$ & $<0.013$ & \\
\hline Aug. & $12 \pm 2$ & $<10.0$ & $1.3 \pm 0.3$ & $<0.4$ & $0.59 \pm 0.09$ & $<0.020$ & \\
\hline Sept. & $<8.6$ & $<8.3$ & $1.0 \pm 0.3$ & $<0.7$ & $0.37 \pm 0.07$ & $<0.016$ & $6.0 \pm 0.5$ \\
\hline Oct. & $31 \pm 3$ & $<8.0$ & $6.5 \pm 0.8$ & $0.6 \pm 0.4$ & $1.4 \pm 0.2$ & $<0.023$ & \\
\hline Nov. & $<9.1$ & $<8.6$ & $0.16 \pm 0.05$ & $<0.4$ & $1.1 \pm 0.1$ & $<0.016$ & \\
\hline Dec. & $9 \pm 2$ & $<8.7$ & $2.7 \pm 0.5$ & $<0.2$ & $1.6 \pm 0.1$ & $<0.012$ & $4.1 \pm 0.2$ \\
\hline Mean & 18 & $<8.7^{b}$ & 5.4 & $<0.5^{b}$ & 5.2 & \multirow[t]{2}{*}{$<0.021^{b}$} & 15.2 \\
\hline \multirow[t]{2}{*}{ Std dev } & 27 & & 12 & & 17.7 & & 24.5 \\
\hline & \multicolumn{6}{|c|}{$\mathrm{pCi} / \mathrm{mL}$} & pCi/dry g \\
\hline Mean & 0.49 & $<0.24^{b}$ & $145 \times 10^{-6}$ & $<14 \times 10^{-6}$ & $140 \times 10^{-6}$ & \multirow[t]{2}{*}{$<0.6 \times 10^{-6}$} & 0.41 \\
\hline \multirow[t]{3}{*}{ Std dev } & 0.71 & & $331 \times 10^{-6}$ & & $480 \times 10^{-6}$ & & 0.66 \\
\hline & & \multicolumn{4}{|c|}{ Annual total discharges by radioisotope } & \multirow{2}{*}{\multicolumn{2}{|c|}{${ }^{239} \mathrm{Pu}$}} \\
\hline & & & ${ }^{3} \mathbf{H}$ & & 137 Cs & & \\
\hline \multicolumn{2}{|l|}{ Bq/y } & \multicolumn{2}{|r|}{$5.7 \times 109^{c}$} & \multicolumn{2}{|c|}{$2.2 \times 10^{6}$} & \multicolumn{2}{|c|}{$1.9 \times 10^{6}$} \\
\hline \multicolumn{2}{|c|}{$\mathrm{Ci} / \mathrm{y}$} & \multicolumn{2}{|r|}{0.15} & \multicolumn{2}{|c|}{$6.0 \times 10^{-5}$} & \multicolumn{2}{|c|}{$5.1 \times 10^{-5}$} \\
\hline Fraction of & & & & & & & \\
\hline DOE & & & 0.000036 & & 0000045 & 0.00 & 0141 \\
\hline Title 17 & & & $15^{d}$ & & & & \\
\hline
\end{tabular}

Note: Radionuclide results are reported $\pm 2 \sigma$; se日 Quality Assurance chapter.

a Sludge from LWRP digesters is dried prior to analysis. The resulting data indicate the plutonium concentration in the sludge prepared by LWRP workers for disposal at the Livermore Sanitary Landfill.

b Due to the large number of nondelects, the median rather than the mean is presented, and the standard deviation from the mean is omitted. See Quality Assurance chapter.

c Not including discharges of $2.2 \times 10^{9} \mathrm{~Bq}(0.06 \mathrm{Ci})$.

d Including ${ }^{137} \mathrm{Cs}$ and ${ }^{239} \mathrm{Pu}$. 
Table 5-4. Metals discharged to sanitary sewer system (in $\mathrm{mg} / \mathrm{L}$ ), 1992 summary.

\begin{tabular}{|c|c|c|c|c|c|c|c|c|c|c|c|c|}
\hline Month & $\mathbf{A g}$ & Al & As & $\mathrm{Be}$ & Cd & $\mathrm{Cr}$ & $\mathrm{Cu}$ & $\mathrm{Fe}$ & $\mathrm{Hg}$ & $\mathrm{Ni}$ & $\mathrm{Pb}$ & $\mathbf{Z n}$ \\
\hline Jan. & 0.022 & 0.58 & $<0.002$ & $<0.0002$ & 0.0014 & 0.033 & 0.05 & 1.14 & $<0.0015$ & 0.014 & $<0.014$ & 0.31 \\
\hline Feb. & 0.012 & 1.75 & $<0.002$ & $<0.0001$ & 0.0013 & 0.030 & $<0.012$ & 1.60 & 0.0020 & 0.016 & $<0.08$ & 0.25 \\
\hline Mar. & 0.010 & 0.37 & $<0.004$ & $<0.0001$ & 0.0011 & 0.061 & 0.10 & 1.03 & 0.0037 & 0.016 & $<0.019$ & 0.36 \\
\hline Apr. & 0.014 & 0.62 & $<0.002$ & $<0.0001$ & 0.0012 & 0.045 & 0.14 & 1.18 & 0.0015 & 0.017 & 0.066 & 0.30 \\
\hline May & 0.010 & 0.39 & $<0.002$ & $<0.0001$ & 0.0008 & 0.028 & 0.08 & 0.89 & $<0.0008$ & 0.005 & 0.016 & 0.22 \\
\hline June & 0.017 & 0.32 & $<0.002$ & $<0.0001$ & 0.0009 & 0.025 & 0.08 & 1.14 & 0.0006 & 0.005 & 0.019 & 0.18 \\
\hline July & 0.009 & 0.24 & $<0.002$ & $<0.0001$ & 0.0012 & 0.020 & 0.05 & 0.65 & $<0.0005$ & 0.004 & 0.023 & 0.11 \\
\hline Aug. & 0.011 & 0.30 & $<0.002$ & $<0.0001$ & 0.0007 & 0.022 & 0.05 & 0.67 & 0.0006 & 0.004 & 0.017 & 0.15 \\
\hline Sep. & 0.010 & 0.27 & $<0.002$ & $<0.0001$ & 0.0015 & 0.024 & 0.06 & 0.77 & $<0.0005$ & 0.004 & 0.016 & 0.15 \\
\hline Oct. & 0.013 & 0.37 & $<0.004$ & $<0.0001$ & 0.0007 & 0.030 & 0.07 & 1.05 & 0.0010 & 0.005 & 0.016 & 0.13 \\
\hline Nov. & 0.016 & 1.00 & $<0.003$ & $<0.0001$ & 0.0012 & 0.028 & 0.08 & 3.07 & 0.0009 & 0.015 & 0.017 & 0.15 \\
\hline Dec. & 0.013 & 0.54 & $<0.002$ & $<0.0001$ & 0.0006 & 0.034 & 0.07 & 1.1 & 0.0015 & 0.010 & 0.011 & 0.22 \\
\hline Mean & 0.013 & 0.57 & $<0.002$ & $<0.0001$ & 0.0010 & 0.031 & 0.08 & 1.19 & 0.0014 & 0.009 & 0.025 & 0.21 \\
\hline SDM & 0.005 & 0.87 & - & - & 0.0007 & 0.019 & 0.03 & 1.2 & 0.0014 & 0.007 & 0.013 & 0.09 \\
\hline DCL'b & 0.2 & $-^{c}$ & 0.06 & $-c$ & 0.14 & 0.62 & 1.0 & $-^{c}$ & 0.01 & 0.62 & 0.2 & 3.0 \\
\hline $\begin{array}{l}\text { Fraction } \\
\text { of } \mathrm{DCL}\end{array}$ & 0.065 & $-c$ & $<0.03$ & $-^{c}$ & 0.007 & 0.05 & 0.08 & $-c$ & 0.14 & 0.014 & 0.13 & 0.07 \\
\hline
\end{tabular}

a The number presented as a mean is the median of the annual values. Due to the large number of nondetects, the standard deviation could not be calculated for these analytes. See Quality Assurance chapter.

b DCL is Discharge Concentration Limit (City of Livermore Ordinance 13.32).

c No established limit for analyte. 
Table 5-5. Positively detected parameter/s in LLNL sanitary sewer effluent, 1992.

\begin{tabular}{|c|c|c|c|c|c|}
\hline Positively detected parameter & $\begin{array}{l}\text { Detection } \\
\text { frequency }\end{array}$ & Minimum & Maximum & Meanb & $\begin{array}{l}\text { Std } \\
\text { devb }\end{array}$ \\
\hline \multicolumn{6}{|l|}{ Physical and chemical (mg/L) } \\
\hline Biochemical oxygen demand & $12 / 12$ & 58 & 630 & 177 & 152 \\
\hline Chemical oxygen demand & $12 / 12$ & 70 & 560 & 300 & 132 \\
\hline Settlable solids (mLL) & $5 / 12$ & $<0.1$ & 17 & $<0.1$ & \\
\hline Total dissolved solids & $12 / 12$ & 160 & 660 & 328 & 134 \\
\hline Total solids & $12 / 12$ & 260 & 630 & 412 & 115 \\
\hline Total suspended solids & $12 / 12$ & 38 & 300 & 80 & 73 \\
\hline Volatile solids & $12 / 12$ & 26 & 294 & 163 & 66 \\
\hline Ammonia nitrogen (as N) & $12 / 12$ & 18 & 43 & 34 & 7 \\
\hline Chloride & $12 / 12$ & 2 & 220 & 56 & 54 \\
\hline Cyanide & $0 / 12$ & $<0.01$ & $<0.02$ & & \\
\hline Nitrate plus nitrite (as N) & $4 / 12$ & $<0.1$ & 5.9 & $<0.1$ & \\
\hline Nitrate plus nitrite (as $\mathrm{NO}_{3}$ ) & $4 / 12$ & $<0.4$ & 26 & $<0.4$ & \\
\hline Nitrite nitrogen (as N) & $7 / 12$ & $<0.01$ & 1.6 & 0.16 & 0.45 \\
\hline Nitrite nitrogen (as $\mathrm{NO}_{2}$ ) & $7 / 12$ & $<0.01$ & 5.3 & 0.53 & 1.5 \\
\hline Sulfate & $12 / 12$ & 15 & 130 & 49 & 39 \\
\hline Bicarbonate alkalinity (as $\mathrm{CaCO}_{3}$ ) & $12 / 12$ & 120 & 210 & 184 & 30 \\
\hline Total Kjeldahl nitrogen (as N) & $12 / 12$ & 22 & 54 & 40 & 8 \\
\hline Total phosphorus (as P) & $12 / 12$ & 2.8 & 8.2 & 5.6 & 1.4 \\
\hline Arsenic & $5 / 12$ & $<0.002$ & 0.0064 & $<0.002$ & \\
\hline Boron & $10 / 12$ & $<0.2$ & 0.54 & 0.33 & 0.12 \\
\hline Cadmium & $11 / 12$ & $<0.0005$ & 0.0015 & 0.0008 & 0.0003 \\
\hline Calcium & $12 / 12$ & 10 & 35 & 18 & 8 \\
\hline Chromium & $12 / 12$ & 0.614 & 0.18 & 0.045 & 0.048 \\
\hline Copper & $12 / 12$ & 0.044 & 0.26 & 0.083 & 0.059 \\
\hline Lead & $12 / 12$ & 0.006 & 0.019 & 0.014 & 0.004 \\
\hline Magnesium & $12 / 12$ & 2.2 & 7.9 & 4.9 & 2.4 \\
\hline Mercury & $11 / 12$ & $<0.0002$ & 0.0021 & 0.0008 & 0.0006 \\
\hline Nickel & $9 / 12$ & $<0.005$ & 0.032 & 0.009 & 0.008 \\
\hline Potassium & $12 / 12$ & 10 & 25 & 17 & 4 \\
\hline Silver & $12 / 12$ & 0.008 & 0.031 & 0.015 & 0.007 \\
\hline Sodium & $12 / 12$ & 26 & 200 & 59 & 47 \\
\hline Zinc & $12 / 12$ & 0.11 & 0.52 & 0.21 & 0.12 \\
\hline \multicolumn{6}{|l|}{ Organic compounds (mg/L) } \\
\hline Oil and grease & $12 / 12$ & 13 & 300 & 79 & 93 \\
\hline Phenolics & $12 / 12$ & 0.01 & 0.12 & 0.05 & 0.03 \\
\hline Total organic carbon & $12 / 12$ & 23 & 84 & 48 & 21 \\
\hline
\end{tabular}


Table 5-5. Positively detected parameters in LLNL sanitary sewer effluent, 1992 (concluded) .

\begin{tabular}{|c|c|c|c|c|c|}
\hline Positively detected parameter & $\begin{array}{l}\text { Detection" } \\
\text { frequency }\end{array}$ & Minimum & Moximum & Meanb & $\begin{array}{l}\text { Std } \\
\text { devb }\end{array}$ \\
\hline \multicolumn{6}{|c|}{ Organochloride pesticides (EPA Method 608, $\mu g \Omega$ ) } \\
\hline Aldrin & $1 / 9$ & $<0.04$ & 0.12 & $<0.04$ & \\
\hline $\mathrm{BHC}$, beta isomer & $1 / 9$ & $<0.03$ & 0.042 & $<0.03$ & \\
\hline BHC, delta isomer & $1 / 9$ & $<0.03$ & 0.13 & $<0.03$ & \\
\hline Endrin & $1 / 12$ & $<0.05$ & 0.062 & $<0.05$ & \\
\hline \multicolumn{6}{|c|}{ Purgeable-extractable pollutants (EPA Methods 624625, $\mu \mathrm{g} /$ ) } \\
\hline 1,1,1-Trichloroethane & $3 / 12$ & $<0.5$ & 1 & $<0.5$ & \\
\hline 1,1-Dichloroethane & $1 / 12$ & $<0.5$ & 32 & $<0.5$ & \\
\hline 1,2-Dichlorobenzene & $2 / 12$ & $<0.5$ & 5.4 & $\varangle 0.5$ & \\
\hline 1,4-Dichlorobenzene & $5 / 12$ & $<0.5$ & 8.8 & $<0.5$ & \\
\hline 2-Butoxyethanol & $1 / 12$ & - & $600 d$ & - & \\
\hline 4-Methylphenol & $8 / 12$ & $<4$ & 70 & 30 & 21 \\
\hline Acetone & $7 / 12$ & $<5$ & 1900 & 173 & 544 \\
\hline Benzene acetic acid & $1 / 12$ & - & $100 d$ & - & \\
\hline Benzoic acid & $8 / 12$ & $<10$ & 750 & 340 & 275 \\
\hline Benzyl alchohol & $5 / 12$ & $<10$ & 200 & $<100$ & \\
\hline Bis(2-ethylhexyl)phthalate & $4 / 12$ & $<4$ & 23 & $<23$ & \\
\hline Bromodichloromethane & $1 / 12$ & $<0.5$ & 0.7 & $<: 0.5$ & \\
\hline Butoxy ethanol phosphate & $3 / 12$ & - & $700 d$ & - & \\
\hline Butylbenzylphthalate & $1 / 12$ & $<3$ & 4 & $<12$ & \\
\hline Butyl carbitol & $1 / 12$ & - & $80^{d}$ & - & \\
\hline Carbon disulfide & $1 / 12$ & $<0.5$ & 180 & $<0.5$ & \\
\hline Chloroform & $12 / 12$ & 4.5 & 32 & 19 & 6.5 \\
\hline Sholestanol & $10 / 12$ & - & $1000^{d}$ & 438 & 309 \\
\hline Cholesten-3-ol & $10 / 12$ & - & $700^{d}$ & 375 & 232 \\
\hline Diethylphthalate & $1 / 12$ & $<3$ & 10 & $<15$ & \\
\hline Freon-113 & $4 / 12$ & $<0.5$ & 150 & $<0.5$ & \\
\hline Methylene chloride & $3 / 12$ & 1.3 & 100 & $<2.3$ & \\
\hline Phenol & $2 / 11$ & $<5$ & 8 & $<14$ & \\
\hline Tetrachloroethene & $1 / 12$ & $<0.5$ & 0.63 & $<0.5$ & \\
\hline Thiobismethane & $3 / 12$ & - & 8 & - & \\
\hline Toluene & Tiiz & $<0.5$ & 51 & 5.2 & 14 \\
\hline Total xylene isomers & $1 / 12$ & $<0.5$ & 1 & $<0.5$ & \\
\hline Trichloroethene & $1 / 12$ & $<0.5$ & 0.5 & $<0.5$ & \\
\hline Trichlorofluoromethane & $2 / 12$ & $<0.5$ & 2.7 & $<0.5$ & \\
\hline
\end{tabular}

a The number of times an analyte was positively identified, followed by the number of samples that were analyzed (generally 12 , one sample for each month of the year).

b Where the detection frequency is less than $50 \%$, the median is presented and the standard deviation from the mean is omitted.

c The samples were analyzed for the full range of EPA Method 608 organochloride compounds. Concentrations of all these compounds except those listed were below detection limits.

- Semiquantified resist. The analytical laboratory soes not usually report a result for this compound, and no detection limit has been established. 
Table 5-6. Radioactive liquid effluent releases from the Livermore site, 19831992.

\begin{tabular}{lcc}
\hline & \multicolumn{2}{c}{ Liquid effluents (GBq) } \\
\cline { 2 - 3 } Year & 3H (LLNL and SNLCA) & $239 \mathrm{Pu}$ \\
\hline 1983 & 63 & $2.5 \times 10^{-4}$ \\
1984 & 63 & $1.2 \times 10^{-4}$ \\
1985 & 133 & $1.8 \times 10^{-4}$ \\
1986 & 74 & $5.5 \times 10^{-4}$ \\
1987 & 52 & $2.6 \times 10^{-2}$ \\
1988 & 56 & $8.1 \times 10^{-4}$ \\
1989 & 59 & $1.8 \times 10^{-4}$ \\
1990 & 25 & $2.3 \times 10^{-4}$ \\
1991 & 32 & $6.1 \times 10^{-4}$ \\
1992 & 8 & $1.9 \times 10^{-3}$ \\
\hline
\end{tabular}

Table 5-7. Summary of contaminant releases detected by the sewer monitoring system, 1992.

\begin{tabular}{lcccc}
\hline Date & Contaminant & $\begin{array}{c}\text { Estimated } \\
\text { duration } \\
(\mathbf{m i n})\end{array}$ & $\begin{array}{c}\text { Instantaneous } \\
\text { concentration } \\
(\mathbf{m g} / \mathbf{L})\end{array}$ & $\begin{array}{c}\text { Discharge } \\
\text { standard" } \\
(\mathbf{m g} / \mathbf{L})\end{array}$ \\
\hline $\mathbf{3 / 1 9}$ & $\mathrm{Ag}$ & 5 & 5.6 & 0.2 \\
$\mathbf{4 / 2}$ & $\mathrm{Zn}$ & 20 & 12.6 & 3.0 \\
$\mathbf{4 / 1 1}$ & $\mathrm{Zn}$ & 5 & 8.8 & 3.0 \\
$\mathbf{4 / 1 3}$ & $\mathrm{Zn}$ & 30 & 19.7 & 3.0 \\
$\mathbf{6 / 1 1}$ & $\mathrm{Ag}$ & 15 & 22.2 & 0.2 \\
$\mathbf{6 / 3 0}$ & $\mathrm{Pb}$ & $-\mathrm{b}$ & 1.0 & 0.2 \\
$\mathbf{7 / 1 1}$ & $\mathrm{Pb}$ & $-\mathrm{b}$ & 0.58 & 0.2 \\
$\mathbf{9 / 2 5}$ & $\mathrm{Pb}$ & 10 & 0.33 & 0.2 \\
$\mathbf{1 0 / 7}$ & $\mathrm{Cr}$ & 30 & 0.99 & 0.62 \\
$\mathbf{1 2 / 1}$ & $\mathrm{Cu}$ & 40 & 1.73 & 1.0 \\
\hline
\end{tabular}

a If a release lasted as long as $\mathbf{2 4}$ hours, concentrations above this level would likely result in disruption of treatment plant operations, or cause the treated wastewater to exceed allowable concentration limits for discharge to the San Francisco Bay.

b Due to fouling of the monitoring equipment by the contaminant, no estimate can be made of the duration of the incident. 


\section{Introduction}

Lawrence Livermore National Laboratory performs surveillance monitoring of waters at the Livermore site, in surrounding regions of the Livermore Valley, and at Site 300 in the Altamont Hills. The waters monitored at the first two locations include reservoirs and ponds, the LLNL swimming pool, rainfall, tap water, storm water runoff, drinking water production wells, and ground water monitoring wells. Surveillance monitoring at Site 300 encompasses on-site and off-site production wells and monitoring wells, spring-fed creeks, and rainfall. Water samples are analyzed for radionuclides and a large range of nonradioactive constituents.

A small fraction of treated effluent from the City of Livermore Water Reclamation Plant (LWRP) is used to irrigate nearby municipal land. This effluent contains low levels of tritium from natural sources and from permitted operational releases to the sanitary sewer system by LLNL and Sandia National Laboratories, California (SNL/CA). [Total radionuclides in liquid effluents are limited to 1 curie $\left(3.7 \times 10^{10} \mathrm{~Bq}\right)$ per year.] Rainfall and storm water runoff in the Livermore Valley also contain small amounts of tritium from natural sources, from past atmospheric tests of nuclear weapons, and from atmospheric emissions by LLNL and SNL/CA. Since 1977, annual tritium measurements have been made on water samples collected from monitoring wells and drinking water wells throughout the Livermore Valley to determine the extent of tritium migration into the ground from the LWRP irrigation water, from tritium washed out of the atmosphere by rain, and from tritium in storm water runoff that flows through the Arroyo Las Positas to Alameda Creek and thence to San Francisco Bay.

LLNL has monitored storm water runoff at the Livermore site since 1975. The original monitoring network was designed to detect pesticides. This monitoring was expanded in 1990 to cover new locations and additional water quality parameters, including radioactivity, metals, and numerous organic compounds. Storm water runoff monitoring now provides a broad measure of the efficacy of LLNL operational procedures that prevent, or contain and remediate, inadvertent spills of hazardous wastes or products onto the ground within the Livermore site. 
The Environmental Report for 1989 (Sims et al. 1990) first reported elevated tritium in storm water samples collected during a March 1989 rain storm at the Livermore site. Although tritium emissions at LLNL are closely monitored, the sampling of rain was expanded in 1990 to understand better the relationship between emissions, rainfall, and storm runoff.

As described in Chapter 7 on Ground Water Protection, LLNL performs additional monitoring at Site 300 to evaluate ground water contaminants originating from historical operations.

\section{Methods}

\section{Livermore Water Reclamation Plant}

Nineteen wells near and at some distance from LWRP are sampled annually for tritium measurement to determine the extent of tritium migration into the ground water (Figure 6-1). Wells used only for monitoring of reclaimed irrigation water are 1H3, 1P2,

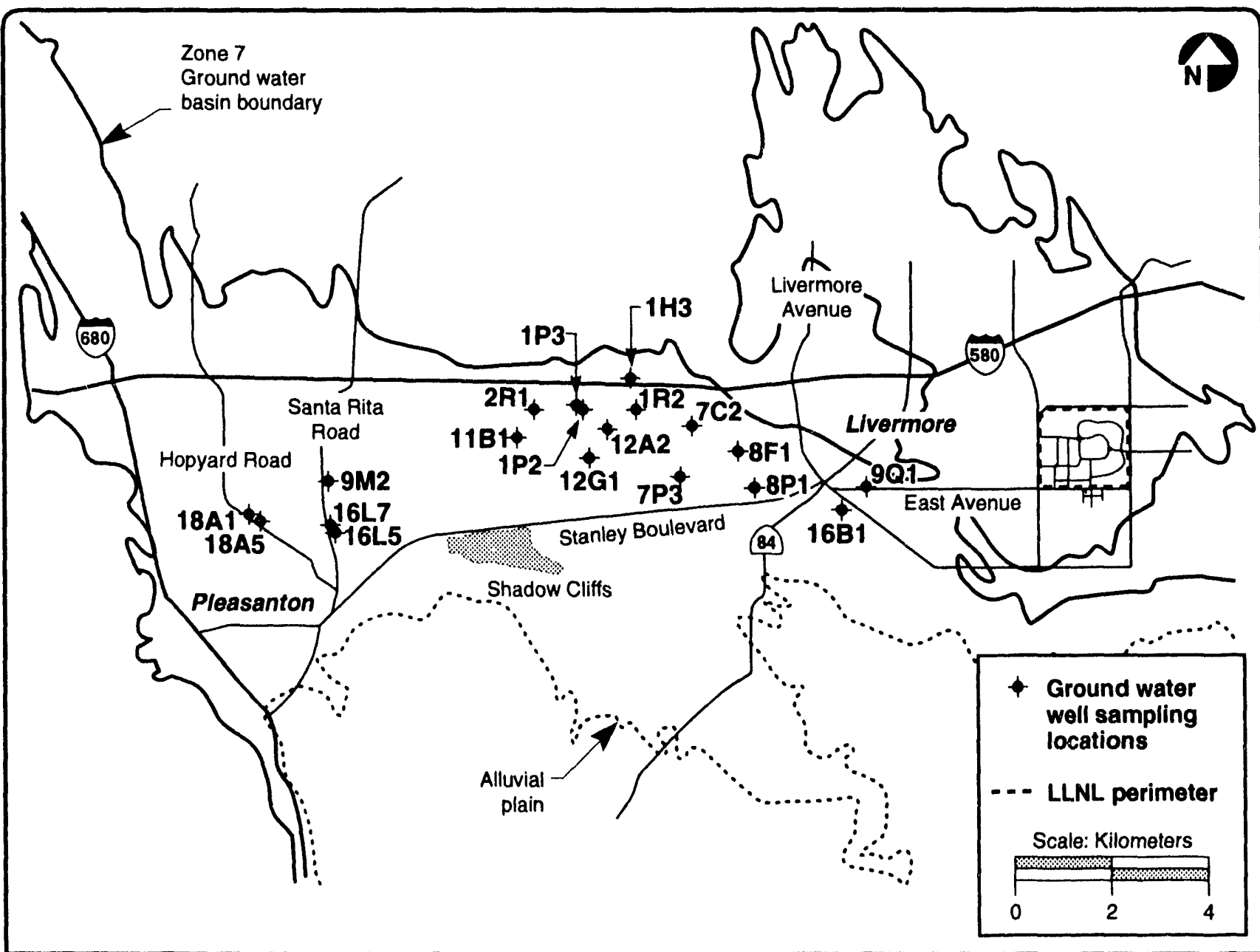

Figure 6-1. Ground water well sampling locations, Livermore Valley, 1992. 


\section{Surveillance Water Monitoring}

1P3, 1R2, 2R1, 7C2, 7P3, 11B1, 12A2, and 12G1. Sampled potable water supply wells serving the City of Livermore are 8F1, 8P1, 9Q1, and 16B1. City of Pleasanton supply wells, which are sampled annually for tritium measurements, are 9M2, 16L7, 16L5, 18A1 and $18 \mathrm{~A} 5$.

\section{Livermore Valley}

LLNL samples both surface water and drinking water in the vicinity of the Livermore site and in the Livermore Valley (Figure 6-2). Locations DEL, ZON7, DUCK, ALAG, SHAD, and CAL are surface water sources. The locations GAS, PALM, and ORCH are drinking water outlets. Samples from these locations are taken quarterly and are measured for gross alpha, gross beta, and tritium. Water samples are obtained with a tethered pail from surface sources. Other locations are sampled directly from the outfall. Samples for tritium analysis are collected in $500-\mathrm{mL}$, argon-flushed glass containers fitted with ground-glass stoppers. Samples for other radiological analyses are collected in $1000-\mathrm{mL}$ polyethylene bottles.

\section{Livermore Site}

The on-site swimming pool and on-site drinking water (POOL, TAP; Figure 6-2) are measured for gross alpha, gross beta, and tritium. The pool is sampled monthly and the drinking water is sampled quarterly.

Rain samples from 19 locations, including 7 on-site, are collected after each major rainstorm and are measured for tritium (Figure 6-3).

The storm water runoff monitoring network includes ten locations (Figure 6-4) that are sampled during active surface water flow following

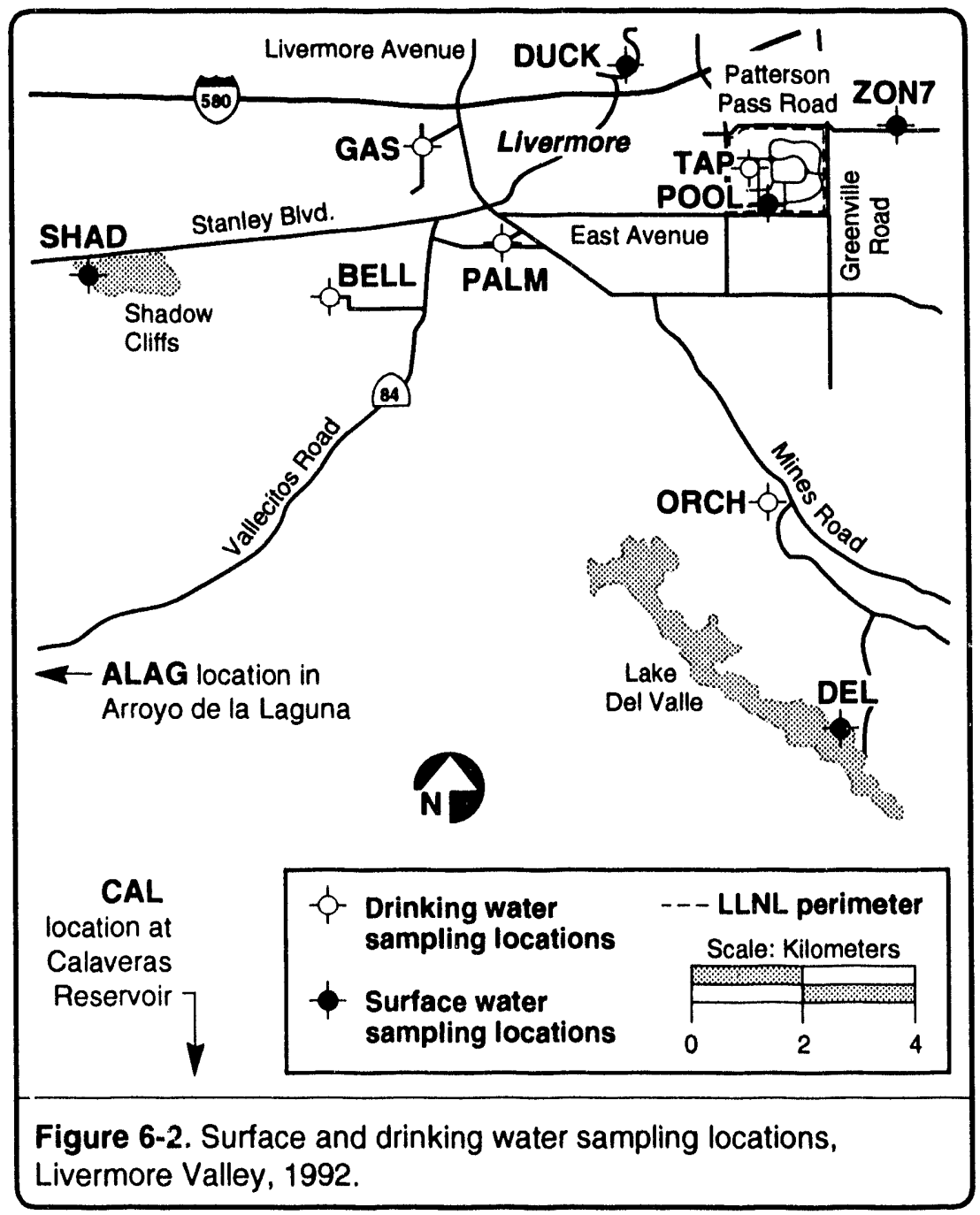
the first major storm of the rainy season. Beginning in January 1993, subsequent to the first major storm, monthly sampling of runoff and dry weather observations will be performed at both the Livermore site and at Site 300 . This is required by a National Pollutant Discharge Elimination System (NPDES) 
General Industrial Activities Storm Water Permit for both sites. This monitoring will supplement the routine surveillance storm water monitoring.

Approximately one-fourth of the storm water runoff generated within the LLNL site drains into the centrally located Central Drainage Basin (CDB; Figure 6-4). The remainder drains either directly, or via a system of storm sewers and ditches, into two arroyos. The arroyos generally drain from east to west. Arroyo Seco cuts across the southwestern corner of the site. Arroyo Las Positas has been diverted from its natural course to follow the northeastern and northern boundaries of the Livermore site. Several long on-site ditches, established to channel storm water runoff, drain from south to north across the site into the Arroyo Las Positas. Of the ten storm water sampling locations at LLNL, five were selected to characterize storm waters either entering the site (influent water) or exiting the site (effluent water). Influent locations are ALPE, GRNE, and ASS2. Effluent

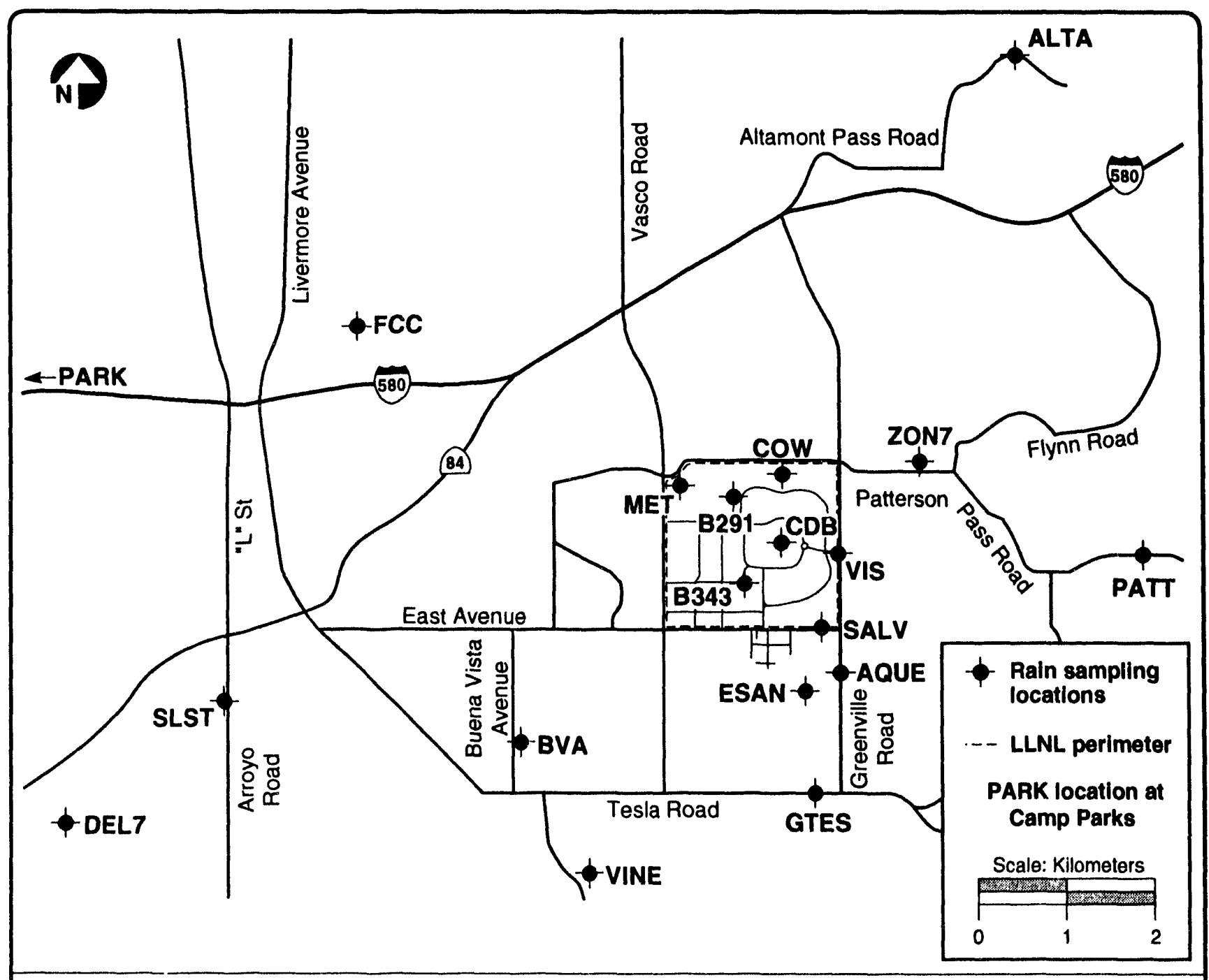

Figure 6-3. Rain sampling locations, Livermore site and Livermore Valley, 1992. 


\section{Surveillance Water Monitoring}

locations are WPDC for the Arroyo Las Positas, and ASW for the Arroyo Seco. Location $\mathrm{CDB}$ measures runoff from the southeastern quadrant of the Livermore site where it enters the Central Drainage Basin. Location ALPN measures outflow from the drainage basin at its confluence point with the Arroyo Las Positas. Location ALPW measures onsite runoff from a ditch along Avenue A where it enters the Arroyo Las Positas. Location 4THA samples runoff from the on-site Plutonium and Tritium Facilities. Location B438 measures storm water runoff from the shops area and the Hazardous Waste Management facilities.

\section{Site 300}

Quarterly surface water samples are collected off site, downstream from Site 300 in the Corral Hollow Creek (GEOCRK) and on site in a small, spring-fed creek near Bunker 812 (812CRK; Figure 6-5). Volatile samples are collected directly from surface flows.

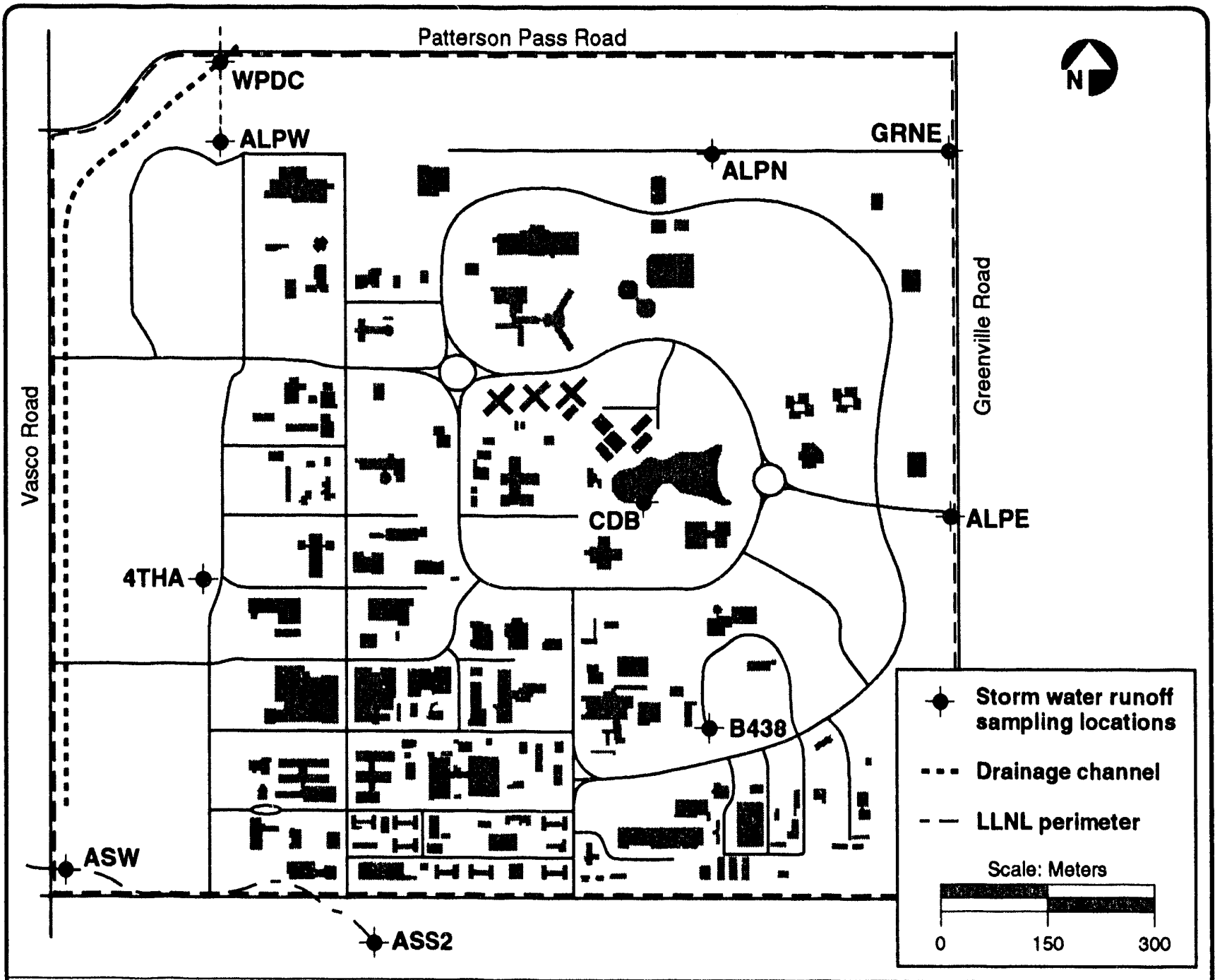

Figure 6-4. Storm water runoff sampling locations, Livermore site and vicinity, 1992. 


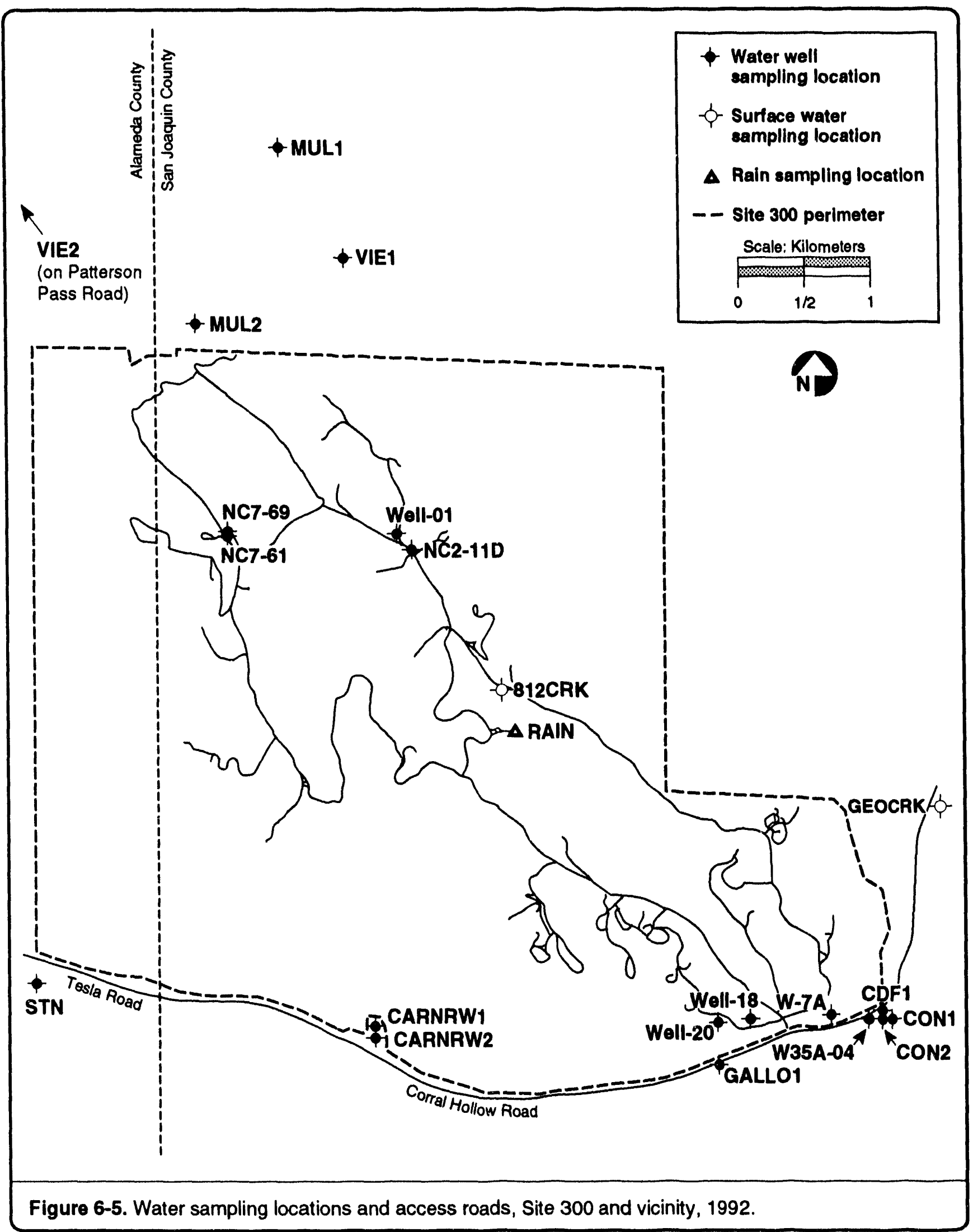


[Samples to be analyzed for tritium are collected in $500-\mathrm{mL}$, argon-flushed glass containers. Samples for gross alpha and beta measurements are collected in 1000-mL polyethylene bottles. Rainfall at one location (RAIN; Figure 6-5) is composited each month and analyzed for tritium.]

Nineteen ground water wells are monitored at Site 300 as part of the ground water surveillance program. There are seven on-site wells: Well 01, Well 18, Well 20, NC2-11D, NC7-61, NC7-69, and W-7A (Figure 6-5). Well 01 supplies fire-suppression water to the Site 300 Fire Department. During 1992, this well was sampled quarterly for certain metals, radioactivity, and organic compounds. Well 20 is the potable water supply well for Site 300 . Well 18 is currently inactive and is maintained as a backup for Well 20. Both these wells are sampled quarterly for certain metals and radioactivity, and monthly for organic compounds. Wells NC2-11D, NC7-61, NC7-69, and W-7A are sampled quarterly for beryllium, chromium, copper, and lead; for organic compounds; and for radioactivity. Additionally, wells NC7-61 and NC7-69 are sampled quarterly for isotopes of uranium.

Twelve off-site water supply wells were sampled and analyzed in 1992. Six were sampled quarterly. Of these, CARNRW1 and CON2 were sampled for organic compounds only, while CARNRW2, CDF1, CON1, and GALLO1 were sampled for a large suite of inorganic and organic compounds (GALLO1 was unavailable for sampling during the third and fourth quarters of 1992). The remaining six wells, MUL1, MUL2, STN, VIE1, VIE2, and W35A-04, are sampled annually for a large suite of inorganic and organic compounds.

Ground water monitoring wells are purged of stagnant water prior to sampling. All sampling equipment is maintained so that it does not contaminate the water sample. Containers and preservatives are used according to established protocols for the analytes of interest. Table 6-1 lists the standard EPA methods used to measure contaminants in water.

\section{Results}

\section{Livermore Site/Livermore Valley, Radioactivity in Water}

Mean gross alpha and gross beta in surface and domestic water sampled during 1992 were very low, averaging less than $11 \%$ and $13 \%$ of the drinking water maximum contaminant levels (MCLs), respectively (Tables 6-2 and 6-3). One storm water location showed elevated gross alpha and gross beta $[0.77 \mathrm{~Bq} / \mathrm{L}$ $(20.8 \mathrm{pCi} / \mathrm{L})$ and $3.82 \mathrm{~Bq} / \mathrm{L}(103.2 \mathrm{pCi} / \mathrm{L})$, respectively]. This sample was taken from the outflow stream of the Central Drainage Basin where it enters the Arroyo Las Positas (location ALPN, Figure 6-4). However, contemporaneous measurements made downstream in the arroyo at location ALPW showed typically low values of gross alpha $(0.04 \mathrm{~Bq} / \mathrm{L}$, or $1 \mathrm{pCi} / \mathrm{L})$ and gross beta $(0.23 \mathrm{~Bq} / \mathrm{L}$, or $6 \mathrm{pCi} / \mathrm{L})$.

Tritium. Mean tritium activity collected from surface and drinking water locations in the Livermore Valley was less than $1 \%$ of the drinking water MCL [ surface water average $=1.55 \mathrm{~Bq} / \mathrm{L}(41.9 \mathrm{pCi} / \mathrm{L})$; drinking water average $=$ 
$0.90 \mathrm{~Bq} / \mathrm{L}$ (24.3 $\mathrm{pCi} / \mathrm{L})$; Table 6-3]. The LLNL swimming pool water had the highest mean value and the highest individual measurement. The mean tritium value for water in the swimming pool for 1992 was $16.96 \mathrm{~Bq} / \mathrm{L}(458 \mathrm{pCi} / \mathrm{L})$, still only $2 \%$ of the drinking water MCL. The highest single observation for location POOL was $22.98 \mathrm{~Bq} / \mathrm{L}$ (621 pCi/L; 3\% of MCL; see Table 6-3).

Rainfall samples were collected at 19 locations from 20 storrns in 1992. The mean rainfall tritium concentration for 1992 was $13.3 \mathrm{~Bq} / \mathrm{L}(359 \mathrm{pCi} / \mathrm{L})$ (Figure 6-3 and Table 6-4), less than $2 \%$ of the drinking water MCL. The highest rainfall tritium activity measured in 1992 was $115.81 \mathrm{~Bq} / \mathrm{L}(3130 \mathrm{pCi} / \mathrm{L} ; 16 \%$ of $\mathrm{MCL}$ ) at the on-site location B291. This rain sampling location is adjacent to an extensively studied subsurface soil zone where tritium-bearing wastewater leaked from a pipe connecting to an underground storage tank. Vegetation above the zone draws small quantities of tritium upwards by capillary action and transpires it directly to the atmosphere.

Storm water runoff was collected at ten on-site locations during 18 rainstorms in 1992 (some storms produced sufficient rainfall for sampling, but did not generate sufficient runoff for sampling). Tritium levels in storm water runoff were low, averaging $10.55 \mathrm{~Bq} / \mathrm{L}(285 \mathrm{pCi} / \mathrm{L})$, less than $2 \%$ of the drinking water MCL (Table 6-2). The highest tritium activity measured in storm water runoff during 1992 was $61.79 \mathrm{~Bq} / \mathrm{L}(1670 \mathrm{pCi} / \mathrm{L})$ at influent location ASS2, less than $10 \%$ of the drinking water MCL.

Well water was collected at 19 locations in the Livermore Valley (Table 6-5). Tritium levels in these wells were at background levels, averaging $2.83 \mathrm{~Bq} / \mathrm{L}$ $(76.5 \mathrm{pCi} / \mathrm{L})$, less than $1 \%$ of the MCL for drinking water. As in previous years, the highest tritium measurement, $16.95 \mathrm{~Bq} / \mathrm{L}(458 \mathrm{pCi} / \mathrm{L})$, was from Well 11B1. However, tritium activity in this well, which is used only for monitoring, has decreased since 1990 and 1991, when it measured $24.1 \mathrm{~Bq} / \mathrm{L}(652 \mathrm{pCi} / \mathrm{L})$ and $23.41 \mathrm{~Bq} / \mathrm{L}(633 \mathrm{pCi} / \mathrm{L})$, respectively.

\section{Livermore Site/Livermore Valley, Nonradioactive Pollutants in Water}

LLNL measures inorganic and organic contaminants in storm water runoff entering and exiting the site (Table 6-6). Of 813 measurements made for potential inorganic contaminants in 1992, only one 450-ppm sulfate value, from influent location ALPE, exceeded the secondary standard (250 ppm) set by the State of California for drinking water. The most commonly detected metals in storm water runoff were arsenic, barium, cadmium, chromium, and lead. During 1992, these metals were found at levels less than the corresponding drinking water MCLs. Other metals, such as beryllium, mercury, selenium, and silver, were observed three or fewer times in storm water runoff. All were found at levels below their respective drinking water MCL. 
Of the nearly 6000 measurements made in 1992 for potential organic contaminants in storm water runoff (Table 6-6), only three measurements of bis(2-ethylhexyl)phthalate exceed the drinking water MCL (4 ppb). The three are from locations ASS2 (37 ppb), ASW (99 ppb), and CDB (23 ppb). The source of this organic compound is unknown. It is a light oil commonly used in the manufacture of PVC plastic. The measurements are suspect since this compound is known to be introduced during laboratory analytical procedures. Other detected organic pollutants include 1,1,1-trichloroethane (4 observations), acetone (6), chloroform (1), Freon-113 (10), toluene (5), trichloroethene (3), and oil and grease (20). A single observation of the herbicide 2,4-D is associated with storm water entering the site at ALPE. Five observations of the pesticide Endrin are associated with runoff from a storm that occurred on February 10, 1992. This pesticide is not used at LLNL and its source is unknown. The concentrations measured are all below the 0.2-ppb MCL for drinking water.

\section{Site 300, Radioactivity in Surface Water}

No measured value of radioactivity in rain or surface water at Site 300 exceeded drinking water MCLs for gross alpha, gross beta, or tritium (Table 6-7). Mean gross alpha and gross beta values at the two surface water locations, GEOCRK and 812 CRK, are less than $43 \%$ of the MCL. Mean tritium activity in the two surface water locations and in rain at Site 300 was very low, less than $1 \%$ of the MCL.

\section{Site 300, Radioactivity in Ground Water}

No measurement of gruss alpha or gross beta in water samples taken from on-site and off-site wells $\epsilon$. iceeded drinking water MCLs (Tables 6-8 to 6-10). The highest measured value of gross alpha is $0.434 \mathrm{~Bq} / \mathrm{L}(11.7 \mathrm{pCi} / \mathrm{L})$ in off-site production well STN (78\% of the MCL). The highest measured gross beta value, $0.336 \mathrm{~Bq} / \mathrm{L}(9.1 \mathrm{pCi} / \mathrm{L})$, was from off-site production well CARNRW2 (18\% of the drinking water MCL). Tritium levels in all but one surveillance well, NC7-61, were far below the drinking water MCL of $740 \mathrm{~Bq} / \mathrm{L}(20,000 \mathrm{pCi} / \mathrm{L})$. Mean tritium activity for the on-site monitoring well NC7-61 was about $9000 \mathrm{~Bq} / \mathrm{L}$ $(243,000 \mathrm{pCi} / \mathrm{L})$ for 1992 , about 12 times the drinking water MCL. This well is located east and downgradient of the Building 850 Area, a known source of tritium (see Chapter 7 on Ground Water Protection for a further discussion).

Site 300, Nonradioactive Pollutants in Water

On-Site Inactive Ground Water Production Wells. The compound $1,1,1$-trichloroethane was detected at $0.6 \mathrm{ppb}$ in a ground water sample collected from Well 01 (Table 6-10). No other organic compounds of concern were detected in this sample. Lead was found in two of four measurements at concentrations of 2 and $3 \mathrm{ppb}$ in Well 01 . These are within the historical range of 
lead values observed in the well ( 1 to $6 \mathrm{ppb})$, and considerably below the MCL of $50 \mathrm{ppb}$ for drinking water.

Sample data from Well 18 (the backup drinking water well) did not exceed any MCL or State Action Level (SAL). Three metals were detected at extremely low concentrations: chromium in Well 18 at $6 \mathrm{ppb}(\mathrm{MCL}=50 \mathrm{ppb})$; copper at $11 \mathrm{ppb}$ (secondary $\mathrm{MCL}=1000 \mathrm{ppb}$ ); and lead in two samples at concentrations of 3 and $4.4 \mathrm{ppb}(\mathrm{MCL}=50 \mathrm{ppb})$.

In 1991, trichloroethene (TCE) was detected in Well 18 at a concentration of $1 \mathrm{ppb}(\mathrm{MCL}=5 \mathrm{ppb})$. Of twelve monthly samples taken from Well 18 in 1992, ten samples contained TCE from 0.5 to $1.1 \mathrm{ppb}$ (Table 6-10). The source(s) of these trace levels of TCE is under investigation. Toluene was detected in one of twelve monthly samples at a concentration of $2 \mathrm{ppb}$. Surveillance monitoring of Well 18 continues on a monthly basis.

On-Site Active Water Supply Well 20. Lead was detected at $2 \mathrm{ppb}$ in one of the quarterly samples of water collected from Well 20, the primary potable water supply well at Site 300 . The primary MCL for lead in drinking water is $50 \mathrm{ppb}$. Although several organic compounds were detected at extremely low concentrations in Well 20 in 1991, the 1992 data reveal no constituents of concern above the detection limits (Table 6-10).

Other On-Site Surveillance Wells. Chromium was detected at $5 \mathrm{ppb}$ in a quarterly sample taken from well NC7-61. Lead was detected at 7.1 ppb in one of four measurements of water from well NC7-69. Beryllium, chromium, copper, and lead were tested for, but not detected, in samples from wells NC2-11D and W-7A.

TCE was detected in all quarterly samples taken from Well W-7A at concentrations ranging from 0.6 to $1.4 \mathrm{ppb}$. Cis-1,2-dichloroethene, a potential product of TCE decomposition, was also detected in one of the quarterly samples taken from well W-7A. This well derives water from the lower water-bearing zone of the Neroly Formation, which serves as the regional aquifer beneath the General Services Area at Site 300. Causes of the low-level TCE contamination are being examined in the continuing Draft Site-Wide Remedial Investigation (SWRI; Webster-Scholten 1993).

Off-Site Water Supply Wells. No primary inorganic compounds of concern were detected above safe drinking water limits in any of the 12 off-site monitoring wells (Table 6-10). Three wells, STN, CON1, and MUL2, exceeded the California Department of Health Services (DHS) secondary (aesthetic) drinking water standards for sulfate, a naturally occurring ground water contaminant in the Altamont Hills. Two wells, CON1 and MUL2, exceeded the secondary standard of 0.05 ppm set for manganese. Manganese is also a naturally occurring ground water contaminant in the Altamont Hills. 
Wells CARNRW1 and CON2 were sampled once in 1992 and analyzed for organic compounds only. Because no compounds of concern were detected, these data are omitted from Table 6-10.

Five trihalomethane (THM) compounds were detected in well CARNRW2 during 1992. These findings result from collecting the sample from a chlorinated water supply rather than from untreated well water. This situation has been corrected by installing a sampling tap at the wellhead. Other organic compounds of concern were detected in CARNRW2 at extremely low levels, including 1,2-dichloroethane, 1,2-dichloropropane, 1,4-dichlorobenzene, ethylbenzene, and xylene isomers (Table 6-10). These contaminants may be associated with well maintenance and chlorination.

Two halocarbon compounds, bromochloromethane and bromoform, were detected in surface water taken from the VIE1 artesian well. The origin of these compounds is unknown. However, the water sampled passed through a surface pool that is highly susceptible to external contamination.

TCE was observed at the lowest possible detection level in two quarterly water samples from surveillance well GALLO1 in January and May 1992. These detections were made after changing the analytical method from EPA 601 to EPA 524.2, which has lower detection levels. The method of analysis was changed because EPA 524.2 is the more common method of analysis for drinking water production wells. The concentrations in both samples, $0.2 \mathrm{ppb}$, were at the EPA 524.2 method detection limit. GALLO1 was not available for sampling during the third and fourth quarters of 1992.

A single detection of TCE at an extremely low concentration of $0.3 \mathrm{ppb}$ occurred in a water sample taken from surveillance well CDF1. This is the first such observation for this well and resulted from changing analytical methods. However, subsequent analyses have failed to confirm TCE in this well. Another detection unique to the sample taken from well CDF1 was carbon disulfide at an extremely low concentration of $0.65 \mathrm{ppb}$.

\section{Environmental Impact}

\section{Livermore Site}

Storm water runoff contained low levels of bis(2-ethylhexyl)phthalate and other organic compounds at various locations, but very few contaminants were detected. Except for bis(2-ethylhexyl)phthalate, nonradiological pollutants above regulatory limits for drinking water were not observed in any storm water samples. Although not all constituents of concern were monitored, storm water runoff from the Livermore site meets the water quality objectives for fresh waters as outlined in the September 9, 1992, amendments to the Water Quality Control Plan-San Francisco Bay Region (California Regional Water Quality Control Board 1991). The detection of the pesticide Endrin at extremely low concentrations in 
runoff has prompted an investigation as to its source. Endrin is not used at the Livermore site and has not previously been detected in soils or in storm water runoff from the site.

The effective dose equivalent (EDE) from tritium is estimated based on the highest tritium activity measured in an off-site water sample. The method used to calculate dose is presented in Appendix B. Of all off-site waters measured, the maximum tritium activity, 53.3 $\mathrm{Bq} / \mathrm{L}(1440 \mathrm{pCi} / \mathrm{L})$, occurred in rain sampled at station AQUE during one storm (Table 6-4). The EDE to an adult who ingested two liters of this rain water per day for one year would be $0.0007 \mathrm{mSv}$ $(0.007 \mathrm{mrem})$, which is less than $1 \%$ of the EPA standard allowable dose of $0.1 \mathrm{mSv} / \mathrm{yr}(10 \mathrm{mrem})$. All other off-site waters, if ingested at the 2-liter-per-day rate, would result in even lower EDEs. The water data collected during 1992 and the estimated potential maximal dose demonstrates a minimal impact of LLNL operations on Valley waters resulting from releases of tritium to the atmosphere and to the sanitary sewer.

\section{Site 300}

The major nonradiological constituents of concern in Site $\mathbf{3 0 0}$ ground waters are the organic solvents, such as TCE, and their decomposition products. These were observed at least once during 1992 in Well 01. In backup water supply Well 18, TCE was observed in ten of twelve monthly samples, with the highest concentration measured at $1.1 \mathrm{ppb}$. This value is $22 \%$ of the 5 -ppb MCL. The implementation of a different method of analysis resulted in the detection of solvents in water samples from off-site production wells CDF1 and GALLO1 at concentrations of 0.3 and $0.2 \mathrm{ppb}$. These detections, which are below the previous detection limit of $0.5 \mathrm{ppb}$, have not been substantiated by subsequent analyses. Investigations as to the extent and sources of organic solvents in Site 300 ground water are discussed in the SWRI (Webster-Scholten 1993).

Halomethane compounds were detected in water samples taken from the offsite active water supply wells CARNRW2 and VIE1. In CARNRW2, they are a result of sampling chlorinated water. No such association can be made for the VIE1 artesian well, but external contamination is likely.

Tritium concentrations in the on-site water supply Well 20 and ten off-site water supply wells were all indistinguishable from background levels and all were less than $1 \%$ of the MCL for drinking water. Mean tritium activity in well NC7-61 decreased $10 \%$ from the previous year $(10,000 \mathrm{~Bq} / \mathrm{L}$ to $9000 \mathrm{~Bq} / \mathrm{L})$. This decrease is double the amount expected from natural decay of tritium and suggests that dilution is occurring within the contaminated zone. Conversely, tritium continues to increase in Well 01, which is near the leading edge of a tritium-bearing ground water plume that has traveled eastward about 1 kilometer from its origin near Building 850. Tritium activities have increased 
from $190 \mathrm{~Bq} / \mathrm{L}(5135 \mathrm{pCi} / \mathrm{L})$ in 1991 to $222 \mathrm{~Bq} / \mathrm{L}(6000 \mathrm{pCi} / \mathrm{L})$ in 1992 , which is equal to $30 \%$ of the drinking water MCL. This well is no longer used as a source of drinking water at Site 300 .

The surveillance monitoring data demonstrate that the off-site radiological and nonradiological impact of LLNL operations at Site 300 are minimal. LLNL is, however, continuing investigations of several areas of ground water contamination within the site.

\section{Special Studies}

\section{Tritium}

Historically LLNL has exhibited elevated tritium levels in both rainfall and storm water runoff. Elevated storm water tritium associated with a rainfall in March 1989 was reported in the Environmental Report for 1989 (Sims et al. 1990). Rain water monitoring was subsequently expanded to 19 locations and the storm water monitoring system was extended to 10 locations to better assess the migration of tritium through the environment.

Tritium in Rain and Storm Water. Tritium levels in rain and storm water dropped significantly during the October 1990 through December 1992 special study period. These observations may be directly related to substantial reductions in the emission of tritiated water vapor (HTO) into the atmosphere by LLNL and SNL/CA (Figure 6-6). In 1989, the total atmospheric HTO emission from these two cources was 82 terabecquerels (TBq). Of this amount, LLNL released $58 \mathrm{TBq}(1560 \mathrm{Ci})$ and $\mathrm{SNL} / \mathrm{CA}$ released $24 \mathrm{TBq}(656 \mathrm{Ci})$. In 1990, annual emissions were reduced to $35 \mathrm{TBq}$. Of this amount, LLNL released $26 \mathrm{TBq}$ $(700 \mathrm{Ci})$ and $\mathrm{SNL} / \mathrm{CA}$ released $9 \mathrm{TBq}(244 \mathrm{Ci})$. A small increase in annual HTO emissions, to $38 \mathrm{TBq}$, occurred in 1991. Of this amount, LLNL released $25 \mathrm{TBq}$ $(678 \mathrm{Ci})$ and $\mathrm{SNL} / \mathrm{CA}$ released $13 \mathrm{TBq}(352 \mathrm{Ci})$. This was followed by another marked reduction in 1992 when HTO emissions totaled 9 TBq. Of this amount, LLNL released $4 \mathrm{TBq}(101 \mathrm{Ci})$ and $\mathrm{SNL} / \mathrm{CA}$ released $5 \mathrm{TBq}(134 \mathrm{Ci})$.

Reductions of tritium emissions by LLNL and SNL/CA appear to have resulted in reductions of tritium precipitated in rain throughout the Livermore Valley (Figure 6-6; note that this figure uses relative units, that is, the $10 \mathrm{~Bq} / \mathrm{L}$ unit used for rain, runoff, and LLNL pool is a trillion times lower than the $10 \mathrm{TBq}$ unit used for emission). The mean of tritium activity measurements in rain for 1990 was $58.9 \mathrm{~Bq} / \mathrm{L}(1590 \mathrm{pCi} / \mathrm{L})$; for 1991 the mean was $42.9 \mathrm{~Bq} / \mathrm{L}(1160 \mathrm{pCi} / \mathrm{L})$; and for 1992 the mean was $13.3 \mathrm{~Bq} / \mathrm{L}(360 \mathrm{pCi} / \mathrm{L})$. This represents more than a fourfold decrease over the past three years.

The mean value of tritium activity in storm water runoff sampled in 1989 was $129 \mathrm{~Bq} / \mathrm{L}(3486 \mathrm{pCi} / \mathrm{L})$. The mean value dropped to $31.3 \mathrm{~Bq} / \mathrm{L}(846 \mathrm{pCi} / \mathrm{L})$ in 1991 , and to $10.6 \mathrm{~Bq} / \mathrm{L}(286 \mathrm{pCi} / \mathrm{L})$ in 1992 (Figure 6-6). This more than tenfold 
decrease in the tritium content of storm water runoff is directly related to the decreased tritium content of rainfall in the Livermore Valley.

Tritium in Other Waters. Other waters, both at the surface and underground, show decreasing tritium activity during the period of this special study (Figure 6-6). Tritium concentrations in the LLNL swimming pool, like those in rain and storm water runoff, appears to have decreased with decreasing emissions from nearby sources. Mean tritium activity of the LLNL pool water decreased from $42 \mathrm{~Bq} / \mathrm{L}(1140 \mathrm{pCi} / \mathrm{L})$ in 1990 to $39 \mathrm{~Bq} / \mathrm{L}(1050 \mathrm{pCi} / \mathrm{L})$ in 1991 and to $17 \mathrm{~Bq} / \mathrm{L}(459 \mathrm{pCi} / \mathrm{L})$ in 1992 . The 1992 level is about $2 \%$ of the MCL for drinking water. Surface waters and drinking waters ("other waters" in Figure 6-6) and wells in the Livermore Valley exhibit very low and consistently decreasing values of tritium activity, equal to approximately one-tenth the values observed for rain and surface runoff. Tritium activities in these waters are below $1 \%$ of the MCL for drinking water. The general decrease observed in all the various waters sampled appears to reflect both the long-term reduction in tritium emissions and the radioactive decay of tritium to helium (half-life equals

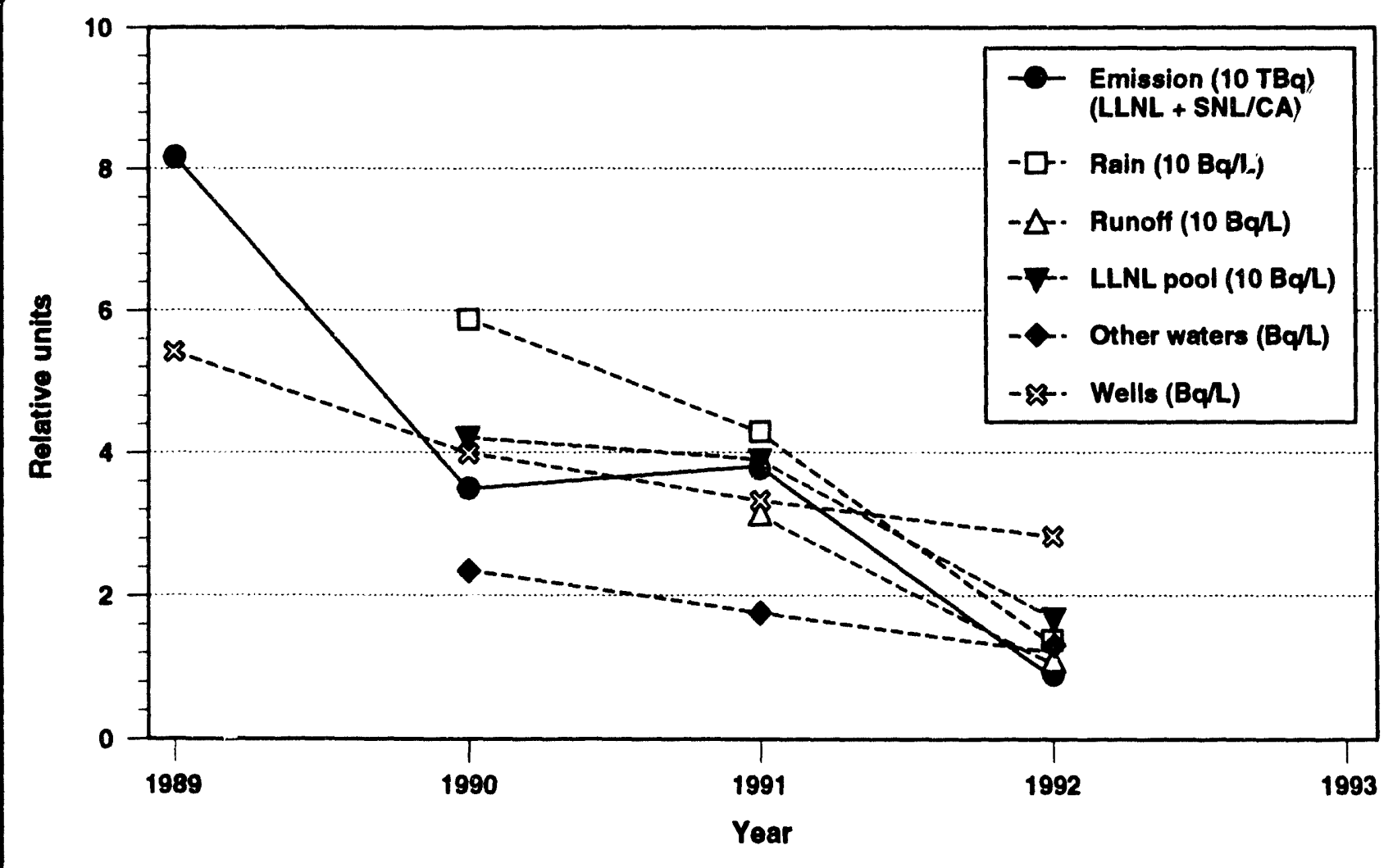

Figure 6-6. Tritium activity in the Livermore Valley, $1989-1992$. 
12.3 years). If no increase in tritium emission occurs, tritium activity will continue to decrease from its present low levels in waters within and beneath the Livermore Valley.

\section{Central Drainage Basin}

The Central Drainage Basin was lined as part of the Livermore site remedial activities. Remedial action studies had indicated that infiltration of storm water from the basin was a cause of increased dispersal of ground water contaminants. Basin lining was completed in March 1992 and LLNL adopted the Drainage Retention Basin Management Plan.

The focus of the Plan was to implement long-term biological monitoring and to address water quality problems through reduction of nutrient loading and bioremediation. Water quality objectives would be met through use of submersed plants to maintain water quality and rooted aquatic plants in the shallow portions of the basin to remove urban runoff pollutants and control erosion of the basin lining cover.

The Plan identified two sources of water to fill and maintain the level of the retention basin. The primary source would be water generated from the ground water treatment units and discharged to the basin through the existing storm water collection system. The second source would be storm water collected during the rainy season. During 1992, storm water runoff was the primary source of basin water, augmented by discharge from a ground water treatment unit.

Regulatory Requirements. The San Francisco Bay Regional Water Quality Control Board (RWQCB) regulates discharge from the basin. The RWQCB issued Waste Discharge Requirements (WDRs) for discharging treated ground water from remedial activities. WDR Order No. 90-091 regulates discharge from Treatment Facility A to help fill the basin. The discharge limitations in this permit, in turn, regulate discharges from the basin into the Livermore-site storm drain. In 1992, LLNL agreed to sample any discharge from the basin monthly and report the results to the RWQCB. In subsequent years, LLNL agreed to sample the initial and one subsequent discharge.

The National Pollution Discharge Elimination System (NPDES) permit CA0029829 for the ground water treatment facility regulates discharges from the basin. Sampling of basin discharge to satisfy requirements of this permit is collected at the basin outfall.

Sampling Program. Sampling is performed to provide information necessary for basin maintenance and to document the effectiveness of nutrient removal. During 1992, the basin was monitored for a suite of management parameters (Table 6-11). Results of sampling performed for NPDES requirements are shown in Table 6-12. 
Sampling Results. During 1992, nitrogen, phosphorus, and chemical oxygen demand (COD) levels exceeded both management objectives and action levels (Table 6-11). Elevated levels of nitrogen were due to high natural levels of nitrate in ground water used to fill the basin. This practice of using ground water in the basin was halted in Fall 1992 and by the end of the year, following fall rains, one of the two measures of nitrogen, ammonia, and nitrate plus nitrite had begun to decline in the basin.

No parameter exceeded any NPDES discharge limit based on three 1992 sampling events (Table 6-12). However, the analytical detection limits for two parameters, copper and thallium, are currently higher than the permitted discharge limits. We will work with our contract analytical labs during 1993 to lower the analytical detection limits for these two parameters to below the discharge limits.

Impacts. There were no significant environmental impacts from the drainage basin. Elevated nutrient levels in the basin were primarily due to use of nitrogen-laden ground water and startup problems with the submersed plants (they died once and were replaced). There were no detected discharges from the basin that exceeded our discharge limits. For two parameters, copper and thallium, there were no detectable amounts in the discharge. Our current detection limit for copper is one-sixth the drinking water MCL. During 1993, the monitoring program will be expanded to assess the efficiency of the retention basin to remediate storm water pollutants. 
Table 6-1. EPA standard methods for water sampling.

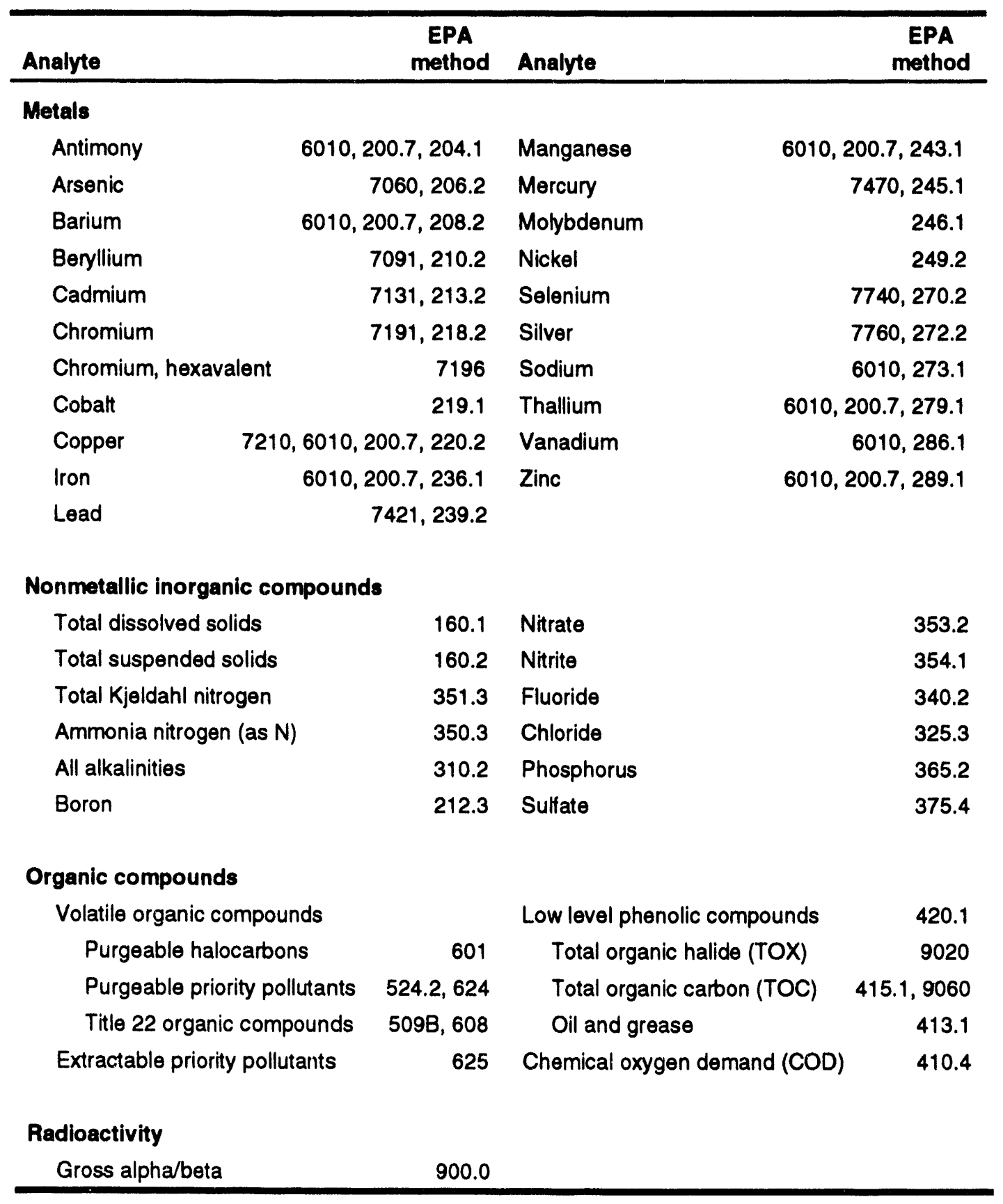




\section{Surveillance Water Monitoring}

Table 6-2. Radioactivity in storm water runoff (in Bq/L), 1992.

\begin{tabular}{|c|c|c|c|c|c|c|c|}
\hline Location & Tritium & Grose alpha & Gross beta & Location & Tritium & Gross alpha & Gross beta \\
\hline \multirow[t]{17}{*}{ ALPE } & $\begin{array}{l}2.18 \pm 1.56 \\
2.38 \pm 1.65\end{array}$ & $\begin{array}{l}0.06 \pm 0.05 \\
0.00 \pm 0.03\end{array}$ & $\begin{array}{l}0.10 \pm 0.06 \\
0.16 \pm 0.07\end{array}$ & 4THA & $\begin{array}{r}1.48 \pm 1.48 \\
20.13 \pm 2.25\end{array}$ & $\begin{array}{l}0.03 \pm 0.04 \\
0.00 \pm 0.03\end{array}$ & $\begin{array}{l}0.05 \pm 0.06 \\
0.11+0.06\end{array}$ \\
\hline & $25.35 \pm 1.93$ & & & & $2.40 \pm 1.65$ & & \\
\hline & $2.58 \pm 1.06$ & & & & $25.12 \pm 1.91$ & & \\
\hline & $7.36 \pm 1.83$ & & & & $8.73 \pm 1.88$ & & \\
\hline & $2.42 \pm 1.37$ & $0.21 \pm 0.29$ & $0.32 \pm 0.25$ & & $18.61 \pm 1.58$ & $0.02 \pm 0.03$ & $0.07 \pm 0.06$ \\
\hline & $1.67 \pm 0.97$ & & & & $4.48 \pm 1.07$ & & \\
\hline & $3.81 \pm 1.07$ & & & & $3.44 \pm 3.26$ & & \\
\hline & $4.07 \pm 1.48$ & $0.05 \pm 0.05$ & $0.09 \pm 0.07$ & & $6.36 \pm 1.13$ & $0.02 \pm 0.03$ & $0.05 \pm 0.07$ \\
\hline & $7.36 \pm 1.13$ & & & & $1.70 \pm 0.96$ & & \\
\hline & $14.50 \pm 1.42$ & & & & $5.55 \pm 1.69$ & & \\
\hline & $5.44 \pm 2.84$ & & & & $44.03 \pm 2.60$ & & \\
\hline & $18.91 \pm 2.23$ & & & & $26.42 \pm 1.90$ & & \\
\hline & $1.75 \pm 1.75$ & $0.00 \pm 0.42$ & $0.22 \pm 0.15$ & & $3.74 \pm 1.83$ & $0.03 \pm 0.37$ & $0.15 \pm 0.16$ \\
\hline & $18.28 \pm 2.52$ & & & & $9.69 \pm 2.28$ & $0.10 \pm 0.06$ & $0.22 \pm 0.05$ \\
\hline & $12.14 \pm 2.39$ & & & & $1.92 \pm 1.92$ & & \\
\hline & $10.73 \div 2.33$ & & & & $1.98 \pm 1.98$ & & \\
\hline & & & & & $1.91 \pm 1.91$ & & \\
\hline \multirow[t]{18}{*}{ GRNE } & $4.63 \pm 1.66$ & $0.10 \pm 0.08$ & $0.21 \pm 0.07$ & & & & \\
\hline & $2.14 \pm 1.65$ & $0.04 \pm 0.05$ & $0.06 \pm 0.06$ & ALPW & $2.96 \pm 1.59$ & $0.04 \pm 0.06$ & $0.23 \pm 0.07$ \\
\hline & $16.47 \pm 2.11$ & & & & $11.03 \pm 1.91$ & $0.04 \pm 0.04$ & $0.06 \pm 0.07$ \\
\hline & $4.37 \pm 1.58$ & & & & $3.56 \pm 1.70$ & & \\
\hline & $5.11 \pm 2.28$ & & & & $21.39 \pm 1.78$ & & \\
\hline & $3.51 \pm 3.51$ & $0.03 \pm 0.16$ & $0.49 \pm 0.27$ & & $14.87 \pm 2.07$ & & \\
\hline & $1.25 \pm 1.23$ & & & & $34.63 \pm 2.22$ & $0.08 \pm 0.07$ & $0.19 \pm 0.08$ \\
\hline & $4.74 \pm 1.52$ & & & & $10.95 \pm 1.74$ & & \\
\hline & $3.57 \pm 1.00$ & $0.00 \pm 0.06$ & $0.10 \pm 0.07$ & & $5.33 \pm 1.15$ & & \\
\hline & $6.62 \pm 1.15$ & & & & $12.28 \pm 1.34$ & $0.05 \pm 0.04$ & $0.08 \pm 0.06$ \\
\hline & $11.91 \pm 1.76$ & & & & $1.34 \pm 0.97$ & & \\
\hline & $3.35 \pm 1.05$ & & & & $7.73 \pm 1.17$ & & \\
\hline & $8.40 \pm 1.22$ & & & & $5.00 \pm 1.05$ & & \\
\hline & $5.00 \pm 2.12$ & $0.07 \pm 0.09$ & $0.52 \pm 0.06$ & & $26.64 \pm 2.45$ & & \\
\hline & $4.81 \pm 2.06$ & & & & $15.24 \pm 2.21$ & $0.24 \pm 0.44$ & $0.46 \pm 0.16$ \\
\hline & $2.25 \pm 2.05$ & & & & $14.47 \pm 2.44$ & $0.00 \pm 0.03$ & $0.16 \pm 0.05$ \\
\hline & $4.66 \pm 2.11$ & & & & $5.33 \pm 2.08$ & $0.07 \pm 0.06$ & $0.20 \pm 0.05$ \\
\hline & & & & & $7.14 \pm 2.22$ & & \\
\hline \multirow[t]{19}{*}{ B438 } & $19.54 \pm 2.11$ & $-0.01 \pm 0.02$ & $0.08 \pm 0.06$ & & $14.02 \pm 2.44$ & & \\
\hline & $2.86 \pm 1.67$ & $0.02 \pm 0.04$ & $0.08 \pm 0.06$ & & & & \\
\hline & $23.87 \pm 3.25$ & & & WPDC & $9.92 \pm 1.82$ & $0.06 \pm 0.04$ & $0.09 \pm 0.07$ \\
\hline & $10.92 \pm 1.33$ & & & & $10.95 \pm 1.96$ & $0.03 \pm 0.04$ & $0.09 \pm 0.07$ \\
\hline & $13.25 \pm 2.03$ & & & & $8.55 \pm 2.56$ & & \\
\hline & $10.25 \pm 1.63$ & $-0.01 \pm 0.03$ & $0.14 \pm 0.07$ & & $8.95 \pm 1.28$ & & \\
\hline & $6.18 \pm 1.16$ & & & & $8.14 \pm 1.86$ & & \\
\hline & $6.11 \pm 1.11$ & & & & $24.20 \pm 1.84$ & $0.01 \pm 0.03$ & $0.09 \pm 0.06$ \\
\hline & $10.21 \pm 1.26$ & $-0.02 \pm 0.02$ & $0.09 \pm 0.06$ & & $12.51 \pm 1.38$ & & \\
\hline & $10.55 \pm 1.32$ & & & & $1.27 \pm 0.97$ & & \\
\hline & $32.93 \pm 2.50$ & & & & $14.32 \pm 1.45$ & $0.00 \pm 0.03$ & $0.07 \pm 0.07$ \\
\hline & $17.09 \pm 1.54$ & & & & $4.00 \pm 1.52$ & & \\
\hline & $44.03 \pm 2.60$ & & & & $8.70 \pm 1.79$ & & \\
\hline & $11.66 \pm 2.11$ & $0.15 \pm 0.46$ & $0.37 \pm 0.17$ & & $2.52 \pm 1.38$ & & \\
\hline & $23.42 \pm 2.69$ & & & & $34.11 \pm 2.18$ & & \\
\hline & $3.62 \pm 2.00$ & & & & $11.21 \pm 2.11$ & $0.31 \pm 0.43$ & $0.40 \pm 0.17$ \\
\hline & $5.37 \pm 2.14$ & & & & $2.48 \pm 1.97$ & & \\
\hline & & & & & $10.21 \pm 2.26$ & & \\
\hline & & & & & $11.77 \pm 2.35$ & & \\
\hline
\end{tabular}

...continued 
Table 6-2. Radioactivity in storm water runoff (in Bq/L), 1992 (concluded).

\begin{tabular}{|c|c|c|c|c|c|c|c|}
\hline Locationa & Tritium & Groses alpha & Grose beta & Location & Tritium & Grose alpha & Groses beta \\
\hline \multirow[t]{18}{*}{ CDB } & $3.61 \pm 1.49$ & $-0.01 \pm 0.02$ & $0.08 \pm 0.06$ & ASS2 & $8.29 \pm 1.78$ & $0.00 \pm 0.04$ & $0.22 \pm 0.09$ \\
\hline & $13.62 \pm 1.47$ & & & & $14.73 \pm 2.09$ & $0.04 \pm 0.05$ & $0.12 \pm 0.06$ \\
\hline & $9.32 \pm 1.75$ & & & & $2.00 \pm 1.63$ & & \\
\hline & $19.50 \pm 2.20$ & & & & $61.79 \pm 3.46$ & & \\
\hline & $5.33 \pm 1.11$ & $0.01 \pm 0.11$ & $-0.14 \pm 0.22$ & & $1.56 \pm 1.56$ & & \\
\hline & $13.54 \pm 1.41$ & & & & $7.62 \pm 1.57$ & $0.03 \pm 0.04$ & $0.13 \pm 0.06$ \\
\hline & $11.77 \pm 1.40$ & & & & $1.88 \pm 1.01$ & & \\
\hline & $13.14 \pm 1.38$ & $0.00 \pm 0.04$ & $0.12 \pm 0.06$ & & $13.28 \pm 1.45$ & & \\
\hline & $28.64 \pm 1.95$ & & & & $3.85 \pm 1.43$ & $0.05 \pm 0.04$ & $0.12 \pm 0.07$ \\
\hline & $8.33 \pm 1.20$ & & & & $0.89 \pm 0.89$ & & \\
\hline & $14.21 \pm 1.46$ & & & & $16.02 \pm 2.05$ & & \\
\hline & $44.03 \pm 2.86$ & & & & $28.75 \pm 2.01$ & & \\
\hline & $11.77 \pm 2.11$ & $0.01 \pm 0.34$ & $0.25 \pm 0.16$ & & $48.84 \pm 2.78$ & & \\
\hline & $8.73 \pm 2.25$ & $0.05 \pm 0.04$ & $0.19 \pm 0.04$ & & $1.75 \pm 1.75$ & $0.06 \pm 0.34$ & $0.19 \pm 0.15$ \\
\hline & $10.40 \pm 2.27$ & & & & $10.77 \pm 2.33$ & $0.06 \pm 0.04$ & $0.25 \pm 0.05$ \\
\hline & $4.03 \pm 2.13$ & & & & $3.10 \pm 2.01$ & & \\
\hline & $3.17 \pm 2.05$ & & & & $2.02 \pm 2.02$ & & \\
\hline & & & & & $1.98 \pm 1.98$ & & \\
\hline \multirow[t]{19}{*}{ ALPN } & $9.55 \pm 1.80$ & $0.07 \pm 0.09$ & $3.82 \pm 0.18$ & & & & \\
\hline & $12.25 \pm 1.90$ & $0.06 \pm 0.05$ & $0.11 \pm 0.06$ & ASW & $9.03 \pm 1.80$ & $0.00 \pm 0.04$ & $0.16 \pm 0.07$ \\
\hline & $1.62 \pm 1.62$ & $0.01 \pm 0.04$ & $0.08 \pm 0.06$ & & $23.38 \pm 2.34$ & $0.02 \pm 0.03$ & $0.12 \pm 0.06$ \\
\hline & $18.46 \pm 2.18$ & & & & $3.48 \pm 1.69$ & & \\
\hline & $2.06 \pm 1.05$ & & & & $29.60 \pm 2.04$ & & \\
\hline & $2.56 \pm 1.66$ & & & & $2.19 \pm 2.19$ & & \\
\hline & $5.29 \pm 1.12$ & $0.07 \pm 0.07$ & $0.17 \pm 0.08$ & & $8.92 \pm 1.26$ & $0.01 \pm 0.04$ & $0.27 \pm 0.07$ \\
\hline & $6.18 \pm 1.54$ & & & & $1.38 \pm 0.96$ & & \\
\hline & $8.62 \pm 1.26$ & & & & $0.95 \pm 0.95$ & & \\
\hline & $4.77 \pm 1.07$ & $0.06 \pm 0.04$ & $0.08 \pm 0.06$ & & $3.39 \pm 1.38$ & $0.06 \pm 0.05$ & $0.01 \pm 0.07$ \\
\hline & $4.51 \pm 1.07$ & & & & $2.31 \pm 1.30$ & & \\
\hline & $3.17 \pm 1.60$ & & & & $6.73 \pm 1.74$ & & \\
\hline & $1.35 \pm 0.96$ & & & & $56.24 \pm 3.04$ & & \\
\hline & $22.53 \pm 1.76$ & & & & $1.75 \pm 1.75$ & $0.06 \pm 0.35$ & $0.31 \pm 0.16$ \\
\hline & $12.28 \pm 2.13$ & $-0.07 \pm 0.30$ & $0.17 \pm 0.16$ & & $18.35 \pm 2.55$ & $0.01 \pm 0.04$ & $0.23 \pm 0.04$ \\
\hline & $6.33 \pm 2.16$ & $0.77 \pm 0.35$ & $0.79 \pm 0.17$ & & $5.07 \pm 2.09$ & & \\
\hline & $10.62 \pm 2.27$ & & & & $2.02 \pm 2.02$ & & \\
\hline & $6.77 \pm 2.21$ & & & & $2.47 \pm 2.02$ & & \\
\hline & $8.36 \pm 2.27$ & & & & & & \\
\hline Mean & 10.55 & 0.06 & 0.24 & & & & \\
\hline Std dev & 10.09 & 0.11 & 0.50 & & & & \\
\hline
\end{tabular}

Note: Results are reported $\pm 2 \sigma$; see Quality Assurance chapter.

Gross alpha and gross beta sampled quarterly; quality assurance duplicates also reported. For very low counts, subtraction of background count may yield a negative number for gross alpha and gross beta (see U.S. Department of Energy 1991).

a See Fig. 6-4 for sampling locations. 
Table 6-3. Radioactivity in water, Livermore Valley (in Bq/L), 1992.

\begin{tabular}{|c|c|c|c|}
\hline Location & Tritium & Gross alpha & Gross beta \\
\hline \multirow[t]{4}{*}{ DEL } & $1.41 \pm 0.11$ & $0.00 \pm 0.08$ & $0.15 \pm 0.07$ \\
\hline & $0.90 \pm 0.09$ & $0.00 \pm 0.08$ & $0.04 \pm 0.06$ \\
\hline & $0.94 \pm 0.13$ & $0.00 \pm 0.08$ & $0.07 \pm 0.06$ \\
\hline & $1.05 \pm 0.13$ & $0.00 \pm 0.07$ & $0.07 \pm 0.06$ \\
\hline \multirow[t]{4}{*}{ ZON7 } & $0.94 \pm 0.08$ & $-0.16 \pm 0.09$ & $9.70 \pm 0.28$ \\
\hline & $0.66 \pm 0.07$ & $0.03 \pm 0.06$ & $0.06 \pm 0.10$ \\
\hline & $0.25 \pm 0.08$ & $0.00 \pm 0.10$ & $0.24 \pm 0.08$ \\
\hline & $0.87 \pm 0.14$ & $0.01 \pm 0.08$ & $0.08 \pm 0.05$ \\
\hline \multirow[t]{3}{*}{ DUCK } & $2.82 \pm 0.18$ & $0.42 \pm 0.39$ & $0.17 \pm 0.17$ \\
\hline & $2.67 \pm 0.20$ & $0.03 \pm 0.27$ & $0.07 \pm 0.16$ \\
\hline & $1.67 \pm 0.14$ & $-0.21 \pm 0.30$ & $0.45 \pm 0.30$ \\
\hline \multirow[t]{4}{*}{ CAL } & $0.92 \pm 0.08$ & $0.03 \pm 0.07$ & $0.07 \pm 0.06$ \\
\hline & $0.61 \pm 0.07$ & $0.03 \pm 0.05$ & $0.08 \pm 0.08$ \\
\hline & $0.75 \pm 0.11$ & $-0.07 \pm 0.06$ & $0.04 \pm 0.06$ \\
\hline & $0.92 \pm 0.13$ & $0.00 \pm 0.05$ & $0.05 \pm 0.05$ \\
\hline \multirow[t]{4}{*}{ ALAG } & $1.00 \pm 0.09$ & $0.01 \pm 0.09$ & $0.11 \pm 0.07$ \\
\hline & $1.15 \pm 0.10$ & $0.00 \div 0.09$ & $-0.01 \pm 0.08$ \\
\hline & $0.94 \pm 0.12$ & $0.02 \pm 0.05$ & $0.00 \pm 0.07$ \\
\hline & $1.17 \pm 0.15$ & $0.05 \pm 0.08$ & $0.10 \pm 0.05$ \\
\hline \multirow[t]{4}{*}{ SHAD } & $3.67 \pm 0.23$ & $0.09 \pm 0.13$ & $0.13 \pm 0.07$ \\
\hline & $3.39 \pm 0.23$ & $0.01 \pm 0.15$ & $0.11 \pm 0.08$ \\
\hline & $3.08 \pm 0.18$ & $0.02 \pm 0.14$ & $0.08 \pm 0.06$ \\
\hline & $3.89 \pm 0.22$ & $0.09 \pm 0.20$ & $-0.01 \pm 0.10$ \\
\hline \multirow[t]{12}{*}{ POOL } & $20.94 \pm 2.18$ & $0.05 \pm 0.13$ & $0.16 \pm 0.08$ \\
\hline & $21.39 \pm 1.78$ & $-0.15 \pm 0.31$ & $0.46 \pm 0.26$ \\
\hline & $22.72 \pm 1.75$ & $-0.06 \pm 0.10$ & $0.06 \pm 0.08$ \\
\hline & $22.57 \pm 1.74$ & $-0.09 \pm 0.11$ & $0.14 \pm 0.09$ \\
\hline & $22.98 \pm 1.79$ & $0.02 \pm 0.14$ & $0.13 \pm 0.07$ \\
\hline & $19.20 \pm 2.15$ & $0.01 \pm 0.31$ & $0.37 \pm 0.15$ \\
\hline & $17.43 \pm 2.09$ & $-0.05 \pm 0.14$ & $0.15 \pm 0.08$ \\
\hline & $15.47 \pm 2.09$ & $-0.69 \pm 0.43$ & $0.39 \pm 0.28$ \\
\hline & $10.99 \pm 2.21$ & $0.10 \pm 0.30$ & $0.48 \pm 0.15$ \\
\hline & $7.81 \pm 1.09$ & $0.08 \pm 0.29$ & $0.16 \pm 0.13$ \\
\hline & $9.36 \pm 1.47$ & $-0.01 \pm 0.30$ & $0.61 \pm 0.17$ \\
\hline & $12.62 \pm 1.99$ & $-b$ & -b \\
\hline
\end{tabular}




\section{Surveillance Water Monitoring}

Table 6-3. Radioactivity in water, Livermore Valley (in Bq/L), 1992 (concluded).

\begin{tabular}{|c|c|c|c|}
\hline Location" & Tritlum & Gross alpha & Gross beta \\
\hline \multirow[t]{4}{*}{ GAS } & $1.66 \pm 0.12$ & $0.43 \pm 0.19$ & $7.43 \pm 0.25$ \\
\hline & $0.75 \pm 0.08$ & $0.01 \pm 0.06$ & $0.09 \pm 0.06$ \\
\hline & $1.33 \pm 0.15$ & $-0.02 \pm 0.10$ & $-0.02 \pm 0.13$ \\
\hline & $1.48 \pm 0.15$ & $0.06 \pm 0.12$ & $0.04 \pm 0.04$ \\
\hline \multirow[t]{8}{*}{ TAPC } & $1.05 \pm 0.08$ & $0.00 \pm 0.03$ & $0.01 \pm 0.08$ \\
\hline & $0.97 \pm 0.14$ & & \\
\hline & $0.23 \pm 0.05$ & $-0.01 \pm 0.04$ & $-0.01 \pm 0.06$ \\
\hline & $0.83 \pm 0.10$ & & \\
\hline & $0.61 \pm 0.11$ & $0.03 \pm 0.04$ & $0.04 \pm 0.07$ \\
\hline & $0.72 \pm 0.13$ & & \\
\hline & $1.02 \pm 0.13$ & $0.01 \pm 0.02$ & $0.00 \pm 0.02$ \\
\hline & $0.90 \pm 0.16$ & & \\
\hline \multirow[t]{4}{*}{ PALM } & $0.97 \pm 0.09$ & $-0.02 \pm 0.08$ & $0.04 \pm 0.06$ \\
\hline & $0.64 \pm 0.08$ & $-0.08 \pm 0.05$ & $0.04 \pm 0.09$ \\
\hline & $0.68 \pm 0.12$ & $0.07 \pm 0.11$ & $0.09 \pm 0.08$ \\
\hline & $0.58 \pm 0.11$ & $0.00 \pm 0.07$ & $0.10 \pm 0.05$ \\
\hline \multirow[t]{4}{*}{ BELL } & $0.93 \pm 0.10$ & $-0.01 \pm 0.05$ & $0.09 \pm 0.06$ \\
\hline & $0.95 \pm 0.63$ & $-0.04 \pm 0.06$ & $0.10 \pm 0.07$ \\
\hline & $0.98 \pm 0.13$ & $0.01 \pm 0.10$ & $0.08 \pm 0.14$ \\
\hline & $1.08 \pm 0.13$ & $-0.02 \pm 0.06$ & $0.07 \pm 0.05$ \\
\hline \multirow[t]{4}{*}{ ORCH } & $1.20 \pm 0.10$ & $-0.01 \pm 0.24$ & $0.09 \pm 0.13$ \\
\hline & $0.94 \pm 0.09$ & $0.05 \pm 0.20$ & $0.06 \pm 0.16$ \\
\hline & $0.49 \pm 0.10$ & $0.20 \pm 0.18$ & $0.08 \pm 0.07$ \\
\hline & $0.51 \pm 0.11$ & $-0.01 \pm 0.18$ & $0.11 \pm 0.12$ \\
\hline Mean & 4.42 & 0.005 & 0.12 \\
\hline Std dev & 6.88 & 0.14 & 0.14 \\
\hline Mean without POOL & 1.22 & 0.02 & 0.08 \\
\hline Std dev & 0.86 & 0.11 & 0.08 \\
\hline Mean of POOL & 16.96 & -0.07 & 0.28 \\
\hline Std dev & 5.56 & 0.22 & 0.18 \\
\hline
\end{tabular}

Note: Results are reported $\pm 2 \sigma$; see Quality Assurance chapter.

For very low counts, subtraction of background values may yield a negative number for gross alpha and gross beta (see U.S. Department of Energy 1991).

a See Fig. 6-2 for sampling locations.

b Sample not taken; seo Quality Assurance chapter.

c Tritium sample twice as frequently as gross alpha and gross beta. 


\section{Surveillance Water Monitoring}

Table 6-4. Tritium in rain, Livermore site and Livermore Valley (in Bq/L), 1992.

\begin{tabular}{|c|c|c|c|c|c|c|c|}
\hline \multirow[b]{2}{*}{ Date } & \multicolumn{7}{|c|}{ Sampling locationa } \\
\hline & B343 & CDB & B291 & VIS & SALV & MET & cow \\
\hline Jan. 7 & $46.99 \pm 2.73$ & $-b$ & $25.16 \pm 2.24$ & $11.77 \pm 1.88$ & $19.39 \pm 2.09$ & $2.30 \pm 1.57$ & $15.39 \pm 2.00$ \\
\hline Jan. 30 & $36.70 \pm 2.68$ & $-^{b}$ & $13.14 \pm 2.04$ & $2.66 \pm 1.65$ & $5.29 \pm 1.77$ & $15.61 \pm 2.11$ & $3.85 \pm 1.62$ \\
\hline Feb. 5 & $79.18 \pm 3.88$ & $-{ }^{b}$ & $54.76 \pm 3.23$ & $17.09 \pm 1.57$ & $40.70 \pm 2.44$ & $10.66 \pm 1.81$ & $16.69 \pm 2.14$ \\
\hline Feb. 8 & $30.49 \pm 2.10$ & $-b$ & $26.90 \pm 1.94$ & $12.58 \pm 1.86$ & $20.05 \pm 1.62$ & $6.03 \pm 1.78$ & $6.44 \pm 1.80$ \\
\hline Feb. 11 & $44.40 \pm 2.75$ & $-b$ & $34.00 \pm 2.52$ & $8.21 \pm 1.76$ & $6.33 \pm 1.68$ & $13.02 \pm 1.93$ & $3.50 \pm 1.01$ \\
\hline Feb. 12 & $31.12 \pm 2.49$ & $9.07 \pm 1.87$ & $13.58 \pm 2.01$ & $5.40 \pm 1.73$ & $8.70 \pm 1.79$ & $7.59 \pm 1.81$ & $3.47 \pm 1.57$ \\
\hline Feb. 18 & $-{ }^{b}$ & $33.67 \pm 2.15$ & $29.23 \pm 2.05$ & $9.92 \pm 1.77$ & $20.87 \pm 1.69$ & $2.31 \pm 1.63$ & $14.43 \pm 2.05$ \\
\hline Feb. 19 & $42.92 \pm 2.75$ & $15.32 \pm 2.01$ & $35.74 \pm 2.57$ & $7.88 \pm 1.76$ & $16.50 \pm 2.03$ & $3.53 \pm 1.59$ & $3.20 \pm 1.59$ \\
\hline Feb. 20 & $48.47 \pm 2.91$ & $35.96 \pm 2.63$ & $115.81 \pm 4.05$ & $23.24 \pm 2.32$ & $13.14 \pm 2.02$ & $1.59 \pm 1.59$ & $23.68 \pm 1.80$ \\
\hline Mar. 3 & $40.70 \pm 2.73$ & $86.58 \pm 3.64$ & $35.48 \pm 2.63$ & $19.54 \pm 2.21$ & $48.84 \pm 2.93$ & $9.92 \pm 1.92$ & $29.86 \pm 2.48$ \\
\hline Mar. 6 & $78.44 \pm 3.45$ & $27.12 \pm 2.39$ & $21.05 \pm 2.21$ & $5.96 \pm 1.76$ & $10.25 \pm 1.90$ & $4.55 \pm 1.71$ & $13.73 \pm 1.98$ \\
\hline Mar. 17 & $53.28 \pm 2.98$ & $43.29 \pm 2.77$ & $51.06 \pm 2.91$ & $22.09 \pm 2.21$ & $37.74 \pm 2.60$ & $1.47 \pm 1.47$ & $12.25 \pm 1.92$ \\
\hline Mar. 26 & $24.75 \pm 1.86$ & $12.47 \pm 1.35$ & $26.38 \pm 1.90$ & $8.18 \pm 1.24$ & $17.09 \pm 2.02$ & $14.87 \pm 1.96$ & $4.81 \pm 1.71$ \\
\hline Apr. 13 & $23.27 \pm 2.33$ & $22.16 \pm 2.28$ & $21.76 \pm 2.28$ & $20.31 \pm 1.63$ & $19.80 \pm 2.22$ & $6.22 \pm 1.13$ & $11.47 \pm 1.33$ \\
\hline Jun. 30 & $41.81 \pm 2.76$ & $14.91 \pm 2.07$ & $110.63 \pm 3.98$ & $10.77 \pm 1.95$ & $24.24 \pm 2.33$ & $1.52 \pm 1.52$ & $3.52 \pm 1.61$ \\
\hline Oct. 21 & $40.33 \pm 3.15$ & $30.64 \pm 3.00$ & $74.00 \pm 4.00$ & $11.06 \pm 2.39$ & $22.83 \pm 2.79$ & $1.99 \pm 1.99$ & $10.88 \pm 2.37$ \\
\hline Nov. 2 & $49.95 \pm 3.50$ & $50.69 \pm 3.50$ & $95.09 \pm 4.47$ & $21.57 \pm 2.76$ & $23.13 \pm 2.80$ & $3.28 \pm 2.14$ & $24.16 \pm 2.75$ \\
\hline Dec. 4 & $12.06 \pm 1.99$ & $3.69 \pm 1.93$ & $9.66 \pm 2.93$ & $4.26 \pm 1.72$ & $4.85 \pm 1.97$ & $6.14 \pm 1.79$ & $4.88 \pm 1.74$ \\
\hline Dec. 10 & $73.26 \pm 3.88$ & $35.26 \pm 3.03$ & $22.76 \pm 2.71$ & $26.79 \pm 2.81$ & $21.68 \pm 2.67$ & $4.81 \pm 2.10$ & $19.83 \pm 2.62$ \\
\hline Dec. 15 & $59.94 \pm 3.66$ & $28.49 \pm 2.05$ & $16.95 \pm 2.32$ & $11.84 \pm 2.38$ & $33.93 \pm 3.05$ & $6.14 \pm 1.44$ & $11.66 \pm 2.11$ \\
\hline Mean & 45.16 & 29.95 & 41.66 & 13.06 & 20.77 & 6.18 & 11.88 \\
\hline Std dev & 18.12 & 20.42 & 32.32 & 7.06 & 11.97 & 4.47 & 7.96 \\
\hline
\end{tabular}

...continued 


\section{Surveillance Water Monitoring}

Table 6-4. Tritium in rain, Livermore site and Livermore Valley (in Bq/L), 1992 (continued).

\begin{tabular}{|c|c|c|c|c|c|c|}
\hline \multirow[b]{2}{*}{ Date } & \multicolumn{6}{|c|}{ Sampling locatlon } \\
\hline & ESAN & AQUE & ZON7 & GTES & BVA & VINE \\
\hline $\operatorname{Jan} .7$ & $-^{b}$ & $8.29 \pm 1.77$ & $-b$ & $1.71 \pm 1.55$ & $1.52 \pm 1.52$ & $1.52 \pm 1.52$ \\
\hline Jan. 30 & $-b$ & $6.51 \pm 1.81$ & $3.11 \pm 1.67$ & $4.92 \pm 1.74$ & $8.03 \pm 1.86$ & $4.00 \pm 1.69$ \\
\hline Feb. 5 & $\square^{b}$ & $16.35 \pm 2.11$ & $18.32 \pm 2.20$ & $4.96 \pm 1.45$ & $9.62 \pm 1.74$ & $15.84 \pm 2.12$ \\
\hline Feb. 8 & $-b$ & $16.10 \pm 1.53$ & $8.03 \pm 1.20$ & $36.30 \pm 2.25$ & $25.75 \pm 1.91$ & $7.03 \pm 2.50$ \\
\hline Feb. 11 & $-^{b}$ & $4.74 \pm 1.63$ & $4.44 \pm 1.46$ & $2.11 \pm 1.52$ & $6.51 \pm 1.70$ & $0.93 \pm 0.92$ \\
\hline Fob. 12 & $-b$ & $12.32 \pm 1.97$ & $3.50 \pm 1.57$ & $8.51 \pm 1.85$ & $4.37 \pm 1.71$ & $1.85 \pm 1.51$ \\
\hline Feb. 18 & $-b$ & $14.54 \pm 1.92$ & $6.99 \pm 2.46$ & $2.01 \pm 1.32$ & $2.38 \pm 1.64$ & $4.66 \pm 1.71$ \\
\hline Feb. 19 & $-^{b}$ & $11.88 \pm 1.90$ & $5.22 \pm 1.66$ & $33.86 \pm 2.51$ & $11.14 \pm 1.86$ & $13.73 \pm 1.95$ \\
\hline Feb. 20 & $-b$ & $27.60 \pm 2.43$ & $14.32 \pm 1.43$ & $3.85 \pm 2.27$ & $1.87 \pm 1.65$ & $0.93 \pm 0.93$ \\
\hline Mar. 3 & $11.66 \pm 1.28$ & $19.68 \pm 2.22$ & $17.58 \pm 2.14$ & $1.37 \pm 1.23$ & $10.73 \pm 2.62$ & $2.85 \pm 1.68$ \\
\hline Mar. 6 & $26.34 \pm 2.37$ & $9.44 \pm 1.87$ & $3.61 \pm 1.62$ & $8.47 \pm 1.84$ & $1.57 \pm 1.57$ & $1.52 \pm 1.52$ \\
\hline Mar. 17 & $37.00 \pm 2.59$ & $53.28 \pm 2.98$ & $12.91 \pm 1.94$ & $1.52 \pm 1.52$ & $2.30 \pm 1.56$ & $2.18 \pm 1.55$ \\
\hline Mar. 26 & $21.02 \pm 1.72$ & $11.21 \pm 1.33$ & $5.96 \pm 1.75$ & $12.69 \pm 1.84$ & $6.51 \pm 1.16$ & $10.40 \pm 1.90$ \\
\hline Apr. 13 & $28.82 \pm 2.48$ & $18.61 \pm 2.20$ & $9.51 \pm 1.26$ & $17.58 \pm 2.16$ & $8.44 \pm 1.26$ & $6.33 \pm 1.15$ \\
\hline Jun. 30 & $51.43 \pm 2.98$ & $26.34 \pm 2.40$ & $6.88 \pm 1.75$ & $-^{b}$ & $1.52 \pm 1.52$ & $1.71 \pm 1.55$ \\
\hline Oct. 21 & $29.56 \pm 2.96$ & $30.86 \pm 2.99$ & $8.03 \pm 2.29$ & $-b$ & $2.03 \pm 2.03$ & - b \\
\hline Nov. 2 & $33.86 \pm 2.74$ & $20.02 \pm 2.72$ & $7.03 \pm 2.21$ & $1.87 \pm 1.81$ & $1.92 \pm 1.92$ & $-^{b}$ \\
\hline Dec. 4 & $34.71 \pm 2.95$ & $5.92 \pm 1.79$ & $1.61 \pm 1.61$ & $1.85 \pm 1.85$ & $6.88 \pm 1.83$ & $6.48 \pm 1.79$ \\
\hline Dec. 10 & $24.86 \pm 2.76$ & $17.80 \pm 2.54$ & $13.73 \pm 2.40$ & $2.04 \pm 2.04$ & $1.94 \pm 1.94$ & $1.98 \pm 1.98$ \\
\hline Dec. 15 & $63.27 \pm 3.73$ & $26.53 \pm 2.04$ & $12.25 \pm 1.58$ & $5.51 \pm 2.14$ & $3.13 \pm 2.06$ & $3.50 \pm 2.07$ \\
\hline Mean & 32.96 & 17.90 & 8.58 & 8.40 & 5.91 & 4.86 \\
\hline Std dev & 14.19 & 11.26 & 4.95 & 10.65 & 5.74 & 4.43 \\
\hline
\end{tabular}




\section{Surveillance Water Monitoring}

Table 6-4. Tritium in rain, Livermore site and Livermore Valley (in Bq/L), 1992 (concluded).

\begin{tabular}{|c|c|c|c|c|c|c|}
\hline \multirow[b]{2}{*}{ Date } & \multicolumn{6}{|c|}{ Sampling location } \\
\hline & PATT & FCC & SLST & ALTA & DEL7 & PARK \\
\hline $\operatorname{Jan} .7$ & $1.53 \pm 1.53$ & $-b$ & $1.49 \pm 1.49$ & $3.44 \pm 1.61$ & $1.50 \pm 1.50$ & $1.48 \pm 1.48$ \\
\hline Jan. 30 & $1.70 \pm 1.62$ & $2.93 \pm 1.67$ & $2.77 \pm 1.67$ & $1.62 \pm 1.62$ & $3.58 \pm 1.69$ & $3.81 \pm 1.70$ \\
\hline Feb. 5 & $1.28 \pm 1.28$ & $2.58 \pm 1.66$ & $4.33 \pm 1.15$ & $2.58 \pm 1.04$ & $2.55 \pm 1.06$ & $2.73 \pm 1.08$ \\
\hline Fob. 8 & $5.88 \pm 5.88$ & $3.92 \pm 2.35$ & $15.87 \pm 1.48$ & $4.63 \pm 1.07$ & $3.01 \pm 1.02$ & $7.03 \pm 2.41$ \\
\hline Feb. 11 & $1.46 \pm 1.46$ & $0.93 \pm 0.93$ & $1.44 \pm 1.44$ & $1.45 \pm 1.45$ & $1.07 \pm 0.97$ & $1.17 \pm 1.17$ \\
\hline Fob. 12 & $1.64 \pm 1.60$ & $1.44 \pm 1.44$ & $2.34 \pm 1.63$ & $2.42 \pm 1.63$ & $1.59 \pm 1.59$ & $1.89 \pm 1.60$ \\
\hline Feb. 18 & $0.95 \pm 0.95$ & $1.59 \pm 1.59$ & $1.59 \pm 1.59$ & $20.13 \pm 1.67$ & $1.60 \pm 1.60$ & $2.09 \pm 1.62$ \\
\hline Fob. 19 & $2.51 \pm 1.55$ & $1.51 \pm 1.51$ & $2.19 \pm 1.54$ & $1.51 \pm 1.51$ & $2.58 \pm 1.56$ & $2.25 \pm 1.55$ \\
\hline Fob. 20 & $3.15 \pm 1.02$ & $1.58 \pm 1.58$ & $1.54 \pm 1.54$ & $2.71 \pm 1.00$ & $1.43 \pm 0.94$ & $1.62 \pm 1.62$ \\
\hline Mar. 3 & $3.96 \pm 1.03$ & $5.99 \pm 1.80$ & $4.88 \pm 1.76$ & $1.41 \pm 1.25$ & $2.18 \pm 2.18$ & $1.57 \pm 1.57$ \\
\hline Mar. 6 & $2.63 \pm 1.64$ & $1.52 \pm 1.52$ & $1.58 \pm 1.58$ & $1.59 \pm 1.59$ & $1.47 \pm 1.47$ & $1.58 \pm 1.58$ \\
\hline Mar. 17 & $3.62 \pm 1.62$ & $1.48 \pm 1.48$ & $1.66 \pm 1.54$ & $4.40 \pm 1.64$ & $1.82 \pm 1.57$ & $1.52 \pm 1.52$ \\
\hline Mar. 26 & $1.23 \pm 1.23$ & $2.58 \pm 1.63$ & $5.25 \pm 1.11$ & $4.44 \pm 1.07$ & $3.70 \pm 1.06$ & $0.94 \pm 0.94$ \\
\hline Apr. 13 & $2.27 \pm 2.27$ & $2.62 \pm 2.62$ & $2.92 \pm 1.04$ & $2.56 \pm 1.67$ & $4.63 \pm 3.19$ & $3.19 \pm 1.32$ \\
\hline Jun. 30 & $1.64 \pm 1.64$ & $1.55 \pm 1.55$ & $4.07 \pm 1.77$ & $-b$ & $2.79 \pm 1.66$ & $1.49 \pm 1.49$ \\
\hline Oct. 21 & $2.51 \pm 2.10$ & $2.20 \pm 2.07$ & $2.30 \pm 2.03$ & $-b$ & $2.04 \pm 2.04$ & $1.98 \pm 1.98$ \\
\hline Nov. 2 & $1.72 \pm 1.72$ & $1.99 \pm 1.99$ & $2.00 \pm 2.00$ & $3.92 \pm 1.83$ & $2.93 \pm 2.12$ & $1.98 \pm 1.98$ \\
\hline Dec. 4 & $2.00 \pm 2.00$ & $1.61 \pm 1.61$ & $3.32 \pm 1.69$ & $1.96 \pm 1.96$ & $1.56 \pm 1.56$ & $2.45 \pm 1.65$ \\
\hline Dec. 10 & $2.03 \pm 2.03$ & $2.00 \pm 2.00$ & $1.93 \pm 1.93$ & $2.75 \pm 2.07$ & $1.95 \pm 1.95$ & $2.92 \pm 2.69$ \\
\hline Dec. 15 & $2.33 \pm 1.27$ & $1.91 \pm 1.91$ & $1.64 \pm 1.64$ & $5.11 \pm 1.89$ & $1.95 \pm 1.95$ & $1.24 \pm 1.24$ \\
\hline Mean & 2.30 & 2.21 & 3.26 & 3.81 & 2.30 & 2.25 \\
\hline Std dev & 1.15 & 1.15 & 3.20 & 4.25 & 0.92 & 1.34 \\
\hline
\end{tabular}

Note: Results are reported $\pm 2 \sigma$; see Quality Assurance chapter.

a See Fig. 6-3 for sampling locations.

b Sample unavailable; 6 samples had insufficient volume, and 18 could not be taken due to missing sample buckets (see Quality Assurance chapter). 
Table 6-5. Tritium activity in wells, Livermore Valley (in Bq/L), 1992.

\begin{tabular}{|c|c|}
\hline Location" & Activity \\
\hline $1 \mathrm{H} 3$ & $0.39 \pm 0.11$ \\
\hline 1P2 & $4.92 \pm 0.27$ \\
\hline $1 \mathrm{P} 3$ & $0.73 \pm 0.11$ \\
\hline $1 \mathrm{R} 2$ & $2.95 \pm 0.18$ \\
\hline $2 \mathrm{R} 1$ & $6.51 \pm 0.31$ \\
\hline $7 \mathrm{C2}$ & $4.22 \pm 0.22$ \\
\hline $7 P 3$ & $0.06 \pm 0.06$ \\
\hline 7P3 duplicate & $0.08 \pm 0.07$ \\
\hline $8 F 1$ & $1.35 \pm 0.13$ \\
\hline $8 P 1$ & $2.29 \pm 0.19$ \\
\hline $9 M 2$ & $1.59 \pm 0.14$ \\
\hline 9M2 duplicate & $1.24 \pm 0.18$ \\
\hline $9 Q 1$ & $0.62 \pm 0.10$ \\
\hline 1181 & $16.95 \pm 0.54$ \\
\hline $12 A 2$ & $2.59 \pm 0.21$ \\
\hline $12 \mathrm{G} 1$ & No sample provided \\
\hline $16 L 5$ & $1.41 \pm 0.15$ \\
\hline $16 \mathrm{~L} 7$ & $0.96 \pm 0.14$ \\
\hline 16L7 duplicate & $0.90 \pm 0.12$ \\
\hline $18 \mathrm{~A} 1$ & $0.92 \pm 0.12$ \\
\hline $18 A 5$ & No sample provided \\
\hline $16 B 1$ & $1.60 \pm 0.15$ \\
\hline Mean & 2.83 \\
\hline \multirow[t]{2}{*}{ Std dev } & 3.91 \\
\hline & PCi/L \\
\hline Mean & 76.5 \\
\hline Std dev & 105.7 \\
\hline
\end{tabular}

Note: Results are reported $\pm 2 \sigma$; see Quality Assurance chapter.

a See Fig. 6-1 for sampling locations. 
Table 6-6. LLNL storm water runoff, nonradioactive parameters, 1992.

\begin{tabular}{|c|c|c|c|c|c|c|c|c|c|c|}
\hline \multirow[b]{2}{*}{ Parameter } & \multicolumn{10}{|c|}{ Sampling location" } \\
\hline & 4THA & ALPE & ALPN & ALPW & ASS2 & ASW & B438 & CDB & GRNE & WPDC \\
\hline \multicolumn{11}{|l|}{ Inorganic chemicals (mg/L) } \\
\hline Alkalinity (Total, as $\mathrm{CaCO}_{3}$ ) & 10 & 30 & 20 & 30 & 30 & 20 & 10 & 10 & 40 & 20 \\
\hline \multirow[t]{5}{*}{ (Bicarbonate $=$ Total $)$} & 10 & 30 & 20 & 20 & 30 & 30 & 20 & zu & 40 & 20 \\
\hline & 20 & 220 & 80 & 30 & 30 & 50 & 20 & 20 & 150 & 40 \\
\hline & 10 & 40 & 20 & 30 & 20 & 20 & 20 & 20 & 100 & 20 \\
\hline & 16 & 47 & 16 & 31 & 19 & 26 & 22 & 15 & 57 & 23 \\
\hline & 15 & & 26 & 15 & 22 & 17 & & & & \\
\hline \multirow[t]{5}{*}{ Arsenic } & 0.003 & 0.002 & 0.02 & 0.002 & & 0.0028 & 0.003 & 0.0044 & 0.005 & 0.003 \\
\hline & 0.002 & 0.005 & & & & & 0.011 & 0.0021 & 0.003 & 0.002 \\
\hline & 0.0024 & & & & & & & & & 0.0049 \\
\hline & 0.002 & & & & & & & & & \\
\hline & 38 nonde & tections a & at $<0.002$ & ppm. & & & & & & \\
\hline \multirow[t]{3}{*}{ Barium } & & 0.10 & 0.1 & 0.055 & & & & & 0.1 & 0.05 \\
\hline & & & & & & & & & 0.05 & \\
\hline & 49 nonde & tections & at $<0.05$ & ppm. & & & & & & \\
\hline Beryllium & 16 nonde & tections : & at $<0.2 \mathrm{pr}$ & $\mathrm{bb}$ and fo & ity at $<0$. & $5 \mathrm{ppb}$. & & & & \\
\hline \multirow[t]{3}{*}{ Cadmium } & 0.0007 & 0.0012 & & & & 0.0008 & 0.0006 & 0.0006 & 0.0008 & \\
\hline & 0.0028 & & & & & & 0.0016 & & & \\
\hline & 47 nonde & tections & at $<0.5 \mathrm{pr}$ & & & & & & & \\
\hline \multirow[t]{6}{*}{ Chemical oxygen demand } & 25 & 25 & 25 & 25 & 25 & 25 & 25 & & 30 & 25 \\
\hline & 50 & 110 & 70 & 80 & 150 & 110 & 110 & 75 & 170 & 75 \\
\hline & 40 & 130 & 40 & 45 & 40 & 90 & 90 & 30 & 100 & 50 \\
\hline & 50 & 60 & 90 & 50 & 110 & 85 & 50 & 80 & 40 & 50 \\
\hline & 150 & 140 & 80 & 180 & 90 & 210 & 160 & 120 & 80 & 130 \\
\hline & 70 & & 50 & 40 & 40 & 30 & & 40 & & \\
\hline \multirow[t]{6}{*}{ Chloride } & 1.2 & 6 & 12 & 18 & 1.2 & 1 & 1 & 1 & 9 & 15 \\
\hline & & 13 & 1 & 13 & & 15 & & & 13 & 4 \\
\hline & & 240 & & 4.7 & & & & & 150 & 1.9 \\
\hline & & 24 & & & & & & & 84 & \\
\hline & & 9 & & & & & & & 30 & \\
\hline & 31 nonde & tections & at $<1 \mathrm{ppn}$ & & & & & & & \\
\hline \multirow[t]{3}{*}{ Chromium } & 0.0061 & 0.0056 & 0.016 & 0.0068 & & 0.005 & 0.0071 & 0.023 & & 0.0083 \\
\hline & & 0.005 & 0.0061 & & & & & 0.0067 & & \\
\hline & 42 nonde & tections & at $<0.005$ & $\mathrm{ppm}$. & & & & & & \\
\hline \multirow[t]{5}{*}{ Fluoride } & 0.1 & 0.9 & & 0.1 & 0.1 & & & & 0.2 & \\
\hline & & 0.1 & & 0.12 & & & & & 0.2 & \\
\hline & & 0.15 & & & & & & & 0.6 & 0.1 \\
\hline & & & & & & & & & 0.4 & \\
\hline & 41 nonde & tections & at $<0.1 \mathrm{p}$ & $\mathrm{om}$. & & & & & 0.16 & \\
\hline
\end{tabular}

...continued 
Table 6-6. LLNL storm water runoff, nonradioactive parameters, 1992 (continued).

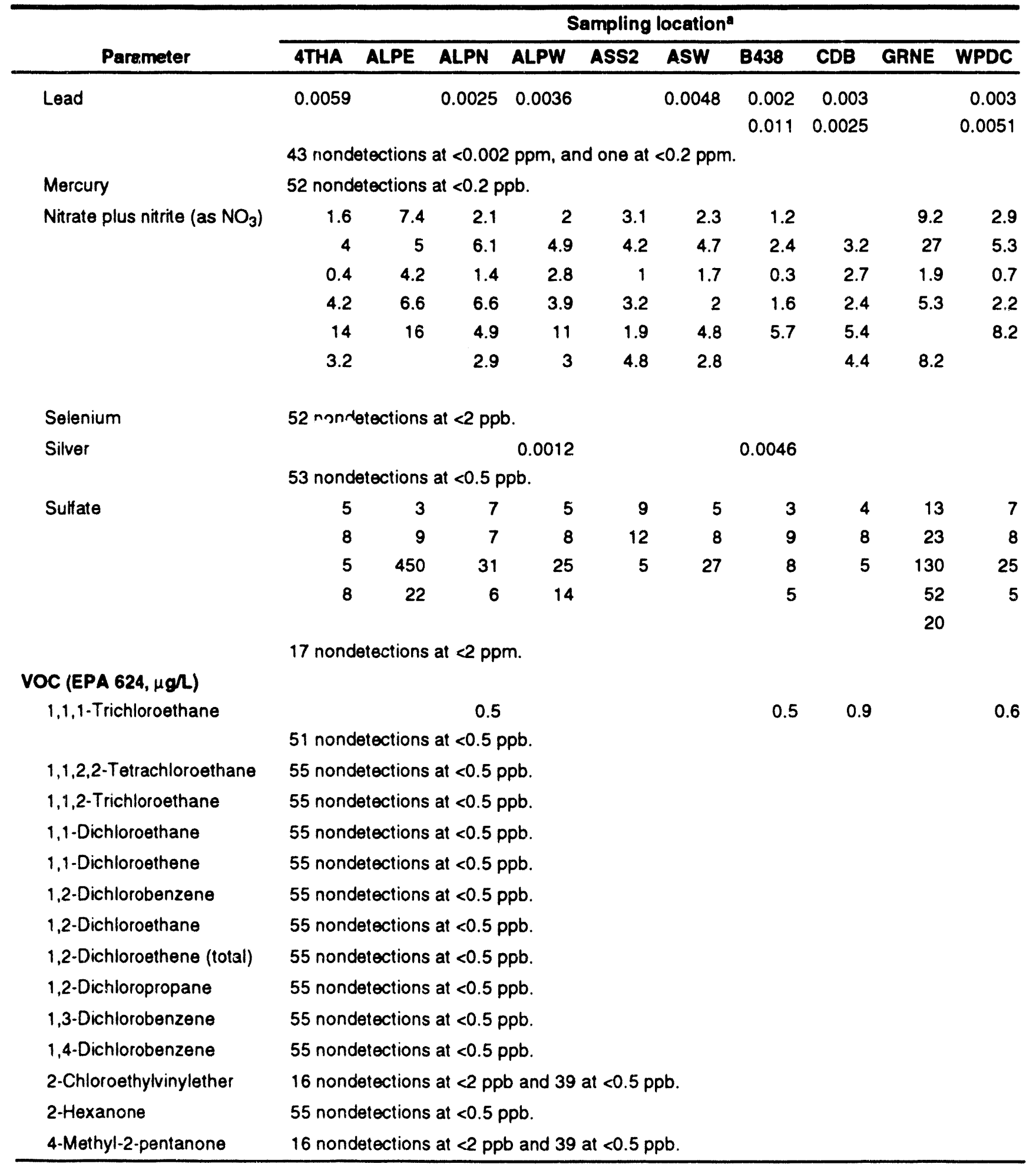


Table 6-6. LLNL storm water runoff, nonradioactive parameters, 1992 (continued).

\begin{tabular}{|c|c|c|c|c|c|c|c|c|c|c|}
\hline \multirow[b]{2}{*}{ Parameter } & \multicolumn{10}{|c|}{ Sampling location } \\
\hline & 4THA & ALPE & ALPN & ALPW & ASS2 & ASW & B438 & CDB & GRNE & WPDC \\
\hline \multirow[t]{2}{*}{ Acetone } & 5.3 & 9.2 & & 5.9 & & 17 & 5.2 & & & 6.9 \\
\hline & \multicolumn{10}{|c|}{49 nondetections at $<5 \mathrm{ppb}$. } \\
\hline Acrolein & \multicolumn{10}{|c|}{16 nondetections at $<10 \mathrm{ppb}$ and 39 at $<5 \mathrm{ppb}$. } \\
\hline Acrylonitrile & \multicolumn{10}{|c|}{55 nondetections at $<5 \mathrm{ppb}$} \\
\hline Benzene & \multicolumn{10}{|c|}{55 nondetections at $<0.5 \mathrm{ppb}$. } \\
\hline Bromodichloromethane & \multicolumn{10}{|c|}{55 nondetections at $<0.5 \mathrm{ppb}$. } \\
\hline Bromoform & \multicolumn{10}{|c|}{55 nondetections at $<0.5 \mathrm{ppb}$. } \\
\hline Bromomethane & \multicolumn{10}{|c|}{55 nondetections at $<0.5 \mathrm{ppb}$. } \\
\hline Carbon disulfide & \multicolumn{10}{|c|}{55 nondetections at $<0.5 \mathrm{ppb}$. } \\
\hline Carbon tetrachloride & \multicolumn{10}{|c|}{55 nondetections at $<0.5 \mathrm{ppb}$. } \\
\hline Chlorobenzene & \multicolumn{10}{|c|}{55 nondetections at $<0.5 \mathrm{ppb}$} \\
\hline Chloroethane & \multicolumn{10}{|c|}{7 nondetections at $<1 \mathrm{ppb}$ and 48 at $<0.5 \mathrm{ppb}$. } \\
\hline \multirow[t]{2}{*}{ Chloroform } & \multicolumn{10}{|c|}{1.4} \\
\hline & \multicolumn{10}{|c|}{54 nondetections at $<0.5 \mathrm{ppb}$. } \\
\hline Chloromethane & \multicolumn{10}{|c|}{55 nondetections at $<0.5 \mathrm{ppb}$. } \\
\hline cis-1,2-Dichloroethene & \multicolumn{10}{|c|}{55 nondetections at $<0.5 \mathrm{ppb}$. } \\
\hline cis-1,3-Dichloropropene & \multicolumn{10}{|c|}{55 nondetections at $<0.5 \mathrm{ppb}$. } \\
\hline Dibromochloromethane & \multicolumn{10}{|c|}{55 nondetections at $<0.5 \mathrm{ppb}$. } \\
\hline Ethylbenzene & \multicolumn{10}{|c|}{55 nondetections at $<0.5 \mathrm{ppb}$. } \\
\hline \multirow[t]{3}{*}{ Freon-113 } & 1.9 & 0.7 & 1.5 & & & 1.5 & 0.8 & 0.7 & 0.7 & 2.1 \\
\hline & & & & & & & 0.5 & & 0.6 & \\
\hline & \multicolumn{10}{|c|}{16 nondetections at $<1 \mathrm{ppb}$ and 29 at $<0.5 \mathrm{ppb}$. } \\
\hline Methyl ethyl ketone & 16 nonde & gtections & at $<5 \mathrm{pp}$ & $b$ and 39 & at $<10 p$ & & & & & \\
\hline Methylene chloride & 16 nonde & etections & at $<0.5$ & $\mathrm{ppb}, 20$ at & $<2 \mathrm{ppb}$ & and 19 at & $<3 \mathrm{ppb}$ & & & \\
\hline Styrene & 55 nond & etections & at $<0.5$ & ppb. & & & & & & \\
\hline Tetrachloroethene & 55 nond & etections & at $<0.5$ & ppb. & & & & & & \\
\hline Toluene & & & 0.8 & & 1.2 & 1.5 & & & & 0.6 \\
\hline & & & & & 0.8 & & & & & \\
\hline & 50 nond & etections & at $<0.5$ & ppb. & & & & & & \\
\hline Total xylene isomers & & & 1.4 & & & & & & & \\
\hline & 54 nond & etections & at $<0.5$ & ppb. & & & & & & \\
\hline trans-1,2-Dichloroethylene & 55 nond & etections & at $<0.5$ & ppb. & & & & & & \\
\hline trans-1,3-Dichloropropene & 55 nond & etections & at $<0.5$ & ppb. & & & & & & \\
\hline Trichloroethene & & & & 0.5 & & & & & & \\
\hline & & & & 0.7 & & & & & & \\
\hline & & & & 1.6 & & & & & & \\
\hline & 52 nond & etections & at $<0.5$ & ppb. & & & & & & \\
\hline
\end{tabular}


Table 6-6. LLNL storm water runoff, nonradioactive parameters, 1992 (continued).

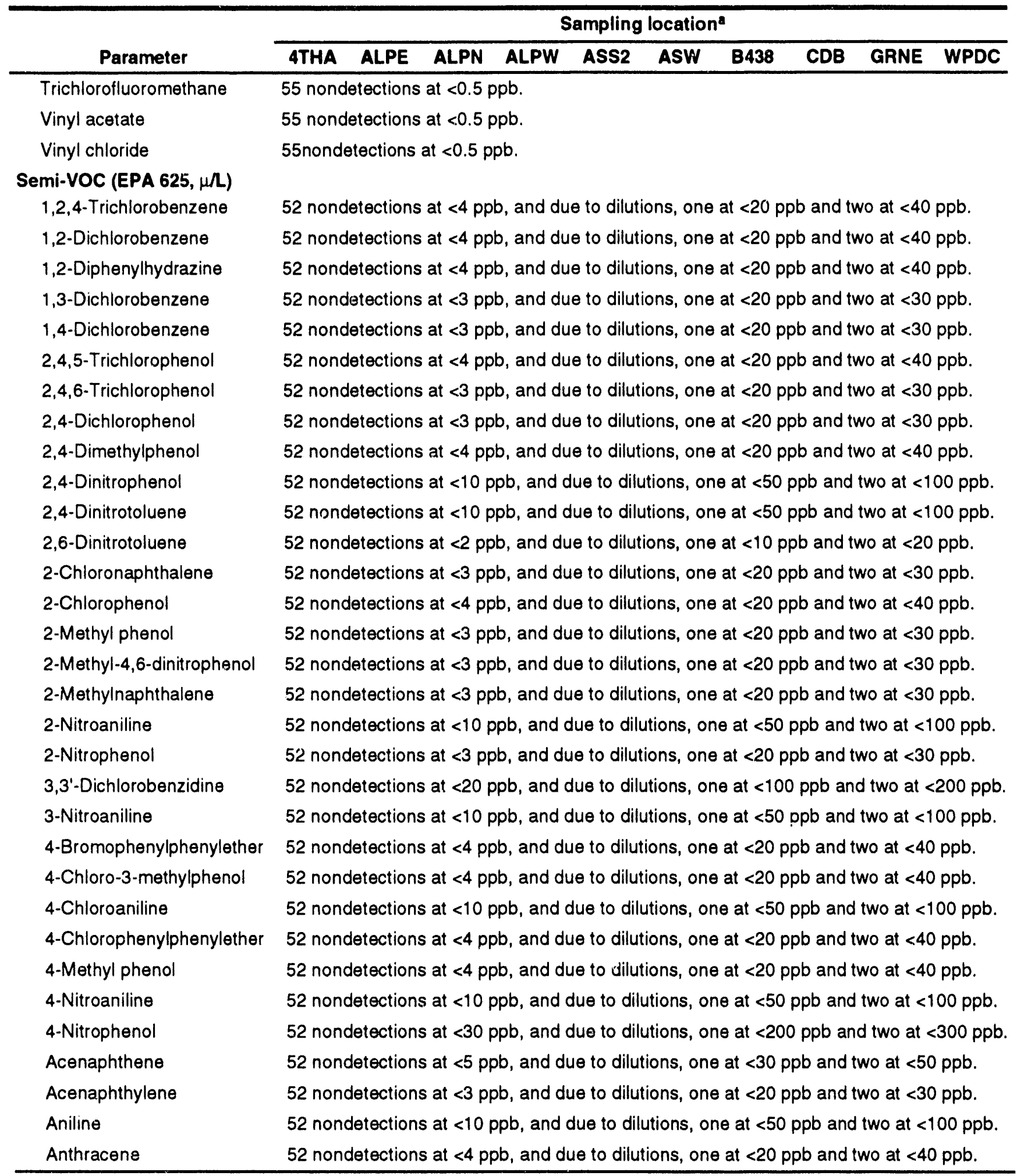




\section{Surveillance Water Monitoring}

Table 6-6. LLNL storm water runoff, nonradioactive parameters, 1992 (continued).

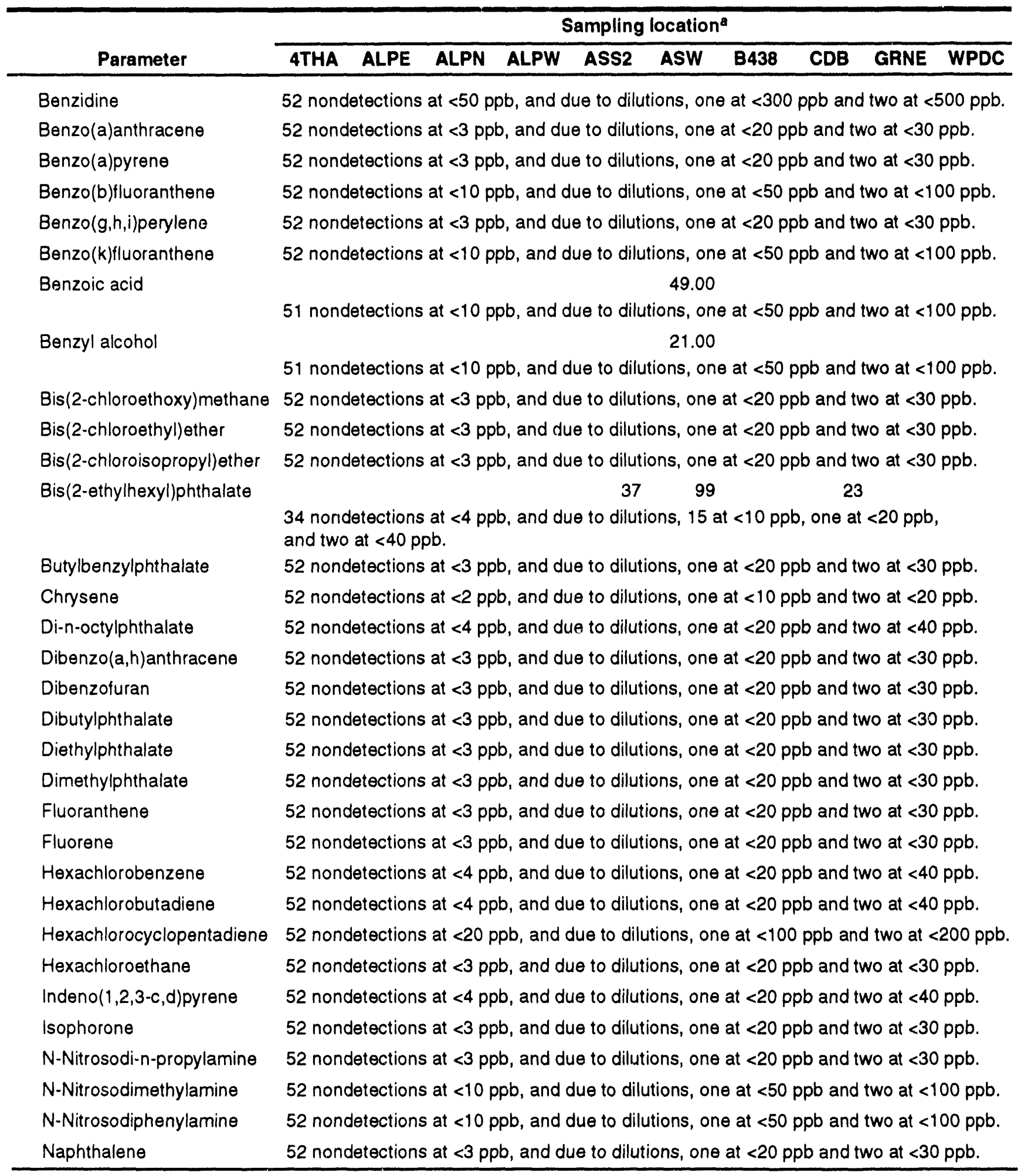

...continued 


\section{Surveillance Water Monitoring}

Table 6-6. LLNL storm water runoff, nonradioactive parameters, 1992 (concluded).

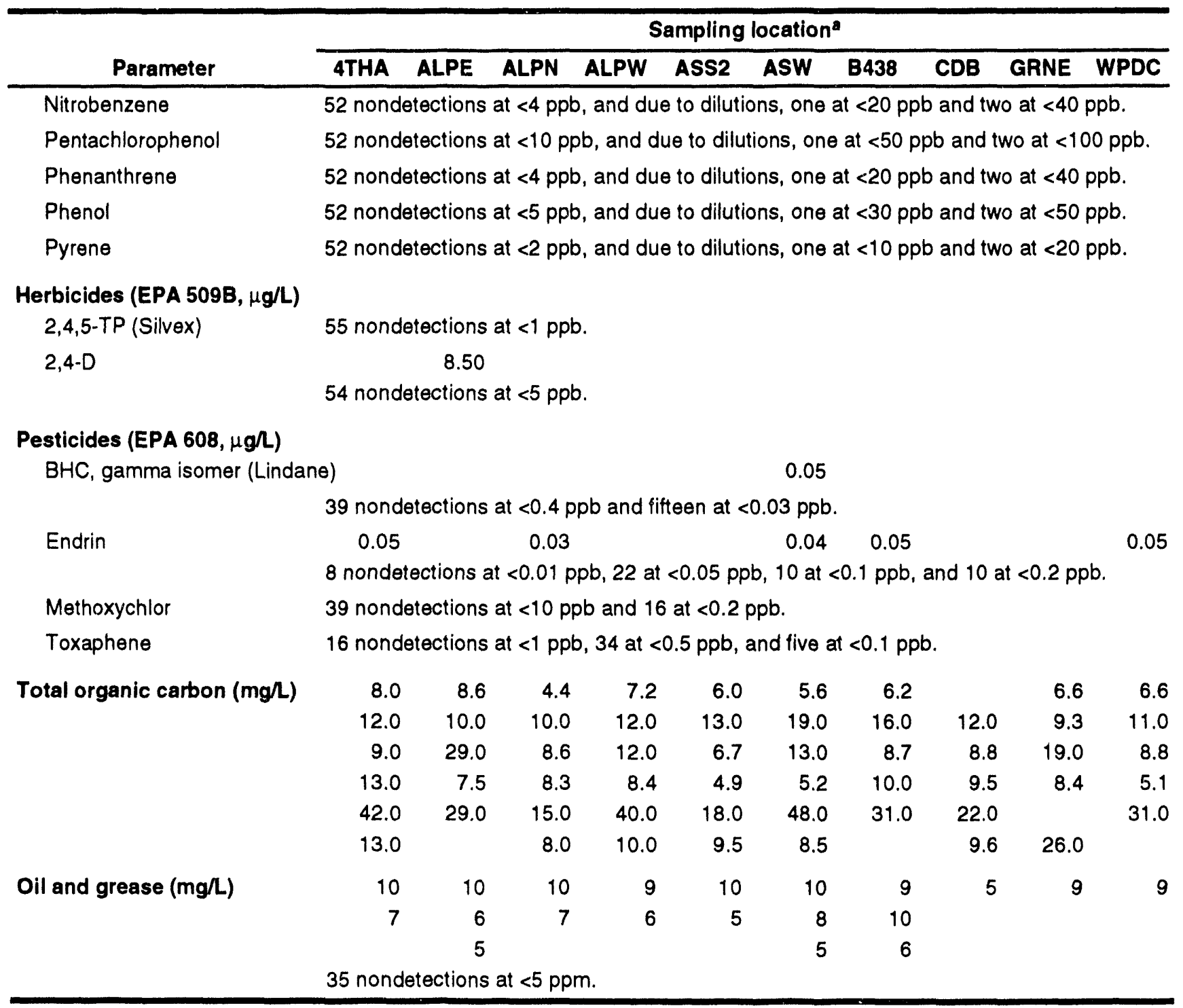

Note: Results are reported $\pm 2 \sigma$; see Quality Assurance chapter.

a See Fig. 6-4 for sampling locations. 
Table 6-7. Quarterly radioactivity in surface water (in Bq/L), Site 300, 1992.

\begin{tabular}{|c|c|c|c|}
\hline & \multicolumn{3}{|c|}{ Sampling location ${ }^{a}$} \\
\hline & GEO & RAIN & 812 CREEK \\
\hline \multicolumn{4}{|l|}{ Tritium } \\
\hline \multirow[t]{2}{*}{ 1st Quarter } & $0.95 \pm 0.08$ & $0.46 \pm 0.11$ & $0.34 \pm 0.20$ \\
\hline & & $0.97 \pm 0.12$ & \\
\hline \multirow[t]{3}{*}{ 2nd Quarter } & $0.98 \pm 0.12$ & $1.20 \pm 0.11$ & $0.33 \pm 0.08$ \\
\hline & & $0.40 \pm 0.10$ & \\
\hline & & $3.60 \pm 0.77$ & \\
\hline 3rd Quarter & $0.89 \pm 0.14$ & & $0.37 \pm 0.07$ \\
\hline \multirow[t]{4}{*}{ 4th Quarter } & $1.17 \pm 0.16$ & $0.47 \pm 0.12$ & $0.48 \pm 0.12$ \\
\hline & & $0.61 \pm 0.61$ & \\
\hline & & $0.56 \pm 0.10$ & \\
\hline & & $0.71 \pm 0.09$ & \\
\hline \multicolumn{4}{|l|}{ Gross alpha } \\
\hline 1st Quarter & $0.07 \pm 0.31$ & & $0.26 \pm 0.16$ \\
\hline 2nd Quarter & $0 \pm 0.24$ & & $0.12 \pm 0.18$ \\
\hline 3rd Quarter & $0.06 \pm 0.31$ & & $0.16 \pm 0.17$ \\
\hline 4th Quarter & $0.24 \pm 0.34$ & & $0.42 \pm 0.30$ \\
\hline \multicolumn{4}{|l|}{ Gross beta } \\
\hline 1st Quarter & $0.17 \pm 0.13$ & & $0.38 \pm 0.08$ \\
\hline 2nd Quarter & $0.19 \pm 0.14$ & & $0.47 \pm 0.09$ \\
\hline 3rd Quarter & $0.24 \pm 0.15$ & & $0.29 \pm 0.08$ \\
\hline 4th Quarter & $0.24 \pm 0.16$ & & $0.48 \pm 0.14$ \\
\hline
\end{tabular}




\section{Surveillance Water Monitoring}

Table 6-8. Analyses of water from supply wells near Site 300, 1992.

\begin{tabular}{|c|c|c|c|c|c|}
\hline \multirow[b]{2}{*}{ Parameter } & \multicolumn{5}{|c|}{ Wella } \\
\hline & STN & CARNRW2 & CDF1 & CON1 & GALLO1b \\
\hline \multicolumn{6}{|l|}{ Inorganic chemicals (mg/L) } \\
\hline Total alkalinity (as $\mathrm{CaCO}_{3}$ ) & 310 & 210 & 230 & 200 & \\
\hline Bicarbonate alk (as $\mathrm{CaCO}_{3}$ ) & 310 & 190 & 150 & 1160 & \\
\hline Carbonate alk (as $\mathrm{CaCO}_{3}$ ) & $<1$ & 20 & 80 & 40 & \\
\hline Arsenic & $<0.002$ & $<0.002$ & 0.003 & $<0.002$ & \\
\hline Barium & $<0.05$ & $<0.05$ & $<0.05$ & $<0.05$ & \\
\hline \multirow[t]{5}{*}{ Beryllium } & $<0.0002$ & $<0.0005$ & $<0.0005$ & $<0.0005$ & $<0.0005$ \\
\hline & & $<0.0005$ & $<0.0005$ & $<0.0005$ & $<0.0005$ \\
\hline & & $<0.0005$ & $<0.0005$ & $<0.0005$ & \\
\hline & & $<0.0005$ & $<.0 .0002$ & $<0.0002$ & \\
\hline & & $<0.0002$ & $<0.0005$ & $<0.0005$ & \\
\hline Cadmium & $<0.0005$ & $<0.0005$ & $<0.0005$ & $<0.0005$ & \\
\hline Calcium & 170 & 23 & 68 & 46 & \\
\hline Chloride & 170 & 100 & 120 & 180 & \\
\hline \multirow[t]{5}{*}{ Chromium } & $<0.005$ & $<0.005$ & $<0.005$ & $<0.005$ & $<0.005$ \\
\hline & & $<0.005$ & $<0.005$ & $<0.005$ & $<0.05$ \\
\hline & & $<0.005$ & $<0.005$ & $<0.005$ & \\
\hline & & $<0.005$ & $<0.005$ & 0.005 & \\
\hline & & $<0.005$ & & & \\
\hline \multirow[t]{5}{*}{ Copper } & $<0.05$ & $<0.005$ & 0.006 & $<0.005$ & $<0.05$ \\
\hline & & $<0.05$ & $<0.05$ & $<0.05$ & $<0.05$ \\
\hline & & 0.008 & $<0.05$ & $<0.05$ & \\
\hline & & $<0.05$ & $<0.05$ & $<0.05$ & \\
\hline & & $<0.05$ & & & \\
\hline Fluoride & 0.37 & 0.4 & 0.4 & 0.4 & \\
\hline Iron & $<0.1$ & $<0.1$ & $<0.1$ & $<0.1$ & \\
\hline \multirow[t]{5}{*}{ Lead } & $<0.002$ & 0.003 & $<0.002$ & $<0.002$ & $<0.002$ \\
\hline & & $<0.002$ & $<0.002$ & $<0.002$ & 0.003 \\
\hline & & $<0.002$ & $<0.002$ & $<0.002$ & \\
\hline & & $<0.002$ & $<0.002$ & $<0.002$ & \\
\hline & & $<0.002$ & 0.004 & 0.003 & \\
\hline Low level phenolics & $<0.005$ & $<0.005$ & $<0.005$ & $<0.005$ & \\
\hline Magnesium & 83 & 4.9 & 29 & 7.8 & \\
\hline Manganese & $<0.05$ & $<0.05$ & $<0.05$ & 0.16 & \\
\hline Mercury & $<0.0002$ & $<0.0002$ & $<0.0002$ & $<0.0002$ & \\
\hline Nitrate plus nitrite (as $\mathrm{NO}_{3}$ ) & 8.6 & $<0.4$ & 10 & $<0.4$ & \\
\hline $\mathrm{pH}$ & 7.2 & 8.4 & 8.2 & 8.2 & \\
\hline Potassium & 5.8 & 8.4 & 6 & 10 & \\
\hline Selenium & $<0.002$ & $<0.002$ & 0.004 & $<0.002$ & \\
\hline Silver & $<0.0005$ & $<0.0005$ & $<0.0005$ & $<0.0005$ & \\
\hline Sodium & 200 & 190 & 150 & 420 & \\
\hline $\begin{array}{l}\text { Specific conductance } \\
(\mu \mathrm{mhos} / \mathrm{cm})\end{array}$ & 50 & 990 & 1300 & 2070 & \\
\hline
\end{tabular}

...continued 
Table 6-8. Analyses of water from supply wells near Site 300, 1992 (continued).

\begin{tabular}{|c|c|c|c|c|c|}
\hline \multirow[b]{2}{*}{ Parameter } & \multicolumn{5}{|c|}{ Wella } \\
\hline & VIE1 & VIE2 & MUL1 & MUL2 & W35A-04 \\
\hline \multicolumn{6}{|l|}{ Inorganic chemicals (mg/L) } \\
\hline Total alkalinity (as $\mathrm{CaCO}_{3}$ ) & 250 & 280 & 200 & 180 & 250 \\
\hline Bicarbonate alk (as $\mathrm{CaCO}_{3}$ ) & 210 & 240 & 200 & 180 & 250 \\
\hline Carbonate alk (as $\mathrm{CaCO}_{3}$ ) & 40 & 40 & $<1$ & $<1$ & $<1$ \\
\hline Arsenic & 0.016 & $<0.002$ & 0.0027 & $<0.002$ & $<0.002$ \\
\hline Barium & 0.051 & $<0.05$ & 0.062 & $<0.05$ & 0.053 \\
\hline Beryllium & $<0.0005$ & $<0.0005$ & $<0.0005$ & $<0.0005$ & $<0.0002$ \\
\hline Cadmium & $<0.0005$ & $<0.0005$ & $<0.0005$ & $<0.0005$ & $<0.0005$ \\
\hline Calcium & 39 & 140 & 71 & 170 & 100 \\
\hline Chloride & 76 & 85 & 46 & 66 & 140 \\
\hline Chromium & $<0.005$ & $<0.005$ & $<0.005$ & $<0.005$ & $<0.005$ \\
\hline Copper & $<0.05$ & $<0.05$ & $<0.05$ & $<0.05$ & $<0.05$ \\
\hline Fluoride & 0.62 & 0.64 & 0.72 & 0.72 & 0.37 \\
\hline Iron & $<0.1$ & $<0.1$ & $<0.1$ & $<0.1$ & $<0.1$ \\
\hline Lead & $<0.002$ & 0.0029 & 0.003 & $<0.002$ & $<0.002$ \\
\hline Low level phenolics & $<0.005$ & $<0.005$ & $<0.005$ & $<0.005$ & $<0.005$ \\
\hline Magnesium & 21 & 18 & 31 & 56 & 48 \\
\hline Manganese & $<0.05$ & $<0.05$ & $<0.05$ & 0.18 & $<0.05$ \\
\hline Mercury & $<0.0002$ & $<0.0002$ & $<0.0002$ & $<0.0002$ & $<0.0002$ \\
\hline Nitrate plus nitrite (as $\mathrm{NO}_{3}$ ) & 6.1 & 13 & 5.4 & 9.4 & 22 \\
\hline $\mathrm{pH}$ & 8.4 & 8.4 & 7.5 & 7.1 & 7.5 \\
\hline Potassium & 8.4 & $<5$ & 6 & 7.5 & $<5.0$ \\
\hline Selenium & 0.0046 & 0.0065 & $0.003 !$ & 0.0075 & 0.0031 \\
\hline Silver & $<0.0005$ & $<0.0005$ & 0.0014 & $<0.0005$ & $<0.0005$ \\
\hline Sodium & 110 & 66 & 78 & 130 & 150 \\
\hline $\begin{array}{l}\text { Specific conductance } \\
(\mu \mathrm{mhos} / \mathrm{cm})\end{array}$ & 770 & 930 & 780 & 1480 & 1410 \\
\hline
\end{tabular}

...continued 


\section{Surveillance Water Monitoring}

Table 6-8. Analyses of water from supply wells near Site 300, 1992 (continued).

\begin{tabular}{|c|c|c|c|c|c|}
\hline \multirow[b]{2}{*}{ Parameter } & \multicolumn{5}{|c|}{ Welf } \\
\hline & STN & CARNRW2 & CDF1 & CONI & GALLO1b \\
\hline Sulfate & 710 & 230 & 320 & 770 & \\
\hline Total dissolved solids (TDS) & 1600 & 740 & 880 & 1500 & \\
\hline Zinc & 0.077 & $<0.05$ & $<0.05$ & $<0.05$ & \\
\hline \multicolumn{6}{|l|}{ Organic chemicalsc } \\
\hline \multicolumn{6}{|l|}{ EPA Method $524.2(\mu \mathrm{g} / \mathrm{L})$} \\
\hline 1,1,1,2-Tetrachloroethane & 1 n.d. $<0.2$ & 5 n.d. $<0.2$ & 4 n.d. $<0.2$ & 4 n.d. $<0.2$ & 2 n.d. $<0.2$ \\
\hline \multirow[t]{2}{*}{ 1,1,1-Trichloroethane } & 1 n.d. $<0.2$ & 4 n.d. $<0.2$ & 3 n.d. $<0.2$ & 3 n.d. $<0.2$ & 2 n.d. $<0.2$ \\
\hline & & 1 n.d. $<0.3$ & 1 n.d. $<0.3$ & 1 n.d. $<0.3$ & \\
\hline 1,1,2,2-Tetrachloroethane & 1 n.d. $<0.2$ & 5 n.d. $<0.2$ & 4 n.d. $<0.2$ & 4 n.d. $<0.2$ & 2 n.d. $<0.2$ \\
\hline \multirow[t]{2}{*}{ 1,1,2-Trichloroethane } & 1 n.d. $<0.2$ & 4 n.d. $<0.2$ & 3 n.d. $<0.2$ & 3 n.d. $<0.2$ & 2 n.d. $<0.2$ \\
\hline & & 1 n.d. $<0.3$ & 1 n.d. $<0.3$ & 1 n.d. $<0.3$ & \\
\hline 1,1-Dichloroethane & 1 n.d. $<0.2$ & 5 n.d. $<0.2$ & 4 n.d. $<0.2$ & 4 n.d. $<0.2$ & 2 n.d. $<0.2$ \\
\hline 1,1-Dichloroethene & \multicolumn{5}{|c|}{ Same as above. } \\
\hline 1,1-Dichloropropene & \multicolumn{5}{|c|}{ Same as above. } \\
\hline 1,2,3-Trichlorobenzene & \multicolumn{5}{|c|}{ Same as above. } \\
\hline 1,2,3-Trichloropropane & \multicolumn{5}{|c|}{ Same as above. } \\
\hline 1,2,4-Trichlorobenzene & \multicolumn{5}{|c|}{ Same as above. } \\
\hline 1,2,4-Trimethylbenzene & \multicolumn{5}{|c|}{ Same as above. } \\
\hline \multirow[t]{2}{*}{ 1,2-Dibromo-3-chloropropane } & 1 n.d. $<2$ & 4 n.d. $<2$ & 3 n.d. $<2$ & 3 n.a. $<2$ & 2 n.d. $<0.2$ \\
\hline & & 1 n.d. $<0.5$ & 1 n.d. $<0.5$ & 1 n.d. $<0.5$ & \\
\hline 1,2-Dibromoethane & 1 n.d. $<0.2$ & 5 n.d. $<0.2$ & 4 n.d. $<0.2$ & 4 n.d. $<0.2$ & 2 n.d. $<0.2$ \\
\hline 1,2-Dichlorobenzene & \multicolumn{5}{|c|}{ Same as above. } \\
\hline 1,2-Dichloroethane & 1 n.d. $<0.2$ & $\begin{array}{c}4 \text { n.d. }<0.2 \\
1\end{array}$ & 4 n.d. $<0.2$ & 4 n.d. $<0.2$ & 2 n.d. $<0.2$ \\
\hline 1,2-Dichloropropane & 1 n.d. $<0.2$ & $\begin{array}{l}4 \text { n.d. }<0.2 \\
0.4\end{array}$ & 4 n.d. $<0.2$ & 4 n.d. $<0.2$ & 2 n.d. $<0.2$ \\
\hline 1,3,5-Trimethylbenzene & 1 n.d. $<0.2$ & 5 n.d. $<0.2$ & 4 n.d. $<0.2$ & 4 n.d. $<0.2$ & 2 n.d. $<0.2$ \\
\hline 1,3-Dichlorobenzene & \multicolumn{5}{|c|}{ Same as above. } \\
\hline 1,3-Dichloropropane & \multicolumn{5}{|c|}{ Same as above. } \\
\hline 1,4-Dichlorobenzene & 1 n.d. $<0.2$ & $\begin{array}{c}4 \text { n.d. }<0.2 \\
0.5\end{array}$ & 4 n.d. $<0.2$ & 4 n.d. $<0.2$ & 2 n.d. $<0.2$ \\
\hline 2,2-Dichloropropane & 1 n.d. $<0.2$ & 5 n.d. $<0.2$ & 4 n.d. $<0.2$ & 4 n.d. $<0.2$ & 2 n.d. $<0.2$ \\
\hline 2-Chlorotoluene & \multicolumn{5}{|c|}{ Same as above. } \\
\hline 4-Chlorotoluene & \multicolumn{5}{|c|}{ Same as above. } \\
\hline Benzene & \multicolumn{5}{|c|}{ Same as above. } \\
\hline Bromobenzene & \multicolumn{5}{|c|}{ Same as above. } \\
\hline Bromochloromethane & \multicolumn{5}{|c|}{ Same as above. } \\
\hline Bromodichloromethane & 1 n.d. $<0.2$ & 3 n.d. $<0.2$ & 4 n.d. $<0.2$ & 4 n.d. $<0.2$ & 2 n.d. $<0.2$ \\
\hline & & 0.8 & & & \\
\hline & & 4.2 & & & \\
\hline
\end{tabular}

...continued 
Table 6-8. Analyses of water from supply wells near Site 300, 1992 (continued).

\begin{tabular}{|c|c|c|c|c|c|}
\hline \multirow[b]{2}{*}{ Parameter } & \multicolumn{5}{|c|}{ Welpa } \\
\hline & VIE1 & VIE2 & MUL1 & MUL2 & W35A-04 \\
\hline Sulfate & 110 & 200 & 240 & 660 & 430 \\
\hline Total dissolved solids (TDS) & 590 & 730 & 580 & 1200 & 1100 \\
\hline Zinc & $<0.05$ & 0.057 & 0.051 & $<0.05$ & $<0.05$ \\
\hline \multicolumn{6}{|l|}{ Organic chemicalsc } \\
\hline \multicolumn{6}{|l|}{ EPA Method $524.2(\mu \mathrm{g} / \mathrm{L})$} \\
\hline 1,1,1,2-Tetrachloroethane & 1 n.d. $<0.2$ & 1 n.d. $<0.2$ & 1 n.d. $<0.2$ & 1 n.d. $<0.2$ & 1 n.d. $<0.2$ \\
\hline 1,1,1-Trichloroethane & 1 n.d. $<0.2$ & 1 n.d. $<0.2$ & 1 n.d. $<0.2$ & 1 n.d. $<0.2$ & 1 n.d. $<0.2$ \\
\hline $1,1,2,2$-Tetrachloroethane & 1 n.d. $<0.2$ & 1 n.d. $<0.2$ & 1 n.d. $<0.2$ & 1 n.d. $<0.2$ & 1 n.d. $<0.2$ \\
\hline 1,1,2-Trichloroethane & 1 n.d. $<0.2$ & 1 n.d. $<0.2$ & 1 n.d. $<0.2$ & 1 n.d. $<0.2$ & 1 n.d. $<0.2$ \\
\hline 1,1-Dichloroethane & 1 n.d. $<0.2$ & 1 n.d. $<0.2$ & 1 n.d. $<0.2$ & 1 n.d. $<0.2$ & 1 n.d. $<0.2$ \\
\hline 1,1-Dichloroethene & \multicolumn{5}{|c|}{ Same as above. } \\
\hline 1,1-Dichloropropene & \multicolumn{5}{|c|}{ Same as above. } \\
\hline 1,2,3-Trichlorobenzene & \multicolumn{5}{|c|}{ Same as above. } \\
\hline 1,2,3-Trichloropropane & \multicolumn{5}{|c|}{ Same as above. } \\
\hline 1,2,4-Trichlorobenzene & \multicolumn{5}{|c|}{ Same as above. } \\
\hline 1,2,4-Trimethylbenzene & \multicolumn{5}{|c|}{ Same as above. } \\
\hline \multicolumn{5}{|l|}{ 3-chloropropane } & 1 n.d. $<2$ \\
\hline \multicolumn{6}{|l|}{ 1,2-Dichlorobenzene } \\
\hline 1,2-Dichloroethane & 1 n.d. $<0.2$ & 1 n.d. $<0.2$ & 1 n.d. $<0.2$ & 1 n.d. $<0.2$ & 1 n.d. $<0.2$ \\
\hline 1,2-Dichloropropane & 1 n.d. $<0.2$ & 1 n.d. $<0.2$ & 1 n.d. $<0.2$ & 1 n.d. $<0.2$ & 1 n.d. $<0.2$ \\
\hline 1,3,5-Trimethylbenzene & 1 n.d. $<0.2$ & 1 n.d. $<0.2$ & 1 n.d. $<0.2$ & 1 n.d. $<0.2$ & 1 n.d. $<0.2$ \\
\hline 1,3-Dichlorobenzene & \multicolumn{5}{|c|}{ Same as above. } \\
\hline 1,3-Dichloropropane & \multicolumn{5}{|c|}{ Same as above. } \\
\hline 1,4-Dichlorobenzene & 1 n.d. $<0.2$ & 1 n.d. $<0.2$ & 1 n.d. $<0.2$ & 1 n.d. $<0.2$ & 1 n.d. $<0.2$ \\
\hline 2,2-Dichloropropane & 1 n.d. $<0.2$ & 1 n.d. $<0.2$ & 1 n.d. $<0.2$ & 1 n.d. $<0.2$ & 1 n.d. $<0.2$ \\
\hline 2-Chlorotoluene & \multicolumn{5}{|c|}{ Same as above. } \\
\hline 4-Chlorotoluene & \multicolumn{5}{|c|}{ Same as above. } \\
\hline Benzene & \multicolumn{5}{|c|}{ Same as above. } \\
\hline Bromobenzene & \multicolumn{5}{|c|}{ Same as above. } \\
\hline Bromochloromethane & 0.4 & & & & \\
\hline Bromodichloromethane & $\begin{array}{l}1 \text { n.d. }<0.2 \\
0.4\end{array}$ & 1 n.d. $<0.2$ & 1 n.d. $<0.2$ & 1 n.d. $<0.2$ & 1 n.d. $<0.2$ \\
\hline
\end{tabular}

...continued 
Table 6-8. Analyses of water from supply wells near Site 300, 1992 (continued).

\begin{tabular}{|c|c|c|c|c|c|}
\hline \multirow[b]{2}{*}{ Paramoter } & \multicolumn{5}{|c|}{ Welp } \\
\hline & STN & CARNRW & $12 \quad$ CDF1 & CON1 & GALLO1b \\
\hline Bromoform & 1 n.d. $<0.2$ & $\begin{array}{c}3 \text { n.d. }<0.2 \\
2.8 \\
23\end{array}$ & 4 n.d $<0.2$ & 4 n.d. $<0.2$ & 2 n.d. $<0.2$ \\
\hline Bromomethane & 1 n.d. $<0.2$ & 5 n.d. $<0.2$ & 4 n.d. $<0.2$ & 4 n.d. $<0.2$ & 2 n.d. $<0.2$ \\
\hline Carbon tetrachloride & 1 n.d. $<0.2$ & $\begin{array}{l}4 \text { n.d. }<0.2 \\
1 \text { n.d. }<0.3\end{array}$ & $\begin{array}{l}3 \text { n.d. }<0.2 \\
1 \text { n.d. }<0.3\end{array}$ & $\begin{array}{l}3 \text { n.d. }<0.2 \\
1 \text { n.d. }<0.3\end{array}$ & 2 n.d. $<0.2$ \\
\hline Chlorobenzene & 1 n.d. $<0.2$ & $\begin{array}{l}4 \text { n.d. }<0.2 \\
0.4\end{array}$ & 4 n.d. $<0.2$ & 4 n.d. $<0.2$ & 2 n.d. $<0.2$ \\
\hline Chloroethane & 1 n.d. $<0.2$ & $\begin{array}{l}4 \text { n.d. }<0.2 \\
1 \text { n.d. }<0.3\end{array}$ & $\begin{array}{l}3 \text { n.d. }<0.2 \\
1 \text { n.d. }<0.3\end{array}$ & $\begin{array}{l}3 \text { n.d. }<0.2 \\
1 \text { n.d. }<0.3\end{array}$ & 2 n.d. $<0.2$ \\
\hline Chloroform & 1 n.d. $<0.2$ & $\begin{array}{c}3 \text { n.d. }<0.2 \\
0.6 \\
1.9\end{array}$ & 4 n.d. $<0.2$ & 4 n.d. $<0.2$ & 2 n.d. $<0.2$ \\
\hline $\begin{array}{l}\text { Chloromethane } \\
\text { cis-1,2-Dichloroethene }\end{array}$ & $\begin{array}{l}1 \text { n.d. }<0.2 \\
\text { Same as abc }\end{array}$ & $\begin{array}{l}5 \text { n.d. }<0.2 \\
\text { ove. }\end{array}$ & 4 n.d. $<0.2$ & 4 n.d. $<0.2$ & 2 n.d. $<0.2$ \\
\hline cis-1,3-Dichloropropene & 1 n.d. $<0.2$ & $\begin{array}{l}4 \text { n.d. }<2 \\
1 \text { n.d. }<0.5\end{array}$ & $\begin{array}{l}3 \text { n.d. }<2 \\
1 \text { n.d. }<0.5\end{array}$ & $\begin{array}{l}3 \text { n.d. }<2 \\
1 \text { n.d. }<0.5\end{array}$ & 2 n.d. $<0.2$ \\
\hline Dibromochloromethane & 1 n.d. $<0.2$ & $\begin{array}{c}3 \text { n.d. }<0.2 \\
1.6 \\
9.8\end{array}$ & 4 n.d. $<0.2$ & 4 n.d. $<0.2$ & 2 n.d. $<0.2$ \\
\hline Dibromomethane & 1 n.d. $<0.2$ & 5 n.d. $<0.2$ & 4 n.d. $<0.2$ & 4 n.d. $<0.2$ & 2 n.d. $<0.2$ \\
\hline Dichlorodifluoromethane & 1 n.d. $<0.2$ & 2 n.d. $<0.2$ & 1 n.d. $<0.2$ & 1 n.d. $<0.2$ & 1 n.d. $<0.2$ \\
\hline Ethylbenzene & 1 n.d. $<0.2$ & $\begin{array}{l}4 \text { n.d. }<0.2 \\
0.5\end{array}$ & 4 n.d. $<0.2$ & 4 n.d. $<0.2$ & 2 n.d. $<0.2$ \\
\hline $\begin{array}{l}\text { Hexachlorobutadiene } \\
\text { Isopropylbenzene }\end{array}$ & $\begin{array}{l}1 \text { n.d. }<0.2 \\
\text { Same as ab }\end{array}$ & $\begin{array}{l}5 \text { n.d. }<0.2 \\
\text { ove. }\end{array}$ & 4 n.d. $<0.2$ & 4 n.d. $<0.2$ & 2 n.d. $<0.2$ \\
\hline $\mathrm{m}$ - and $\mathrm{p}$-Xylene isomers & 1 n.d. $<0.2$ & $\begin{array}{l}4 \text { n.d. }<0.2 \\
0.2\end{array}$ & 4 n.d. $<0.2$ & 4 n.d. $<0.2$ & 2 n.d. $<0.2$ \\
\hline Methylene chloride & 1 n.d. $<1$ & $\begin{array}{l}4 \text { n.d. }<1 \\
1 \text { n.d. }<0.2\end{array}$ & $\begin{array}{l}3 \text { n.d. }<1 \\
1 \text { n.d. }<0.2\end{array}$ & $\begin{array}{l}3 \text { n.d. }<1 \\
1 \text { n.d. }<0.2\end{array}$ & 2 n.d. $<1$ \\
\hline n-Butylbenzene & 1 n.d. $<0.2$ & 5 n.d. $<0.2$ & 4 n.d. $<0.2$ & 4 n.d. $<0.2$ & 2 n.d. $<0.2$ \\
\hline n-Propylbenzene & Same as abc & ove. & & & \\
\hline Naphthalene & Same as abc & ove. & & & \\
\hline $0-X y l e n \theta$ & Same as abc & ove. & & & \\
\hline p-Isopropyl toluene & Same as abc & ove. & & & \\
\hline sec-Butylbenzene & Same as abc & ove. & & & \\
\hline $\begin{array}{l}\text { Styrene } \\
\text { tert-Butylbenzene }\end{array}$ & $\begin{array}{l}\text { Same as abc } \\
\text { Same as abc }\end{array}$ & ove. & & & \\
\hline Tetrachloroethene & Same as abc & ove. & & & \\
\hline Toluene & Same as abc & ove. & & & \\
\hline $\begin{array}{l}\text { trans-1,2-Dichloroethylene } \\
\text { trans-1,3-Dichloropropene }\end{array}$ & $\begin{array}{l}\text { Same as abc } \\
\text { Same as abc }\end{array}$ & & & & \\
\hline
\end{tabular}

...continued 
Table 6-8. Analyses of water from supply wells near Site 300, 1992 (continued).

\begin{tabular}{|c|c|c|c|c|c|}
\hline \multirow[b]{2}{*}{ Paramoter } & \multicolumn{5}{|c|}{ Wolp } \\
\hline & VIE1 & VIE2 & MUL1 & MUL2 & W35A-04 \\
\hline Bromoform & 0.8 & 1 n.d. $<0.2$ & 1 n.d. $<0.2$ & 1 n.d. $<0.2$ & 1 n.d. $<0.2$ \\
\hline $\begin{array}{l}\text { Bromomethane } \\
\text { Carbon tetrachloride }\end{array}$ & $\begin{array}{l}1 \text { n.d. }<0.2 \\
1 \text { n.d. }<0.2\end{array}$ & $\begin{array}{l}1 \text { n.d. }<0.2 \\
1 \text { n.d. }<0.2\end{array}$ & $\begin{array}{l}1 \text { n.d. }<0.2 \\
1 \text { n.d. }<0.2\end{array}$ & $\begin{array}{l}1 \text { n.d. }<0.2 \\
1 \text { n.d. }<0.2\end{array}$ & $\begin{array}{l}1 \text { n.d. }<0.2 \\
1 \text { n.d. }<0.2\end{array}$ \\
\hline Chlorobenzene & 1 n.d. $<0.2$ & 1 n.d. $<0.2$ & 1 n.d. $<0.2$ & 1 n.d. $<0.2$ & 1 n.d. $<0.2$ \\
\hline Chloroethane & 1 n.d. $<0.2$ & 1 n.d. $<0.2$ & 1 n.d. $<0.2$ & 1 n.d. $<0.2$ & 1 n.d. $<0.2$ \\
\hline Chloroform & 1 n.d. $<0.2$ & 1 n.d. $<0.2$ & 1 n.d. $<0.2$ & 1 n.d. $<0.2$ & 1 n.d. $<0.2$ \\
\hline $\begin{array}{l}\text { Chloromethane } \\
\text { cis-1,2-Dichloroethene } \\
\text { cis-1,3-Dichloropropene }\end{array}$ & $\begin{array}{l}1 \text { n.d. }<0.2 \\
\text { Same as abc } \\
1 \text { n.d. }<0.2\end{array}$ & $\begin{array}{l}1 \text { n.d. }<0.2 \\
\text { ove. } \\
1 \text { n.d. }<0.2\end{array}$ & $\begin{array}{l}1 \text { n.d. }<0.2 \\
1 \text { n.d. }<0.2\end{array}$ & $\begin{array}{l}1 \text { n.d. }<0.2 \\
1 \text { n.d. }<0.2\end{array}$ & $\begin{array}{l}1 \text { n.d. }<0.2 \\
1 \text { n.d. }<0.2\end{array}$ \\
\hline Dibromochloromethane & 1 n.d. $<0.2$ & 1 n.d. $<0.2$ & 1 n.d. $<0.2$ & 1 n.d. $<0.2$ & 1 n.d. $<0.2$ \\
\hline $\begin{array}{l}\text { Dibromomethane } \\
\text { Dichlorodifluoromethane }\end{array}$ & 1 n.d. $<0.2$ & 1 n.d. $<0.2$ & 1 n.d. $<0.2$ & 1 n.d. $<0.2$ & 1 n.d. $<0.2$ \\
\hline Ethylbenzene & 1 n.d. $<0.2$ & 1 n.d. $<0.2$ & 1 n.d. $<0.2$ & 1 n.d. $<0.2$ & 1 n.d. $<0.2$ \\
\hline $\begin{array}{l}\text { Hexachlorobutadiene } \\
\text { Isopropylbenzene }\end{array}$ & $\begin{array}{l}1 \text { n.d. }<0.2 \\
\text { Same as abc }\end{array}$ & $\begin{array}{l}1 \text { n.d. }<0.2 \\
\text { ove. }\end{array}$ & 1 n.d. $<0.2$ & 1 n.d. $<0.2$ & 1 n.d. $<0.2$ \\
\hline $\mathrm{m}$ - and $\mathrm{p}$-Xylene isomers & 1 n.d. $<0.2$ & 1 n.d. $<0.2$ & 1 n.d. $<0.2$ & 1 n.d. $<0.2$ & 1 n.d. $<0.2$ \\
\hline Methylene chloride & 1 n.d. $<1$ & 1 n.d. $<1$ & 1 n.d. $<1$ & 1 n.d. $<1$ & 1 n.d. $<1$ \\
\hline n-Butylbenzene & 1 n.d. $<0.2$ & 1 n.d. $<0.2$ & 1 n.d. $<0.2$ & 1 n.d. $<0.2$ & 1 n.d. $<0.2$ \\
\hline n-Propylbenzene & Same as abc & ove. & & & \\
\hline Naphthalene & Same as abc & ove. & & & \\
\hline $0-X y l e n \theta$ & Same as abc & ove. & & & \\
\hline p-lsopropyl toluene & Same as abc & ove. & & & \\
\hline sec-Butylbenzene & Same as abc & ove. & & & \\
\hline Styrene & Same as abc & ove. & & & \\
\hline $\begin{array}{l}\text { tert-Butylbenzene } \\
\text { Tetrachloroethene }\end{array}$ & $\begin{array}{l}\text { Same as abc } \\
\text { Same as abc }\end{array}$ & ove. & & & \\
\hline $\begin{array}{l}\text { Toluene } \\
\text { trans-1,2-Dichloroethylene } \\
\text { trans-1,3-Dichloropropene }\end{array}$ & $\begin{array}{l}\text { Same as ab } \\
\text { Same as ab } \\
\text { Same as ab }\end{array}$ & $\begin{array}{l}\text { ove. } \\
\text { ove. } \\
\text { ove. }\end{array}$ & & & \\
\hline
\end{tabular}


Table 6-8. Analyses of water from supply wells near Site 300, 1992 (continued).

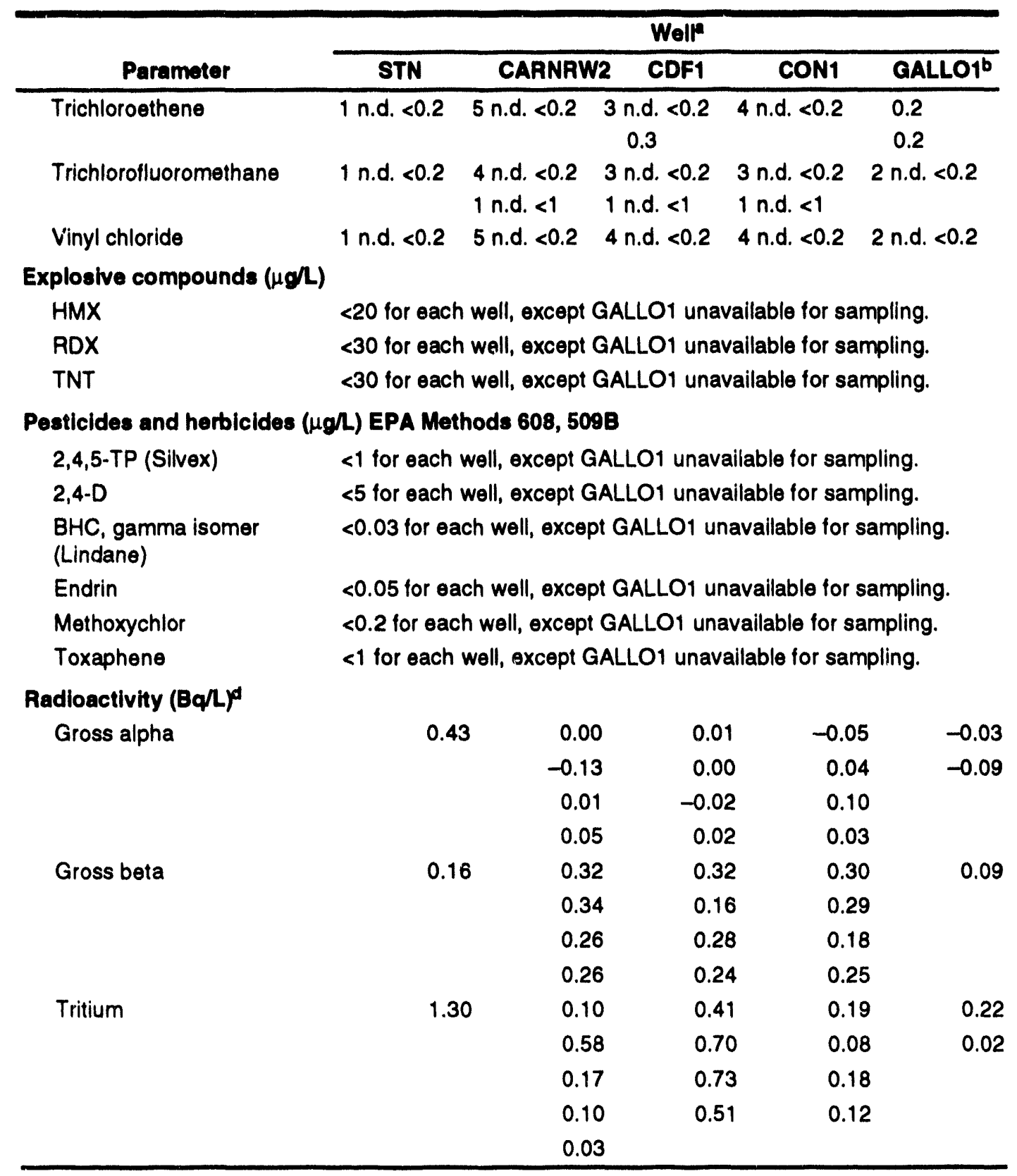


Table 6-8. Analyses of water from supply wells near Site 300, 1992 (concluded).

\begin{tabular}{|c|c|c|c|c|c|}
\hline \multirow[b]{2}{*}{ Parameter } & \multicolumn{5}{|c|}{ Wolla } \\
\hline & VIE1 & VIE2 & MULI & MUL2 & W35A-04 \\
\hline Trichloroethene & 1 n.d. $<0.2$ & 1 n.d. $<0.2$ & 1 n.d. $<0.2$ & 1 n.d. $<0.2$ & 1 n.d. $<0.2$ \\
\hline Trichlorofluoromethane & 1 n.d. $<0.2$ & 1 n.d. $<0.2$ & 1 n.d. $<0.2$ & 1 n.d. $<0.2$ & 1 n.d. $<0.2$ \\
\hline Vinyl chloride & 1 n.d. $<0.2$ & 1 n.d. $<0.2$ & 1 n.d. $<0.2$ & 1 n.d. $<0.2$ & 1 n.d. $<0.2$ \\
\hline \multicolumn{6}{|c|}{ Explosive compounds $(\mu \mathrm{g} / \mathrm{L})$} \\
\hline$H M X$ & \multicolumn{5}{|c|}{$<20$ for each well. } \\
\hline RDX & \multicolumn{5}{|c|}{$<30$ for each well. } \\
\hline TNT & \multicolumn{5}{|c|}{$<30$ for each well. } \\
\hline \multicolumn{6}{|c|}{ Pesticides and herblcides ( $\mu \mathrm{g} / L$ ) EPA Methods 608, 509B } \\
\hline 2,4,5-TP (Silvex) & \multicolumn{5}{|c|}{$<1$ for each well. } \\
\hline 2,4-D & \multicolumn{5}{|c|}{$<5$ for each well. } \\
\hline $\begin{array}{l}\text { BHC, gamma isomer } \\
\text { (Lindane) }\end{array}$ & \multicolumn{5}{|c|}{$<0.03$ for each well. } \\
\hline Endrin & \multicolumn{5}{|c|}{$<0.05$ for each well. } \\
\hline Methoxychlor & \multicolumn{5}{|c|}{$<0.2$ for each well. } \\
\hline Toxaphene & \multicolumn{5}{|c|}{$<1$ for each well. } \\
\hline \multicolumn{6}{|l|}{ Radioactivity (Bq/L) ${ }^{d}$} \\
\hline Gross alpha & \multicolumn{2}{|c|}{0.11} & 0.14 & -0.01 & 0.07 \\
\hline Gross beta & \multicolumn{2}{|c|}{0.29} & 0.15 & 0.15 & 0.19 \\
\hline Tritium & \multicolumn{2}{|c|}{0.14} & 0.17 & 0.12 & 1.39 \\
\hline
\end{tabular}

Notes: Results are reported $\pm 2 \sigma$; see Quality Assurance chapter.

The abbreviation "n.d." stands for nondetection.

a See Fig. 6-5 for sampling locations.

b Inaccessible for sampling in third and fourth quarters.

c Less sensitive EPA Methods 601 and 624 were run in addition to EPA Method 524.2 on duplicate samples from wells STN, CDF1, and CON1. All measurements were below detection limits except for carbon disulfide $(0.65 \mathrm{ppb})$ in well CDF1 and chloroform (1.4 ppb) in well STN.

d For very low counts, subtraction of background count may yield a negative number for gross alpha and gross beta (see U.S. Department of Energy 1991). 
Table 6-9. Quarterly analyses of water from surveillance monitoring wells, Site 300, 1992.

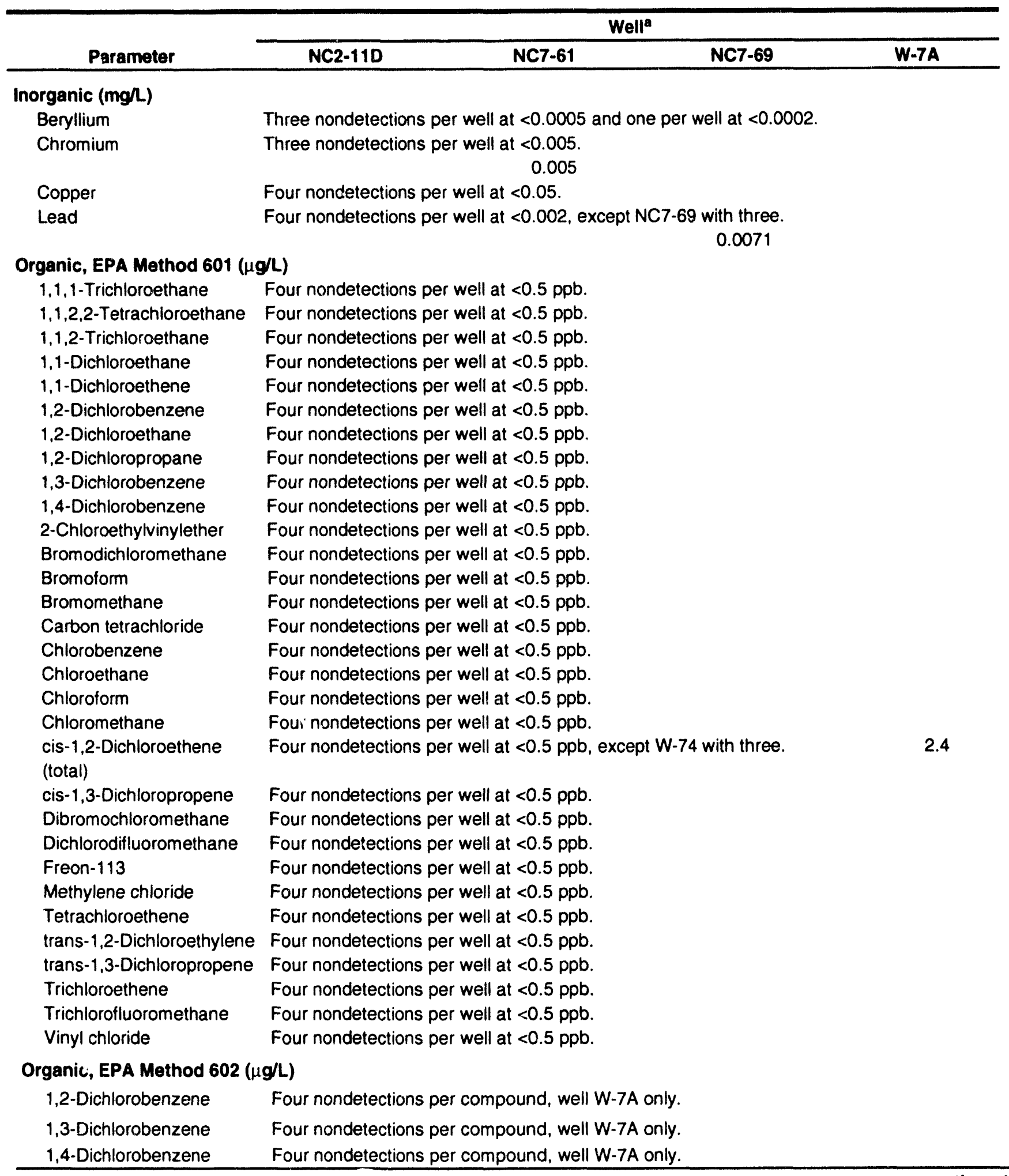


Table 6-9. Quarterly analyses of water from surveillance monitoring wells, Site 300, 1992 (concluded).

\begin{tabular}{|c|c|c|c|c|}
\hline \multirow[b]{2}{*}{ Parameter } & \multicolumn{4}{|c|}{ Wella } \\
\hline & NC2-11D & NC7.61 & NC7-69 & W-7A \\
\hline \multicolumn{5}{|c|}{ Organic, EPA Method $602(\mu \mathrm{g} / \mathrm{L})$} \\
\hline Benzene & \multicolumn{4}{|c|}{ Four nondetections per compound, well W-7A only. } \\
\hline Chlorobenzene & \multicolumn{4}{|c|}{ Four nondetections per compound, well W-7A only. } \\
\hline Ethylbenzene & \multicolumn{4}{|c|}{ Four nondetections per compound, well W-7A only. } \\
\hline Toluene & \multicolumn{4}{|c|}{ Four nondetections per compound, well W-7A only. } \\
\hline Total xylene isomers & \multicolumn{4}{|c|}{ Four nondetections per compound, well W-7A only. } \\
\hline \multicolumn{5}{|l|}{ Radioactivity (Bq/L) } \\
\hline \multirow[t]{4}{*}{ Gross alpha } & $0.12 \pm 0.13$ & $0.10 \pm 0.11$ & $0.02 \pm 0.08$ & $0.00 \pm 0.07$ \\
\hline & $0.05 \pm 0.11$ & $0.14 \pm 0.13$ & $0.0004 \pm 0.10$ & $0.00 \pm 0.15$ \\
\hline & $0.20 \pm 0.13$ & $0.06 \pm 0.13$ & $-0.02 \pm 0.08$ & $0.04 \pm 0.19$ \\
\hline & $0.19 \pm 0.13$ & $0.09 \pm 0.16$ & $0.00 \pm 0.08$ & $0.00 \pm 0.15$ \\
\hline \multirow[t]{4}{*}{ Gross beta } & $0.11 \pm 0.07$ & $0.16 \pm 0.08$ & $0.13 \pm 0.08$ & $0.19 \pm 0.07$ \\
\hline & $0.15 \pm 0.09$ & $0.12 \pm 0.07$ & $0.09 \pm 0.08$ & $0.22 \pm 0.07$ \\
\hline & $0.12 \pm 0.07$ & $0.19 \pm 0.07$ & $0.11 \pm 0.07$ & $0.22 \pm 0.07$ \\
\hline & $0.20 \pm 0.05$ & $0.21 \pm 0.08$ & $0.18 \pm 0.06$ & $0.30 \pm 0.11$ \\
\hline Radium-226 & $0.002 \pm 0.017$ & & & \\
\hline \multirow[t]{4}{*}{ Tritium } & $69.56 \pm 4.17$ & $9509.00 \pm 48$ & $0.05 \pm 0.04$ & $0.17 \pm 0.06$ \\
\hline & $67.71 \pm 1.63$ & $9102.00 \pm 36$ & $0.06 \pm 0.06$ & $0.02 \pm 0.02$ \\
\hline & & $8917.00 \pm 36$ & $0.15 \pm 0.05$ & $0.10 \pm 0.06$ \\
\hline & & $8510.00 \pm 306$ & $0.13 \pm 0.08$ & $0.10 \pm 0.10$ \\
\hline \multirow[t]{4}{*}{ Uranium-233, -234 } & & $0.11 \pm 0.02$ & $0.0004 \pm 0.002$ & \\
\hline & & $0.10 \pm 0.01$ & $0.002 \pm 0.002$ & \\
\hline & & $0.17 \pm 0.01$ & $0.08 \pm 0.01$ & \\
\hline & & $0.10 \pm 0.01$ & $0.01 \pm 0.00$ & \\
\hline \multirow[t]{4}{*}{ Uranium-235 } & & $0.005 \pm 0.003$ & $0.0004 \pm 0.0009$ & \\
\hline & & $0.002 \pm 0.002$ & $-0.0004 \pm 0.0009$ & \\
\hline & & $0.003 \pm 0.001$ & $0.0003 \pm 0.0009$ & \\
\hline & & $0.00 \pm 0.00$ & $0.00 \pm 0.00$ & \\
\hline \multirow[t]{4}{*}{ Uranium-238 } & & $0.07 \pm 0.01$ & $0.00 \pm 0.0007$ & \\
\hline & & $0.07 \pm 0.01$ & $0.001 \pm 0.002$ & \\
\hline & & $0.09 \pm 0.01$ & $0.0009 \pm 0.0007$ & \\
\hline & & $0.08 \pm 0.01$ & $0.00 \pm 0.00$ & \\
\hline
\end{tabular}

Note: Results are reported $\pm 2 \sigma$; see Quality Assurance chapter.

a See Fig. 6-5 for sampling locations. 
Table 6-10. Analyses of water from supply wells, Site 300, 1992.

\begin{tabular}{|c|c|c|c|}
\hline \multirow{2}{*}{ Parameter } & \multicolumn{3}{|c|}{ Wella $^{\mathbf{a}}$} \\
\hline & Well 01 & Well 18 & Well 20 \\
\hline \multicolumn{4}{|l|}{ Inorganic chemicals (mg/L) } \\
\hline \multirow[t]{2}{*}{ Total alkalinity (as $\mathrm{CaCO}_{3}$ ) } & & & 230 \\
\hline & & & 220 \\
\hline \multirow[t]{2}{*}{ Bicarbonate alk (as $\mathrm{CaCO}_{3}$ ) } & & & 200 \\
\hline & & & 220 \\
\hline \multirow[t]{2}{*}{ Carbonate alk (as $\mathrm{CaCO}_{3}$ ) } & & & 30 \\
\hline & & & $<1$ \\
\hline \multirow[t]{4}{*}{ Beryllium } & $<0.0005$ & $<0.0005$ & $<0.0005$ \\
\hline & $<0.0005$ & $<0.0005$ & $<0.0005$ \\
\hline & $<0.0002$ & $<0.0002$ & $<0.0002$ \\
\hline & $<0.0005$ & $<0.0005$ & $<0.0005$ \\
\hline \multirow[t]{2}{*}{ Calcium } & & & 6.2 \\
\hline & & & 6.2 \\
\hline \multirow[t]{2}{*}{ Chloride } & & & 78 \\
\hline & & & 74 \\
\hline \multirow[t]{4}{*}{ Chromium } & $<0.005$ & $<0.005$ & $<0.005$ \\
\hline & $<0.005$ & $<0.005$ & $<0.005$ \\
\hline & $<0.005$ & 0.006 & $<0.005$ \\
\hline & $<0.005$ & $<0.005$ & $<0.005$ \\
\hline \multirow[t]{6}{*}{ Copper } & $<0.005$ & $<0.005$ & $<0.005$ \\
\hline & $<0.05$ & $<0.05$ & $<0.05$ \\
\hline & $<0.05$ & $<0.05$ & $<0.05$ \\
\hline & $<0.05$ & $<0.05$ & $<0.05$ \\
\hline & & $<0.05$ & $<0.05$ \\
\hline & & 0.011 & $<0.005$ \\
\hline \multirow[t]{2}{*}{ Iron } & & & $<0.1$ \\
\hline & & & 0.4 \\
\hline \multirow[t]{4}{*}{ Lead } & $<0.002$ & $<0.002$ & 0.002 \\
\hline & 0.002 & 0.0044 & $<0.002$ \\
\hline & $<0.002$ & 0.003 & $<0.002$ \\
\hline & 0.003 & $<0.002$ & $<0.002$ \\
\hline \multirow[t]{2}{*}{ Magnesium } & & & $<0.5$ \\
\hline & & & $<0.5$ \\
\hline \multirow[t]{2}{*}{ Manganese } & & & $<0.05$ \\
\hline & & & $<0.05$ \\
\hline \multirow[t]{2}{*}{ Nitrate plus nitrite (as $\mathrm{NO}_{3}$ ) } & & & 2.7 \\
\hline & & & $<0.4$ \\
\hline \multirow[t]{2}{*}{ Potassium } & & & 8.1 \\
\hline & & & 8 \\
\hline \multirow[t]{2}{*}{ Sodium } & & & 220 \\
\hline & & & 230 \\
\hline \multirow[t]{2}{*}{ Sulfate } & & & 200 \\
\hline & & & 190 \\
\hline \multirow[t]{2}{*}{ Total dissolved solids (TDS) } & & & 720 \\
\hline & & & 730 \\
\hline
\end{tabular}


Table 6-10. Analyses of water from supply wells, Site 300, 1992 (continued).

\begin{tabular}{|c|c|c|c|}
\hline \multirow[b]{2}{*}{ Parameter } & \multicolumn{3}{|c|}{ Welp } \\
\hline & Well 01 & Well 18 & Well 20 \\
\hline Zinc & & & $\begin{array}{l}<0.05 \\
<0.05\end{array}$ \\
\hline Specific conductance ( $\mu \mathrm{mhos} / \mathrm{cm}$ ) & & & $\begin{array}{r}990 \\
1080\end{array}$ \\
\hline \multirow[t]{2}{*}{$\mathrm{pH}$} & & & 8.4 \\
\hline & & & 8.3 \\
\hline \multicolumn{4}{|c|}{ Organic chemicals EPA Method $524.2\left(\mu g^{\prime} L\right)$} \\
\hline \multirow[t]{2}{*}{ 1,1,1,2-Tetrachloroethane } & 2 n.d. $<0.2$ & 6 n.d. $<0.5$ & 10 n.d. $<0.2$ \\
\hline & & 6 n.d. $<0.2$ & \\
\hline \multirow[t]{2}{*}{ 1,1,1-Trichloroethane } & 0.6 & 6 n.d. $<0.5$ & 3 n.d. $<0.3$ \\
\hline & 1 n.d. $<0.3$ & 6 n.d. $<0.2$ & 7 n.d. $<0.2$ \\
\hline \multirow[t]{2}{*}{ 1,1,2,2-Tetrachloroethane } & 2 n.d. $<0.2$ & 6 n.d. $<0.5$ & 10 n.d. $<0.2$ \\
\hline & & 6 n.d. $<0.2$ & \\
\hline \multirow[t]{2}{*}{ 1,1,2-Trichloroethane } & 1 n.d. $<0.2$ & 6 n.d. $<0.5$ & 3 n.d. $<0.3$ \\
\hline & 1 n.d. $<0.3$ & 6 n.d. $<0.2$ & 7 n.d. $<0.2$ \\
\hline \multirow[t]{2}{*}{ 1,1-Dichloroesthane } & 2 n.d. $<0.2$ & 6 n.d. $<0.5$ & 10 n.d. $<0.2$ \\
\hline & & 6 n.d. $<0.2$ & \\
\hline \multirow[t]{2}{*}{ 1,1-Dichloroethene } & 2 n.d. $<0.2$ & 6 n.d. $<0.5$ & 10 n.d. $<0.2$ \\
\hline & & 6 n.d. $<0.2$ & \\
\hline \multirow[t]{2}{*}{ 1,1-Dichloropropene } & 2 n.d. $<0.2$ & 6 n.d. $<0.5$ & 10 n.d. $<0.2$ \\
\hline & & 6 n.d. $<0.2$ & \\
\hline \multirow[t]{2}{*}{ 1,2,3-Trichlorobenzene } & 2 n.d. $<0.2$ & 6 n.d. $<0.5$ & 10 n.d. $<0.2$ \\
\hline & & 6 n.d. $<0.2$ & \\
\hline \multirow[t]{2}{*}{ 1,2,3-Trichloropropane } & 2 n.d. $<0.2$ & 6 n.d. $<0.5$ & 10 n.d. $<0.2$ \\
\hline & & 6 n.d. $<0.2$ & \\
\hline \multirow[t]{2}{*}{ 1,2,4-Trichlorobenzene } & 2 n.d. $<0.2$ & 6 n.d. $<0.5$ & 10 n.d. $<0.2$ \\
\hline & & 6 n.d. $<0.2$ & \\
\hline \multirow[t]{2}{*}{ 1,2,4-Trimethy:"Jenzene } & 2 n.d. $<0.2$ & 6 n.d. $<0.5$ & 10 n.d. $<0.2$ \\
\hline & & 6 n.d. $<0.2$ & \\
\hline \multirow[t]{2}{*}{ 1,2-Dibromo-3-chloropropane } & 1 n.d. $<2$ & 6 n.d. $<0.5$ & 3 n.d. $<0.5$ \\
\hline & 1 n.d. $<0.5$ & 6 n.d. $<2.0$ & 7 n.d. $<2.0$ \\
\hline 1,2-Dibromoethane & 2 n.d. $<0.2$ & 6 n.d. $<0.2$ & 10 n.d. $<0.2$ \\
\hline \multirow[t]{2}{*}{ 1,2-Dichlorobenzene } & 2 n.d. $<0.2$ & 6 n.d. $<0.5$ & 10 n.d. $<0.2$ \\
\hline & & 6 n.d. $<0.2$ & \\
\hline \multirow[t]{2}{*}{ 1,2-Dichloroethane } & 2 n.d. $<0.2$ & 6 n.d. $<0.5$ & 10 n.d. $<0.2$ \\
\hline & & 6 n.d. $<0.2$ & \\
\hline 1,2-Dichloroethene (total) & 2 n.d. $<0.2$ & 6 n.d. $<0.5$ & \\
\hline
\end{tabular}

...continued 


\section{Surveillance Water Monitoring}

Table 6-10. Analyses of water from supply wells, Site 300, 1992 (continued).

\begin{tabular}{|c|c|c|c|}
\hline \multirow[b]{2}{*}{ Parameter } & \multicolumn{3}{|c|}{ Welp } \\
\hline & Well 01 & Well 18 & Well 20 \\
\hline \multirow[t]{2}{*}{ 1,2-Dichloropropane } & 2 n.d. $<0.2$ & 6 n.d. $<0.5$ & 10 n.d. $<0.2$ \\
\hline & & 6 n.d. $<0.2$ & \\
\hline \multirow[t]{2}{*}{ 1,3,5-Trimethylbenzene } & 2 n.d. $<0.2$ & 6 n.d. $<0.5$ & 10 n.d. $<0.2$ \\
\hline & & 6 n.d. $<0.2$ & \\
\hline \multirow[t]{2}{*}{ 1,3-Dichlorobenzene } & 2 n.d. $<0.2$ & 6 n.d. $<0.5$ & 10 n.d. $<0.2$ \\
\hline & & 6 n.d. $<0.2$ & \\
\hline \multirow[t]{2}{*}{ 1,3-Dichloropropane } & 2 n.d. $<0.2$ & 6 n.d. $<0.5$ & 10 n.d. $<0.2$ \\
\hline & & 6 n.d. $<0.2$ & \\
\hline \multirow[t]{2}{*}{ 1,4-Dichlorobenzene } & 2 n.d. $<0.2$ & 6 n.d. $<0.5$ & 10 n.d. $<0.2$ \\
\hline & & 6 n.d. $<0.2$ & \\
\hline \multirow[t]{2}{*}{ 2,2-Dichloropropane } & 2 n.d. $<0.2$ & 6 n.d. $<0.5$ & 10 n.d. $<0.2$ \\
\hline & & 6 n.d. $<0.2$ & \\
\hline \multirow[t]{2}{*}{ 2-Chlorotoluene } & 2 n.d. $<0.2$ & 6 n.d. $<0.5$ & 10 n.d. $<0.2$ \\
\hline & & 6 n.d. $<0.2$ & \\
\hline \multirow[t]{2}{*}{ 4-Chlorotoluene } & 2 n.d. $<0.2$ & 6 n.d. $<0.5$ & 10 n.d. $<0.2$ \\
\hline & & 6 n.d. $<0.2$ & \\
\hline \multirow[t]{2}{*}{ Benzene } & 2 n.d. $<0.2$ & 6 n.d. $<0.5$ & 10 n.d. $<0.2$ \\
\hline & & 6 n.d. $<0.2$ & \\
\hline \multirow[t]{2}{*}{ Bromobenzene } & 2 n.d. $<0.2$ & 6 n.d. $<0.5$ & 10 n.d. $<0.2$ \\
\hline & & 6 n.d. $<0.2$ & \\
\hline \multirow[t]{2}{*}{ Bromochloromethane } & 2 n.d. $<0.2$ & 6 n.d. $<0.5$ & 10 n.d. $<0.2$ \\
\hline & & 6 n.d. $<0.2$ & \\
\hline \multirow[t]{2}{*}{ Bromodichloromethane } & 2 n.d. $<0.2$ & 6 n.d. $<0.5$ & 11 n.d. $<0.2$ \\
\hline & & 6 n.d. $<0.2$ & \\
\hline \multirow[t]{2}{*}{ Bromoform } & 2 n.d. $<0.2$ & 6 n.d. $<0.5$ & 11 n.d. $<0.2$ \\
\hline & & 6 n.d. $<0.2$ & \\
\hline \multirow[t]{2}{*}{ Bromomethane } & 2 n.d. $<0.2$ & 6 n.d. $<0.5$ & 10 n.d. $<0.2$ \\
\hline & & 6 n.d. $<0.2$ & \\
\hline \multirow[t]{2}{*}{ Carbon tetrachloride } & 1 n.d. $<0.2$ & 6 n.d. $<0.5$ & 3 n.d. $<0.3$ \\
\hline & 1 n.d. $<0.3$ & 6 n.d. $<0.2$ & 7 n.d. $<0.2$ \\
\hline \multirow[t]{2}{*}{ Chlorobenzene } & 2 n.d. $<0.2$ & a n.d. $<0.5$ & 10 n.d. $<0.2$ \\
\hline & & 6 n.d. $<0.2$ & \\
\hline \multirow[t]{2}{*}{ Chloroethane } & 1 n.d. $<0.2$ & 6 n.d. $<0.5$ & 3 n.d. $<0.3$ \\
\hline & 1 n.d. $<0.3$ & 6 n.d. $<0.2$ & 7 n.d. $<0.2$ \\
\hline \multirow[t]{2}{*}{ Chloroform } & 2 n.d. $<0.2$ & 6 n.d. $<0.5$ & 11 n.d. $<0.2$ \\
\hline & & 6 n.d. $<0.2$ & \\
\hline
\end{tabular}

...continued 
Table 6-10. Analyses of water from supply wells, Site 300, 1992 (continued).

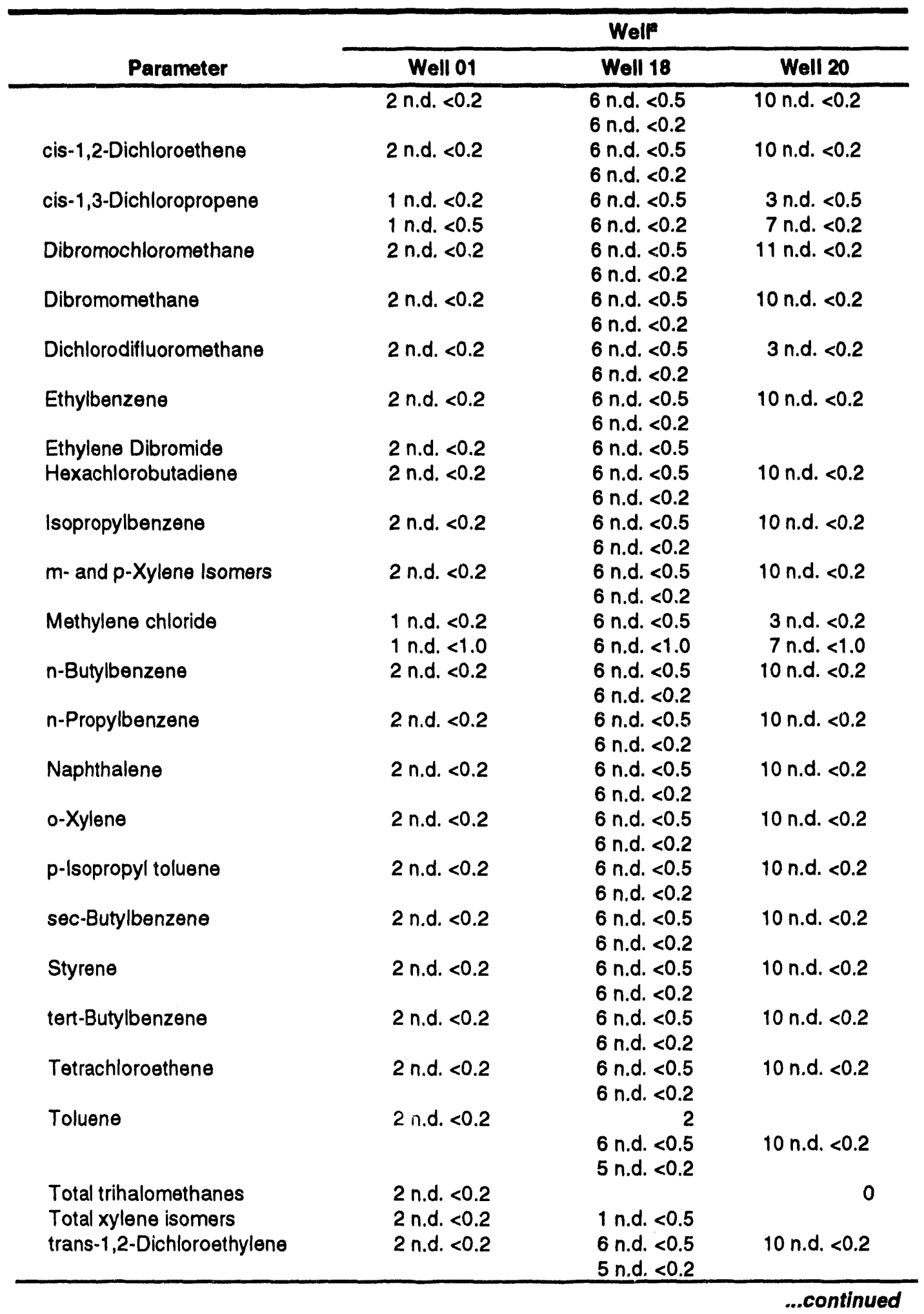




\section{Surveillance Water Monitoring}

Table 6-10. Analyses of water from supply wells, Site 300, 1992 (continued).

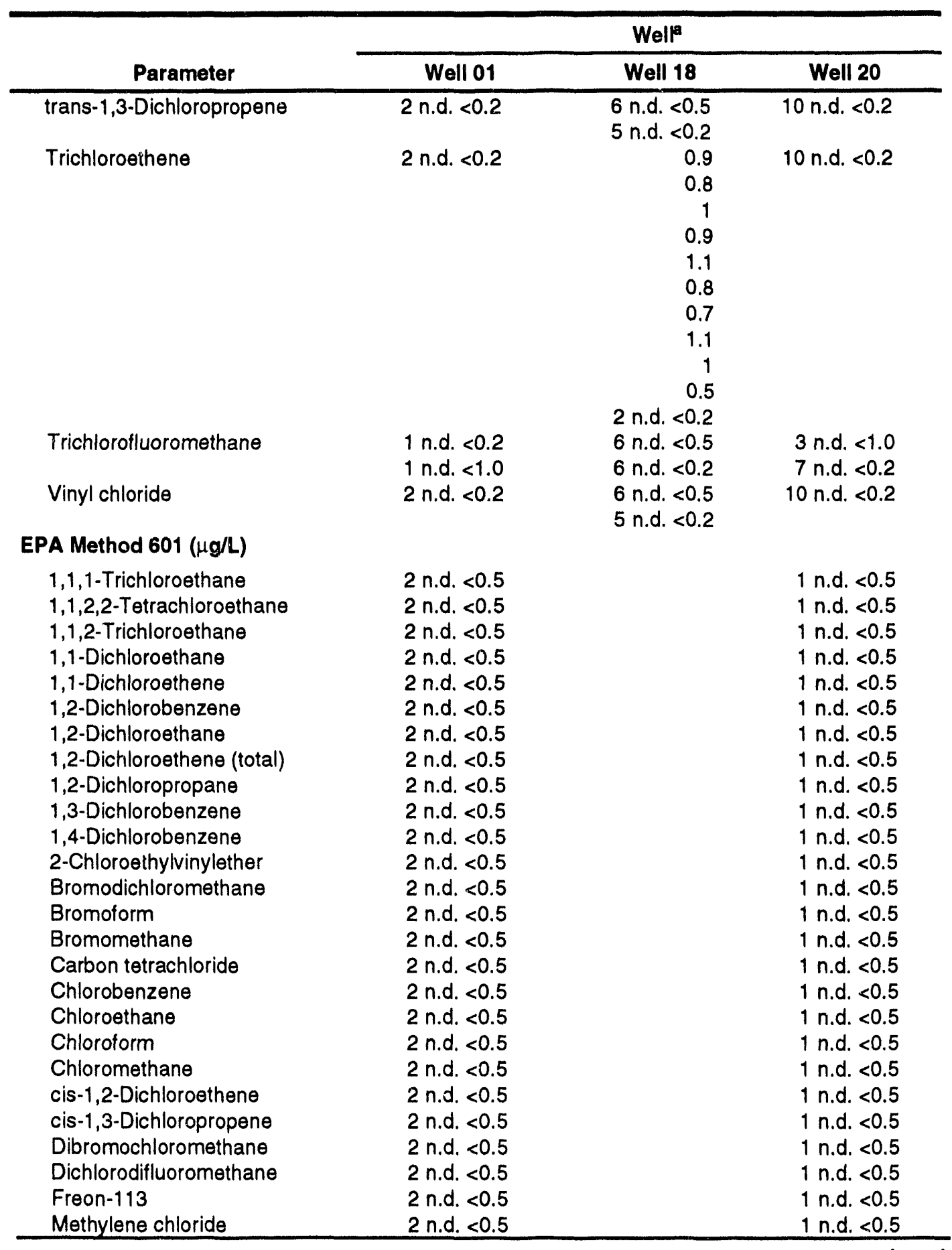


Table 6-10. Analyses of water from supply wells, Site 300, 1992 (concluded).

\begin{tabular}{|c|c|c|c|}
\hline \multirow[b]{2}{*}{ Parameter } & \multicolumn{3}{|c|}{ Wella } \\
\hline & Well 01 & Well 18 & Well 20 \\
\hline Tetrachloroethene & 2 n.d. $<0.5$ & & 1 n.d. $<0.5$ \\
\hline trans-1,2-Dichloroethene & 2 n.d. $<0.5$ & & 1 n.d. $<0.5$ \\
\hline trans-1,3-Dichloropropene & 2 n.d. $<0.5$ & & 1 n.d. $<0.5$ \\
\hline Trichloroethene & 2 n.d. $<0.5$ & & 1 n.d. $<0.5$ \\
\hline Trichlorofluoromethane & 2 n.d. $<0.5$ & & 1 n.d. $<0.5$ \\
\hline Vinyl chloride & 2 n.d. $<0.5$ & & 1 n.d. $<0.5$ \\
\hline \multicolumn{4}{|l|}{ EPA Method $602(\mu \mathrm{g} / \mathrm{L})$} \\
\hline 1,2-Dichlorobenzene & & & 1 n.d. $<0.5$ \\
\hline 1,3-Dichlorobenzene & & & 1 n.d. $<0.5$ \\
\hline 1,4-Dichlorobenzene & & & 1 n.d. $<0.5$ \\
\hline Benzene & & & 1 n.d. $<0.5$ \\
\hline Chlorobenzene & & & 1 n.d. $<0.5$ \\
\hline Ethylbenzene & & & 1 n.d. $<0.5$ \\
\hline Toluene & & & 1 n.d. $<0.5$ \\
\hline Total xylene isomers & & & 1 n.d. $<0.5$ \\
\hline \multicolumn{4}{|c|}{ Bacteria and trihalomethanes $(\mu \mathrm{g} / \mathrm{L})$} \\
\hline Bromodichloromethane & & & 1 n.d. $<0.5$ \\
\hline Bromoform & & & 1 n.d. $<0.5$ \\
\hline Chloroform & & & 1 n.d. $<0.5$ \\
\hline Dibromochloromethane & & & 1 n.d. $<0.5$ \\
\hline Total coliform (MPN/100mL) & & & 1 n.d. $<2.2$ \\
\hline Total trihalomethanes & & & 0 \\
\hline Plate count (CFU/mL) & & & 73 \\
\hline \multicolumn{4}{|l|}{ Radioactivity (Bq/L) } \\
\hline \multirow[t]{4}{*}{ Gross alpha } & $0.03 \pm 0.09$ & $0.03 \pm 0.10$ & $-0.04 \pm 0.08$ \\
\hline & $0.08 \pm 0.10$ & $0.00 \pm 0.15$ & $-0.03 \pm 0.09$ \\
\hline & $0.06 \pm 0.09$ & $0.00 \pm 0.26$ & $-0.06 \pm 0.10$ \\
\hline & $0.12 \pm 0.10$ & $0.04 \pm 0.19$ & $-0.05 \pm 0.12$ \\
\hline Gross beta & $\begin{array}{l}0.12 \pm 0.06 \\
0.11 \pm 0.08 \\
0.11 \pm 0.07 \\
0.16 \pm 0.05 \\
200 \pm 11\end{array}$ & $\begin{array}{l}0.22 \pm 0.07 \\
0.15 \pm 0.08 \\
0.00 \pm 0.26 \\
0.26 \pm 0.07 \\
0.14 \pm 0.04\end{array}$ & $\begin{array}{l}0.21 \pm 0.07 \\
0.22 \pm 0.09 \\
0.29 \pm 0.09 \\
0.20 \pm 0.05 \\
0.15 \pm 0.12\end{array}$ \\
\hline Tritium & $\begin{array}{l}231 \pm 13 \\
195 \pm 10 \\
235 \pm 13\end{array}$ & $\begin{array}{l}0.13 \pm 0.11 \\
0.12 \pm 0.12 \\
0.66 \pm 0.66\end{array}$ & $\begin{array}{l}0.10 \pm 0.03 \\
0.09 \pm 0.03 \\
0.13 \pm 0.12 \\
0.07 \pm 0.07\end{array}$ \\
\hline
\end{tabular}

Notes: Results are reported $\pm 2 \sigma$; see Quality Assurance chapter. The abbreviation "n.d." stands for nondetection.

a See Fig. 6-5 for sampling locations. 
Table 6-11. Central Drainage Basin management results (in mg/L), 1992.

\begin{tabular}{|c|c|c|c|c|c|c|}
\hline Analyte & May 4 & Jul. 31 & Nov. 16 & Dec. 18 & Objective & $\begin{array}{l}\text { Action } \\
\text { level }\end{array}$ \\
\hline Ammonia nitrogen (N) & 0.03 & 0.05 & 0.17 & 0.086 & $<0.025$ & $>0.1$ \\
\hline Boron & $-{ }^{a}$ & $<0.2$ & $-a$ & -a & $<0.5$ & $>0.7$ \\
\hline Chemical oxygen demand & $<10$ & 25 & 28 & 20 & $<20$ & $>20^{b}$ \\
\hline Chlorophyll a & $-\mathbf{a}$ & 0.0024 & 0.0028 & 0.0017 & $<10$ & $>10$ \\
\hline Fecal collform (MPN100mL) & $一^{a}$ & 130 & $-^{a}$ & 50 & $<200$ & $>400$ \\
\hline Nitrate plus nitrite (N) & $<0.1$ & $<0.1$ & 1.5 & 1.9 & $<0.2$ & $>0.2$ \\
\hline Oil and grease & $-a$ & 5 & - $^{a}$ & $<5$ & $<15$ & $>15$ \\
\hline Total alkalinity $\left(\mathrm{CaCO}_{3}\right)$ & 40 & 80 & 160 & 130 & $>75$ & $<50$ \\
\hline Total collform (MPN/100mL) & - & 500 & -a & 700 & $<2000$ & $>5000$ \\
\hline Total dissolved solids & 97 & 110 & 240 & 200 & $<400$ & $>350$ \\
\hline Total phosphorus (as $\mathrm{PO}_{4}$ ) & 0.23 & 0.17 & 0.06 & $一^{a}$ & $<0.02$ & $>0.02$ \\
\hline Total VOCs $(\mu \mathrm{g} / \mathrm{L})$ & $-^{c}$ & $-^{c}$ & $-^{c}$ & $-^{c}$ & $<5$ & $>5$ \\
\hline
\end{tabular}

Note: Results are reported $\pm 2 \sigma$; see Quality Assurance chapter.

a Sample not tested for this constituent.

b Action level for BOD.

c All analytes were found below the detection limits listed for EPA Method 601 in 40 CFR Part 136. 
Table 6-12. Central Drainage Basin discharge results (in $\mu \mathrm{g} / \mathrm{L}$ ), 1992.

\begin{tabular}{|c|c|c|c|c|}
\hline Analyte & Dec. 9 & Dec. 16 & Dec. 30 & Discharge limit ${ }^{\mathrm{a}}$ \\
\hline 1,2-Dibromo-3-chloropropane & $<0.5$ & $<0.5$ & $<0.5$ & 0.02 \\
\hline 1,2-Dibromoethane & $<0.2$ & $<0.2$ & $<0.2$ & 0.02 \\
\hline Antimony (mg/L) & $<0.2$ & $<0.2$ & $<0.2$ & 1.46 \\
\hline Aquatic bioassay, survival (\%) & 90 & & & $90 \%$ survival \\
\hline Arsenic (mg/L) & 0.0027 & $<0.002$ & $<0.002$ & 0.02 \\
\hline Benzene & $<0.2$ & $<0.2$ & $<0.2$ & 0.70 \\
\hline Beryllium (mg/L) & $<0.0002$ & $<0.0002$ & $<0.0002$ & 0.0007 \\
\hline Boron (mg/L) & 0.35 & 0.38 & 0.24 & 7.00 \\
\hline Cadmium (mg/L) & $<0.0005$ & $<0.0005$ & $<0.0005$ & 0.005 \\
\hline Chromium (mg/L) & 0.006 & $<0.005$ & $<0.005$ & 0.05 \\
\hline Copper (mg/L) & $<0.05$ & $<0.05$ & $<0.05$ & 0.02 \\
\hline Iron (mg/L) & $<0.1$ & $<0.1$ & 0.65 & 3.00 \\
\hline Lead (mg/L) & $<0.002$ & $<0.002$ & $<0.002$ & 0.0056 \\
\hline Manganese & $<0.05$ & $<0.05$ & $<0.05$ & 0.50 \\
\hline Mercury (mg/L) & $<0.0002$ & $<0.0002$ & $<0.0002$ & 0.001 \\
\hline Nickel (mg/L) & 0.0072 & $<0.005$ & $<0.005$ & 0.0071 \\
\hline $\mathrm{pH}$ & 8.4 & 8.1 & 8 & $>6.5$ and $<8.5$ \\
\hline Selenium (mg/L) & $<0.002$ & $<0.002$ & $<0.002$ & 0.10 \\
\hline Silver $(\mathrm{mg} / \mathrm{L})$ & $<0.0005$ & $<0.0005$ & $<0.0005$ & 0.0023 \\
\hline Tetrachloroethene & $<0.2$ & $<0.2$ & $<0.2$ & 4 \\
\hline Thallium & $<0.3$ & $<0.4$ & $<0.4$ & 0.13 \\
\hline Vinyl chloride & $<0.2$ & $<0.2$ & $<0.2$ & 2 \\
\hline Zinc (mg/L) & $<0.05$ & $<0.05$ & $<0.05$ & 0.058 \\
\hline Total VOCs & $-b$ & $-b$ & $-^{b}$ & 5 \\
\hline Total petroleum hydrocarbons & $-^{c}$ & $一^{c}$ & $-^{c}$ & 50 \\
\hline PAHS & $-^{d}$ & $-^{d}$ & $-d$ & 15 \\
\hline Base/neutral acid and pesticides & $-^{\theta}$ & -e & -9 & 5 \\
\hline
\end{tabular}

Note: Results are reported $\pm 2 \sigma$; see Quality Assurance chapter

a Discharge limits established in NPDES permit CA0029829.

b All analytes were found below the detection limits listed for EPA Method 601 in 40 CFR Part 136.

c All analytes were found below the detection limits listed for EPA Method 8015 in EPA SW 846.

d All analytes were found below the detection limits listed for EPA Method 610 in 40 CFR Part 136.

e All analytes were found below the detection limits listed for EPA Method 625 in 40 CFR Part 136. 
Katrina G. Fischer

Albert L. Lamarre

Richard G. Blake

Donald H. MacQueen

William G. Hoppes

\section{Introduction}

Ground water protection activities at LLNL are performed as part of the investigation, study, monitoring, and remediation of contamination caused by past practices and as a safeguard against new contamination. The existing ground water monitoring network at the Livermore site was installed to assess ground water quality and investigate contamination in response to the Comprehensive Environmental Response, Compensation, and Liability Act of 1980 (CERCLA).

In addition to these ground water remediation activities, DOE Order 5400.1 establishes requirements for environmental protection programs at DOE operations to assure compliance with local, state, and federal laws and regulations. Section 9, Chapter IV, of the DOE Order addresses environmental monitoring programs and sets forth requirements for a ground water protection plan that builds upon monitoring programs implemented for compliance with the Resource Conservation and Recovery Act of 1976 (RCRA). Development of the monitoring portion of this protection plan for the Livermore site continued during 1992.

LLNL conducts ground water monitoring at Site 300 under two programs. One program is designed to meet regulatory commitments established for specific site investigations under CERCLA, such as the investigation of trichloroethene (TCE), tritium, and high-explosives (HE) compounds in ground water at Site 300. Details of this program are described in the LLNL Site 300 Environmental Investigations Quarterly Reports (Carlsen 1991a, b) and in the Draft Remedial Investigation of the Building 833 Area, Lawrence Livermore National Laboratory Site 300 (Webster-Scholten et al. 1991), Investigation of Holocene Faulting Near Decommissioned Landfill Pit 6, Lawrence Livermore National Laboratory Site 300 (Carpenter et al. 1991), and Draft Feasibility Study for Landfill Pit 6, Lawrence Livermore National Laboratory Site 300 (Taffet et al. 1991); the program is summarized in this chapter.

The second program consists of compliance ground water monitoring at landfill and wastewater surface impoundment units, as required by the California Regional Water Quality Control Board (RWQCB), Central Valley 
Region; California Department of Toxic Substances Control (DTSC); and the U.S. Environmental Protection Agency (EPA). Details of this program are described in the LLNL Site 300 Ground Water Monitoring Program quarterly reports (Fischer et al. $1992 \mathrm{a}-\mathrm{d})$.

\section{Livermore Site}

\section{Ground Water Remediation}

In the 1940s, before it became a research laboratory, the U.S. Navy used the Livermore site as a flight training base and aircraft rework facility. An asphaltpaved landing mat was located near the center of the site, with peripheral taxiways and a concrete parking apron. The Navy used some unpaved areas within the landing mat for aircraft maintenance and for storage and disposal of fuels, solvents, and other related materials. After the Livermore Naval Air Station was closed in the late 1940s, short-term users of the site included the California Research and Development Corporation and Pratt and Whitney.

During the operation of the naval station, volatile organic compounds (VOCs) were used routinely in aircraft and engine assembly, repair, and overhaul operations, and in paint stripping. Much of this activity was performed in the southeast portion of the site and in the unpaved areas adjacent to the landing mat. In 1954, LLNL (known then as the University of California Radiation Laboratory) stored organic solvents and stored and treated hazardous waste on a portion of the East Taxi Strip. From 1956 to the present, LLNL has engaged in research and other activities that involve the storage, use, and disposal of VOCs, fuel hydrocarbons, and other hazardous materials. Since the late 1970s, LLNL has taken many steps to eliminate discharges of hazardous materials to the environment.

LLNL began a ground water investigation in December 1983, after detecting tetrachloroethene (PCE) and TCE in on-site monitoring wells and in several nearby off-site domestic and agricultural wells. During preliminary ground water investigations from 1980 to 1983 made under the requirements of the RWQCB, LLNL completed, logged, sampled, and hydraulically tested over a dozen monitoring wells. (By 1992 over 300 monitoring wells had been installed.) Data from this sampling have been interpreted in conjunction with results of record searches to determine the extent and possible sources of ground water contamination. The sources of much of the VOCs in the ground water are believed to be related directly to the solvent use and disposal practices employed by the U.S. Navy during its occupation of the site. There is also evidence that localized spills, leaking tanks, and impoundments contributed VOCs, fuel hydrocarbons (FHCs), chromium, and tritium to ground water and unsaturated sediments in the post-Navy era (Ziagos 1992). 
Since being placed on the National Priorities List, LLNL has structured its Ground Water Project to conform with specific EPA CERCLA guidance and the negotiated Federal Facility Agreement (U.S. Department of Energy 1988). The environmental restoration effort encompasses numerous activities to be performed over several years to investigate, evaluate, and eventually remediate potentially hazardous compounds that may have affected soil, sediment, and ground water quality. The Ground Water Project also encompasses air and surface water as these environmental media relate to the investigation and remediation efforts. Because the impact of past releases to the air and surface water have been relatively minor, most of the emphasis is placed on investigating the soil, sediment, and ground water.

The remedial activities include investigation of the hydrogeology of the site, including known and suspected sources of contamination; installation of piezometers and monitoring and extraction wells; soil, sediment, and ground water sampling; evaluation and closure of potential conduit wells; aquifer testing; and development of computer models and a conceptual site model to aid ground water studies and to design remediation strategies. In addition, LLNL continues to search historical records to identify potential sources of contamination; assess potential risks to public health resulting from the identified contaminants in soil, sediment, and ground water; provide project information to the public and coordinate community involvement in the remedial process; evaluate numerous studies designed to aid site remediation; prepare reports documenting the investigation, evaluation, and remediation; and implement the appropriate technologies to accomplish environmentally responsive cleanup of the site.

Several documents related to the environmental restoration effort have been submitted to the regulatory agencies. The Work Plan (Webster-Scholten and Hall 1988) outlines the above activities and other related tasks associated with the formal Remedial Investigation (RI; Thorpe et al. 1990) and Feasibility Study (FS; Isherwood et al. 1991) reports, and resultant remedial action. The RI report provides significant detail regarding the environmental investigation of potential sources of contamination. A Community Relations Plan (CRP; Anderson and Post 1988) was submitted and, as provided in this plan, persoruel in the LLNL Livermore-site Ground Water Project hold community meetings, publish an informational pamphlet, and have formed a Community Work Group to keep the public informed and provide a pathway for public input to the remedial action process. The Quality Assurance Project Plan (QAPP; Rice 1988) outlines methods and processes to ensure that the project data are precise, accurate, complete, representative, and can be compared, and that project data are maintained at an acceptable level. 
The FS report presents alternatives for remediating contaminants present in ground water and unsaturated sediments on the Livermore site, and the Proposed Remedial Action Plan (PRAP; Dresen et al. 1991) selects the alternatives that best meet all appropriate requirements. The PRAP was presented for public comment in the last quarter of 1991, and the Record of Decision and Responsiveness Summary (Ziagos 1992), which documents LLNL's responses to public comments regarding the PRAP, has been submitted to the regulatory agencies and DOE.

During 1992, LLNL completed numerous environmental restoration tasks at the Livermore site; the most important efforts include:

- LLNL investigated potential sources of ground water contamination in the East Taxi Strip-Trailer 5475 area, Building 292 area, northeast boundary area, and MW-501 area.

- The Infiltration Experiment Site in the northwest part of the site and the Gasoline Spill Area in the southern part of the site were investigated as part of the Vadose Zone Investigation Program. This program was initiated to better understand potential impacts to ground water by contaminants in the unsaturated zone.

- At the Gasoline Spill Area, steam injection wells, electrical heating wells, and associated monitoring wells were installed for the Dynamic Underground Stripping Demonstration Project (DUSDP), which uses steam and electrical heating to remove free-phase fuel hydrocarbons. The DUSDP began in November 1992 and will continue into 1993.

- Treatment Facilities A and B (TFA, TFB) continued operation during 1992 to remove VOCs from extracted ground water.

CERCLA documents submitted to the regulatory agencies include:

- Record of Decision (Ziagos 1992), issued on August 5, 1992,

- Remedial Design Report 1 (Boegel et al. 1993), issued on October 9, 1992, and

- Draft Remedial Action Implementation Plan (Dresen et al. 1992), issued on November 6, 1992.

In addition, work began on a Community Relations Plan (Anderson and Post 1988); DOE/LLNL met four times with the Community Work Group and produced and distributed issues of the Ground Water Update, an informational bulletin for interested parties.

Other accomplishments include (1) further development of the computer code NUFT to simulate the vadose zone contaminant transport; (2) measuring physical, chemical, and hydraulic properties on vadose zone sediment samples; 
(3) conducting five constant discharge pumping tests in extraction wells and piezometers in the Gasoline Spill Area and in the vicinity of TFA and TFB; and (4) drilling, sampling, and conducting hydraulic tests on monitoring wells.

As part of the RI, LLNL continues to drill additional wells, sample ground water, sediment, and soil, and perform hydraulic tests. These activities enable LLNL to refine its understanding of the hydrogeologic system, determine how and when contaminants are transported, and determine the extent of VOCs present. To facilitate the preparation of the FS, LLNL executed pilot studies for extraction and treatment of ground water in the off-site area, as well as ground water and soil vapor in the Gasoline Spill Area (Figure 7-1). Using the acquired data, LLNL is developing both analytic and numeric models to assist in the design of optimal remediation strategies and to satisfy other CERCLA requirements.

Following is a more detailed discussion, updated based on 1992 investigations, on the presence of contaminants in unsaturated sediment and ground water at the Livermore site.

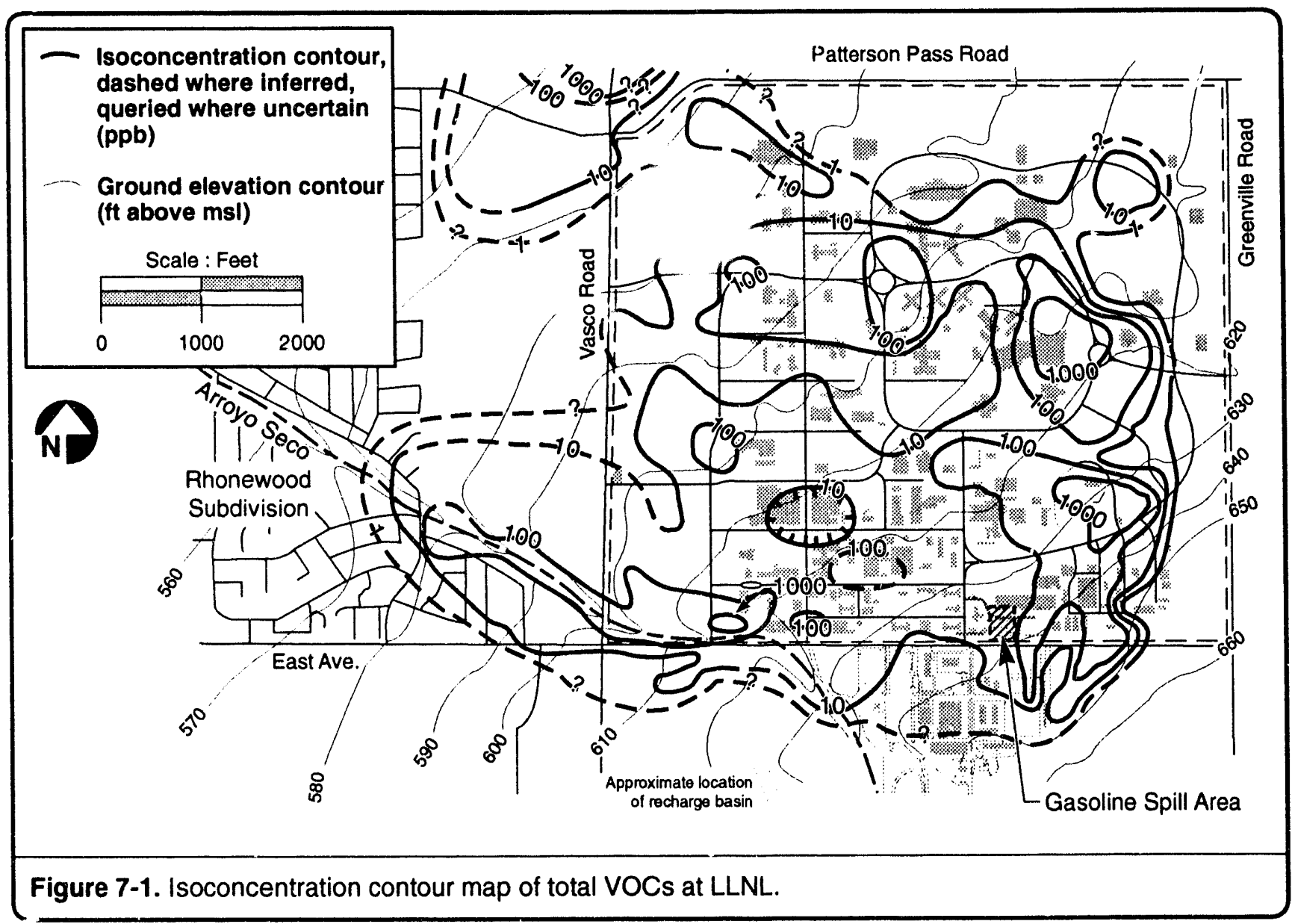


VOCs in Unsaturated Sediment. Present data indicate that total VOC concentrations exceeding one part per million (ppm) in unsaturated sediment, high enough to possibly contaminate ground water, occur in five areas: the east traffic circle, the vicinity of Building 518, Eastgate Drive, the vicinity of Building 514, and the East Taxi Strip in the southeastern portion of LLNL (Isherwood et al. 1991). Sediment samples from other potential source areas contained total VOC concentrations ranging from less than 5 parts per billion (ppb) to less than $500 \mathrm{ppb}$, with most containing less than $100 \mathrm{ppb}$, below levels that might contaminate ground water.

VOCs in Ground Water. Eight chlorinated solvents-PCE, TCE, 1,1-dichloroethene (1,1-DCE), 1,2-dichloroethene (1,2-DCE), 1,2-dichloroethane (1,2-DCA), 1,1-dichloroethane (1,1-DCA), carbon tetrachloride, and chloroformhave been detected in ground water in the Livermore-site area in concentrations exceeding federal or State Action Levels or maximum contaminant levels (MCLs). TCE is the most abundant VOC and occurs primarily in the eastern portion of Livermore site in concentrations exceeding $1 \mathrm{ppm}$ near the historic sources. PCE occurs mainly in the southwest part of Livermore site and in the adjacent off-site area to the west. PCE also is present in the eastern portion of Livermore site, at concentrations lower than that of TCE. Although generally at levels below their respective MCLs, the presence of Freon-11, Freon-113, and 1,1,1-trichloroethane (1,1,1-TCA) is being considered in the design of the treatment system for the areas in which those contaminants occur.

Fuel Hydrocarbons (FHCs). Significant concentrations of FHCs occur only in the Gasoline Spill Area (Figure 7-1), where about 64,000 liters of leaded gasoline leaked prior to 1979 and have contaminated both the unsaturated sediments and ground water.

Radiological Parameters. Tritium is the only radiological substance present in ground water in concentrations that exceed an MCL. In the southeast part of the Livermore site, MW-206 (Figure 7-2) currently contains tritium above the MCL of $740 \mathrm{~Bq} / \mathrm{L}(20,000 \mathrm{pCi} / \mathrm{L})$. At the time of the RI (Thorpe et al. 1990), 11 other LLNL monitoring wells reportedly contained tritium activities above $37 \mathrm{~Bq} / \mathrm{L}(1,000 \mathrm{pCi} / \mathrm{L})$. The most recent sampling data indicate that 10 of these original 11 wells still have tritium activities greater than $37 \mathrm{~Bq} / \mathrm{L}(1,000 \mathrm{pCi} / \mathrm{L})$. By using analytical methods having lower detection limits in subsequent investigations, tritium was detected at activities from 7 to $560 \mathrm{~Bq} / \mathrm{L}$ (200 to $15,100 \mathrm{pCi} / \mathrm{L}$ ) in 32 other monitoring wells (Macdonald et al. 1993).

\section{Ground Water Protection}

Ground water protection monitoring at and near the Livermore site is required by DOE Order 5400.1, which establishes environmental protection 


\section{Ground Water Protection}

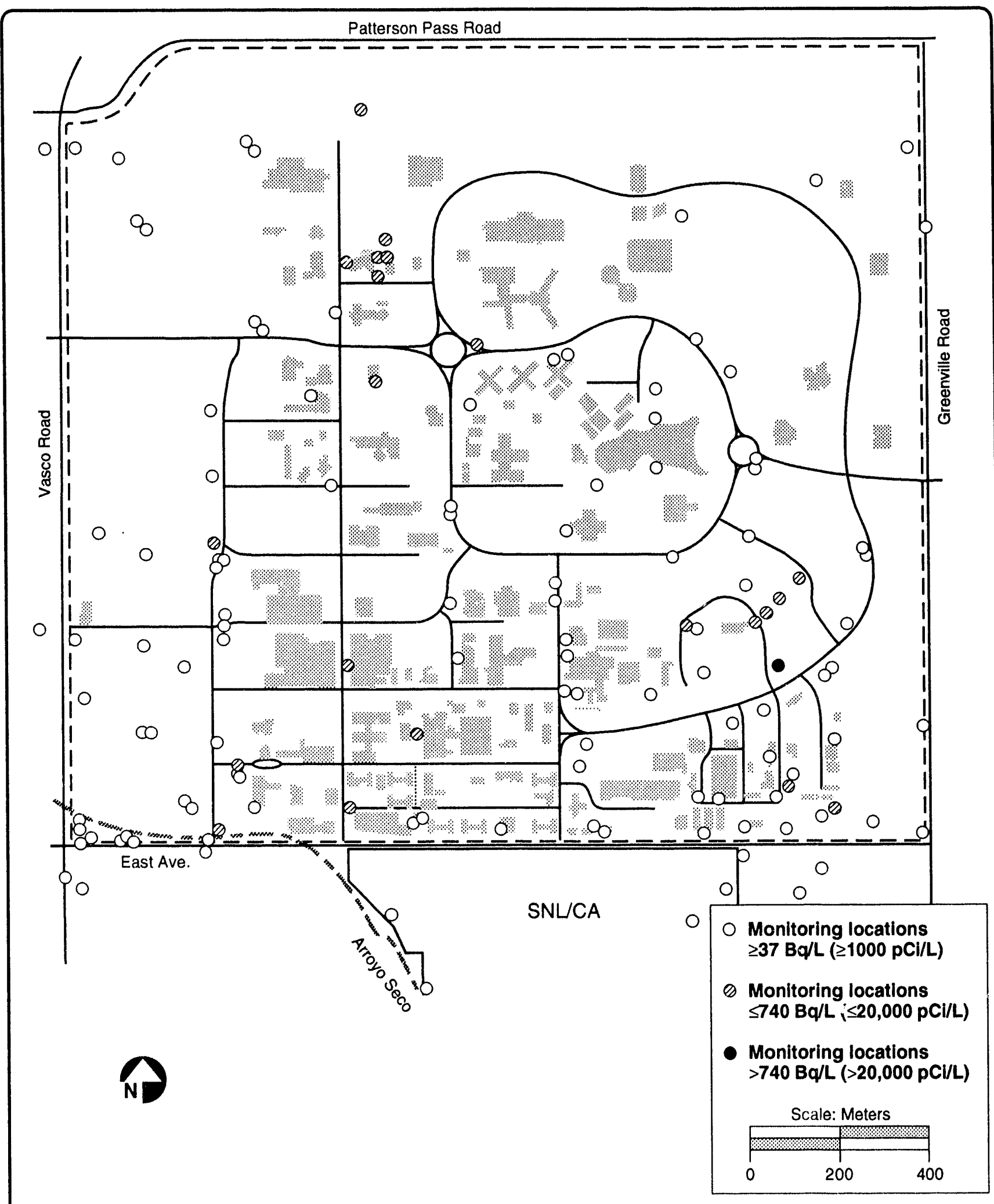

Figure 7-2. Ground water monitoring locations with tritium concentrations exceeding $37 \mathrm{~Bq} / \mathrm{L}(1000 \mathrm{pCi} / \mathrm{L})$ at LLNL, January 1992. 
program requirements to assure that DOE operations comply with local, state, and federal environmental protection laws and regulations; federal Executive Orders; and internal DOE policies. Section 9, Chapter IV, of DOE Order 5400.1 also addresses requirements for a Ground Water Protection plan, which must be a part of the Environmental Protection Implementation Plan. The Ground Water Protection Plan augments existing monitoring programs implemented to comply with RCRA (40 CFR 264 and 265) and other programs developed to monitor pollutant sources. The DOE Order also states that documentation for compliance with the Safe Drinking Water Act, RCRA, and CERCLA may be used in whole or in part to satisfy this requirement.

The goal of the Ground Water Protection Plan is to provide LLNL the opportunity to detect and mitigate, before ground water has been impacted, any releases or spills that occur as a result of current operations. For this reason, and because ground water travels at such a slow rate at the Livermore site, ground water monitoring is not the most appropriate mechanism for detecting the potential of LLNL operations to affect it. The protection of ground water at the Livermore site relies most heavily on monitoring other environmental media. The timely detection of potential contaminants from current operations requires that sediment, soil, and storm water runoff be monitored for indications of a release or spill.

The Ground Water Protection Plan is under development for the Livermore site. Developing this plan is a four-step process: (1) identify geologic areas susceptible to ground water contamination, (2) identify existing sources of contaminants, (3) assess existing monitoring programs, and (4) develop and implement any new monitoring that is needed.

In addition to our existing efforts in soil and storm water monitoring, the major effort in strengthening the Ground Water Protection Plan during 1992 involved identifying and controlling sources of contaminants. The potential sources of contaminants to the ground water beneath LLNL include underground storage tanks (both for wastewater retention and hazardous product storage); the sanitary sewer system; discharges of wastewater to storm sewers or ground; hazardous, radioactive, and other waste and chemicals temporarily stored in buildings on site. During 1992, four existing and new programs produced results that are being incorporated into the overall Ground Water Protection Plan.

Underground Storage Tanks. To comply with local, state and federal tank and clean water regulations and ordinances, LLNL implements a tank testing and permitting process; provides guidance for the proper construction and operation of tank systems; and oversees and implements closure of old or out-of-use tank systems. LLNL is presently implementing the Tank Upgrade Project to bring all LLNL tank systems into compliance with current environmental regulations 
promulgated to prevent the accidental release of hazardous substances to the environment.

The primary objective of the Tank Upgrade Project is to remove, modify, reclassify, or replace all hazardous waste and product tanks to meet all current state and federal regulations. The present scope of work includes 214 tank systems-roughly 100 for upgrading, including replacement (22 aboveground and 28 below-ground waste retention tank systems, and 13 aboveground and 37 below-ground petroleum product tank systems), and 24 for closure only. In addition, there are approximately 90 tanks and/or transformers designated as requiring corrective actions to meet Spill Prevention Control and Countermeasures (SPCC) regulations.

Spill Prevention Control and Countermeasures Plans. These plans were distributed to LLNL operators, supervisors, and managers having responsibility for oil-containing tanks, containers, and equipment. The SPCC Plans provide guidance and procedures to prevent and mitigate spills of oil to navigable waters from equipment and tanks. An SPCC training plan was developed during the 1992 calendar year and classes were given to recipients of the SPCC Plans in February and March of 1993.

Building Drain Investigation/Sanitary Sewer Rehabilitation. Two key efforts to directly reduce potential contamination of ground water during 1992 were the Sanitary Sewer Rehabilitation Project and the Building Drain Line Investigation. The Sanitary Sewer Rehabilitation Project is a 3-year effort to rehabilitate the aged and somewhat deteriorated sanitary sewer system and correct past construction deficiencies. Staff used closed-circuit television to identify and locate problems. Directly replacing old pipe or installing sewer pipe inversion linings eliminates any significant potential for infiltration of storm water or exfiltration of wastewater.

On the basis of current environmental regulations, LLNL must certify that a building's wastewater discharge lines are properly connected to the appropriate retention tank system, sewer, or storm lines. The Building Drain Line Investigation documented all building and trailer drain lines at both the Livermore site and Site 300. Each building drain line was dye-tested or otherwise verified to confirm the discharge destination. The investigation resulted in the identification of required repair actions to bring the sanitary and storm sewer systems into compliance with regulatory requirements.

Storm Water Pollution Prevention Plans. LLNL initiated a major effort during 1992 to control potential contamination of ground water from pollutants in storm water runoff. This effort complied with the California RWQCB General Storm Water Permit for Industrial Discharges and for Construction activities. The Industrial Discharges Permit prohibits discharges of unpermitted nonstorm water to the storm drainage system. As part of this effort, LLNL inventoried and 
described all potential sources of pollutants to storm water, as well as existing storm water management practices, including pollution prevention personnel, preventive maintenance, good housekeeping practices, spill prevention and response, sediment and erosion prevention, employee training, and inspection practices. The Construction Permit seeks to reduce, through "best management practices," the discharge of sediment in storm water from construction sites, and eliminate, to the extent feasible, any discharges that are not uncontaminated storm water. These efforts are described in storm water pollution prevention plans (SWPPPs). During 1992, SWPPPs were prepared for three construction projects, a building (B132) at the Livermore site, a road improvement project at Site 300 , and the closure of two hazardous waste disposal pits, also at Site 300.

This ongoing effort is a major step in establishing a Ground Water Protection Plan because it eliminates all nonpermitted discharges, identifies potential sources of storm water pollutants, and documents LLNL storm water pollution prevention practices. Infiltration from the storm sewer system provides the most probable pathway to ground water.

Further efforts to strengthen LLNL's Ground Water Protection Plan will continue in 1993.

\section{Site 300}

\section{Ground Water Remediation}

Ground water at Site 300 moves via pores and fractures. The regional ground water table lies within sandstone and conglomerate beds, and ground water generally follows the dip of the bedrock under the influence of head differences. Three known perched ground water bodies exist locally beneath the site. Site 300 drinking and process water is supplied by a separate deep confined aquifer.

Areas of investigation at Site 300 include the HE Process Area, the General Services Area, the East and West Firing Areas, the Building 834 Complex, and the Pit 6 Area (Figure 7-3). Early activities associated with the investigation and remediation of these areas included researching the site hydrogeology; installing monitoring wells, extraction wells, and piezometers; testing aquifers; surveying soil vapor to identify areas that could be sources of contamination; sampling saturated and unsaturated sediment from boreholes and wells; developing closure and post-closure plans for solid-waste landfills and the HE Burn Pit facility; identifying and investigating former water supply wells; evaluating, sealing, and abandoning specific inactive water supply wells; investigating and closing underground tanks that may have leaked; sampling ground and surface waters; developing computer models and a conceptual site hydrogeologic model to aid ground water studies; evaluating how contaminants are transported and what eventually happens to them; continuing to search historical records to 
identify potential sources of contamination; and submitting reports to regulatory agencies to document these activities.

More recent activities performed for this project include (1) preparing a Draft Site-Wide Remedial Investigation Report (SWRI) (Webster-Scholten 1993) which compiles all ground water and soil investigation information for the entire site; (2) assessing potential public health risks and ecological risks resulting from contamination of soil, sediment, and ground water; (3) involving the public in the ground water remediation process and supplying public information materials; (4) capping two landfills; and (5) performing extraction and cleanup of contaminated ground water.

Ground water investigations began in 1981 under the regulatory authority of the Central Valley RWQCB. In August 1990, Site 300 was placed on EPA's

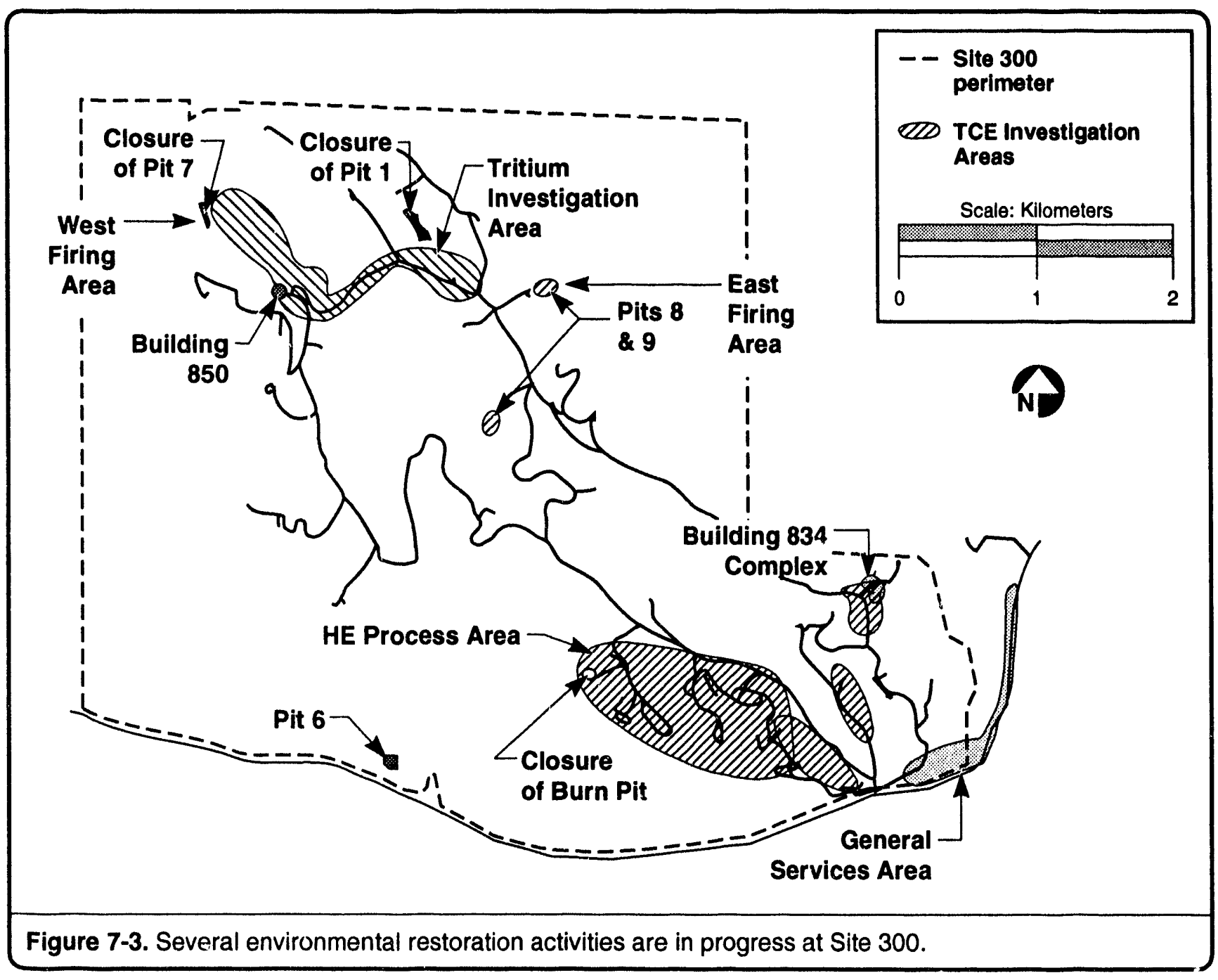


National Priorities List under CERCLA. In June 1992, the DOE and LLNL negotiated a Federal Facility Agreement, pursuant to CERCLA, which describes the ground water and soil investigations to be conducted and specifies their due dates.

The compounds addressed in the investigations at Site 300 include primarily TCE, tritium, and two HE compounds [cyclotetra-methyltetramine (HMX) and hexahydro-1,3,5-trinitro-1,3,5-triazine (RDX)]. To date, no other major environmental contaminants have been identified for these areas.

During 1992, a number of environmental restoration tasks were accomplished at Site 300 . These tasks, some of which have been under way for several years, include:

- Closure of landfill Pits 1 and 7 with approval by the EPA, RWQCB, and DTSC.

- Conducting ground water "pump and treat" operations at the central and eastern General Services Area as CERCLA removal actions.

- Preparing the SWRI (Webster-Scholten 1993).

The following sections, updated based on 1992 activities, summarize the investigation activities at Site 300 since the early 1980s. Summary results are presented in the accompanying figures detailing the isoconcentrations of the contaminant for each investigation.

TCE Investigation. In 1982, LLNL began initial assessment of the environmental effects of TCE released in several areas of the site. By 1987, ten locations within the site were found to have TCE present in soil, sediment, and ground water. TCE investigations have continued, emphasizing the southeast part of the site (Figure 7-3), the Building 834 Complex, the HE Process Area, the General Services Area, and the Pit 6 Area. Recent monitoring has also revealed trace amounts of TCE in ground water near the Pit 7 Complex (in the West Firing Area) and at Pit 8 (in the East Firing Area). RI reports have been written for each area (Taffet et al. 1989b, Taffet et al. 1992). Feasibility studies have been written or begun for each area.

HE Compounds Investigation. Discharges of rinse water from buildings within the HE Process Area historically have been disposed of in unlined lagoons adjacent to the processing buildings. Use of these lagoons was terminated in 1985; the lagoons were closed and capped with impermeable clay in 1989. Sporadic, but generally low, concentrations of HE compounds, metals, and VOCs were identified in the vadose zone beneath some of the lagoons, but these contaminants have not dispersed to the underlying ground water (Webster-Scholten 1993). During field investigations of ground water, low concentrations of TCE and $\mathrm{HE}$ compounds were discovered in two perched water-bearing zones within 
the HE Process Area. The full extent of the contamination has been determined, and remedial alternatives are being studied.

Tritium Investigation. Debris from HE tests historically has been disposed of in landfills at the northern portion of Site 300. In 1981, the Hazardous Waste Assessment study of the hydrology, geology, and ground water chemistry associated with Site 300 landfills was initiated. As part of this project, monitoring wells were installed at the landfills, and a program of periodic ground water monitoring was initiated. In 1984, tritium activities in water from four of the wells rose above the California MCL for drinking water. A tritium investigation was initiated, and two areas that have tritium in ground water have been delineated: (1) the Pit 7 Complex and (2) Building 850, Doall Road, and Elk Ravine in the West and East Firing areas.

Landfill Closures. Until November 8, 1988, LLNL operated two Class II solidwaste landfills (Pits 1 and 7) at Site 300, which received firing-table debris from explosives testing. The landfills are subject to RCRA regulation and were operated under RCRA interim status. In March 1988, partly because the landfills were nearly full, LLNL decided not to pursue its RCRA Part B permit application for land disposal. Disposal of wastes at Pits 1 and 7 was discontinued in November 1988. LLNL submitted a closure and post-closure plan for the landfills to the regulatory agencies, which was approved early in 1991. LLNL completed most of the capping and closure efforts at these pits in 1992. Closure is expected in 1993.

\section{Ground Water Protection Monitoring}

Monitoring of Pit 1, Pit 6, Pit 7, and the B-817 HE Process Area surface impoundments is reported quarterly as set forth in the Department of Health Services (DHS) Interim Status Document (ISD) CA 2890090002 (California DHS 1981), which currently is administered by the California EPA's Department of Toxic Substances Control (DTSC); and Waste Discharge Requirements (WDR) Order Nos. 80-184 (1980) and 85-188 (1985), administered by the Central Valley RWQCB. Wells in the Pit 2 and Pit 8 areas are also monitored for a variety of metals, minerals, organic compounds, and radionuclides.

The compliance sampling program encompasses 38 wells that monitor the ground water beneath the inactive landfills. The wells are located in four geographic areas of Site 300:

- Portions of the East Firing Area (17 wells and 6 Barcad samplers). Included in the area are the Building 801 and 802 firing tables and inactive landfill Pits $1,2,8$, and 9 .

- Portions of the West Firing Area (10 wells). This area includes the Building 850 and 851 firing tables and the Pit 7 Complex. 
- Another inactive landfill Pit 6 (six wells), located south along the Site 300 boundary bordering Corral Hollow Road.

- The Building 817 HE Process Area, which has a network of four wells and one piezometer to monitor the HE wastewater surface impoundments.

Detection limits and analytical parameters for the ground water compliance monitoring wells (see Figure 6-1 in Chapter 6 on Surveillance Water Monitoring for monitoring locations) are presented in Tables 7-1 and 7-2.

On April 15, 1992, in accordance with California Code of Regulations (CCR) Title 23 requirements for a revised monitoring plan, LLNL submitted a Draft Report of Waste Discharge (ROWD) covering Pit 1, Pit 7, and the B817 HE Process Area surface impoundments (Hoppes et al. 1992). However, ground water monitoring performed in 1992 was not substantially changed from 1991, pending implementation and approval of new Waste Discharge Requirements issued by the State Water Resources Control Board (SWRCB).

As requested by the Central Valley RWQCB, the ROWD was submitted as a draft to allow for preliminary review and comments. The final report for Pits 1 and 7 was to be submitted upon signing the Federal Facility Agreement (effective as of June 29, 1992). The final report for the HE Process Area surface impoundments was to be submitted by June 30,1992 . LLNL did not receive a request for preliminary changes and, therefore, the draft ROWD was submitted as LLNL's proposed revision to the existing compliance ground water plan for those areas (draft updated to final status on July 5, 1992). These changes will be implemented upon approval by the Central Valley RWQCB and modification of existing WDR Order Nos. 80-184 and 85-188.

During the 1992 third quarter monitoring period, closure activities at Site 300 included capping of landfill Pits 1 and 7. Pit 1 upgradient wells K1-01C and K1-07, along with the newly added Pit 7 downgradient well NC7-48, were inaccessible during the capping of these landfills for about a month, preventing collecting three samples from the Pit 1 wells and four samples from well NC7-48. These locations were restored and sampling resumed during the fourth quarter.

Radiological Results. Details of the quarterly sampling and monitoring results can be found in the LLNL Site 300 Ground Water Monitoring Program quarterly reports (Fischer et al. 1992a-d). Tables C-1 through C-8 in Appendix C contain supporting monitoring data.

Well NC2-07: This well is the most downgradient monitoring point for Pits 1, $2,7,8$, and 9 and is sampled for radiological compounds, gross alpha, gross beta, and tritium. The highest observed gross alpha and gross beta activities did not exceed $35 \%$ and $15 \%$, respectively, of the MCL. Tritium activities in NC2-07 did not exceed $5 \%$ of the drinking water MCL during 1992 (Table C-1). 


\section{Ground Water Protection}

Pit 1: Gross alpha, gross beta, and tritium activities in Pit 1 wells were well below corresponding drinking water MCLs. The highest average gross alpha and gross beta values for Pit 1 wells (Figure 7-4, Table C-2) did not exceed 33\% and $10 \%$ of the EPA and DTSC MCLs, respectively (U.S. Environmental Protection Agency 1977, 22 CCR 64443). The highest gross alpha and gross beta activities [0.224 and $0.238 \mathrm{~Bq} / \mathrm{L}$, respectively $(1.38$ and $6.43 \mathrm{pCi} / \mathrm{L})]$ were both observed in Pit 1 well K1-01C. These values were $40 \%$ and 13\% of the MCL. The highest average tritium activities for Pit 1 wells did not exceed $10 \%$ of the EPA and DTSC MCL.

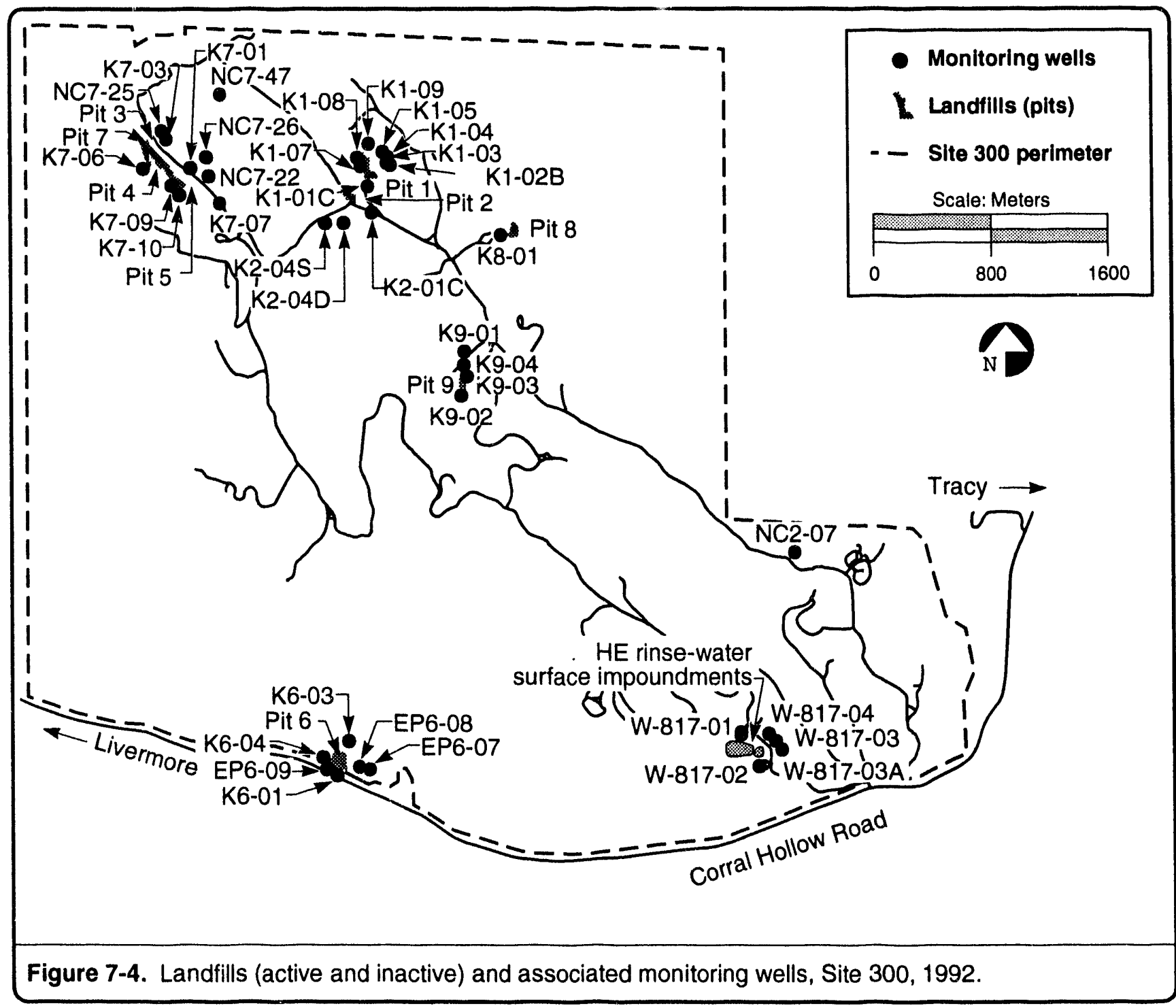


Pit 2: Gross alpha, gross beta, and tritium activities in Pit 2 monitor wells were higher than those observed at Pit 1 but, with one exception, below applicable MCLs (Table C-3). The exception was the one sample collected from well $\mathrm{K} 2-04 \mathrm{~S}$, which resulted in an unusually high gross alpha activity of $0.67 \mathrm{~Bq} / \mathrm{L}(18.1 \mathrm{pCi} / \mathrm{L})$, which is above the MCL of $0.56 \mathrm{~Bq} / \mathrm{L}$, compared to other Pit 2 wells; however, the other three quarterly samples collected from this location averaged only $21 \%$ of the MCL. Other gross alpha and gross beta results from Pit 2 wells were all an average of $10 \%$ to $30 \%$ of the MCLs.

Pit 6: Average gross alpha and gross beta activities for Pit 6 wells did not exceed $20 \%$ of the MCLs. Tritium activities averaged less than $1 \%$ of the MCL (Table C-4).

Pit 7 Complex: We observed elevated levels of ${ }^{234} U$ and ${ }^{238} U$ in the third quarter samples from well K7-06. Results for other quarters were more in line with historic activities. Levels of all radionuclides except ${ }^{226} \mathrm{Ra}$ were somewhat elevated in well NC7-26 compared to results reported in the Environmental Report for 1991 (Gallegos et al. 1992a; see Table C-5). Gross alpha and total uranium activities continue to occur above MCLs in well NC7-25. During the second quarter of 1992, well NC7-48 was added to the Pit 7 Complex compliance ground water monitoring network. NC7-48 is located immediately downgradient of Pit 7 and upgradient of landfill Pit 3. Thus, it is the only monitoring well that samples ground water that could be aftected only by Pit 7. Gross alpha activity at well NC7-48 was as high as $0.57 \mathrm{~Bq} / \mathrm{L}(15.4 \mathrm{pCi} / \mathrm{L})$, slightly above the MCL, but averaged only $55 \%$ of the MCL. Radium-226 and total uranium activities were also elevated above the respective MCLs in the two samples collected in 1992. Too few data have been collected from well NC7-48 to permit statistical analysis or trending.

Tritium activities continued to be observed above the MCL $(740 \mathrm{~Bq} / \mathrm{L})$ in samples from three routine monitoring wells: K7-01 [1438 to $1882 \mathrm{~Bq} / \mathrm{L}$ $\left(3.88 \times 10^{4}\right.$ to $\left.\left.5.08 \times 10^{4} \mathrm{pCi} / \mathrm{L}\right)\right], \mathrm{K} 7-03\left[1435\right.$ to $6424 \mathrm{~Bq} / \mathrm{L}\left(3.88 \times 10^{4}\right.$ to $17.36 \times$ $\left.\left.10^{4} \mathrm{pCi} / \mathrm{L}\right)\right]$, and NC7-25 [8531 to $11,689 \mathrm{~Bq} / \mathrm{L}\left(2.3 \times 10^{5} \mathrm{pCi} / \mathrm{L}\right.$ to $31.6 \times$ $\left.\left.10^{5} \mathrm{pCi} / \mathrm{L}\right)\right]$. Tritium activities in the other five Pit 7 wells all averaged less than $20 \%$ of the MCL (Figure 7-5).

Pit 8: Gross alpha and gross beta values from well K8-01 we 2 less than $25 \%$ of the MCL. Tritium activities were low, averaging less than $1 \%$ of the MCL (Table C-6). Uranium activities at K8-01 were less than $35 \%$ of the MCL; ${ }^{226} \mathrm{Ra}$ was not detected.

HE Rinse Water Surface Impoundments: Water samples are only analyzed for tritium in the Building 817 area. During 1992, average tritium activities from the $\mathrm{B} 817$ area wells did not exceed $0.5 \%$ of the MCL (Table C-7).

Pit 9: The highest gross alpha value $[0.028 \mathrm{~Bq} / \mathrm{L}(0.76 \mathrm{pCi} / \mathrm{L})$ at well $\mathrm{K} 9-02]$ from Pit 9 wells was less than $5 \%$ of the MCL. The average gross beta values 
were below $20 \%$ of the MCL. Tritium activities at Pit 9 wells averaged $5 \%$ or less of the MCL (Table C-8).

Nonradiological Results. Nonradiological results are summarized according to the geographical areas of Site 300. Tables C-1 through C-8 in Appendix C contain monitoring data for all four geographical areas.

NC2-07: Analyses at this location do not include metals. No volatile organic compounds were detected in well NC2-07 by EPA Method 601 (Table C-1).

Pit 1: Several metals were observed in 1992 samples at background concentrations. None exceeded 25\% of the respective MCL (Table C-2; Fischer et al.

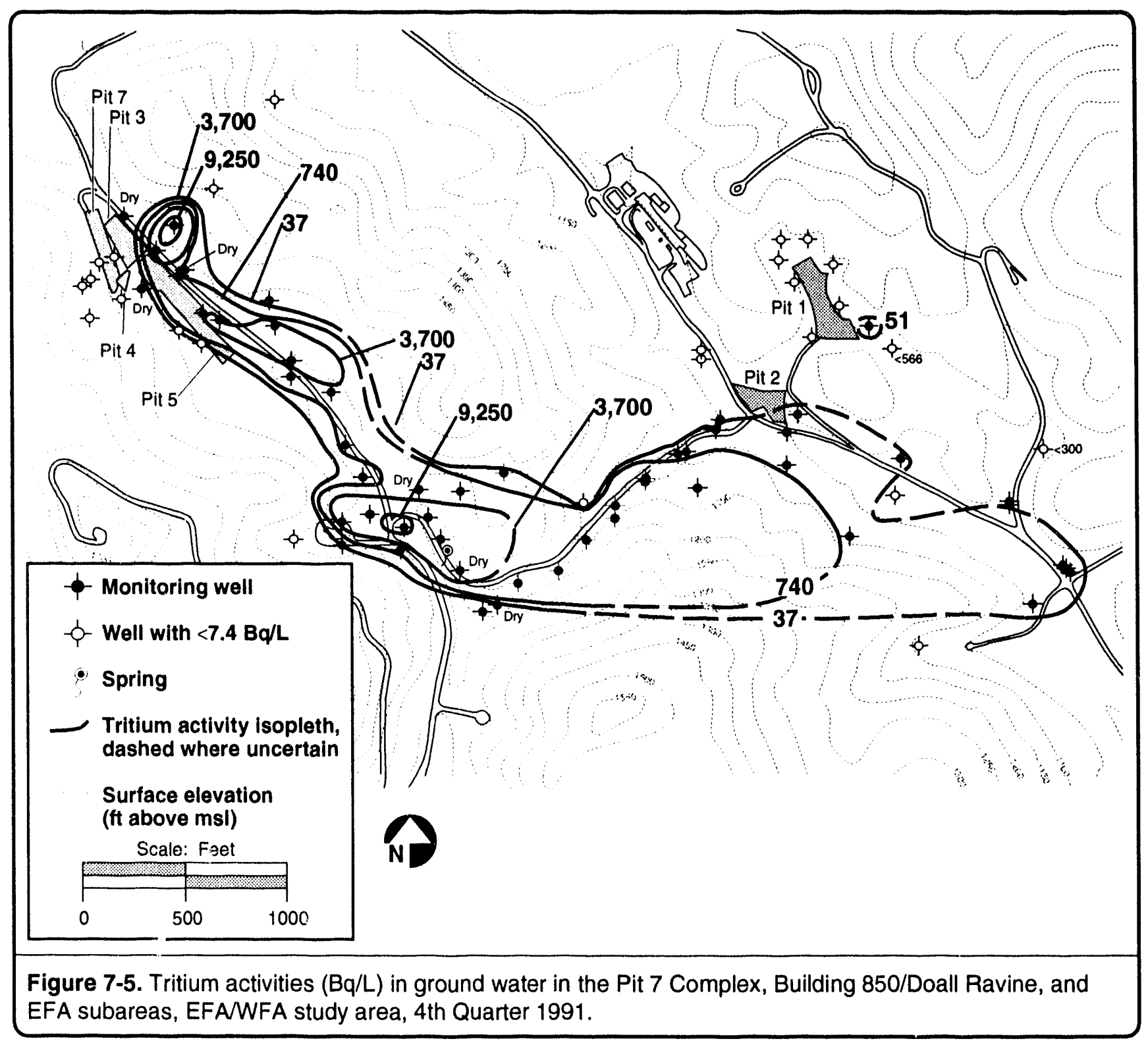


1992a-d). Single nickel activities at well K1-0-1C $(0.046 \mathrm{mg} / \mathrm{L}$, less than one-half the MCL) and well K1-08 (0.2 mg/L, twice the MCL) were not confirmed by subsequent sampling and analysis and we now believe that they resulted from analytical laboratory error.

Well K1-02B (downgradient of Pit 1) continues to show a significantly higher specific conductance than the average of samples from wells K1-01C and K1-07, which are upgradient Pit 1 wells (Fischer et al. 1992a-d). A study of correlations was performed during the second quarter (Fischer et al. 1992b) between specific conductance and calcium, chloride, sodium, sulfate, and total dissolved solids (TDS). The results of this study indicate that spatial variation in the Pit 1 wells' specific conductance values can be largely attributed to variation in calcium, chloride, and sulfate. Additionally, as the temporal variation of specific conductance is common to all eight Pit 1 Area wells (upgradient and downgradient), the variation is not likely caused by leakage of contaminants from Pit 1.

Low concentrations of Freon-113 (1,1,2-trichloro-1,2,2-trifluoroethane) continue to be observed in wells K1-05, K1-08, and K1-09. The highest concentration observed during 1992, which was $115 \mu \mathrm{g} / \mathrm{L}$ in $\mathrm{K} 1-09$, is less than $10 \%$ of the recommended State Action Level (SAL) but is more than the highest concentration $(35 \mu \mathrm{g} / \mathrm{L})$ observed in 1991 in the same well.

Pit 2: Metal analyses at well K2-01C, K2-04D, and K2-04S are limited to beryllium and lead. One of four quarterly samples from well K2-01C contained lead at $0.011 \mathrm{mg} / \mathrm{L}$, still below the $\mathrm{MCL}(0.05 \mathrm{mg} / \mathrm{L})$. This concentration, much higher than historical lead values at this well, was not confirmed by subsequent sampling and analysis (Table C-3) and may be the result of analytical laboratory error. Dichloro-difluoromethane (Freon-12) was the only VOC detected in the Pit 2 area wells (one time detection of $0.6 \mu \mathrm{g} / \mathrm{L}$ at well $\mathrm{K} 2-01 \mathrm{C}$; detection level of $0.5 \mu \mathrm{g} / \mathrm{L})$.

Although chloroform $(0.9 \mu \mathrm{g} / \mathrm{L}$; detection level of $0.5 \mu \mathrm{g} / \mathrm{L})$ was detected in well K8-01, this common laboratory contaminant was detected only once. Trichloroethene (TCE) was detected at concentrations as high as $6 \mu \mathrm{g} / \mathrm{L}$ (MCL of $5 \mu \mathrm{g} / \mathrm{L}$, Table C-6) but was otherwise below the MCL. Additional characterization of the ground water in this vicinity is being conducted as part of the Site 300 TCE investigations (Webster-Scholten 1993).

Pit 6: Arsenic, iron, and selenium vere observed in Pit 6 wells at concentrations consistent with those of natural constituents of the area g ound water (Buddemier et al. 1987, Carlsen et al. 1987).

Pit 7 Complex: Samples from Pit 7 Complex wells were analyzed for four metals: barium, beryllium, lead, and vanadium. In 1992, all four metals were observed at background concentrations (Table C-5).

TCE continued to be observed in wells K7-01 and K7-03 at concentrations near or exceeding the MCL. Also 1,1-dichloroethene was detected in samples 
from wells K7-01 and K7-03. The Pit 7 Complex ground water investigation of TCE is discussed in the LLNL SWRI (Webster-Scholten 1993). There were firsttime and one-time detections of 1,1,1-TCA and chloroform in well NC7-26 $(1.2 \mu \mathrm{g} / \mathrm{L}$ and $0.6 \mu \mathrm{g} / \mathrm{L}$, respectively; detection limit of $0.5 \mu \mathrm{g} / \mathrm{L})$; and a one-time detection of $0.9 \mu \mathrm{g} / \mathrm{L}$ of trichlorofluoromethane (Freon-11; detection limit of $0.5 \mu \mathrm{g} / \mathrm{L}$ ) in NC7-48. Subsequent samples did not confirm these three observations; thus, they may be the result of analytical laboratory error. No other VOCs were detected in any other Pit 7 ground water protection monitoring well samples (Table C-4).

Pit 8: Chromium was the most commonly detected metal from well K8-01. The average concentrations $(0.024 \mathrm{mg} / \mathrm{L})$ were one-half the MCL $(0.05 \mathrm{mg} / \mathrm{L})$. Nitrate plus nitrite (as nitrate) was detected at an average concentration of $9 \mathrm{mg} / \mathrm{L}$, just below the MCL of $10 \mathrm{mg} / \mathrm{L}$. Similarly, higher values of nitrate plus nitrite (as nitrogen) were detected, at an average concentration of $40 \mathrm{mg} / \mathrm{L}$, below the MCL of $45 \mathrm{mg} / \mathrm{L}$. The nitrate levels likely occur naturally in the ground water near Pit 8 , as these results are consistent with historical values for this location (Table C-6). As in Pit 2, these observations are considered consistent with natural constituents of the area ground water (Buddemeier et al. 1987, Carlsen et al. 1987).

HE Surface Impoundments: Elevated levels of nitrate, selenium, and arsenic, all exceeding the corresponding drinking water MCL, have been consistently detected in the wells of this area since the wells were installed in 1985. These observations are considered to be natural constituents of the area ground water (Carlsen et al. 1987). There were also observations, at or near the detection limit, of chromium, iron, vanadium, zinc, and lead (Table C-7).

During 1992, TCE continued to be observed in ground water samples collected from three of the four wells downgradient of the B817 surface impoundments. For the fourth downgradient well (W-817-02), TCE had been detected in a fourth quarter 1991 ground water sample. However, TCE was not detected in any of the four ground water samples collected in 1992. The TCE breakdown product cis-1,2-dichloroethene was again observed throughout 1992 in well W-817-03A (Table C-7). Ongoing investigation of VOCs in the HE Process Area is being conducted by LLNL, and progress of this investigation has been described in the SWRI (Webster-Scholten 1993).

Except in samples collected in April 1992, RDX continued to be detected in concentrations from $<30 \mu \mathrm{g} / \mathrm{L}$ to $116 \mu \mathrm{g} / \mathrm{L}$ (Table C-6) in ground water samples collected from well W-817-01. Although HMX was reported in the second quarter 1991 in W-817-04, no HE compounds were detected in any ground water samples collected in 1992. HE compounds in the HE Process Area are being investigated as described in the SWRI (Webster-Scholten 1993). 
HE compounds in the B817 HE Process Area ground water are being investigated and will be discussed in Chapter 13 of the SWRI, to be submitted at a later date.

Pit 9: Concentrations of metals in the Pit 9 wells were within historical values, and no VOCs were detected (Table C-8).

\section{Environmental Impact}

\section{Livermore Site}

The primary environmental impact to ground water at the Livermore site is from contamination by nonradiological constituents of unsaturated sediments and ground water and is a result of past practices.

Nonradiological Impacts. Significant VOC concentrations (exceeding $1 \mathrm{ppm}$ ) in unsaturated sediment occur in five areas: the east traffic circle, the vicinity of Building 518, Eastgate Drive, the vicinity of Building 514, and the former East Taxi Strip in the southeastern portion of LLNL. At only two of these locations, the East Taxi Strip and Building 518, are VOC concentrations high enough to possibly affect ground water (Isherwood et al. 1991).

VOCs occur in large, diffuse, ground water plumes 9 to 30 meters thick, which underlie about $85 \%$ of the Livermore site (Figure 7-1). VOCs are seldom found below a depth of 60 meters, and the calculated volume of VOCs in ground water is less than 760 liters. VOCs have migrated off site in two areas, one about 750 meters west of Vasco Road under private property, and the second about 250 meters south of southeastern LLNL under DOE property at Sandia National Laboratories, California (SNL/CA). Investigations have determined that the ground water plume near Vasco Road appears to be migrating from the southwest corner of the site to the west-northwest, at a maximum rate of about 20 meters per year (Figure 7-1). Three California Water Service municipal supply wells are situated within 3 kilometers of this plume. Moving at the rate of 20 meters per year, this plume would reach these wells in about 150 year's if, in fact, the wells are in the direct flow path, which is questionable. Chemical and hydraulic data strongly indicate that a TCE plume identified northwest of LLI JL originates on private property (Figure 7-1). That plume currently is under investigation by the RWQCB and property owners in that area. For more detailed information, see Iovenitti et al. (1991).

Detailed geologic and chemical mapping of the Gasoline Spill Area shows that the highest concentrations of fuel hydrocarbons (FHCs) in the unsaturated sediments (above $10 \mathrm{ppm}$ ) are mainly restricted to the immediate spill point, within a radius of approximately 15 meters, and decrease rapidly away from the spill senter. The FHCs appear to have moved vertically downward through the vadose zone, spreading laterally immediately upon entering the water table. 
Petroleum hydrocarbons (as gasoline concentrations exceeding $10 \mathrm{ppm}$ in ground water) form a north-south trending, oval-shaped plume, which extends approximately 30 meters away from the spill center along its long axis. Benzene concentrations above the $0.001-\mathrm{ppm} \mathrm{MCL}$ level are restricted to an area within 90 meters of the leak point. FHCs in ground water are not present below about 50 meters. The only other FHC reported in concentrations exceeding an MCL is ethylene dibromide, which recent analyses suggest is present in five monitoring wells in the immediate vicinity of the gasoline spill point.

Remediation. Treatment Facility A (TFA) treated more than 71 million liters of ground water during 1992, removing and destroying approximately 10 kilograms (6.3 liters) of VOCs. About 200 million liters of ground water have been treated since TFA began operating. Treatment Facility B (TFB) treated about 9.4 million liters of water in 1992, removing and destroying approximately 1.5 kilograms ( 0.9 liter) of VOCs from ground water. More than 15 million liters have been treated since TFB began operating.

Construction activities for Treatment Facility F (TFF), the treatment system for the steam injection/ground water and vapor extraction experiments, occurred at the Gasoline Spill Area throughout 1992. TFF will support the DOEsponsored Dynamic Underground Stripping Project as well as sediment and ground water remediation. Remediation operations at TFF during 1992 were limited to four 10-hour soil vapor extraction tests conducted between August and December. Vapor extraction occurred for a total of about 40 hours at a cornbined vapor extraction rate of about 50 standard cubic feet per minute (scfm). Tc.tal fuel hydrocarbon concentrations measured in extracted air ranged from about 4000 to 6000 parts per million by volume (ppmv), resulting in a new fuel hydrocarbon removal in 1992 of about 76 liters of gasoline.

Radiological Impacts. The most significant site of radiological contamination involves tritium in ground water beneath the former East Taxi Strip area. The migration of tritium from this area has been evaluated, and the tritium is expected to decay to levels below the MCL (or to background) long before affecting any drinking water supplies. Therefore, impact to human health is expected to be minimal.

Tritium has not been detected above $37 \mathrm{~Bq} / \mathrm{L}(1000 \mathrm{pCi} / \mathrm{L})$ in off-site wells. The natural half-life for tritium (12.3 years) and the slow movement of ground water ensure that the observed tritium will have decayed to well below the MCL long before migrating off site (Isherwood et al. 1991).

\section{Site 300}

The primary environmental impacts to Site 300 ground water arise from contamination from past practices that has reached the ground water in perched aquifers beneath disposal sites, rinse water lagoons, and buildings; and the low 
concentration of TCE in ground water extending off site south and northeast of Site 300 along Corral Hollow Creek.

Nonradiological Impacts. TCE is present in soil, sediment, or ground water at eleven locations at Site 300. The most significant areas of contamination include the Building 834 Complex, the HE Process Area, the General Services Area, Pit 6 Area, Pit 7 Complex, and the Building 865 Advanced Testing Accelerator upgradient of Pit 1. Following is an assessment of several areas that evidenced changes in the ground water concentration during 1992.

General Services Area: The General Services Area is located in the southeastern corner of Site 300 . Since 1982 LLNL has conducted an intensive investigation in the General Services Area and off-site areas to locate VOC release points and to define the vertical and horizontal distribution of VOCs, primarily TCE and PCE, in the sediment and ground water. Total VOC concentrations in excess of $1 \mathrm{ppb}$ extended about 1 kilometer off site from the eastern General Services Area along Corral Hollow Creek in 1991, but has since been partially remediated. From startup of the system in July 1991 through December 1992, approximately 33 million liters of TCE-containing ground water were treated.

Building 865 Area: Trending analysis conducted on the Freon-113 observations from Pit 1 wells indicated that concentrations are increasing rapidly (Figure 7-6) but are still well below the MCL. Pit 1 has no record of Freon-113 disposal and is not believed to be its source. Although these wells are upgradient (K1-05) and cross gradient (K1-08 and K1-09) from the Pit 1 landfill, they are all located downgradient from Building 865, the Advanced Test Accelerator, which will be investigated by LLNL. The SWRI (Webster-Scholten 1993) indicates that Freon-113 has been detected in ground water from several wells downgradient of Building 865 .

Pit 6: TCE concentrations in well EP6-09 have increased, with some fluctuation, since 1987. As shown in Figure 7-7, this pattern continued in 1992. Our experience suggests that as the main body of the TCE plume reaches this well, the concentration will level off. Tetrachloroethene (PCE) was detected for the first time in compliance well EP6-08 $(0.92 \mu \mathrm{g} / \mathrm{L} ; \mathrm{MCL}$ is $5 \mu \mathrm{g} / \mathrm{L})$, although it has been detected before in Pit 6 wells that are not part of the Pit 6 monitoring network. The compound 1,2-dichloroethane (1,2-DCA) was detected at $0.6 \mu \mathrm{g} / \mathrm{L}$ in well EP6-09 (Table C-4). No other VOCs were detected in the Pit 6 area. Ongoing investigation of VOCs in the ground water at Pit 6 are discussed in the SWRI (Webster-Scholten 1993).

Pit 7: As reported in Fischer et al. (1992b), TCE concentrations (average $11.0 \mu \mathrm{g} / \mathrm{L}$ range 10 to $12 \mu \mathrm{g} / \mathrm{L}, \mathrm{n}=4$ ) in $\mathrm{K} 7-01$, which already exceed the MCL, have been increasing at a rate of greater than $10 \%$ per year. 
HE Process Area: Trend analysis of TCE levels in HE surface impoundment wells indicate that TCE concentrations are decreasing in W-817-03A at about $10 \%$ per year $\left(F_{(1,21)}=7.15, p<0.01, r^{2}=0.25\right)$ (Figure 7-8).

Building 834 Complex: Remediation has begun at the Building 834 Complex, where about 300 kilograms of TCE have been removed from the unsaturated sediment and ground water by extraction and treatment.

Radiological Impacts. Two areas exhibit significant impact to ground water from tritium contamination. These are the Pit 7 Complex and the area of Building 850, Doall Road, and Elk Ravine. Computer modeling indicates that by the time the tritiated water from sites of known ground water contamination reaches the Site 300 boundary, the tritium will have decayed to near or at background activities. LLNL has submitted draft RI/FS reports of the tritium investigation to the regulatory agencies.

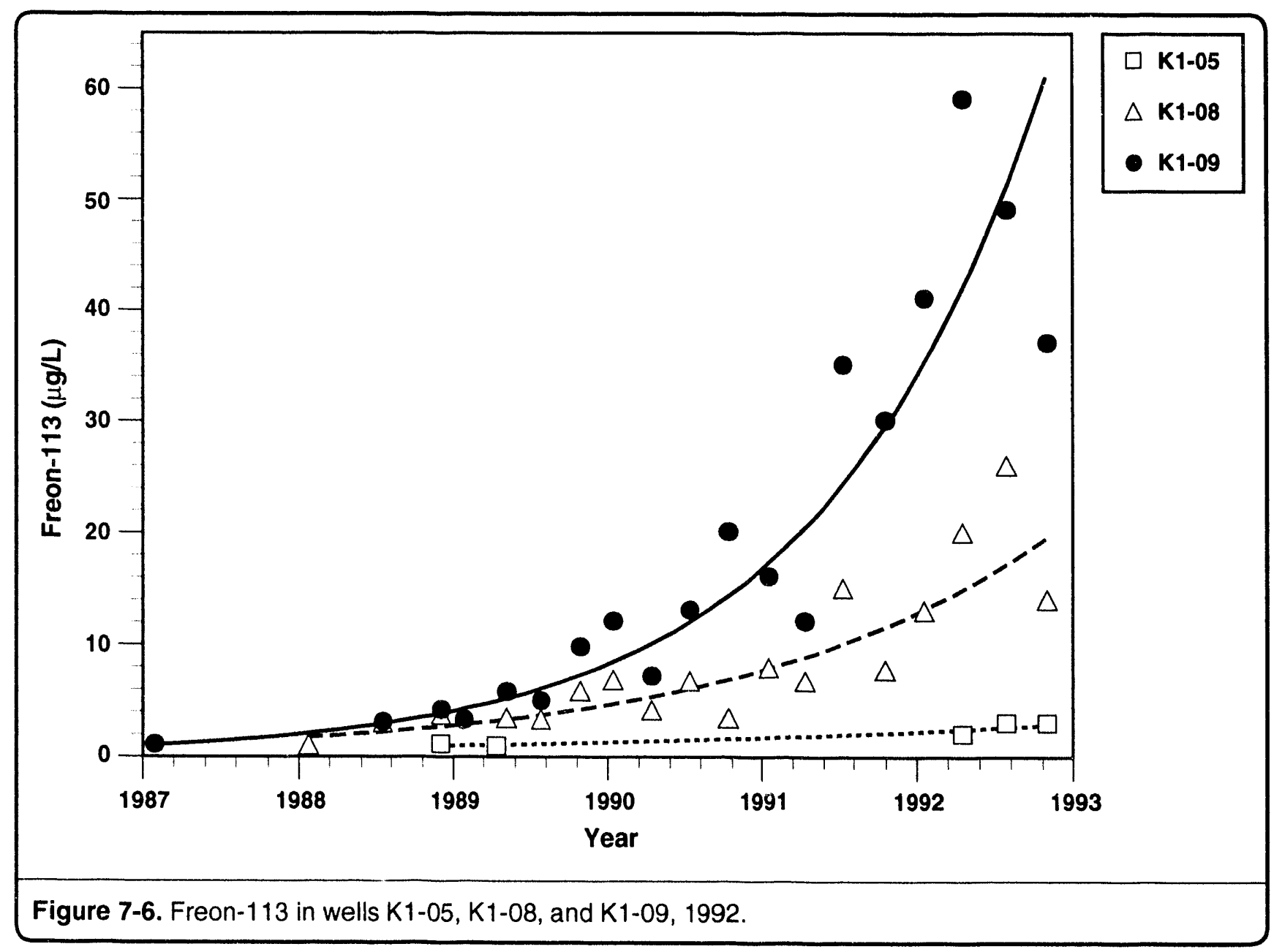


Details of the Remedial Investigation for the East and West Firing Areas Study Area are discussed in the SWRI (Webster-Scholten 1993). The SWRI indicates that at the Building 850 firing tables, tritium was released to the subsurface by percolation of rainfall runoff and dust control water. Tritium from the Building 850 area has recently migrated to the area of Pit 1 . Tritium was released to ground water from Pits 3 and 5 in the Pit 7 Complex, due to heavy rains in $1982-83$ and the resulting rising water table.

The highest tritium activity $[84.4 \mathrm{~Bq} / \mathrm{L}(2279 \mathrm{pCi} / \mathrm{L})]$ observed in a Pit 1 well (K1-02B) increased from the value observed in 1991 [51.4 Bq/L $(1388 \mathrm{pCi} / \mathrm{L})]$. This increase results from the arrival of the leading edge of the ground water tritium plume emanating from Building 850 (Figure 7-4).

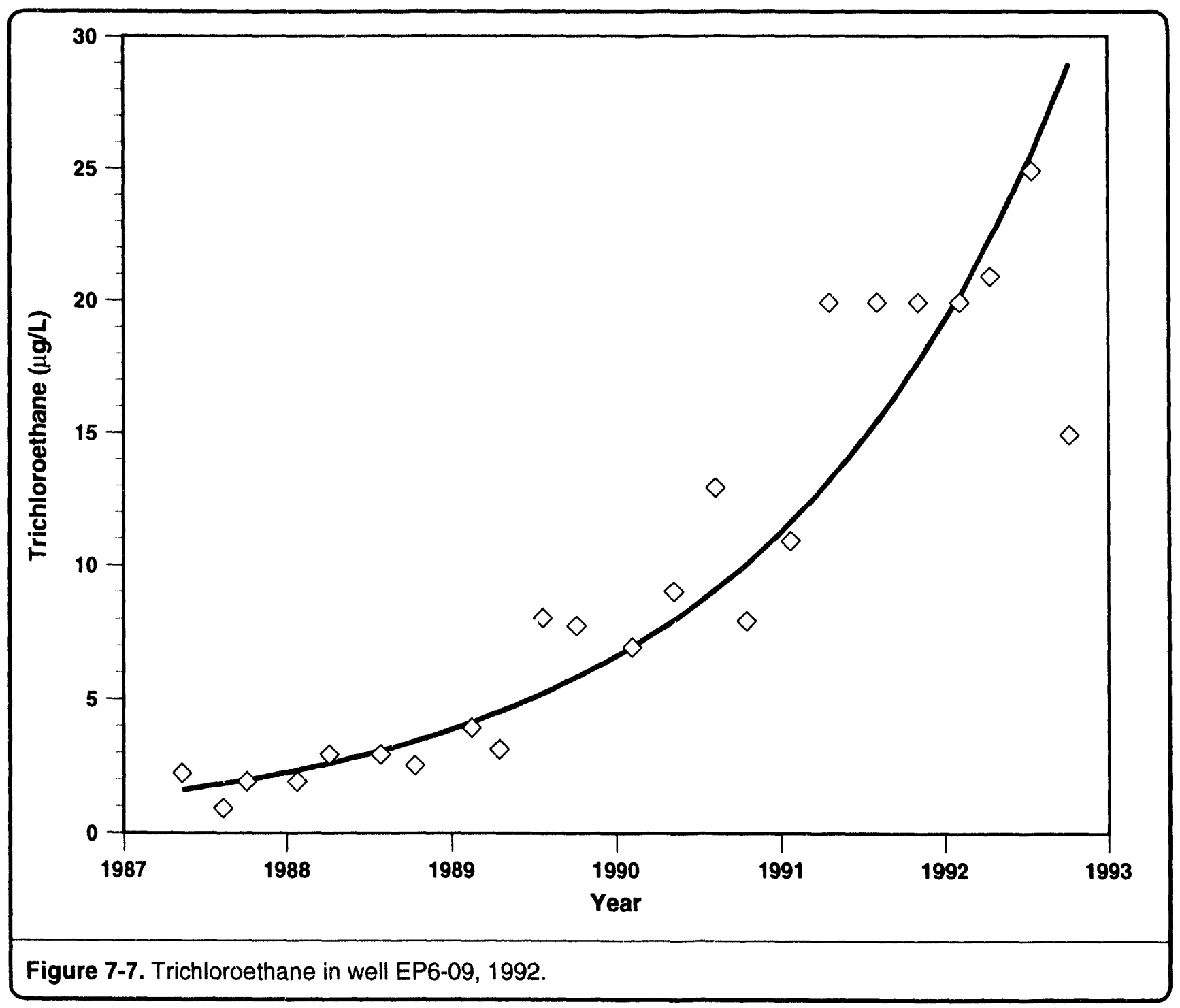


We performed a trend analysis of tritium activities in well K1-02B (Figure 7-9). The estimated model fit to the data is statistically significant $(p=0.0001)$. These estimates indicate that tritium activity levels at well K1-02B are increasing by about $40 \%$ per year, or doubling about every 23 months. We anticipate that this trend will level off when the main body of the plume arrives at $\mathrm{K} 1-02 \mathrm{~B}$.

Pit 2: The Building 850 tritium plume also appears to be impacting ground water at Pit 2. Average tritium values for Pit 2 wells increased from those observed during 1991 as a result of the Building 850 tritium plume, but did not exceed $70 \%$ of the EPA and DTSC MCLs.

Pit 7: We do not believe that the elevated uranium levels seen in Pit 7 wells are the result of contamination. Naturally occurring uranium nominally contains $99.2830 \%$ by weight ${ }^{238} \mathrm{U}, 0.7110 \%{ }^{235} \mathrm{U}$, and $0.0054 \%{ }^{234} \mathrm{U}$. Studies show that the percentage weight of ${ }^{235} \mathrm{U}$ in natural uranium varies by as much as $0.1 \%$,

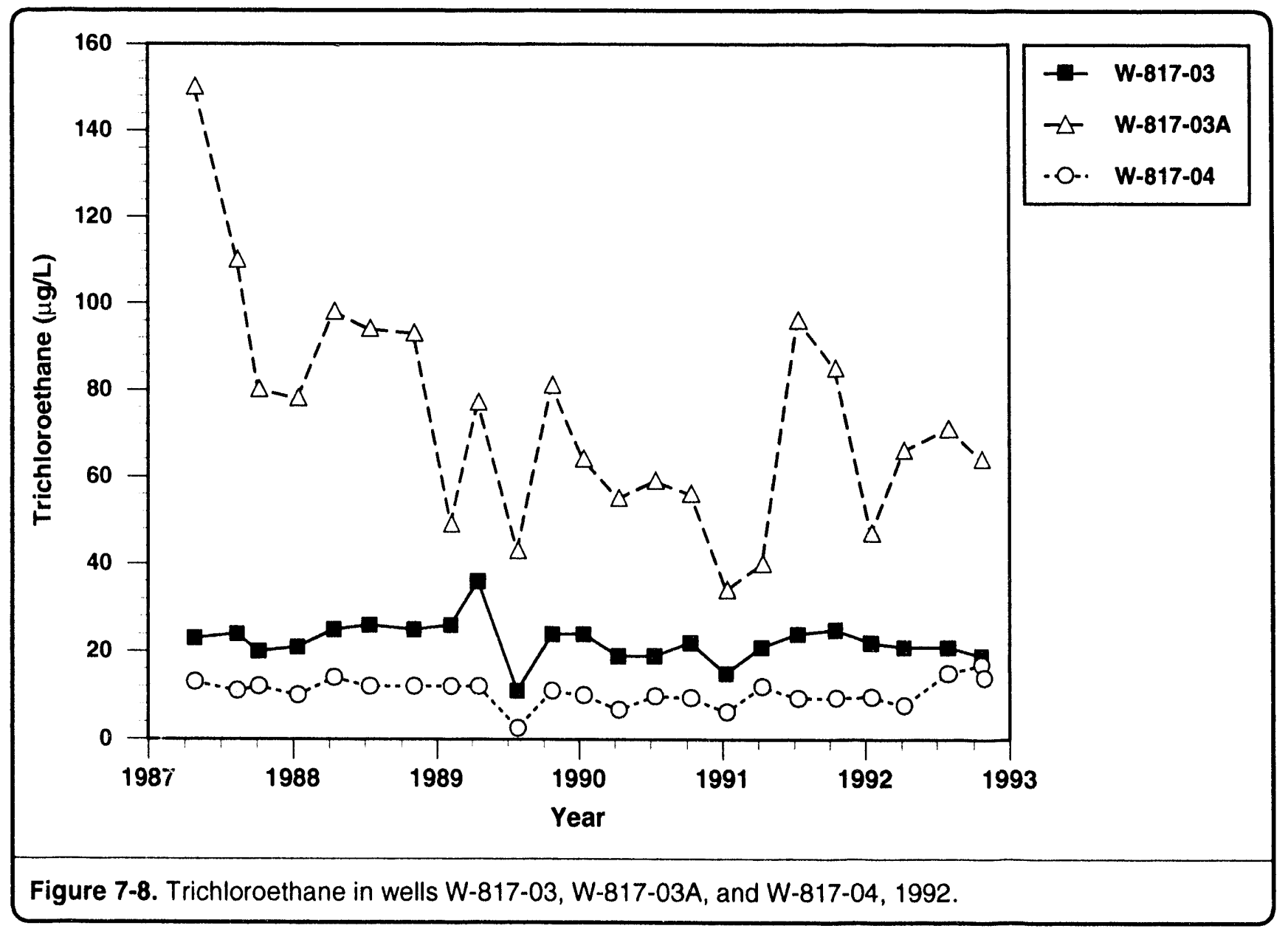


depending on the source (Weast et al. 1982). Adding the $0.1 \%$ variation, we expect a range of 0.611 to $0.811 \% 235 \mathrm{U}$ in natural uranium, and consider a value less than $0.611 \%$ indicative of depleted uranium. Our analysis of uranium ${ }^{235} \mathrm{U}$ ratio of $0.814 \%$, very close to the upper range expected from natural uranium, and well above that of depleted uranium.

\section{Summary}

The primary environmental impacts from Livermore site and Site 300 ground water arise from contaminants released by past practices affecting ground water aquifers beneath disposal sites, rinse water lagoons, and buildings. Although concentrations of contaminants at some localized areas are high, modeling has shown that this contamination is unlikely to migrate to any extent that would significantly affect human health at any on-site or off-site location.

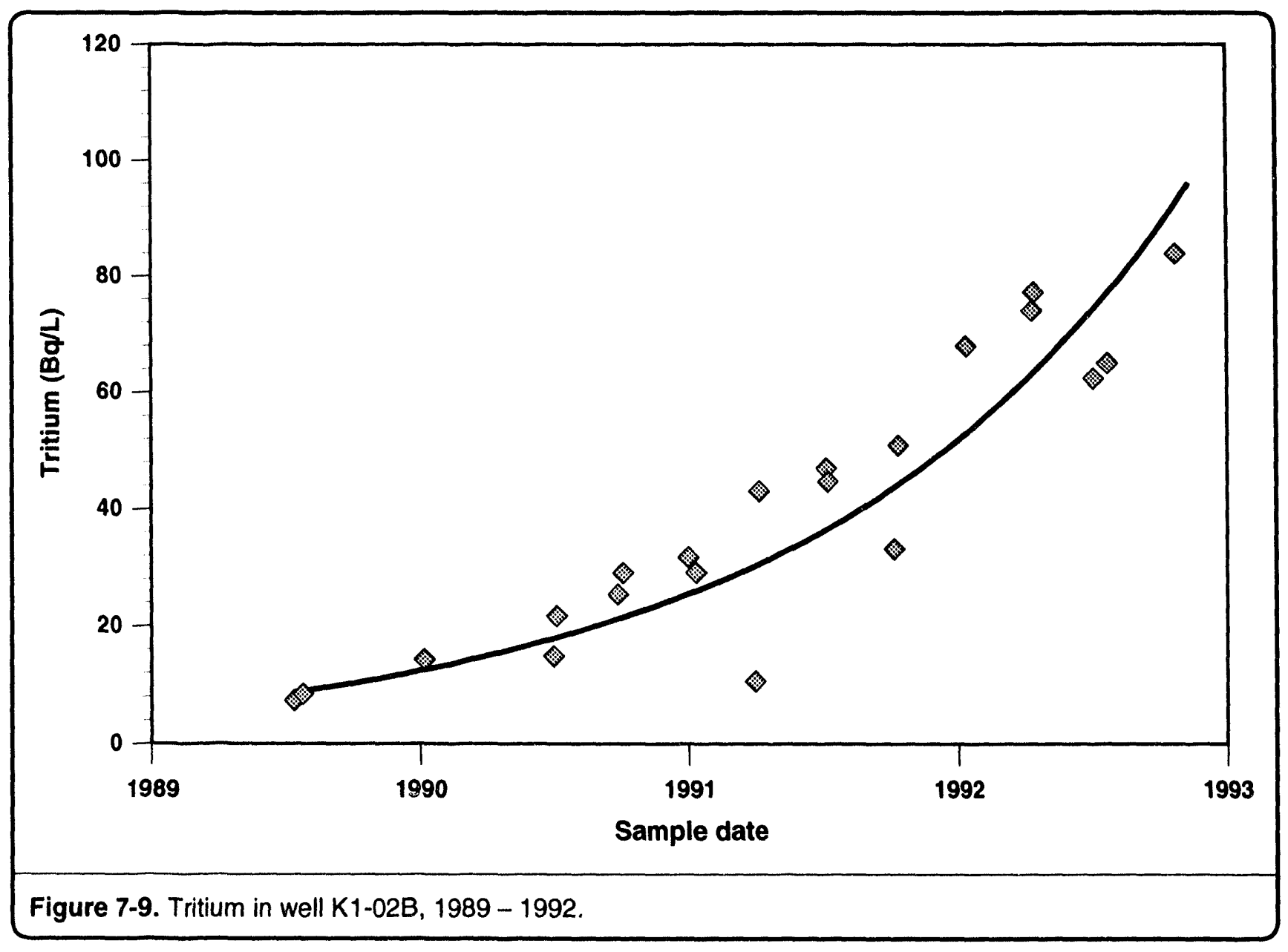


At the Livermore site, areas of contamination are well known, and either active remediation is under way, or modeling has shown that concentrations will drop to near background by the time plumes leave the site boundary.

The one area of off-site contamination at Site 300 is the low concentration of TCE in a ground water plume extending off site along Corral Hollow Creek. Remediation efforts are under way to mitigate any potential impacts of this plume in the eastern General Services Area and off-site concentrations have significantly decreased. Detections of TCE in off-site ground water production wells are discussed in Chapter 6 on Surveillance Water Monitoring.

In summary, during 1992 there were no significant impacts to the environment or public health arising from ground water contamination at the Livermore site or Site 300 , nor any indication of recent releases affecting ground water. 
Table 7-1. Water quality parameters and detection limits for monitoring wells, Site 300, 1992.

\begin{tabular}{|c|c|c|}
\hline Parameter & Pitsa & Detection limitb \\
\hline Physical and chemical & & (mg/L) \\
\hline Aluminum & 8 & 0.2 \\
\hline Ammonia nitrogen (as N) & $1,2,6,8,9$ & 0.02 \\
\hline Antimony & $\mathrm{HE}$ & 0.2 \\
\hline Arsenic & $1,6,8,9, \mathrm{HE}$ & 0.002 \\
\hline Barium & $1,6,7,8,9, \mathrm{HE}$ & 0.05 \\
\hline Beryllium & $1,2,6,7,8,9, \mathrm{HE}$ & 0.0005 \\
\hline Bicarbonate alkalinity (as $\mathrm{CaCO}_{3}$ ) & $1,6,8,9, \mathrm{HE}$ & $-c$ \\
\hline Cadmium & $1,6,8,9, \mathrm{HE}$ & 0.0005 \\
\hline Calcium & $1,9, \mathrm{HE}$ & 0.5 \\
\hline Carbonate alkalinity (as $\mathrm{CaCO}_{3}$ ) & $1,6,8,9, \mathrm{HE}$ & $-c$ \\
\hline Chloride & $1,6,8,9, \mathrm{HE}$ & 1 \\
\hline Chromium & $1,6,8,9, \mathrm{HE}$ & 0.005 \\
\hline Cobalt & HE & 0.05 \\
\hline Conductivity ( $\mu \mathrm{mhos} / \mathrm{cm}$ ) & $1,6,8,9, \mathrm{HE}$ & $-c$ \\
\hline Copper & $1,9, \mathrm{HE}$ & 0.05 \\
\hline Filterable residue (TDS) & $1,6,8,9, \mathrm{HE}$ & 10 \\
\hline Fluoride & $1,6,8,9$ & 0.1 \\
\hline Hydroxide alkalinity (as $\mathrm{CaCO}_{3}$ ) & $1,6,8,9, \mathrm{HE}$ & $-c$ \\
\hline Iron & 1,6. HE & 0.1 \\
\hline Lead & $1,2,6,7,8,9, \mathrm{HE}$ & 0.002 \\
\hline Magnesium & $1,9, \mathrm{HE}$ & 0.5 \\
\hline Manganese & $1,6,9, \mathrm{HE}$ & 0.05 \\
\hline Mercury & $1,6,8,9, \mathrm{HE}$ & 0.0002 \\
\hline Molybdenum & $\mathrm{HE}$ & 0.1 \\
\hline Nickel & $1,6,9, \mathrm{HE}$ & 0.005 \\
\hline Nitrate (as $\left.\mathrm{NO}_{3}\right)$ & $1,2,6,8,9, \mathrm{HE}$ & 0.4 \\
\hline Nitrate (as N) & $1,2,6,8,9, \mathrm{HE}$ & 0.1 \\
\hline Nitrite (as N) & $1,2,6,8,9, \mathrm{HE}$ & 0.01 \\
\hline Nitrite (as $\left.\mathrm{NO}_{2}\right)$ & $1,2,6,8,9, \mathrm{HE}$ & 0.03 \\
\hline Nonfilterable residue (TSS) & $1,6,9$ & 1 \\
\hline $\mathrm{pH}$ (units) & $1,6,8,9, \mathrm{HE}$ & $-c$ \\
\hline Potassium & $1,9, \mathrm{HE}$ & 1 \\
\hline Selenium & $1,6,8,9, \mathrm{HE}$ & 0.002 \\
\hline Silver & $1,6,8,9, \mathrm{HE}$ & 0.0005 \\
\hline Sodium & $1,6,8,9, \mathrm{HE}$ & 5 \\
\hline Sulfate & $1,6,8,9, \mathrm{HE}$ & 1 \\
\hline Thallium & $\mathrm{HE}$ & 0.2 \\
\hline Total alkalinity (as $\mathrm{CaCO}_{3}$ ) & $1,6,8,9, \mathrm{HE}$ & $-c$ \\
\hline Total Kjeldahl nitrogen & $1,2,6,8,9$ & 0.5 \\
\hline Vanadium & $1,6,7,9, \mathrm{HE}$ & 0.05 \\
\hline Zinc & $1,9, \mathrm{HE}$ & 0.05 \\
\hline
\end{tabular}


Table 7-1. Water quality parameters and detection limits for monitoring wells, Site 300, 1992 (concluded).

\begin{tabular}{|c|c|c|}
\hline Parameter & Pitsa & Detection limitb \\
\hline Purgeable halocarbons (EPA Method 601) & & $(\mu g / L)$ \\
\hline All analytes & $1,2,6,7,8, \mathrm{HE}$ & 0.5 \\
\hline \multicolumn{3}{|l|}{ Title 22 Organics } \\
\hline 2,4,5-TP (Silvex) & $1,6,9$ & 1 \\
\hline $2,4-D$ & $1,6,9$ & 5 \\
\hline Endrin & $1,6,9$ & 0.05 \\
\hline Lindane & $1,6,9$ & 0.03 \\
\hline Methoxychlor & $1,6,9$ & 0.2 \\
\hline Toxaphene & $1,6,9$ & 0.5 \\
\hline \multicolumn{3}{|l|}{ Purgeable priority pollutants (EPA Method 624) } \\
\hline Acetone & $7,8,9$ & 5 \\
\hline Acrolein & $7,8,9$ & 5 \\
\hline Acrylonitrile & $7,8,9$ & 5 \\
\hline Methyl ethyl ketone & $7,8,9$ & 5 \\
\hline All other analytes & $7,8,9$ & 0.5 \\
\hline \multicolumn{3}{|l|}{ High explosives } \\
\hline HMX & $1,6,8,9, \mathrm{HE}$ & 20 \\
\hline RDX & $1,6,8,9, \mathrm{HE}$ & 30 \\
\hline TNT & $1,6,8,9, \mathrm{HE}$ & 30 \\
\hline Other organics & & $(\mathrm{mg} / \mathrm{L})$ \\
\hline Low-level phenolics & $1,6,8,9$ & 0.005 \\
\hline TOC & $1,6,8,9, \mathrm{HE}$ & 0.5 \\
\hline TOX & $1,6,8,9, \mathrm{HE}$ & 0.02 \\
\hline Radiological parameters & & $(\mathrm{Bq} / \mathrm{L})$ \\
\hline Gross alpha & $1,2,6,7,8,9$ & 0.064 \\
\hline Gross beta & $1,2,6,7,8,9$ & 0.058 \\
\hline Tritium & $1,2,6,7,8,9, \mathrm{HE}$ & 0.062 \\
\hline Uranium-238 & $1,6,7,8,9$ & 0.0006 \\
\hline Uranium-235 & $1,6,7,8,9$ & 0.0005 \\
\hline Uranium-233, -234 & $1,6,7,8,9$ & 0.0004 \\
\hline Radium-226 & $1,6,7,8,9$ & 0.004 \\
\hline
\end{tabular}

a $\mathrm{HE}=$ High Explosives Process-Water Surface Impoundment wells.

b Detection limits may vary from sample to sample depending on such items as matrix interference, instrument sensitivity, or requested method. The limits shown are the lowest detection limits reported.

c Not applicable. 
Table 7-2. Parameters sampled in Pits 1 and 2 Barcad samplers, Site 300, 1992.

\begin{tabular}{|c|c|c|c|c|}
\hline \multirow[b]{2}{*}{ Parameter } & \multicolumn{4}{|c|}{ Barcad sampler } \\
\hline & K1-01A & K1-01B & K1-02A & K2-01A \\
\hline \multicolumn{5}{|c|}{ Physical and chemical (mg/L) } \\
\hline Arsenic & 0.0050 & 0.0100 & 0.0120 & $<0.0020$ \\
\hline Barium & 3 n.d. at $<0.0500$ & $\begin{array}{r}0.0600 \\
0.0500 \\
1 \text { n.d. at }<0.0500\end{array}$ & 4 n.d. at $<0.0500$ & 4 n.d. at $<0.0500$ \\
\hline Beryllium & 3 n.d. at $<0.0005$ & 3 n.d. at $<0.0005$ & $\begin{array}{l}3 \text { n.d. at }<0.0005 \\
1 \text { n.d. at }<0.0002\end{array}$ & $\begin{array}{l}3 \text { n.d. at }<0.0005 \\
1 \text { n.d. at }<0.0002\end{array}$ \\
\hline Cadmium & $<0.0005$ & $<0.0005$ & $<0.0005$ & $<0.0005$ \\
\hline Chromium & $<0.0050$ & $<0.0050$ & $<0.0050$ & $<0.0050$ \\
\hline Iron & $<0.1000$ & $<0.1000$ & $<0.1000$ & $<0.1000$ \\
\hline Lead & 2 n.d. at $<\begin{array}{r}0.0020 \\
0.0020\end{array}$ & 2 nd. at $<\begin{array}{r}0.0970 \\
0.0020\end{array}$ & 4 n.d. at $<0.0020$ & 4 n.d. at $<0.0020$ \\
\hline Manganese & $<0.0500$ & $<0.0500$ & $<0.0500$ & $<0.0500$ \\
\hline Mercury & $<0.0002$ & $<0.0002$ & $<0.0002$ & $<0.0002$ \\
\hline Nickel & $<0.1000$ & $<0.1000$ & $<0.1000$ & $<0.1000$ \\
\hline Selenium & $<0.0020$ & $<0.0020$ & $<0.0020$ & $<0.0020$ \\
\hline Silver & $<0.0005$ & $<0.0005$ & $<0.0005$ & $<0.0005$ \\
\hline Vanadium & $<0.0500$ & $<0.0500$ & $<0.0500$ & $<0.0500$ \\
\hline \multicolumn{5}{|c|}{ Radiological (Bq/L) } \\
\hline Gross alpha & $\begin{array}{r}0.036 \pm 0.095 \\
-0.006 \pm 0.101 \\
-0.009 \pm 0.087\end{array}$ & $\begin{array}{l}0.025 \pm 0.081 \\
0.003 \pm 0.079 \\
0.001 \pm 0.082\end{array}$ & $\begin{array}{r}0.0 \pm 0.076 \\
-0.031 \pm 0.096 \\
0.002 \pm 0.079 \\
0.023 \pm 0.089\end{array}$ & $\begin{array}{c}0.091 \pm 0.104 \\
0.013 \pm 0.057 \\
0.1 \pm 0.1 \\
0.061 \pm 0.108\end{array}$ \\
\hline Gross beta & $\begin{array}{r}0.206 \pm 0.071 \\
0.158 \pm 0.085 \\
0.0676 \pm 0.077\end{array}$ & $\begin{array}{l}0.055 \pm 0.062 \\
0.094 \pm 0.07 \\
0.077 \pm 0.072\end{array}$ & $\begin{array}{c}0.102 \pm 0.062 \\
0.15 \pm 0.07 \\
0.133 \pm 0.079 \\
0.14 \pm 0.05\end{array}$ & $\begin{array}{l}0.167 \pm 0.068 \\
0.089 \pm 0.064 \\
0.207 \pm 0.074 \\
0.153 \pm 0.068\end{array}$ \\
\hline Tritium & $\begin{array}{l}-0.46 \pm 3.42 \\
-1.47 \pm 3.35 \\
-2.07 \pm 2.35\end{array}$ & $\begin{array}{r}-1.32 \pm 3.38 \\
-0.40 \pm 3.37 \\
0.15 \pm 2.46\end{array}$ & $\begin{array}{r}-0.77 \pm 3.41 \\
0.74 \pm 3.35 \\
0.01 \pm 2.43 \\
3.81 \pm 4.48\end{array}$ & $\begin{array}{r}-0.22 \pm 3.43 \\
2.01 \pm 3.50 \\
0.64 \pm 2.39 \\
2.29 \pm 3.89\end{array}$ \\
\hline Uranium-234 & $\begin{array}{l}0.023 \pm 0.004 \\
0.021 \pm 0.005 \\
0.041 \pm 0.008\end{array}$ & $\begin{array}{r}0 \pm 0.002 \\
0.002 \pm 0.002 \\
0.026 \pm 0.005\end{array}$ & $\begin{array}{l}0.011 \pm 0.003 \\
0.006 \pm 0.003 \\
0.017 \pm 0.004 \\
0.014 \pm 0.004\end{array}$ & $\begin{array}{l}0.005 \pm 0.002 \\
0.003 \pm 0.002 \\
0.033 \pm 0.007 \\
0.013 \pm 0.003\end{array}$ \\
\hline Uranium-235 & $\begin{aligned} 0.0002 & \pm 0.0009 \\
0.001 & \pm 0.001 \\
0.0004 & \pm 0.0008\end{aligned}$ & $\begin{aligned} 0 & \pm 0.001 \\
0 & \pm 0.001 \\
0.0003 & \pm 0.0006\end{aligned}$ & $\begin{aligned} 0.0002 & \pm 0.0005 \\
0.001 & \pm 0.001 \\
0 & \pm 0.0006 \\
0 & \pm 0.001\end{aligned}$ & $\begin{array}{c}0 \pm 0.0007 \\
0 \pm 0.001 \\
0.001 \pm 0.002 \\
0.001 \pm 0.001\end{array}$ \\
\hline Uranium-238 & $\begin{array}{r}0.01 \pm 0.003 \\
0.011 \pm 0.003 \\
0.008 \pm 0.003\end{array}$ & $\begin{array}{r}0 \pm 0.001 \\
0.001 \pm 0.001 \\
0.001 \pm 0.001\end{array}$ & $\begin{array}{l}0.006 \pm 0.002 \\
0.003 \pm 0.002 \\
0.006 \pm 0.003 \\
0.007 \pm 0.003\end{array}$ & $\begin{array}{l}0.006 \pm 0.002 \\
0.001 \pm 0.001 \\
0.016 \pm 0.005 \\
0.007 \pm 0.002\end{array}$ \\
\hline Radium-226 & $\begin{aligned} 0.010 & \pm 0.019 \\
0.0035 & \pm 0.02 \\
0 & \pm 0.037\end{aligned}$ & $\begin{array}{r}-0.011 \pm 0.018 \\
-0.009 \pm 0.024 \\
0.021 \pm 0.024\end{array}$ & $\begin{aligned} 0 & \pm 0.016 \\
-0.006 & \pm 0.026 \\
0.014 & \pm 0.014 \\
0.0017 & \pm 0.017\end{aligned}$ & $\begin{array}{c}0.01 \pm 0.02 \\
0.018 \pm 0.015 \\
0.002 \pm 0.022 \\
0.008 \pm 0.014\end{array}$ \\
\hline
\end{tabular}


Table 7-2. Parameters sampled in Pits 1 and 2 Barcad samplers, Site 300, 1992 (concluded).

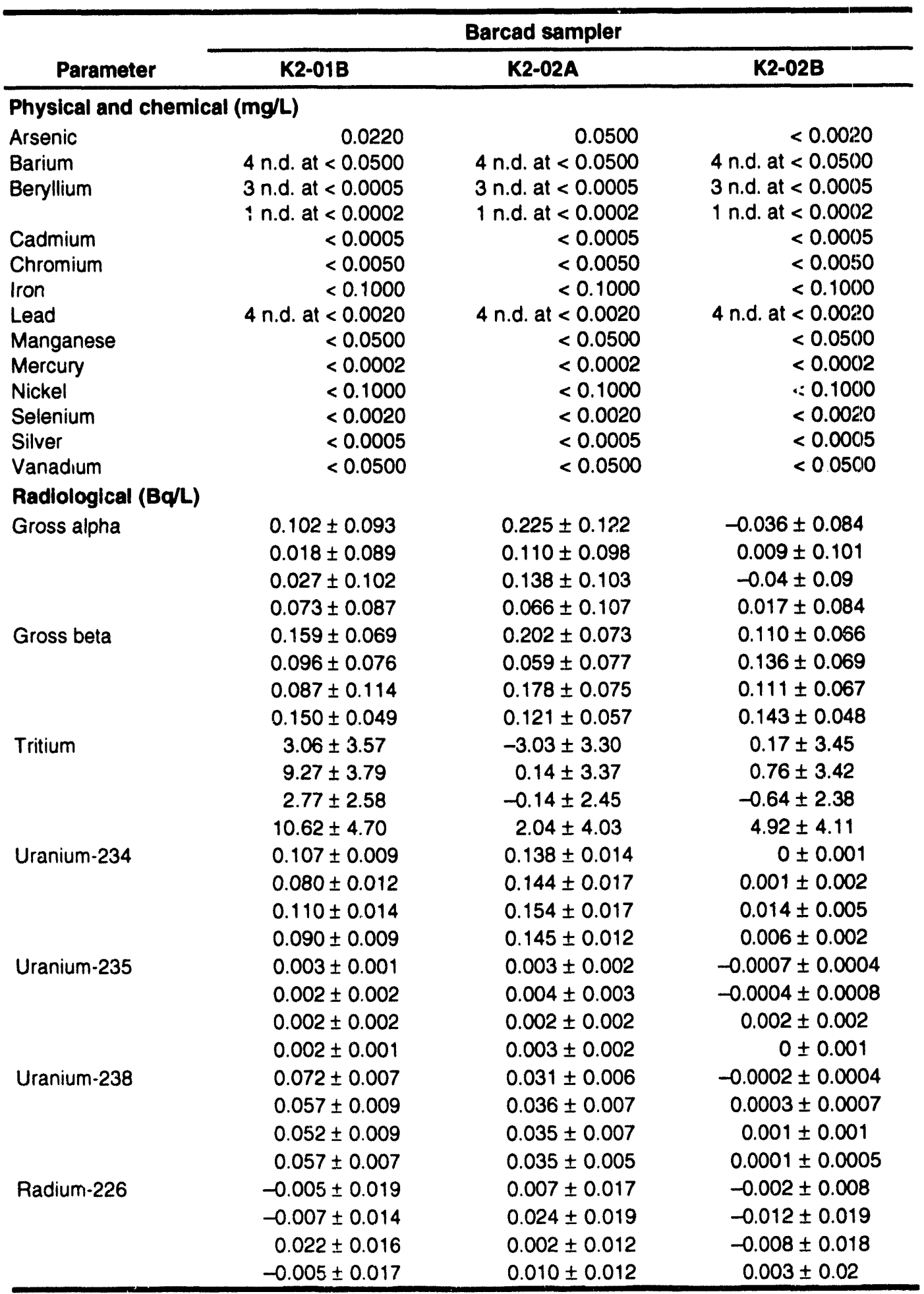

Note: The abbreviation "n.d." stands for nondelection. 


\section{Introduction}

Soil, defined as weathered materials near the surface of the earth, suitable for the growth of plants, is an integrating medium that can contain pollutants originally released directly to the ground, to the air, or through liquid effluents. The U.S. Department of Energy guidance, on which much of LLNL environmental monitoring is based (U.S. Department of Energy 1991), states that "periodic sampling and analysis of indicator materials, such as soil...should be performed to determine if there is measurable long-term buildup of radionuclides in the terrestrial environment. ... Soil sampling and analysis should be used to evaluate the long-term accumulation trends and to estimate environmental radionuclide inventories." The guidance specifies that nuclides in use at the facility, as well as naturally occurring nuclides, should be monitored. In particular, the guidance states that "it is desirable to assess, document, and periodically reassess the distribution and fate of radionuclides in the environment, especially plutonium in soil samples." Particulate radionuclides are of major interest in the LLNL soil monitoring program because airborne particulate releases are the most likely potential pathway for LLNL-induced soil contamination.

Sediments are defined as finely divided solid materials that have settled out of a liquid stream or standing water. In a geologic sense, the top 1000 meters or more at LLNL is sediment. To evaluate current conditions, LLNL samples stream sediments in currently active drainages. Sedimentary materials also can accumulate cuntaminants. According to U.S. Department of Energy (1991), "the sampling of sedimentary material from streams or ponds can provide an indication of the accumulation of undissolved radionuclides in the aquatic environment. The accumulation of radioactive materials in sediment can lead to exposure of humans through ingestion of aquatic species, through sediment resuspension into drinking water supplies, or as an external radiation source... ." U.S. Department of Energy (1991) also states that sampling and analysis of sediments is a more sensitive indicator of waterborne radionuclides than direct water sampling because in sedimentary material there is the potential for the accumulation of contaminants over a period of time. Sediments also have the potential to concentrate nonradioactive constituents, such as metals and organic compounds, that are present in storm water runoff. Stream sediment sampling, followed by radiological and chemical analyses, helps LLNL determine if 
significant buildups of radioactive or nonradioactive pollutants have occurred in nearby arroyos and other drainage areas.

Since 1971, surface soil sampling in the vicinity of LLNL and Site 300 has been part of a continuing LLNL monitoring program designed to measure any changes in environmental levels of radioactivity and to evaluate any increase in radioactivity that might have resulted from LLNL operations. Similarly, sediment samples have been collected from selected arroyos and other drainage areas at and around LLNL since 1988; these locations largely coincide with selected storm water sampling locations. Analyses have been conducted on both radioactive and nonradioactive constituents of sediment samples every year since the inception of sediment monitoring.

Because high-explosive tests at Site 300 occasionally involve the use of depleted uranium $\left({ }^{238} \mathrm{U}\right)$, one purpose of the annual soil sampling is to determine if these tests perturb the natural uranium content of the soil. The inclusion of the other naturally occurring nuclides $\left({ }^{40} \mathrm{~K},{ }^{226} \mathrm{Ra},{ }^{228} \mathrm{Ra},{ }^{232} \mathrm{Th}\right.$, and ${ }^{235} \mathrm{U}$ ) and the long-lived fission product ${ }^{137} \mathrm{Cs}$ provides background information and baseline data on global fallout. In 1991, LLNL began analyzing surface soil samples for beryllium, a potentially toxic metal used at both the Livermore site and Site 300.

Location maps for soil and sediment sampling conducted during 1992 are provided in Figures 8-1 through 8-3. LLNL established soil sampling locations in all possible directions around the Livermore site, included areas selected to represent background concentrations (distant locations unlikely to be affected by LLNL operations), and included areas with known contaminants, such as plutonium at the Livermore Water Reclamation Plant (LWRP). In general, Site 300 soil sampling locations were established around firing tables and other areas of potential soil contamination. Stream sediment sampling locations were chosen to coincide with major LLNL storm water drainages. All soil and sediment sampling locations have permanent location markers for reference and are suitable for emergency response applications, depending on the nature of the emergency incident. Other locations can be selected and sampled using existing written procedures published with the Environmental Monitoring Plan (Gallegos et al. 1992b). It is unlikely that LLNL operations could have any large-scale impacts on soil and sediment that would not be detected by the current monitoring networks.

The soil and sediment monitoring networks are subject to continuing evaluation and reassessment. Changes and additions to the soil and sediment monitoring networks since 1988 are evidence of an LLNL goal to monitor more adequately the soils and sediments that potentially could be affected by LLNL operations. Soil and sediment monitoring at LLNL has increased to meet increasing regulatory requirements and self-imposed monitoring and assessment goals. 


\section{Methods}

\section{Soils}

Prior to 1988, soil samples were collected at sites selected at random from Livermore Valley locations previously sampled for a 1971-1972 study. That earlier study was conducted to determine background concentrations of radionuclides in area soils. In 1988, Livermore Valley surveillance soil sampling locations were chosen to coincide with air sampling locations or to give coverage to areas with contaminants from past incidents or of other special concern. In 1991, five additional soil sampling locations associated with air sampling locations were established. The 1992 soil samples were collected from the same locations as those in 1991. During 1992, soil samples were collected from the same fourteen Site 300 sampling locations as in 1990 and 1991. The use of constant sampling locations from year to year allows more meaningful trending of data.

Choices of soil sampling locations were based on the following criteria: (1) proximity to LLNL and

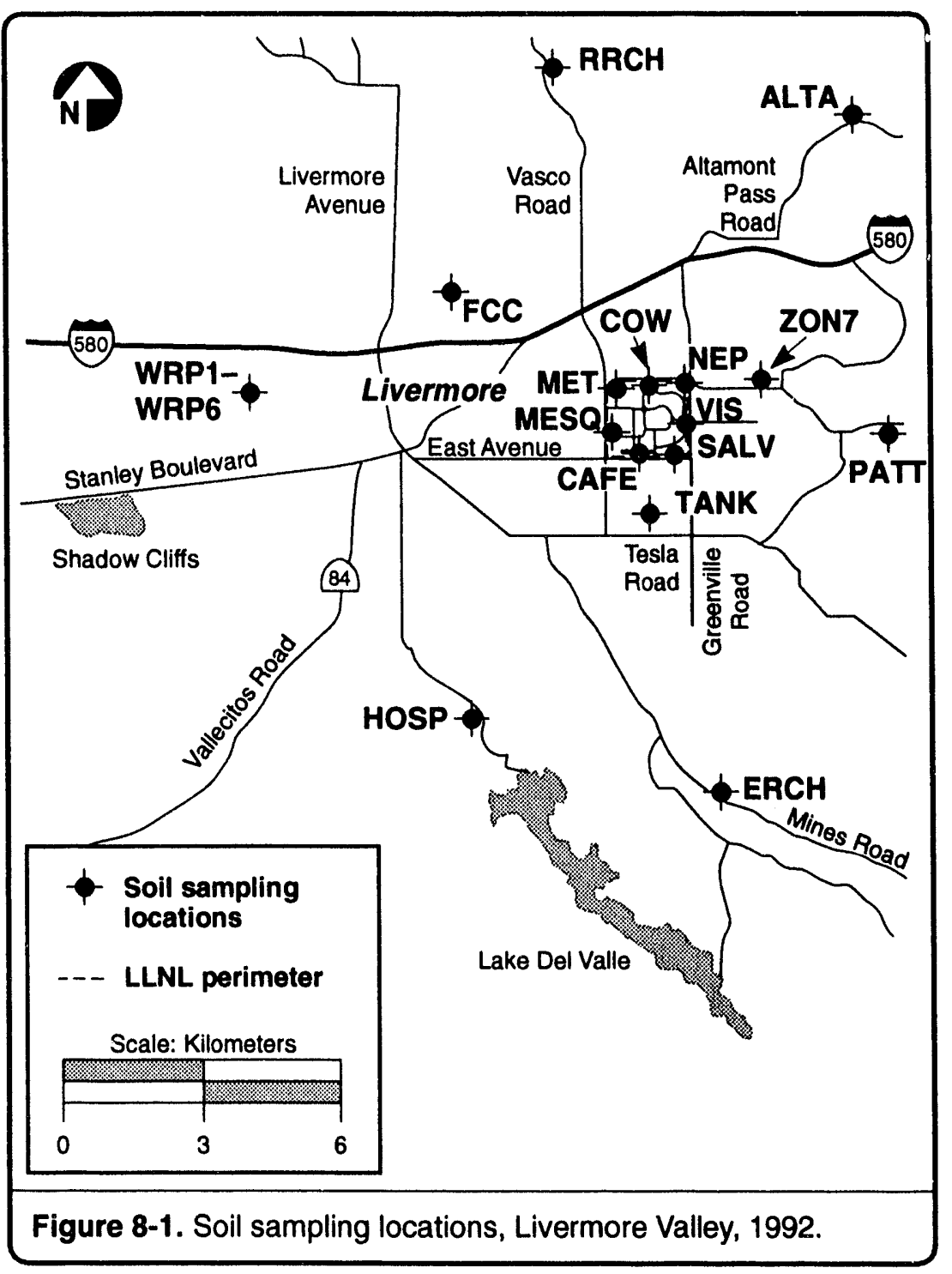
the potential for being affected by LLNL operations, (2) background locations with geologically similar substrates as those near LLNL, but unlikely to be affected by LLNL operations, and (3) areas of known or suspected LLNL-induced contamination. Additionally, soil sampling locations frequently were located near air monitoring locations, for comparative purposes; pollution released to the air is the most likely potential source for surface soil contaminants. Frequently tilled or disturbed areas, or locations near buildings or other obstructions, were avoided. Sampling locations were also chosen to avoid areas with unusual wind or precipitation influences. Other considerations included access during inclement weather and personnel safety in vehicle operation, vehicle parking, or sample collection.

Sampling locations at areas with known or suspected contaminants were monitored to delimit the extent of the contaminants and to track the contaminants from year to year. 


\section{Soil and Sediment Monitoring}

For example, six soil sampling locations were located near LWRP to monitor soils that contain elevated plutonium levels originating from a 1967 accidental release to the sewer.

Soil sampling is conducted according to written, standardized procedures contained in Gallegos et al. (1992b). Samples are collected from undisturbed areas in the proximity of the permanent sampling location marker. These areas generally are level, free of rocks, and are unsheltered by trees or buildings. The sampling technician chooses a one-meter square from which to collect the sample, and records how far away and in what direction from the permanent marker the sample is collected. Each sample is a composite consisting of five subsamples collected individually, with a core sampler, at the four corners and the center of the square.

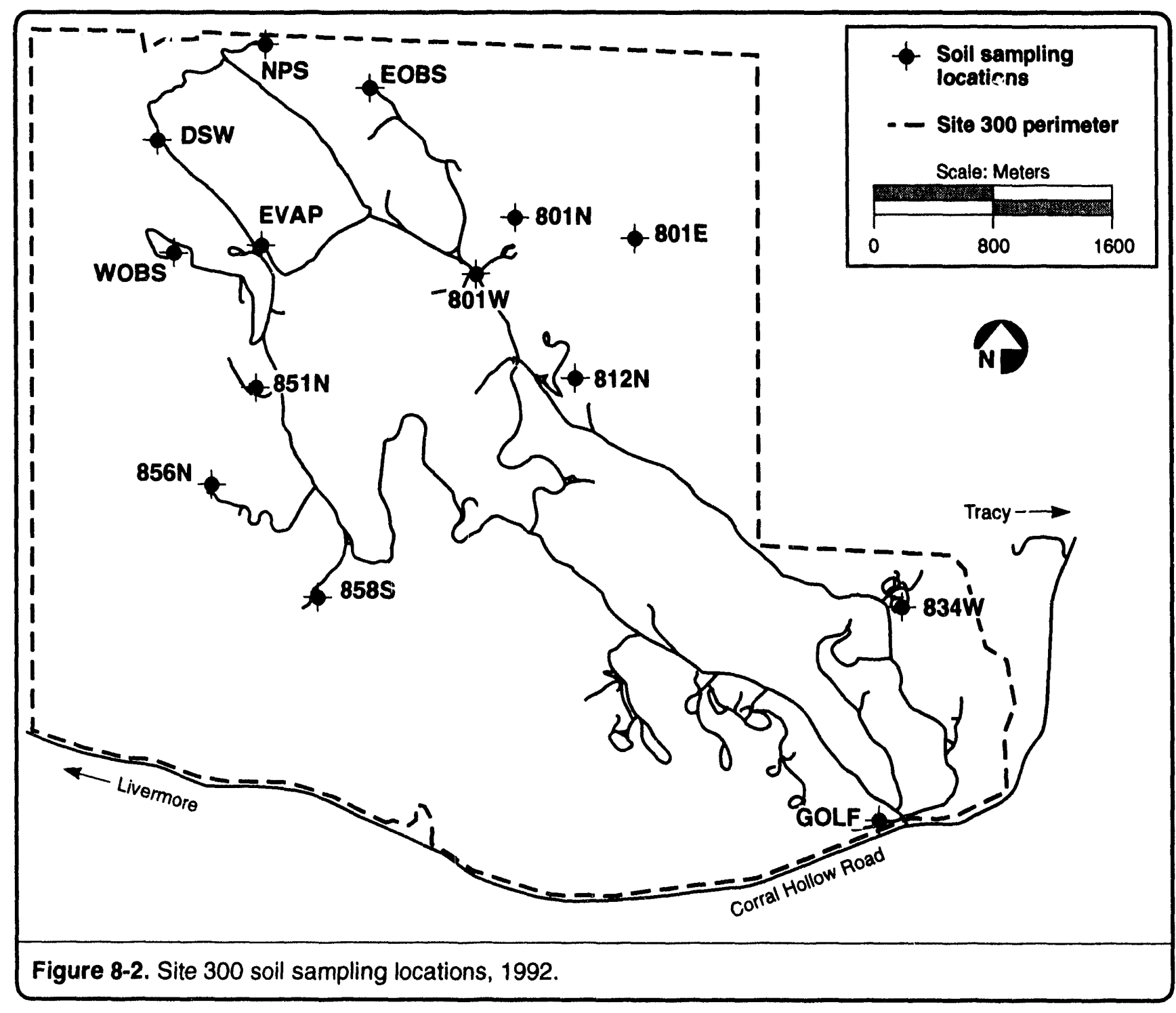


Surface vegetation is cleared away from the sampling area. Before handling any samples, sample colleztors put on clean, disposable, vinyl gloves so the sample does not become contaminated. A stainless steel core sampler $(8.25-\mathrm{cm}$ diameter), designed at LLNL, is driven into the ground to a depth of $5 \mathrm{~cm}$ for each subsample, and the five subsamples are composited in a plastic bag. All subsamples are collected from the top $5 \mathrm{~cm}$ of soil because surface deposition from the air is the primary pathway for potential contamination. An aliquot (approximately $10 \mathrm{~g}$ ) of soil is removed, placed in a plastic vial, and submitted for beryllium analysis. The remainder of the sample is double-bagged to avoid sample contamination, a sample identification tag is attached to the outside bag, and the sample is submitted for analyses.

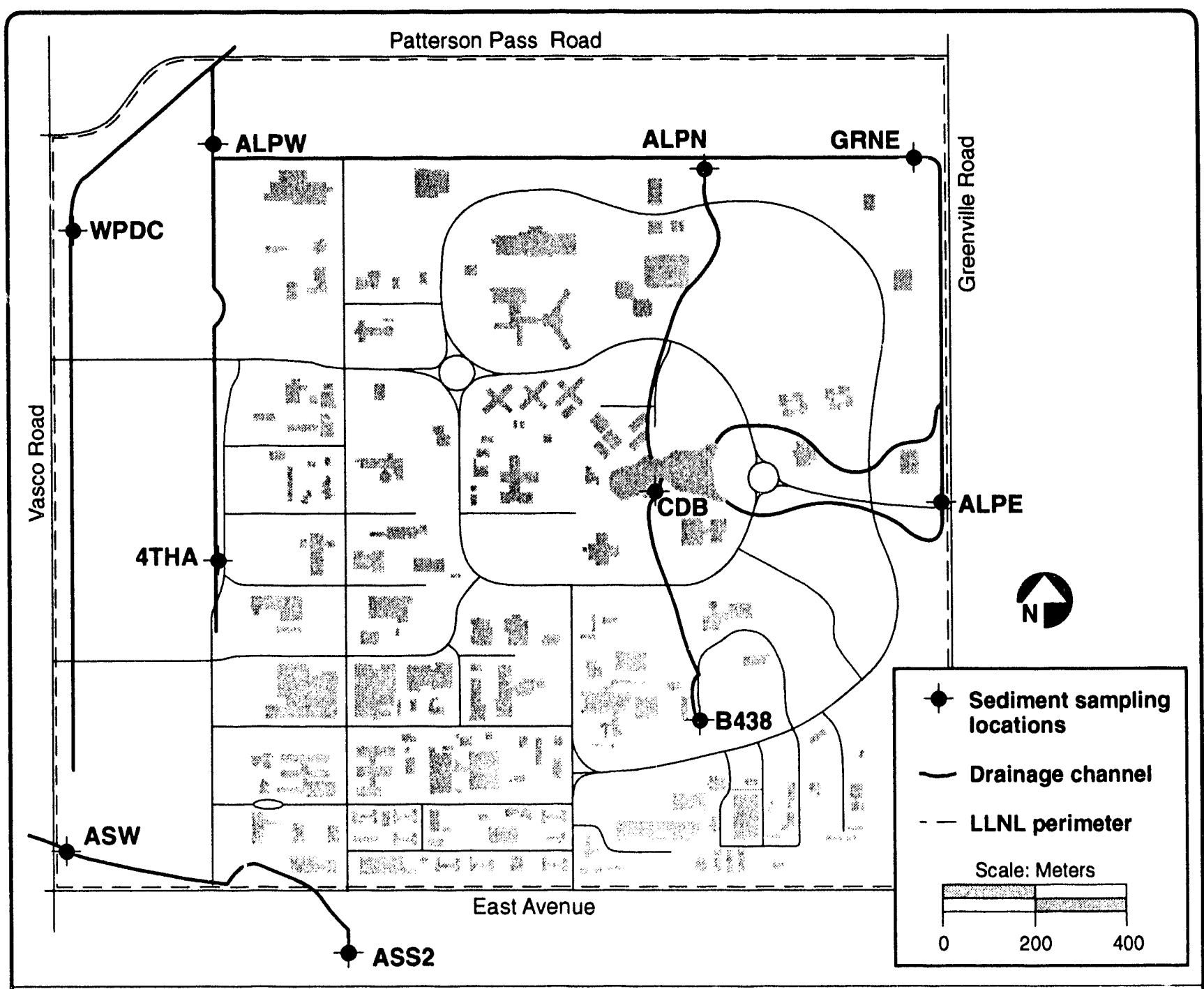

Figure 8-3. Arroyo and drainage basin sediment sampling locations, 1992. 
Quality assurance (QA) samples are submitted with each batch of soil samples. At locations chosen for duplicate samples, two identical samples are collected. Adjacent cores are collected from the corners and center of the sampling square. Separate composites of five cores each are made, and the two samples are identified with unique sample identifier codes. Similarly, duplicate $10-\mathrm{g}$ aliquots for beryllium analyses are produced. The sampling locations of QA samples are not identified on the sample bags, the sample identification tags, or vial labels, so the analytical laboratory does not know where the QA samples originated. However, this information is recorded on Field Tracking Forms.

New gloves are worn at each sampling location. After all subsamples are collected from a location, the core sampler is decontaminated by washing it thoroughly with deionized water and a low-phosphate detergent. After decontamination, the core sampler is kept in a plastic bag to avoid subsequent contamination.

Samples are delivered on the day of collection to LLNL's Radiation Analytical Sciences laboratory for analyses. Soil samples are dried, ground, sieved, and blended. The plutonium content of a sample aliquot is determined by alpha spectroscopy, following four hours of acid leaching in a mixture of concentrated $\mathrm{HNO}_{3}$ and $\mathrm{HCl}$ and separation and purification by ion exchange/electroplating. Other sample aliquots $(300 \mathrm{~g})$ are analyzed for more than 150 radionuclides by gamma spectroscopy, using a Ge ( $\mathrm{Li}$ ) detector (Garrison et al. 1985). The 10-g subsamples for beryllium analyses are sent to a contract analytical laboratory and are analyzed by graphite-furnace atomic absorption spectroscopy. Chain-of-custody procedures are followed throughout the sampling, delivery, and analytical processes.

The samples collected for the special sediment study were collected using these soil sampling techniques.

\section{Stream Sediments}

Annual stream sediment surveillance samples from drainages at and around the Livermore site are collected after the cessation of spring runoff and are analyzed for a variety of constituents. The concentrations of radionuclides (including tritium and plutonium), heavy metals, volatile and semivolatile organic compounds, pesticides, and polychlorinated biphenyls (PCBs) are determined.

The number and location of sediment sampling locations have been reevaluated and increased since sediment sampling began in 1988. Sediment was sampled from ten major LLNL drainages, including four locations first sampled during 1991 (4THA, at Fourth and A Streets; B438, adjacent to Building 438; GRNE, at the northeast influent to Arroyo Las Positas just off Greenville Road; and WPDC, in the west-perimeter drainage channel). Location CDB2, a location near the middle of the Central Drainage Basin, was not sampled because construction activities placing clay and rock in the drainage basin were under way in 1992. The sediment sampling locations coincided with storm water runoff sampling locations so it would be possible to compare the sampling results from these two related media.

A culvert, bridge, or other permanent marker serves as a reference point for each sampling location. Three samples are collected at randomly selected distances from the 
permanent marker of each sampling location. The sample collection technicians record how far away and in what direction from the permanent marker the samples are actually collected.

As described below, an individual sediment sample is collected as two subsamples, from varying depths, in two steps. The first subsample is collected with a hand auger and is submitted for radiological analyses. A split-spoon sampler is used to colleci the second subsample from the bottom of the same hole. That subsample is analyzed for metals and organic compounds.

Before handling a sample, the technicians put on clean, disposable, vinyl gloves so the sample does not become contaminated. Using a hand auger, the technician removes sediment to a depth of $0.30 \mathrm{~m}$. The auger is used again to collect an additional $0.15 \mathrm{~m}$ of sediment from the hole, and this subsample is placed in a glass container. A sample identification label is attached to the sample container, which is placed on ice in an ice chest and delivered to the Radiation Analytizal Sciences laboratory. The lab analyzes the sample for tritium and other radiological constituents.

To prepare for the collection of the second subsample, all loose sediment is cleared from the sample hole. Using a slide hammer, the technician drives the split-spoon sampier an additional $0.15 \mathrm{~m}$ into the ground, extracts it, and disassembles it, revealing the internal sampling tubs containing the intact, untouched sediment sample. The sample tube is sealed with Totlon and protective plastic caps, a sample identification label is attached, and the sample tube is placed on ice in an ice chest. This subsample is analyzed for metals and organic compounds by a contract analytical laboratory.

QA samples are submitted with each batch of sediment samples. In reality, the three samples taken at each sediment sampling location are duplicate samples. However, blind duplicates are also collected. A fourth sample is taken from each of three designated sampling locations, and is submitted blind, as a QA sample, to the analytical laboratories. The sampling locations of QA samples are not identified on the sample containers or Chain-of-Custody Record Forms. However, the locations are recorded on Field Tracking Forms

New gloves are worn when each sample is collected. Before collecting additional samples, either at the same location or at another location, the auger, split-spoon sampler, and other sampling equipment are thoroughly decontaminated by washing them with deionized water and a low-phosphate detergent.

Subsamples that are collected with the auger and placed in glass sample containers are delivered on the same day to the Radiation Analytical Sciences laboratory for analysis. Using freeze-drying techniques, water is recovered from the samples, and the tritium content of the water is determined by liquid-scintillation counting. The plutonium content of a sample aliquot is determined by alpha spectroscopy, following the sample preparation procedures outlined above for soil samples. Other sample aliquots are analyzed for more than 150 radionuclides using gamma spectroscopy as described above for soil samples. Subsamples, collected with the split-spoon sampler and contained in the sample tubes, are submitted to a contract laboratory for analysis. The sediment samples are analyzed for metals by inductively coupled plasma (ICP) emission spectroscopy, following extraction in water, for comparison to regulatory limits (22 CCR 66261.24). 
Standard EPA methods (8010 for halogenated volatile organic compounds, Method 8020 for aromatic volatile organic compounds, and Method 8080 for pesticides and PCBs) are used to analyze the samples for organic compounds. Chain-of-custody procedures are followed throughout the sampling, delivery, and analytical processes.

The radioanalytical methods employed in the soil and sediment monitoring programs by the Radiation Analytical Sciences laboratory are state-of-the-art; the methods enable detection of concentrations at levels more sensitive than regulatory limits. Nonradiological analyses are performed by State of California certified analytical laboratories using state-of-the-art technology.

\section{Results}

\section{Livermore Site}

Table 8-1 presents data on the concentrations of ${ }^{239} \mathrm{Pu},{ }^{40} \mathrm{~K},{ }^{60} \mathrm{Co},{ }^{137} \mathrm{Cs}$, ${ }^{226} \mathrm{Ra},{ }^{228} \mathrm{Ra},{ }^{232} \mathrm{Th},{ }^{235} \mathrm{U},{ }^{238} \mathrm{U}$, and ${ }^{241} \mathrm{Am}$ in surface soils from the Livermore Valley sampling locations. These were the only positively detected radionuclides from the over 150 that were included in the sample analysis.

The concentrations and distributions of all observed radionuclides in soil for 1992 are within the ranges reported in previous years and generally reflect worldwide fallout and naturally occurring concentrations; the ratio of $235 \mathrm{U}$ to $238 \mathrm{U}$ reflects the natural ratio of $0.7 \%$. The source of the low but positively detected level of ${ }^{60} \mathrm{Co}$ at WRP6 (at the Livermore Water Reclamation Plant, hereafter LWRP) could not be determined; this isotope is widely used in a variety of medical, technical, and research applications.

Plutonium-239 was detected at a higher level than background, $3.2 \times$ $10^{-3} \mathrm{~Bq} / \mathrm{g}\left(8.5 \times 10^{-8} \mu \mathrm{Ci} / \mathrm{g}\right)$ at location ZON7. Although last year's sample from this location was within background, since 1973 soil samples in this area have generally shown ${ }^{239} \mathrm{Pu}$ values that are higher than background. The slightly higher values have been attributed to the operation of solar evaporators located on the Livermore site in the early 1970s nearly due west of the sampling locations (Silver et al. 1974).

Elevated levels of ${ }^{239} \mathrm{Pu}$, resulting from an estimated $32-\mathrm{mCi}$ plutonium release to the sewer in 1967 and first observed in soils near LWRP during the early 1970s, again were detected in locations WRP1 to WRP6. The highest value of $110 \times 10^{-3} \mathrm{~Bq} / \mathrm{g}\left(3.0 \times 10^{-6} \mu \mathrm{Ci} / \mathrm{g}\right)$ measured at LWRP during 1992 represents $22 \%$ of the proposed EPA surface soil screening level of $7400 \mathrm{~Bq} / \mathrm{m}^{2}$ $\left(0.2 \mu \mathrm{Ci} / \mathrm{m}^{2}\right)$, or $0.5 \mathrm{~Bq} / \mathrm{g}\left(13 \times 10^{-6} \mu \mathrm{Ci} / \mathrm{g}\right)$, assuming average Livermore Valley soil densities of $1.5 \mathrm{~g} / \mathrm{cm}^{3}$ and a potential resuspension depth of $1.0 \mathrm{~cm}$ (42 Federal Register 230, 1977; U.S. Environmental Protection Agency 1977, 1978). The proposed EPA screening level for surface soil contamination was derived using very conservative assumptions and mathematical models that considered both the inhalation and ingestion pathways. According to the proposed EPA 


\section{Soil and Sediment Monitoring}

guidance, areas that do not exceed the screening level are generally said to be in compliance and need no further investigation for possible remediation (42 Federal Register 230, 1977; U.S. Environmental Protection Agency 1977, 1978). Historical plots of average ${ }^{239} \mathrm{Pu}$ concentrations in soil in the Livermore Valley, at Site 300 and at LWRP are shown in Figure 8-4. Livermore Valley and Site 300 concentrations have remained relatively constant over the past ten years and are indicative of worldwide fallout. The apparent increase in ${ }^{239} \mathrm{Pu}$ at LWRP is a result of a high value in 1992 . The ${ }^{239} \mathrm{Pu}$ is likely to be present in discrete particles, so the presence or absence of the particles will dominate the measured ${ }^{239} \mathrm{Pu}$ in any given sample. The high value found in 1992 caused the mean to be high, while a low value in 1989 caused the mean to be low. Further statistical analysis showed that all LWRP ${ }^{239} \mathrm{Pu}$ soils data are lognormally distributed, and there is no general increase or decrease in ${ }^{239} \mathrm{Pu}$ values with time. Moreover, all measured concentrations, regardless of location and year, have been a small fraction of the proposed EPA screening level, which is also shown in Figure 8-4 for comparison.

At WRP5, in the same area that exhibited elevated ${ }^{239} \mathrm{Pu}$ levels, and at WRP2, ${ }^{241} \mathrm{Am}$ was detected at a low level; it probably is caused by the natural decay of the trace concentrations of ${ }^{241} \mathrm{Pu}$ that are typically present with ${ }^{239} \mathrm{Pu}$.

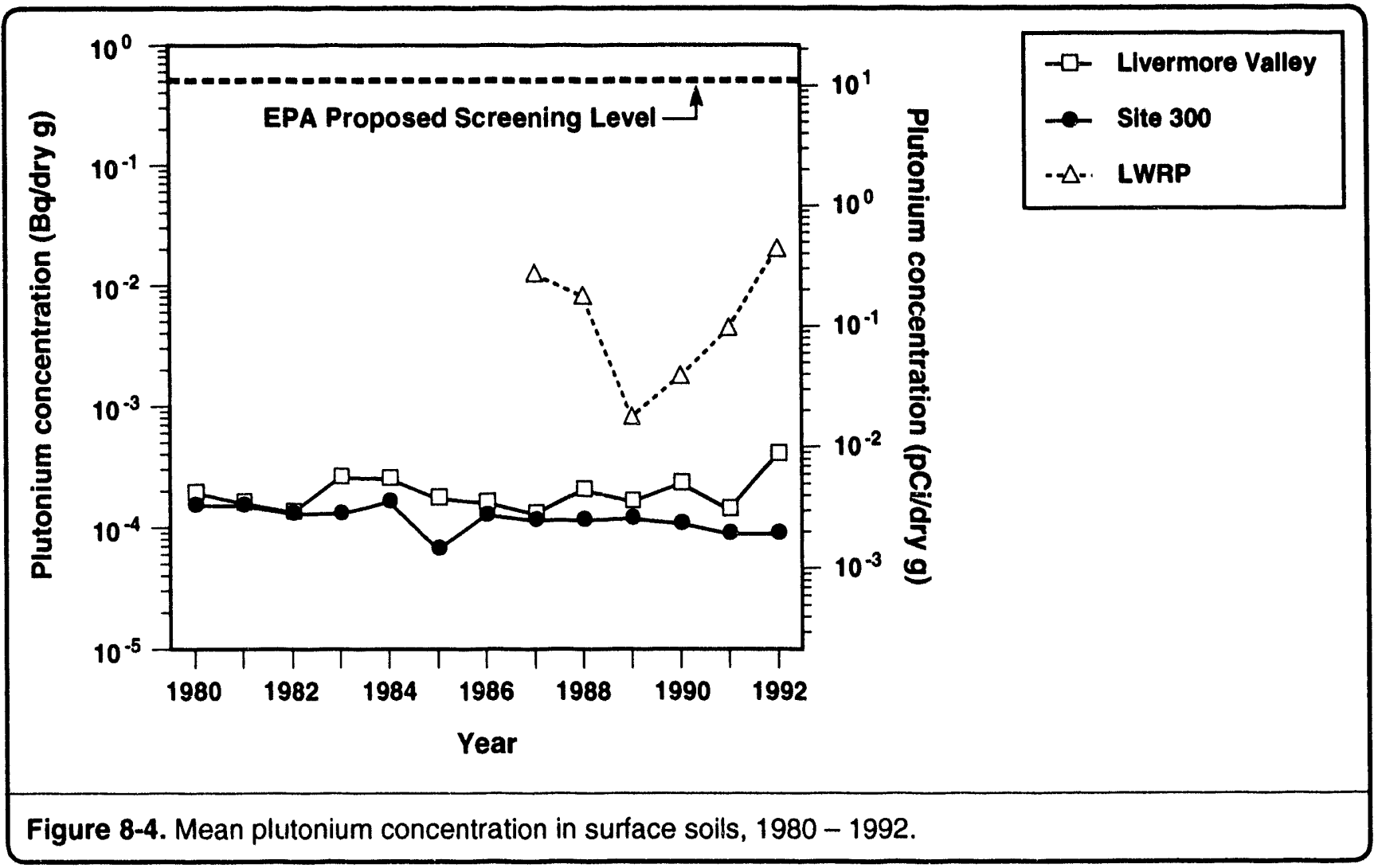


Table 8-1 also shows data on the concentrations of beryllium in surface soils from Livermore Valley sampling locations. Beryllium levels in soil samples from the Livermore Valley were comparable to the normal range of background concentrations (Wilber 1980).

Table 8-2 presents data on radionuclides detected in the sediment samples. Again, only positively detected levels are shown. The levels of ${ }^{239} \mathrm{Pu}$ were generally at background concentrations, reflective of worldwide fallout; the slightly higher values at ALPE, ALPW, and CDB may be attributed to historic activities at I' NL. Most other radionuclides were detected at levels similar to those reported from 1988 through $1991:{ }^{137} \mathrm{Cs}$, a fission product, was found at worldwide background concentrations; and ${ }^{40} \mathrm{~K},{ }^{226} \mathrm{Ra},{ }^{228} \mathrm{Ra},{ }^{232} \mathrm{Th},{ }^{235} \mathrm{U}$, and 238U-naturally occurring radionuclides-were detected at background concentrations. Tritium concentrations were below those reported from 1988 through 1991. The location CDB showed the highest tritium and ${ }^{239} \mathrm{Pu}$ values. This location is a drainage point over which one-third of Livermore-site surface area discharge flows; therefore it is expected that materials collected at this point could be higher than at other locations.

Table 8-3 presents data on metals that were detected in the sediment samples. There are no specific regulatory limits for metals in sediments, except for purposes of disposal; however, comparison of these values to disposal limits found in $22 \mathrm{CCR} \S 66261.24$ provides a means to distinguish analytes of concern. No concentrations above levels normally encountered in sediments were detected. As in 1991, most mean (or median) metal concentrations were less than $1 \%$, and all were less than $10 \%$ of the standards set in 22 CCR $\S 66261.24$.

Table 8-4 presents data on positively detected organic compounds in the sediment samples. Current techniques for detecting these compounds are very sensitive; therefore, traces of a variety of compounds (many of which were undetectable before 1989) were observed in some samples. Only a few positively detected analytes were found in most samples, and many of these were concentrations at, or marginally above, the analytical detection limits. A few samples contained organic compound concentrations slightly greater than background concentrations (for example, BHC, beta isomer, one time only, at ASW; dieldrin, two times at 4THA and one time at ASW; total xylene isomers, one time only, at ASS2). Toluene was detected at all locations except GRNE; however, the mean toluene concentration in 1992 was <0.014, while in 1991 it was $<0.023$. These concentrations are not of regulatory or health concern, but will be monitored during sediment sampling scheduled for 1993.

Most of the detected organic compounds in sediments are typical of fuel constituents and are expected in locations, such as the arroyos where samples are taken, that concentrate storm water runoff from roads, parking lots, and fields on site. Others are typical laboratory solvents. As for metals, there are no specific 
regulatory standards for organic compounds in sediments, so again regulatory levels for disposal are used for comparison (Marshack 1986; 22 CCR $\S 66261.24$, $\S 66268.43$ ). For those positively detected organic compounds that have published regulatory limits for disposal, all mean concentrations in sediments were less than $5 \%$, and most were less than $0.1 \%$ of those limits.

\section{Site 300}

Table 8-5 presents data on the concentrations of ${ }^{239} \mathrm{Pu},{ }^{40} \mathrm{~K},{ }^{137} \mathrm{Cs},{ }^{226} \mathrm{Ra}$, ${ }^{228} \mathrm{Ra},{ }^{232} \mathrm{Th},{ }^{235} \mathrm{U}$, and ${ }^{238} \mathrm{U}$ in soil from the Site 300 sampling locations; only positively detected radionuclides are shown. The concentrations and distributions of all observed radionuclides in Site 300 soil for 1992 remain similar to the levels measured from 1988 through 1991, lie within the ranges reported in all years since monitoring began, and generally reflect worldwide fallout and naturally occurring concentrations; the ratio of ${ }^{235} \mathrm{U}$ to ${ }^{238} \mathrm{U}$ reflects the natural ratio of $0.7 \%$.

Historical trends of ${ }^{238} \mathrm{U}$ concentrations from both the Livermore Valley and Site 300 are shown in Figure 8-5. Mean values have remained relatively constant for both places; however, Site 300 mean values are slightly greater than ihose from the Livermore Valley because of naturally occurring uranium in Site 300 soil. Maximum values at Site 300 represent perturbations to the natural levels caused by the use of depleted uranium in high-explosive tests. Since 1988, these levels have been reduced by the cleanup activities around firing tables and landfills.

As in 1991, but in contrast to previous years, no 1992 soil samples, including those from regions near the firing tables and disposal areas, exhibited markedly greater-than-background concentrations of ${ }^{238} \mathrm{U}$. The highest value of $12 \mu \mathrm{g} / \mathrm{g}$ (about 3 times greater than average background) was significantly lower than values reported prior to 1991 (Figure 8-5). In 1989, a maximum value of 98.2 $\mu \mathrm{g} / \mathrm{g}$ was detected in the vicinity of an inactive firing table (soil sampling location $812 \mathrm{~N}$ ), and in 1990 a maximum value of $62.2 \mu \mathrm{g} / \mathrm{g}$ was detected in the vicinity of an on-site landfill (soil sampling location DSW). In 1991 and 1992, the soil samples from these locations contained ${ }^{238} \mathrm{U}$ at background concentrations.

Table 8-5 also presents data on concentrations of beryllium in soil from Site 300 sampling locations in 1992. Beryllium content of soil samples from Site 300 were comparable to the normal range of background concentrations (Wilber 1980). However, Site 300 beryllium concentrations were approximately twice that from the Livermore Valley. Whether this difference is attributable to natural variability in background beryllium levels or because beryllium has been used at Site 300 in high-explosive tests is unknown. However, Shinn et al. (1989) stated that "the maximum deposition of beryllium (from high-explosive tests) contributed a very small amount of beryllium to the soil compared to the natural 
background. The maximum observed deposition would amount to less than $3 \%$ of the natural background... ."

\section{Environmental Impact}

\section{Livermore Site}

Routine soil and sediment sample analyses indicate that the impact of LLNL operations on these media in 1992 has not changed from previous years and remains insignificant. The apparent increase in ${ }^{239} \mathrm{Pu}$ at the LWRP is a result of a high value in 1992 and low value in 1989 affecting the mean; further statistical analysis of all LWRP data shows no significant increase or decrease of ${ }^{239} \mathrm{Pu}$ over time. Most analytes of interest or concern were detected at background concentrations, in trace amounts, or could not be measured above detection limits. Even where certain analytes are detected, concentrations are far below regulatory standards. Sampling of soils and sediments will continue on an annual basis.

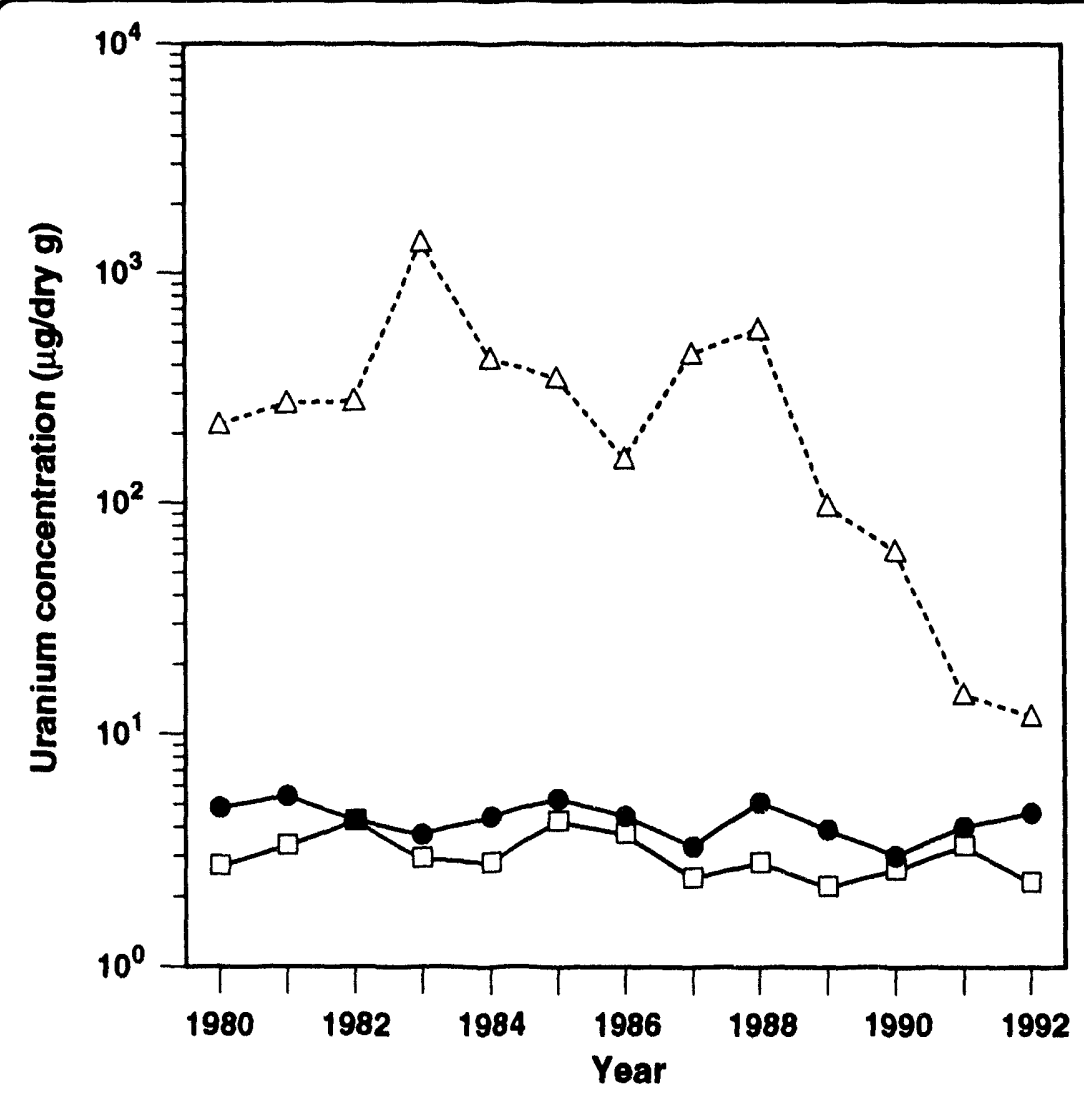

$\rightarrow$ Livermore Valley (mean)

Site 300 (mean)

$-\Delta-$ Site 300 (maximum)

Figure 8-5. Uranium concentration in surface soils, $1980-1992$. 
Site 300

With the exception of a single sample exhibiting slightly elevated ${ }^{238} \mathrm{U}$ (below those reported for previous years), the concen:rations of radionuclides and beryllium observed in soil samples collected at Site 300 are representative of background or naturally occurring levels. In the past, ${ }^{238} \mathrm{U}$-contaminated gravel from the firing tables inas removed to on-site landfills. These landfills at Site 300 have been inactive since 1988. The 1992 analyses did not detect significantly higher concentrations of ${ }^{238} \mathrm{U}$ in soil in areas adjacent to firing bunkers and near the landfills, in contrast to previous years (Gudiksen et al. 1973, Holland et al. 1987, Holland and Brekke 1988, Sims et al. 1991). The level of 238U has been reduced to near background levels, probably because of the bunker cleanup operations that removed contaminated gravel to the landfills.

\section{Special Projects: Shallow Sediment Sampling}

Sediment samples have been taken at LLNL for the past five years. Samples for analysis of radionuclides are collected at 0.30-0.45 m deep, and samples for analysis of metals are taken from $0.45-0.60 \mathrm{~m}$ deep. This year, we also sampled sediments at $0.05 \mathrm{~m}$ (the depth at which soils are sampled), to ascertain if there are any differences in metal or radionuclide concentrations for shallow or deeper samples. The results for the shallow samples are reported in Tables 8-6 and 8-7; the results for the routine deeper samples are shown in Tables 8-2 and 8-3. Most of the analytes show similar concentrations at both depths. Beryllium-7 was only detected in shallow samples. Because ${ }^{7} \mathrm{Be}$ is generated from cosmic rays and has a short half-life (53.4 days), there is little chance for it to migrate to deeper levels before decaying. Thorium-232 was found at lower concentrations at the shallower depth; this may be due to weathering in this rather dense, naturally occurring radionuclide. Of the metals, only zinc and copper had higher concentrations at shallower depths. Both of these could be attributed to the operation of motor vehicles. Copper is found in brake pads and diesel fuel, and zinc is present in tires; both are regarded as sources of storm water pollution (Mangarella 1992). The sediments samples are taken in storm water drainage channels, and motor vehicles would provide constant addition of such pollutants. 


\section{Soil and Sediment Monitoring}

Table 8-1. Positively detected radionuclides and beryllium in soil sampled at depths from 0 to $50 \mathrm{~mm}$, Livermore Valley, 1992.

\begin{tabular}{|c|c|c|c|c|c|c|}
\hline \multirow[b]{2}{*}{ Location" } & $241 \mathrm{Am}$ & ${ }^{60} \mathrm{Co}$ & ${ }^{239} \mathrm{Pu}$ & 232Th & $235 \mathrm{U}$ & 238U \\
\hline & \multicolumn{3}{|c|}{$\left(10^{-3} \mathrm{~Bq} / \mathrm{dry} \mathrm{g}\right)$} & \multicolumn{3}{|c|}{$(\mu g / d r / g)$} \\
\hline ALTA & $<1.92$ & $<0.11$ & $0.18 \pm 0.02$ & $8.8 \pm 0.3$ & $0.024 \pm 0.005$ & $<2.5$ \\
\hline CAFE & $<0.93$ & $<0.07$ & $0.70 \pm 0.04$ & $5.5 \pm 0.2$ & $0.017 \pm 0.004$ & $2.5 \pm 2.1$ \\
\hline cow & $<0.89$ & $<0.11$ & $0.09 \pm 0.01$ & $7.2 \pm 0.2$ & $0.020 \pm 0.005$ & $<2.5$ \\
\hline ERCH & $<2.18$ & $<0.07$ & $0.25 \pm 0.04$ & $5.4 \pm 0.2$ & $0.022 \pm 0.005$ & $2.2 \pm 1.1$ \\
\hline FCC & $<0.59$ & $<0.07$ & $0.09 \pm 0.01$ & $5.9 \pm 0.1$ & $0.017 \pm 0.005$ & $2.4 \pm 1.6$ \\
\hline HOSP & $<1.59$ & $<0.07$ & $0.14 \pm 0.02$ & $4.7 \pm 0.1$ & $0.014 \pm 0.005$ & $1.9 \pm 1.2$ \\
\hline MESQ & $<0.85$ & $<0.11$ & $0.03 \pm 0.01$ & $7.3 \pm 0.2$ & $0.020 \pm 0.005$ & $<1.1$ \\
\hline MET & $<0.52$ & $<0.07$ & $0.05 \pm 0.01$ & $6.9 \pm 0.2$ & $0.021 \pm 0.004$ & $<1.5$ \\
\hline NEP & $<0.96$ & $<0.07$ & $0.09 \pm 0.01$ & $6.1 \pm 0.2$ & $0.017 \pm 0.005$ & $2.4 \pm 1.9$ \\
\hline PATT & $<0.41$ & $<0.11$ & $0.02 \pm 0.005$ & $8.9 \pm 0.3$ & $0.032 \pm 0.009$ & $2.9 \pm 2.6$ \\
\hline RRCH & $<0.48$ & $<0.07$ & $0.28 \pm 0.02$ & $6.4 \pm 0.2$ & $0.019 \pm 0.005$ & $<1.2$ \\
\hline SALV & $<0.37$ & $<0.07$ & $0.20 \pm 0.02$ & $7.1 \pm 0.3$ & $0.023 \pm 0.005$ & $2.3 \pm 2.0$ \\
\hline TANK & $<1.63$ & $<0.11$ & $0.006 \pm 0.003$ & $6.0 \pm 0.1$ & $0.017 \pm 0.004$ & $<1.8$ \\
\hline VIS & $<1.96$ & $<0.07$ & $0.60 \pm 0.04$ & $7.1 \pm 0.1$ & $0.023 \pm 0.005$ & $2.5 \pm 2.3$ \\
\hline ZON7 & $<1.00$ & $<0.22$ & $3.2 \pm 0.18$ & $6.6 \pm 0.2$ & $0.020 \pm 0.005$ & $4.4 \pm 2.9$ \\
\hline WRP1 & $<1.63^{b}$ & $<0.07$ & $3.9 \pm 0.18^{b}$ & $6.1 \pm 0.1$ & $0.017 \pm 0.004$ & $2.2 \pm 1.7$ \\
\hline WRP2 & $1.92 \pm 0.92^{b}$ & $<0.07$ & $2.8 \pm 0.12^{b}$ & $8.0 \pm 0.2$ & $0.021 \pm 0.005$ & $2.7 \pm 2.4$ \\
\hline WRP3 & $<0.89^{b}$ & $<0.11$ & $3.20 \pm 0.15^{b}$ & $7.5 \pm 0.2$ & $0.024 \pm 0.006$ & $<2.3$ \\
\hline WRP4 & $<1.81^{b}$ & $<0.07$ & $0.84 \pm 0.05^{b}$ & $6.6 \pm 0.1$ & $0.018 \pm 0.005$ & $2.7 \pm 2.4$ \\
\hline WRP5 & $31.45 \pm 4.09^{b}$ & $1.26 \pm 0.20$ & $110 \pm 6.0^{b}$ & $6.2 \pm 0.2$ & $0.025 \pm 0.006$ & $3.3 \pm 2.0$ \\
\hline WRP6 & $<1.74^{b}$ & $<0.07$ & $0.34 \pm 0.05^{b}$ & $5.5 \pm 0.2$ & $0.160 \pm 0.050$ & $1.9 \pm 1.7$ \\
\hline Mean & $<1.00^{c}$ & $<0.07^{c}$ & $0.40^{d} 20^{\circ}$ & 6.7 & 0.027 & $<2.3$ \\
\hline \multirow[t]{2}{*}{ Std dev } & -1 & -1 & $0.80^{d}\left(44^{\circ}\right)$ & 1.1 & 0.031 & -1 \\
\hline & \multicolumn{3}{|c|}{ (10-3 pci/dry g) } & & & \\
\hline Mean & $<27^{c}$ & $<1.9^{c}$ & $11^{d}\left(540^{\circ}\right)$ & & & \\
\hline Std dev & -1 & -1 & $22^{d}\left(1200^{\circ}\right)$ & & & \\
\hline
\end{tabular}

...continued 
Table 8-1. Positively detected radionuclides and beryllium in soil sampled at depths from 0 to $50 \mathrm{~mm}$, Livermore Valley, 1992 (concluded).

\begin{tabular}{|c|c|c|c|c|c|}
\hline \multirow[b]{2}{*}{ Location" } & ${ }^{40} K$ & ${ }^{226} \mathrm{Ra}$ & ${ }^{228} \mathrm{Ra}$ & \multirow{2}{*}{$\frac{{ }^{137} \mathrm{Cs}}{\left(10^{-3} \mathrm{~Bq} / \text { dry g) }\right.}$} & \multirow{2}{*}{$\frac{\mathrm{Be}}{(\mathrm{mg} / \mathrm{kg})}$} \\
\hline & \multicolumn{3}{|c|}{ (Bq/dry g) } & & \\
\hline ALTA & $0.60 \pm 0.02$ & $0.025 \pm 0.0005$ & $0.031 \pm 0.0013$ & $8.0 \pm 0.32$ & 0.91 \\
\hline CAFE & $0.47 \pm 0.02$ & $0.019 \pm 0.0006$ & $0.019 \pm 0.0010$ & $3.4 \pm 0.24$ & 0.35 \\
\hline cow & $0.54 \pm 0.02$ & $0.024 \pm 0.0005$ & $0.025 \pm 0.0010$ & $3.4 \pm 0.34$ & 0.48 \\
\hline ERCH & $0.34 \pm 0.01$ & $0.019 \pm 0.0004$ & $0.021 \pm 0.0006$ & $11 \pm 0.33$ & 0.33 \\
\hline FCC & $0.40 \pm 0.01$ & $0.022 \pm 0.0004$ & $0.021 \pm 0.0008$ & $3.6 \pm 0.33$ & 0.50 \\
\hline HOSP & $0.35 \pm 0.01$ & $0.017 \pm 0.0005$ & $0.017 \pm 0.0009$ & $6.6 \pm 0.26$ & 0.40 \\
\hline MESQ & $0.50 \pm 0.01$ & $0.024 \pm 0.0005$ & $0.027 \pm 0.0011$ & $0.81 \pm 0.32$ & 0.66 \\
\hline MET & $0.54 \pm 0.02$ & $0.021 \pm 0.0006$ & $0.024 \pm 0.0007$ & $2.2 \pm 0.24$ & 0.46 \\
\hline NEP & $0.49 \pm 0.02$ & $0.021 \pm 0.0006$ & $0.021 \pm 0.0011$ & $3.4 \pm 0.24$ & 0.47 \\
\hline PATT & $0.52 \pm 0.02$ & $0.045 \pm 0.0014$ & $0.032 \pm 0.0016$ & $1.0 \pm 0.30$ & 0.66 \\
\hline RRCH & $0.41 \pm 0.02$ & $0.022 \pm 0.0007$ & $0.022 \pm 0.0011$ & $9.7 \pm 0.39$ & 0.63 \\
\hline SALV & $0.41 \pm 0.01$ & $0.027 \pm 0.0005$ & $0.027 \pm 0.0008$ & $0.81 \pm 0.21$ & 0.52 \\
\hline TANK & $0.37 \pm 0.01$ & $0.018 \pm 0.0004$ & $0.021 \pm 0.0009$ & $0.22 \pm 0.19$ & 0.49 \\
\hline VIS & $0.44 \pm 0.01$ & $0.025 \pm 0.0005$ & $0.025 \pm 0.0008$ & $5.5 \pm 0.33$ & 0.49 \\
\hline ZON7 & $0.57 \pm 0.02$ & $0.022 \pm 0.0007$ & $0.024 \pm 0.0010$ & $10 \pm 0.31$ & 0.57 \\
\hline WRP1 & $0.41 \pm 0.01$ & $0.019 \pm 0.0004$ & $0.021 \pm 0.0006$ & $1.3 \pm 0.21$ & 0.48 \\
\hline WRP2 & $0.47 \pm 0.01$ & $0.026 \pm 0.0005$ & $0.027 \pm 0.0011$ & $2.2 \pm 0.28$ & 0.42 \\
\hline WRP3 & $0.45 \pm 0.01$ & $0.024 \pm 0.0005$ & $0.026 \pm 0.0010$ & $2.6 \pm 0.33$ & 0.52 \\
\hline WRP4 & $0.41 \pm 0.01$ & $0.022 \pm 0.0004$ & $0.024 \pm 0.0010$ & $2.2 \pm 0.28$ & 0.47 \\
\hline WRP5 & $0.40 \pm 0.01$ & $0.022 \pm 0.0004$ & $0.021 \pm 0.0011$ & $11 \pm 0.33$ & 0.93 \\
\hline WRP6 & $0.35 \pm 0.01$ & $0.017 \pm 0.0005$ & $0.019 \pm 0.0008$ & $1.2 \pm 0.17$ & 0.33 \\
\hline Mean & 0.45 & 0.023 & 0.024 & 4.3 & 0.53 \\
\hline \multirow[t]{2}{*}{ Std dev } & 0.08 & 0.006 & 0.004 & 3.6 & 0.16 \\
\hline & \multicolumn{3}{|c|}{ (pCi/dry g) } & $\left(10^{-3} \mathrm{pCi} /\right.$ dry $\left.\mathrm{g}\right)$ & \\
\hline Mean & 12.2 & 0.62 & 0.65 & 116 & \\
\hline Std dev & 2.2 & 0.16 & 0.11 & 97 & \\
\hline
\end{tabular}

Note: Radionuclide results are reported $\pm 2 \sigma$; see Quality Assurance chapter.

a See Fig. 8-1 for sampling locations.

b Samples from areas of known contamination.

c Median value; see Quality Assurance chapter.

d Summary statistics for samples from uncontaminated areas.

c Summary statistics for samples from areas of know contamination.

1 No measure of dispersion calculated; see Quality Assurance chapter. 


\section{Soil and Sediment Monitoring}

Table 8-2. Positively detected radionuclides in arroyo sediments near the Livermore site, 1992.

\begin{tabular}{|c|c|c|c|c|c|}
\hline \multirow{3}{*}{$\begin{array}{l}\text { Location } \\
\text { 4THA }\end{array}$} & ${ }^{137} \mathrm{Cs}$ & ${ }^{239} \mathrm{Pu}$ & ${ }^{40} K$ & ${ }^{226} \mathrm{Ra}$ & ${ }^{228} \mathbf{R a}$ \\
\hline & \multicolumn{2}{|c|}{$\left(10^{-3} \mathrm{~Bq} / \mathrm{dry} \mathrm{g}\right)$} & \multicolumn{3}{|c|}{ (Bq/dry g) } \\
\hline & $5.4 \pm 0.40$ & $0.16 \pm 0.028$ & $0.42 \pm 0.01$ & $0.021 \pm 0.0004$ & $0.022 \pm 0.0009$ \\
\hline & $1.3 \pm 0.23$ & $0.054 \pm 0.013$ & $0.50 \pm 0.02$ & $0.026 \pm 0.0008$ & $0.029 \pm 0.0012$ \\
\hline & $8.9 \pm 0.36$ & $0.21 \pm 0.034$ & $0.46 \pm 0.01$ & $0.023 \pm 0.0007$ & $0.024 \pm 0.0012$ \\
\hline \multirow[t]{3}{*}{ ALPE } & $<0.11$ & $<0.001$ & $0.43 \pm 0.01$ & $0.027 \pm 0.0005$ & $0.029 \pm 0.0009$ \\
\hline & $<0.11$ & $0.0065 \pm 0.003$ & $0.49 \pm 0.01$ & $0.028 \pm 0.0006$ & $0.029 \pm 0.0009$ \\
\hline & $<0.11$ & $<0.0022$ & $0.50 \pm 0.01$ & $0.028 \pm 0.0006$ & $0.030 \pm 0.0009$ \\
\hline \multirow[t]{3}{*}{ ALPN } & $<0.11$ & $0.0027 \pm 0.002$ & $0.59 \pm 0.01$ & $0.023 \pm 0.0005$ & $0.026 \pm 0.0010$ \\
\hline & $<0.07$ & $0.0076 \pm 0.003$ & $0.56 \pm 0.02$ & $0.021 \pm 0.0004$ & $0.024 \pm 0.0007$ \\
\hline & $<0.11$ & $0.0024 \pm 0.002$ & $0.53 \pm 0.02$ & $0.019 \pm 0.0006$ & $0.024 \pm 0.0012$ \\
\hline \multirow[t]{3}{*}{ ALPW } & $1.2 \pm 0.33$ & $0.27 \pm 0.017$ & $0.55 \pm 0.02$ & $0.021 \pm 0.0006$ & $0.027 \pm 0.0014$ \\
\hline & $2.0 \pm 0.28$ & $1.02 \pm 0.055$ & $0.45 \pm 0.01$ & $0.019 \pm 0.0004$ & $0.020 \pm 0.0008$ \\
\hline & $1.4 \pm 0.39$ & $0.19 \pm 0.019$ & $0.46 \pm 0.01$ & $0.019 \pm 0.0006$ & $0.021 \pm 0.0015$ \\
\hline \multirow[t]{3}{*}{ ASS2 } & $0.67 \pm 0.23$ & $0.025 \pm 0.006$ & $0.42 \pm 0.01$ & $0.017 \pm 0.0005$ & $0.017 \pm 0.0009$ \\
\hline & $0.63 \pm 0.18$ & $0.027 \pm 0.006$ & $0.49 \pm 0.02$ & $0.018 \pm 0.0007$ & $0.018 \pm 0.0011$ \\
\hline & $0.67 \pm 0.20$ & $0.014 \pm 0.003$ & $0.49 \pm 0.01$ & $0.019 \pm 0.0004$ & $0.019 \pm 0.0008$ \\
\hline \multirow[t]{3}{*}{ ASW } & $0.63 \pm 0.27$ & $0.015 \pm 0.003$ & $0.41 \pm 0.01$ & $0.020 \pm 0.0006$ & $0.021 \pm 0.0011$ \\
\hline & $2.4 \pm 0.26$ & $0.084 \pm 0.008$ & $0.45 \pm 0.01$ & $0.015 \pm 0.0005$ & $0.016 \pm 0.0010$ \\
\hline & $0.56 \pm 0.21$ & $0.012 \pm 0.003$ & $0.47 \pm 0.02$ & $0.024 \pm 0.0005$ & $0.022 \pm 0.0009$ \\
\hline \multirow[t]{3}{*}{ B438 } & $<0.07$ & $0.0059 \pm 0.002$ & $0.54 \pm 0.02$ & $0.023 \pm 0.0005$ & $0.028 \pm 0.0008$ \\
\hline & $<0.11$ & $0.0037 \pm 0.002$ & $0.48 \pm 0.01$ & $0.023 \pm 0.0005$ & $0.025 \pm 0.0008$ \\
\hline & $0.19 \pm 0.14$ & $0.086 \pm 0.007$ & $0.49 \pm 0.01$ & $0.024 \pm 0.0005$ & $0.025 \pm 0.0007$ \\
\hline \multirow[t]{3}{*}{ CDB } & $0.37 \pm 0.23$ & $0.68 \pm 0.032$ & $0.50 \pm 0.02$ & $0.029 \pm 0.0006$ & $0.030 \pm 0.0009$ \\
\hline & $<0.19$ & $0.56 \pm 0.029$ & $0.48 \pm 0.02$ & $0.024 \pm 0.0010$ & $0.027 \pm 0.0014$ \\
\hline & $0.44 \pm 0.20$ & $0.99 \pm 0.044$ & $0.46 \pm 0.01$ & $0.027 \pm 0.0005$ & $0.028 \pm 0.0011$ \\
\hline \multirow[t]{3}{*}{ GRNE } & $<0.07$ & $0.0012 \pm 0.001$ & $0.62 \pm 0.02$ & $0.027 \pm 0.0005$ & $0.026 \pm 0.0010$ \\
\hline & $<0.11$ & $0.00073 \pm 0.0006$ & $0.56 \pm 0.02$ & $0.025 \pm 0.0005$ & $0.027 \pm 0.0011$ \\
\hline & $<0.11$ & $<0.00041 \pm 0.0005$ & $0.59 \pm 0.01$ & $0.027 \pm 0.0005$ & $0.029 \pm 0.0009$ \\
\hline \multirow[t]{3}{*}{ WPDC } & $<0.07$ & $<0.00032 \pm 0.0004$ & $0.59 \pm 0.02$ & $0.033 \pm 0.0007$ & $0.035 \pm 0.0011$ \\
\hline & $<0.11$ & $0.0011 \pm 0.001$ & $0.59 \pm 0.02$ & $0.029 \pm 0.0006$ & $0.035 \pm 0.0010$ \\
\hline & $<0.11$ & $<0.00005$ & $0.56 \pm 0.02$ & $0.027 \pm 0.0005$ & $0.034 \pm 0.0010$ \\
\hline Mean & $<0.19^{b}$ & $<0.15$ & 0.50 & 0.024 & 0.026 \\
\hline Std dev & $-^{c}$ & $>0.28$ & 0.059 & 0.0042 & 0.0050 \\
\hline
\end{tabular}

$\left(10^{-3} \mathrm{pCi} /\right.$ dry g)

\begin{tabular}{lrr} 
& \multicolumn{2}{c}{$\left(10^{-3}\right.$ pcl/dry g) } \\
\cline { 2 - 3 } Mean & $<5.1^{\mathrm{b}}$ & $<4.1$ \\
Std dev & $-^{\mathrm{c}}$ & $>7.6$ \\
\hline
\end{tabular}

\begin{tabular}{lll}
\multicolumn{3}{c}{ (pCi/dryg) } \\
\hline 14 & 0.65 & 0.70 \\
1.6 & 0.11 & 0.14 \\
\hline
\end{tabular}

...continued 
Table 8-2. Positively detected radionuclides in arroyo sediments near the Livermore site, 1992 (concluded).

\begin{tabular}{|c|c|c|c|c|}
\hline \multirow[b]{2}{*}{ Locationa } & \multirow{2}{*}{$\frac{{ }^{3} H}{(B q / L)^{d}}$} & 232Th & $235 \mathrm{U}$ & ${ }^{238} \mathrm{U}$ \\
\hline & & \multicolumn{3}{|c|}{$(\mu g / d r y g)$} \\
\hline \multirow[t]{3}{*}{ 4THA } & \pm 1.5 & $6.7 \pm 0.2$ & $0.018 \pm 0.0050$ & $<2.4$ \\
\hline & \pm 1.6 & $8.5 \pm 0.3$ & $0.023 \pm 0.0058$ & $<1.4$ \\
\hline & \pm 1.6 & $6.8 \pm 0.3$ & $0.017 \pm 0.0056$ & $<1.3$ \\
\hline \multirow[t]{3}{*}{ ALPE } & \pm 1.4 & $8.3 \pm 0.2$ & $0.023 \pm 0.0049$ & $<2.2$ \\
\hline & \pm 1.5 & $8.7 \pm 0.2$ & $0.018 \pm 0.0046$ & $<1.3$ \\
\hline & $16 \pm 2.0$ & $8.6 \pm 0.2$ & $0.017 \pm 0.0035$ & $<2.0$ \\
\hline \multirow[t]{3}{*}{ ALPN } & $7.8 \pm 1.2$ & $7.4 \pm 0.3$ & $0.012 \pm 0.0038$ & $<1.9$ \\
\hline & $5.0 \pm 1.5$ & $7.0 \pm 0.1$ & $0.018 \pm 0.0046$ & $<1.5$ \\
\hline & $5.7 \pm 1.1$ & $6.8 \pm 0.2$ & $0.016 \pm 0.0053$ & $<2.4$ \\
\hline \multirow[t]{3}{*}{ ALPW } & $14 \pm 1.4$ & $8.2 \pm 0.3$ & $0.024 \pm 0.0071$ & $3.9 \pm 3.3$ \\
\hline & \pm 1.4 & $5.7 \pm 0.1$ & $0.018 \pm 0.0043$ & $3.1 \pm 2.7$ \\
\hline & $7.2 \pm 1.2$ & $6.4 \pm 0.3$ & $0.013 \pm 0.0087$ & $<1.0$ \\
\hline \multirow[t]{3}{*}{ ASS2 } & $5.2 \pm 1.5$ & $5.0 \pm 0.2$ & $0.016 \pm 0.0045$ & $3.3 \pm 1.8$ \\
\hline & $6.8 \pm 1.1$ & $5.6 \pm 0.2$ & $0.015 \pm 0.0054$ & $<1.9$ \\
\hline & $4.7 \pm 1.1$ & $5.7 \pm 0.2$ & $0.017 \pm 0.0048$ & $2.6 \pm 2.3$ \\
\hline \multirow[t]{3}{*}{ ASW } & $2.7 \pm 1.0$ & $6.1 \pm 0.2$ & $0.017 \pm 0.0060$ & $<2.4$ \\
\hline & $3.2 \pm 1.0$ & $4.7 \pm 0.1$ & $0.014 \pm 0.0042$ & $2.7 \pm 2.7$ \\
\hline & $2.6 \pm 1.0$ & $6.7 \pm 0.2$ & $0.016 \pm 0.0046$ & $<1.9$ \\
\hline \multirow[t]{3}{*}{ B438 } & $27 \pm 1.9$ & $7.3 \pm 0.3$ & $0.027 \pm 0.0065$ & $2.3 \pm 1.4$ \\
\hline & $19 \pm 1.7$ & $7.3 \pm 0.1$ & $0.018 \pm 0.0048$ & $<2.1$ \\
\hline & $7.9 \pm 1.2$ & $6.5 \pm 0.3$ & $0.024 \pm 0.0058$ & $1.7 \pm 1.2$ \\
\hline \multirow[t]{3}{*}{ CDB } & $96 \pm 4.6$ & $8.5 \pm 0.3$ & $0.022 \pm 0.0052$ & $<1.1$ \\
\hline & $78 \pm 3.9$ & $7.7 \pm 0.3$ & $0.020 \pm 0.0087$ & $4.6 \pm 3.4$ \\
\hline & $85 \pm 4.2$ & $8.1 \pm 0.2$ & $0.019 \pm 0.0046$ & $4.0 \pm 2.2$ \\
\hline \multirow[t]{3}{*}{ GRNE } & $4.7 \pm 1.0$ & $7.5 \pm 0.2$ & $0.015 \pm 0.0052$ & $2.0 \pm 1.7$ \\
\hline & $6.4 \pm 1.5$ & $8.1 \pm 0.2$ & $0.018 \pm 0.0057$ & $2.9 \pm 2.6$ \\
\hline & $4.3 \pm 1.5$ & $8.4 \pm 0.2$ & $0.022 \pm 0.0054$ & $<2.1$ \\
\hline \multirow[t]{3}{*}{ WPDC } & $7.4 \pm 1.2$ & $9.3 \pm 0.4$ & $0.028 \pm 0.0067$ & $3.1 \pm 1.4$ \\
\hline & $7.8 \pm 1.2$ & $9.4 \pm 0.4$ & $0.031 \pm 0.0075$ & $2.8 \pm 1.5$ \\
\hline & $6.8 \pm 1.2$ & $9.3 \pm 0.3$ & $0.029 \pm 0.0047$ & $2.9 \pm 2.5$ \\
\hline Mean & 18 & 7.3 & 0.020 & $<2.3^{b}$ \\
\hline \multirow[t]{2}{*}{ Std dev } & 24 & 1.3 & 0.0048 & $-^{c}$ \\
\hline & (pCi/L) & & & \\
\hline Mean & 490 & & & \\
\hline Std dev & 650 & & & \\
\hline
\end{tabular}

Note: Radionuclide results are reported $\pm 2 \sigma$; see Quality Assurance chapter.

a See Fig. 8-3 for sampling locations.

b Median value; see Quality Assurance chapter.

c No measure of dispersion calculated; see Quality Assurance chapter.

d Activity in recovered water. 


\section{Soil and Sediment Monitoring}

Table 8-3. Positively detected metals ${ }^{a}$ (in $\mathrm{mg} / \mathrm{L}$ ) in arroyo sediments near the Livermore site, 1992.

\begin{tabular}{|c|c|c|c|c|c|c|c|c|c|c|c|}
\hline Location ${ }^{b}$ & As & $\mathbf{B a}$ & Cd & Total $\mathrm{Cr}$ & Co & $\mathrm{Cu}$ & $\mathrm{Pb}$ & $\mathrm{Hg}$ & $\mathrm{Ni}$ & v & $\mathrm{Zn}$ \\
\hline \multirow[t]{3}{*}{ 4THA } & 0.03 & 9.6 & $<0.05$ & 0.05 & 0.48 & 0.17 & 0.3 & $<0.002$ & 0.7 & 0.24 & 8.1 \\
\hline & 0.06 & 9.5 & $<0.05$ & 0.10 & 0.51 & 0.22 & 0.4 & $<0.002$ & 0.8 & 0.24 & 4.4 \\
\hline & 0.04 & 11 & $<0.05$ & 0.08 & 0.58 & 0.24 & 0.5 & $<0.002$ & 1.0 & 0.28 & 6.2 \\
\hline \multirow[t]{3}{*}{ ALPE } & 0.03 & 11 & $<0.05$ & 0.08 & 0.77 & 0.08 & $<0.2$ & $<0.002$ & 1.4 & 0.65 & 0.31 \\
\hline & $<0.02$ & 9.9 & $<0.05$ & 0.07 & 0.55 & 0.11 & $<0.2$ & $<0.002$ & 0.9 & 0.49 & 0.43 \\
\hline & $<0.02$ & 9.3 & $<0.05$ & 0.08 & 0.63 & 0.07 & $<0.2$ & $<0.002$ & 1.0 & 0.56 & 0.35 \\
\hline \multirow[t]{3}{*}{ ALPN } & $<0.02$ & 9.9 & $<0.05$ & $<0.05$ & 0.47 & $<0.05$ & $<0.2$ & $<0.002$ & 0.5 & 0.43 & 0.08 \\
\hline & $<0.02$ & 12 & $<0.05$ & $<0.05$ & 0.49 & 0.06 & $<0.2$ & $<0.002$ & 0.6 & 0.34 & 0.08 \\
\hline & 0.02 & 11 & $<0.05$ & $<0.05$ & 0.47 & $<0.05$ & $<0.2$ & $<0.002$ & 0.7 & 0.46 & 0.07 \\
\hline \multirow[t]{3}{*}{ ALPW } & 0.08 & 7.7 & $<0.05$ & 0.19 & 0.43 & 0.18 & 0.3 & $<0.002$ & 0.4 & 0.72 & 1.1 \\
\hline & 0.23 & 5.4 & $<0.05$ & 0.17 & 0.53 & 0.61 & $<0.2$ & 0.002 & 0.4 & 0.36 & 4.8 \\
\hline & 0.05 & 6.4 & $<0.05$ & 0.11 & 0.37 & 1.0 & $<0.2$ & $<0.002$ & 0.3 & 0.32 & 2.0 \\
\hline \multirow[t]{3}{*}{ ASS2 } & 0.02 & 6.8 & 0.24 & 0.08 & 0.22 & 0.11 & 3.4 & $<0.002$ & 0.4 & 0.21 & 0.48 \\
\hline & 0.03 & 6.7 & $<0.05$ & 0.06 & 0.23 & 0.10 & $<0.2$ & $<0.002$ & 0.4 & 0.23 & 0.66 \\
\hline & 0.03 & 7.2 & $<0.05$ & $<0.05$ & 0.17 & 0.12 & $<0.2$ & $<0.002$ & 0.4 & 0.18 & 0.43 \\
\hline \multirow[t]{3}{*}{ ASW } & 0.03 & 5.7 & $<0.05$ & 0.11 & 0.23 & 0.24 & 0.4 & $<0.002$ & 0.4 & 0.21 & 0.82 \\
\hline & 0.04 & 5.5 & $<0.05$ & 0.10 & 0.18 & 0.33 & 0.8 & $<0.002$ & 0.3 & 0.20 & 1.1 \\
\hline & 0.06 & 7.5 & $<0.05$ & 0.09 & 0.23 & 0.41 & 0.4 & $<0.002$ & 0.4 & 0.24 & 1.4 \\
\hline \multirow[t]{3}{*}{ B438 } & $<0.02$ & 8.1 & $<0.05$ & $<0.05$ & 0.46 & 0.08 & $<0.2$ & $<0.002$ & 0.5 & 0.20 & 0.10 \\
\hline & $<0.02$ & 7.3 & $<0.05$ & $<0.05$ & 0.36 & 0.06 & $<0.2$ & $<0.002$ & 0.2 & 0.18 & 0.10 \\
\hline & $<0.02$ & 6.9 & $<0.05$ & $<0.05$ & 0.36 & 0.14 & $<0.2$ & $<0.002$ & 0.4 & 0.20 & 1.1 \\
\hline \multirow[t]{3}{*}{ CDB } & $<0.02$ & 8.9 & $<0.05$ & $<0.05$ & 0.45 & 0.09 & $<0.2$ & $<0.002$ & 0.7 & 0.24 & 0.14 \\
\hline & $<0.02$ & 10 & $<0.05$ & $<0.05$ & 0.51 & 0.11 & $<0.2$ & $<0.002$ & 0.7 & 0.30 & 0.57 \\
\hline & $<0.02$ & 7.9 & $<0.05$ & $<0.05$ & 0.36 & 0.06 & $<0.2$ & $<0.002$ & 0.6 & 0.21 & 0.09 \\
\hline \multirow[t]{3}{*}{ GRNE } & $<0.02$ & 10 & $<0.05$ & $<0.05$ & 0.51 & 0.11 & $<0.2$ & $<0.002$ & 0.4 & 0.38 & 0.06 \\
\hline & 0.03 & 13 & $<0.05$ & 0.05 & 0.52 & 0.10 & $<0.2$ & $<0.002$ & 0.6 & 0.66 & 0.16 \\
\hline & 0.05 & 8.3 & 0.06 & 0.22 & 0.52 & 0.18 & $<0.2$ & $<0.002$ & 0.5 & 0.60 & 0.32 \\
\hline \multirow[t]{3}{*}{ WPDC } & 0.02 & 11 & $<0.05$ & 0.07 & 0.47 & 0.13 & $<0.2$ & $<0.002$ & 0.6 & 0.36 & 0.08 \\
\hline & $<0.02$ & 13 & $<0.05$ & $<0.05$ & 0.55 & 0.13 & $<0.2$ & $<0.002$ & 0.5 & 0.43 & 0.08 \\
\hline & $<0.02$ & 13 & $<0.05$ & 0.05 & 0.53 & 0.15 & $<0.2$ & $<0.002$ & 0.6 & 0.36 & 0.06 \\
\hline Mean & $0.02^{c}$ & 9.0 & $<0.05^{c}$ & $0.06^{c}$ & 0.44 & $<0.18$ & $<0.2^{c}$ & $<0.002^{c}$ & 0.6 & 0.35 & 1.2 \\
\hline Std dev & $-^{d}$ & 2.3 & $-^{d}$ & -d & 0.14 & $>0.19$ & - d $^{d}$ & - $^{d}$ & 0.3 & 0.16 & 2.0 \\
\hline
\end{tabular}

a Other metals for which analysis was performed include beryllium, molybdenum, antimony, selenium, thallium, and silver. However, all values for those analyses were below detection limits.

b See Fig. 8-3 for sampling locations.

c Median value; see Quality Assurance chapter.

d No measure of dispersion calculated; see Quality Assurance chapter. 


\section{Soil and Sediment Monitoring}

Table 8-4. Positively detected organic compounds (in $\mathrm{mg} / \mathrm{kg}$ ) in arroyo sediments at the Livermore site, 1992.

\begin{tabular}{|c|c|c|c|c|c|c|c|c|c|}
\hline Location & $\begin{array}{c}\text { BHC } \\
\text { alpha } \\
\text { isomer }\end{array}$ & $\begin{array}{c}\text { BHC } \\
\text { beta } \\
\text { isomer }\end{array}$ & $\begin{array}{c}\text { Carbon } \\
\text { tetra- } \\
\text { chloride }\end{array}$ & $\begin{array}{c}\text { Chloro- } \\
\text { form }\end{array}$ & $\begin{array}{l}\text { Dichloro- } \\
\text { difluoro- } \\
\text { methane }\end{array}$ & Dieldrin & $\begin{array}{l}\text { Endo- } \\
\text { sulfan I }\end{array}$ & Endrin & $\begin{array}{l}\text { Endrin } \\
\text { aldehyde }\end{array}$ \\
\hline \multirow[t]{3}{*}{ 4THA } & 0.003 & $<0.002$ & $<0.0002$ & $<0.0002$ & $<0.0002$ & $<0.004$ & $<0.002$ & $<0.004$ & $<0.006$ \\
\hline & 0.002 & $<0.002$ & $<0.0002$ & $<0.0002$ & $<0.0002$ & $<0.004$ & $<0.002$ & $<0.004$ & $<0.006$ \\
\hline & 0.002 & $<0.002$ & $<0.0002$ & 0.0016 & $<0.0002$ & 0.008 & $<0.002$ & $<0.004$ & $<0.006$ \\
\hline \multirow[t]{3}{*}{ ALPE } & $<0.002$ & $<0.002$ & $<0.0002$ & $<0.0002$ & $<0.0002$ & $<0.004$ & $<0.002$ & $<0.004$ & $<0.006$ \\
\hline & $<0.001$ & $<0.001$ & $<0.0002$ & $<0.0002$ & $<0.0002$ & $<0.002$ & $<0.001$ & $<0.002$ & $<0.003$ \\
\hline & $<0.001$ & $<0.001$ & $<0.0002$ & $<0.0002$ & $<0.0002$ & $<0.002$ & $<0.001$ & $<0.002$ & $<0.003$ \\
\hline \multirow[t]{3}{*}{ ALPN } & $<0.001$ & $<0.001$ & $<0.0002$ & 0.0002 & $<0.0002$ & $<0.002$ & $<0.001$ & $<0.002$ & $<0.003$ \\
\hline & $<0.001$ & $<0.001$ & $<0.0002$ & $<0.0002$ & 0.0002 & $<0.002$ & $<0.001$ & $<0.002$ & $<0.003$ \\
\hline & $<0.001$ & $<0.001$ & $<0.0002$ & $<0.0002$ & $<0.0002$ & $<0.002$ & $<0.001$ & $<0.002$ & $<0.003$ \\
\hline \multirow[t]{3}{*}{ ALPW } & $<0.001$ & $<0.001$ & $<0.0002$ & $<0.0002$ & 0.0002 & $<0.002$ & 0.002 & 0.003 & $<0.003$ \\
\hline & $<0.001$ & $<0.001$ & $<0.0002$ & $<0.0002$ & 0.0002 & $<0.002$ & $<0.001$ & $<0.002$ & $<0.003$ \\
\hline & $<0.002$ & $<0.002$ & $<0.0002$ & 0.0013 & $<0.0002$ & $<0.004$ & $<0.002$ & $<0.004$ & $<0.006$ \\
\hline \multirow[t]{3}{*}{ ASS2 } & $<0.001$ & $<0.001$ & 0.0002 & 0.0002 & $<0.0002$ & $<0.002$ & $<0.001$ & $<0.002$ & $<0.003$ \\
\hline & $<0.001$ & $<0.001$ & 0.0002 & 0.0002 & $<0.0002$ & $<0.002$ & $<0.001$ & $<0.002$ & $<0.003$ \\
\hline & $<0.001$ & $<0.001$ & $<0.0002$ & $<0.0002$ & $<0.0002$ & $<0.002$ & $<0.001$ & $<0.002$ & $<0.003$ \\
\hline \multirow[t]{3}{*}{ ASW } & $<0.001$ & 0.002 & $<0.0002$ & $<0.0002$ & $<0.0002$ & $<0.002$ & $<0.001$ & $<0.002$ & $<0.003$ \\
\hline & $<0.001$ & $<0.001$ & $<0.0002$ & 0.0007 & $<0.0002$ & 0.004 & $<0.001$ & 0.005 & 0.003 \\
\hline & $<0.001$ & $<0.001$ & $<0.0002$ & $<0.0002$ & 0.0003 & $<0.002$ & $<0.001$ & $<0.002$ & $<0.003$ \\
\hline \multirow[t]{3}{*}{ B438 } & $<0.001$ & $<0.001$ & $<0.0002$ & $<0.0002$ & $<0.0002$ & $<0.002$ & $<0.001$ & $<0.002$ & $<0.003$ \\
\hline & $<0.001$ & $<0.001$ & $<0.0002$ & $<0.0002$ & $<0.0002$ & $<0.002$ & $<0.001$ & $<0.002$ & $<0.003$ \\
\hline & $<0.001$ & $<0.001$ & $<0.0002$ & $<0.0002$ & $<0.0002$ & $<0.002$ & $<0.001$ & $<0.002$ & $<0.003$ \\
\hline \multirow[t]{3}{*}{ CDB } & $<0.001$ & $<0.001$ & $<0.0002$ & $<0.0002$ & $<0.0002$ & $<0.002$ & $<0.001$ & $<0.002$ & $<0.003$ \\
\hline & $<0.001$ & $<0.001$ & $<0.0002$ & $<0.0002$ & $<0.0002$ & $<0.002$ & $<0.001$ & $<0.002$ & $<0.003$ \\
\hline & $<0.001$ & $<0.001$ & $<0.0002$ & $<0.0002$ & $<0.0002$ & $<0.002$ & $<0.001$ & $<0.002$ & $<0.003$ \\
\hline \multirow[t]{3}{*}{ GRNE } & $<0.001$ & $<0.001$ & $<0.0002$ & $<0.0002$ & $<0.0002$ & $<0.002$ & $<0.001$ & $<0.002$ & $<0.003$ \\
\hline & $<0.001$ & $<0.001$ & $<0.0002$ & $<0.0002$ & $<0.0002$ & $<0.002$ & $<0.001$ & $<0.002$ & $<0.003$ \\
\hline & $<0.001$ & $<0.001$ & $<0.0002$ & $<0.0002$ & $<0.0002$ & $<0.002$ & $<0.001$ & $<0.002$ & $<0.003$ \\
\hline \multirow[t]{3}{*}{ WPDC } & $<0.001$ & $<0.001$ & $<0.0002$ & $<0.0002$ & $<0.0002$ & $<0.002$ & $<0.001$ & $<0.002$ & $<0.003$ \\
\hline & $<0.001$ & $<0.001$ & $<0.0002$ & $<0.0002$ & $<0.0002$ & $<0.002$ & $<0.001$ & $<0.002$ & $<0.003$ \\
\hline & $<0.001$ & $<0.001$ & $<0.0002$ & $<0.0002$ & $<0.0002$ & $<0.002$ & $<0.001$ & $<0.002$ & $<0.003$ \\
\hline Median & $<0.001$ & $<0.001$ & $<0.0002$ & $<0.0002$ & $<0.0002$ & $<0.002$ & $<0.001$ & $<0.002$ & $<0.003$ \\
\hline Std dev & -b & $-b$ & $-b$ & -b & $-b$ & $-^{b}$ & $-^{b}$ & $-b$ & $-b$ \\
\hline
\end{tabular}


Table 8-4. Positively detected organic compounds (in $\mathrm{mg} / \mathrm{kg}$ ) in arroyo sediments at the Livermore site, 1992 (concluded).

\begin{tabular}{|c|c|c|c|c|c|c|c|c|c|}
\hline Location ${ }^{a}$ & $\begin{array}{c}\text { Freon- } \\
113\end{array}$ & $\begin{array}{c}\text { Methylene } \\
\text { chloride }\end{array}$ & $p, p-D D E^{c}$ & $\begin{array}{l}\text { Tetra- } \\
\text { chloro- } \\
\text { ethene }\end{array}$ & Toluene & $\begin{array}{c}\text { Total } \\
\text { xylene } \\
\text { Isomers }\end{array}$ & $\begin{array}{l}1,1,1- \\
\text { TCA }\end{array}$ & $\begin{array}{l}\text { Trichloro- } \\
\text { ethene }\end{array}$ & $\begin{array}{l}\text { Trichloro- } \\
\text { fluoro- } \\
\text { methane }\end{array}$ \\
\hline \multirow[t]{3}{*}{ 4THA } & $<0.0002$ & $<0.0002$ & $<0.004$ & $<0.0002$ & 0.005 & $<0.002$ & $<0.0002$ & $<0.0002$ & $<0.0002$ \\
\hline & $<0.0002$ & $<0.0002$ & $<0.004$ & $<0.0002$ & 0.010 & $<0.002$ & $<0.0002$ & $<0.0002$ & $<0.0002$ \\
\hline & 0.0009 & 0.0004 & 0.007 & 0.0002 & 0.027 & $<0.002$ & 0.0004 & $<0.0002$ & 0.0076 \\
\hline \multirow[t]{3}{*}{ ALPE } & $<0.0002$ & $<0.0002$ & $<0.004$ & $<0.0002$ & 0.004 & $<0.002$ & $<0.0002$ & $<0.0002$ & $<0.0002$ \\
\hline & $<0.0002$ & $<0.0002$ & $<0.002$ & $<0.0002$ & 0.010 & $<0.002$ & $<0.0002$ & $<0.0002$ & $<0.0002$ \\
\hline & $<0.0002$ & $<0.0002$ & $<0.002$ & $<0.0002$ & 0.014 & $<0.002$ & 0.0002 & $<0.0002$ & $<0.0002$ \\
\hline \multirow[t]{3}{*}{ ALPN } & $<0.0002$ & $<0.0002$ & $<0.002$ & $<0.0002$ & 0.015 & $<0.002$ & $<0.0002$ & $<0.0002$ & $<0.0002$ \\
\hline & $<0.0002$ & $<0.0002$ & $<0.002$ & $<0.0002$ & 0.008 & $<0.002$ & $<0.0002$ & $<0.0002$ & $<0.0002$ \\
\hline & $<0.0002$ & $<0.0002$ & $<0.002$ & $<0.0002$ & 0.003 & $<0.002$ & $<0.0002$ & $<0.0002$ & $<0.0002$ \\
\hline \multirow[t]{3}{*}{ ALPW } & $<0.0002$ & 0.0002 & $<0.002$ & $<0.0002$ & 0.090 & $<0.002$ & $<0.0002$ & $<0.0002$ & $<0.0002$ \\
\hline & $<0.0002$ & $<0.0002$ & $<0.002$ & $<0.0002$ & 0.027 & $<0.002$ & 0.0002 & 0.0003 & $<0.0002$ \\
\hline & $<0.0002$ & $<0.0002$ & $<0.004$ & $<0.0002$ & 0.028 & $<0.002$ & $<0.0002$ & $<0.0002$ & $<0.0002$ \\
\hline \multirow[t]{3}{*}{ ASS2 } & $<0.0002$ & 0.0066 & $<0.002$ & $<0.0002$ & 0.004 & $<0.002$ & $<0.0002$ & $<0.0002$ & $<0.0002$ \\
\hline & $<0.0002$ & 0.0064 & 0.003 & $<0.0002$ & 0.011 & $<0.002$ & $<0.0002$ & $<0.0002$ & 0.0004 \\
\hline & $<0.0002$ & 0.0020 & $<0.002$ & $<0.0002$ & 0.004 & 0.004 & $<0.0002$ & $<0.0002$ & $<0.0002$ \\
\hline \multirow[t]{3}{*}{ ASW } & $<0.0002$ & $<0.0002$ & 0.004 & $<0.0002$ & 0.012 & $<0.002$ & $<0.0002$ & $<0.0002$ & $<0.0002$ \\
\hline & $<0.0002$ & 0.0002 & $<0.002$ & 0.0034 & 0.033 & $<0.002$ & 0.0022 & 0.0006 & $<0.0002$ \\
\hline & $<0.0002$ & $<0.0002$ & 0.003 & $<0.0002$ & 0.006 & $<0.002$ & $<0.0002$ & $<0.0002$ & $<0.0002$ \\
\hline \multirow[t]{3}{*}{ B438 } & $<0.0002$ & $<0.0002$ & $<0.002$ & $<0.0002$ & $<0.002$ & $<0.002$ & $<0.0002$ & $<0.0002$ & $<0.0002$ \\
\hline & $<0.0002$ & $<0.0002$ & $<0.002$ & $<0.0002$ & 0.004 & $<0.002$ & $<0.0002$ & $<0.0002$ & $<0.0002$ \\
\hline & $<0.0002$ & $<0.0002$ & $<0.002$ & $<0.0002$ & 0.004 & $<0.002$ & $<0.0002$ & $<0.0002$ & $<0.0002$ \\
\hline \multirow[t]{3}{*}{ CDB } & $<0.0002$ & 0.0002 & $<0.002$ & 0.0002 & 0.043 & $<0.002$ & 0.0003 & 0.0011 & $<0.0002$ \\
\hline & 0.0004 & $<0.0002$ & $<0.002$ & 0.0032 & 0.012 & $<0.002$ & $<0.0002$ & 0.0006 & 0.0003 \\
\hline & $<0.0002$ & $<0.0002$ & $<0.002$ & $<0.0002$ & 0.008 & $<0.002$ & $<0.0002$ & 0.0003 & $<0.0002$ \\
\hline \multirow[t]{3}{*}{ GRNE } & $<0.0002$ & $<0.0002$ & $<0.002$ & $<0.0002$ & $<0.002$ & $<0.002$ & $<0.0002$ & $<0.0002$ & $<0.0002$ \\
\hline & $<0.0002$ & $<0.0002$ & $<0.002$ & $<0.0002$ & $<0.002$ & $<0.002$ & $<0.0002$ & $<0.0002$ & $<0.0002$ \\
\hline & $<0.0002$ & $<0.0002$ & $<0.002$ & $<0.0002$ & $<0.002$ & $<0.002$ & $<0.0002$ & $<0.0002$ & $<0.0002$ \\
\hline \multirow[t]{3}{*}{ WPDC } & $<0.0002$ & $<0.0002$ & $<0.002$ & $<0.0002$ & 0.004 & $<0.002$ & $<0.0002$ & $<0.0002$ & $<0.0002$ \\
\hline & $<0.0002$ & $<0.0002$ & $<0.002$ & $<0.0002$ & 0.012 & $<0.002$ & $<0.0002$ & $<0.0002$ & $<0.0002$ \\
\hline & $<0.0002$ & $<0.0002$ & $<0.002$ & $<0.0002$ & 0.002 & $<0.002$ & $<0.0002$ & $<0.0002$ & $<0.0002$ \\
\hline Median & $<0.0002$ & $<0.0002$ & $<0.002$ & $<0.0002$ & $<0.014^{\circ}$ & $<0.002$ & $<0.0002$ & $<0.0002$ & $<0.0002$ \\
\hline Std dev & - b & $-^{b}$ & $-{ }^{b}$ & $-b$ & $>0.018$ & $-b$ & $-^{b}$ & $-b$ & $-^{\mathbf{b}}$ \\
\hline
\end{tabular}

a See Fig 8-3 for sampling locations.

b No measure of dispersion calculated; see Quality Assurance chapter.

c $p, p^{\prime}-D D E ; p, p^{\prime}$-dichlorodiphenyldichloroethylene; or 2,2-bis(4-chlorophenyl)-1,1-dichloroethylene.

d 1,1,1-Trichioroethane.

- Mean value; see Quality Assurance chapter. 


\section{Soil and Sediment Monitoring}

Table 8-5. Positively detected radionuclides and beryllium in soil sampled at depths from 0 to $50 \mathrm{~mm}$, Site 300, 1992.

\begin{tabular}{|c|c|c|c|c|c|c|c|}
\hline \multirow[b]{2}{*}{ Locationa } & ${ }^{137} \mathrm{Cs}$ & & ${ }^{39} \mathrm{Pu}$ & & ${ }^{40} K$ & ${ }^{226} \mathbf{R a}$ & ${ }^{228} \mathrm{Ra}$ \\
\hline & \multicolumn{3}{|c|}{$\left(10^{-3} \mathrm{~Bq} / \mathrm{dry} \mathrm{g}\right)$} & \multicolumn{4}{|c|}{ (Bq/dry g) } \\
\hline $801 E$ & $3.4 \pm 0.3$ & 0.070 & \pm 0.008 & 0.53 & \pm 0.02 & $0.038 \pm 0.0008$ & $0.034 \pm 0.001$ \\
\hline $801 \mathrm{~N}$ & $4.7 \pm 0.2$ & 0.12 & \pm 0.011 & 0.39 & \pm 0.01 & $0.032 \pm 0.0006$ & $0.029 \pm 0.001$ \\
\hline $801 W$ & $1.6 \pm 0.3$ & 0.073 & \pm 0.008 & 0.54 & \pm 0.02 & $0.044 \pm 0.0009$ & $0.044 \pm 0.001$ \\
\hline 812N & $0.3 \pm 0.2$ & 0.011 & \pm 0.003 & 0.33 & \pm 0.01 & $0.014 \pm 0.0004$ & $0.014 \pm 0.001$ \\
\hline $834 W$ & $0.5 \pm 0.2$ & 0.016 & \pm 0.004 & 0.50 & \pm 0.02 & $0.038 \pm 0.0008$ & $0.041 \pm 0.002$ \\
\hline $851 N$ & $3.8 \pm 0.3$ & 0.10 & \pm 0.012 & 0.49 & \pm 0.02 & $0.043 \pm 0.0009$ & $0.050 \pm 0.002$ \\
\hline $856 \mathrm{~N}$ & $3.0 \pm 0.2$ & 0.078 & \pm 0.010 & 0.39 & \pm 0.01 & $0.025 \pm 0.0005$ & $0.033 \pm 0.001$ \\
\hline $858 \mathrm{~S}$ & $3.2 \pm 0.3$ & 0.080 & \pm 0.011 & 0.43 & \pm 0.01 & $0.030 \pm 0.0006$ & $0.033 \pm 0.001$ \\
\hline DSW & $8.9 \pm 0.4$ & 0.22 & \pm 0.018 & 0.47 & \pm 0.01 & $0.032 \pm 0.0006$ & $0.030 \pm 0.001$ \\
\hline EOBS & $<0.1^{b}$ & 0.066 & \pm 0.010 & 0.51 & \pm 0.01 & $0.024 \pm 0.0005$ & $0.035 \pm 0.001$ \\
\hline EVAP & $4.5 \pm 0.3$ & 0.060 & \pm 0.007 & 0.39 & \pm 0.02 & $0.030 \pm 0.0006$ & $0.032 \pm 0.001$ \\
\hline GOLF & $2.8 \pm 0.3$ & 0.0085 & $5 \pm 0.003$ & 0.49 & \pm 0.01 & $0.028 \pm 0.0006$ & $0.028 \pm 0.001$ \\
\hline NPS & $3.7 \pm 0.3$ & 0.092 & \pm 0.009 & 0.63 & \pm 0.02 & $0.028 \pm 0.0006$ & $0.030 \pm 0.001$ \\
\hline WOBS & $9.5 \pm 0.4$ & 0.25 & \pm 0.022 & 0.42 & \pm 0.01 & $0.023 \pm 0.0005$ & $0.026 \pm 0.001$ \\
\hline Mean & $<3.6$ & 0.089 & & 0.47 & & 0.031 & 0.033 \\
\hline \multirow[t]{2}{*}{ Std dev } & $>2.8$ & 0.070 & & 0.078 & & 0.0082 & 0.0085 \\
\hline & \multicolumn{3}{|c|}{$\left(10^{-3} \mathrm{pCi} / \mathrm{dry} \mathrm{g}\right)$} & \multicolumn{4}{|c|}{ (pCi/dry g) } \\
\hline Mean & $>97$ & 2.4 & & 13 & & 0.84 & 0.89 \\
\hline Std dev & $<76$ & 1.9 & & 2.1 & & 0.22 & 0.23 \\
\hline
\end{tabular}


Table 8-5. Positively detected radionuclides and beryllium in soil sampled at depths from 0 to $50 \mathrm{~mm}$, Site 300, 1992 (concluded).

\begin{tabular}{|c|c|c|c|c|}
\hline & 232Th & $235 U$ & $238 \mathrm{U}$ & Be \\
\hline Location" & & ( $\mu g /$ dry $g)$ & & (mg/kg) \\
\hline B01E & $10 \pm 0.3$ & $0.030 \pm 0.0090$ & $6.7 \pm 3.8$ & 1.28 \\
\hline $801 N$ & $7.5 \pm 0.4$ & $0.024 \pm 0.0077$ & $2.7 \pm 1.3$ & 2.50 \\
\hline $801 W$ & $13 \pm 0.3$ & $0.027 \pm 0.0079$ & $5.1 \pm 4.1$ & 1.61 \\
\hline $812 N$ & $4.0 \pm 0.1$ & $0.035 \pm 0.0045$ & $12 \pm 2.0$ & 0.34 \\
\hline $834 W$ & $12 \pm 0.4$ & $0.020 \pm 0.0071$ & $3.0 \pm 2.2$ & 1.00 \\
\hline $851 N$ & $14 \pm 0.3$ & $0.030 \pm 0.0069$ & $6.4 \pm 3.5$ & 1.07 \\
\hline $856 \mathrm{~N}$ & $8.6 \pm 0.3$ & $0.029 \pm 0.0068$ & $2.7 \pm 2.0$ & 1.09 \\
\hline 8585 & $9.2 \pm 0.3$ & $0.026 \pm 0.0073$ & $4.7 \pm 2.4$ & 1.11 \\
\hline DSW & $8.7 \pm 0.2$ & $0.033 \pm 0.0049$ & $5.1 \pm 2.5$ & 0.63 \\
\hline EOBS & $10 \pm 0.2$ & $0.019 \pm 0.0047$ & $<3.1$ & 0.73 \\
\hline EVAP & $9.1 \pm 0.3$ & $0.030 \pm 0.0054$ & $4.8 \pm 2.0$ & 0.54 \\
\hline GOLF & $7.7 \pm 0.2$ & $0.020 \pm 0.0044$ & $2.4 \pm 2.1$ & 0.77 \\
\hline NPS & $8.3 \pm 0.2$ & $0.025 \pm 0.0046$ & $2.4 \pm 2.3$ & 0.48 \\
\hline WOBS & $7.8 \pm 0.2$ & $0.017 \pm 0.0049$ & $<3.0$ & 0.51 \\
\hline Mean & 9.3 & 0.026 & $<4.6$ & 0.98 \\
\hline Std dev & 2.5 & 0.0055 & $>2.6$ & 0.56 \\
\hline
\end{tabular}

Note: Radionuclide results are reported $\pm 2 \sigma$; see Quality Assurance chapter.

a See Fig. 8-2 for sampling locations.

b A duplicate sample taken at this location yielded the same result. 


\section{Soil and Sediment Monitoring}

Table 8-6. Positively detected radionuclides in arroyo sediments (0 to $50 \mathrm{~mm}$ ) near the Livermore site, 1992.

\begin{tabular}{|c|c|c|c|c|c|c|}
\hline \multirow[b]{2}{*}{ Locationa } & ${ }^{7} \mathrm{Be}$ & ${ }^{137} \mathrm{Cs}$ & ${ }^{239} \mathrm{Pu}$ & ${ }^{40} K$ & ${ }^{226} \mathrm{Ra}$ & ${ }^{228} \mathrm{Ra}$ \\
\hline & \multicolumn{3}{|c|}{$\left(10^{-3} \mathrm{~Bq} / \mathrm{dry} \mathrm{g}\right)$} & \multicolumn{3}{|c|}{ (Bq/dry g) } \\
\hline 4THA & $<2.3$ & $4.0 \pm 0.28$ & $0.23 \pm 0.015$ & $0.48 \pm 0.01$ & $0.024 \pm 0.0005$ & $0.024 \pm 0.0010$ \\
\hline ALPE & $<2.6$ & $0.74 \pm 0.24$ & $0.038 \pm 0.005$ & $0.34 \pm 0.01$ & $0.016 \pm 0.0003$ & $0.015 \pm 0.0007$ \\
\hline ALPN & $5.7 \pm 4.6$ & $<0.074$ & $0.0059 \pm 0.002$ & $0.54 \pm 0.02$ & $0.013 \pm 0.0004$ & $0.013 \pm 0.0007$ \\
\hline ALPW & $<2.4$ & $0.89 \pm 0.24$ & $0.041 \pm 0.006$ & $0.54 \pm 0.02$ & $0.025 \pm 0.0005$ & $0.026 \pm 0.0010$ \\
\hline ASS2 & $10 \pm 6.4$ & $1.0 \pm 0.23$ & $0.025 \pm 0.004$ & $0.53 \pm 0.01$ & $0.020 \pm 0.0004$ & $0.020 \pm 0.0008$ \\
\hline ASW & $4.5 \pm 2.9$ & $0.41 \pm 0.10$ & $0.018 \pm 0.004$ & $0.31 \pm 0.01$ & $0.011 \pm 0.0003$ & $0.011 \pm 0.0006$ \\
\hline B438 & $<7.8$ & $<0.52$ & $0.37 \pm 0.022$ & $0.47 \pm 0.01$ & $0.020 \pm 0.0006$ & $0.023 \pm 0.0009$ \\
\hline CDB & $4.1 \pm 3.6$ & $0.74 \pm 0.16$ & $1.5 \pm 0.062$ & $0.52 \pm 0.02$ & $0.026 \pm 0.0005$ & $0.026 \pm 0.0011$ \\
\hline GRNE & $<2.3$ & $1.0 \pm 0.19$ & $0.029 \pm 0.004$ & $0.47 \pm 0.01$ & $0.021 \pm 0.0006$ & $0.022 \pm 0.0011$ \\
\hline WPDC & $<2.5$ & $<0.11$ & $0.0019 \pm 0.001$ & $0.51 \pm 0.02$ & $0.029 \pm 0.0006$ & $0.032 \pm 0.0010$ \\
\hline Mean & $<3.4^{b}$ & $0.74^{b}$ & 0.23 & 0.47 & 0.021 & 0.021 \\
\hline \multirow[t]{2}{*}{ Std dev } & $-c$ & $-c$ & 0.46 & 0.082 & 0.0058 & 0.0065 \\
\hline & \multicolumn{3}{|c|}{$\left(10^{-3} \mathrm{pCi} / \mathrm{dry} \mathrm{g}\right)$} & \multicolumn{3}{|c|}{ (pCi/dry g) } \\
\hline Mean & $<92^{b}$ & $20^{\mathrm{b}}$ & 6.2 & 13 & 0.57 & 0.57 \\
\hline Std dev & $-c$ & $-^{c}$ & 12 & 2.2 & 0.16 & 0.18 \\
\hline
\end{tabular}

\begin{tabular}{|c|c|c|c|}
\hline \multirow[b]{2}{*}{ Locationa } & ${ }^{232} \mathrm{Th}$ & $235 U$ & ${ }^{238} \mathrm{U}$ \\
\hline & \multicolumn{3}{|c|}{$(\mu g /$ dry $g)$} \\
\hline 4THA & $6.8 \pm 0.2$ & $0.020 \pm 0.0040$ & $3.03 \pm 3.00$ \\
\hline ALPE & $4.4 \pm 0.1$ & $0.011 \pm 0.0038$ & $<1.2$ \\
\hline ALPN & $3.6 \pm 0.1$ & $0.012 \pm 0.0034$ & $<1.6$ \\
\hline ALPW & $7.3 \pm 0.3$ & $0.021 \pm 0.0053$ & $2.8 \pm 2.3$ \\
\hline ASS2 & $5.9 \pm 0.1$ & $0.018 \pm 0.0040$ & $<2.0$ \\
\hline ASW & $2.8 \pm 0.2$ & $0.015 \pm 0.0051$ & $0.7 \pm 0.6$ \\
\hline B438 & $6.6 \pm 0.2$ & $0.018 \pm 0.0053$ & $<1.5$ \\
\hline CDB & $6.9 \pm 0.3$ & $0.027 \pm 0.0064$ & $3.5 \pm 2.2$ \\
\hline GRNE & $5.9 \pm 0.2$ & $0.020 \pm 0.0049$ & $<1.6$ \\
\hline WPDC & $8.9 \pm 0.3$ & $0.028 \pm 0.0050$ & $3.8 \pm 2.9$ \\
\hline Mean & 5.9 & 0.019 & $<1.8^{b}$ \\
\hline Std dev & 1.8 & 0.006 & $-c$ \\
\hline
\end{tabular}

Note: Radionuclide results are reported $\pm 2 \sigma$; see Quality Assurance chapter.

a See Fig. 8-3 for sampling locations.

b Median value; see Quality Assurance chapter.

c No measure of dispersion calculated; see Quality Assurance chapter. 
Table 8-7. Positively detected metals ${ }^{a}$ (in $\mathrm{mg} / \mathrm{L}$ ) in arroyo sediments (0 to $50 \mathrm{~mm}$ depth) near the Livermore site, 1992.

\begin{tabular}{lcccccccccc}
\hline Location $^{b}$ & As & Ba & Cd & Total Cr & Co & Cu & Pb & NI & V & Zn \\
\hline 4THA & 0.095 & 7.5 & 0.12 & 0.26 & 0.55 & 2.5 & 4.1 & 0.62 & 0.26 & 26 \\
ALPE & $<0.02$ & 8.3 & $<0.05$ & 0.17 & 0.30 & 0.22 & 1.2 & 0.43 & 0.15 & 9.1 \\
ALPN & $<0.02$ & 4.6 & $<0.05$ & $<0.05$ & 0.34 & 0.078 & $<0.2$ & 0.41 & 0.17 & 1.8 \\
ALPW & 0.056 & 10 & $<0.05$ & 0.12 & 0.61 & 0.24 & $<0.2$ & 0.71 & 0.44 & 1.1 \\
ASS2 & $<0.02$ & 7.6 & $<0.05$ & $<0.05$ & 0.37 & 0.18 & $<0.2$ & 0.4 & 0.22 & 2.4 \\
ASW & $<0.02$ & 4.9 & $<0.05$ & $<0.05$ & 0.25 & 0.15 & $<0.2$ & 0.31 & 0.13 & 3.7 \\
B438 & $<0.02$ & 9.2 & $<0.05$ & 0.064 & 0.54 & 0.34 & $<0.2$ & 0.79 & 0.22 & 6.9 \\
CDB & 0.031 & 10 & $<0.05$ & $<0.05$ & 0.58 & 0.37 & $<0.2$ & 0.68 & 0.38 & 3.1 \\
GRNE & 0.045 & 11 & $<0.05$ & $<0.05$ & 0.42 & 0.44 & 2.2 & 0.54 & 0.42 & 9.7 \\
WPDC & $<0.02$ & 14 & $<0.05$ & 0.05 & 0.70 & 0.15 & $<0.2$ & 0.96 & 0.39 & 0.25 \\
& & & & & & & & & & \\
Mean & $<0.02 \mathrm{c}$ & $\mathbf{8 . 7}$ & $<0.05^{\mathrm{c}}$ & $<0.05^{\mathrm{c}}$ & $\mathbf{0 . 4 7}$ & $\mathbf{0 . 4 7}$ & $<0.2^{\mathrm{c}}$ & $\mathbf{0 . 6}$ & $\mathbf{0 . 2 8}$ & $\mathbf{6 . 4}$ \\
Std dov & $-\mathrm{d}$ & $\mathbf{2 . 8}$ & $-\mathrm{d}$ & $-\mathrm{d}$ & $\mathbf{0 . 1 5}$ & $\mathbf{0 . 7 2}$ & $-\mathrm{d}$ & $\mathbf{0 . 2}$ & $\mathbf{0 . 1 2}$ & $\mathbf{7 . 6}$ \\
\hline
\end{tabular}

a Other metals for which analysis was performed include beryllium, molybdenum, antimony, selenium, thallium, and silver. However, all values for those analyses were below detection limits.

b See Fig. 8-3 for sampling locations.

c Median value; see Quality Assurance chapter.

d No measure of dispersion calculated; see Quality Assurance chapter. 


\section{Introduction}

Because vegetation can be a biological end point for pollutants originally released to the soil, to the air, or through liquid effluents, the sampling and analysis of native vegetation can provide information about the presence and movement of radionuclides in the environment. Contaminated vegetation can contribute radiation dose to humans through direct ingestion or indirectly through the products from animals that have consumed it.

DOE guidance states that "periodic sampling and analysis of indicator materials, such as soil or vegetation, should be performed to determine if there is measurable long-term buildup of radionuclides in the terrestrial environment." Terrestrial foods are specifically mentioned in the Environmental Regulatory Guide for Radiological Effluent Monitoring and Environmental Surveillance (U.S. Department of Energy 1991) as appropriate for surveillance sampling and analysis, even if the potential annual effective dose equivalent from ingestion is below $0.01 \mathrm{mSv}$ (1.0 mrem). The DOE guidance also calls for pathway analyses, and particularly mentions the need for analyses of milk and other representative samples of pathway-significant agricultural products grown in the proximity of the LLNL site (within 16 kilometers).

Since 1972, vegetation and foodstuff sampling in the vicinity of LLNL and Site 300 has been part of a continuing LLNL monitoring program designed to measure any changes in environmental levels of radioactivity, to evaluate any increase in radioactivity that might have resulted from LLNL operations, and to calculate potential human doses resulting from direct and indirect ingestion of these items. During 1992, LLNL collected and analyzed samples of vegetation, milk, honey, and wine. Using the monitoring data and dose models presented in Appendix B, LLNL calculated potential human doses from all of these substances except honey. Dose assessments were not made for honey because there is very limited information regarding its consumption.

Tritium is the nuclide of major interest in the LLNL vegetation and foodstuff monitoring program because LLNL has historically released tritium to the air both accidentally and in the course of routine operations. Tritium has the propensity to move into the environment as tritiated water, and as such can be assimilated easily into vegetation and foodstuff. In this manner, it can contribute to human radiation dose burdens if it is ingested directly or indirectly. 
All LLNL environmental surveillance monitoring networks are subject to constant evaluation. Changes and additions to the vegetation and foodstuff monitoring networks since 1988, including those instituted during 1992, are evidence of the LLNL goal to more adequately monitor all media potentially affected by L.LNL operations. Vegetation and foodstuff monitoring has increased to meet increasing regulatory requirements and self-imposed monitoring and assessment goals. Moreover, the radioanalytical methods used in the LLNL vegetation and foodstuff monitoring program are state-of-the-art and, in fact, detect radionuclides at levels far more sensitive than any required by regulatory limits.

Because of the importance of food to human life, the potential effects of LLNL operations on local foodstuffs and agricultural products are often the subject of public and regulatory concern. This concern justifies the current level of monitoring and the use of sophisticated analytical techniques. It is unlikely that any realistic significant impacts to vegetation and local foodstuff could be produced by LLNL operations and not be detected by the current monitoring networks.

\section{Methods}

\section{Vegetation}

LLNL surveillance vegetation samples, usually annual grasses, were collected quarterly from fixed locations in the Livermore Valley, the San Joaqui Valley, the San Ramon Valley, and Site 300 and then analyzed for tritium. Sampling locations in 1992 were the same as those sampled during 1991; location maps are provided in Figures 9-1 and 9-2. These locations were originally selected so samples would represent vegetation that (1) had proximity to LLNL and the potential for being affected by LLNL operations, (2) was from background locations where vegetation was similar to that which grows near LLNL, but unlikely to be affected by LLNL operations, and (3) was from areas of known or suspected LLNL-induced contamination.

All vegetation sampling was conducted according to written and approved standardized procedures (Gallegos et al. 1992b). For all vegetation sample collections, frequently tilled or disturbed areas and locations near buildings or other obstructions were avoided. The selected areas were unshaded and exhibited native vegetation for much of the year. Areas with unusual wind or precipitation/irrigation influences also were avoided. Practical considerations also tempered the location selections. These included access during inclement weather, personnel safety in vehicle operation, vehicle parking, or sample collection requirements.

The routine vegetation sampling locations are in areas designated with permanent location markers. Consistent use of the same general sampling locations allows for more meaningful trending of data and closer monitoring of areas of concern. Such is the case regarding Site 300 vegetation sampling activities; vegetation from areas where tritium is known to be present in the subsurface soil are examined closely each year. 
In 1992, vegetation samples usually consisted of the green leaves and green stems of annual grasses. Other herbaceous vegetation or even perennial vegetation was sampled if grasses were not available. Approximately 0.5 to 1 kilogram of vegetation was collected for analysis. Two subsamples of vegetation comprised a single sample. The laboratory analyzed one subsample and archived the other in a freezer in case the sample had to be reanalyzed due to loss of sample or a questionable analytical result.

Before handling any sample, technicians put on clean, disposable vinyl gloves so the sample would not be contaminated. Shears (decontaminated between samples) were used to trim the vegetation near the base of the plants. Any obviously dead leaves or stems were removed, and any root material was trimmed and discarded. Samples were double-bagged in plastic to avoid sample contamination, placed on dry ice in an ice chest, and kept frozen (to minimize water loss to the bag's surface) until analysis.

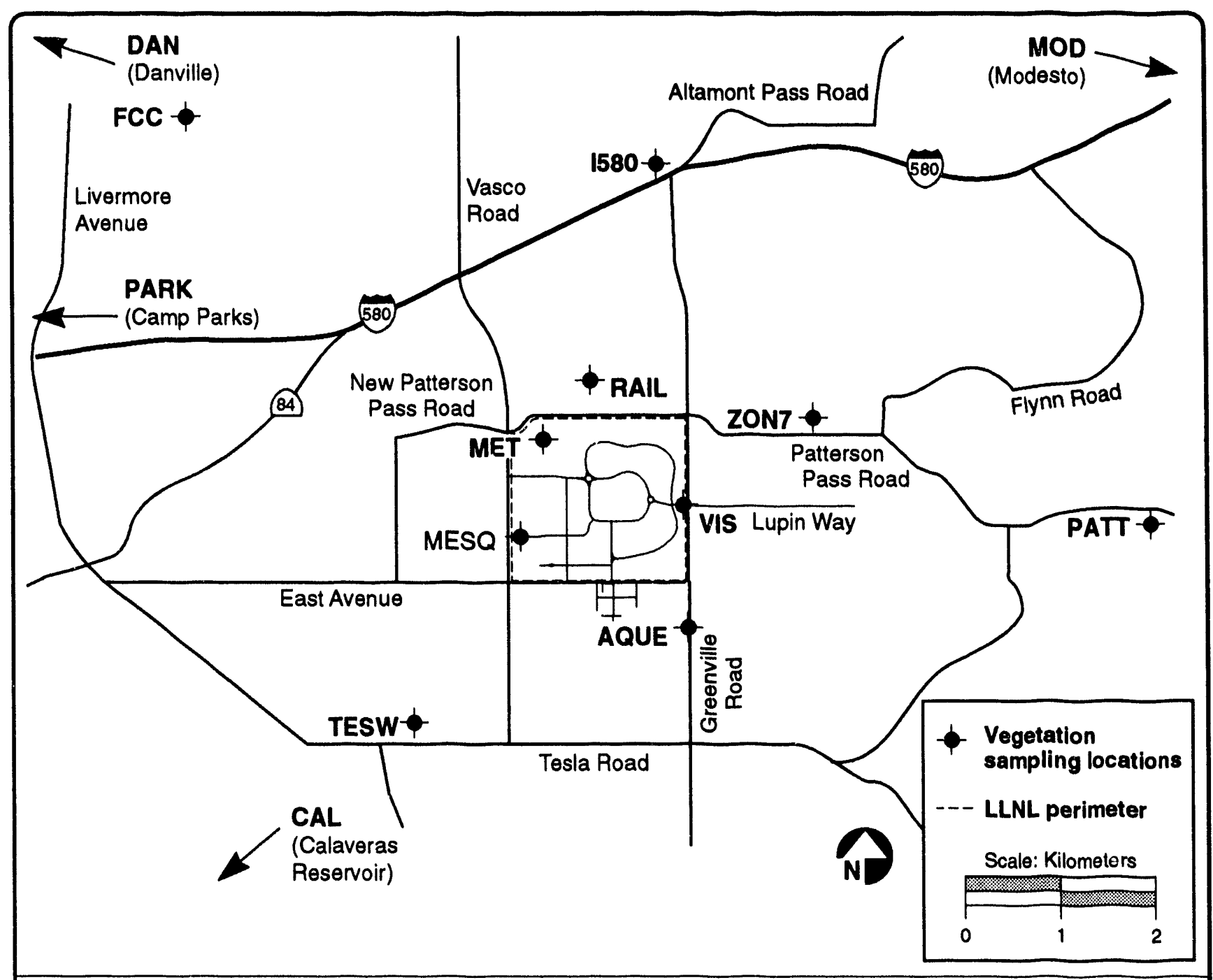

Figure 9-1. Livermore Valley vegetation sampling locations, 1992. 
Samples were identified with a tag on which a sample identification code, which includes the location designator, was written. This code was recorded on a Field Tracking Form, along with the distance and direction of the actual sampling location from the permanent sampling location marker. Other pertinent information about the sample, such as the species (if known) or generalized plant group (e.g., annual grass, herb, perennial shrub, tree) was also recorded. Standard chain-of-custody procedures were followed.

Samples were delivered on the day of collection to LLNL's Radiation Analytical Sciences laboratory, and were kept frozen prior to processing. Water from the vegetation was collected using freeze-drying techniques (lyophilization), and the tritium content of the water was determined by liquid-scintillation counting.

Approximately $10 \%$ of the sites were sampled in duplicate to comply with quality assurance protocols (Holland 1987). Duplicate collections consisted of four subsamples of vegetation collected from a sampling location using identical methodology. Two of the

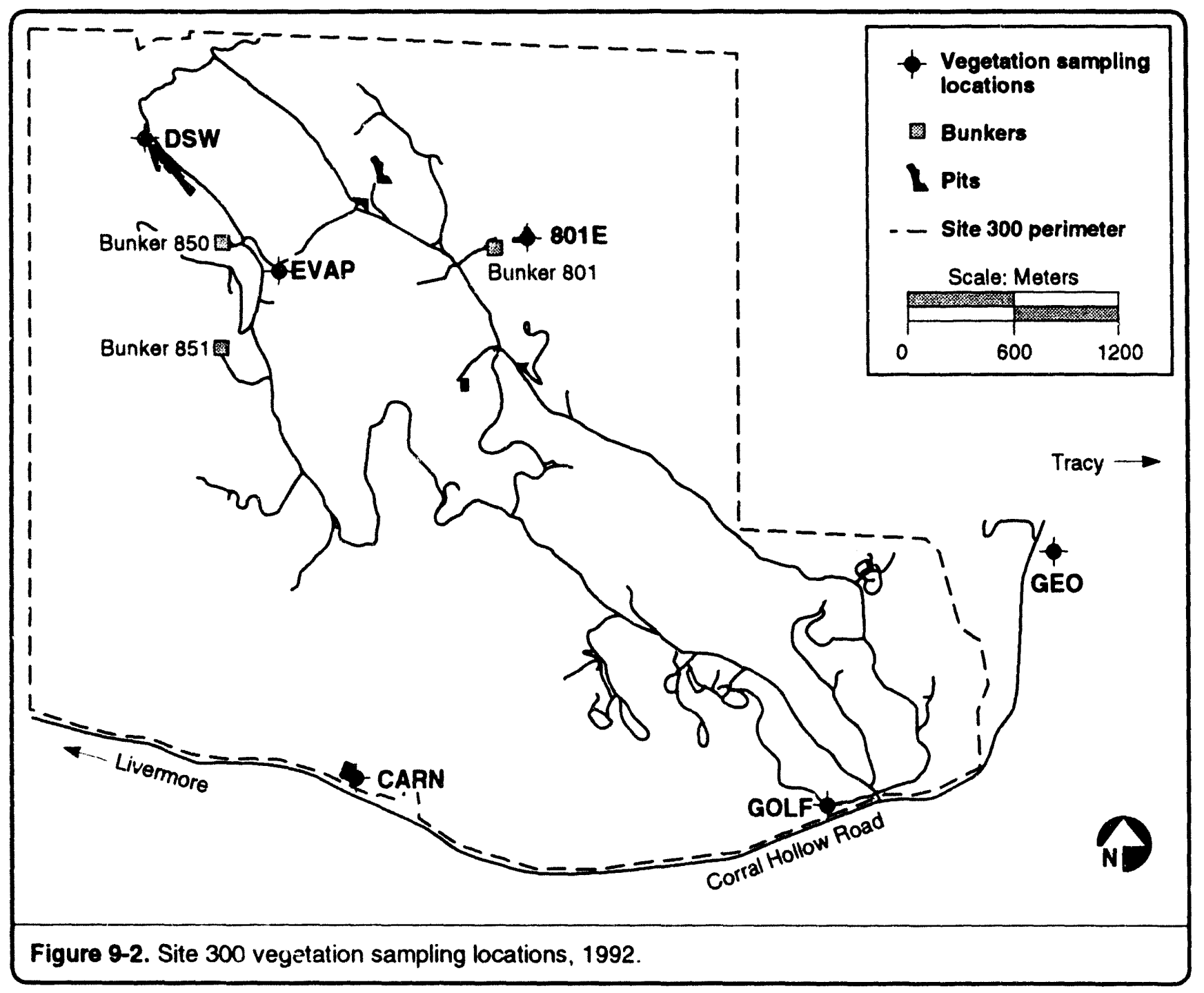


subsamples served as the official sample, while the other two subsamples were considered to be duplicates. Duplicate samples were preserved, stored, processed, and analyzed with methods identical to those employed for all other samples.

Milk

Milk is widely consumed by all age groups, and therefore the U.S. Department of Energy (1991) considers it important as one of the foods most frequently contributing radiation dose to people when dairy animals are pastured near a nuclear site. DOE guidance on milk sampling states that the number of sampling locations depends on how many milk production facilities there are, but should include at a minimum one background location and one potentially affected location. Milk sampling locations around LLNL are limited by the fact that there is only one cow milk facility near LLNL. As a result, LLNL obtained goat milk samples from producers in the Livermore Valley (potentially affected locations, all within 5 kilometers of LLNL) and from the Central Valley of California (to represent background locations for comparative purposes). Milk samples generally were collected where the goats were being raised (Figure 9-3). However, one set of samples was purchased from a grocery store, and another was purchased at a goat-milk plant in the Central Valley. A cow milk sample became available during mid-1992 and was collected and analyzed for comparative purposes.

Milk samples were collected monthly except during the months of December and January, when the goat's milk production was limited or absent. Milk production was variable, so samples were obtained as available. All of the milk samples were obtained in precooled, one-gallon sample containers provided by LLNL and were kept refrigerated until analysis.

The samples generally were delivered on the day of collection to LLNL's Radiation Analytical Sciences laboratory. If not delivered on the same day, samples were kept secure and refrigerated until they could be delivered. Chain-of-custody procedures were followed throughout the delivery and analytical process.

The laboratory analyzed the samples for tritium and gamma-emitting isotopes (to determine background milk doses from fallout and naturally occurring radionuclides). A one-liter aliquot of each milk sample was preserved with formalin at the laboratory and was analyzed, in a Marinelli beaker with a $\mathrm{Ge}(\mathrm{Li})$ spectrometer, for gamma-emitting isotopes. The remainder of each sample was freeze-dried (lyophilization) and the resultant water was analyzed for tritium by liquid-scintillation counting. At least one milk location was sampled in duplicate each month to comply with quality assurance protocols (Holland 1987).

\section{Honey}

Honey is another local agricultural product that potentially can be affected by LLNL operations. Bees collecting pollen and nectar from contaminated vegetation may pass the contamination along to their honey. Human ingestion of contaminated honey can be a pathway contributing to total radiation dose. As was the case for milk, the honey sampling network design was limited by the number of the honey producers in the sampling 
area. During 1992, LLNL obtained three honey samples with a variety of flower sources from beekeepers in the Livermore Valley and three samples from background locations.

Honey samples were collected during September and October. We contacted the sample providers in advance to ensure that the honey collected was from 1992 and was from the desired general location. Each sample consisted of one to three pounds of honey. The honey was transferred to standard sample containers that were labeled for identification. Information provided by the honey producers was recorded along with other information about the samples, such as where the honey was actually produced, where the bees gathered pollen, and what flower species were involved.

Chain-of-custody procedures were followed when delivering samples to LLNL's Radiation Analytical Sciences laboratory and throughout the analytical process. Honey samples were analyzed for tritium by combusting the samples in a Parr bomb and performing liquid-scintillation counting of the water that was produced during combustion.

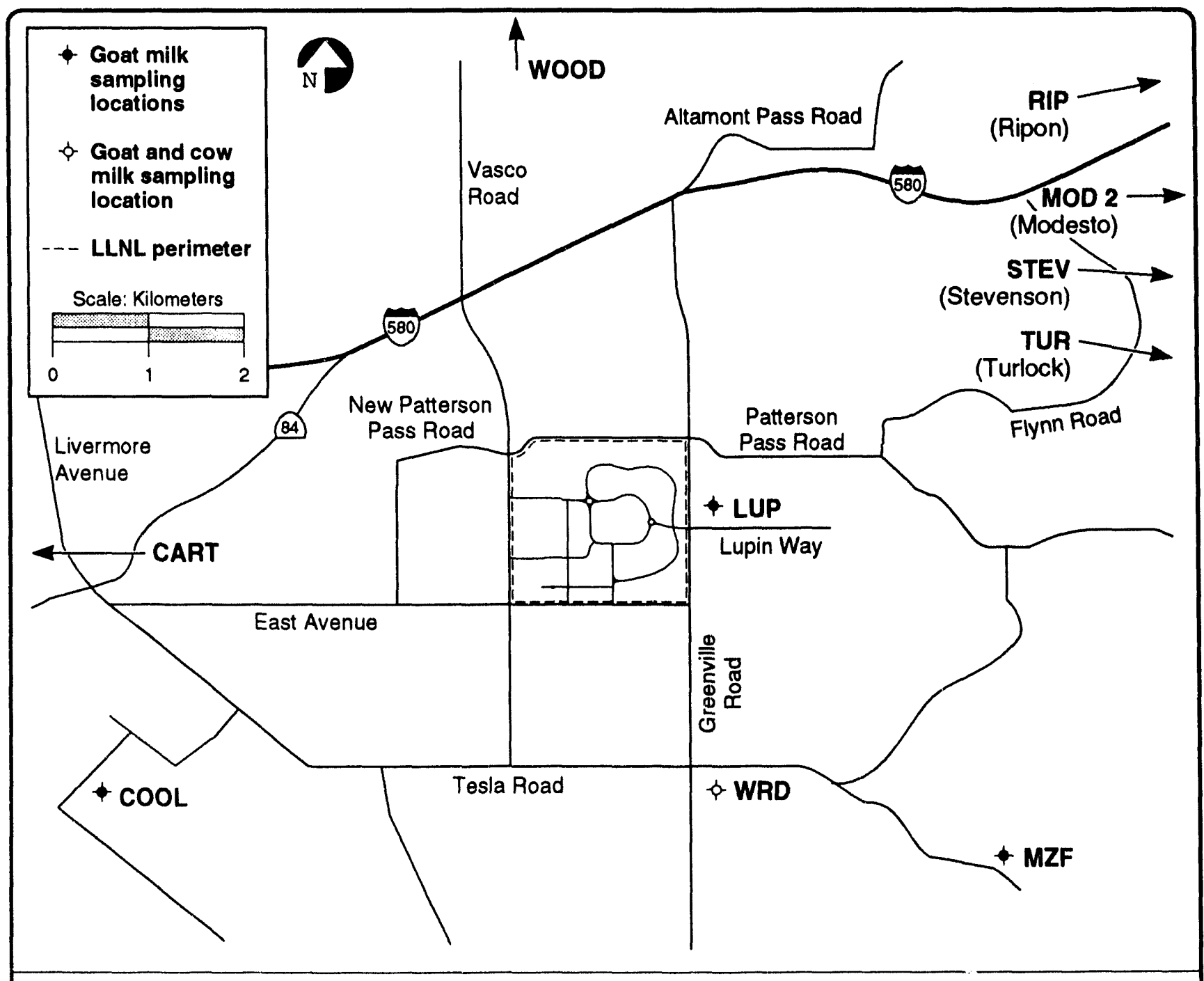

Figure 9-3. Goat and cow milk sampling locations, 1992. 
Honey-sample submittals complied with quality assurance protocols (Holland 1987).

Wine

Wine is the most important agricultural product in the Livermore Valley, representing an approximately $\$ 30$-million annual industry. Data since monitoring began have indicated that although tritium concentrations in all wines are low, Livermore Valley wines contain statistically more tritium than do their California counterparts.

Wine samples of three types were collected and analyzed for tritium concentrations: wine produced from grapes grown in the Livermore Valley, wines produced from grapes grown in California outside the Livermore Valley, and wines produced from grapes grown in Europe (France, Germany, and Italy). The latter two groups were divided into eight and thirteen wine-producing regions, respectively, and were used as comparative samples.

The wine samples were purchased from local retailers in a variety of vintages and thus reflect the body of wines available to the general public during 1992. The resulting analytical data can be used to estimate the potential tritium dose received by consumers during the year of purchase. The 1992 sampling data cannot, however, be used to indicate how the year's operations at LLNL affected wines produced in 1992. Some time-in some cases, several years-will have elapsed between the harvest of the grapes and the release of the vintage. However, wine sample data can be decay-corrected to its original tritium concentrations (given the number of months that have elapsed between wine production and LLNL analysis) to determine trends and to help determine the impact of LLNL operations during a particular vintage year.

Wine samples were purchased in 750-milliliter to 1-liter bottles. One wine from four of the eight non-Livermore, California wine growing regions and one wine from six of the thirteen European wine growing regions was purchased and submitted for tritium analyses. The selection of samples from all the wines available within a geographic area was random. Any estate wine from a designated area was considered representative of that area. The most recent vintages available were collected, with an equal mix of red and white wines. Approximately $10 \%$ of the total complement of wines were sampled in duplicate to comply with quality assurance protocols (Holland 1987). Because of the importance of the wine sampling network, LLNL sampled and analyzed as many of the available Livermore Valley wines as possible. Twelve Livermore Valley estate wines not previously sampled were purchased and analyzed.

The wine samples were submitted for analysis unopened, to avoid airborne tritium contamination. Chain-of-custody procedures were followed when delivering samples and throughout the analytical process. Wines were analyzed for tritium using ${ }^{3} \mathrm{He}$ mass spectrometry in the LLNL Nuclear Chemistry Noble Gas Mass Spectrometry Laboratory (Surano et al. 1991). We used this highly sensitive method for our wine analysis so that we could determine differences in the tritium content of the samples. Had less sensitive methods been used, such as those employed by commercial analytical laboratories, the tritium content of all samples would be near or below detection limits and no differences would be apparent. 


\section{Results}

\section{Livermore}

Vegetation. Table 9-1 shows 1992 tritium data for vegetation collected in the Livermore-site vegetation monitoring program. In general, vegetation tritium concentrations were lower than found in previous years.

An analysis of variance (ANOVA) indicated that location VIS had statistically greater vegetation tritium concentrations than all other locations except AQUE and MESQ. AQUE and MESQ, in turn, had greater tritium concentrations than all other locations except RAIL and MET. All other locations exhibited tritium concentrations in vegetation that were not statistically different.

Three logical groups of vegetation locations can be examined. The first group, "Near," consists of locations at or within 1 kilometer of the Livermore-site perimeter (AQUE, RAIL, MESQ, MET, and VIS). The "Intermediate" group consists of locations in the Livermore Valley, removed from the site (1 to 5 kilometers from the Livermore-site perimeter), but close enough and often downwind so that they are still potentially under the influence of tritium releases at the site. The Intermediate locations are 1580, TESW, ZON7, and PATT. The third group, "Background," represents locations unlikely to be affected by LLNL operations. Three of these locations (MOD, DAN, CAL) are far removed from the Livermore site; all are more than 25 kilometers away. The other two (FCC, PARK) are in the Livermore Valley, but are sufficiently removed from the Livermore site (greater than 5 kilometers) and are generally upwind so they are unlikely to be affected by LLNL operations. A statistical evaluation using ANOVA performed on these groups of data indicated that the Near group had significantly higher plant water tritium concentrations [mean of $25 \mathrm{~Bq} / \mathrm{L}$ $(666 \mathrm{pCi} / \mathrm{L})$ ] than either the Intermediate or the Background groups [means of $9 \mathrm{~Bq} / \mathrm{L}(243 \mathrm{pCi} / \mathrm{L})$ and $3 \mathrm{~Bq} / \mathrm{L}(81 \mathrm{pCi} / \mathrm{L})$, respectively]. The differences between the Intermediate and Background groups were not significant at the $95 \%$ confidence level. The plant water tritium concentrations in vegetation from the Near and Intermediate groups were about half those reported for 1991; Background group concentrations remained essentially unchanged from 1991.

Wine. The results from the 1992 wine tritium analyses are shown in Table 9-2. The sample numbers shown in the table were assigned randomly to wine samples from each region and are not related to sample numbers used in previous years. Tritium concentrations were within the range of those reported in previous years, and they remained low in wines from all areas.

The data for the 1992 sampling year were analyzed using ANOVA. The statistical analysis showed that the mean tritium concentration of the Livermore wines sampled was statistically greater than that of the California (other than Livermore) wines sampled. However, the mean tritium concentration of the 
Livermore wines sampled was not significantly different from that of the European wines sampled. The statistical analyses also indicated that there was no significant difference between the means of European and California wines sampled. Multiple comparison tests indicated that the mean levels of the 1992 sampling year data from California and Europe were not statistically different from those reported for the 1990 and 1991 sampling years. For Livermore wines, the 1992 values were statistically lower than those reported in 1991, but were not statistically different from those reported for 1990. Figure 9-4, which shows the results of the wine analyses by sampling year since monitoring began, also shows that 1992 tritium concentrations are among the lowest for all Livermore wines since monitoring began.

Regression analyses and ANOVA of the wine data (when decay-corrected) grouped by vintage year showed tritium concentrations have statistically decreased for all areas since monitoring began, and since 1980 .

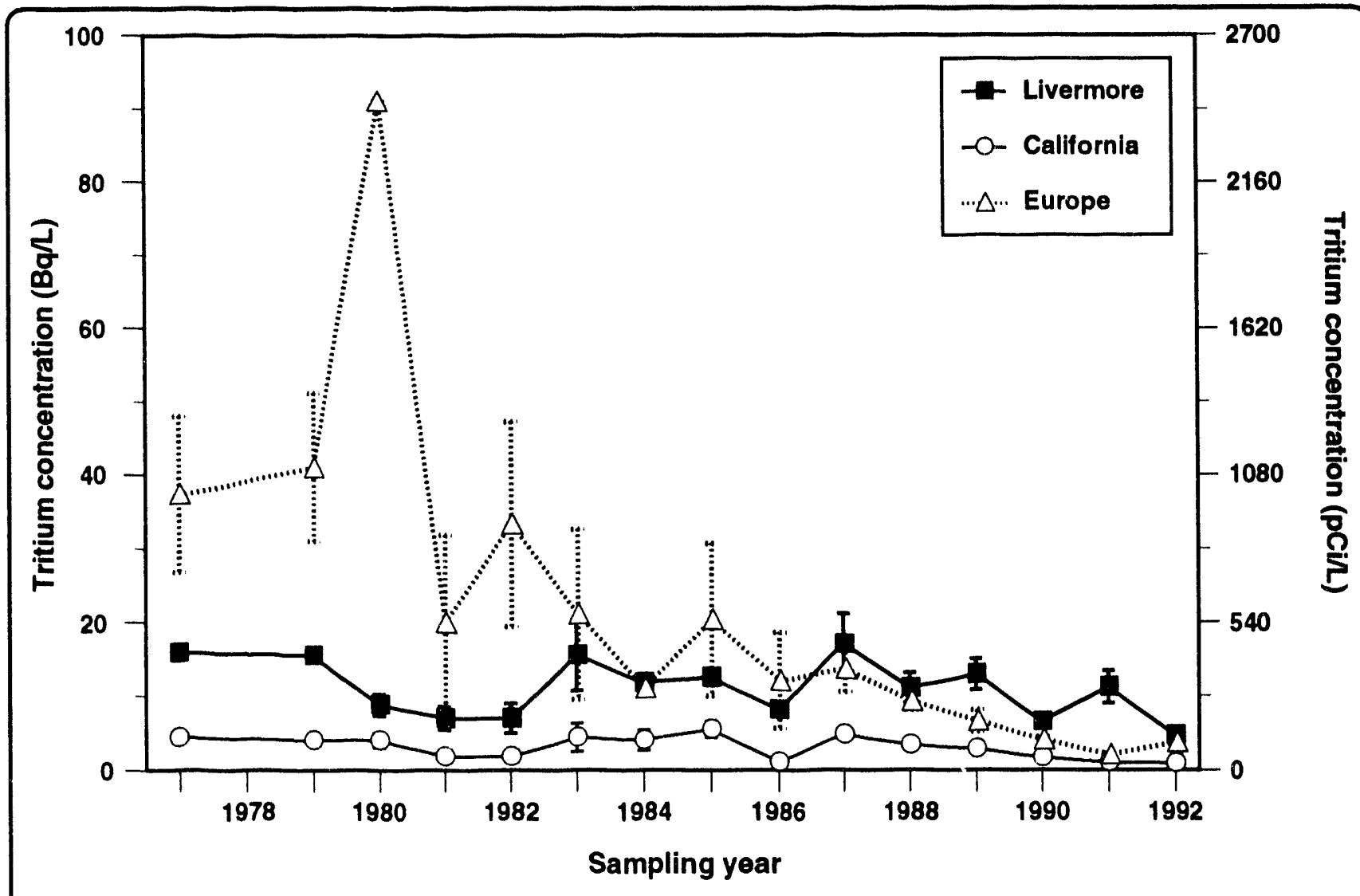

Figure 9-4. Mean tritium in retail wines, 1977 - 1992, plotted by sampling year (error bars are \pm 1 standard error). 
Last year we reported that Livermore wines, examined by vintage year, had statistically greater tritium concentrations since 1980 than European wines. This is particularly apparent since 1986 (Figure 9-5). The 1991 vintage tritium concentrations from these two areas are similar. However, even with these similar 1991 vintage data used in the statistical comparisons, the tritium concentration of vintage Livermore wines since 1980 remains statistically greater than that of either European or other California wines. Vintage wines from both Livermore and Europe continue to exhibit statistically higher tritium concentrations than vintage wines from other California areas during the same period, indicating three distinct data sets still exist.

Honey. Table 9-3 shows tritium data for honey collected from producers in the Livermore Valley and from other producers in California during 1992.

Although the mean tritium concentration of Livermore Valley honey is greater than that of honey from other areas of California, the difference is not statistically significant, as determined by ANOVA. The single high value of note

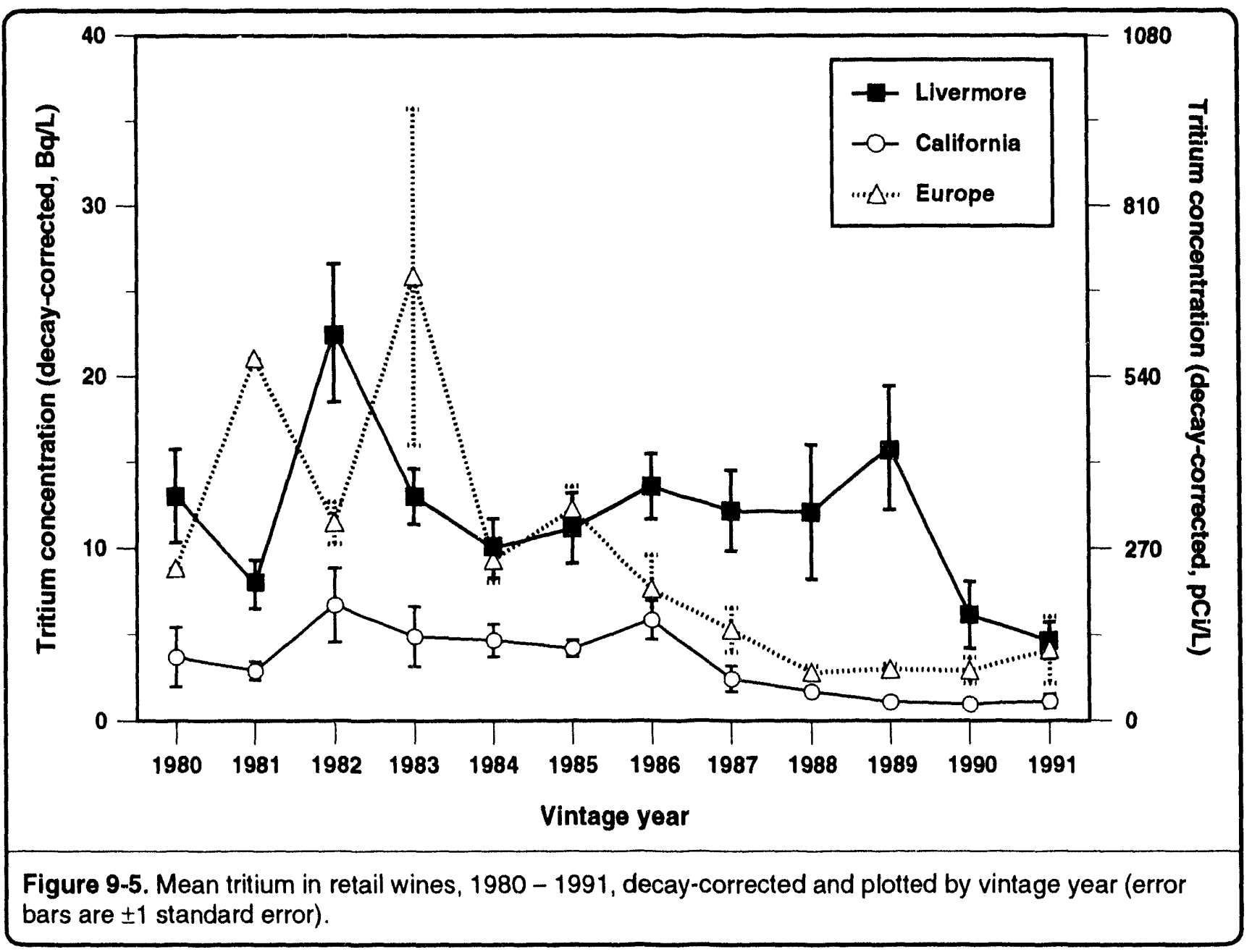


from the Livermore Valley [at $28 \mathrm{~Bq} / \mathrm{L}(749 \mathrm{pCi} / \mathrm{L})$, from hives kept in Livermore] was corroborated by a replicated analysis. Similar values from both Livermore and other areas of California have been reported in past years, and the large variance of tritium values in honey is not unusual (Griggs et al. 1984, Brekke et al. 1989, Sims et al. 1990). Tritium concentrations in honey are not necessarily indicative of the specific locations where the hives are kept; however, they can generally be indicative of regional tritium sources. This is because bees can have extensive ranges and their hives are often transported from place to place to fulfill pollination needs.

ANOVA also indicated that the tritium concentrations in Livermore Valley honey have not changed significantly during the past five years. The statistical analyses also indicated that 1992 California tritium values in honey were not significantly different from those reported from 1988 through 1991. Figure 9-6 shows the mean values for Livermore Valley honey collected from 1979 through 1992, and other California honey collected from 1980 through 1992. The graph shows that the tritium content of honey fluctuates significantly from year to year, and although the mean Livermore Valley concentration is usually somewhat higher than that of other California honey, the differences are rarely

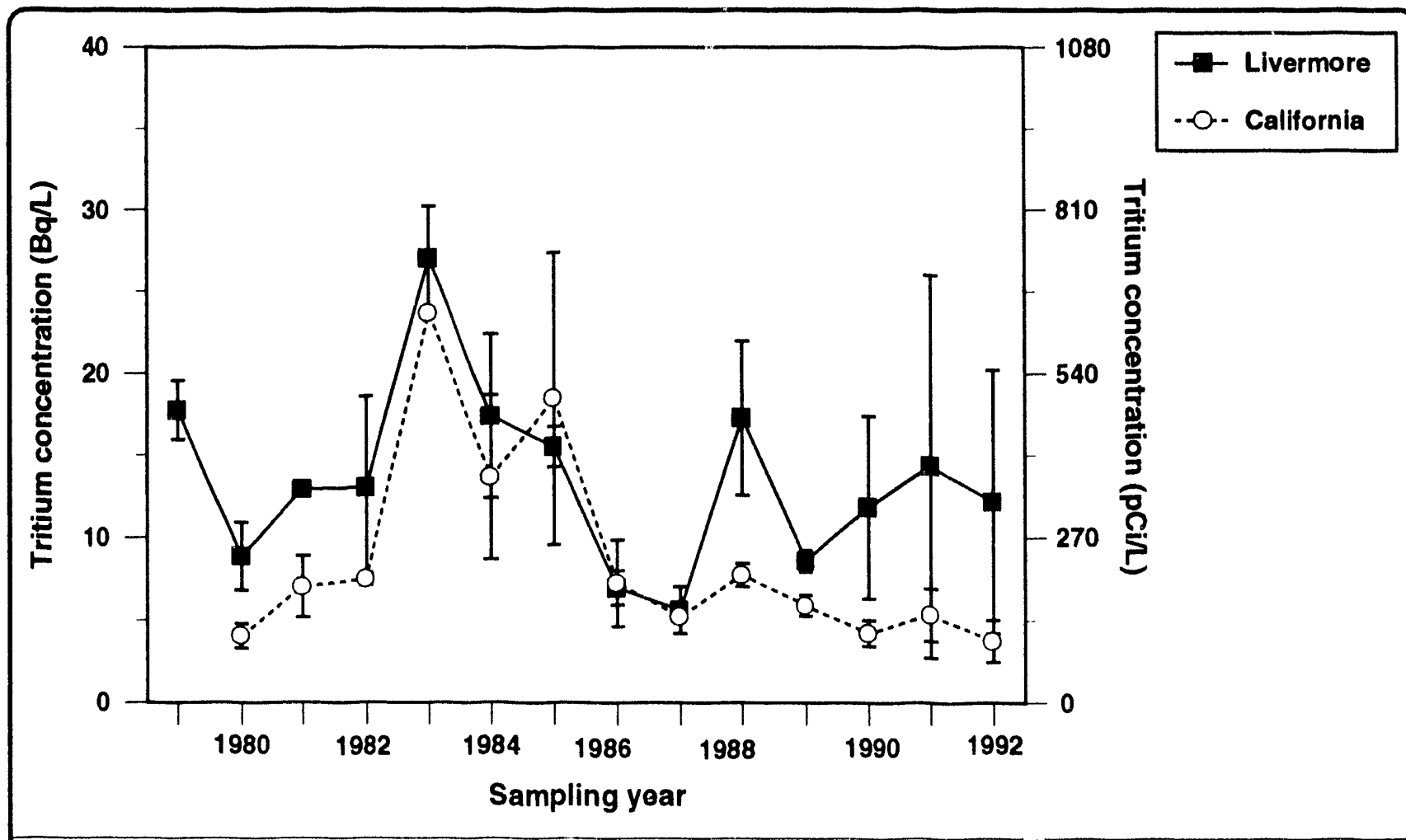

Figure 9-6. Tritium concentration in honey (error bars are \pm 1 standard error). 
statistically significant. Large variances in both Livermore Valley and California tritium concentrations in honey are often seen in the sample data, as evidenced by the large standard-error bars for many of the points.

Goat Milk. The activities of tritium, ${ }^{40} \mathrm{~K}$ (potassium-40, a naturally occurring radionuclide), and ${ }^{137} \mathrm{Cs}$ (cesium-137, a fallout product) in goat-milk samples collected during 1992 are shown in Tables 9-4 through 9-6. No other radionuclides were present at detectable levels; ${ }^{137} \mathrm{Cs}$ and ${ }^{40} \mathrm{~K}$ were detected at background levels.

ANOVA showed that tritium values for Livermore Valley goat milk as a whole are not significantly different (at $95 \%$ confidence) from the background concentrations. Furthermore, there were no significant differences among any of the Livermore Valley sampling locations. The cow-milk samples contained nearly the same amount of tritium as all the goat-milk samples. Dose calculations in Table 9-4 are based on a goat-milk intake of $310 \mathrm{~L} / \mathrm{y}$, tritium dose factors provided by DOE (U.S. Department of Energy 1988), and on models described in Appendix B. Because ${ }^{40} \mathrm{~K}$ is not an effluent at LLNL and because values from all goat-milk samples were similar, individual doses from ${ }^{40} \mathrm{~K}$ were not calculated for each sampling location.

\section{Site 300}

Vegetation. Table 9-7 shows tritium data for vegetation collected at Site 300 during 1992. With the exception of a single location, most values are at or near detection limits and are similar to those reported from 1988 through 1991. As was the case in 1990 and 1991, vegetation samples from location DSW contained the highest tritium values detected. As determined by multiple comparison ANOVA, location DSW exhibited plant water tritium concentrations that were significantly greater than the other locations. Tritium concentrations in vegetation from another area of potential concern, EVAP, were not significantly elevated during 1992.

Tritium has been observed in the vegetation of the DSW sampling location since 1971; it is in an area presently being investigated under CERCLA for tritium contamination of ground water. This sampling location is adjacent to a landfill that contain debris contaminated with tritium from past experiments, tritium is no longer used in explosives tests. As a result of seasonal rains, tritium has spread from this debris and is present in the local soil and ground water. The landfill area is under continued investigation for soil and ground water tritium, as described in reports published as part of LLNL's Environmental Restoration Program (see, e.g., Lamarre 1989a-c; Taffet et al. 1989a, b; Taffet et al. 1991; Carlsen 1991a, b; Webster-Scholten 1993).

The effective dose equivalents shown in Table 9-7 were derived using doseconversion factors provided by DOE (U.S. Department of Energy 1988) and a 
dose-pathway model from NRC Regulatory Guide 1.109 (U.S. Nuclear Regulatory Commission 1977). Appendix B provides a detailed discussion of dose-calculation methods.

\section{Environmental Impact}

\section{Livermore Site}

LLNL impacts on vegetation in the Livermore Valley in 1992 remain minimal. Tritium levels detected in the Livermore Valley vegetation were generally reduced from those observed in previous years. The reduction is probably the result of reduced tritium emissions from both LLNL and SNL/CA. The effective dose equivalents shown in Table 9-1 were derived using the doseconversion factors provided by DOE (U.S. Department of Energy 1988) and the dose-pathway model from NRC Regulatory Guide 1.109 (U.S. Nuclear Regulatory Commission 1977). Based on the conservative assumption that an adult's diet includes vegetables with the highest observed mean tritium concentration, and meat and milk derived from livestock fed on grasses with the same concentration, the maximum potential dose is $0.00012 \mathrm{mSv} / \mathrm{y}(0.012 \mathrm{mrem} / \mathrm{y})$.

There are no health standards for radionuclides in wine. However, all the wine tritium levels were far below drinking water standards. In fact, even the highest detected Livermore Valley value $[8.84 \mathrm{~Bq} / \mathrm{L}(239 \mathrm{pCi} / \mathrm{L})]$ represents only $1.2 \%$ of the California drinking-water standard [741 Bq/L $(20,000 \mathrm{pCi} / \mathrm{L})]$. Doses from wine consumption can be calculated according to methods for water ingestion, which are detailed in Appendix B.

The corresponding annual dose of the highest detected Livermore Valley tritium value in wine $[8.84 \mathrm{~Bq} / \mathrm{L}(239 \mathrm{pCi} / \mathrm{L})]$, based on the very conservative assumption that wine is consumed in the same quantities as water $(730 \mathrm{~L} / \mathrm{y}$ or $2 \mathrm{~L} / \mathrm{d})$, is $0.00011 \mathrm{mSv}(0.011 \mathrm{mrem})$. Using a more realistic wine consumption factor $(52 \mathrm{~L} / \mathrm{y}$ or $1 \mathrm{~L} / \mathrm{wk}$ of wine from a single area), and the mean tritium values detected in wines from the three sampling areas, the annual dose from Livermore wine would be $0.0000041 \mathrm{mSv}(0.00041 \mathrm{mrem})$, from European wine would be $0.0000033 \mathrm{mSv}(0.00033 \mathrm{mrem})$, and from California wine would be $0.0000008 \mathrm{mSv}(0.00008 \mathrm{mrem})$. Compared with an annual background dose of approximately $3 \mathrm{mSv}$ ( $300 \mathrm{mrem})$, which includes radon, and a $0.1-\mathrm{mSv}$ (10-mrem) dose from a typical chest $x$ ray (Shleien and Terpilak 1984), the potential dose from consuming wine from any area is minute. Therefore, although Livermore wines contained statistically more tritium than wines produced in other areas of California, the effects of the tritium are negligible.

The tritium concentrations in goat milk produced in the Livermore Valley were statistically the same as those of goat milk from other areas of California. Associated doses, like those for wine, were negligible. Regardless of sampling location, the annual ${ }^{40} \mathrm{~K}$ dose received from goat-milk consumption has been 
Table 9-1. Tritium in vegetation (in $\mathrm{Bq} / \mathrm{L}$ of moisture content), 1992 (concluded).

\begin{tabular}{|c|c|c|c|c|}
\hline & \multicolumn{4}{|c|}{ Sampling location } \\
\hline & DAN & PARK & CAL & VIS \\
\hline First quarter & $2.01 \pm 0.83$ & $11.29 \pm 1.17$ & $2.28 \pm 0.85$ & $12.73 \pm 1.20$ \\
\hline Second quarter & $2.62 \pm 1.19$ & $<1.65$ & $1.44 \pm 1.15$ & $17.72 \pm 2.16$ \\
\hline Third quarter & $2.94 \pm 1.64$ & $1.94 \pm 1.59$ & $4.07 \pm 1.69$ & $58.83 \pm 3.24$ \\
\hline Fourth quarter & $<2.17$ & $<2.10$ & $<2.18$ & $56.98 \pm 3.76$ \\
\hline Mean & $<2.43$ & $<2.02^{\circ}$ & $<2.49$ & 36.57 \\
\hline Std dev & $>0.42$ & $-d$ & $>1.12$ & 24.74 \\
\hline \multirow[t]{2}{*}{ Dose $(\mathrm{mSv} / \mathrm{y})^{\mathrm{b}}$} & $<1.2 \times 10^{-5}$ & $<9.8 \times 10^{-6}$ & $<1.2 \times 10^{-5}$ & $1.8 \times 10^{-4}$ \\
\hline & \multicolumn{4}{|c|}{ (pCin) } \\
\hline Mean & $<66$ & $<55^{\circ}$ & $<67$ & 988 \\
\hline Std dev & $>11$ & -d & $>30$ & 669 \\
\hline Dose (mrem/y) & $<1.2 \times 10^{-3}$ & $<9.8 \times 10^{-4}$ & $<1.2 \times 10^{-3}$ & $1.8 \times 10^{-2}$ \\
\hline
\end{tabular}

Note: Radionuclide results are reported $\pm 2 \sigma$, in $\mathrm{Bq} / \mathrm{L}$; see Quality Assurance chapter.

a See Fig. 9-1 for sampling locations.

b This dose represents the total effective dose equivalent from the forage-cow-milk pathway; see Appendix B.

c Median value; see Quality Assurance chapter.

d No measure of dispersion calculated; see Quality Assurance chapter. 
Table 9-2. Tritium in retail wine (in Bq/L), 1992.a

\begin{tabular}{|c|c|c|c|}
\hline \multirow[b]{2}{*}{ Sample } & \multicolumn{3}{|c|}{ Area of production } \\
\hline & Livermore Valley & California & Europe \\
\hline 1 & $2.29 \pm 0.18$ & $1.44 \pm 0.13$ & $2.24 \pm 0.14$ \\
\hline 2 & $8.60 \pm 0.37$ & $0.61 \pm 0.12$ & $2.63 \pm 0.16$ \\
\hline 3 & $3.22 \pm 0.23$ & $0.91 \pm 0.13$ & $1.84 \pm 0.14$ \\
\hline 4 & $8.84 \pm 0.48$ & $0.68 \pm 0.12$ & $3.20 \pm 0.17$ \\
\hline 5 & $7.96 \pm 0.56$ & & $10.94 \pm 1.11$ \\
\hline 6 & $8.43 \pm 0.38$ & & $1.43 \pm 0.12$ \\
\hline 7 & $4.21 \pm 0.26$ & & \\
\hline 8 & $3.10 \pm 0.26$ & & \\
\hline 9 & $2.94 \pm 0.17$ & & \\
\hline 10 & $1.07 \pm 0.17$ & & \\
\hline 11 & $2.75 \pm 0.19$ & & \\
\hline 12 & $2.62 \pm 0.60$ & & \\
\hline Mean & 4.67 & 0.91 & 3.71 \\
\hline \multirow[t]{2}{*}{ Std dev } & 2.89 & 0.38 & 3.60 \\
\hline & \multicolumn{3}{|c|}{$(\mathrm{pCl} / 2)$} \\
\hline Mean & 126 & 25 & 100 \\
\hline Std dev & 78 & 10 & 97 \\
\hline
\end{tabular}

Note: Radionuclide results are reported $\pm 2 \sigma$, in $\mathrm{Bq} / \mathrm{L}$; see Quality Assurance chapter.

a Wines from a variety of vintages were purchased and analyzed in 1992. The concentrations shown are not decay-corrected to vintage year.

Table 9-3. Tritium in honey (in Bq/L in recovered water), 1992.

\begin{tabular}{lcc}
\hline & \multicolumn{2}{c}{ Sampling location } \\
\cline { 2 - 3 } Sample & California & Livermore Valley \\
\hline 1 & $1.65 \pm 0.56$ & $27.71 \pm 1.33$ \\
2 & $6.03 \pm 0.83$ & $1.10 \pm 0.69$ \\
3 & $3.50 \pm 0.90$ & $7.73 \pm 0.84$ \\
& & \\
Mean & 3.73 & 12.18 \\
Std dev & 2.20 & 13.85 \\
& & \\
Mean & 101 & 329 \\
Std dev & 59 & $\mathbf{3 7 4}$ \\
\hline
\end{tabular}

Note: Radionuclide results are reported $\pm 2 \sigma$, in $\mathrm{Bq} / \mathrm{L}$; see Quality Assurance chapter. 


\section{Vegetation and Foodstuff Monitoring}

Table 9-4. Tritium in goat's milk (in Bq/L), 1992.

\begin{tabular}{|c|c|c|c|c|}
\hline \multirow[b]{2}{*}{ Month } & \multicolumn{4}{|c|}{ Sampling location } \\
\hline & WRD (cow) & WRD' & MZFb & LUPb \\
\hline Feb. & $-^{c}$ & $-d$ & $4.81 \pm 1.44$ & $4.96 \pm 1.08$ \\
\hline Mar. & $-c$ & $1.61 \pm 1.60$ & $<1.58$ & $2.56 \pm 1.64$ \\
\hline Apr. & $-c$ & $<1.61$ & - & $<2.31$ \\
\hline May & $-c$ & $1.51 \pm 1.21$ & - & $1.92 \pm 0.96$ \\
\hline June & $-^{c}$ & $2.78 \pm 1.73$ & - $\theta$ & $3.26 \pm 1.78$ \\
\hline July & $-^{c}$ & $<1.64$ & —e & $2.08 \pm 1.69$ \\
\hline Aug. & $2.07 \pm 1.83$ & $2.56 \pm 1.85$ & - $\theta$ & - \\
\hline Sept. & $<1.89$ & $<1.91$ & - $\theta$ & - \\
\hline Oct. & $<2.01$ & $<2.01$ & $-^{\theta}$ & - \\
\hline Nov. & - & $<1.62$ & $-^{\theta}$ & - \\
\hline Mean & $<2.01^{f}$ & $<1.64$ & $<3.20^{\prime}$ & $<2.85$ \\
\hline Std dev & $\rightarrow$ & o & $\rightarrow$ & $>1.13$ \\
\hline \multirow[t]{2}{*}{ Dose $(\mathrm{mSv} / \mathrm{y})^{k}$} & $<1.1 \times 10^{-5}$ & $<8.7 \times 10^{-6}$ & $<1.7 \times 10^{-5}$ & $<1.5 \times 10^{-5}$ \\
\hline & \multicolumn{4}{|c|}{$(\mathrm{pCln})$} \\
\hline Mean & $<54^{\prime}$ & $<44^{\prime}$ & $<86^{f}$ & $<77$ \\
\hline Std dev & -9 & $\rightarrow$ & -9 & $>31$ \\
\hline \multirow[t]{2}{*}{ Dose (mrem/y) } & $<1.1 \times 10^{-3}$ & $<8.7 \times 10^{-4}$ & $<1.7 \times 10^{-3}$ & $<1.5 \times 10^{-3}$ \\
\hline & \multicolumn{4}{|c|}{ Sampling location } \\
\hline Month & $\mathrm{COOL}^{\mathrm{b}}$ & MOD21 & STEV & TUA \\
\hline Feb. & $3.74 \pm 1.05$ & $-c$ & $-c$ & $5.44 \pm 1.11$ \\
\hline Mar. & $<1.54$ & -c & $-c$ & $2.76 \pm 1.64$ \\
\hline Apr. & $2.26 \pm 1.66$ & $<1.59$ & $-c$ & $2.42 \pm 1.67$ \\
\hline May & $2.96 \pm 0.99$ & $3.60 \pm 1.06$ & $-c$ & $1.55 \pm 1.28$ \\
\hline June & $<1.65$ & $2.60 \pm 1.73$ & $-c$ & $<2.41$ \\
\hline July & $<1.66$ & $<1.65$ & $-c$ & $1.76 \pm 1.68$ \\
\hline Aug. & $<1.78$ & - & $3.48 \pm 1.88$ & $2.92 \pm 1.85$ \\
\hline Sept. & $<1.88$ & - $\theta$ & $3.35 \pm 1.98$ & $<1.89$ \\
\hline Oct. & $<1.99$ & $-^{\theta}$ & $2.47 \pm 2.04$ & $2.92 \pm 2.05$ \\
\hline Nov. & $-d$ & - & $1.82 \pm 1.63$ & $1.78 \pm 1.63$ \\
\hline Mean & $<1.88^{h}$ & $<2.13^{h}$ & 2.78 & $<2.59$ \\
\hline Std dev & -9 & $\rightarrow$ & 0.78 & $>1.12$ \\
\hline \multirow[t]{2}{*}{ Dose $(m S v / y)^{h}$} & $<9.9 \times 10^{-6}$ & $<1.1 \times 10^{-5}$ & $1.5 \times 10^{-5}$ & $<1.4 \times 10^{-5}$ \\
\hline & \multicolumn{4}{|c|}{$(\mathrm{pc} / \mathrm{L})$} \\
\hline Mean & $<51^{t}$ & $<57^{\prime}$ & 75 & $<70$ \\
\hline Std dev & -9 & -9 & 21 & $>30$ \\
\hline Dose (mrem/y) & $<9.9 \times 10^{-4}$ & $<1.1 \times 10^{-3}$ & $1.5 \times 10^{-3}$ & $<1.4 \times 10^{-3}$ \\
\hline
\end{tabular}


Table 9-4. Tritium in goat's milk (in Bq/L), 1992 (concluded).

\begin{tabular}{|c|c|c|c|}
\hline \multirow[b]{2}{*}{ Month } & \multicolumn{3}{|c|}{ Sampling location } \\
\hline & RIPI & WOOD' & CARTI \\
\hline Feb. & $-d$ & $-c$ & $2.01 \pm 0.99$ \\
\hline Mar. & $3.67 \pm 1.68$ & $-c$ & $<1.58$ \\
\hline Apr. & - & $-c$ & $2.76 \pm 1.69$ \\
\hline May & $-^{\circ}$ & $-^{c}$ & $3.01 \pm 1.02$ \\
\hline June & $-\theta$ & $-c$ & $<1.70$ \\
\hline July & $\ldots$ & $-^{c}$ & $2.67 \pm 1.70$ \\
\hline Aug. & -0 & $-c$ & $3.92 \pm 1.90$ \\
\hline Sept. & $-\theta$ & $<1.87$ & $<1.92$ \\
\hline Oct. & - & $2.26 \pm 2.04$ & $2.91 \pm 2.06$ \\
\hline Nov. & $\boldsymbol{-}^{\theta}$ & $<1.60$ & $<1.57$ \\
\hline Mean & 3.67 & $<1.87^{\circ}$ & $<2.34^{\dagger}$ \\
\hline Std dev & 0.00 & $\rightarrow$ & $0.61^{k}$ \\
\hline \multirow[t]{2}{*}{ Dose (mSv/y)h } & $1.9 \times 10^{-5}$ & $<9.9 \times 10^{-6}$ & $<1.2 \times 10^{-5}$ \\
\hline & \multicolumn{3}{|c|}{ (pcin) } \\
\hline Mean & 99 & $<51^{1}$ & $<63^{\prime}$ \\
\hline Std dev & 0 & $\rightarrow$ & $16^{k}$ \\
\hline Dose (mrem/y) & $1.9 \times 10^{-3}$ & $<9.9 \times 10^{-4}$ & $<1.2 \times 10^{-3}$ \\
\hline
\end{tabular}

Note: Radionuclide results are reported $\pm 2 \sigma$, in $\mathrm{Bq} / \mathrm{L}$; see Quality Assurance chapter.

a See Fig. 9-3 for sampling locations.

b Livermore Valley sampling location.

c Participation in monitoring program began during mid-year.

d No sample obtained; see Quality Assurance chapter.

- Provider discontinued participation in monitoring program.

1 Median value; seo Quality Assurance chapter.

$g$ No measure of dispersion calculated; se日 Quality Assurance chapter.

$h$ This dose is the effective dose equivalent; see Appendix B.

1 Califomia (non-Livermore Valley) sampling location.

1 Sample purchased in carton from store.

k Median absolute deviation; see Quality Assurance chapter. 
Table 9-5. Potassium-40 in milk (in Bq/L), 1992.

\begin{tabular}{|c|c|c|c|c|}
\hline \multirow[b]{2}{*}{ Month } & \multicolumn{4}{|c|}{ Sampling location" } \\
\hline & WRD (cow) & WRD & $M Z F^{\circ}$ & LUPb \\
\hline Feb. & $-c$ & $-d$ & $53.1 \pm 1.1$ & $56.0 \pm 1.7$ \\
\hline Mar. & $-c$ & $63.7 \pm 1.9$ & $59.5 \pm 1.8$ & $75.3 \pm 1.5$ \\
\hline Apr. & $-^{c}$ & $62.4 \pm 1.9$ & - & $56.4 \pm 1.7$ \\
\hline May & $-c$ & $69.0 \pm 2.1$ & $-\theta$ & $63.3 \pm 1.9$ \\
\hline June & $-c$ & $67.6 \pm 2.0$ & $-^{\theta}$ & $60.8 \pm 1.2$ \\
\hline July & $-^{c}$ & $68.1 \pm 2.0$ & -0 & $67.4 \pm 1.3$ \\
\hline Aug. & $46.5 \pm 0.9$ & $67.2 \pm 1.3$ & -0 & $-^{0}$ \\
\hline Sept. & $57.0 \pm 1.7$ & $58.5 \pm 1.8$ & - & -0 \\
\hline Oct. & $53.4 \pm 1.6$ & $55.7 \pm 2.2$ & $-\theta$ & $-\theta$ \\
\hline Nov. & $-^{d}$ & $68.4 \pm 2.1$ & -0 & - \\
\hline Mean & 52.3 & 64.5 & 56.3 & 63.2 \\
\hline \multirow[t]{2}{*}{ Std dev } & 5.3 & 4.8 & 4.5 & 7.3 \\
\hline & \multicolumn{4}{|c|}{$(\mathrm{pCi} / \mathrm{L})$} \\
\hline Mean & 1413 & 1744 & 1522 & 1708 \\
\hline \multirow[t]{2}{*}{ Std dev } & 144 & 130 & 122 & 197 \\
\hline & \multicolumn{4}{|c|}{ Sampling location" } \\
\hline Month & COOL ${ }^{b}$ & MOD2 & STEV & TUR' \\
\hline Feb. & $52.9 \pm 1.6$ & $-c$ & $-^{c}$ & $63.6 \pm 1.9$ \\
\hline Mar. & $53.1 \pm 1.6$ & $-^{c}$ & $-c$ & $62.6 \pm 1.9$ \\
\hline Apr. & $57.8 \pm 1.2$ & $65.6 \pm 2.0$ & $-c$ & $63.2 \pm 1.3$ \\
\hline May & $58.8 \pm 1.8$ & $71.1 \pm 2.1$ & $-^{c}$ & $65.2 \pm 2.0$ \\
\hline June & $58.1 \pm 2.3$ & $66.9 \pm 2.0$ & $-^{c}$ & $63.6 \pm 2.5$ \\
\hline Juhy & $63.4 \pm 1.9$ & $67.6 \pm 2.0$ & $-^{c}$ & $68.6 \pm 1.4$ \\
\hline Aug. & $63.5 \pm 1.9$ & - & $68.1 \pm 2.0$ & $68.5 \pm 2.1$ \\
\hline Sept. & $55.5 \pm 1.7$ & - $^{0}$ & $66.2 \pm 2.0$ & $64.4 \pm 1.9$ \\
\hline Oct. & $49.1 \pm 1.5$ & $--^{\circ}$ & $59.5 \pm 1.8$ & $67.7 \pm 2.0$ \\
\hline Nov. & $-^{d}$ & -0 & $52.8 \pm 2.1$ & $63.8 \pm 1.9$ \\
\hline Mean & 56.9 & 67.8 & 61.7 & 65.1 \\
\hline \multirow[t]{2}{*}{ Std dev } & 4.8 & 2.3 & 7.0 & 2.3 \\
\hline & \multicolumn{4}{|c|}{ (pCin) } \\
\hline Mean & 1538 & 1832 & 1666 & 1760 \\
\hline Std dev & 130 & 63 & 189 & 61 \\
\hline
\end{tabular}


Table 9-5. Potassium-40 in milk (in Bq/L), 1992 (concluded).

\begin{tabular}{|c|c|c|c|}
\hline \multirow[b]{2}{*}{ Month } & \multicolumn{3}{|c|}{ Sampling location } \\
\hline & RIPf & WOOD & CARTS \\
\hline Feb. & $-d$ & $-^{c}$ & $61.6 \pm 1.8$ \\
\hline Mar. & $67.6 \pm 2.0$ & $-c$ & $60.4 \pm 1.8$ \\
\hline Apr. & - & $-c$ & $61.7 \pm 1.9$ \\
\hline May & $-^{\theta}$ & $-^{c}$ & $61.5 \pm 1.2$ \\
\hline June & - $^{\circ}$ & $-^{c}$ & $61.3 \pm 1.8$ \\
\hline July & $-^{\circ}$ & $-^{c}$ & $67.4 \pm 1.3$ \\
\hline Aug. & -0 & $-^{c}$ & $62.2 \pm 1.2$ \\
\hline Sept. & - & $64.2 \pm 1.9$ & $63.1 \pm 1.9$ \\
\hline Oct. & $\boldsymbol{\theta}^{\theta}$ & $61.4 \pm 1.8$ & $66.1 \pm 1.3$ \\
\hline Nov. & -0 & $62.5 \pm 1.9$ & $64.9 \pm 1.9$ \\
\hline Mean & 67.6 & 62.7 & 63.0 \\
\hline \multirow[t]{2}{*}{ Std dev } & $-{ }^{n}$ & 1.4 & 2.3 \\
\hline & \multicolumn{3}{|c|}{ (pCin) } \\
\hline Mean & 1827 & 1694 & 1704 \\
\hline Std dev & 0 & 38 & 63 \\
\hline
\end{tabular}

Note: Radionuclide results are reported $\pm 2 \sigma$, in Bq/L; see Quality Assurance chapter.

a See Fig. 9-3 for sampling locations.

b Livermore Valiey sampling location.

c Participation in monitoring program began during mid-year.

- No sample obtained; see Quality Assurance chapter.

- Provider discontinued participation in monitoring program.

1 Califomia (non-Livermore Valley) sampling location.

g Sample purchased in carton from store.

h No standard deviation calculated; see Quality Assurance chapter. 
Table 9-6. Cesium-137 in milk (in Bq/L), 1992.

\begin{tabular}{|c|c|c|c|c|}
\hline \multirow[b]{2}{*}{ Month } & \multicolumn{4}{|c|}{ Sampling location" } \\
\hline & WRD (cow) & WRD & MzF & LUPb \\
\hline Feb. & $-^{c}$ & $-d$ & $0.125 \pm 0.048$ & $0.030 \pm 0.024$ \\
\hline Mar. & $-c$ & $<0.006$ & $0.189 \pm 0.044$ & $0.034 \pm 0.029$ \\
\hline Apr. & $-c$ & $<0.006$ & - & $<0.005$ \\
\hline May & $-c$ & $<0.007$ & -e & $<0.015$ \\
\hline June & $-c$ & $<0.032$ & $-\theta$ & $<0.005$ \\
\hline July & $-c$ & $<0.014$ & $-\theta$ & $<0.005$ \\
\hline Aug. & $<0.006$ & $0.044 \pm 0.032$ & $-\theta$ & -0 \\
\hline Sept. & $<0.014$ & $<0.005$ & -e & $-\theta$ \\
\hline Oct. & $<0.018$ & $<0.012$ & - & - \\
\hline Nov. & $-d$ & $<0.006$ & $-\theta$ & - \\
\hline Median & $<0.014$ & $<0.007$ & $0.157^{t}$ & $<0.010$ \\
\hline \multirow[t]{2}{*}{ Std dev } & - & -9 & 0.045 & - \\
\hline & \multicolumn{4}{|c|}{ (pCin) } \\
\hline Median & $<0.38$ & $<0.19$ & 4.25 & $<0.27$ \\
\hline Std dev & -9 & $\rightarrow$ & 1.22 & $\rightarrow$ \\
\hline
\end{tabular}

\begin{tabular}{|c|c|c|c|c|}
\hline \multirow[b]{2}{*}{ Month } & \multicolumn{4}{|c|}{ Sampling location } \\
\hline & $\mathrm{COOL}^{\mathrm{b}}$ & MOD2h & STEVh & TUR $^{h}$ \\
\hline Feb. & $0.081 \pm 0.044$ & $-^{c}$ & $-c$ & $<0.006$ \\
\hline Mar. & $0.088 \pm 0.048$ & $-^{c}$ & $-c$ & $<0.006$ \\
\hline Apr. & $0.048 \pm 0.031$ & $<0.007$ & $-^{c}$ & $<0.033$ \\
\hline May & $<0.010$ & $<0.005$ & $-^{c}$ & $<0.006$ \\
\hline June & $<0.005$ & $<0.006$ & $-^{c}$ & $<0.005$ \\
\hline July & $<0.016$ & $<0.006$ & $-^{c}$ & $<0.005$ \\
\hline Aug. & $<0.012$ & $-\theta$ & $<0.007$ & $<0.006$ \\
\hline Sept. & $<0.005$ & - & $<0.006$ & $0.019 \pm 0.017$ \\
\hline Oct. & $<0.014$ & $-\theta$ & $<0.006$ & $0.035 \pm 0.022$ \\
\hline Nov. & $-^{d}$ & - & $<0.005$ & $<0.006$ \\
\hline Median & $<0.014$ & $<0.006$ & $<0.006$ & $<0.006$ \\
\hline \multirow[t]{2}{*}{ Std dev } & -g & $-g$ & -9 & -9 \\
\hline & \multicolumn{4}{|c|}{ (pCin) } \\
\hline Median & $<0.38$ & $<0.16$ & $<0.16$ & $<0.16$ \\
\hline Std dev & -9 & $\rightarrow$ & $-g$ & $\rightarrow$ \\
\hline
\end{tabular}


Table 9-6. Cesium-137 in milk (in Bq/L), 1992 (concluded).

\begin{tabular}{|c|c|c|c|}
\hline \multirow[b]{2}{*}{ Month } & \multicolumn{3}{|c|}{ Sampling location ${ }^{\mathrm{a}}$} \\
\hline & RIPh & WOODh & CART' \\
\hline Feb. & $-{ }^{d}$ & $-^{c}$ & $<0.031$ \\
\hline Mar. & $<0.006$ & $-^{c}$ & $<0.006$ \\
\hline Apr. & $-\theta$ & $-^{c}$ & $<0.012$ \\
\hline May & $-\theta$ & $-{ }^{c}$ & $<0.005$ \\
\hline Jun. & $-e$ & $-^{c}$ & $<0.006$ \\
\hline Jul. & - $^{\circ}$ & $-^{c}$ & $<0.005$ \\
\hline Aug. & $-^{\theta}$ & $-^{c}$ & $<0.005$ \\
\hline Sept. & $-\theta$ & $<0.006$ & $<0.005$ \\
\hline Oct. & - $\theta$ & $0.020 \pm 0.017$ & $<0.005$ \\
\hline Nov. & $-\theta$ & $<0.005$ & $<0.033$ \\
\hline Median & $<0.006$ & $<0.006$ & $<0.006$ \\
\hline \multirow[t]{2}{*}{ Std dev } & -9 & -9 & -9 \\
\hline & \multicolumn{3}{|c|}{$(\mathrm{pCi} / \mathrm{L})$} \\
\hline Median & $<0.16$ & $<0.16$ & $<0.16$ \\
\hline Std dev & $-g$ & $\rightarrow$ & -g \\
\hline
\end{tabular}

Note: Radionuclide results are reported $\pm 2 \sigma$, in $B q / L ;$ see Quality Assurance chapter.

a See Fig. 9-3 for sampling locations.

b Livermore Valley sampling location.

c Participation in monitoring program began during mid-year.

d No sample obtained; see Quality Assurance chapter.

- Provider discontinued participation in monitoring program.

1 Mean value; see Quality Assurance chapter.

g No measure of dispersion calculated; see Quality Assurance chapter.

h California (non-Livermore Valley) sampling location.

i Sample purchased in carton from store. 
Table 9-7. Tritium in vegetation (in Bq/L of moisture content), Site 300, 1992.

\begin{tabular}{|c|c|c|c|}
\hline \multirow[b]{2}{*}{ Quarter } & \multicolumn{3}{|c|}{ Sampling location ${ }^{a}$} \\
\hline & CARN & GOLF & GEO \\
\hline First quarter & $<1.57$ & $16.91 \pm 2.96$ & $16.98 \pm 2.11$ \\
\hline Second quarter & $2.76 \pm 1.02$ & $1.91 \pm 0.98$ & $2.95 \pm 1.39$ \\
\hline Third quarter & $<1.63$ & $<1.65$ & $<1.62$ \\
\hline Fourth quarter & $<1.94$ & $<1.87$ & $<1.92$ \\
\hline Mean & $<1.78^{b}$ & $<1.89 b$ & $<2.43^{b}$ \\
\hline Std dev & $-c$ & $-c$ & $-c$ \\
\hline \multirow[t]{2}{*}{ Dose $(m S v / y)^{d}$} & $<8.6 \times 10^{-6}$ & $<9.1 \times 10^{-6}$ & $<1.2 \times 10^{-5}$ \\
\hline & & (pCin) & \\
\hline Mean & $<48^{b}$ & $<51^{b}$ & $<66^{b}$ \\
\hline Std dev & $-c$ & $-^{c}$ & $-c$ \\
\hline Dose $(m r e m / y) d$ & $<8.6 \times 10^{-4}$ & $<9.1 \times 10^{-4}$ & $<1.2 \times 10^{-3}$ \\
\hline
\end{tabular}

\begin{tabular}{lccc}
\hline & \multicolumn{3}{c}{ Sampling locationa } \\
\cline { 2 - 4 } \multicolumn{1}{c}{ Quarter } & DSW & $801 \mathrm{E}$ & EVAP \\
\hline First quarter & $14.73 \pm 2.03$ & $1.66 \pm 1.59$ & $3.00 \pm 0.88$ \\
Second quarter & $210.90 \pm 8.86$ & $3.81 \pm 1.39$ & $4.11 \pm 1.72$ \\
Third quarter & $297.11 \pm 6.24$ & $2.92 \pm 1.72$ & $4.00 \pm 1.76$ \\
Fourth quarter & $279.72 \pm 6.71$ & $<2.49$ & $7.77 \pm 2.18$ \\
Mean & 200.61 & $<2.72$ & 4.72 \\
Std dev & 129.40 & $>0.90$ & 2.09 \\
Dose (mSv/y) & & $<1.3 \times 10^{-5}$ & $2.3 \times 10^{-5}$ \\
& $9.7 \times 10^{-4}$ & $(\mathrm{pCi} / \mathrm{L})$ & 128 \\
Mean & & $<74$ & 57 \\
Std dev & 5422 & $>24$ & $2.3 \times 10^{-3}$ \\
Dose (mrem/y) & 3497 & $<1.3 \times 10^{-3}$ & \\
\hline
\end{tabular}

Note: Radionuclide results are reported $\pm 2 \sigma$, in $\mathrm{Bq} / \mathrm{L} ;$ see Quality Assurance chapter.

a See Fig. 9-2 for sampling locations.

b Median value; see Quality Assurance chapter.

c No measure of dispersion calculated; see Quality Assurance chapter.

d This dose represents the total effective dose equivalent from the forage-cow-milk pathway; see Appendix B. 
Barbara C. Fields

Kris A. Surano

\section{Introduction}

A wide variety of radioisotopes are used at LLNL, including transuranics, biomedical tracers, tritium, mixed fission products, and other radioisotopes for general research and nuclear weapons research. LLNL conducts direct gamma and neutron radiation monitoring to establish background radiation levels in its vicinity and to determine the environmental radiological impact of its operations in accordance with federal regulations (40 CFR 58.20, 40 CFR 61), DOE Orders 5400.1 and 5400.5, and California Code of Regulations Title 17, Article 1, Section 30250. Because environmental radiological monitoring is used to assess the potential direct radiation dose the public receives as the result of LLNL operations, LLNL has developed an extensive radiological monitoring network for its Livermore-site perimeter, the Livermore Valley, and the Site 300 perimeter. LLNL has measured both gamma and neutron radiation at the Livermore-site perimeter since 1973. LLNL implemented an environmental direct radiation monitoring program at Site 300 in 1988.

Gamma radiation is measured using thermoluminescent dosimeters (TLDs); it cannot be collected on filters nor chemically trapped. The TLDs provide a measure of the total amount of gamma radiation at a particular location. Gamma radiation results from natural background sources of geologic/terrestrial or cosmic origin, or from man-made sources, such as fallout from past nuclear weapons testing and any contribution from LLNL operations. Neutron radiation is measured with ${ }^{235} \mathrm{U}$ track-etch detectors (modified neutron rem-meters).

In addition to the routine monitoring, a special gamma radiation study was begun off site near Site 300 in 1991 and was expanded to other areas of the San Francisco Bay Area in 1992. The special study was initiated to determine if the geology at Site 300 or the work conducted at Site 300 were contributing factors in slightly elevated dose levels found at some locations at and near Site 300.

\section{Methods}

External doses from gamma radiation are monitored at 16 Livermore-site perimeter locations (Figure 10-1), 49 Livermore Valley locations (Figure 10-2), 12 Site 300 perimeter locations, and 2 locations in the area surrounding Site 300 (Figure 10-3). Off-site TLDs are located in areas surrounding the Livermore site and in nearby residential areas. These are considered background locations and they are used for comparison with perimeter measurements. Six new locations, also shown in Figure 10-3, were added in 
1991 in the area near Site 300 as part of the special study described above. In 1992, additional monitoring locations were established in the San Francisco Bay Area to expand this special study (Figure 10-4). The special study is described in detail below.

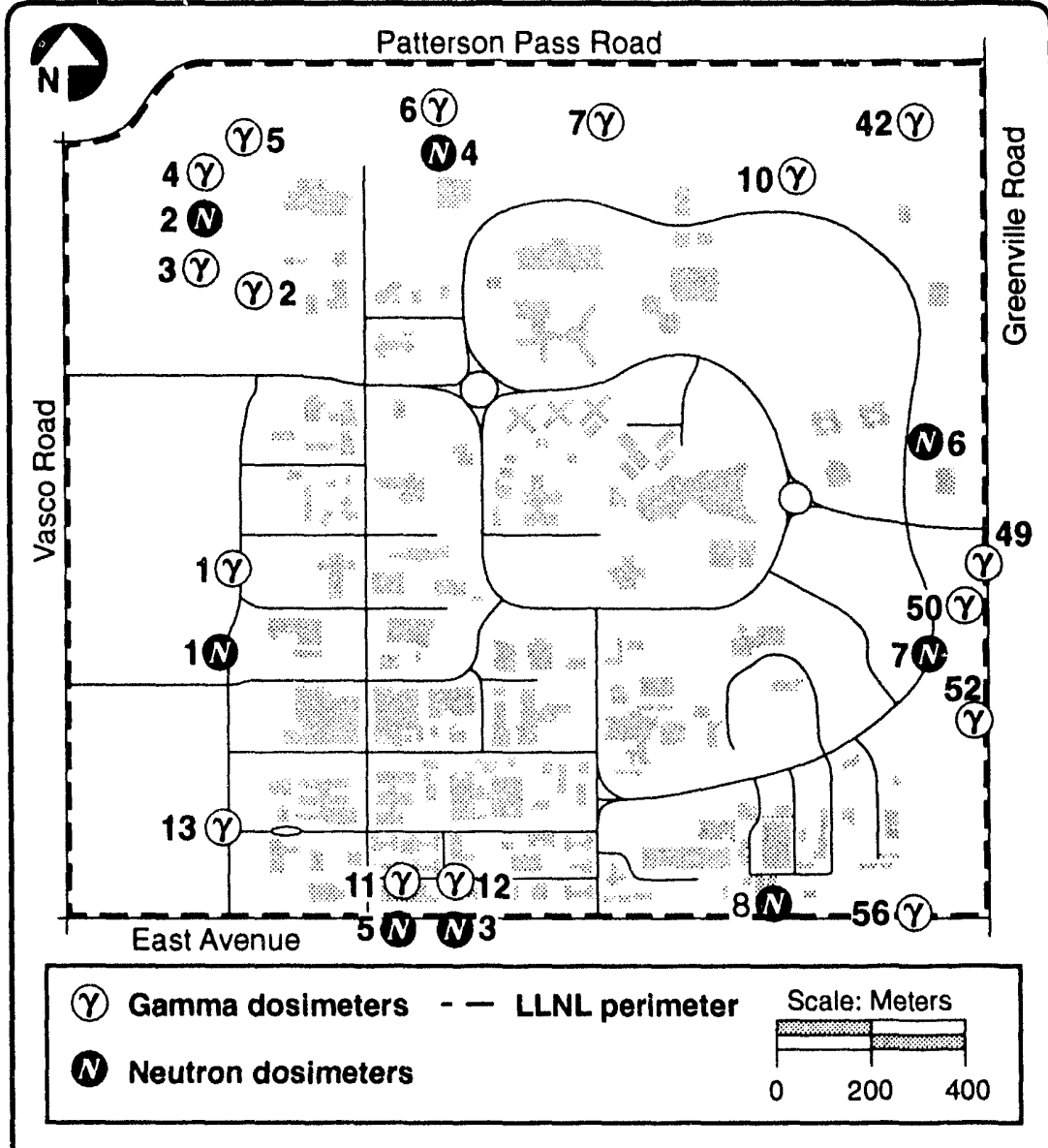

Figure 10-1. Gamma and neutron dosimeter locations, Livermore site, 1992.

Direct gamma radiation doses are measured with reusable TLDs mounted in the field on existing structures (such as fence lines) at approximately 1 meter above ground level. Each TLD is heatsealed in a $8.9 \mathrm{~cm} \times 5.7 \mathrm{~cm}$, 4.5-mm-thick foil pouch for protection against light and moisture. Each quarter, the TLDs are exchanged and read, data are analyzed, and the dose rates are subsequently calculated.

TLDs contain three elements of thallium-activated calcium sulfate $\left(\mathrm{CaSO}_{4}\right)$ and one element of lithium borate $\left(\mathrm{Li}_{2} \mathrm{~B}_{4} \mathrm{O}_{7}\right)$. Energy is stored when these compounds are exposed to gamma radiation. Impurities in the TLD crystal form low-temperature trapping sites for electrons excited to higher energy states by gamma radiation at normal ambient temperatures. When the TLDs are heated in the analytical laboratory, the electrons return to lower energy states and light (photons) is emitted. The light intensity, measured with a photomultiplier tube, is proportional to the original absorbed dose. After the TLD is read, it is heated again and reread. This second reading should be near zero, indicating that all the stored energy in the traps has been released and measured. This process, called annealing, also verifies that the TLD is again ready for field deployment.

Direct gamma radiation exposures are measured in milliroentgens $(\mathrm{mR})$. The measured exposure is converted to dose by calibrating the dosimeters against sources that deliver a known absorbed dose and then applying a quality factor for a beta/gamma radiation field. The resultant dose equivalents ( $\mathrm{mSv}$ or mrem) are compared to DOE Order 5400.5 radiation protection standards. The doses at the site boundaries are also compared to background measurements to determine the contribution, if any, from LLNL operations. To ensure accuracy in TLD measurements, some TLDs are irradiated each quarter to specific exposures for calibration purposes. Once each quarter, other 
TLDs are irradiated to specific exposures to serve as quality control accuracy checks. Duplicate TLDs are co-located in the field at several locations each quarter to assess TLD measurement precision.

Environmental neutron dose measurements are made using ${ }^{235} \mathrm{U}$ track-etch detectors (modified neutron rem-meters) at eight Livermore-site perimeter locations (Figure 10-1). A detailed description of the detector and spark-count procedure may be found in Fisher (1976). The neutron rem-meters are read each quarter. To ensure accuracy of measurements, quality control checks are conducted on two rem-meters selected at random each quarter.

\section{Results}

\section{Livermore Site}

Table 10-1 shows the quarterly and annual TLD gamma radiation dose equivalent for the Livermore-site perimeter locations, and Table 10-2 presents data for Livermore Valley off-site locations. The mean 1992 dose equivalent from

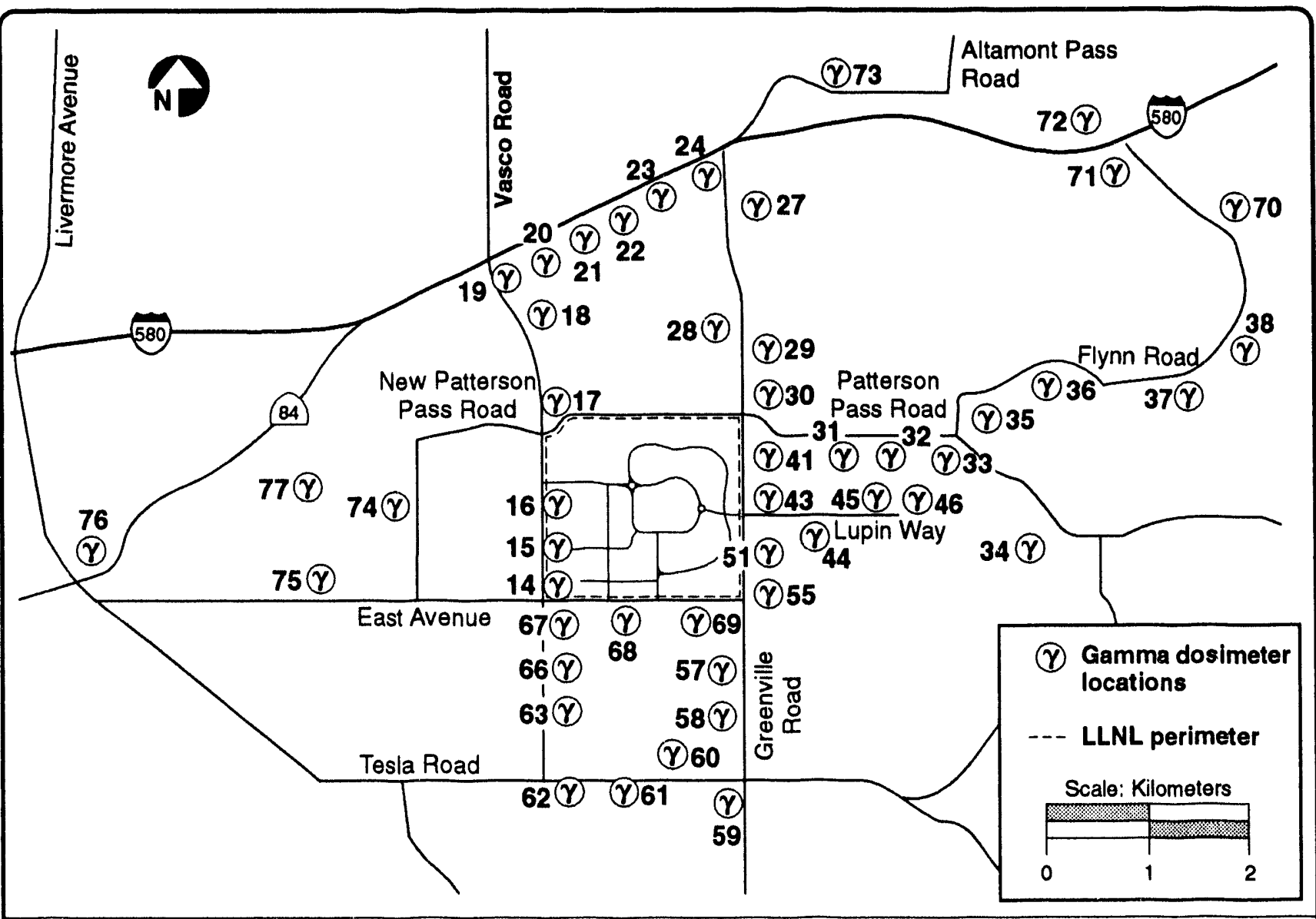

Figure 10-2. Gamma dosimeter locations, Livermore Valley, 1992. 
external direct radiation exposure at the Livermore-site perimeter, $0.66 \mathrm{mSv}$ (66 mrem), is the same as background external dose measured in the Livermore Valley $(0.66 \mathrm{mSv} ; 66 \mathrm{mrem})$. This annual total dose equivalent is typical when compared to that of previous years, i.e., $1987,0.64 \mathrm{mSv}$ (64 mrem); $1988,0.63 \mathrm{mSv}$ (63 mrem); 1989, $0.63 \mathrm{mSv}$ (63 mrem); 1990, $0.65 \mathrm{mSv}$ (65 mrem); and 1991, $0.65 \mathrm{mSv}$ (65 mrem).

Table 10-3 presents the data for neutron monitoring at the Livermore-site perimeter. The annual average neutron radiation dose at the Livermore-site perimeter for 1992 was $0.058 \mathrm{mSv}$ (5.8 mrem), similar to values in $1987,0.050 \mathrm{mSv}$ (5.0 mrem); $1988,0.042 \mathrm{mSv}(4.2 \mathrm{mrem}) ; 1989,0.053 \mathrm{mSv}$ (5.3 mrem); 1990, $0.046 \mathrm{mSv}$ (4.6 mrem); and 1991, $0.047 \mathrm{mSv}$ (4.7 mrem). The neutron doses measured at the site boundary are consistent with those expected from natural background radiation (NCRP 1976a, b). An absorbed dose rate of $0.047 \mathrm{mSv}$ (4.7 mrem) per year due to cosmic rays was estimated for LLNL from a plot in Natural Background Radiation in the United States (NCRP 1976b). This is based

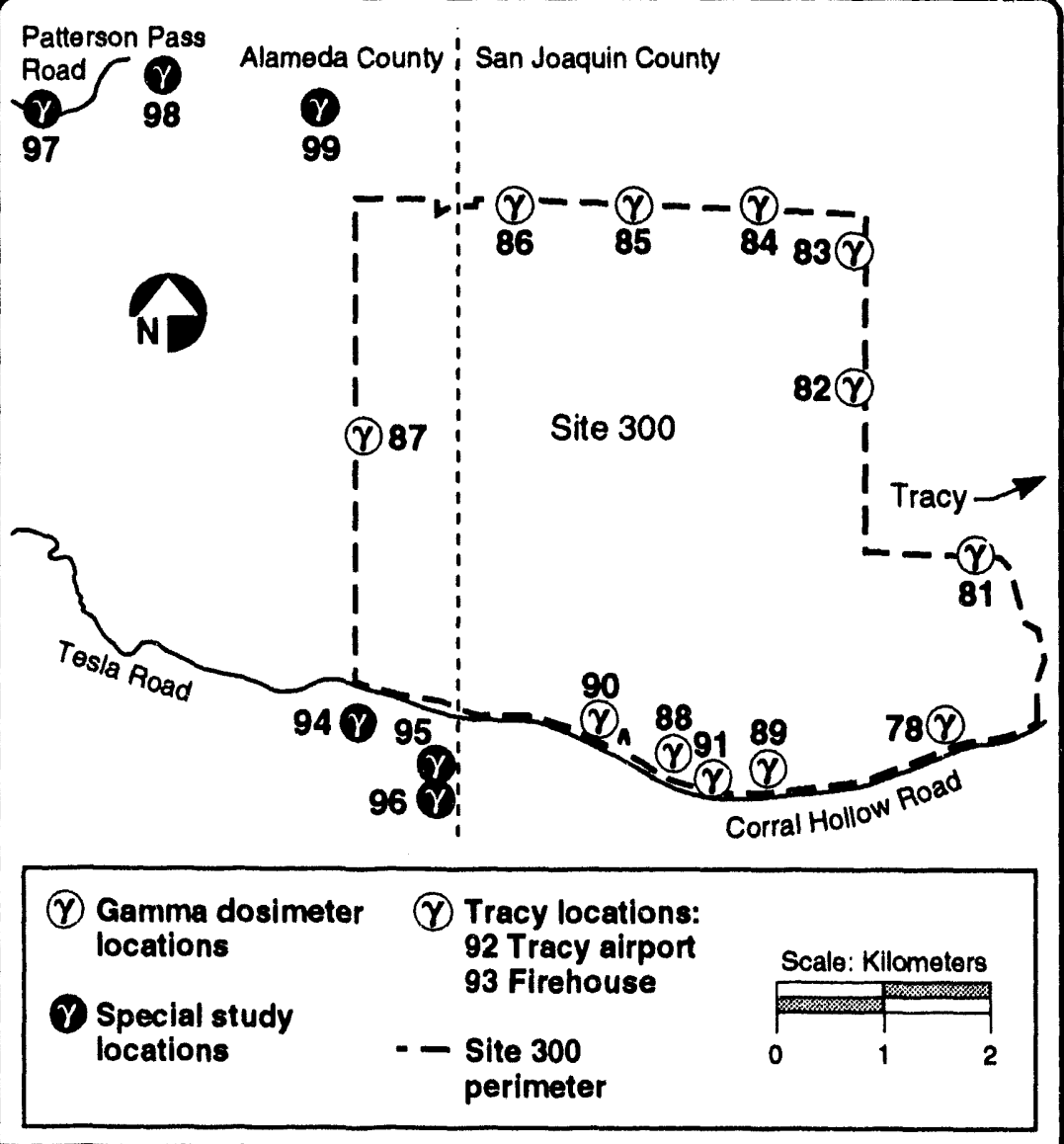

Figure 10-3. Gamma dosimeter locations, Site 300, 1992. upon an energy deposition rate of $0.043 \mu \mathrm{rad} / \mathrm{h}$ and a quality factor of 10 . The dose rate was also derived from information in NCRP 50 (NCRP 1976a) as $0.087 \mathrm{mSv}$ (8.7 mrem), which is based on an energy deposition rate of $0.1 \mu \mathrm{rad} / \mathrm{h}$. These dose rates are consistent with the 1992 monitored neutron dose at the LLNL perimeter.

Site 300

Table 10-4 presents the TLD data for routine monitoring at Site 300 during 1992, expressed as dose equivalent. The perimeter average measured dose in 1992 was $0.77 \mathrm{mSv}$ (77 mrem), ranging from 0.71 to $0.85 \mathrm{mSv}$ (71 to $85 \mathrm{mrem})$. The off-site measured doses were 0.60 and $0.71 \mathrm{mSv}$ (60 and $71 \mathrm{mrem}$ ) at Locations 92 and 93 (in and near Tracy, CA). 


\section{Environmental Impact}

\section{Livermore Site}

Based on past measurements (Lindeken et al. 1973), environmental terrestrial (geologic) radiation doses in the Livermore Valley vary from 0.30 to $0.60 \mathrm{mSv} / \mathrm{y}$ ( 30 to $60 \mathrm{mrem} / \mathrm{y}$ ). Cosmic radiation, as calculated for the local elevation and geomagnetic latitude according to the data of Lowder and Beck (1966), is about $0.35 \mathrm{mSv} / \mathrm{y}(35 \mathrm{mrem} / \mathrm{y})$. This combination results in a typical total direct radiation dose level of 0.65 to $0.95 \mathrm{mSv} / \mathrm{y}(65$ to $95 \mathrm{mrem} / \mathrm{y})$. Direct radiation doses measured at the LLNL perimeter in 1992 fall within these predicted values and are statistically equivalent to the off-site doses, which are considered natural background levels. This indicates that there is no contribution from LLNL operations to the direct radiation dose at or beyond the Livermore-site perimeter.

\section{Site 300}

The initial TLD network design limited monitoring to the Site 300 perimeter and two locations in and near the city of Tracy. These off-site locations were chosen to represent background radiation levels. However, the Tracy locations are located on a geological substrate different from that at Site 300 . The 1992 average measured dose at the Site 300 perimeter was $0.77 \mathrm{mSv}(77 \mathrm{mrem})$, and the average measured doses near the city of Tracy were 0.60 and $0.71 \mathrm{mSv}$ (60 and $71 \mathrm{mrem})$, respectively. The doses at the site perimeter fall within the expected range for background locations (Lindeken et al. 1973). The doses measured at the Tracy locations are lower than would be expected for background locations of geologic origin like that at Site 300.

\section{Special Study}

Tables 10-5 and 10-6 present results from the special gamma radiation study conducted at and near Site 300 and in the San Francisco Bay Area to determine if the geology of Site 300 (the Neroly rock formation) or the work conducted at Site 300 contribute to slightly higher dose levels measured at some locations. When the direct radiation monitoring program was implemented at the Site 300 perimeter, LLNL observed consistently higher dose levels at some locations. A special study was started in 1991 to monitor gamma radiation at six locations on the Neroly Formation but away from the Site 300 perimeter. Comparisons of data from these locations to those on the Site 300 perimeter were inconclusive. Three locations south of Site 300 exhibited dose levels higher than those from the Site 300 perimeter, and three locations north of Site 300 exhibited doses that were lower. Therefore, the study was expanded during 1992 to investigate variations in background radiation levels of the Neroly Formation. By reviewing geological maps, we identified Neroly Formation rock 40 to 50 kilometers away from 


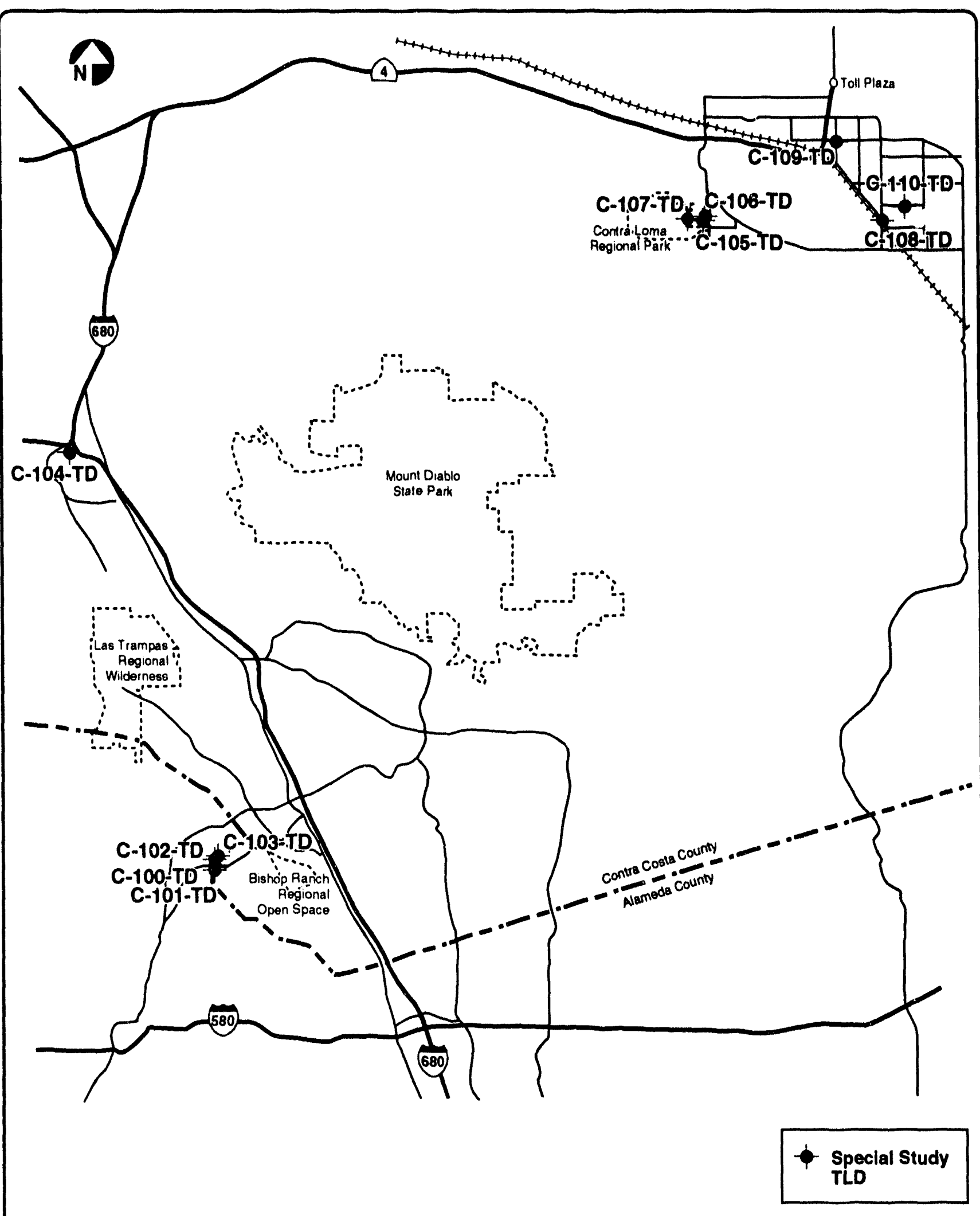

Figure 10-4. Gamma dosimeter locations, San Francisco Bay Area, 1992. 
Site 300. We conducted TLD monitoring at locations in these to Neroly Formation rocks for a one-year period (see Figure 10-5).

An analysis of variance (ANOVA) was performed on the data. The data were grouped as follows: Site 300 perimeter, Site 300 south, Site 300 north, and San Francisco Bay Area. The mean estimate at the Bay Area location is $0.68 \pm$ $0.11 \mathrm{mSV}$ (68 $\pm 11 \mathrm{mrem}$ ), compared to $0.77 \pm 0.05 \mathrm{mSv}$ (77 $\pm 5 \mathrm{mrem}$ ) at Site 300 perimeter, $0.72 \pm 0.04 \mathrm{mSv}(72 \pm 4 \mathrm{mrem})$ at Site 300 north location, and $0.90 \pm 0.04(90 \pm 4 \mathrm{mrem})$ at Site 300 south. The dose levels at the Site 300 perimeter and the Bay Area locations show a significant difference by ANOVA. There is no significant difference between the Bay Area and Site 300 off-site north, and there is no significant difference between Site 300 south and Site 300. However, some Bay Area locations had dose levels equivalent to those at Site 300 perimeter and Site 300 south.

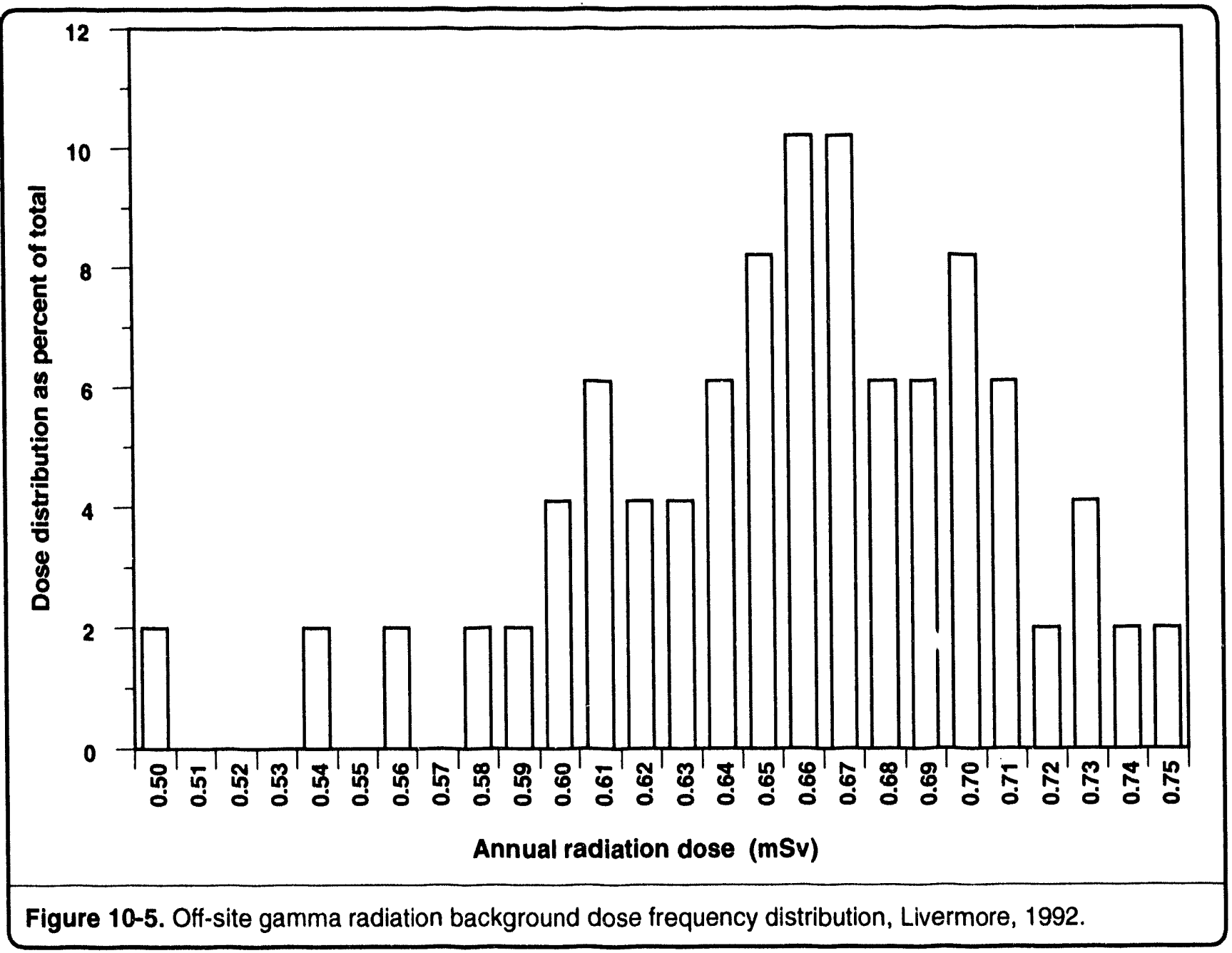


Variability of gamma measurements in the Neroly Formation rock has been investigated by LLNL (Webster-Scholten 1993) in a study of variable uranium concentrations in ground water. This study concluded "that volcaniclastic Neroly Formation rocks are derived from extrusive rocks formed in what is now the Sierra Nevada region. Clastic material from the erosion of these igneous rocks was transported into the area of Site 300 and lithified to form the Neroly Formation. Uranium distribution in the original magma was variable and consequently resulted in variable uranium content to the subsequent derived formation. Also, volcanic ash containing uranium covered large areas near Site 300. Some of the ash was deposited in the Neroly Formation. However, volcanic ash falls vary in intensity and accumulation, also resulting in variable distribution uranium." Therefore, variability in background radiation levels such as those we have observed is not surprising.

The gamma radiation measurements at Site 300 fall within the range of readings from the suite of locations sampled in this special study. This indicates that the Site 300 gamma readings could result from natural uranium within the Neroly Formation, although a more detailed study of the rock itself would be necessary to verify this finding. LLNL has discontinued the San Francisco Bay Area special study; however, LLNL will continue gamma measurements on the north and south sides of Site 300 . 
Table 10-1. TLD environmental radiation measurements (in $\mathrm{mSv}$ ), Livermoresite perimeter, 1992.

\begin{tabular}{cccccc}
\hline Locationa & Jan.-Mar. & Apr.June & July-Sept. & Oct.-Dec. & Annual Total \\
\hline 1 & 0.18 & 0.18 & 0.17 & 0.19 & 0.72 \\
2 & 0.16 & 0.16 & 0.15 & 0.18 & 0.65 \\
3 & 0.18 & 0.18 & 0.17 & 0.19 & 0.72 \\
4 & 0.18 & 0.18 & 0.17 & 0.23 & 0.76 \\
5 & 0.18 & 0.18 & 0.17 & 0.19 & 0.72 \\
6 & 0.18 & 0.17 & 0.14 & 0.20 & 0.69 \\
7 & 0.17 & 0.18 & 0.16 & 0.19 & 0.70 \\
10 & 0.16 & 0.17 & 0.16 & 0.18 & 0.67 \\
11 & 0.14 & 0.13 & 0.12 & 0.14 & 0.53 \\
12 & 0.15 & 0.14 & 0.14 & 0.17 & 0.60 \\
13 & 0.14 & 0.15 & 0.14 & 0.16 & 0.59 \\
42 & 0.16 & 0.16 & 0.15 & 0.18 & 0.65 \\
47 & 0.16 & 0.16 & 0.15 & 0.18 & 0.65 \\
50 & 0.16 & 0.15 & 0.14 & 0.17 & 0.62 \\
52 & 0.16 & 0.15 & 0.14 & 0.17 & 0.62 \\
56 & 0.16 & 0.16 & 0.15 & 0.17 & 0.64 \\
& & & & & \\
Mean & 0.16 & 0.16 & 0.15 & 0.18 & 0.66 \\
Std Dev & 0.01 & 0.02 & 0.01 & 0.02 & 0.06 \\
& & & & & \\
Mean & 16 & 16 & 15 & 18 & 6 \\
Std Dev & 1 & 2 & 1 & 2 & \\
\hline & & & & & 6 \\
\hline
\end{tabular}

a See Fig. 10-1 for sampling locations. 
Table 10-2. TLD environmental radiation measurements (in $\mathrm{mSv}$ ), Livermore Valley, 1992.

\begin{tabular}{|c|c|c|c|c|c|}
\hline Location" & Jan.-Mar. & Apr.-June & July-Sept. & Oct-Dec. & Annual Total \\
\hline 14 & 0.16 & 0.15 & 0.15 & 0.18 & 0.64 \\
\hline 15 & 0.16 & 0.15 & 0.14 & 0.18 & 0.63 \\
\hline 16 & 0.17 & 0.16 & 0.15 & 0.18 & 0.66 \\
\hline 17 & 0.17 & 0.16 & 0.15 & 0.18 & 0.66 \\
\hline 18 & 0.13 & 0.12 & 0.12 & 0.13 & 0.50 \\
\hline 19 & 0.17 & 0.16 & 0.15 & $-b$ & $0.64^{c}$ \\
\hline 20 & 0.16 & 0.15 & 0.14 & -b & $0.60^{c}$ \\
\hline 21 & 0.16 & 0.15 & 0.14 & - & $0.60^{c}$ \\
\hline 22 & 0.18 & 0.17 & 0.16 & 0.19 & 0.70 \\
\hline 23 & 0.18 & 0.17 & 0.16 & 0.19 & 0.70 \\
\hline 24 & 0.17 & 0.17 & 0.17 & $-b$ & $0.68^{c}$ \\
\hline 27 & 0.18 & 0.18 & 0.17 & 0.20 & 0.73 \\
\hline 28 & 0.18 & 0.19 & 0.17 & 0.21 & 0.75 \\
\hline 29 & 0.17 & 0.18 & 0.17 & 0.19 & 0.71 \\
\hline 30 & 0.16 & 0.17 & 0.16 & 0.18 & 0.67 \\
\hline 31 & 0.16 & $-b$ & 0.15 & 0.17 & $0.64^{c}$ \\
\hline 32 & 0.16 & $-b$ & 0.15 & 0.18 & $0.65^{c}$ \\
\hline 33 & 0.17 & 0.18 & b & 0.19 & $0.72^{c}$ \\
\hline 34 & 0.18 & - b & 0.17 & 0.20 & $0.73^{c}$ \\
\hline 35 & 0.17 & 0.17 & 0.16 & 0.19 & 0.69 \\
\hline 36 & 0.17 & 0.18 & 0.18 & b- & $0.66^{c}$ \\
\hline 37 & 0.16 & 0.17 & 0.15 & 0.18 & 0.66 \\
\hline 38 & 0.17 & 0.19 & 0.16 & 0.18 & 0.70 \\
\hline 41 & 0.16 & 0.16 & - & 0.18 & $0.67^{c}$ \\
\hline 43 & 0.17 & 0.17 & 0.16 & 0.19 & 0.69 \\
\hline 44 & $-b$ & 0.18 & 0.17 & $-b$ & $0.61^{c}$ \\
\hline 45 & 0.16 & 0.15 & 0.14 & 0.16 & 0.61 \\
\hline 46 & 0.16 & 0.15 & 0.14 & 0.17 & 0.62 \\
\hline 51 & 0.17 & $-b$ & 0.14 & 0.18 & $0.65^{c}$ \\
\hline 55 & 0.16 & 0.15 & 0.14 & 0.17 & 0.62 \\
\hline 57 & 0.17 & 0.18 & 0.16 & 0.20 & 0.71 \\
\hline 58 & 0.17 & 0.16 & 0.15 & 0.18 & 0.66 \\
\hline 59 & 0.17 & 0.16 & 0.16 & 0.18 & 0.67 \\
\hline
\end{tabular}


Table 10-2. TLD environmental radiation measurements (in $\mathrm{mSv}$ ), Livermore Valley, 1992 (concluded).

\begin{tabular}{|c|c|c|c|c|c|}
\hline Location & Jan.-Mar. & Apr.-June & July-Sept. & Oct.-Dec. & Annual Total \\
\hline 60 & 0.17 & 0.16 & 0.16 & 0.18 & 0.67 \\
\hline 61 & 0.15 & 0.14 & 0.13 & 0.16 & 0.58 \\
\hline 62 & 0.15 & 0.14 & 0.14 & 0.16 & 0.59 \\
\hline 63 & 0.16 & 0.16 & 0.16 & 0.18 & 0.66 \\
\hline 66 & 0.18 & 0.16 & 0.16 & 0.19 & 0.69 \\
\hline 67 & 0.15 & 0.16 & 0.15 & 0.17 & 0.63 \\
\hline 68 & 0.16 & 0.16 & 0.15 & 0.18 & 0.65 \\
\hline 69 & 0.16 & 0.15 & 0.14 & 0.16 & 0.61 \\
\hline 70 & 0.17 & 0.15 & 0.15 & 0.18 & 0.65 \\
\hline 71 & $-^{b}$ & 0.15 & $-^{b}$ & 0.19 & 0.68 \\
\hline 72 & 0.19 & 0.18 & 0.17 & 0.20 & 0.74 \\
\hline 73 & $-^{b}$ & 0.16 & 0.16 & 0.18 & $0.67^{c}$ \\
\hline 74 & 0.15 & 0.15 & 0.14 & 0.17 & 0.61 \\
\hline 75 & 0.14 & 0.13 & 0.12 & 0.15 & 0.54 \\
\hline 76 & 0.12 & 0.12 & 0.13 & 0.19 & 0.56 \\
\hline 77 & $-^{b}$ & $\square^{b}$ & $-b$ & 0.17 & $0.68^{c}$ \\
\hline Mean & 0.16 & 0.16 & 0.15 & 0.18 & 0.66 \\
\hline \multirow[t]{2}{*}{ Std dev } & 0.01 & 0.02 & 0.01 & 0.01 & 0.05 \\
\hline & \multicolumn{5}{|c|}{ [mrem] } \\
\hline Mean & 16 & 16 & 15 & 18 & 66 \\
\hline Std dev & 1 & 2 & 1 & 1 & 5 \\
\hline
\end{tabular}

a See Fig. 10-2 for sampling locations.

b No data available for these samples; see Quality Assurance chapter.

c When TLD is missing, annual dose is calculated as four times the mean quarterly dose, as determined from available data. 
Table 10-3. Neutron environmental radiation measurements (in $\mathrm{mSv}$ ), Livermore-site perimeter, 1992.

\begin{tabular}{cccccc}
\hline Locationa & Jan.-Mar. & Apr.-June & July-Sept. & Oct.-Dec. & Annual Total \\
\hline 1 & 0.016 & 0.015 & 0.013 & 0.013 & 0.057 \\
2 & 0.038 & 0.014 & 0.012 & 0.008 & 0.072 \\
3 & 0.015 & 0.026 & 0.013 & 0.010 & 0.064 \\
4 & 0.018 & 0.018 & 0.007 & 0.013 & 0.056 \\
5 & 0.015 & 0.017 & $-\mathrm{b}$ & 0.011 & $0.057 \mathrm{c}$ \\
6 & 0.009 & 0.009 & 0.011 & 0.011 & 0.040 \\
7 & 0.009 & 0.017 & 0.017 & 0.014 & 0.057 \\
8 & 0.007 & 0.017 & 0.029 & 0.008 & 0.061 \\
& & & & & \\
Mean & 0.016 & 0.017 & 0.015 & 0.011 & 0.058 \\
Std dev & 0.010 & 0.005 & 0.007 & 0.002 & 0.009 \\
& & & & & \\
Mean & & & [mrem] & & 1.1 \\
Std dev & 1.6 & 1.7 & 1.5 & 5.8 \\
\hline
\end{tabular}

a See Fig. 10-1 for sampling locations.

b No data available for these samples; see Quality Assurance chapter.

c When neutron dosimeter data are missing, the sannual dose is calculated as four times the average quarterly dose, as determined from available data. 
Table 10-4. TLD environmental radiation measurements (in mSv), Site 300 perimeter and off-site locations, 1992.

\begin{tabular}{|c|c|c|c|c|c|}
\hline Location ${ }^{a}$ & Jan.-Mar. & Apr.-June & July-Sept. & Oct.-Dec. & Annual Total \\
\hline \multicolumn{6}{|c|}{ Site perimeter } \\
\hline 78 & 0.17 & 0.18 & $-b$ & 0.18 & $0.71^{\mathrm{c}}$ \\
\hline 81 & 0.20 & 0.21 & 0.20 & 0.24 & 0.85 \\
\hline 82 & 0.18 & 0.18 & 0.18 & $-b$ & $0.72^{c}$ \\
\hline 83 & 0.19 & 0.20 & $-b$ & $-b$ & $0.78^{c}$ \\
\hline 84 & 0.18 & 0.19 & 0.18 & - & $0.73^{c}$ \\
\hline 85 & 0.18 & 0.19 & $-b$ & $-b$ & $0.74^{c}$ \\
\hline 86 & 0.18 & 0.20 & 0.17 & $-b$ & $0.73^{c}$ \\
\hline 87 & 0.20 & 0.24 & 0.21 & $-b$ & $0.87^{c}$ \\
\hline 88 & 0.18 & 0.2 .0 & 0.17 & 0.21 & 0.76 \\
\hline 89 & 0.19 & 0.19 & 0.19 & 0.23 & 0.80 \\
\hline 90 & 0.18 & 0.19 & 0.18 & 0.22 & 0.77 \\
\hline 91 & 0.18 & 0.19 & 0.17 & 0.21 & 0.75 \\
\hline Mean & 0.18 & 0.20 & 0.18 & 0.22 & 0.77 \\
\hline \multirow[t]{2}{*}{ Std dev } & 0.01 & 0.02 & 0.01 & 0.02 & 0.05 \\
\hline & \multicolumn{5}{|c|}{ [mrem] } \\
\hline Mean & 18 & 20 & 18 & 22 & 77 \\
\hline Std dev & 1 & 2 & 1 & 2 & 5 \\
\hline \multicolumn{6}{|l|}{ Off site } \\
\hline 92 & 0.18 & 0.18 & 0.16 & 0.19 & 0.71 \\
\hline 93 & 0.15 & 0.15 & 0.14 & 0.16 & 0.60 \\
\hline Mean & 0.17 & 0.17 & 0.15 & 0.18 & 0.66 \\
\hline Std dev & 0.02 & 0.02 & 0.01 & 0.02 & 0.08 \\
\hline & \multicolumn{5}{|c|}{ [mrem] } \\
\hline Mean & 17 & 17 & 15 & 18 & 66 \\
\hline Std dev & 2 & 2 & 1 & 2 & 8 \\
\hline
\end{tabular}

a See Fig. 10-3 for sampling locations.

b No data available for these samples; see Quality Assurance chapter.

c When TLD data are missing, the annual dose is calculated as four times the average quarterly dose, as determined from available data. 
Table 10-5. TLD environmental radiation measurements (in mSv), special study, Site 300 vicinity, 1992.

\begin{tabular}{cccccc}
\hline Locationa $^{\mathrm{a}}$ & Jan.-Mar. & Apr.June & July-Sept. & Oct.-Dec. & Annual Total \\
\hline 94 & 0.22 & $-b$ & 0.24 & 0.28 & $0.99^{\mathrm{c}}$ \\
95 & 0.20 & 0.22 & 0.21 & 0.23 & 0.86 \\
96 & 0.20 & 0.21 & 0.21 & 0.25 & 0.87 \\
97 & $-\mathrm{b}$ & $-b$ & 0.17 & $-b$ & $0.68^{\mathrm{C}}$ \\
98 & $-\mathrm{b}$ & 0.18 & 0.17 & 0.21 & $0.75^{\mathrm{c}}$ \\
99 & 0.18 & 0.18 & 0.17 & 0.20 & 0.73 \\
& & & & & \\
Mean & 0.20 & 0.20 & 0.20 & 0.23 & 0.81 \\
Std dev & 0.02 & 0.02 & 0.03 & 0.03 & 0.11 \\
& & & & & \\
Mean & 20 & 20 & 20 & 23 & 81 \\
Std dev & 2 & 2 & 3 & 3 & 11 \\
\hline
\end{tabular}

a Soe Fig. 10-3 for sampling locations.

b No data available for these samples; see Quality Assurance chapter.

c When TLD data are missing, the annual dose is calculated as four times the average quarterly dose, as determined from available data. 
Table 10-6. TLD environmental radiation measurements (in $\mathrm{mSv}$ ), special study, San Francisco Bay Area, 1992-1993.

\begin{tabular}{|c|c|c|c|c|c|}
\hline Location ${ }^{a}$ & $\begin{array}{l}\text { Apr.-Jun. } \\
1992\end{array}$ & $\begin{array}{c}\text { July-Sept. } \\
1092\end{array}$ & $\begin{array}{c}\text { Oct.-Dec. } \\
1992\end{array}$ & $\begin{array}{c}\text { Jan.-Mar. } \\
1993\end{array}$ & $\begin{array}{c}\text { Annual } \\
\text { Total }\end{array}$ \\
\hline C.100-TD & 0.15 & 0.14 & 0.17 & 0.15 & 0.61 \\
\hline C-101-TD & 0.15 & 0.15 & 0.16 & 0.14 & 0.60 \\
\hline C.102-TD & 0.15 & 0.14 & $-^{b}$ & 0.13 & 0.56 \\
\hline C-103-TD & 0.14 & 0.14 & 0.15 & 0.14 & 0.57 \\
\hline C-104-TD & 0.21 & 0.22 & 0.23 & 0.20 & 0.86 \\
\hline C-105-TD & 0.14 & 0.13 & 0.16 & 0.13 & 0.56 \\
\hline C-106-TD & 0.17 & 0.18 & 0.19 & 0.16 & 0.70 \\
\hline C-107-TD & 0.19 & $-b$ & 0.20 & 0.16 & 0.73 \\
\hline C-108-TD & 0.16 & 0.16 & 0.17 & 0.14 & 0.63 \\
\hline C-109-TD & 0.21 & 0.20 & 0.23 & 0.18 & $0.82^{c}$ \\
\hline C-110-TD & 0.20 & 0.20 & 0.23 & 0.19 & $0.82^{c}$ \\
\hline Mean & 0.17 & 0.17 & 0.19 & 0.16 & 0.68 \\
\hline \multirow[t]{2}{*}{ Std Dev } & 0.03 & 0.03 & 0.03 & 0.02 & 0.11 \\
\hline & \multicolumn{5}{|c|}{ [mrem] } \\
\hline Mean & 17 & 17 & 19 & 16 & 68 \\
\hline Std Dev & 3 & 3 & 3 & 2 & 11 \\
\hline
\end{tabular}

a See Fig. 10-4 for sampling locations.

b No data available for these samples; see Quality Assurance chapter.

c When TLD data are missing, the annual dose is calculated as four times the average quarterly dose, as determined from available data. 
Robert J. Harrach

Kris A. Surano

\section{Introduction}

Radiological doses to the public are due to both natural and man-made radiation. The total radiological dose to different populations from these sources is determined by extensive measurements and calculations. This chapter describes LLNL's radiological dose assessments, made to determine the impact of LLNL operations, and contains a discussion of the analysis we performed to establish LLNL's compliance with the National Emission Standards for Hazardous Air Pollutants (NESHAPs).

Because this report is distributed outside the scientific community, we have included a brief preliminary discussion to enable the nontechnical reader to understand more easily the radiological dose assessment information we report. For more information the reader is referred to Radiation: Doses, Effects, Risks (U.N. Environment Programme 1985).

\section{Natural and Man-Made Radiation}

By far the greatest part of radiation received by the world's population comes from natural sources-primarily cosmic rays that impinge on Earth's atmosphere from space, and radionuclides naturally present in our environment, such as radioactive materials in soil and rocks. Among these terrestrial sources are carbon-14, potassium-40, rubidium-87, uranium-238, thorium-232, and the radioactive elements, such as radon, that arise following decay of uranium and thorium. The source of human exposure to natural radiation can be external (from substances staying outside the body) or internal (from substances inhaled in air or ingested in food and water). Individual doses vary with location. The level of cosmic radiation increases with altitude, because there is less air overhead to act as a shield, and the earth's poles receive more cosmic radiation than the equatorial regions, because the earth's magnetic field diverts the radiation. The levels of terrestrial radiation differ from place to place around the United States and around the world mainly due to variations in soil and rock composition.

Adding to this pervasive natural or background radiation is man-made radiation from radionuclides used in medicine, consumer products, the production of energy, and the production of nuclear weapons. Exposure to manmade sources can be controlled more readily than exposure to most natural sources. However, nuclear explosives tested in the atmosphere in the 1950s- 
1960s spread radioactivity across the surface of the globe, and nuclear reactor accidents such as that at Chernobyl affected large areas. At present, medical treatment is the largest common source of public exposure to man-made radiation. Individual medical doses vary enormously-someone who has never had an x-ray examination may receive zero dose while patients undergoing treatment for cancer may receive many thousand times the annual average dose from natural radiation. Another source of public exposure to man-made radiation is consumer products, including luminous-dial watches, smoke detectors, airport $x$-ray baggage inspection systems, optical lenses, false teeth, and tobacco products.

\section{Radioactivity}

Generally, naturally occurring isotopes are stable, but notable exceptions include carbon-14, potassium-40, uranium-235, and uranium-238, which are naturally occurring but radioactive. Nuclear decay divides into three main categories: alpha, beta, and gamma. Alpha decay is the spontaneous emission of an alpha particle (a bound state of two protons and two neutrons-the nucleus of a helium atom) from a nucleus containing a large number of protons (82 or more most commonly). Beta decay is the spontaneous conversion of a neutron to a proton in the nucleus with the emission of an electron, and gamma decay is the spontaneous emission of high-energy photons (high-frequency light) by nuclei.

Radioisotopes decay at quite different rates; their "half-life," or length of time for half of the atoms to decay, spans a wide range from fractions of a second to millions of years. For example, tritium (the radioactive form of hydrogen) has a 12.3-year half-life, compared to 24,131 years for plutonium-239.

Some radioisotopes undergo a decay chain, forming radioisotopes that decay into other radioisotopes until a stable state is achieved. As an example, an atom of uranium-238 can undergo alpha decay, leaving behind a daughter, thorium-234, which is also radioactive. The transformations of the decay chain continue, ending with the formation of lead-206, which is a stable isotope.

Radioactivity can be hazardous because radiation (alpha particle, beta particle, or gamma rays) is released with great energy. It is capable of altering the electronic configuration of atoms and molecules, especially by stripping one or more electrons off the atoms of the irradiated material, thereby disrupting the chemical activity in living cells. If the disruption is severe enough to overwhelm the normal restorative powers of the cell, the cell may die or become permanently damaged. Cells are exposed to many naturally occurring sources of chemical disruption, including toxic chemicals in food, microbes that cause disease, high-energy radiation from outer space (cosmic rays), heat and light (including the sun's rays, which can cause sunburn and skin cancer). Consequently, cells and living organisms have evolved with the ability to survive 


\section{Radiological Dose Assessment}

limited amounts of damage, including that due to naturally occurring radioactivity.

Three main factors determine the radiation-induced damage that might be caused to living tissue: the number of radioactive nuclei that are present, the rate they give off energy, and the effectiveness of energy transfer to the host medium, i.e., how the radiation interacts with the tissue. Alpha radiation can be halted by a piece of paper and can scarcely penetrate the dead outer layers of skin. Radioisotopes that give off alpha radiation are generally not health hazards unless they get inside the body through an open wound, or are ingested or inhaled. In those cases alpha radiation can be especially damaging, because its disruptive energy can be deposited within a small distance, resulting in significant energy deposited in a few cells. Beta radiation from nuclear decay typically penetrates a centimeter or two of living tissue. It therefore deposits energy over many cells, decreasing the insult to any single cell. Gamma radiation is extremely penetrating and can pass through most materials, only being significantly attenuated by thick slabs of dense materials, such as lead.

\section{Measurement of Radioactivity and Dose}

The rate that a nucleus decays is expressed in units of becquerels, abbreviated $\mathrm{Bq}$, where one becquerel is one decay per second, or alternatively in curies, $\mathrm{Ci}$, where one curie equals $3.7 \times 10^{10}$ (37 billion) decays per second, or $3.7 \times 10^{10} \mathrm{~Bq}$ (approximately equal to the decay rate of $1 \mathrm{gram}$ of pure radium). Becquerels and curies are not measures of the effect of radiation on living tissue. This depends on the efficiency of energy deposition as the radiation traverses matter.

The amount of energy deposited in living tissue is called the "dose." The amount of radiation energy absorbed per gram of tissue is called the "absorbed dose," and is expressed in units of rads or grays (G), where $1 \mathrm{G}$ equals 100 rads. Because an absorbed dose produced by alpha radiation is more damaging to living tissue than the same dose produced by beta or gamma radiation, the absorbed dose is multiplied by a "quality factor" to give the dose equivalent. The quality factor for alpha radiation is 20; for beta and gamma, 1 . The dose equivalent is measured in units of rem or sievert (Sv); $1 \mathrm{~Sv}$ equals $100 \mathrm{rem}$. Also commonly used are millirem (mrem) and millisievert (mSv), which are onethousandth of a rem and sievert, respectively.

Just as one type of radiation can be more damaging than others, some parts of the body are more vulnerable to radiation damage than others, so the different parts of the body are given weightings. For example, a given radiation dose is more likely to cause cancer in the lung than in the thyroid. The reproductive organs are of particular concern, because of the potential risk of genetic damage. Once particular organs are weighted appropriately, the dose equivalent becomes the "effective dose equivalent" (EDE), also expressed in rem or sievert. 
The effective dose equivalent describes doses to individuals. When individual effective dose equivalents received by a group of people are summed, the result is called the "collective effective dose equivalent" and is expressed in person-sievert or person-rem. Finally, to account for the long-term effects of radionuclides as they continue to decay and affect generations of people, we calculate the dose over many years, summing the effect over time. This is termed the "collective effective dose equivalent commitment." Most of our discussion in this chapter deals with the effective dose equivalent and the collective effective dose equivalent.

\section{Sources of Natural Radioactivity}

The average radiation dose from natural sources in the United States is $3.0 \mathrm{mSv} / \mathrm{y}(300 \mathrm{mrem} / \mathrm{y})$. Approximately $0.3 \mathrm{mSv} / \mathrm{y}(30 \mathrm{mrem} / \mathrm{y})$ of this exposure comes from high-energy radiation from outer space (cosmic rays). Terrestrial sources, mainly radionuclides in rock and soil, account for approximately $0.3 \mathrm{mSv} / \mathrm{y}(30 \mathrm{mrem} / \mathrm{y})$ of the average natural dose. Another significant part of the dose comes from radionuclides we ingest through food and drink, resulting in approximately $0.4 \mathrm{~m} \mathrm{~Sv} / \mathrm{y}(40 \mathrm{mrem} / \mathrm{y})$. Potassium-40 and carbon-14 are common radionuclides in food.

The remaining $67 \%$ of the average dose from natural sources in the United States comes from radon gas. Radon is one of the major radionuclides produced by uranium decay, and our inhalation dose is dominated by the short-lived decay products of radon. The dose varies with geographic location, but the average U.S. citizen receives approximately $2 \mathrm{mSv} / \mathrm{y}(200 \mathrm{mrem} / \mathrm{y})$ of natural exposure from radon.

Radon gas seeps out of the earth worldwide. Radon in water and natural gas provide additional, but less important, sources of radon in homes. Consumption of water high in radon is not the main exposure source; a greater exposure is believed to arise from inhalation of radon in water vapor from showers. The U.S. Environmental Protection Agency (EPA) has instituted a major program to educate the public regarding the effects of naturally occurring radon (U.S. Environmental Protection Agency 1986).

Medical treatment is the largest common source of public exposure to manmade radiation, and most of it is from medical $x$ rays. These contribute $0.39 \mathrm{mSv}$ ( $39 \mathrm{mrem}$ ) to the average whole-body dose in the United States, but individual doses vary enormously. For example, a typical dental $x$-ray series results in a skin dose (not whole body) of approximately $2.5 \mathrm{mSv}$ (250 mrem). Nuclear medicine contributes $0.14 \mathrm{mSv}$ ( $14 \mathrm{mrem}$ ) to the average, and consumer products add $0.1 \mathrm{mSv}$ (10 mrem). For a typical member of the public, radiation from medical procedures and consumer products result in a dose of approximately $0.63 \mathrm{mSv} / \mathrm{y}(63 \mathrm{mrem} / \mathrm{y})$. The average dose from other man-made sources, 
including fallout from nuclear testing, is about $0.02 \mathrm{mSv}$ ( $2 \mathrm{mrem}$ ). Figure 11-1 shows the distribution of annual radiation doses from various sources.

\section{Radiation Sources, Control Measures, and Standards}

\section{Radionuclides at LLNL}

A wide variety of radioisotopes is used at LLNL, including transuranics, biomedical tracers, tritium, mixed fission products, and other radioisotopes for general research and nuclear weapons research. Radioisotope handling procedures and work enclosures are determined for each project, depending on the isotope, the quantity being used, and the type of operations being performed. Radioisotope handling and working environments include glove boxes, exhaust hoods and laboratory bench tops. Exhaust paths to the atmosphere range from triple-filtered stacks and direct small hood roof vents at the Livermore site, to direct dispersal of depleted uranium during non-nuclear explosives testing at Site 300.

\section{LLNL's Radiation Control Program}

Protection of employees and the public from the uncontrolled release of radioactive materials into the environment is a primary consideration of LLNL. This effort consists of four stages. First, when an operation or facility is designed, a thorough

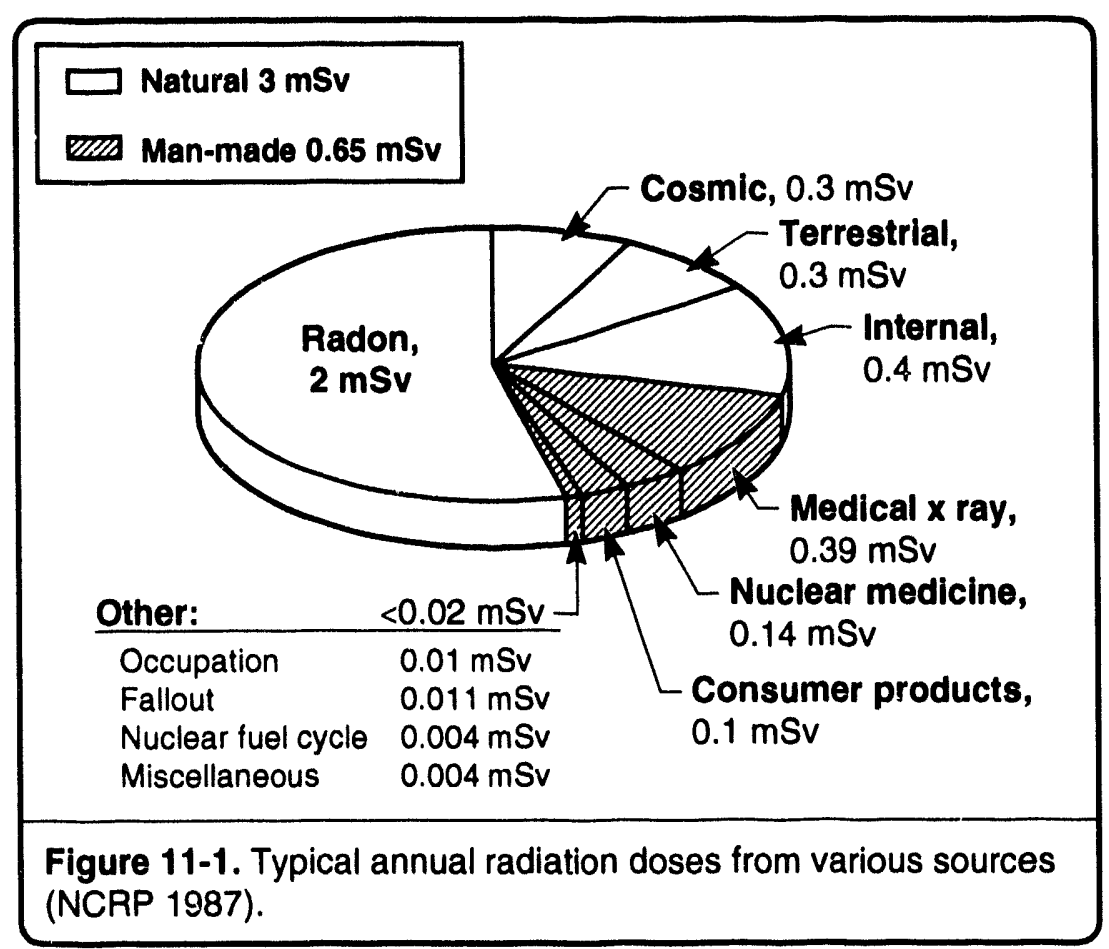
assessment of potential radiation hazards is conducted, and engineering controls are specified to minimize exposure and release of dangerous quantities of radioactivity. These controls might include, but are not limited to, physical access control, shielding, and remote handling equipment. Facility Safety Analysis Reports and Facility Safety Procedures are written to document the need for these measures and to specify the requirements for maintenance, training, emergency response, and other administrative control measures.

The other three stages of the radiation control program come into play when a facility is occupied for use. The second part of the program takes place when, prior to the conduct of an operation in the facility, an Operational Saffty 
Procedure (OSP) is written that specifies the actions to be taken in conducting a research or development project. This procedure is reviewed by analysts in the Environmental Protection Department and by industrial hygienists and health physicists in the Hazards Control Department. These reviews assess the safety of the operation, its compliance with current occupational health and environmental standards, and the adequacy of proposed engineering and administrative controls.

The OSP also specifies training requirements for personnel performing the procedure. This third part of the control program enables LLNL personnel who work with radiation and radioactivity to recognize and prevent the execution of unsafe operations.

The last stage of the radiation control program involves direct monitoring of the work environment and physical sampling of air and surfaces in facilities where radioactive materials are handled. Additionally, personal dosimetry and bioassay programs monitor for potential worker exposure to direct radiation and exposure to radioactive isotopes. This monitoring program measures the effectiveness of a facility's radiation control program, as well as providing information on worker exposures.

\section{Radiation Protection Standards}

DOE environmental radiation protection standards are provided in DOE Order 5400.5, Radiation Protection of the Public and the Environment, which incorporates standards for controlling exposures to the public from operations at DOE facilities. These standards are based on recommendations by the International Commission on Radiological Protection (ICRP 1977, 1980) and the National Council on Radiation Protection and Measurements (NCRP 1976a, b). The primary DOE radiation standards for protection of the public are $1 \mathrm{mSv} / \mathrm{y}$ (100 mrem/y) effective dose equivalent for prolonged exposure, and $5 \mathrm{mSv} / \mathrm{y}$ (500 mrem/y) effective dose equivalent for occasional exposure. These limits are based on the dose to the maximally exposed individual in an uncontrolled area.

The limits apply to the sum of the effective dose equivalent from external radiation and the committed (50-y) effective dose equivalent from radioactive materials that may be ingested or inhaled during a calendar year, and that may therefore remain in the body for many years.

DOE and LLNL also comply with the EPA's standard for radiation protection. This EPA radiation dose standard, which applies to air emissions, is defined in 40 CFR 61 . It limits to $0.1 \mathrm{mSv} / \mathrm{y}(10 \mathrm{mrem} / \mathrm{y})$ the whole-body effective dose equivalent to members of the public from nOE activities. Before December 15,1989 , the standard was $0.25 \mathrm{mSv}$; $y(25 \mathrm{mrem} / \mathrm{y})$ dose equivalent for whole-body exposures from the air pathway, and $0.75 \mathrm{mSv} / \mathrm{y}(75 \mathrm{mrem} / \mathrm{y})$ dose equivalent for exposure of any organ from the air pathway. 
Because the EPA standard is small compared to natural exposures to radioactivity, it would be difficult to prove compliance with the standard by measuring the effects of the dispersal of radioactivity in the atmosphere. EPA has therefore developed computer codes that implement its approved dosimetry model, and mandated that these codes be used to calculate potential doses to the public for compliance demonstrations. Calculations reported here used either the EPA's AIRDOS or CAP88-PC code. The latter is a recently developed, improved and expanded computer code, incorporating a modified version of AIRDOS as one of its component codes. The models used in these codes to evaluate radiological doses and consequent risk contain conservative assumptions that are expected to result in calculated doses larger than ones actually received by members of the public.

\section{Radiological Doses from Air Emissions}

In accordance with DOE environmental protection orders and other federal and state requirements, LLNL assessed the radiological impact from operations at the Livermore site and Site 300 during 1992. Small amounts of radioactive materials from LLNL operations were discharged to the environment with air and sewer effluents (see Chapter 4 on Air Monitoring and Chapter 5 on Sewage Monitoring). Because sewer effluents are not consumed, they do not represent an ingestion or inhalation pathway for radiation exposure. Therefore, doses are calculated only for material that enters the environment via air releases.

Potential radiological doses to the public are either determined from measurements of radionuclides in the environment or calculated using EPAapproved computer codes. These calculations of the potential public dose use theoretical models for transport of radionuclides through the environment, including dispersion in air, into water and food, and finally into human beings through inhalation or ingestion. Although LLNL seeks to obtain sufficient samples of the local environment to assure that its impacts are well understood, sampling for radioactivity cannot occur at all locations. The theoretical calculations are important because they set an absolute upper bound on the potential radiological impacts of LLNL operations. The radionuclide source terms used in the codes are based on measured effluents and facility inventories.

The results of the measurements and calculations reported in this chapter are an important indicator of the success of LLNL's discharge control program. Development of the Livermore Valley and the San Joaquin Vailey have decreased the distance between sources of emissions and the residents and workers that might be exposed. In all directions, people live and work within several hundred meters of LLNL's boundaries. It is therefore vital that our assessments provide the best information possible regarding the impact of LLNL operations. 


\section{Monitored Releases of Radioactive Materials}

General surveillance air monitoring for tritium and for radioactive particulates has been in place since the 1970 s and will continue. The data from this monitoring network provide continuous measures of the concentrations of radionuclides present in the air at the Livermore site, Site 300, and in the surrounding areas. This network allows for direct measurements of the overall impact of LLNL operations, as discussed in detail in Chapter 4, Air Monitoring. Beyond the general monitoring, site-specific surveillance air monitors are placed in the vicinity of "diffuse" emission sources on site, such as those (described below) associated with Buildings 292, 331,514, and 612, and in and around the southeast quadrant of the Livermore site. The data from these monitoring networks reveal the concentrations of radionuclides present in the air near these sources and allow a direct and accurate determination of their environmental impact. This practice will continue at these locations and will be instituted for any newly identified diffuse sources.

Radionuclide concentrations measured in environmental media are pre sented in the chapters in this report covering each specific medium. In particular, measurements of external direct radiation reported in Chapter 10, Environmental Radiation Monitoring, show no difference between doses measured in the immediate environs of LLNL's Livermore site and Site 300, as compared to measurements made at remote locations. While the Livermore-site boundary measurements of external gamma doses averaged $0.65 \mathrm{mSv}$ (65 mrem), Livermore Valley locations averaged $0.66 \mathrm{mSv}$ (66 mrem). Site 300 boundary locations average $0.78 \mathrm{mSv}(78 \mathrm{mrem})$, while off-site samples showed 0.65 to $0.81 \mathrm{mSv}$ ( 65 to $81 \mathrm{mrem}$ ). These measurements indicate that operations at the two sites did not significantly increase the possibility for public exposure to gamma radiation.

Routine LLNL operations during 1992 released a total of $6.55 \times 10^{12} \mathrm{~Bq}$ $(177 \mathrm{Ci})$ of tritium to the atmosphere; of that, $3.7 \times 10^{12} \mathrm{~Bq}(100 \mathrm{Ci})$ was in the form of tritiated water. An additional $9.77 \times 10^{12} \mathrm{~Bq}(264 \mathrm{Ci})$ was released by Sandia National Laboratories, California (SNL/CA), with approximately $4.81 \times 10^{12} \mathrm{~Bq}(130 \mathrm{Ci})$ in the form of tritiated water. LLNL's electron-positron linear accelerator was not activated in 1992, thereby eliminating the releases of the short half-life radioisotopes ${ }^{13} \mathrm{~N}$ (half-life of ten minutes) and ${ }^{15} \mathrm{O}$ (half-life of two minutes) that we reported in previous years. The amount of radioactivity released from LLNL during 1992 was less than during 1991, and was also below the range of previous years (see tables in Chapter 4, Air Monitoring).

There were no accidental radionuclide releases to the atmosphere at either the Livermore site or Site 300 during 1992. 


\section{Air Emissions Data}

Reported annual releases are based on inventory data and unabated EPA potential release fractions (discussed below) for unmonitored sources, and on actual emission measurements for continuously monitored facilities. The continuously monitored facilities at LLNL are Buildings 175, 231, 251, 419,490 , and 491. Many of the monitored facilities show zero emissic data are based on limit-of-sensitivity (LOS) results from the analysis oi .. samples. Use of zero values for LOS data in this case can be justified $b_{c}:^{\prime} a$ facility knowledge; the use of tested, multiple-stage, high efficiency particulate air (HEPA) filters (see next paragraph) in all significant release pathways; and isotopic analyses of selected filters, by alpha spectroscopy, which demonstrate that measured activity on filters comes from naturally occurring radionuclides such as radon daughters and polonium. In any case, given the level of HEPA filtration, it is likely that actual concentrations are closer to zero than they are to the LOS. Furthermore, even if the LOS values are substituted in screening dose calculations (a very conservative assumption), the total dose attributable to LLNL activities is not significantly affected.

HEPA filters are used in many LLNL facilities to control particulate emissions. For some discharge points, scrubbers and electrostatic precipitators aid the control of emissions. No stack emission control systems are implemented for tritium. The operational performanc? ' 11 HEPA filtration systems is tested on a routine basis. The efficiency, le-stage HEPA filter is $99.97 \%$. Double-staged filter systems are in pla me discharge points. Triple-stage HEPA filters are used on glove-box ventulation systems in the Building 332 Plutonium Facility and in a portivn of Building 251.

Some LLNL facilities have intermittent monitoring systems that function when operations are taking place. Other facilities have no effluent monitoring systems. All effective dose equivalents (EDEs) for unmonitored and noncontinuously monitored facilities are based on radionuclide inventories and unabated emissions using EPA methodology. Such reported EDEs overestimate the actual LLNL impact. EDEs associated with continuously monitored facilities are based on the actual air emission monitoring data and are therefore more realistic estimators of potential dose.

\section{LLNL Buildings with Radionuclide Release Potential}

We have reviewed all LLNL buildings that contain radioactive materials management areas (RMMAs), i.e., areas in which radionuclides are used or activation preducts potentially occur. There are 56 buildings containing RMMAs48 on the Livermore site ard 8 at Site 300 . Table 11-1 lists these buildings, gives the number of potential radionuclide discharge points, the types of operations occurring in each facility, the calculated maximum effective dose equivalent that 
a member of the public could experience at or beyond the site boundary due to discharge points modeled for each facility, and cites the NESHAPs quarterly report(s) where the pertinent dose calculations were presented.

In Table 11-1, detailed information is given for all point source emissions from 43 Livermore-site buildings. Three Livermore-site facilities (Buildings 363, 365 , and 366) are referenced, but operations using radionuclides no longer occur in those buildings, or the radionuclides are encapsulated or sealed. Two Livermore-site facilities (Buildings 514 and 612) are treated as area sources with detailed information given in the discussion of "diffuse sources" in the following section; three other diffuse sources also are discussed in that section. Table 11-1 also includes detailed information for three Site 300 explosive testing facilities: Buildings 801, 850, and 851 and their associated firing tables. Five other Site 300 facilities are referenced but radionuclides are not in use in those buildings, are encapsulated, or are sealed.

\section{Calculations of Effective Dose Equivalents}

\section{Description of Dose Model, Input Parameters, and Defined Terms}

The EPA-mandated AIRDOS or CAP88-PC models were used to carry out our radiological dose assessments, as noted above. Early last year, when CAP88PC became available, we changed from AIRDOS to almost exclusive use of CAP88-PC to take advantage of the significant improvements made in the model. Separate determinations of the EDE, in particular at the location of the "site-wide maximally exposed individual" as defined below, from all potential discharge points at LLNL singly and collectively, were made for the Livermore site and Site 300. The CAP88-PC and AIRDOS codes tabulate EDE in mrem/y.

Required input parameters for the AIRDOS/CAP88-PC model runs include isotope type, emission rate in curies per year $\left(1 \mathrm{Ci}=3.7 \times 10^{10} \mathrm{~Bq}\right)$, and stack characteristics including height, diameter, and emission velocity of the effluent gas. Meteorological data input for all model runs were actual Livermore-site or Site 300 annual average data, as pertinent, acquired from the respective on-site meteorological towers. No EPA meteorological default files were used.

Because AIRDOS and CAP88-PC do not contain all the radionuclides present at LLNL, surrogate radionuclides were used in some cases to estimate EDEs. In selecting the surrogates, we used the most restrictive lung class (whether clearance from the lungs takes place in days, weeks, or years). When possible, we used a surrogate radionuclide with similar lung class chemistry and similar values for "annual limits of intake via inhalation and derived air concentration." CAP88-PC contains a library of considerably more nuclides than AIRDOS. By rerunning calculations in CAP88-PC previously modeled with AIRDOS, we have found that the use of surrogates in the calculations result in conservative estimates of EDEs. 
Calculations for noncontinuously monitored facilities were based on assumptions concerning the fraction of the annual inventory that potentially could be released. If the material was a sealed gas, then the release fraction 0.01 generally was used; for liquids and powders, 0.001 was used; and for solids, 0.000001 was used; all in accordance with 40 CFR part 61, Appendices D and E. In addition, no credit was taken for radionuclide emission control devices; emission inputs into the models were assumed to be unabated. Hence, when control devices were in use, actual emissions and exposures were much lower than reported.

For monitored facilities, actual measured emissions were used as input variables into the codes. The use of actual monitoring data is much more direct and more accurate than using assumptions based on inventory and release fractions.

Modeling releases to the atmosphere from explosive tests using depleted uranium at Site 300 requires special attention compared to conventional stack or area sources. During experiments, the explosive device being tested is placed on an open-air firing table and detonated. We have limited data to characterize the initial state of the cloud of explosive decomposition products created by the detonation because properties of the cloud are not typically measured in the experiments. Empirical scaling laws can, however, be used to define the cloud using the radionuclide and high-explosive inventories. We assume all of the depleted uranium is dispersed into the cloud, and the median particle size is assumed to be the CAP88-PC default value of 1 micrometer. The masses of the three uranium isotopes (with atomic weights 238,235 , and 234) are multiplied by their respective specific activities to get the total number of curies for each isotope in the cloud. CAP88-PC then simulates each shot as a low-level, steadystate, stack-type emission occurring over one year. EPA directed LLNL to use the CAP88-PC code for these calculations, despite the recognized difficulties in modeling explosive events.

Another category of sources requiring special attention is "diffuse emissions," including "fugitive emissions." Diffuse, or nonpoint, sources are difficult to quantify, and dose calculations associated with these types of sources are left to the discretion of the DOE facility. Four different modeling approaches were used for diffuse sources at LLNL in 1992. Elevated tritium levels in soil moisture near Building 292 required a calculation of the source term and the use of CAP88-PC; a small amount of tritium-contaminated equipment outside Building 331 required facility personnel knowledge to estimate emissions; for Building 514, housing the Hazardous Waste Management "tank farm," and Building 612, adjacent to waste processing and storage yards, standard CAP88-PC modeling techniques were used; and elevated levels of plutonium in the southeast quadrant of the Livermore site used direct ambient air monitoring to get data on which to base dose calculations. A description of each such source 
and the assumptions made regarding their emissions are given in our NESHAPs Annual Report for 1992 (Surano et al. 1993).

Doses are evaluated for the site-wide maximally exposed individual (SW-MEI), defined as the hypothetical member of the public (individual receptor) who could receive the greatest LLNL-induced EDE from all sources at a single location. At the Livermore site, the SW-MEI is located at the UNCLE Credit Union, about 10 meters outside the controlled eastern perimeter of the site. This location lies 948 meters from the principal radionuclide source, the Tritium Facility (Building 331), in an east-northeast direction. At Site 300, the SW-MEI is located in an experimental area termed "Bunker 2" operated by Physics International. Bunker 2 lies about 300 meters outside the east-central boundary of Site 300 . This bunker is 2.38 kilometers southeast of the principal firing table at Building 801. Some of our calculations preceded the choice of SWMEI and may not include a dose value for that specific location. The EDE values we quote in this document are either those at the location of the SW-MEI or the maximum to any member of the public, in any direction.

Appendix $B$ gives an expanded discussion of the doses of major interest, and presents examples of explicit dose calculations for different exposure pathways.

\section{Calculated Results Summary-Livermore Site and Site 300, 1992}

Table 11-2 summarizes our calculations of the annual radiation dose from airborne radionuclides emitted by LLNL operations in 1992. These air pathway doses, expressed as EDEs, were calculated using CAP88-PC and as such include exposures from inhalation, air immersion, ingestion, and ground surface irradiation. CAP88-PC was run using specific meteorology for the Livermore site and Site 300, as appropriate. Table 11-2 lists the principal facilities, i.e., those accounting for $97 \%$ or more of the total EDE, and shows the dominant radionuclide(s) for each facility. The listed EDE is equal to or greater than that which would be experienced by the SW-MEI.

For the Livermore site, the contribution to the total dose calculated for the SW-MEI from point source emissions is $0.69 \mu \mathrm{Sv}(0.069 \mathrm{mrem})$ and from diffuse emissions is $0.1 \mu \mathrm{Sv}(0.010 \mathrm{mrem})$. Summing the contributions from both point and diffuse sources yields a total dose of $0.79 \mu \mathrm{Sv}(0.079 \mathrm{mrem})$ for the Livermore site in 1992. The main contributions to this total were $0.37 \mu \mathrm{Sv}(0.037$ mrem) or $47 \%$ from the LLNL Tritium Facility (Building 331) emissions, and $0.12 \mu \mathrm{Sv}$ $(0.012 \mathrm{mrem})$ or $15 \%$ from one discharge point in Building 177 . Directly measured emissions (i.e., those for the eight continuously monitored facilitiesBuildings $175,231,251,331,332,419,490$, and 491-and the diffuse emissions from the southeast quadrant) comprised $49 \%$ of the total for the Livermore site. The remainder is estimated by CAP88-PC model runs assuming unabated emissions. This Livermore-site dose represents less than $1 \%$ of the EPA limit of 
$0.1 \mathrm{mSv} / \mathrm{y}(10 \mathrm{mrem} / \mathrm{y})$ for airborne emissions. Compared to data of previous years, this value is much reduced: dose values reported for 1991 and 1990 were $2.34 \mu \mathrm{Sv}(0.234 \mathrm{mrem})$ and $2.40 \mu \mathrm{Sv}(0.240 \mathrm{mrem})$, respectively. Reduced emissions of tritium from Building 331 accounts for much of this decline.

The dose to the site-wide maximally exposed member of the public at Site 300 during 1992 was calculated to be $0.21 \mu \mathrm{Sv}(0.021 \mathrm{mrem})$ EDE. Explosive tests at the Building 801 firing table accounted for nearly all of this dose. Emissions from other potential discharge points at Site 300 were negligible in 1992. For example, the firing table associated with Building 851 contributed only $6.5 \times 10^{-6} \mu \mathrm{Sv}$ $\left(6.5 \times 10^{-7} \mathrm{mrem}\right)$, and radioactive materials in Site 300 buildings are either encapsulated or sealed; no operations are performed within these facilities that have the potential to release radionuclides to the atmosphere under normal operating conditions. The dose contributed by firing table operations to the SW-MEI at Site 300 has been steadily declining: in 1991 it was $0.44 \mu \mathrm{Sv}$ (0.044 mrem), and in 1990 it was $0.57 \mu \mathrm{Sv}(0.057 \mathrm{mrem})$.

Site 300 remains an isolated facility. Except for similar test facilities to the east and south and a vehicular recreation area, the land is devoted to wind farming and livestock husbandry. The population density within several kilometers of the site is extremely low.

The principal radionuclides contributing to these doses are tritium in the tritiated water form (HTO)-which accounts for about $50 \%$ of the $0.79 \mu \mathrm{Sv}$ ( $0.079 \mathrm{mrem})$ Livermore-site dose-and the isotopes of uranium with atomic weights 238, 234, and 235, accounting for all of the Site 300 dose and about $36 \%$ of the Livermore-site dose. The relative significance of inhalation and ingestion via the air pathway is different for these nuclides. Ingestion is slightly more important than inhalation in the case of tritium, with a ratio of about $52 / 48$, while inhalation is far more important for uranium, contributing about $95 \%$ versus $5 \%$ for ingestion.

Table 11-3 compares the radiation doses due to atmospheric emissions at LLNL to other sources of radioactivity to which the U.S. population is exposed. The dose resulting from Livermore-site operations is lower by a factor of 3000 for the maximally exposed individual. Table 11-3 shows that, among the sources of background radiation, radon emissions rank highest. Doses from LLNL operations are at the typical ambient outside levels for radon, with concentrations of $4 \times 10^{-3}$ to $2 \times 10^{-2} \mathrm{~Bq}(0.1$ to $0.5 \mathrm{pCi})$ per liter of air. The EPA radon screening level is $0.1 \mathrm{~Bq}(4 \mathrm{pCi})$ per liter of air. To place the DOE radiation protection limit, $1 \mathrm{mSv} / \mathrm{y}(100 \mathrm{mrem} / \mathrm{y})$, into perspective, during the same period the average resident of the Livermore Valley receives a dose of approximately $3.0 \mathrm{mSv} / \mathrm{y}(300 \mathrm{mrem} / \mathrm{y})$ from natural background radiation, including the dose from radon. 
The CAP88-PC code was used to calculate the collective population dose. The collective effective dose equivalent for the population is the sum of the estimated doses to all individuals residing within an $80-$ kilometer radius of LLNL. The calculated collective EDE for the total population (which is estimated to be 6.3 million within 80 kilometers of the Livermore site, based on 1990 census information) was 0.015 person-Sv (1.5 person-rem) from Livermore-site emissions during 1992. The collective EDE due to emissions from the relatively isolated Site 300 was, surprisingly, nearly five times higher, estimated to be 0.071 person-Sv (7.1 person-rem) for the 5.4 million people within 80 kilometers of Site 300. The main reason is that the Site 300 explosion effluent gets thrown much higher into the air than conventional stack emissions, and the Gaussian dispersion model in CAP88-PC predicts that the concentration drops off very slowly with distance. In any case, these collective EDEs from the two LLINL sites are quite small compared to that from natural sources of radiation. Using $3.0 \mathrm{mSv}(300 \mathrm{mrem})$ as a typical annual average from natural sources, the collective EDE due to natural sources during the same period was estimated to be $1.9 \times 10^{4}$ person-Sv $\left(1.9 \times 10^{6}\right.$ person-rem $)$.

\section{Summary and Conclusion}

Calculations of effective dose equivalents for all Livermore site and Site 300 facilities having the potential to release radionuclides to the atmosphere have been completed. The annual dose from actual emissions (after control devices) of all facilities was found to be well below the applicable standards for radiation protection of the public, in particular the $100 \mu \mathrm{Sv} / \mathrm{y}(10 \mathrm{mrem} / \mathrm{y})$ NESHAPs standard. Three facilities at the Livermore site (Buildings 331, 332, and the hardened portion of Building 251) will maintain continuous monitoring systems, based on their potential for emissions exceeding $1 \mu \mathrm{Sv} / \mathrm{y}(0.1 \mathrm{mrem} / \mathrm{y})$ without emission control devices. No other facilities at either the Livermore site or Site 300 were found to require continuous monitoring systems, though five additional facilities have continuous monitoring in place.

Using the EPA-mandated computer models and actual LLNL meteorology, the 1992 calculated EDE to the individual site-wide maximally exposed member of the public from Livermore site radionuclide air emissions was $0.79 \mu \mathrm{Sv}$ $(0.079 \mathrm{mrem})$. The main contribution, $0.37 \mu \mathrm{Sv}(0.037 \mathrm{mrem})$, was from the LLNL Tritium Facility (Building 331) emissions, which constituted $47 \%$ of the Livermore site EDE. The only other emission point contributing greater than $10 \%$ of the EDE was one discharge point in Building 177, whose emissions accounted for $15 \%$ of the EDE.

The EDE to the individual site-wide maximally exposed member of the public at Site 300 was calculated to be $0.21 \mu \mathrm{Sv}$ (0.021 mrem). Building 801 firing 
table experiments accounted for nearly all of this EDE. No other Site 300 operations resulted in significant dose contributions.

The collective effective dose equivalent for LLNL 1992 operations was 0.015 person-Sv (1.5 person-rem) from the Livermore site and 0.071 person-Sv (7.1 person-rem) from Site 300, including all pathways (ingestion, air immersion, surface exposure, and inhalation pathways). The dose was calculated out to a distance of 80 kilometers from both sites in all directions using 1990 census population data comprising 6.3 million people for the Livermore site and 5.4 million for Site 300 .

These potential radiological doses from LLNL operations were approximately three thousand times less than the doses normally received by this population from natural background radiation sources, even though highly conservative assumptions were used in the calculations. Thus, the maximum credible doses show that LLNL's use of radionuclides had no significant impact on public health during 1992. 
Table 11-1. LLNL buildings containing RMMAs.

\begin{tabular}{|c|c|c|c|c|c|}
\hline Bldg & Facility & $\begin{array}{l}\text { Potential } \\
\text { discharge } \\
\text { points }\end{array}$ & $\begin{array}{c}\operatorname{Max}_{\mathrm{EDE}^{\mathrm{a}}} \\
(\mu \mathrm{Sv} / \mathrm{y})\end{array}$ & $\begin{array}{l}\text { Quarter } \\
\text { reported }\end{array}$ & Operations \\
\hline 131 & Engineering & 2 & $4.0 \times 10^{-3}$ & Dec. 91 & $\begin{array}{l}\text { Handling, storing, machining, characterizing, } \\
\text { assembling, sorting, and transferring materials, } \\
\text { repackaging of waste }\end{array}$ \\
\hline 151 & Nuclear Chemistry & 23 & $2.1 \times 10^{-2}$ & $\begin{array}{l}\text { Sept. } 91 \\
\text { Dec. } 92\end{array}$ & $\begin{array}{l}\text { Chemical separation, crushing/dissolving, aliquot } \\
\text { preparation and storage, gas analysis, } \\
\text { radiochemical separations, preparation of } \\
\text { radioactive counting standards }\end{array}$ \\
\hline 171 & Lasers & 1 & $3.1 \times 10^{-8}$ & Sept. 92 & Melting of uranium under vacuum \\
\hline 175 & MARS & $6^{c}$ & $2.4 \times 10^{-1}$ & Dec. 92 & Cleaning/disassembly of uranium parts \\
\hline 177 & Lasers & 6 & $1.2 \times 10^{-1}$ & $\begin{array}{l}\text { May } 93 \\
\text { Annual }\end{array}$ & Vaporization and coating of uranium \\
\hline 179 & Lasers & 2 & $1.3 \times 10^{-3}$ & Sept. 92 & Melting of uranium under vacuum \\
\hline 190 & Physics & 1 & 0.0 & Mar. 92 & Accelerator \\
\hline 194 & Physics & 2 & $3.2 \times 10^{-2}$ & $\begin{array}{l}\text { Dec. } 91 \\
\text { Dec. } 92\end{array}$ & Accelerator \\
\hline 212 & Physics & 2 & $1.8 \times 10^{-9}$ & Sept. 92 & $\begin{array}{l}\text { Environmental, safety, and health surveillance for } \\
\text { shutdown of accelerator }\end{array}$ \\
\hline 222 & Chemistry & 16 & $2.8 \times 10^{-2}$ & $\begin{array}{l}\text { Sept. } 91 \\
\text { Dec. } 92\end{array}$ & Radioanalytical analyses and tracer use \\
\hline 226 & Chemistry & 1 & $8.1 \times 10^{-8}$ & Mar. 92 & Radioactive and mixed waste chemical analyses \\
\hline 227 & Chemistry & 3 & $8.2 \times 10^{-5}$ & Sept. 91 & Uranium bonding and testing \\
\hline \multirow[t]{2}{*}{231} & $\begin{array}{l}\text { Mechanical } \\
\text { Engineering }\end{array}$ & 5 & $1.5 \times 10^{-4}$ & Mar. 92 & $\begin{array}{l}\text { Materials research and testing, plastics shop work, } \\
\text { electron beam welding }\end{array}$ \\
\hline & $\begin{array}{l}\text { Mechanical } \\
\text { Engineering Vault }\end{array}$ & $1^{c}$ & 0.0 & Sept. 92 & Storage, handling, and shipping of radionuclides \\
\hline 235 & WMRDF & 8 & $2.7 \times 10^{-6}$ & Dec. 91 & Welding, actinide and uranium catalyst research \\
\hline 241 & Physics & 2 & $2.1 \times 10^{-5}$ & Sept. 92 & Materials development, measurement, and testing \\
\hline 243 & Energy Research & 1 & 0.0 & Mar. 92 & Earth sciences and geological studies \\
\hline \multirow[t]{3}{*}{251} & Heavy Elements & & & & Heavy-element research \\
\hline & hardened area & $4^{c}$ & $-^{d}$ & Dec. 92 & \\
\hline & unhardened areas & $44^{c}$ & $7.8 \times 10^{-1}$ & Dec. 92 & \\
\hline 253 & Hazards Control & 1 & $6.3 \times 10^{-14}$ & Sept. 92 & Radiochemical analyses \\
\hline 254 & Hazards Control & 1 & 0.0 & Sept. 92 & Radiochemical analyses of bioassays \\
\hline 255 & Hazards Control & 1 & $4.1 \times 10^{-6}$ & Sept. 92 & Instrument calibration \\
\hline 2581 & Test Program & 1 & $1.0 \times 10^{-7}$ & Sept. 92 & Foil testing \\
\hline 281 & Nuclear Chemistry & 4 & $5.1 \times 10^{-3}$ & Sept. 91 & $\begin{array}{l}\text { Preparation and storage of radiochemical stock } \\
\text { solutions }\end{array}$ \\
\hline 282 & Nuclear Chemistry & 1 & $8.2 \times 10^{-3}$ & $\begin{array}{l}\text { Sept. } 91 \\
\text { Mar. } 92\end{array}$ & Use of tritium gas in spectrometer experiment \\
\hline
\end{tabular}


Table 11-1. LLNL buildings containing RMMAs (continued).

\begin{tabular}{|c|c|c|c|c|c|}
\hline Bldg & Facility & $\begin{array}{l}\text { Potential } \\
\text { discharge } \\
\text { points }\end{array}$ & $\begin{array}{c}\text { Max } \\
\text { EDEa }^{a} \\
(\mu S v / y)\end{array}$ & $\begin{array}{l}\text { Quarter } \\
\text { reportedb }\end{array}$ & Operations \\
\hline 292 & Physics & 3 & $7.8 \times 10^{-7}$ & Sept. 92 & Tritium contamination from prior operations \\
\hline 298 & Laser Fusion & 2 & $1.8 \times 10^{-3}$ & Sept. 92 & $\begin{array}{l}\text { Handling and assembly of tritium-filled targets; } \\
\text { sputtering uranium }\end{array}$ \\
\hline 321 & $\begin{array}{l}\text { Materials } \\
\text { Fabrication }\end{array}$ & 5 & $4.0 \times 10^{-3}$ & Dec. 91 & Machining \\
\hline 331 & Tritium & $2^{c}$ & $1.9 \times 10^{-1 d}$ & $\begin{array}{l}\text { May } 93 \\
\text { Annual }\end{array}$ & $\begin{array}{l}\text { Cryogenic tritium experiments and cryogenic } \\
\text { experiments on deuterium-tritium }\end{array}$ \\
\hline 332 & Plutonium & $11^{c}$ & $-^{d}$ & Dec. 92 & Machining and metallurgy \\
\hline 341 & Physics & 1 & $4.2 \times 10^{-6}$ & Mar. 92 & High-energy research \\
\hline 361 & $\begin{array}{l}\text { Biomedical } \\
\text { Research }\end{array}$ & 16 & $1.1 \times 10^{-5}$ & $\begin{array}{l}\text { Sept. } 91 \\
\text { Dec. } 92\end{array}$ & $\begin{array}{l}\text { Radiolabeling and use of tracers for biomedical } \\
\text { research }\end{array}$ \\
\hline 363 & $\begin{array}{l}\text { Biomedical } \\
\text { Research }\end{array}$ & $--^{\theta}$ & 0.0 & Sept. 91 & Biomedical research \\
\hline 365 & $\begin{array}{l}\text { Biomedical } \\
\text { Research }\end{array}$ & 4 & 0.0 & Sept. 91 & $\begin{array}{l}\text { Radiolabeling and use of tracers for biomedical } \\
\text { research }\end{array}$ \\
\hline 366 & $\begin{array}{l}\text { Biomedical } \\
\text { Research }\end{array}$ & $-{ }^{\theta}$ & 0.0 & Sept. 91 & Biomedical research \\
\hline 377 & $\begin{array}{l}\text { Environmental } \\
\text { Research }\end{array}$ & 3 & $8.8 \times 10^{-5}$ & Mar. 92 & $\begin{array}{l}\text { Radiolabeling and use of tracers for environmental } \\
\text { research }\end{array}$ \\
\hline 378 & $\begin{array}{l}\text { Environmental } \\
\text { Research }\end{array}$ & 2 & $1.6 \times 10^{-8}$ & Mar. 92 & $\begin{array}{l}\text { Radiolabeling and use of tracers for environmental } \\
\text { research }\end{array}$ \\
\hline 381 & Laser Research & 2 & $1.9 \times 10^{-4}$ & Sept. 92 & Neutron generation \\
\hline 391 & NOVA & 3 & $2.0 \times 10^{-3}$ & Sept. 92 & Vaporization of targets \\
\hline 412 & $\begin{array}{l}\text { Environmental } \\
\text { Research }\end{array}$ & $-^{\theta}$ & 0.0 & $\begin{array}{l}\text { Mar. } 92 \\
\text { Dec. } 92\end{array}$ & Environmental monitoring of soils and other media \\
\hline 419 & Decontamination & $2^{c}$ & 0.0 & Dec. 92 & $\begin{array}{l}\text { Decontamination of equipment (in process of } \\
\text { being closed) }\end{array}$ \\
\hline 490 & $\begin{array}{l}\text { Laser Isotope } \\
\text { Separation }\end{array}$ & $4^{c}$ & $4.6 \times 10^{-2}$ & $\begin{array}{l}\text { Mar. } 92 \\
\text { Dec. } 92\end{array}$ & $\begin{array}{l}\text { Melting and vaporization of uranium, isotope } \\
\text { separation }\end{array}$ \\
\hline 491 & $\begin{array}{l}\text { Laser Isotope } \\
\text { Separation }\end{array}$ & $1^{c}$ & 0.0 & Mar. 92 & Refurbishment of separator components \\
\hline 493 & Support Facility & $-\theta$ & 0.0 & Dec. 92 & Isotope storage \\
\hline 513 & $\begin{array}{l}\text { Hazardous Waste } \\
\text { Management }\end{array}$ & 3 & $6.3 \times 10^{-4}$ & Sept. 91 & Storage, solidification, and shredding of waste \\
\hline 514 & $\begin{array}{l}\text { Hazardous Waste } \\
\text { Management }\end{array}$ & 1 & $5.0 \times 10^{-2}$ & $\begin{array}{l}\text { Sept. } 91 \\
\text { Dec. } 92\end{array}$ & Waste treatment \\
\hline 594 & $\begin{array}{l}\text { Environmental } \\
\text { Research }\end{array}$ & $-e$ & 0.0 & Dec. 92 & Tracer experiments for atmospheric pollution \\
\hline 612 & $\begin{array}{l}\text { Hazardous Waste } \\
\text { Management }\end{array}$ & 5 & $5.6 \times 10^{-4}$ & Sept. 91 & $\begin{array}{l}\text { Lab packing, bulking, compacting, baling, and } \\
\text { handling of waste; drum crushing }\end{array}$ \\
\hline
\end{tabular}


Table 11-1. LLNL buildings containing RMMAs (concluded).

\begin{tabular}{|c|c|c|c|c|c|}
\hline Bldg & Facility & $\begin{array}{c}\text { Potential } \\
\text { discharge } \\
\text { points }\end{array}$ & $\begin{array}{c}\text { Max } \\
\text { EDEa } \\
(\mu S v / y)\end{array}$ & $\begin{array}{l}\text { Quarter } \\
\text { reported }\end{array}$ & Operations \\
\hline 614 & $\begin{array}{l}\text { Hazardous Waste } \\
\text { Management }\end{array}$ & $-\theta$ & 0.0 & Dec. 92 & Storage of waste \\
\hline 625 & $\begin{array}{l}\text { Hazardous Waste } \\
\text { Management }\end{array}$ & $-\theta$ & 0.0 & Dec. 92 & Container storage \\
\hline \multirow[t]{2}{*}{801} & Site 300 & $\boldsymbol{\theta}^{\theta}$ & 0.0 & Mar. 93 & $\begin{array}{l}\text { Electron beam accelerator/ } \\
\text { machining/firing table }\end{array}$ \\
\hline & $\begin{array}{l}\text { Site } 300 \text { Firing } \\
\text { Table at } 801\end{array}$ & -1 & $2.1 \times 10^{-1}$ & $\begin{array}{l}\text { May } 93 \\
\text { Annual }\end{array}$ & Detonation of explosives \\
\hline 804 & Site 300 & - & 0.0 & Mar. 93 & Low-level-waste storage area \\
\hline $810 A$ & Site 300 & - & 0.0 & Mar. 93 & Storage of parts \\
\hline $810 B$ & Site 300 & $-e$ & 0.0 & Mar. 93 & Storage of parts \\
\hline $822 B$ & Site 300 & $-\theta$ & 0.0 & Mar. 93 & Storage of parts \\
\hline \multirow[t]{2}{*}{850} & Site 300 & $-\theta$ & 0.0 & Mar. 93 & Firing table bunker \\
\hline & $\begin{array}{l}\text { Site } 300 \text { Firing } \\
\text { Table at } 850\end{array}$ & -1 & 0.0 & $\begin{array}{l}\text { May } 93 \\
\text { Annual }\end{array}$ & Detonation of explosives \\
\hline \multirow[t]{2}{*}{851} & Site 300 & $-\theta$ & 0.0 & Mar. 93 & Electron beam accelerator/firing table \\
\hline & $\begin{array}{l}\text { Site } 300 \text { Firing } \\
\text { Table at } 851\end{array}$ & - & $6.5 \times 10^{-6}$ & $\begin{array}{l}\text { May } 93 \\
\text { Annual }\end{array}$ & Detonation of explosives \\
\hline $854 \mathrm{~F}$ & Site 300 & $-\theta$ & 0.0 & Mar. 93 & Storage of mixed fission products \\
\hline
\end{tabular}

a The maximum effective dose equivalent occurring at or beyond the site boundary from a single discharge point, among all discharge points modeled for a facility.

b Date of quarterly or annual NESHAPs report submission to EPA.

c Facility has an air effluent sampling system on at least one discharge point.

d The effluents from the facility are and will continue to be monitored.

- Radionuclides are not in use, are encapsulated or sealed, or the quantities fall below the screening levels for modeling.

1 Open air dispersal in 1992. 


\section{Radiological Dose Assessment}

Table 11-2. Annual radiation dose via airborne emissions, 1992.

\begin{tabular}{|c|c|c|c|}
\hline \multirow{2}{*}{$\begin{array}{l}\text { Facility or } \\
\text { operation }\end{array}$} & \multirow{2}{*}{$\begin{array}{l}\text { Dominant } \\
\text { radionuclide(s) }\end{array}$} & \multicolumn{2}{|c|}{ EDE at SW-MEI } \\
\hline & & $\mu \mathrm{Sv} / \mathrm{y}$ & mrem/y \\
\hline \multicolumn{4}{|l|}{ Livermore site } \\
\hline B331/Tritium Facility & ${ }^{3} \mathrm{H}$ & 0.37 & 0.037 \\
\hline B177/Lasers & $238 U,{ }^{234} U,{ }^{235} U$ & 0.166 & 0.0166 \\
\hline B151/Nuclear Chemistry & $\begin{array}{c}{ }^{239} \mathrm{Pu}_{\mathrm{u}}{ }^{240} \mathrm{Pu}_{1}{ }^{152} \mathrm{Eu}, \\
{ }^{141} \mathrm{Ce},{ }^{210} \mathrm{~Pb}\end{array}$ & 0.078 & 0.0078 \\
\hline $\mathrm{B} 222$ & ${ }^{232} \mathrm{Th},{ }^{238} \mathrm{U},{ }^{141} \mathrm{C} \in$ & 0.028 & 0.0028 \\
\hline B321 & $238 \mathrm{U}$ & 0.017 & 0.0017 \\
\hline $\mathrm{B} 282$ & ${ }^{3} \mathrm{H}$ & 0.016 & 0.0016 \\
\hline$B 514^{b}$ & ${ }^{238} \cup,{ }^{235} U$ & 0.069 & 0.0069 \\
\hline SE Quadrant ${ }^{b}$ & ${ }^{239} \mathrm{Pu}$ & 0.019 & 0.0019 \\
\hline $\mathrm{B} 331^{\mathrm{b}}$ & ${ }^{3} \mathrm{H}$ & 0.0089 & 0.00089 \\
\hline \multirow[t]{2}{*}{ Sum of other sources } & various & 0.018 & 0.0018 \\
\hline & & Total $=0.79^{c, d}$ & $0.079^{c, d}$ \\
\hline \multicolumn{4}{|l|}{ Site 300} \\
\hline B801/firing table & ${ }^{238} \cup,{ }^{234} \cup,{ }^{235} \cup$ & $0.21^{c}$ & $0.021^{c}$ \\
\hline
\end{tabular}

a The facilities cited here are discussed in the text of this report, and in more detail in NESHAPs quarterly and annual reports.

b Diffuse sources (see text).

c These Livermore site and Site 300 totals represent $0.8 \%$ and $0.2 \%$, respectively, of the federal standard.

d Directly measured emissions (mainly those from B331 stacks and SE quadrant diffuse emissions) comprise $49 \%$ of this total; the remainder is estimated by CAP88.PC calculations assuming unabated emissions (see text). 
Table 11-3. Comparison of radiation doses, LLNL, 1992.

\begin{tabular}{|c|c|c|c|c|}
\hline \multirow[b]{2}{*}{ Location/source } & \multicolumn{2}{|c|}{ Individual dose" } & \multicolumn{2}{|c|}{ Population doseb } \\
\hline & (mSv) & (mrem) & $\begin{array}{c}\text { (person- } \\
\text { Sv) }\end{array}$ & $\begin{array}{l}\text { (person- } \\
\text { rem) }\end{array}$ \\
\hline \multicolumn{5}{|l|}{ Livermore-site sources } \\
\hline Atmospheric emissions & 0.00079 & 0.079 & 0.015 & 1.5 \\
\hline \multicolumn{5}{|l|}{ Site 300 sources } \\
\hline Atmospheric emissions & 0.00021 & 0.021 & 0.071 & 7.1 \\
\hline \multicolumn{5}{|l|}{ Other sources ${ }^{c}$} \\
\hline \multicolumn{5}{|l|}{ Natural radioactivityd } \\
\hline Cosmic radiation & 0.3 & 30 & 1,900 & 190,000 \\
\hline Terrestrial radiation & 0.3 & 30 & 1,900 & 190,000 \\
\hline Internal (food consumption) & 0.4 & 40 & 2,500 & 250,000 \\
\hline Radon & 2.0 & 200 & 12,500 & $1,250,000$ \\
\hline Medical radiation (diagnostic procedures) ${ }^{\ominus}$ & 0.53 & 53 & 3,300 & 330,000 \\
\hline Weapons test fallout ${ }^{\theta}$ & 0.011 & 1.1 & 68 & 6,800 \\
\hline Nuclear fuel cycle ${ }^{\theta}$ & 0.004 & 0.4 & 25 & 2,500 \\
\hline
\end{tabular}

a This dose represents that experienced by the site-wide maximally exposed individual member of the public.

b The population dose is the collective (combined) dose for all individuals residing with an $80-\mathrm{km}$ radius of LLNL (approximately 6.3 million people for the Livermore site and 5.4 million for Site 300 ), calculated with respect to distance and direction from each site.

c From National Council on Radiation Protection (NCRP 1987).

d These values vary with location.

- This dose is an average over the U.S. population. 


\section{Introduction}

The previous chapters on environmental media monitored by LLNL presented data from the monitoring activities, and Chapter 11 discusses the radiological dose assessments. In this chapter, the data on nonradiological pollutants are summarized graphically and compared to regulatory limits. These results demonstrate LLNL's continued compliance with regulatory requirements, as well as the effectiveness of LLNL's operational controls.

\section{Air Monitoring}

LLNL conducts surveillance air monitoring, which involves measurement of particles collected on filters, to determine if airborne hazardous materials are being released by Laboratory operations. To comply with the Clean Air Act National Emissions Standards for Hazardous Air Pollutants, concentrations of beryllium are monitored at the Livermore-site perimeter, at off-site locations near the Livermore site, and at Site 300. Concentrations of lead are also monitored at Site 300 . The annual average concentration of airborne beryllium at the Livermore site is $23.5 \mathrm{pg} / \mathrm{m}^{3}$ and is less than $0.3 \%$ of the monthly ambient concentration guide of $10,000 \mathrm{pg} / \mathrm{m}^{3}$ established by the Bay Area Air Quality Management District (BAAQMD). These levels are attributed to suspension of surface soil containing naturally occurring beryllium. At Site 300 , the annual average airborne beryllium concentration is $26.3 \mathrm{pg} / \mathrm{m}^{3}$ and is also less than $0.3 \%$ of the monthly ambient concentration guide. Figure 12-1 shows the monthly mean concentration of beryllium on air filters at the Livermore-site perimeter and at Site 300 .

The estimated release from exempt and permitted sources of air pollutants at the Livermore site can be compared with the most recent estimate of 1992 daily release of air pollutants for the entire Bay Area. For example, the total estimated oxides of nitrogen released in the Bay Area is 500 metric tons per day, compared to LLNL's 0.079 metric tons per day ( $0.02 \%$ of total Bay Area emissions). The BAAQMD estimate for reactive organic emissions (ROG) is 743 metric tons/day, 
versus Livermore site's 0.013 metric tons/day $(0.002 \%$ of total Bay Area emissions) in 1992.

\section{Sewage Monitoring}

LLNL discharges wastewater to the Livermore Water Reclamation Plant (LWRP) under a permit that limits the concentrations of certain contaminants to prevent disruption of the treatment plant operations. To demonstrate compliance with those conditions, LLNL conducts a self-monitoring program that includes metals, organic toxins, and water quality indicators. A flow-proportional sampling system is used to acquire the necessary samples. Weekly composites of these samples are analyzed for metals, and once each month special samples are analyzed for a broad range of water quality parameters.

The data gathered in 1992 are presented in Chapter 5, Sewage Monitoring, and summarized in Figure 12-2. The results of the weekly metals analyses show that, on average, metals concentrations remained well below the discharge guideline. The results for the quarterly water-quality and toxic chemicals monitoring were also generally unexceptional.
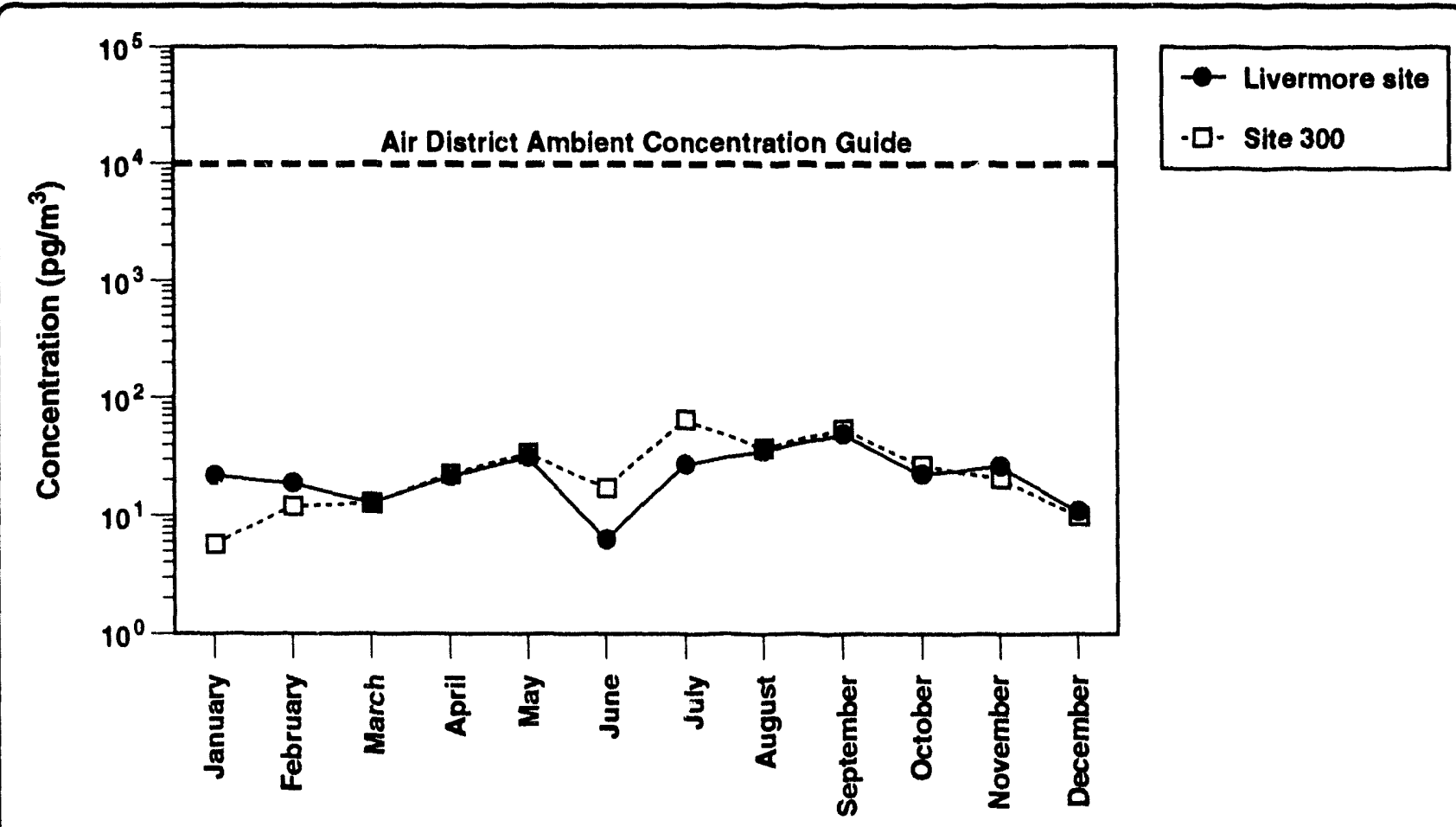

Month

Figure 12-1. Monthly mean concentrations of beryllium on air filters, Livermore site and Site 300, 1992. 
LWRP issued one Notice of Violation to LLNL during 1992, for discharges of oil and grease above the permit limit of $100 \mathrm{mg} / \mathrm{L}$. Because the LWRP uses an oil and grease separator to prevent buildup of grease in sensitive components of the treatment process, no enforcement action was pursued beyond the issuance of a formal Notice of Violation. However, the LWRP recommended changes to the LLNL sampling protocol. Prior to August 1992, the oil and grease analysis was performed on a single grab sample of LLNL's effluent, so the results represented concentrations only at that single point in time. The LWRP suggested that LLNL take a number of grab samples during a 24-hour period and average the sample results to obtain a daily average oil and grease value, providing a more representative measure of LLNL's impact on the LWRP. Finally, LLNL reviewed discharge controls at facilities that are known to generate large quantities of oil and grease (three on-site cafeterias), identifying potential problems and specifying improvements to be made to those operations. These improvements are planned for implementation during FY93.

LLNL maintains equipment that continuously monitors for high concentrations of radioactivity, metals, and acids or caustics. Although less sensitive than

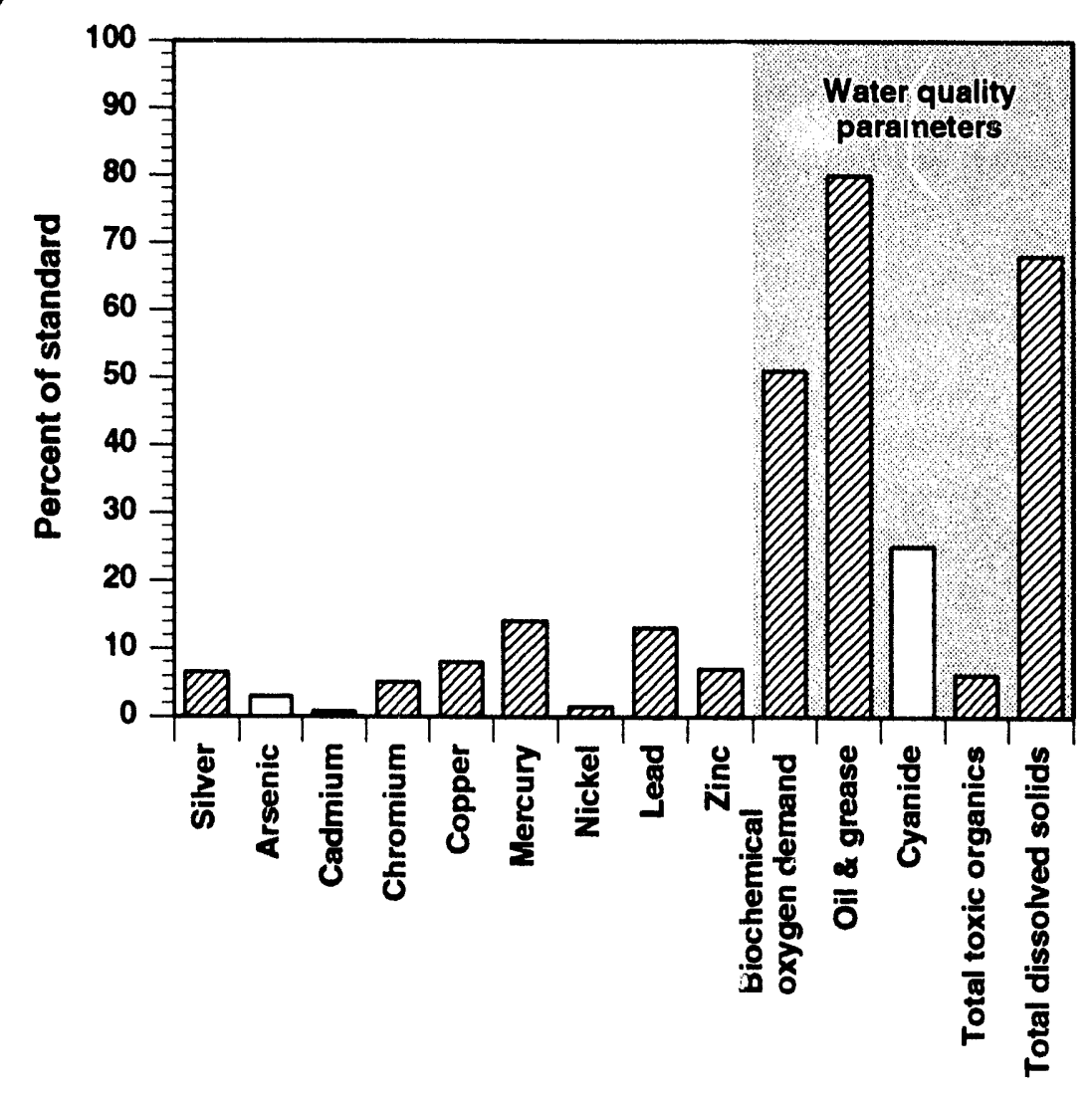

Average value of substance

Detection limit of substance

Figure 12-2. Surnmary of monthly average results for nonradiological substances in the sanitary sewer. 
the off-line chemical analysis, this system is capable of detecting discharges that might have a serious impact on LWRP operations. In such circumstances, LLNL's Sewer Diversion Facility (SDF) is automatically activated, thereby preventing release of all except the first few minutes of the affected sewage. During 1992, ten possible problem releases were identified but none represented a threat to LWRP operations. Following analysis to make certain the retained sewage met discharge requirements, effluent diverted into the SDF was returned to the sanitary sewer.

\section{Water Monitoring}

Water sources that are analyzed for nonradiological constituents include ground water, surface water, and domestic water supplies. Samples are analyzed using standard EPA methods for solvents, metals, and pesticides.

Of the various types of water sources tested, specific regulatory concentration limits exist only for drinking water supplies (22 CCR 64443). At Site 300, on-site water supply Wells 18 and 20 are sampled quarterly for metals (beryllium, chromium, copper, and lead) and monthly for volatile organic compounds (VOCs). The locations of these wells are shown in Figure 6-5 in Chapter 6 on Surveillance Water Monitoring. Figure 12-3 shows that the only analytes detected in 1992 were chromium, copper, lead, TCE, and 1,1,1-trichloroethane. None of the observed concentrations of positively detected analytes exceed $30 \%$ of the applicable primary drinking water standard.

Off-site drinking water supply Wells CARNRW2, CDF1, CON1, and GALLO1 are sampled quarterly for metals (beryllium, chromium, copper, and lead) and for VOCs; these wells are sampled concurrently with the two on-site wells during each quarter as part of routine ground water surveillance monitoring. As shown in Figure 12-3, the concentrations of positively detected analytes were all $40 \%$ or less of their applicable primary or secondary drinking water standard. Trihalomethanes were detected in CARNRW2 during 1992, caused by sample contamination from the well chlorination system; in 1993, the procedures were changed to eliminate this problem. The elevated levels of selenium and arsenic in wells in the Site 300 area are believed to result from natural rather than man-made causes (Buddemeier et al. 1987).

Annual samples are collected from five wells located near Site 300. Although these wells are used primarily to supply livestock and fire protection water, human consumption of the water from these wells is not precluded. Therefore, primary drinking water standards are used as the most conservative standard for comparison. The average concentrations of detected analytes were all below $75 \%$ of the standard (see Figure 12-3). Again, the elevated levels of selenium and arsenic are believed to occur from natural geologic conditions in the area. 


\section{Soil and Sediment Monitoring}

Sediment samples from the arroyos and other drainages at and near LLNL were collected in 1992, continuing a monitoring effort begun in 1988. Analyses were conducted for a full suite of nonradiological constituents, including metals, pesticides, PCBs, VOCs, and semivolatile organic compounds, to determine if significant buildup of pollutants had occurred. Because there are no standards directly written for sediments, the standards against which the results are compared are state standards for waste (22 CCR Chapters 11 and 18).

Small amounts of metals and organic compounds were detected in sediments. In general, the levels detected were trace amounts, often at or near the analytical detection limit; these levels are indicative of background concentrations. Current analytical techniques for detection of organic compounds are much improved compared to those in the past. Therefore, traces

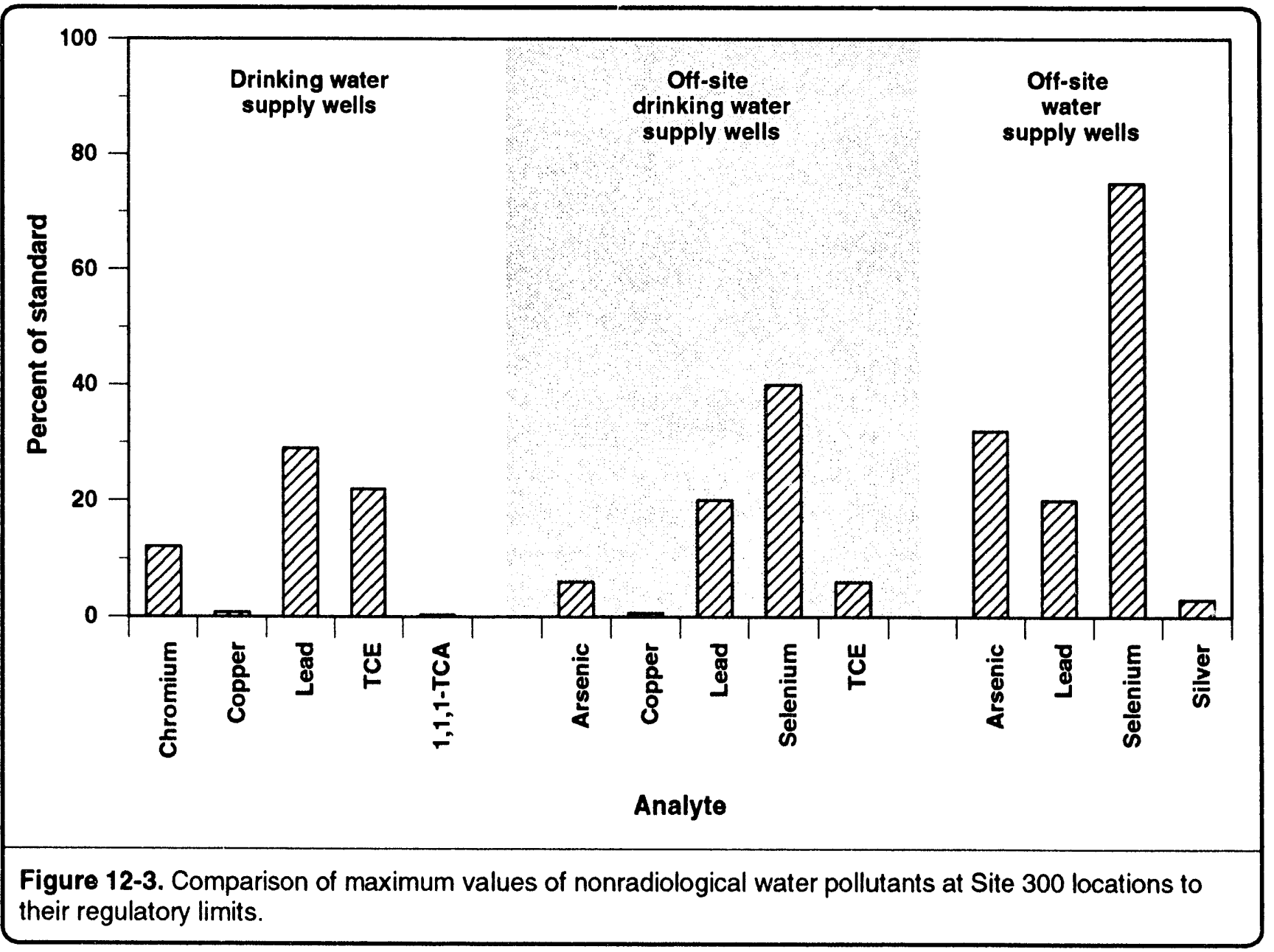




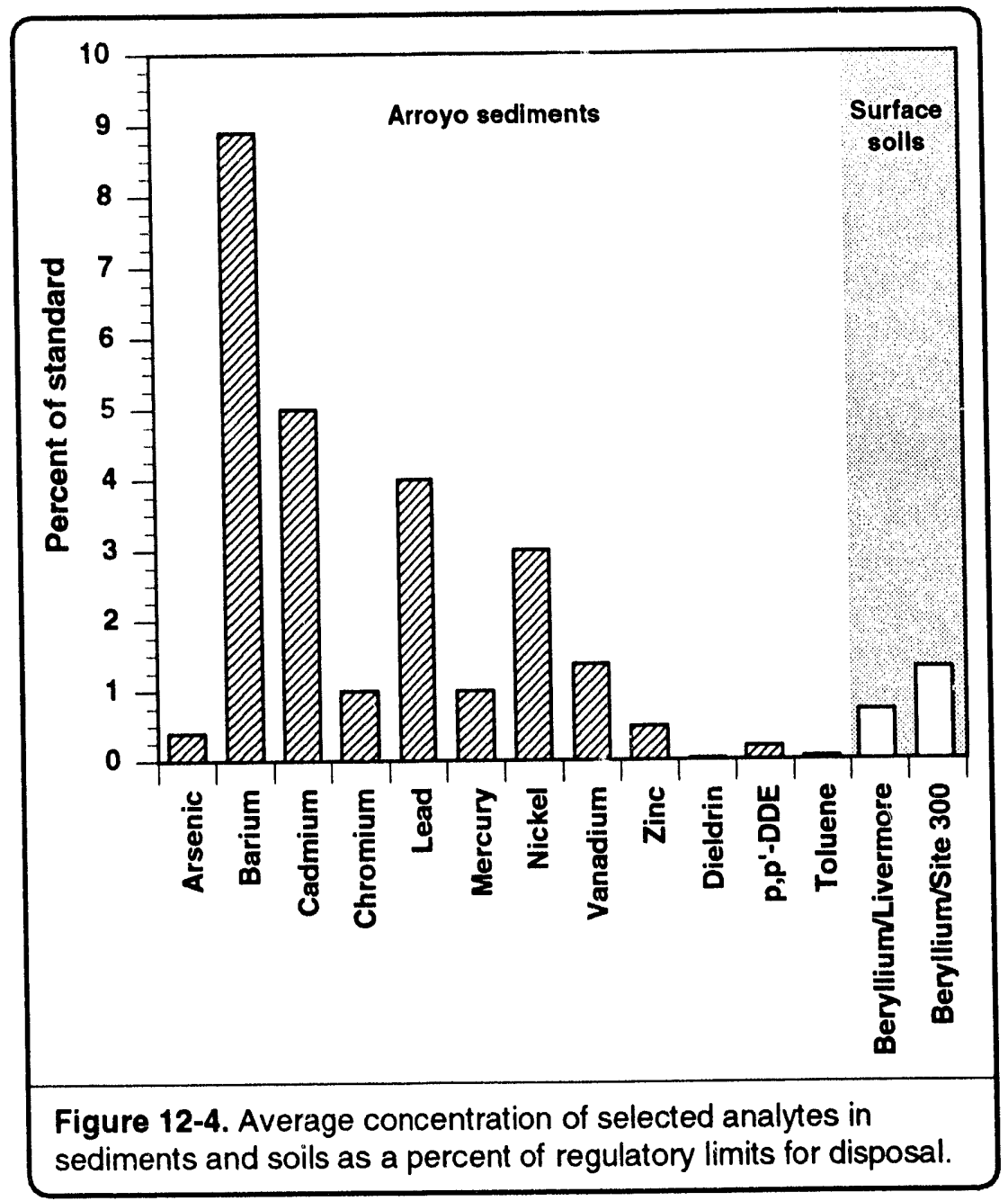

of a variety of compounds (many of which were undetectable before 1989) were observed in some samples. When compared with regulatory limits, all the measured concentrations were small fractions of their respective standards (Figure 12-4). Most of the measured concentrations were less than $1 \%$ of their respective standard, and all concentrations were less than $10 \%$ of the standard. Also, the average values (means and medians) used to generate the comparisons included analytical detection limit values even when the results were reported as less than the detection limit. This means that the averages are conservative, and that actual concentrations were likely to be lower.

The metals and organic compounds that were detected in arroyo sediments generally can be expected near any urban area. Many of the organic compounds are common solvents (or fuel constituents) and are expected in areas that collect storm water runoff, such as in the LLNL drainages and arroyos.

Also during 1992, surface soil samples collected from the Livermore Valley and at Site 300 were analyzed for beryllium. Beryllium, a potentially toxic metal that is used at both the Livermore site and Site 300, is also a naturally occurring metal in soils worldwide. The beryllium concentrations detected in surface soils in the Livermore Valley and at Site 300 are within the range of worldwide natural background concentrations, although the concentrations in samples from Site 300 were approximately twice that of samples from the Livermore Valley. The mean concentrations from both areas were small fractions of the beryllium regulatory limit for waste (Figure 12-4); neither mean concentration was above 0.01 of the regulatory limit.

In summary, nonradiological analyses of sediments and surface soils indicate that the impact of LLNL operations on the nonradiological constituents in these 
media during 1992 did not change from previous years and remains insignificant. All analytes were detected at background concentrations, in trace amounts, or could not be detected at all.

\section{Noise Monitoring}

On the basis of meteorological measurements made prior to a test, a limit is set on the weight of high explosives (HE) that can be detonated without noise impact on populated areas. To monitor this limit, six microbarograph sensors are maintained in or near the City of Tracy.

LLNL conducted 269 high-explosives tests at Site 300 during 1992. Microbarograph measurements in the vicinity of Site 300 and the City of Tracy demonstrated that the peak noise limit of $126 \mathrm{~dB}$ (400 $\mu$ bar) was not exceeded during any of these tests. There were no complaints of noise or overpressure from residents in the vicinity of Site 300 , and none of the 269 tests produced noise levels above $120 \mathrm{~dB}$ $(200 \mu$ bar $)$ at any of the six stations. (See Figure 12-5 for noise monitoring locations.)

\section{Environmental Occurrences}

Notification of environ-

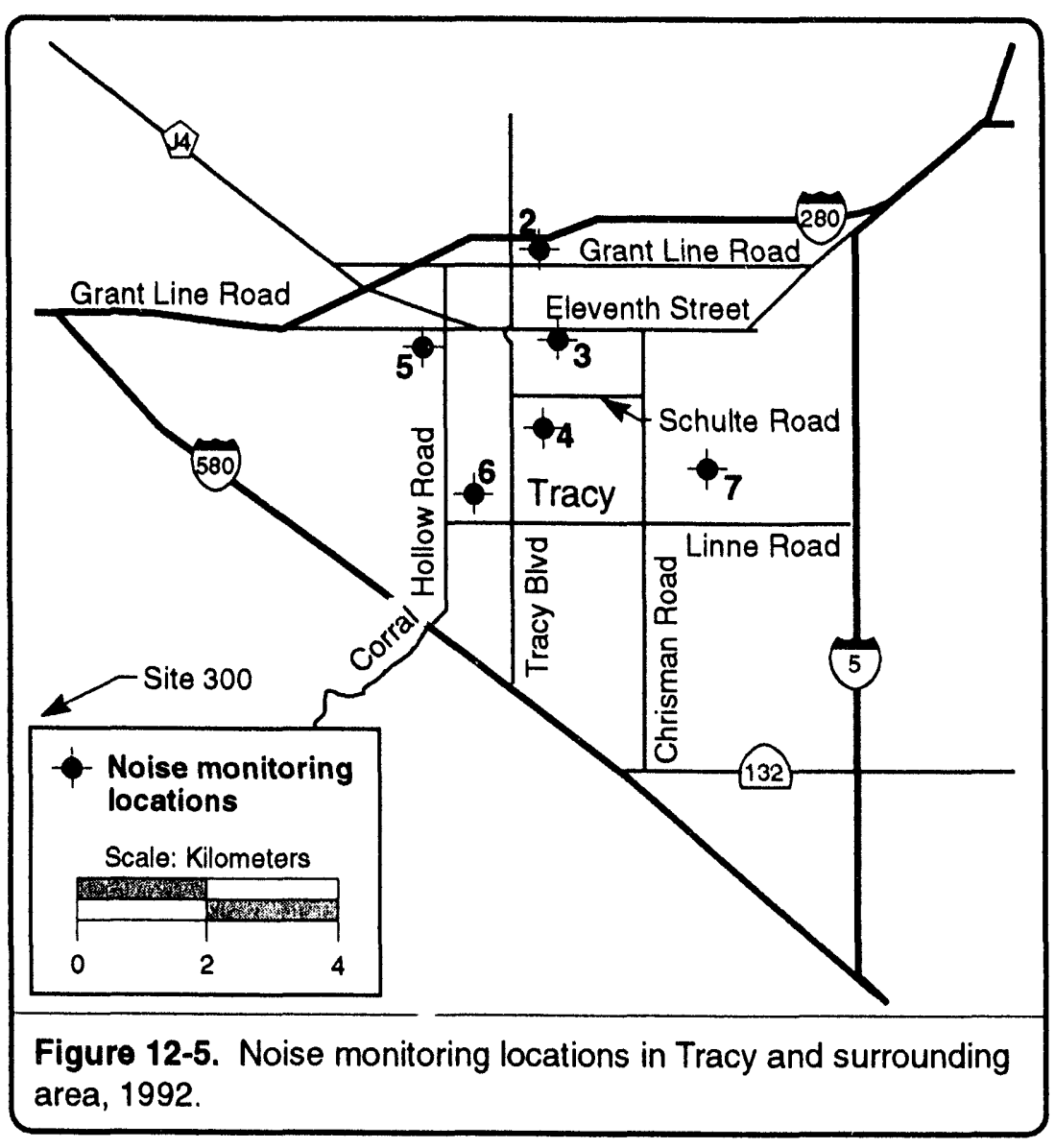
mental occurrences is required under a number of environmental laws and regulations, including the 5000 series of DOE Orders, DOE Order 5000.3A, Occurrence Reporting and Processing of Operations Information, and DOE Order 5484.1, Environmental Protection, Safety, and Health Protection Information Reporting Requirements. DOE Order 5000.3A, issued in May 1990, provides guidelines for contractor facilities to categorize and report environmental occurrences to DOE. The Order divides occurrences into three categories: emergencies, unusual occurrences, and 
off-normal occurrences. (DOE Order 5000.3B has been issued with an effective date of February 22, 1993; however, the contract between the University of California and DOE requires a 60 -day review period for $U C$ to respond to DOE Orders; therefore, LLNL will continue to refer to DOE Order 5000.3A until further notice.)

The Environmental Protection Department (EPD) responds to all reports of spills or other environmental occurrences through a well-established reporting process. EPD has established a 7-days-a-week, 24-hours-a day, on-call, rotational position called the Environmental Duty Officer (EDO), who can be reached by pager or by cellular phone at any time. The EDO determines the reporting requirements, works with environmental analysts and with Laboratory management on the process of notifying local, state, and federal regulatory agencies and DOE, and provides advice on immediate cleanup and monitoring necessary to protect the environment.

EPD responded to 11 incidents during 1992 through the first quarter of 1993 that required agency notification. Two of these incidents qualified as an unusual occurrence in the DOE Order 5000.3A reporting scheme; the other incidents were reported as off-normal occurrences (any incident that requires notification of an environmental regulatory agency is considered an off-normal occurrence). None of these incidents caused any adverse impact to human health or the environment. These incidents are described below.

A blocked sewage line on February 22, 1992, resulted in a 95-liter sewage release into the storm system between Trailers 3725 and 3726 . The sewage line was cleared and the area was sprayed with disinfectant. The San Francisco Bay Regional Water Quality Control Board was notified.

On April 21, 1992, 5680 liters of low-conductivity cooling water (LCW) were released in Building 341, Room 1304, when a leak occurred in the LCW line. A portion of the spill flowed under the door of Room 1304 and out onto the asphalt and gravel north of the building. The source of the leak was identified and repaired. Approximately 500 liters of water inside the building were recovered and disposed of in the sanitary sewer following verification of sewerability.

On May 22, 1992, wastewater containing lead, chrome, and copper was observed to be leaking from a 55-gallon drum stored in the Building 612 Receiving, Segregation, and Container Storage Unit. Between 15 and 23 liters of wastewater were recovered from the secondary containment basin for the unit. The wastewater in the drum was transferred to a new container and the secondary containment basin was decontaminated. This was reported as an unusual occurrence.

On July 14, 1992, oil from a leaking hydraulic press spilled into a nearby sump that was connected to the storm drain system. Approximately 26 liters of oil were recovered from the storm drain manhole and disposed of as hazardous 
waste. In addition, the storm drain line leading from the sump to the storm drain manhole was decontaminated.

On July 15, 1992, up to 8 liters of industrial wastewater, containing sewerable and nonhazardous levels of trace metals, sprayed out of a damaged underground retention system line onto the soil outside Building 231 . The sink that discharged to the damaged pipe was closed off. The liquid in the underground collection tank was pumped to an aboveground tank to prevent additional liquid from leaking.

On August 18, 1992, 1890 liters of fire suppression water were released from open lines during installation of the fire suppression system in Building 153. A small volume escaped the building and spilled onto the ground outside the building. Water supplying the lines was shut off. Water in the building was mopped up and vacuumed. This water was inadvertently dumped onto the ground outside the building.

On August 25, 1992, between 190 to 380 liters of rinse water, generated during the rinsing of a tanker truck that previously contained sewerable wastewater, were released onto the asphalt yard of the Area 612 Facility. The rinse water flowed across the yard and entered an earthen storm drain ditch. Absorbent was used to dam off the entrance to the storm drain ditch, thereby preventing any additional rinse water from leaving the facility. All standing water was collected using wet/dry vacuums. Absorbent was used to absorb any remaining liquid in the facility yard and the drainage ditch.

On September 10,1992, a release of 60 liters of treated wastewater occurred when water was being pumped from the tank farm to portable tanks at Building 514. Water spilled onto the asphalt and soil. Standing water was collected using a wet/dry vacuum and absorbent. The water was determined to be sewerable.

On September 17, 1992, a cap blew off a photoprocessing filter unit in Building $551 \mathrm{~W}$ basement, discharging approximately 38,000 liters of uncontaminated filtered drinking water into utility trenches located on the floor of the basement. To avoid a potential electrical hazard, the water was pumped from the trenches to the outside parking lot, which drained to the Central Drainage Basin.

On September 21, 1992, 60 liters of liquid sewage were released to the ground southwest of Trailer 5627 as a result of a pump failure. No liquid sewage migrated off site, and the pump was subsequently repaired.

On October 22, 1992, between 19 to 38 liters of lead-contaminated oil spilled from a honing machine during transport near the intersection of East Gate Drive and South Outer Loop Road, south of Building 411. Absorbent was used to clean up residual oil spilled on the asphalt. All spent absorbent was disposed of as hazardous waste. 
Agencies notified of the incidents described above included the DOE, the Alameda County Department of Health Services, the U.S. Environmental Protection Agency, the California Environmental Protection Agency Department of Toxic Substances Control, the San Francisco Regional Water Quality Control Board, Office of Emergency Services, and the National Response Center. None of the incidents caused any adverse impact to human health or the environment.

\section{Superfund Amendments and Reauthorization Act (SARA) Title III Reporting Requirements}

SARA Title III also is known as the Emergency Planning and Community Right-to-Know Act. It requires owners/operators of facilities that have certain hazardous chemicals on site to provide information on the manufacture, use, and storage of those chemicals to organizations responsible for emergency-response planning. In California, this information must be provided to the California Office of Emergency Services, the county, and the local fire department.

An initial physical inventory of all chemicals subject to reporting under SARA Title III was conducted during November and December 1988. The inventory included the chemical name, location, weight, type of container, and physical state of the substance. The inventory included the Livermore site, Site 300, and leased space off site. In early 1989, the data were entered into a computerized database system and reports were generated for local emergency planning agencies. In subsequent years, EPD continued periodic updates of the inventory to reflect changes in chemicals handled on site. 


\section{Introduction}

Quality Assurance (QA) requirements for environmental monitoring of Department of Energy (DOE) facilities are mandated by a number of DOE documents: DOE Order 5400.1 identifies QA requirements for radiological effluent and surveillance monitoring and specifies that a QA program consistent with DOE Order $5700.6 \mathrm{~B}$ be established; DOE Order $5700.6 \mathrm{~B}$ sets forth policy, requirements, and responsibilities for the establishment and maintenance of plans and actions that assure quality achievement in DOE programs; finally, the DOE Environmental Regulatory Guide for Radiological Effluent Monitoring and Environmental Surveillance (U.S. Department of Energy 1991) requires the preparation of an Environmental Monitoring Plan containing a QA section that discusses the applicable elements of the American National Standards Institute/American Society of Mechanical Engineers (ANSI/ASME) NQA-1, Quality Assurance Program Requirements for Nuclear Facilities (ASME 1989).

During 1992, LLNL conducted QA activities at the Livermore site and Site 300 in accordance with a plan based on DOE Order 5700.6B and ANSI/ ASME NQA-1 (Holland 1987). Sampling was conducted according to procedures published in the LLNL Environmental Monitoring Plan (Gallegos et al. 1992b).

Environmental monitoring samples are analyzed by LLNL or commercial laboratories using EPA standard methods when available. When EPA standard methods are not available, custom analytical procedures, usually developed at LLNL, are used. The radiochemical methods used by LLNL's Radiation Analytical Sciences laboratory are described in their procedures. When analyses are performed by independent contractors, LLNL requires that their laboratories be certified by the State of California. In addition, LLNL requires all analytical laboratories to maintain adequate quality assurance programs and documentation of methods. Laboratory audits are performed annually as required by the QA plan (Holland 1987). Furthermore, both internal and independent audits of LLNL's monitoring program are performed annually. 


\section{Deviations and Changes to the Sampling Program}

The sections below describe changes to the environmental sampling effort made during 1992, deviations from planned environmental sampling, and regularly scheduled samples for which data are not reported because they could not be collected or were lost during analysis.

\section{Sampling Location Designators}

Tables 13-1 and 13-2 decode sampling location designators and provide a cross-reference for current designators and those used in previous years.

\section{Air Sampling Program}

One Quality Assurance duplicate sampler was added to the air tritium network in July 1992. No other changes were made to either the air tritium or the air particulate network. During 1992, 546 of 561 possible air particulate samples and 482 of 498 possible air tritium samples were collected as part of the routine monitoring program. These represent sample recovery rates of $97 \%$ for both matrices. [The Quality Assurance Plan (Holland 1987) sets a minimum sample recovery rate of $85 \%$.] Lost air particulate samples were usually caused by sampler malfunction or loss of electric power. Air tritium sample loss was due to sampler malfunction or breakage of sample flasks.

\section{Sewage Monitoring Program}

Modest changes were made to the sewer monitoring network during 1992. The sampling location for Sandia National Laboratories, California (SNL/CA) effluent was moved when, as part of a planned upgrade, SNL/CA re-routed its sewage flow to combine with LLNL sewerage just west of LLNL Building 111. The new location became operational in May. Secondly, LLNL altered the methodology for sampling the combined LLNL and SNL/CA effluent as it enters the City of Livermore collection system. Previously, flow-proportional compliance samples were collected from the flow that runs through the continuous monitoring system. The Livermore Water Reclamation Plant (LWRP) felt that this sample was inappropriate for compliance metals analysis because maceration and chlorination were performed before the flow reached the compliance sampler. An upstream sampler was installed in October. The location designators for sewer sampling were updated in 1992 as indicated in Table 13-1.

Sample and data capture in the compliance sampling program was high: 913 of 927 samples were acquired and analyzed, for a total data capture of $98 \%$. Missed samples resulted from sampling equipment failure and personnel turnover at the LWRP where City of Livermore employees collect some samples for LLNL. The sampler used to acquire daily composites of SNL/CA's discharges to LLNL was unreliable and resulted in a low data capture at that location: only 
161 of 366 samples, or $44 \%$. The sampler was replaced in November with a more reliable model.

\section{Routine Water Sampling Program}

Routine water sampling networks include Livermore Valley wells, other Livermore Valley waters, Livermore-site storm water runoff, and Livermore Valley rain. A sample for these networks is defined to be one bottle, even though many bottles may be taken at the same time and location in order to determine the presence of different analytes.

Valley wells are sampled annually at 19 locations and two duplicate samples are taken for quality control purposes. Samples are obtained and given to LLNL by water providers in the Livermore Valley. Of 21 planned samples, 19 were collected and analyzed in 1992, resulting in a sample recovery rate of $90 \%$.

Other Livermore Valley waters are sampled quarterly at 11 locations and monthly at the LLNL swimming pool. Two duplicate quality control samples are taken. A total of 128 samples was planned for 1992; 124 were collected and analyzed, resulting in a $97 \%$ recovery rate. Location DUCK was dry during the third quarter sampling window (two samples lost) and two planned LLNL pool samples were overlooked in December.

Livermore-site storm water runoff was sampled at 10 locations for 18 different storms during 1992. Duplicate samples were taken at one location for each storm for quality control purposes. Nineteen bottles were taken at each sampling location, resulting in a total planned sample size of 3762. Six locations could not be sampled because of insufficient water flow, resulting in the loss of 114 samples. Total sample recovery was $97 \%$.

Livermore Valley rain was sampled at 19 locations for 20 storms during 1992. Duplicate quality control samples were taken at two locations during each storm. One sample was planned at each location for each storm, resulting in 420 total possible samples. The samples that were successfully collected and analyzed numbered 396, for a recovery rate of $94 \%$. Of the 24 samples lost, 6 were of insufficient volume for analysis, and 18 could not be taken because the sampling buckets were missing.

\section{Vegetation and Foodstuff Sampling Program}

No changes were made to the vegetation sampling program in 1992. All 92 planned samples were collected and analyzed, giving a $100 \%$ recovery rate.

The sampling program for wine was reduced due to budgetary constraints. A decrease in the number of California wines sampled was justified by consistency in results over the years and across geographical areas. Wine samples included 12 Livermore wines (down from 14 in 1991), 4 other California wines (down from 8 in 1991), and 5 European wines (down from 13 in 1991). He-3 mass spectrometry was used exclusively as the analytical method for tritium. A total of 
26 wine samples was planned and all of them were successfully collected and analyzed.

Due to the seven-year California drought, it was difficult to locate honey producers in 1992, and no honey sample was taken from the on-site location. Other honey sampling locations, the sampling method, and the analytical methods remained the same as those used in previous years. A total of seven honey samples was submitted-three from Livermore, three from other California locations, and one duplicate of one of the non-Livermore samples. All submitted samples were successfully analyzed.

Three goat's milk suppliers went out of business in 1992. Location MZF went out of business in April and was not replaced. Location LUP went out of business in August 1992 and was replaced by WOOD. Location RIP went out of business and was replaced by MOD2 in April 1992. In August 1992, the MOD2 goats were moved to Stevenson and the location was renamed STEV. An existing goat's milk supplier, WRD, began supplying cow's milk during 1992 and three cow's milk samples were collected and analyzed. As occurs every year, goat's milk was not available from any providers in January and December because goats do not produce milk during these months.

Out of 65 planned milk samples, 61 were collected and analyzed, giving a recovery rate of $94 \%$.

\section{Environmental Radiation Monitoring Program}

No major changes were made to the environmental radiation monitoring program in 1992. A recovery of $100 \%$ was achieved for the site perimeter thermoluminescent dosimeters, $91 \%$ for Livermore Valley locations (179 out of 196 samples), and $83 \%$ for Site 300 (66 out of 80 samples). Dosimeter loss usually is a result of construction activities, destruction by cows, or vandalism. Of the 24 planned neutron probes, 23 were collected and analyzed, giving a $96 \%$ recovery rate.

\section{Meteorology Monitoring Program}

Data recovery from the meteorological monitoring program during 1992 was $99 \%$, both at the Livermore site and at Site 300. Missing data were caused by routine maintenance or modem problems. Equipment at both meteorological towers was operational for the entire year. Calibrated sensors (traceable to National Institute for Standards and Technology) for air temperature, wind speed, wind direction, and relative humidity were routinely replaced with newly calibrated instruments twice during the year.

\section{Soils/Sediments Monitoring Program}

Soils were sampled at 21 locations at the Livermore site and in Livermore Valley and at 14 locations at Site 300 . All planned samples were obtained and 
successfully analyzed for radionuclides and beryllium. No changes to soil sampling locations were made during 1992.

Ten of eleven planned sediment samples at the main site were collected and analyzed for radionuclides, metals, and organic compounds, giving a recovery rate of $91 \%$. One location, located at the center of the LLNL Central Drainage Basin, could not be sampled because of construction at the site. As a special study, additional samples of sediment material were taken from a more shallow depth at each of the ten locations sampled.

\section{Compliance Ground Water Sampling Program}

Upgradient wells $\mathrm{K}$ ! $-01 \mathrm{C}$ and $\mathrm{K} 1-07$ were temporarily buried during Pit 1 landfill closure capping activities, and downgradient well NC7-48 was temporarily buried during Pit 7 landfill closure capping activities in the third quarter of 1992, rendering them inaccessible for sample collection. All of these wells were restored and sampling resumed during the fourth quarter.

Well K7-09 was analyzed for metals (barium, beryllium, lead, and vanadium) and radiological compounds (gross alpha, gross beta, tritium, and uranium isotopes) during the first quarter of 1992. As these analytes had not been regularly monitored at this location, additional analyses were performed to provide both comparison and consistency with other wells in the Pit 7 Complex.

Monitoring of well K7-09 was discontinued in the second quarter of 1992. Volatile organic compounds have not been detected in this well since April of 1989 and have never been detected in well K7-10, which monitors the shallower water-bearing zone in the same area.

Compliance well K7-07 and piezometers NC7-22 and -37 have been dry since 1987. In the future, results from these locations will be reported only when water levels are sufficient for sample collection; however, the water level at these locations will continue to be monitored quarterly.

Well NC7-48 was added to the Pit 7 complex monitoring network during the second quarter. Analyses performed on samples from this location include metals (beryllium, barium, lead, and vanadium) along with EPA Method 624 organic compounds and radiological compounds (gross alpha and beta, tritium, uranium isotopes, and also ${ }^{226} \mathrm{Ra}$ if gross alpha exceeds $5 \mathrm{pCi} / \mathrm{L}$ ). Because additional Method 624 compounds were not detected, samples in subsequent quarters were analyzed by EPA Method 601, which is both sufficient for well NC7-48 and consistent with the analyses performed on samples from other Pit 7 Complex area wells.

Previously used analytical methods for determining the presence of Title 22 pesticides and herbicides provided a level of detection (LOD) above the respective Maximum Contaminant Limits (MCLs). During the third quarter of 1992, these methods were replaced with EPA-approved methods providing 
lower levels of detection. Total suspended solids (TSS) were not analyzed by the analytical laboratory as requested for Pit 1 wells during the third quarter of 1992.

\section{Routine Ground Water Monitoring}

Analytical method improvements to the drinking water wells (Well 01, Well 18, and Well 20) were made during the first quarter of 1992. These wells are now analyzed by EPA Method 524.2, which offers a lower level of detection for volatile compounds than the previously used EPA Methods 601 and 602.

\section{Quality Assurance Activities}

A full-time Quality Assurance/Training Coordinator was added to the environmental monitoring program in 1992, facilitating the completion and distribution of 24 of $60 \mathrm{QA}$ and sampling procedures. These procedures cover the areas of monitoring and sampling methods, sampling locations, and instrument calibrations for each medium; emergency monitoring and response; dose assessment; data management and analysis; document preparation and archiving; reporting; training; and other quality assurance activities. Work is continuing in this area, and it is expected that the balance of the procedures, as well as a revised environmental monitoring QA plan based on the new DOE Order 5700.6C, will be completed and implemented during 1993.

A total of 123 Nonconformance Reports (NCRs) was written during 1992. The major source of NCRs was the analytical laboratories used to evaluate environmental samples. One step taken to address the problems with the laboratories was to build teams consisting of representatives from the environmental monitoring program and from an analytical laboratory, which met to improve communications and clearly define both groups' expectations. Other significant sources of NCRs include equipment failure and vandalism (e.g., power turned off or missing samples).

During 1992, the environmental monitoring program was audited by the LLNL Assurance Review Office (ARO), by the DOE Environmental, Safety, \& Health Progress Assessment, and by DOE's Technical Safety Appraisal revisit. Major areas of concern in these audits include training, quality assurance, air monitoring, and the Environmental Monitoring Plan (EMP). LLNL expects to complete and begin to implement a revised environmental monitoring QA plan and related procedures as well as a new training plan in 1993. A technical review and revision of the EMP are also planned for 1993.

\section{Participation in Laboratory Intercomparison Studies}

During 1992, LLNL's Radiation Analytical Sciences (RAS) laboratory and the Hazards Control Department's analytical laboratory both participated in the EPA's Environmental Monitoring Systems Laboratory Intercomparison Studies Program. Table 13-3 shows the comparison of analyses by these laboratories 
with known values. The normalized deviation from the known value is considered acceptable if it falls between -3 and +3 . However, normalized deviations that fall between -3 and -2 and those which fall between +2 and +3 are considered suspect and could indicate a potential problem with laboratory accuracy. For the RAS results, one value during 1992, the Octcber value for tritium in water, is in the suspect range. RAS personnel are aware of this potential problem, and will take further action if future results are outside the -3 to +3 range. The Hazards Control laboratory's results for gross beta analysis fell within the suspect range in January and outside of specified control limits in May and September of 1992. Upon further inquiry, it was discovered that the EPA had changed the calibration standard it was using for gross beta. The laboratory is in the process of further investigation of the problem and determining appropriate corrective action.

The Hazards Control laboratory participated in two California Department of Health Services Environmental Laboratory Accreditation Program (ELAP) Water Pollution Studies for metals during 1992. The Hazards Control analytical laboratory measures metals in sewage effluent for the LLNL environmental monitoring program. All ELAP intercomparison values for these metals were found to be acceptable in studies performed in June and December of 1992 and the data are presented in Table 13-4.

RAS also participated in the 1992 intercomparison studies by the DOE Environmental Measurements Laboratory (EML). The data from this study are presented in Table 13-5. September results for ${ }^{239} \mathrm{Pu}$ in air, soil, and water as originally reported to EML were incorrect due to samples being mislabeled. This led to unacceptable results. When the problem was recognized by the laboratory, the values were recalculated. Revised, acceptable results appear directly below the original results in Table 13-5.

Additional problems with EML results occurred for tritium in water, both in March and in September. RAS experienced significant staff turnover during 1992 and both tritium analyses were performed by chemists who were in the process of being trained to do the analyses required for the EML studies.

Because of the problems mentioned above, RAS has established a system of independent checks at each stage of its measurement and data reduction system. Analysis, measurement, and data reduction processes are checked by a coworker at each step of the procedure. In addition, the laboratory worksheet has been modified to ensure that all pertinent data are recorded. It is expected that these steps, along with the completion of training for RAS chemists, will eliminate future errors in these analyses.

Contract laboratories are also required to participate in laboratory intercomparison programs; however, permission to publish their results for comparison purposes has not been granted. 


\section{Duplicate Analyses}

Table 13-6 presents selected data generated by the duplicate sampling regime. Samples from both the Livermore site and Site 300 are included to provide a larger data set for statistical analysis. Only data pairs with at least one value above the detection limit are used in this analysis. If there are fewer than eight suitable data pairs, the ratios of the individual duplicate sample pairs are averaged. The average ratio should be between 0.7 and 1.3. When eight or more suitable data pairs are available, precision and regression analyses are performed. The confidence interval limits for precision should be within $\pm 50 \%$. The slope of the regression line should be between 0.7 and 1.3 , and the intercept should be \pm the detection limit. The coefficient of determination $\left(\mathrm{r}^{2}\right)$ should be $>0.8$.

The analyses for these sample media show reasonable agreement between routine samples and quality assurance duplicates, with the exception of ${ }^{3} \mathrm{H}$ in ground water, sewage and air, and ${ }^{239} \mathrm{Pu}$ in air. The ${ }^{3} \mathrm{H}$ deviation in ground water is due to a single routine and duplicate sample pair for which the agreement is poor. Results excluding this pair are given in parentheses in Table 13-6. The ${ }^{239} \mathrm{Pu}$ deviations, also seen in 1989 through 1991, are thought to be caused by the proximity of the levels to the detection limit.

\section{Statistical Methods}

Statistical methods used in this report have been implemented pursuant to the DOE Environmental Regulatory Guide for Radiological Effluent Monitoring and Environmental Surveillance (U.S. Department of Energy 1991). These methods reduce the large volumes of monitoring data to summary concentration estimates that are suitable for both temporal and spatial comparisons. Attention is given to estimating accuracy, bias, and precision of all data.

Data review and analyses are conducted in accordance with the environmental monitoring Quality Assurance Plan (Holland 1987) and the Environmental Monitoring Plan (Gallegos et al. 1992b). Both documents contain detailed information regarding the acceptability of data and the procedures that are followed for the identification, notification, and correction of suspect data.

\section{Radiological Data}

The precision of radiological analytical results is displayed in the data tables as $2 \sigma$ counting errors. The counting errors are not used in any summary statistic calculations. By convention, any radiological result exhibiting a $2 \sigma$ counting error greater than $100 \%$ is said to be below the detection criterion and is presented in the tables with a less-than symbol $(<)$ to indicate its status. No value of error is reported for values below the detection criterion. Calculation of 
summary statistics uses the repurted value in accordance with the procedures described below.

\section{Nonradiological Data}

Nonradiological data that are reported as being below the analytical detection limit also are displayed in the tables with a less-than symbol $(<)$. As with the radiological data, the actual detection limit values are used in the calculation of summary statistics as explained below.

\section{Statistical Comparisons}

Standard comparison techniques (regression, $t$-test, and ANOVA) have been used where appropriate to determine the statistical significance of trends or differences between means. All such tests of significance have been performed at the 0.05 level. When such a comparison is made, it is explicitly stated in the text as being "statistically significant" or "not statistically significant." Other uses of the word "significant" in the text do not imply that statistical tests have been performed. These uses instead relate to the concept of practical significance, and are based on professional judgment.

\section{Summary Statistics}

Determinations of measures of central tendency and associated measures of dispersion are calculated according to DOE Environmental Regulatory Guide for Radiological Effluent Monitoring and Environmental Surveillance (U.S. Department of Energy 1991) protocols. For data sets not containing values below the detection criterion, measures of central tendency are reported as the arithmetic mean plus or minus one standard deviation.

For data sets containing one-third or fewer values below the detection criterion, measures of central tendency are reported as less than the arithmetic mean. If no values below the detection criterion are above the calculated mean, dispersion is reported as greater than one calculated standard deviation. If any values below the detection criterion are above the calculated mean, dispersion is not reported. Statistics are calculated using the reported detection limit value for nonradiological data or the reported value for radiological data.

For data sets containing more than one-third but less than one half values below the detection criterion, the central tendency is reported as the median value. If all values below the detection criterion are sufficiently below the median value (determined on a case-by-case basis), dispersion is reported as the median absolute deviation. If calculation of the absolute median deviation is not appropriate, dispersion is not reported. Statistics are calculated using the reported detection limit value for nonradiological data or the reported value for radiological data. 
For data sets containing greater than or equal to one half values below the detection criterion, the central tendency is reported as less than the median value. Dispersion is not reported.

\section{Radiation Units}

Data for 1992 have been reported in SI units, to conform with standard scientific practices and federal law. Values in the text are reported in becquerel per liter ( $\mathrm{Bq} / \mathrm{L}$ ) and millisievert (mSv); equivalent values in picocuries ( $\mathrm{pCi}$ ) and millirem (mrem) are given in parentheses, as these were the reporting units used in previous environmental reports, and will be useful for making data comparisons. Values in graphic figures are given only in SI units; SI units are used in tables with summary statistics including values for both sets of units. 
Table 13-1. LLNLLivermore Valley sampling location designators.

\begin{tabular}{|c|c|c|}
\hline Medium/location & $\begin{array}{c}\text { Current } \\
\text { designator }\end{array}$ & $\begin{array}{c}\text { Previous } \\
\text { designator(8) }\end{array}$ \\
\hline \multicolumn{3}{|l|}{ Air particulate } \\
\hline NW Perimeter (Met Tower) & L-MET & $90-13$ \\
\hline Cowbarn & L.COW & $90-15$ \\
\hline E Perimeter (Visitors Center) & L-VIS & $90-14$ \\
\hline East \& Greenville Aves. (Salvage) & L-SALV & $90-01$ \\
\hline South Cafeteria (East Ave.) & L-CAFE & $90-12$ \\
\hline Mesquite Way & L-MESQ & $90-02$ \\
\hline UNCLE Credit Union & L-CRED & - \\
\hline Near Building 531 & L-B531 & - \\
\hline East Ave. Firehouse & L-FIRE & $90-17$ \\
\hline Sandia Tanks & L-TANK & $90-03$ \\
\hline Zone 7 & L-ZON7 & $90-04$ \\
\hline Patterson Pass & L-PATT & $90-05$ \\
\hline Altamont Pass & L-ALTA & $90-07$ \\
\hline Residence (N. Vasco Rd.) & L-RRCH & $90-06$ \\
\hline FCC Station & L-FCC & $90-08$ \\
\hline Livermore City Corp. Yard & L-LCCY & $90-09$ \\
\hline LWRP Sewer Plant & L-LWRP & $90-16$ \\
\hline Livermore VA Hospital & L-HOSP & $90-10$ \\
\hline Residence (Mines Rd.) & L-ERCH & $90-11$ \\
\hline \multicolumn{3}{|l|}{ Air tritium } \\
\hline NW Perimeter (Met Tower) & L-MET & $93-13$ \\
\hline Cowbarn & L-COW & $93-15$ \\
\hline E Perimeter (Visitors Center) & L-VIS & $93-14$ \\
\hline East \& Greenville Aves. (Salvage) & L.SALV & $93-01$ \\
\hline East Ave. "South" Cafeteria & L-CAFE & $93-12$ \\
\hline West Parking Lot (Mesquite Way) & L-MESQ & $93-02$ \\
\hline LLNL Pool & L-POOL & - \\
\hline Building 331 Yard & L-B331 & - \\
\hline Building 292 Area & L-B292 & - \\
\hline Building 514 Yard & L-B514 & - \\
\hline Building 624 (612 Yard) & L-B624 & - \\
\hline East Ave. Firehouse & L-FIRE & $93-17$ \\
\hline West of Sandia (Veterinarian) & L-VET & $93-52$ \\
\hline East of Sandia (Crossroad) & L-XRDS & $93-51$ \\
\hline Zone 7 & L-ZON7 & $93-04$ \\
\hline Altamont Pass & L-ALTA & $93-07$ \\
\hline Livermore City Corp. Yard & L-LCCY & $93-09$ \\
\hline
\end{tabular}


Table 13-1. LLNL/Livermore Valley sampling location designators (continued).

\begin{tabular}{|c|c|c|c|}
\hline Medium/location & $\begin{array}{l}\text { Current } \\
\text { designator }\end{array}$ & $\begin{array}{c}\text { Previous } \\
\text { designator(s) }\end{array}$ & \\
\hline \multicolumn{4}{|l|}{ Cow's milik } \\
\hline Residence (Greenville Rd.) & L-WRD & - & \\
\hline \multicolumn{4}{|l|}{ Goat's milk } \\
\hline Residence (Greenville Rd.) & L-WRD & $91-05$ & \\
\hline Fesidence (Edwards) & L-COOL & - & \\
\hline Cartoned Milk & C-CART & $91-97$ & \\
\hline Pre-Pasturized (Turlock) & C-TUR & - & \\
\hline Residence (Stevenson) & C-STEV & - & repliced C-MOD2 \\
\hline Residence (Brentwood) & C-WOOD & - & replaced L-LUP \\
\hline Residence (Tesla Rd.) & L-MZF & $91-07$ & abandoned \\
\hline Residence (Modesto) & C-MOD & - & abandoned \\
\hline Residence (Fii,Jon) & C-RIP & - & replaced \\
\hline Residence (Modesto) & C-MOD2 & - & replaced \\
\hline Residence (Lupin Way) & L-LUP & $91-13$ & replaced \\
\hline \multicolumn{4}{|l|}{ Vegetation } \\
\hline Patterson Pass & L-PATT & $95-04$ & \\
\hline Zone 7 & L-ZON7 & $95-15$ & \\
\hline I-580 \& Greenville Rd. & $L-1580$ & $95-20$ & \\
\hline Aqueduct & L-AQUE & $95-23$ & \\
\hline N of LLNL (Railroad Tracks) & L-RAIL & $95-29$ & \\
\hline Tesla Rd. (West) & L-TESW & $95-32$ & \\
\hline FCC Station & L-FCC & $95-33$ & \\
\hline Met Tower & L-MET & - & replaced L-VASW \\
\hline Calaveras Reservoir & L-CAL & - & \\
\hline Camp Parks & L-PARK & - & \\
\hline Visitors Center & L-VIS & - & \\
\hline Mesquite Way & L-MESQ & - & replaced L-VASW \\
\hline Residence (Danville) & L-DAN & - & \\
\hline Residence (Modesto) & C-MOD & - & \\
\hline Vasco Rd. (W of LLNL) & L-VASW & $95-31$ & replaced \\
\hline \multicolumn{4}{|l|}{ Arroyo sediment } \\
\hline Central Drainage Basin & L-CDB & $\mathrm{CDB}$ & \\
\hline Arroyo Las Positas East & L-ALPE & ALPE & \\
\hline Arroyo Las Positas West & L-ALPW & ALPW & \\
\hline
\end{tabular}

continued 
Table 13-1. LLNL/Livermore Valley sampling location designators (continued).

\begin{tabular}{|c|c|c|c|}
\hline Medlum/location & $\begin{array}{l}\text { Current } \\
\text { designator }\end{array}$ & $\begin{array}{c}\text { Previous } \\
\text { designator(s) }\end{array}$ & \\
\hline \multicolumn{4}{|l|}{ Arroyo sediment } \\
\hline Arroyo Seco West & L-ASW & L-ASN & renamed \\
\hline Arroyo Seco South \#2 & L-ASS2 & - & replaced L-ASS \\
\hline 4th and $A$ Streets & L-4THA & - & \\
\hline Arroyo Las Positas North & L-ALPN & - & \\
\hline Greenville Rd., NE Perimeter & L-GRNE & - & \\
\hline Central Drainage Basin 2 & L-CDB2 & - & \\
\hline East of Bı illding 438 & L-438E & - & \\
\hline W Perimeter Drainage Channel & L-WPDC & - & \\
\hline Arroyo Seco South & L-ASS & ASS & replaced \\
\hline Arroyo Seco East & L-ASE & ASE & abandoned \\
\hline \multicolumn{4}{|l|}{ Soil } \\
\hline Mesquite Way & L-MESQ & L.02 & \\
\hline Sandia Tanks & L-TANK & L-03 & \\
\hline Zone 7 & L-ZON7 & L-04 & \\
\hline Patterson Pass & L-PATT & L-05 & \\
\hline FCC Station & L-FCC & L-08 & \\
\hline Livermore VA Hospital & L-HOSP & $L-10$ & \\
\hline NW Perimeter (Met Tower) & L-MET & $L-13$ & \\
\hline E Perimeter (Visitors Center) & L-VIS & $L-14$ & \\
\hline Cowbarn & L-COW & L-15 & \\
\hline NE Corner Perimeter Fence & L-NEP & $L-18$ & \\
\hline LWRP Sewer Plant (1/3 N) & L-WRP1 & L-19 & \\
\hline LWRP Sewer Plant (2/3 N) & L-WRP2 & L-20 & \\
\hline LWRP Sewer Plant (NW) & L-WRP3 & L-21 & \\
\hline LWRP Sewer Plant ( $1 / 3$ W) & L-WRP4 & $\mathrm{L}-22$ & \\
\hline LWRP Sewer Plant (2/3 W) & L-WRP5 & L-23 & \\
\hline LWRP Sewer Plant (SW) & L-WRP6 & L-24 & \\
\hline Salvage & L-SALV & - & \\
\hline South Cafeteria (East Ave.) & L-CAFE & - & \\
\hline Altamont & L-ALTA & - & \\
\hline Residence (Mines Rd.) & L-ERCH & - & \\
\hline Residence (N. Vasco Rd.) & L-RRCH & - & \\
\hline
\end{tabular}


Table 13-1. LLNL/Livermore Valley sampling location designators (continued).

\begin{tabular}{|c|c|c|c|}
\hline Medium/location & $\begin{array}{c}\text { Current } \\
\text { designator }\end{array}$ & $\begin{array}{c}\text { Prevlous } \\
\text { designator(s) }\end{array}$ & \\
\hline \multicolumn{4}{|l|}{ Sewage } \\
\hline Building 196 (daily composite) & L-B196 & LLNL & \\
\hline Building 196 (weekly composite) & L-C196 & - & replaced L-LWRP \\
\hline LWRP (effluent) & L-WRPE & - & \\
\hline LWRP (digestor) & L-WRD1 & - & \\
\hline LWRP (digestor) & L-WRD2 & - & \\
\hline Manhole 40B & L-M40 & - & \\
\hline Manhole 51A & L-M51 & - & \\
\hline Manhole 53A & L-M53 & - & \\
\hline Manhole $59 \mathrm{~A}$ & L-M69 & - & \\
\hline Manhole 86B & L-M86 & - & \\
\hline Manhole 125C & L-M125 & - & \\
\hline Manhole 163A (Sandia) & $L-163 A$ & - & \\
\hline Manhole 177E & L-M177 & - & \\
\hline Manhole 185F & L-M185 & - & \\
\hline Manhole 231A & L-M231 & - & \\
\hline Manhole 238C & L-M238 & - & \\
\hline LWRP & L-LWRP & LWRP & replaced \\
\hline \multicolumn{4}{|l|}{ Run-Off } \\
\hline Arroyo Las Positas (E of LLNL) & L-ALPE & 01 & \\
\hline Central Drainage Basin & L-CDB & 02 & \\
\hline Arroyo Las Positas (NW boundary) & L-ALPW & 03 & \\
\hline Arroyo Seco West (Vasco/East Ave.) & L-ASW & L-ASN;06 & renamed \\
\hline East of B-438 & L-B438 & - & \\
\hline Arroyo Las Positas ( $\mathrm{N}$ at cowbarn) & L-ALPN & - & \\
\hline 4th and $A$ Streets & L-4THA & - & \\
\hline West Perimeter Drainage Channel & L-WPDC & - & \\
\hline Arroyo Seco South \#2 & L-ASS2 & - & replaced L-ASS \\
\hline Greenville Road (NE Perimeter) & L-GRNE & - & \\
\hline Arroyo Seco East (influent to Sandia) & L-ASE & 04 & abandoned \\
\hline Arroyo Seco South (West Parking Lot) & L-ASS & 05 & replaced \\
\hline
\end{tabular}


Table 13-1. LLNL/Livermore Valley sampling location designators (concluded).

\begin{tabular}{|c|c|c|}
\hline Medlum/location & $\begin{array}{l}\text { Current } \\
\text { designator }\end{array}$ & $\begin{array}{c}\text { Previous } \\
\text { designator(8) }\end{array}$ \\
\hline \multicolumn{3}{|l|}{ Rain } \\
\hline Zone 7 & L-ZON7 & - \\
\hline Cowbarn & L-COW & - \\
\hline FCC Station & L-FCC & - \\
\hline DelValle/Zone 7 & L-DEL7 & - \\
\hline Camp Parks & L-PARK & - \\
\hline Vineyard & L-VINE & - \\
\hline NW Perimeter (Met Tower) & L-MET & - \\
\hline Residence (South L St.) & L-SLST & - \\
\hline Residence (Buena Vista Ave.) & L-BVA & - \\
\hline Visitors Center & L-VIS & - \\
\hline Building 291 & L-B291 & - \\
\hline Central Drainage Basin & L.CDB & - \\
\hline Greenville and Tesla Rds. & L.-GTES & - \\
\hline East of Sandia & L-ESAN & - \\
\hline Building 343 & L-B343 & - \\
\hline Aqueduct & L-AQUE & - \\
\hline East \& Greenville Aves. (Salvage) & L-SALV & - \\
\hline Altamont & L-ALTA & 一 \\
\hline Patterson Pass & L-PATT & - \\
\hline \multicolumn{3}{|l|}{ Water } \\
\hline Central Drainage Basin & L-CDB & - \\
\hline Del Valle Lake & L-DEL & $92-11$ \\
\hline Zone 7 & L-ZON7 & $92-15$ \\
\hline Springtown Duck Pond & L-DUCK & $92-16$ \\
\hline Gas Station Tap Water & L-GAS & $92-19$ \\
\hline Arroyo de Laguna (Sunol) & L-ALAG & $92-24$ \\
\hline Shadow Cliffs & L-SHAD & $92-26$ \\
\hline Calaveras Reservoir & L-CAL & $92-29$ \\
\hline Building 151 Tap Water & L-TAP & $92-30$ \\
\hline Residence (Palm Ave.) & L-PALM & $92-31$ \\
\hline Private Well & L-ORCH & $92-34$ \\
\hline Residence (Bell Ave.) & L-BELL & $92-37$ \\
\hline LLNL Pool & L-POOL & $92-43$ \\
\hline
\end{tabular}


Table 13-2. Site 300 sampling location designators.

\begin{tabular}{|c|c|c|}
\hline Medium/location & $\begin{array}{c}\text { Current } \\
\text { designator }\end{array}$ & $\begin{array}{c}\text { Previous } \\
\text { designator(s) }\end{array}$ \\
\hline \multicolumn{3}{|l|}{ Air particulate } \\
\hline East Observation Point & 3-EOBS & $40-01$ \\
\hline East Control Post & 3-ECP & $40-02$ \\
\hline West Control Post & 3-WCP & $40-03$ \\
\hline Linac Rd. & 3-LIN & $40-04$ \\
\hline Near Well \#4 (Golf Course) & 3-GOLF & $40-05$ \\
\hline Tracy Firehouse & 3-TFIR & $40-06$ \\
\hline North Power Station & 3-NPS & $40-08$ \\
\hline West Observation Point & 3-WOBS & $40-09$ \\
\hline East of Building 801 & 3-801E & $40-10$ \\
\hline \multicolumn{3}{|l|}{ Soll } \\
\hline West of Building 834 & $3-834 W$ & 3ESEI01 or 1103 \\
\hline Near Building 858 & 3-858S & 3WSWI01 or 1104 \\
\hline North of Building 856 & $3-856 \mathrm{~N}$ & $3 W \times X K 01$ or 1106 \\
\hline North of Road to Building 851 & $3-851 N$ & 3WNWI01 or 1107 \\
\hline North of East Observation Point & 3-EOBS & 3NNWL01 or 1112 \\
\hline North of Well \#8 (ERD Evaporator) & 3-EVAP & 3WNWK01 or 1109 \\
\hline North Power Station & 3-NPS & $3 N W X P 01$ or 1110 \\
\hline West Landfill (Disp. Site West) & 3-DSW & 3NWXP02 or 1111 \\
\hline West of Building 801 & $3-801 W$ & 3NNWG01 or 1113 \\
\hline East of Building 801 & 3-801E & $3 \mathrm{NXXHO1}$ or 1114 \\
\hline Behind Building 812 & $3-812 N$ & $3 N \times X C 01$ or 1115 \\
\hline West of Cafeteria (Golf Course) & 3-GOLF & 3SEXL01 or 1116 \\
\hline West Observation Point & 3-WOBS & $3 W N W N 01$ or 1108 \\
\hline North of Building 801 & $3-801 \mathrm{~N}$ & 1117 \\
\hline \multicolumn{3}{|l|}{ Vegetation } \\
\hline Carnegie & 3-CARN & $45-01$ \\
\hline Golf Course & 3-GOLF & $45-02$ \\
\hline Geodetic & 3-GEO & $45-03$ \\
\hline Disposal Site West & 3-DSW & $45-06$ \\
\hline Behind Building 801 & $3-801 E$ & $45-12$ \\
\hline Near Well \#8 (ERD Evaporator) & 3-EVAP & $45-13$ \\
\hline
\end{tabular}

...continued 
Table 13-2. Site 300 sampling location designators (concluded).

\begin{tabular}{llc}
\hline \multicolumn{1}{c}{ Mediumllocation } & \multicolumn{1}{c}{$\begin{array}{c}\text { Current } \\
\text { designator }\end{array}$} & $\begin{array}{c}\text { Previous } \\
\text { designator(s) }\end{array}$ \\
\hline Water & & \\
Well 1 & 3-WELL01 & $42-01$ \\
Private Well & 3-CON1 & $42-07$ \\
Private Well & 3-CON2 & - \\
Well 18 & 3-WELL18 & $42-22$ \\
Geodetic Creek & 3-GEOCRK & $42-14$ \\
Rain & 3-RAIN & $42-20$ \\
812 Creek & 3-812CRK & $42-21$ \\
Carnegie Retention Well 1 & 3-CARNRW1 & $42-23$ \\
Carnegie Retention Well 2 & 3-CARNRW2 & $42-24$ \\
Well 20 & 3-WELL20 & $42-31$ \\
Private Well & 3-GALLO1 & $42-28$ \\
Private Well & 3-GALLO2 & - \\
CDF Well & 3-CDF1 & $42-27$ \\
Private Well & 3-MUL1 & - \\
Private Well & 3-MUL2 & - \\
Private Well & 3-VIE1 & - \\
Private Well & 3-VIE2 & - \\
Private Well & 3-STN & - \\
\hline
\end{tabular}




\section{$Y$}

Table 13-3. Results from the EPA Environmental Monitoring Systems Laboratory (EMSL) Intercomparison Program, 1992.

\begin{tabular}{|c|c|c|c|c|c|c|}
\hline Analysis & Date & Medlum & Units & LLNL & Known & $\begin{array}{c}\text { Normallzed } \\
\text { deviation } \\
\text { from the } \\
\text { known } \\
\text { value a }^{\text {a }}\end{array}$ \\
\hline \multirow[t]{3}{*}{${ }^{3} \mathrm{H}^{\mathrm{b}}$} & February & water & $\mathrm{pCi} / \mathrm{L}$ & 7985.67 & 7904.0 & 0.18 \\
\hline & June & & $\mathrm{pCi} / \mathrm{L}$ & 2184.00 & 2125.0 & 0.29 \\
\hline & October & & pCilL & 6818.00 & 5962.0 & $2.49^{\mathrm{a}}$ \\
\hline \multirow[t]{2}{*}{${ }^{239} \mathrm{Pu}^{\mathrm{b}}$} & January & water & $\mathrm{pCi} / \mathrm{L}$ & 15.90 & 16.8 & -0.92 \\
\hline & August & & $\mathrm{pCi} / \mathrm{L}$ & 9.07 & 9.0 & 0.13 \\
\hline \multirow[t]{2}{*}{${ }^{137}$ Cs $^{b}$} & March & air & pCi/filter & 13.00 & 10.0 & 1.04 \\
\hline & August & & $\mathrm{pCi} /$ filter & 19.67 & 18.0 & 0.58 \\
\hline \multirow{2}{*}{$\begin{array}{l}\text { Total } K^{b} \\
131 j^{b}\end{array}$} & April & milk & $\mathrm{mg} / \mathrm{L}$ & 1776.67 & 1710.0 & 1.34 \\
\hline & & & $\mathrm{pCi} / \mathrm{L}$ & 83.67 & 78.0 & 1.23 \\
\hline${ }^{137} \mathrm{Cs}^{\mathrm{b}}$ & & & pCi/L & 40.67 & 39.0 & 0.58 \\
\hline \multirow[t]{3}{*}{ Gross alphac } & January & water & pCi/L & 26.00 & 30.0 & -0.87 \\
\hline & May & & $\mathrm{pCi} / \mathrm{L}$ & 16.00 & 15.0 & 0.35 \\
\hline & September & & $\mathrm{pCi} / \mathrm{L}$ & 41.67 & 45.0 & -0.52 \\
\hline \multirow[t]{3}{*}{ Gross beta ${ }^{c}$} & January & water & pCi/L & 22.33 & 30.0 & $-2.66^{a}$ \\
\hline & May & & pCi/L & 56.33 & 44.0 & $4.27^{a}$ \\
\hline & September & & pCi/l & 39.00 & 50.0 & $-3.81^{a}$ \\
\hline
\end{tabular}

a Deviations are acceptable if they are between -3 and +3 ; however, deviations between +2 and +3 or between -2 and -3 are indicative of potential problems with laboratory accuracy.

b All data were provided by LLNL's Radiation Analytical Sciences laboratory.

c Data were provided by LLNL's Hazards Control analytical laboratory. 
Table 13-4. Results from the California Department of Health Services Environmental Laboratory Accreditation Program (ELAP) Water Pollution Studies. ${ }^{a}$

\begin{tabular}{|c|c|c|c|c|c|c|}
\hline Analysis & Date & Sample & $\begin{array}{l}\text { LLNL } \\
\text { value }^{b}\end{array}$ & $\begin{array}{c}\text { True } \\
\text { value }^{b}\end{array}$ & $\begin{array}{c}\text { Acceptable } \\
\text { limits }\end{array}$ & $\begin{array}{l}\text { Warning } \\
\text { limits }\end{array}$ \\
\hline \multirow[t]{4}{*}{ Aluminum } & $6 / 22 / 92$ & 1 & 2080 & 2100 & $1790-2420$ & $1870-2340$ \\
\hline & & 2 & 954 & 950 & $778-1130$ & $823-1090$ \\
\hline & $12 / 21 / 92$ & 1 & 70.5 & 65.9 & $40.1-100$ & 47.7-92.6 \\
\hline & & 2 & 563 & 548 & $435-645$ & $462-619$ \\
\hline \multirow[t]{4}{*}{ Arsenic } & $6 / 22 / 92$ & 1 & 108 & 100 & $71.8-127$ & $78.7-120$ \\
\hline & & 2 & 458 & 430 & $348-508$ & $368-488$ \\
\hline & $12 / 21 / 92$ & 1 & 142 & 140 & $113-168$ & $120-161$ \\
\hline & & 2 & 15.1 & 15.0 & $11.1-19.4$ & $12.2-18.4$ \\
\hline \multirow[t]{4}{*}{ Beryllium } & $6 / 22 / 92$ & 1 & 96.1 & 100 & $74.9-126$ & $81.4-120$ \\
\hline & & 2 & 38.8 & 40 & $30.0-50.9$ & $32.6-48.2$ \\
\hline & $12 / 21 / 92$ & 1 & 15.7 & 16.8 & $12.4-21.4$ & $13.5-20.2$ \\
\hline & & 2 & 630 & 609 & $497-714$ & $525-686$ \\
\hline \multirow[t]{4}{*}{ Cadmium } & $6 / 22 / 92$ & 1 & 242 & 250 & $210-290$ & $220-280$ \\
\hline & & 2 & 38.3 & 40 & $32.8-48.7$ & $34.8-46.7$ \\
\hline & $12 / 21 / 92$ & 1 & 16.6 & 16.8 & $12.2-21.5$ & $13.4-20.4$ \\
\hline & & 2 & 381 & 380 & $319-440$ & $334-425$ \\
\hline \multirow[t]{4}{*}{ Chromium } & $6 / 22 / 92$ & 1 & 758 & 800 & $658-927$ & $691-893$ \\
\hline & & 2 & 307 & 315 & $206-371$ & $279-358$ \\
\hline & $12 / 21 / 92$ & 1 & 599 & 620 & $516-717$ & $541-691$ \\
\hline & & 2 & 8.90 & 8.11 & $3.37-12.3$ & $4.49-11.2$ \\
\hline \multirow[t]{4}{*}{ Copper } & $6 / 22 / 92$ & 1 & 776 & 790 & $714-877$ & $735-856$ \\
\hline & & 2 & 127 & 130 & $113-144$ & $117-140$ \\
\hline & $12 / 21 / 92$ & 1 & 5.80 & 6.33 & $3.68-8.58$ & $4.30-7.97$ \\
\hline & & 2 & 242 & 241 & $213-267$ & $219-260$ \\
\hline \multirow[t]{4}{*}{ Iron } & $6 / 22 / 92$ & 1 & 1430 & 1500 & $1310-1690$ & $1360-1650$ \\
\hline & & 2 & 515 & 540 & $471-613$ & $489-595$ \\
\hline & $12 / 21 / 92$ & 1 & 696 & 711 & $625-800$ & $647-778$ \\
\hline & & 2 & 86.6 & 83.0 & $68.2-97.7$ & $71.9-94.0$ \\
\hline \multirow[t]{2}{*}{ Mercury } & $6 / 22 / 92$ & 1 & 4.70 & 5.30 & $3.89-6.65$ & $4.24-6.30$ \\
\hline & & 2 & 0.628 & 0.330 & $0.0869-0.620$ & $0.154-0.552$ \\
\hline \multirow[t]{4}{*}{ Nickel } & $6 / 22 / 92$ & 1 & 730 & 740 & $660-814$ & $680-794$ \\
\hline & & 2 & 2200 & 2200 & $1970-2410$ & $2020-2360$ \\
\hline & $12 / 21 / 92$ & 1 & 31.1 & 31.7 & $25.5-37.4$ & $27.0-35.9$ \\
\hline & & 2 & 580 & 569 & $498-632$ & $515-616$ \\
\hline
\end{tabular}


Table 13-4. Results from the California Department of Health Services Environmental Laboratory Accreditation Program (ELAP) Water Pollution Studies $^{a}$ (concluded).

\begin{tabular}{|c|c|c|c|c|c|c|}
\hline Analysis & Date & Sample & $\begin{array}{l}\text { LLNL } \\
\text { valueb }\end{array}$ & $\begin{array}{c}\text { True } \\
\text { value }^{b}\end{array}$ & $\begin{array}{l}\text { Acceptable } \\
\text { limits }\end{array}$ & $\begin{array}{l}\text { Warning } \\
\text { limits }\end{array}$ \\
\hline \multirow[t]{4}{*}{ Lead } & $6 / 22 / 92$ & 1 & 544 & 550 & $474-623$ & $493-604$ \\
\hline & & 2 & 142 & 150 & $120-179$ & $127-172$ \\
\hline & $12 / 21 / 92$ & 1 & 956 & 959 & $831-1080$ & $862-1050$ \\
\hline & & 2 & 1560 & 1500 & $1300-1680$ & $1350-1630$ \\
\hline \multirow[t]{4}{*}{ Zinc } & $6 / 22 / 92$ & 1 & 364 & 360 & $318-406$ & $329-395$ \\
\hline & & 2 & 1430 & 1400 & $1230-1570$ & $1270-1520$ \\
\hline & $12 / 21 / 92$ & 1 & 17.6 & 17.0 & $11.3-22.9$ & $12.8-21.4$ \\
\hline & & 2 & 625 & 612 & $536-689$ & $555-670$ \\
\hline \multirow[t]{4}{*}{ Silver } & $6 / 22 / 92$ & 1 & 0.82 & 1.03 & $0.652-1.40$ & $0.748-1.30$ \\
\hline & & 2 & 9.87 & 10.6 & $8.45-12.6$ & $8.98-12.1$ \\
\hline & $12 / 21 / 92$ & 1 & 5.58 & 5.59 & $4.20-7.01$ & $4.56-6.66$ \\
\hline & & 2 & 14.1 & 14.0 & $11.1-16.8$ & $11.8-16.1$ \\
\hline
\end{tabular}

a All data were provided by LLNL's Hazards Control analytical laboratory.

b All results reported in micrograms per liter. 
Table 13-5. Results from the DOE Environmental Measurements Laboratory (EML) Quality Assurance Program, 1992.a

\begin{tabular}{|c|c|c|c|c|c|}
\hline Analysis & Date & Medium & LLNL value & EML value & Ratio \\
\hline \multirow[t]{2}{*}{$7_{\mathrm{Be}}$} & Mar. & air & 30.9 & 28.6 & 1.08 \\
\hline & Sept. & (Bq/filter) & 321 & 308 & 1.04 \\
\hline \multirow[t]{2}{*}{${ }^{54} \mathrm{Mn}$} & Mar. & & 6.33 & 5.97 & 1.06 \\
\hline & Sept. & & 25.7 & 25.9 & 0.99 \\
\hline \multirow[t]{2}{*}{${ }^{57} \mathrm{Co}$} & Mar. & & 7.89 & 7.93 & 0.99 \\
\hline & Sept. & & 6.03 & 6.40 & 0.94 \\
\hline \multirow[t]{2}{*}{${ }^{60} \mathrm{Co}$} & Mar. & & 5.83 & 5.81 & 1.00 \\
\hline & Sept. & & 3.25 & 3.06 & 1.06 \\
\hline \multirow[t]{2}{*}{${ }^{134} C_{8}$} & Mar. & & 5.09 & 4.44 & 1.15 \\
\hline & Sept. & & 3.73 & 3.72 & 1.00 \\
\hline \multirow[t]{2}{*}{${ }^{137} \mathrm{Cs}$} & Mar. & & 6.26 & 5.76 & 1.09 \\
\hline & Sept. & & 5.94 & 5.82 & 1.02 \\
\hline \multirow[t]{2}{*}{${ }^{144} \mathrm{Ce}$} & Mar. & & 74.1 & 63.9 & 1.16 \\
\hline & Sept. & & 38.1 & 43.3 & 0.88 \\
\hline \multirow[t]{3}{*}{${ }^{238} \mathrm{Pu}$} & Mar. & & 0.309 & 0.270 & 1.14 \\
\hline & Sept. & & 0.426 & 0.0420 & $10.14^{b}$ \\
\hline & Sept. & & $0.0364^{c}$ & 0.0420 & $0.87^{c}$ \\
\hline \multirow[t]{3}{*}{${ }^{239} \mathrm{Pu}$} & Mar. & & 0.318 & 0.285 & 1.12 \\
\hline & Sept. & & 0.148 & 0.0450 & $3.29^{b}$ \\
\hline & Sept. & & $0.0430^{c}$ & 0.0450 & $0.96^{\mathrm{C}}$ \\
\hline${ }^{238} U$ & Mar. & & 0.208 & 0.200 & 1.04 \\
\hline \multirow[t]{2}{*}{${ }^{40} K$} & Mar. & soil & 704 & 719 & 0.98 \\
\hline & Sept. & $(\mathrm{Bq} / \mathrm{kg})$ & 380 & 384 & 0.99 \\
\hline \multirow[t]{2}{*}{${ }^{137} \mathrm{Cs}$} & Mar. & & 5.96 & 5.23 & 1.14 \\
\hline & Sept. & & 312 & 285 & 1.09 \\
\hline \multirow[t]{3}{*}{${ }^{239} \mathrm{Pu}$} & Mar. & & 50 & 25.5 & $1.96^{b}$ \\
\hline & Sept. & & 1.99 & 7.76 & $0.26^{b}$ \\
\hline & Sept. & & $6.86^{c}$ & 7.76 & $0.88^{c}$ \\
\hline \multirow[t]{3}{*}{${ }^{3} \mathbf{H}$} & Mar. & water & 156 & 227 & $0.69^{b}$ \\
\hline & Sept. & $(B q / L)$ & 158 & 118 & $1.34^{b}$ \\
\hline & Sept. & & $135^{d}$ & 118 & $1.14^{\mathrm{d}}$ \\
\hline \multirow[t]{2}{*}{${ }^{137} \mathrm{Cs}$} & Mar. & & 86.2 & 84.6 & 1.02 \\
\hline & Sept. & & 31.2 & 29.0 & 1.08 \\
\hline \multirow[t]{3}{*}{${ }^{239} \mathrm{Pu}$} & Mar. & & 0.666 & 0.580 & 1.15 \\
\hline & Sept. & & 2.01 & 0.238 & $8.45^{b}$ \\
\hline & Sept. & & $0.245^{\mathrm{C}}$ & 0.238 & $1.03^{C}$ \\
\hline
\end{tabular}

a All data were provided by LLNL's Radiation Analytical Sciences Section.

b These values are outside the required tolerance levels of $\pm 2 \sigma$.

c Recalculated LLNL results and ratios. Original results as published by EML were incorrect due to labeling errors. Corrected results and ratios are shown as second set of September data.

d Recalculated LLNL results and ratio. Original results as published by EML were incorrect due to calculation errors. Corrected results and ratios are shown as second set of September data. 
Table 13-6. Quality assurance duplicate sampling, selected parameters, 1992.a

\begin{tabular}{|c|c|c|c|c|c|c|}
\hline Media & Parameter & $\begin{array}{c}\text { Precision } \\
(\%)\end{array}$ & $\begin{array}{l}\text { Coefficient of } \\
\text { determination }\end{array}$ & Intercept & Slope & $\begin{array}{l}\text { Mean } \\
\text { ratioc }\end{array}$ \\
\hline \multicolumn{7}{|l|}{ Sewage } \\
\hline & ${ }^{3} \mathrm{H}(\mu \mathrm{Ci} / \mathrm{mL})$ & -136 to 221 & 0.999 & $2.9 \times 10^{-8}$ & 0.93 & \\
\hline & Zinc (mgL) & -4 to 4 & 0.95 & 1.3 & 0.95 & \\
\hline \multicolumn{7}{|l|}{ Water } \\
\hline \multirow[t]{4}{*}{ Ground } & ${ }^{3} H(p C i / L)$ & $\begin{array}{c}-1404 \text { to } 2231 \\
(-23 \text { to } 20)^{d}\end{array}$ & 0.99 & -3526 & 1.06 & \\
\hline & $\mathrm{TOC}(\mathrm{mg} / \mathrm{L})$ & -34 to 34 & 0.86 & -0.13 & 1.15 & \\
\hline & $\begin{array}{l}\text { Conductivity } \\
(\mu \mathrm{mhos} / \mathrm{cm})\end{array}$ & -9109 & 0.98 & 20.3 & 0.98 & \\
\hline & As (mg/L) & -16 to 10 & 0.98 & -0.0009 & 1.02 & \\
\hline Surface & ${ }^{3} \mathrm{H}(\mathrm{pCi} / \mathrm{L})$ & -32 to 29 & 0.999 & -4.01 & 1.16 & \\
\hline Rain & ${ }^{3} \mathrm{H}(\mathrm{pCi} / \mathrm{L})$ & -87 to 120 & 0.98 & -2.59 & 0.97 & \\
\hline Runoff & ${ }^{3} \mathrm{H}(\mathrm{pCi} / \mathrm{L})$ & -62 to 72 & 0.99 & 0.93 & 0.94 & \\
\hline \multicolumn{7}{|l|}{ Vegetation } \\
\hline & ${ }^{3} \mathrm{H}(\mathrm{pCi} / \mathrm{L})$ & -72 to 168 & 0.99 & 41.0 & 0.96 & \\
\hline \multicolumn{7}{|l|}{ Air } \\
\hline & ${ }^{3} \mathrm{H}(\mathrm{pCi} / \mathrm{L})$ & -183 to 272 & 0.66 & 0.002 & 1.09 & \\
\hline & $\begin{array}{l}{ }^{239} \mathrm{Pu} \\
(\mu \mathrm{Ci} / \mathrm{mL})\end{array}$ & -94 to 29 & 0.09 & $1.65 \times 10^{-19}$ & 0.46 & \\
\hline & $\begin{array}{l}\text { Beryllium } \\
\text { (pG/m) }\end{array}$ & -80 to 99 & 0.74 & 1.89 & 0.89 & \\
\hline \multicolumn{7}{|l|}{ Soll } \\
\hline & ${ }^{239} \mathrm{Pu}(\mu \mathrm{Ci} / \mathrm{g})$ & & & & & 0.66 \\
\hline & ${ }^{228} \mathrm{Ra}(\mathrm{pCi} / \mathrm{g})$ & & & & & 0.98 \\
\hline
\end{tabular}

a Only calculated for data sets with more than eight valid data pairs.

b Calculated according the methodology in Quality Assurance Handbook for Air Pollution Measurement Systems (U.S. Environmental Protection Agency 1979) for data sets with more than eight valid data pairs.

c Only used for data sets with fewer than eight valid data pairs.

d Indicates result with a single highly influentlal data pair removed. 
American Society of Mechanical Engineers (ASME) (1989), Quality Assurance Program Requirements for Nuclear Facilities, American Society of Engineers, New York, NY (ANSI / ASME NQA-1-1989).

Anderson, K. L., and P. V. Post (1988), Community Relations Plan for the Lawrence Livermore National Laboratory Superfund Site Located in Alameda County, California, Lawrence Livermore National Laboratory, Livermore, CA (UCAR-10249).

Biermann, A. H., and K. C. Lamson (1992a), LLNL NESHAPs Project Quarterly Progress Report, June 30, 1992, Lawrence Livermore National Laboratory, Livermore, CA (UCRL-AR-108419-92-2).

Biermann, A. H., and K. C. Lamson (1992b), LLNL NESHAPs Project Quarterly Progress Report, September 30, 1992, Lawrence Livermore National Laboratory, Livermore, CA (UCRL-AR-108419-92-3).

Biermann, A. H., K. C. Lamson, R. J. Harrach, E. Christofferson, and R. L. Berger (1992), LLNL NESHAPs Project Quarterly Progress Report, December 31, 1992, Lawrence Livermore National Laboratory, Livermore, CA (UCRL-AR108419-92-4).

Biermann, A. H., R. J. Harrach, R. L. Berger, and K. A. Surano (1993), LLNL NESHAPs Project Quarterly Progress Report, March 31, 1993, Lawrence Livermore National Laboratory, Livermore, CA (UCRL-AR-108419-93-1).

Boegel, A. J., M. D. Dresen, E. Folsom, P. Thiry, J. P. Ziagos, L. L. Berg, and J. K. Macdonald, Eds. (1993), Remedial Design Report No. 1 for Treatment Facilities $A$ and $B$, Lawrence Livermore National Laboratory Livermore Site, Lawrence Livermore National Laboratory, Livermore, CA (UCRLAR-110576)

Brekke, D. D., R. C. Holland, M. G. Brown, and T. M. Carlsen (1989), Environmental Monitoring at the Lawrence Livermore National Laboratory, 1988 Annual Report, Lawrence Livermore National Laboratory, Livermore, CA (UCRL-50027-88).

Bryn, S. M., R. K. Landgraf, and S. E. Booth (1990), Draft Remedial Investigation and Feasibility Study for the Lawrence Livermore National Laboratory Site 300 Building 834 Complex, Lawrence Livermore National Laboratory, Livermore, CA (UCRL-ID-103963 Vols. 1 and 2 DR).

Buddemeier, R. W., D. H. Armstrong, and M. G. Brown (1987), LLNL Site 300 Groundivater Monitoring Program Quarterly Report, April-June 1987, Lawrence Livermore National Laboratory, Livermore, CA (UCAR10191-87-2). 
California Department of Health Services (DHS) (1981), Interim Status Document, CA 2890090002, effective March 30, 1981, for Lawrence Livermore National Laboratory Site 300, Corral Hollow Road, Alameda County, Livermore, CA 94550, operated by Regents of the University of California, P.O. Box 808, L-520, Livermore, CA 94550.

California Regional Water Quality Control Board, San Francisco Bay Region (1991), Water Quality Control Plan, San Francisco Bay Basin Region (2), State of California, Environmental Protection Agency.

Carlsen, T. M. (1991a), LLNL Site 300 Environmental Investigations Quarterly, January-March 1991, Lawrence Livermore National Laboratory, Livermore, CA (UCAR-10194-91-1).

Carlsen, T. M. (1991b), LLNL Site 300 Environmental Investigations Quarterly, AprilJune 1991, Lawrence Livermore National Laboratory, Livermore, CA (UCAR-10194-91-2).

Carlsen, T. M., M. G. Brown, and D. H. Armstrong (1987), LLNL Site 300 Groundwater Monitoring Program Quarterly Report, October-December 1987, Lawrence Livermore National Laboratory, Livermore, CA (UCAR10191-87-4).

Carpenter, D. W., J. J. Sweeney, P. W. Kasameyer, N. R. Burkhard, K. G. Knauss, and R. J. Shelmon (1984), Geology of the Lawrence Livermore National Laboratory Site and Adjacent Areas, Lawrence Livermore National Laboratory, Livermore, CA (UCRL-53316).

Carpenter, D. W., J. R. Copland, A. L. Lamarre, R. S. Mateik, M. J. Taffet, and W. M. Wade (1991), Investigation of Holocene Faulting Near Decommissioned Landfill Pit 6, Lawrence Livermore National Laboratory Site 300, Lawrence Livermore National Laboratory, Livermore, CA (UCRL-ID-106316).

Crow, N. B., and A. L. Lamarre (1990), Remedial Investigation of the High-Explosives (HE) Process Areas, LLNL Site 300, Lawrence Livermore National Laboratory, Livermore, CA (UCID-21920).

Dresen, M. D., E. M. Nichols, R. O. Devany, D. Rice, Jr., F. Yukic, G. Howard, P. Cederwall, B. Qualheim, R. S. Lawson, and W. Isherwood (1989a), LLNL Ground Water Project, Monthly Progress Report, January 1989, Lawrence Livermore National Laboratory, Livermore, CA (UCAR-10160-89-2).

Dresen, M. D., R. O. Devany, F. A. Yukic, D. Rice, Jr., G. Howard, P. Cederwall, R. S. Lawson, P. Post, and W. Isherwood (1989b), LLNL Ground Water Project, Monthly Progress Report, February 1989, Lawrence Livermore National Laboratory, Livermore, CA (UCAR-10160-89-3).

Dresen, M. D., R. S. Lawson, J. L. Iovenitti, R. O. Devany, P. D. Weiler, D. W. Rice, Jr., P. Cederwall, A. J. Boegel, and W. F. Isherwood (1989c), LLNL Ground Water Project, Monthly Progress Report, May 1989, Lawrence Livermore National Laboratory, Livermore, CA (UCAR-10160-89-6). 
Dresen, M. D., R. S. Lawson, P. F. McKereghan, R. O. Devany, P. D. Weiler, P. Post, J. L. Iovenitti, D. W. Rice, Jr., P. Cederwall, A. J. Boegel, and W. F. Isherwond (1989d), LLNL Ground Water Project, Monthly Progress Report, June 1989, Lawrence Livermore National Laboratory, Livermore, CA (UCAR-10160-89-7).

Dresen, M. D., R. S. Lawson, D. W. Rice, Jr., P. Cederwall, A. J. Boegel, and W. F. Isherwood (1989e), LLNL Ground Water Project, Monthly Progress Report, July 1989, Lawrence Livermore National Laboratory, Livermore, CA (UCAR-10160-89-8).

Dresen, M. D., R. O. Devany, D. W. Rice, Jr., P. D. Weiler, R. S. Lawson, D. Layton, E. M. Nichols, P. Cederwall, G. Howard, and W. F. Isherwood (1989f), LLNL Ground Water Project 1988 Annual Report, Lawrence Livermore National Laboratory, Livermore, CA (UCAR-10160-89-1).

Dresen, M. D., W. F. Isherwood, and J. P. Ziagos (1991), Proposed Remedial Action Plan for the Lawrence Livermore National Laboratory, Livermore Site, Livermore, CA, Lawrence Livermore National Laboratory, Livermore, CA (UCRL-AR-105577).

Dresen, M. D., J. P. Ziagos, A. J. Boegel, and E. M. Nicols (1992), Draft Remedial Action Implementation Plan for the LLNL Livermore Site, Livermore, California, Lawrence Livermore National Laboratory, Livermore, CA (UCRL-AR-110532 DR).

Federal Register (1977), "Notice of Proposed Federal Radiation Protection Guidance," Vol. 42, No. 230, pp. 60956-9 (November 30, 1977).

Federal Register (1990), "National Environmental Policy Act Implementing Procedures," Vol. 55, No. 213, p. 46444 (November 2, 1990).

Ferry, R. A., A. L. Lamarre, and R. K. Landgraf (1990), Draft Feasibility Study of the General Services Area (GSA), LLNL Site 300, December 1990, Lawrence Livermore National Laboratory, Livermore, CA (UCRL-AR-104510 DR).

Fischer, K.G., W.G. Hoppes, and D.H. MacQueen (1992a), LLNL Site 300 Groundwater Monitoring Program Quarterly Report, October-December 1991, Lawrence Livermore National Laboratory, Livermore, CA (UCAR10191-91-4).

Fischer, K.G., W.G. Hoppes, and D.H. MacQueen (1992b), LLNL Site 300 Groundwater Monitoring Program Quarterly Report, January-March 1992, Lawrence Livermore National Laboratory, Livermore, CA (UCAR10191-92-1).

Fischer, K.G., W.G. Hoppes, and D.H. MacQueen (1992c), LLNL Site 300 Groundwater Monitoring Program Quarterly Report, April-June 1992, Lawrence Livermore National Laboratory, Livermore, CA (UCAR10191-92-2).

Fischer, K.G., W.G. Hoppes, and D.H. MacQueen (1992d), LLNL Site 300 Groundwater Monitoring Program Quarterly Report, July-September 1992, Lawrence Livermore National Laboratory, Livermore, CA (UCAR 10191-92-3). 
Fisher, J. C., Jr. (1976), Calibration of Anderson-Braun Remmeters with Track Etch Detectors, Hazards Control Progress Report No. 52, Lawrence Livermore National Laboratory, Livermore, CA (UCRL-50007-76-1).

Gallegos, G. M., B. K. Balke, K. A. Surano, W. G. Hoppes, P. J. Tate, J. C. Steenhoven, B. C. Fields, L. M. Garcia, and K. C. Lamson (1992a), Environmental Report for 1991, Lawrence Livermore National Laboratory, Livermore, CA (UCRL-50027-91).

Gallegos, G., K. Surano, K. Lamson, P. Tate, B. Balke, A. Biermann, W. Hoppes, B. Fields, F. Gouveia, R. Berger, F. Miller, D. Rueppel, and J. Sims (1992b), Environmental Monitoring Plan, Lawrence Livermore National Laboratory, Livermore, CA (UCRL-ID-106132).

Garrison, J., S. MacLean, R. Bocanegra, and R. W. Buddemeier (1985), Radiochemical Methods of the Site Environmental Monitoring Program, Lawrence Livermore National Laboratory, Livermore, CA (M-122, Rev. 1).

Golder Associates, Inc. (1993), Construction Quality Assurance Closure Report, Lawrence Livermore National Laboratory Site 300 Pits 1 and 7, Lawrence Livermore National Laboratory, Livermore, CA (UCRL-CR-112802 Vols. I and II).

Grandfield, C. H. (1989), Guidelines for Discharges to the Sanitary-Sewer System, Lawrence Livermore National Laboratory, Livermore, CA (UCAR-10235).

Grayson, A. R. (1992), Wastewater Discharge Permit Application 1992-1993, Lawrence Livermore National Laboratory, Livermore, CA (UCRL-AR106905-92).

Grayson, A. R., and S.L. Brigdon (1992), Wastewater Point-Source Monitoring at LLNL, Livermore Site Semiannual Report June-November 1991, Lawrence Livermore National Laboratory, Livermore, CA (UCAR-10204-91-2).

Griggs, K. S., D. S. Myers, and R. W. Buddemeier (1984), Environmental Monitoring at the Lawrence Livermore National Laboratory 1983 Annual Report Lawrence Livermore National Laboratory, Livermore, CA (UCRL50027-83).

Gudiksen, P. H., C. L. Lindeken, J. W. Meadows, and K. O. Hamby (1973), Environmental Levels of Radioactivity in the Vicinity of the Lawrence Livermore Laboratory, 1972 Annual Report, Lawrence Livermore National Laboratory, Livermore, CA (UCRL-51333).

Holland, R. C. (1987), Environmental Quality Verification Group Quality Assurance Plan, Lawrence Livermore National Laboratory, Livermore, CA (UCAR-10203).

Holland, R. C., and D. D. Brekke (1988), Environmental Monitoring at the Lawrence Livermore National Laboratory, 1987 Annual Report, Lawrence Livermore National Laboratory, Livermore, CA (UCRL-50027-87).

Hoppes, W.G., D.H. MacQueen, and K.G. Fischer, Draft Report of Waste Discharge, LLNL Site 300, Lawrence Livermore National Laboratory, Livermore, CA (UCRL-AR-110071 DR). 
Intersociety Committee for a Manual of Methods (ICMM) for Ambient Air Sampling and Analysis (1972), Methods of Air Sampling and Analysis, American Public Health Association, Washington, DC.

International Commission on Radiological Protection (ICRP) (1977), Recommendations of the International Commission on Radiological Protection, Publication 26 (Pergamon Press, New York, NY).

International Commission on Radiological Protection (ICRP) (1980), Limits for Intakes of Radionuclides by Workers, Publication 30 (Pergamon Press, New York, NY).

Iovenitti, J. L., J. K. Macdonald, M. D. Dresen, W. F. Isherwood, and J. P. Ziagos (1991), Possible Sources of VOCs in the Vasco Road-Patterson Pass Road Area, Livermore, California, Lawrence Livermore National Laboratory, CA (UCRL-AR-106898).

Isherwood, W. F., C. H. Hall, M. D. Dresen, and A. J. Boegel (1991), CERCLA Feasibility Study Report for the LLNL Livermore Site, Lawrence Livermore National Laboratory, Livermore, CA (UCRL-AR-104040).

Lamarre, A. L., Ed. (1989a), LLNL Site 300 Environmental Investigations Quarterly, July-September 1989, Lawrence Livermore National Laboratory, Livermore, CA (UCAR-10194-89-3).

Lamarre, A. L., Ed. (1989b), LLNL Site 300 Environmental Investigations Quarterly, October-December 1989, Lawrence Livermore National Laboratory, Livermore, CA (UCAR-10194-89-4).

Lamarre, A. L. (1989c), Lawrence Livermore National Laboratory Site 300 Environmental Restoration Work Plan, Lawrence Livermore National Laboratory, Livermore, CA (UCAR-10247, Rev. 1).

Lamson, K. (1992), LLNL NESHAPs Project Quarterly Progress Report, March 31, 1992, Lawrence Livermore National Laboratory, Livermore, CA (UCRLAR-108419-92-1).

Lawrence Livermore National Laboratory (1991), Essential Features of the LLNL Environmental, Safety, \& Health Program, Lawrence Livermore National Laboratory, Livermore, CA (UCRL-AR-104719).

Lindeken, C. L., R. O. Morgin, and K. F. Petrock (1963), “Collection Efficiency of Whatman-41 Filter Paper for Submicron Aerosols," Health Physics 9, 305-308.

Lindeken, C. L., P. H. Gudiksen, J. W. Meadows, K. O. Hamby, and L. R. Anspaugh (1973), Environmental Levels of Radioactivity in Livermore Valley Soils, Lawrence Livermore National Laboratory, Livermore, CA (UCRL-74424).

Lowder, W. M., and H. L. Beck (1966), "Cosmic Ray Ionization in the Lower Atmosphere," Journal of Geophysical Research 71, 4661-4668.

Macdonald, J. K., R. W. Bainer, E. M. Nichols, M. D. Dresen, J. P. Ziagos, and R. G. Blake (1993), LLNL Ground Water Project Monthly Progress Report, November 1992, Lawrence Livermore National Laboratory, Livermore, CA (UCAR-10160-92-11). 
Mangarella, P. (1992), Source Identification and Control, Santa Clara Valley Nonpoint Source Pollution Control Program, Woodward-Clyde Consultants, presentation to meeting of American Public Works Association and California State Water Resources Control Board, Sacramento, CA, February 12, 1993.

Marshack, J. D. (1986), Designated Levels in a Solid for the Protection of Ground Water Quality, Central Valley Regional Water Quality Control Board, Sacramento, CA.

Mcllvride, W. A., R. A. Ferry, S. P. Vonder Haar, W. M. Wade, and L. L. Glick (1990), Remedial Investigation of the General Services Area (GSA), Lawrence Livermore National Laboratory Site 300, May 1990, Lawrence Livermore National Laboratory, Livermore, CA (UCRL-AR-103161).

National Council on Radiation Protection and Measurements (NCRP) (1976a), Environmental Radiation Measurements, Report No. 50, National Council on Radiation Protection and Measurements, Washington, DC.

National Council on Radiation Protection and Measurements (NCRP) (1976b), Natural Background Radiation in the United States, Report No. 45, National Council on Radiation Protection and Measurements, Washington, DC.

National Council on Radiation Protection and Measurements (NCRP) (1987), Ionizing Radiation Exposure of the Population of the United States, Report No. 93, National Council on Radiation Protection and Measurements, Washington, DC.

Parks, B. S. (1992), User's Guide for CAP88-PC, Version 1.0, U.S. Environmental Protection Agency, Office of Radiation Programs, Las Vegas, NV (EPA 402-B-92-001).

Peterson, K. R., T. W. Crawford, and L. A. Lawson (1976), CPS: A ContinuousPoint-Source Computer Code for Plume Dispersion and Deposition Calculations, Lawrence Livermore National Laboratory, Livermore, CA (UCRL-52049).

Rice, D. W., Jr. (1989) Quality Assurance Project Plan, LLNL Ground Water Project, Lawrence Livermore National Laboratory, Livermore, CA (UCAR-10219 Rev. 1.2).

Shinn, J. H., R. T. Cederwall, K. C. Lamson, and C. S. Mitchell (1989), Beryllium Dispersion Near Explosive Firing Tables: A Comparison of Computed and Observed Results, Lawrence Livermore National Laboratory, Livermore, CA (UCID-21682).

Shleien, B., and M. S. Terpilak (1984), The Health Physics and Radiological Health Handbook (Nucleon Lectern Associates, Inc., Olney, MD).

Silver, W. J., C. L. Lindeken, J. W. Meadows, W. H. Hutchin, and D. R. McIntyre (1974), Environmental Levels of Radioactivity in the Vicinity of the Lawrence Livermore Laboratory, 1973 Annual Report, Lawrence Livermore National Laboratory, Livermore, CA (UCRL-51547).

Sims, J. M., K. A. Surano, K. C. Lamson, and M. G. Brown (1990), Environmental Report for 1989, Lawrence Livermore National Laboratory, Livermore, CA (UCRL-50027-89). 
Sims, J. M., K. A. Surano, K. C. Lamson, B. K. Balke, and J. C. Steenhoven (1991), Environmental Report for 1990, Lawrence Livermore National Laboratory, Livermore, CA (UCRL-50027-90).

Stone, R., and M. R. Ruggieri (1983), Ground-Water Quality and Movement at Lawrence Livermore National Laboratory, Lawrence Livermore National Laboratory, Livermore, CA (UCRL-53474).

Surano, K. A., G. B. Hudson, R. A. Failor, J. M. Sims, R. C. Holland, S. C. MacLean, and J. C. Garrison (1991), Helium-3 Mass Spectrometry for Low-Level Tritium Analysis of Environmental Samples, prepared for the American Nuclear Society Second International Topical Conference; Methods and Applications of Radioanalytical Chemistry, Kona, Hawaii, April 1991, Lawrence Livermore National Laboratory, Livermore, CA (UCRL-JC-107042).

Surano, K. A., R. A. Failor, A. H. Biermann, R. L. Berger, and R. J. Harrach (1993), LLNL NESHAPs Project 1992 Annual Report, Lawrence Livermore National Laboratory, Livermore, CA (UCRL-ID-113867-93).

Taffet, M. J. (1990), Draft Remedial Investigation of Landfill Pit 6, Lawrence Livermore National Laboratory Site 300, Lawrence Livermore National Laboratory, Livermore, CA (UCRL-ID-103961 DR).

Taffet, M. J., A. L. Lamarre, and W. A. Mcllvride (1989a), LLNL Site 300 Environmental Investigations Quarterly, January-March 1989, Lawrence Livermore National Laboratory, Livermore, CA (UCAR-10194-89-1).

Taffet, M. J., J. A. Oberdorfer, and W. A. Mcllvride (1989b), Remedial Investigation and Feasibility Study for the Lawrence Livermore National Laboratory Site 300 Pit 7 Complex, Lawrence Livermore National Laboratory, Livermore, CA (UCID-21685,

Taffet, M. J., J. R. Copland, and R. A. Ferry (1991), Draft Feasibility Study for Landfill Pit 6, Lawrence Livermore National Laboratory Site 300, Lawrence Livermore National Laboratory, Livermore, CA (UCRL-AR-106307 DR).

Taffet, M. J., and J. A. Oberdorfer (1991), Draft Feasibility Study of the Building 850/East Firing Area, LLNL Site 300, Lawrence Livermore National Laboratory, Livermore, CA (UCRL-AR-107033 DR).

Taffet, M. J., J. A. Oberdorfer, T. M. Carlsen, W. R. Dugan, and R. S. Mateik (1992), Draft Remedial Investigation of the Building 850/East Firing Area, Lawrence Livermore National Laboratory Site 300, Lawrence Livermore National Laboratory, Livermore, CA (UCRL-ID-104335, Volumes 1, 2, and $3 \mathrm{DR})$.

Thorpe, R. K., W. F. Isherwood, M. D. Dresen, and C. P. Webster-Scholten (1990), CERCLA Remedial Investigation Report for the LLNL Livermore Site, Lawrence Livermore National Laboratory, Livermore, CA (UCAR-10299, Volumes 1 through 5).

U.N. Environment Programme (1985), Radiation: Doses, Effects, Risks, U.N. Environment Programme, Nairobi, Kenya (Sales No. E.86.III.D.4). 
U.S. Department of Energy (1988), External Dose-Rate Conversion Factors for Calculation of Dose to the Public, U.S. Department of Energy, Washington, DC (DOE/EH-0070).

U.S. Department of Energy (1989), Federal Facility Agreement under CERCLA Section 120, U.S. Environmental Protection Agency, California Department of Health Services, and California Regional Water Quality Control Board.

U.S. Department of Energy (1990), Environment, Safety, and Health, Tiger Team Assessment of the Lawrence Livermore National Laboratory, U.S. Department of Energy, Washington, DC (DOE/EH-0142).

U.S. Department of Energy (1991), Environmental Regulatory Guide for Radiological Effluent Monitoring and Environmental Surveillance, U.S. Department of Energy, Washington, DC (DOE/EH-0173T).

U.S. Department of Energy and University of California (1992a), Draft Environmental Impact Statement and Environmental Impact Report Executive Summary, Lawrence Livermore National Laboratory, Livermore, CA (DOE/EIS-0157, SCH90030847, EXE SUM, Vols. I-III).

U.S. Department of Energy and University of California (1992b), Final Environmental Impact Statement and Environmental Impact Report Executive Summary, Lawrence Livermore National Laboratory, Livermore, CA (DOE/EIS-0157 EXE SUM, SCH90030847, Vols.1-V).

U.S. Environmental Protection Agency (1977), Dose Limits for Persons Exposed to Transuranium Elements in the General Environment, U.S. Environmental Protection Agency (Technical Report 520/4-77-016).

U.S. Environmental Protection Agency (1978), Response to Comments: Guidance on Dose Limits for Persons Exposed to Transuranium Elements in the General Environment, U.S. Environmental Protection Agency (Technical Report 520/4-78-010).

U.S. Environmental Protection Agency (1979), Quality Assurance Handbook for Air Pollution Measurement Systems, U.S. Environmental Protection Agency, Office of Research and Development, Quality Assurance Division, Environmental Monitoring Systems Laboratory, Research Triangle Park, NC (EPA-60019-76-005).

U.S. Environmental Protection Agency and U.S. Department of Health and Human Services (1986), A Citizen's Guide to Radon: What It Is and What To Do About It, U.S. Environmental Protection Agency, Office of Air Public Awareness, Washington, DC (Pamphlet Number OPA 86-004).

U.S. Nuclear Regulatory Commission (1977), Calculation of Annual Doses to Man from Routine Releases of Reactor Effluent for the Purpose of Evaluation Compliance with 10 Code of Federal Regulations, Part 50, Appendix 1, Washington, DC (Regulatory Guide 1.109).

Weast, R.C., ed. (1982), Handbook of Chemistry and Physics, CRC Press, Inc., Boca Raton, FL. 
Webster-Scholten, C. P., and C. H. Hall (1988), Work Plan, Lawrence Livermore National Laboratory, Livermore Site: CERCLA/SARA Remedial Investigations/ Feasibility Studies, Lawrence Livermore National Laboratory, Livermore, CA (UCAR-10225).

Webster-Scholten, C. P., T. M. Carlsen, J. R. Copland, R. A. Ferry, S. P. Vonder Haar, and W.M. Wade (1991), Draft Remedial Investigation of the Building 833 Area, Lawrence Livermore National Laboratory Site 300, Lawrence Livermore National Laboratory, Livermore, CA (UCRL-AR107377 DR).

Webster-Scholten, C. P., ed. (1993), Draft Site-Wide Remedial Investigation Report, LLNL Site 300, Lawrence Livermore National Laboratory, Livermore, CA (UCRL-AR-108131 DR).

Wilber, C.G. (1980), Beryllium-A Potential Environmental Contaminant, Charles C. Thomas, Springfield, IL.

William Self Associates, Documentation and Assessment of the History of the LLNL Livermore Facility, and Site CA-SJO-173H, the Carnegie Town Site at LLNL Site 300, Alameda and San Joaquin Counties, CA, Lawrence Livermore National Laboratory, Livermore, CA (UCRL-CR-110610).

Ziagos, J. P., (1992), Record of Decision for the Lawrence Livermore National Laboratory Livermore Site, Lawrence Livermore National Laboratory, Livermore, CA (UCRL-AR-109105). 
A Absorbed dose

Absorption

Accuracy

ACEHS

ACG

Action Level

ALARA

Alluvium

Alpha particle

Ambient air

Analyte

Anion
The amount of energy deposited by radiation in a given amount of material. The unit of absorbed dose is the rad.

The process by which the number and energy of particles or photons entering a body of matter is reduced by interaction with the matter.

The closeness of the result of a measurement to the true value of the quantity measured.

Alameda County Environmental Health Services.

Ambient concentration guide.

Defined by regulatory agencies, it is the level of pollutants which, if exceeded, requires regulatory action.

As Low As Reasonably Achievable. A U.S. Department of Energy (DOE) and Nuclear Regulatory Commission policy for radiation exposures. It is an approach to radiation exposure control or management where the exposures and resulting doses are maintained as far below the limit specified for the appropriate circumstances as social, economic, technical, and practical considerations permit.

Sediment deposited by flowing water.

A positively charged particle emitted from the nucleus of an atom. It has a mass and charge equal to those of a helium nucleus (two protons and two neutrons).

The surrounding atmosphere, usually the outside air, as it exists around people, plants, and structures. It is not considered to include the air immediately adjacent to emission sources.

A constituent that is being analyzed.

A negatively charged ion. 
ANOVA Analysis of variance. A test of whether two or more sample means could have been obtained from the same statistical population.

ANSI

Aquifer

Aquitard

ASME

ATA

Atom

Atomic absorption spectroscopy

AVLIS

B BAAQMD

BAT

Beta particle

Blank sample
American National Standards Institute.

A saturated layer of rock or soil below the ground surface that can supply usable quantities of ground water to wells and springs. Aquifers can be a source of water for domestic, agricultural, and industrial uses.

A geologic unit that functions as an upper or lower boundary of an aquifer.

American Society of Mechanical Engineers.

Advanced Test Accelerator.

The smallest particle of an element capable of entering into a chemical reaction.

Chemical analysis performed by vaporizing a sample and measuring the absorbance of light by the vapor. Abbreviated AA.

Atomic Vapor Laser Isotope Separation.

Bay Area Air Quality Management District. The local agency responsible for regulating stationary air emission sources (including the Livermore site) in the San Francisco Bay Area.

Best available technology (economically achievable).

A negatively charged particle emitted from the nucleus of an atom. It has a mass and charge equal to those of an electron.

A control sample that is identical, in principle, to the sample of interest, except that the substance being analyzed is absent. The measured value or signals in blanks for the substance being analyzed is believed to be due to artifacts and should be subtracted from the measured value to give a net result reflecting the amount of substance in the sample of interest. 
Blind sample A control sample of known concentration in which the expected values of the constituent are unknown to the analyst.

Biota

The animal and plant life of a particular region considered as a total ecological entity.

BOD Biochemical (biological) oxygen demand. A measure of the amount of oxygen in biological processes that break down organic matter in water; a measure of the organic pollutant load. It is used as an indicator of water quality.

$\mathrm{Bq}$

Becquerel. The SI unit of activity of a radionuclide, equal to the activity of a radionuclide having one spontaneous nuclear transition per second.

C CAP88

Computer code required by the EPA for modeling air emissions.

Cation

A positively charged ion.

CCR

California Code of Regulations.

CEQA

California Environmental Quality Act of 1970. CEQA requires that all California state, local, and regional agencies document, consider, and disclose to the public the environmental implications of their actions. CEQA also requires that adverse environmental impacts be mitigated through mitigation measures or project alternatives.

CERCLA Comprehensive Environmental Response, Compensation and Liability Act of 1980. Administered by EPA, this program, also known as Superfund, requires private parties to notify the EPA after the release of hazardous substances and undertake short-term removal and longterm remediation. If conditions exist that could create the threat of hazardous substances being released, the Act also requires the remediation of those conditions. In 1986, the Superfund Amendments and Reauthorization Act (SARA) was enacted, which amended and reauthorized CERCLA for five years at a total funding level of $\$ 8.5$ billion.

CFR Code of Federal Regulations. A codification of all regulations promulgated by federal government agencies. 
Chain-of-custody A method for documenting the history and possession of a sample from the time of its collection, through its analysis and data reporting, to its final disposition.

Chlorocarbon

A compound of carbon and chlorine, or carbon, hydrogen, and chlorine, such as carbon tetrachloride, chloroform, and tetrachloroethylene.

COD

Chemical oxygen demand. A gross analytical test for the presence of organic compounds in water.

Collective dose equivalent

The sums of the dose equivalents of all individuals in an exposed population within a certain radius, and expressed in units of person-rem (or person-sievert).

Collective

effective dose equivalent

The sums of the effective dose equivalents of all individuals in an exposed population within a certain radius, and expressed in units of person-rem (or person-sievert).

Committed dose equivalent

The predicted total dose equivalent to a tissue or organ over a 50-year period after known intake of a radionuclide into the body. It does not include contributions from external dose. Committed dose equivalent is expressed in units of sievert (or rem).

Committed effective dose equivalent

The sum of the committed dose equivalents to various tissues, each multiplied by the appropriate weighting factor. Committed effective dose equivalent is expressed in units of sievert (or rem).

Cosmic radiation Radiation with very high energies, originating outside the earth's atmosphere. Cosmic radiation is one source contributing to natural background radiation.

CRP

Community Relations Plan.

CRWQCB California Regional Water Quality Control Board.

CRWQCBCVR. California Regional Water Quality Control Board, Central Valley Region.

Curie (Ci)

A unit of measurement of radioactivity, defined as the amount of radionuclide in which the decay rate is $2.22 \times$ $10^{12}$ disintegrations per minute $\left(3.7 \times 10^{10}\right.$ disintegrations per second), which is approximately equal to the decay rate of one gram of pure radium 
D Daughter nuclide A nuclide formed by the radioactive decay of another nuclide, which is called the parent.

DCG

Derived Concentration Guide. Concentrations of radionuclides in water and air that could be continuously consumed or inhaled (365 days/y) and not exceed the DOE primary radiation protection standard to the public (100 mrem/y effective dose equivalent).

DCL

Discharge Concentration Limit (City of Livermore Ordinance 13.32).

Disintegration (nuclear)

DOE

Dose

Dose commitment

Dose equivalent

Dosimeter

Dosimetry

DTSC
A spontaneous nuclear transformation (radioactivity) characterized by the emission of energy and/or mass from the nucleus of an atom.

U.S. Department of Energy. The federal agency that is responsible for conducting energy research and regulating nuclear materials used for weapons production.

The energy imparted to matter by ionizing radiation. The unit of absorbed dose is the rad, equal to 0.01 joules per kilogram for irradiated material in any medium.

The dose which an organ or tissue would receive during a specified period of time (e.g., 50 or 100 years) as a result of intake of one or more radionuclides from one year's release.

The product of the absorbed dose (rad) in tissue and a quality factor. Dose equivalent is expressed in units of rem (or sievert). The dose equivalent to an organ, tissue, or whole body in a year will be that received from the direct exposure plus the committed dose equivalent received from radionuclides taken into the body during the year.

A portable detection device for measuring the total accumulated exposure to ionizing radiation.

The theory and application of the principles and techniques involved in the measurement and recording of radiation doses. Its practical aspect is concerned with the use of various types of radiation measurement instruments.

California Environmental Protection Agency, Department of Toxic Substances Control. 
DWS Drinking water standard. A standard established by the State of California and found in Title 22 of the California Code of Regulations.

E EA

EDE

EDO

EFA

Effluent

EIR

EIS

EMAD

EML
Environmental Assessment. An environmental review document that identifies environmental impacts from any federally approved or funded project. If an EA shows significant impact, an EIS is required.

Effective dose equivalent. An estimate of the total risk of potential effects from radiation exposure. It is the sum of the committed effective dose equivalent from internal deposition and the effective dose equivalent from external penetrating radiation received during a calendar year. The committed effective dose equivalent is the sum of the individual organ committed dose equivalents multiplied by weighting factors that represent the proportion of the total random risk that each organ would receive from uniform irradiation of the whole body.

Environmental Duty Officer.

East Firing Area (LLNL Site 300).

A liquid or gaseous waste discharged to the environment.

Environmental Impact Report. A detailed report, required by the California Environmental Quality Act, on the environmental impacts from any action carried out, approved, or funded by a California state, regional, or local agency.

Environmental Impact Statement. A detailed report, required by the National Environmental Policy Act, on the environmental impacts from a federally approved or funded project. An EIS must be prepared by a federal agency when a "major" federal action that will have "significant" environmental impacts is planned.

Environmental Monitoring and Analysis Division (LLNL).

U.S. Department of Energy Environmental Measurements Laboratory. 
EMS Environmental Monitoring Section in the Environmental Monitoring and Analysis Division of the Environmental Protection Department (at LLNL).

EPA

Environmental Protection Agency. The federal agency responsible for enforcing federal environmental laws. Although some of this responsibility may be delegated to state and local regulatory agencies, EPA retains oversight authority to ensure protection of human health and the environment.

EPD Environmental Protection Department (LLNL).

EPSRU Electrical Power System Replacements and Upgrades.

ERD Environmental Restoration Division of the Environmental Protection Department at LLNL.

ES\&H Environmental, Safety, and Health.

ETA Environmental Test Area (at LLNL).

ETA II Experimental Test Accelerator II.

F Federal facility A facility that is owned or operated by the federal government. Federal facilities are subject to the same requirements as other responsible parties once placed on the Superfund National Priorities List.

Federal Register A document published daily by the federal government containing notification of government agency actions. The Federal Register contains notification of EPA and DOE actions, including notification of EPA and DOE decisions concerning permit applications and rulemaking.

FFA Federal Facility Agreement. A negotiated agreement that specifies required actions at a federal facility as agreed upon by various agencies (e.g., EPA, DHS, RWQCB, and DOE).

FFCA Federal Facilities Compliance Agreement.

FHC Fuels and aromatic hydrocarbon.

Fissile Any material fissionable by neutrons of all energies (e.g., 235 $\mathrm{U}$ and ${ }^{239} \mathrm{Pu}$ ). 
Fluvial

FONSI

FS

G $\mathrm{g}$

Gamma ray

Gray $(\mathrm{G})$

GWP

H Half-life

(radiological)
Produced by the action of a stream or river.

Finding of No Significant Impact.

Feasibility Study. A study based on a Remedial Investigation to evaluate and develop remedial action alternatives to prevent, or mitigate, the migration or the release of hazardous substances or contaminants.

Gram, the standard metric measure of weight approximately equal to 0.035 ounce.

High-energy, short-wavelength electromagnetic radiation emitted from the nucleus of an atom. Gamma radiation frequently accompanies the emission of alpha or beta particles. Gamma rays are identical to $x$ rays except for the source of the emission.

The SI unit of measure for absorbed dose. It is the quantity of energy imparted by ionizing radiation to a unit mass of matter such as tissue. One gray corresponds to one joule per kilogram.

Ground Water Project.

The time required for one-half the radioactive atoms in a given amount of material to decay. After one half-life, 50 out of 100 atoms will have changed; during the next halflife, 25 more will decay, and so on, exponentially.

Hazardous waste Wastes exhibiting any of the following characteristics: ignitability, corrosivity, reactivity, or EP-toxicity (yielding toxic constituents in a leaching test). In addition, EPA has listed as hazardous other wastes that do not necessarily exhibit these characteristics. Although the legal definition of hazardous waste is complex, the term more generally refers to any waste that EPA believes could pose a threat to human health and the environment if managed improperly.

HE

High explosives. Materials that release large amounts of energy when detonated.

HLW

High-level radioactive waste.

HMX

Cyclotetramethyltetramine, a high-explosive compound. 
HPLC High-pressure liquid chromatograph. A technique used to separate and quantify chemicals.

HT

Tritiated hydrogen gas. Tritium is the hydrogen isotope with one proton and two neutrons in the nucleus. It emits a low-energy beta particle and has a half-life of 12.3 years.

HTO Tritiated water (and water vapor). (See HT.)

HWCA California Hazardous Waste Control Act. This legislation specifies requirements for the management of hazardous wastes in California.

HWM

Hazardous Waste Management.

HWMD

Hazardous Waste Management Division at LLNL.

Hydraulic

gradient

In an aquifer, the rate of change of total head (water-level elevation) per unit distance of flow at a given point and in a given direction.

Hydrology The science dealing with the properties, distribution, and circulation of natural water systems.

I ICP

Inductively coupled plasma emission spectroscopy-An analytical technique generally used to quantify metals, such as chromium, copper, and zinc.

ICRP International Commission on Radiological Protection. An international organization that studies radiation, including its measurement and effects.

Inorganic Compounds that either do not contain carbon or do not compounds contain hydrogen along with carbon. Inorganic compounds include metals, salts, and various carbon oxides (carbon monoxide, carbon dioxide).

In situ

A term that can be used to refer to the treatment of contaminated areas without excavation or other removal, as in the in situ treatment of soils through biodegradation of contaminants on site. 
Interim status

Ion

Isopleth

Isotopes

J Joule

L L

Land Ban

LEDO

LEL

Less than

detection limits

LLD

LLNL
A legal classification that applies to hazardous waste incinerators or other hazardous waste management facilities that were under construction or in operation by November 19, 1980, and can meet other interim status requirements. Interim status facilities may operate while EPA considers their permit application.

An atom or compound that carries an electrical charge.

A line on a map connecting points at which a given variable has a specified constant value, such as topographic contour lines.

Forms of an element having the same number of protons in their nuclei but differing numbers of neutrons.

The SI unit of work or energy equal to 107 ergs or approximately 0.7375 footpound. (A foot-pound is the amount of energy to move one pound the distance of one foot.)

Liter, the SI measure of capacity approximately equal to 1.057 quart.

A regulatory program that identifies hazardous wastes that are restricted from land disposal. The regulations incorporate a phasing-in of restrictions in three stages.

Laboratory Emergency Duty Officer. A senior LLNL management official with authority to commit LLNL resources on the behalf of the Director during an emergency.

Lower explosive limit.

A phrase indicating that a chemical constituent was either not identified or not quantified at the lowest level of sensitivity of the analytical method being employed by the laboratory. Therefore, the chemical constituent either is not present in the sample, or it is present in such a small concentration that it cannot be measured by the analytical procedure.

Lower limit of detection. The smallest concentration or amount of analyte that can be detected in a sample at a $95 \%$ confidence level.

Lawrence Livermore National Laboratory. 
LLW Low-level waste.

LOD Level of detection.

LWRP Livermore Water Reclamation Plant. The City of Livermore's municipal wastewater treatment plant, which accepts discharges from the LLNL Livermore site.

M MAD

Median absolute deviation-the median of the differences of all data values from the median.

MATHEW/ Transport and diffusion computer codes used to deterADPIC codes mine the concentration of pollutants at a distance from the source.

$\mathrm{MCL}$

Maximum contaminant level in drinking water established by EPA or DTSC.

MDL

Minimum detection limit.

MOCVD

Metallorganic chemical vapor deposition.

$\mathrm{mR}$

Milliroentgens-A unit of measurement used to express radiation exposure.

mrem

Millirem-A unit of measurement used to express radiation dose to a person-equal to 0.00001 sievert.

msl

Mean Sea Level-The average sea surface level for all stages of the tide over a 19-year period. This is usually determined by hourly height readings from a fixed reference level.

MTL

Multi-user Tandem Laboratory.

N NAAQS

National Ambient Air Quality Standards. Air standards established pursuant to the Clean Air Act to protect human health and the environment.

NCRP

National Council on Radiation Protection.

NEPA

National Environmental Policy Act. This federal legislation, enacted in 1969, requires all federal agencies to document and consider environmental impacts from federally funded or approved projects. DOE is responsible for NEPA compliance at LLNL. 
NESHAPs National Emission Standards for Hazardous Air Pollutants. These standards are found in the Clean Air Act and set limits for arsenic, asbestos, beryllium, mercury, radionuclides, vinyl chloride, and benzene.

NIST

National Institute for Standards and Technology. The federal agency, formerly known as the National Bureau of Standards, responsible for reference materials against which laboratory materials are calibrated.

Nonpoint source Any nonconfined area from which pollutants are discharged into a body of water (e.g., agricultural runoff, construction runoff, and parking-lot drainage).

NOI Notice of Intent.

NOP Notice of Preparation.

NOV Notice of Violation.

NPDES National Pollutant Discharge Elimination System. This federal regulation, under the Clean Water Act, requires permits for discharges into surface waterways.

NPL National Priorities List. EPA's list of the top-priority hazardous waste sites in the country that are subject to the Superfund program.

NRC Nuclear Regulatory Commission. The federal agency charged with oversight of nuclear power and nuclear machinery and applications not regulated by DOE or the Department of Defense.

NTS Nevada Test Site (DOE). The facility in the United States where nuclear weapons are tested.

Nuclide A species of atom characterized by the constitution of its nucleus. The nuclear constitution is specified by the number of protons, number of neutrons, and energy content; or, alternatively, by the atomic number, mass number, and atomic mass. To be regarded as a distinct nuclide, the atom must be capable of existing for a measurable length of time.

O Off site

Outside the boundaries of the LLNL Livermore site and Site 300 properties. 
On site

ORAD

OSHA

OSP

P Part B permit

Performance standards

Piezometer

$\mathrm{pH}$

POC

Point source

Porter-Cologne

Act

POTW

PPAP

$\mathrm{ppb}$
Within the boundaries of the LLNL Livermore site or Site 300 properties.

Operations and Regulatory Affairs Division (LLNL).

Occupational Safety and Health Act.

Operational Safety Procedure.

The second, narrative section submitted by generators in the RCRA permitting process. It covers in detail the procedures followed at a facility to protect human health and the environment.

Specific regulatory requirements established by EPA limiting the concentrations of designated organic compounds, particulate matter, and hydrogen chloride in incinerator emissions.

Generally, a small-diameter, nonpumping well used to measure the elevation of the water table or potentiometric surface.

A measure of hydrogen-ion concentration in an aqueous solution. Acidic solutions have a $\mathrm{pH}$ from 0 to 6 , basic solutions have a $\mathrm{pH}$ greater than 7 , and neutral solutions have a $\mathrm{pH}$ of 7 .

Precursor organic compound.

Any confined and discrete conveyance (e.g., pipe, ditch, well, or stack).

The California law that was enacted to protect water quality in California and to protect the beneficial uses of California water.

Publicly Owned Treatment Works. A general term used for sewage treatment plants. The Livermore Water Reclamation Plant is the POTW that accepts sewage from the Livermore site.

Pollution Prevention Awareness Plan.

Parts per billion. A unit of measure for the concentration of a substance in its surrounding medium. For example, one billion grams of water containing one gram of salt has a salt concentration of one part per billion. 
ppm

PRAP

Precision

Pretreatment

Pretreatment regulations

Priority pollutants

Public comment period

Public hearing

Public notice

Q QA

QAPP
Parts per million. A unit of measure for the concentration of a substance in its surrounding medium. For example, one million grams of water containing one gram of salt has a salt concentration of one part per million.

Proposed Remedial Action Plan.

The closeness of approach of a value of similar or replicate results to a common value in a series of measurements.

Any process used to reduce a pollutant load before it enters the sewer system.

National wastewater pretreatment regulations, adopted by EPA in compliance with the 1977 amendments to the Clean Water Act, which required that EPA establish pretreatment standards for existing and new industrial sources.

A set of organic and inorganic chemicals identified by EPA as indicators of environmental contamination.

A specified amount of time allowed for members of the public to express their views and concerns regarding an action by a public agency.

A formal gathering of officials and the public where the views and concerns of members of the public are verbally expressed regarding a public agency's action; public comments may be written or oral. The agency is required to consider the comments in its evaluation of the action being taken.

Notification by an agency informing the public of agency actions (e.g., the issuance of a draft permit).

Quality assurance. A system of activities whose purpose is to provide the producer or user of a product or service the assurance that it meets defined standards of quality with a stated level of confidence.

Quality Assurance Project Plan-An LLNL plan that outlines methods and processes to ensure that the precision, accuracy, completeness, representativeness, and compatibility of project data are known and maintained at an acceptable level. 
QC Quality control.

Quality factor The factor by which the absorbed dose (rad) is multiplied to obtain a quantity that expresses, on a common scale for all ionizing radiation, the biological damage to exposed persons. It is used because some types of radiation, such as alpha particles, are more biologically damaging than others.

R rad

Radioactive decay

Radioactivity

Radionuclide

The unit of absorbed dose. It is the quantity of energy imparted by ionizing radiation to a unit mass of matter such as tissue. One rad equals 0.01 joule per kilogram.

RADRISK EPA computerized simulation model.

RAIP

Remedial Action Implementation Plan.

RCRA

Resource Conservation and Recovery Act of 1976. RCRA is a program of federal laws and regulations that govern the management of hazardous wastes. RCRA is applicable to all entities that manage hazardous wastes.

RDX

Hexahydro-1,3,5-trinitro-1,3,5-triazine, a high-explosive compound.

Reagent

Any substance used in a chemical reaction to detect or measure another substance, or to convert one substance into another by means of the reaction which it causes.

rem

Radiological unit of dose equivalent. This is the product of the absorbed dose (rad), quality factor (Q), distribution factor, and other necessary modifying factors. The unit rem describes the effectiveness of various radiations to produce biological effects ( 1 rem $=0.01$ sievert). 
Response to comments

RI

Riparian

Risk assessment

RMMA

ROD

Roentgen

ROV

RTRF

RWQCB

S SAL
A document that addresses all significant public comments received by EPA during the public comment period on a proposed permit or action. The document includes a summary of each comment, as well as EPA's response to each comment.

Remedial Investigation. An investigation conducted to fully assess the nature and extent of the release, or threat of release, of hazardous substances, pollutants, or contaminants and to gather necessary data to support the corresponding feasibility study.

Inhabiting the bank area along or about a body of water.

The use of established methods to measure the risks posed by an activity such as hazardous waste treatment. Risk assessments evaluate (1) the relationship between exposure to toxic substances and the subsequent occurrence of health effects, and (2) the potential for that exposure.

Radioactive materials management areas.

Record of Decision.

A unit of exposure dose of $x$ - or gamma-radiation such that the electrons and positrons liberated by this radiation produce, in air, when stopped completely, ions carrying positive and negative charges of $2.58 \times 10^{-4}$ coulomb per kilogram of air. Abbreviated $R$.

Report of Violation.

Real Time Radiography Facility.

Regional Water Quality Control Board. The California regional agency responsible for water quality standards and the enforcement of state water quality laws within its jurisdiction. California is divided into a number of RWQCBs; the Livermore site is regulated by the San Francisco Bay Region, and Site 300 is regulated by the Central Valley Region.

State Action Level. See Action Level. 
Sampling and Analysis Plan

Saturated zone

SARA

Screen zone

Sensitivity

Sewerage

SFRWQCB

SI

Sievert

Site 300

SDM
A detailed document describing the procedures used to collect, handle, and analyze groundwater samples for detection or assessment-monitoring parameters. The plan details quality control measures that will be implemented to ensure that sample-collection, analysis, and data-presentation activities meet the prescribed requirements.

A subsurface zone below which all rock pore-space is filled with water; also called the phreatic zone.

Superfund Amendments and Reauthorization Act of 1986. This act modifies and reauthorizes CERCLA. Title III of this act is also known as the Emergency Planning and Community Right-to-Know Act of 1986.

In well construction, the section of a formation that contains the screen, or perforated pipe that allows water to enter the well.

The capability of methodology or instrumentation to discriminate between samples having differing concentrations or containing varying amounts of analyte.

The system of sewers.

San Francisco Regional Water Quality Control Board.

Système International d'Unités. An international system of physical units. Units of measure in this system include meters (length), kilogram (mass), kelvin (temperature), becquerel (radioactivity), gray (radioactive dose), and sievert (dose equivalent).

The SI unit of dose equivalent. This is the product of the absorbed dose (gray), quality factor (Q), distribution factor, and other necessary modifying factors. The unit Sv describes the effectiveness of various radiations to produce biological effects; $1 \mathrm{~Sv}=\mathrm{Gy} \times \mathrm{Q} \times \mathrm{N}=100 \mathrm{rem}$.

LLNL's high-explosives test facility, located approximately 15 miles east of the Livermore site.

Standard deviation of the mean. (See standard deviation.) 
SJCHD

SJCPHS

SJVUAPCD

$\mathrm{SNL} / \mathrm{CA}$

SPPP

Std dev

STLC

Superfund

Surface impoundment

SWRCB

T TCLP
San Joaquin County Health District. The local agency that enforces underground-tank regulations in San Joaquin County, including Site 300.

San Joaquin County Public Health Services.

San Joaquin Valley Unified Air Pollution Control District. The local agency responsible for regulating stationary air emission sources (including Site 300) in San Joaquin County.

Sandia National Laboratories, California.

Stormwater Pollution Prevention Plan.

Standard deviation. An indication of the dispersion of a set of results around their average (mean).

Soluble Threshold Limit Concentration. A value that can be used to determine if a waste is hazardous.

The common name used for the Comprehensive Environmental Response, Compensation, and Liability Act of 1980 (CERCLA). California has also established a "State Superfund" under provisions of the California Hazardous Waste Control Act.

A facility or part of a facility that is a natural topographic depression, man-made excavation, or diked area formed primarily of earthen materials, although it may be lined with man-made materials. The impoundment is designed to hold an accumulation of liquid wastes, or wastes containing free liquids, and is not an injection well. Examples of surface impoundments are holding, storage, settling and aeration pits, ponds, and lagoons.

State Water Resources Control Board. The state agency that develops and adopts statewide water quality plans and policies, issues permits to use water, and acts as an appellate agency for actions of the Regional Water Quality Control Boards.

Toxicity Characteristic Leaching Procedure. An analytical method designed to determine the mobility of both organic and inorganic compounds present in liquid, solid, and multiphase wastes. It is used to determine applicability of Land Ban to a waste. 
TDS Total Dissolved Solids. The portion of solid material in a waste stream that is dissolved and passed through a filter.

TFA

TFB

TFF

TLD

TOC

TOX

TPH-G

Tritium

TRU

TSCA

T-test

TTO

TTU

Tuff

Turbidity

U U-AVLIS

UC
Treatment Facility A.

Treatment Facility B.

Treatment Facility F.

Thermoluminescent dosimeter. A device used to measure external gamma radiation levels.

Total organic carbon-The sum of the organic material present in a sample.

Total organic halides-The sum of the organic halides present in a sample.

Total petroleum hydrocarbons as gasoline.

Tritium is the hydrogen isotope with one proton and two neutrons in the nucleus. It emits a low-energy beta particle and has a half-life of 12.3 years.

Transuranic waste.

Toxic Substances Control Act. The law governing the manufacture, processing, and use of chemical substances.

A type of ANOVA that determines the significance of the difference between two sample means.

Total toxic organic compounds. A list of organic compounds for which EPA has established discharge limits for specific processes or industries.

Transportable Treatment Unit.

A fragmental rock consisting of the smaller kinds of volcanic detritus, usually more or less stratified.

A measure of the concentration of sediment or suspended particles in solution.

Uranium-Atomic Vapor Laser Isotope Separation.

University of California. 
Unsaturated zone That portion of the subsurface in which the pores are only partially filled with water. The direction of water flow is vertical in this zone; which is also referred to as the vadose zone.

USGS

U.S. Geological Survey. The federal agency responsible for maintaining maps of the United States.

UST

Underground storage tank. A stationary device designed to contain an accumulation of hazardous materials or waste. A tank is constructed primarily of nonearthen material, but the entire surface area of the tank is totally below the surface of, and covered by, the ground.

V Vadose zone The partially saturated or unsaturated region above the water table that does not yield water to wells.

VHS

Volatile halogenated solvent. A term used by LLNL for analysis of the solvents detectable by EPA Method 601 .

VOC Volatile organic compound. Liquid or solid organic compounds that have a tendency to spontaneously pass into the vapor state.

VPL

Vacuum Process Laboratory.

VSI

Visual Site Inspection. An inspection required by EPA as part of the RCRA permit process to identify solid waste management units that could have had, or continue to have, releases of hazardous constituents to the environment.

W WAA

Waste Accumulation Area. An officially designated area that meets current environmental standards and guidelines for temporary (less than 90 days) storage of hazardous waste before pickup by the Hazardous Waste Management Division for off-site disposal.

WFA

Wastewater treatment system
West Firing Area (LLNL Site 300).

A collection of treatment processes and facilities designed and built to reduce the amount of suspended solids, bacteria, oxygen-demanding materials, and chemical constituents in wastewater. 
Water table The water level surface below the ground at which the unsaturated zone ends and the saturated zone begins. It is the level to which a well that is screened in the unconfined aquifer would fill with water.

WDR

Waste Discharge Requirements. Issued by the California Regional Water Quality Control Board.

Weighting factor A value used to calculate dose equivalents. It is tissuespecific and represents the fraction of the total health risk resulting from uniform, whole-body irradiation that could be contributed to that particular tissue. The weighting factors used in this report are recommended by the ICRP (Publication 26).

Wind rose

A diagram that shows the frequency and intensity of wind from different directions at a particular place.

WMP

Waste Minimization Project.

X XRFA

X-Ray Fluorescence Analysis. The identification of elements through characteristic $x$ rays generated by excitation. The sewer-monitoring system uses this technology for metal detection.

Z $\quad$ Zone 7

The common name for the Alameda County Flood Control and Water Conservation District. Zone 7 is the water management agency for the Livermore-Amador Valley with responsibility for water treatment and distribution. Zone 7 is also responsible for management of agricultural and surface water and the groundwater basin. 
Anderson, K.L., and H.F. Heffner, Ground Water Project Update, Lawrence Livermore National Laboratory, Livermore, CA (UCAR-10280-92-1).

Bainer, R.W., and M. Adams, Characterization of the Detailed Study Area Utilizing Shallow Three-Dimensional High Resolution Seismic Reflection Technology for Remediation Optimization, prepared for the Technology Information Exchange Workshop, Pleasanton, CA, November 17-19, 1992, Lawrence Livermore National Laboratory, Livermore, CA (UCRL-JC-110593 ABS).

Bainer, R.W., M.D. Dresen, and J.P. Ziagos, Environmental Restoration at the Lawrence Livermore National Laboratory Livermore Site, prepared for the American Institute of Hydrology's Second USA/USSR Joint Conference of Environmental Hydrology and Hydrogeology, Washington, DC, May 1620, 1993, Lawrence Livermore National Laboratory, Livermore, CA (UCRL-JC-109493 ABS).

Bainer, R.W., D.W. Peifer, and J.D. Hoffman, Optimization of Remediation of an Active Source Area at Lawrence Livermore National Laboratory, prepared for the Technology Information Exchange Workshop, Pleasanton, CA, November 17-19, 1992, Lawrence Livermore National Laboratory, Livermore, CA (UCRL-JC-110592 ABS).

BeLue, A., E. Hess, and K.C. Lamson, Sampling and Analysis Plan for Contaminated Soils, Lawrence Livermore National Laboratory, Livermore, CA (UCRLAR-111744).

Biermann, A.H., and K.C. Lamson, LLNL NESHAPs Project Quarterly Progress Report, June 30, 1992, Lawrence Livermore National Laboratory, Livermore, CA (UCRL-AR-108419-92-2).

Biermann, A.H., and K.C. Lamson, LLNL NESHAPs Project Quarterly Progress Report, September 30, 1992, Lawrence Livermore National Laboratory, Livermore, CA (UCRL-AR-108419-92-3).

Bishop, D.J., J.L. Iovenitti, J.J. Nitao, and D.W. Rice, Jr., Vadose Zone Investigation Program to Provide Field and Laboratory Data for Critical Parameters Needed for Development of Conceptual and Numerical Models, prepared for the American Geophysical Union Fall Meeting, San Francisco, CA, December 7-11, 1992, Lawrence Livermore National Laboratory, Livermore, CA (UCRL-JC110615 ABS).

Bishop, D.J., J.L. Iovenitti, and D.W. Rice, Jr., Vadose Characterization Parameters and Methodologies Critical to the Development of Conceptual and Computational Models, prepared for the Technical Information Exchange Workshop, Albuquerque, NM, May 19-21, 1992, Lawrence Livermore National Laboraiory, Livermore, CA (UCRL-JC-110429 ABS). 
Bishop, D.J., S.D. Nelson, D.W. Rice, Jr., P.W. Krauter, M.C. Jovanovich, and K. Lee, Characterization of Subsurface Sediments at a Site of Gasoline Contamination, prepared for the Spectrum '92 Nuclear and Hazardous Waste Management International Topical Meeting, Boise, ID, August 2327, 1992, Lawrence Livermore National Laboratory, Livermore, CA (UCRL-JC-109676).

Bishop, D.J., J.J. Nitao, J.L. Iovenitti, and D.W. Rice, Jr., Field and Laboratory Characterization of Vadose Sediments for Development of Conceptual and Computational Models, prepared for the Technology Information Exchange Workshop, Pleasanton, CA, November 17-19, 1992, Lawrence Livermore National Laboratory, Livermore, CA (UCRL-JC-110589 ABS).

Bishop, D.J., D.W. Rice, Jr., and S.D. Nelson, Dynamic Underground Stripping Project Characterization Report, Lawrence Livermore National Laboratory, Livermore, CA (UCRL-AR-108707).

Boegel, A.J., Field Experiment for Solar Detoxification of Volatile Organic Compounds in Ground Water, prepared for the Technology Information Exchange Workshop, Pleasanton, CA, November 17-19, 1992, Lawrence Livermore National Laboratory, Livermore, CA (UCRL-JC-110612 ABS).

Bowers, J.S., S.M. Painter, and K.C. Lamson, Sampling and Analysis Plan for Stabilized Wastes, Lawrence Livermore National Laboratory, Livermore, CA (UCRL-AR-111745).

Camp, D.W., Soil Gas Composition Provides Evidence of In Situ Biodegradation of Organic Compounds, prepared for HAZMACON '92, Long Beach, CA, March 30-April 2, 1992, Lawrence Livermore National Laboratory, Livermore, CA (UCRL-JC-108422).

Campbell, B.E., and D.A. Graser, Engineering Assessment and Certification of Integrity of the Area 514 Storage Tank 514-R501, Lawrence Livermore National Laboratory, Livermore, CA (UCRL-ID-109101).

Campbell, B.E., and D.A. Graser, Engineering Assessments and Certification of Integrity of the Area 514 Wastewater Tank Farm Unit Tank Systems 514-R5A1, 514-R5A2, 514-R5A3, 514-R5A4, 514-R5A5, and 514-R5A6, Lawrence Livermore National Laboratory, Livermore, CA (UCRL-ID-109123).

Canales, T.W., V.M. Johnson, M.P. Maley, P.F. McKereghan, E.M. Nichols, A.F.B. Tompson, and J.P. Ziagos, PLANET, An Interactive Computer System for the Rapid Generation and Evaluation of Ground Water Pump and Treat Scenarios, prepared for the Spectrum '92 Nuclear and Hazardous Waste Management International Topical Meeting, Boise, ID, August 23-27, 1992, Lawrence Livermore National Laboratory, Livermore, CA (UCRL-JC-109674).

Carley, J.F., Analysis of Radioactivity-Swipe Data on Retired Fluorescent Tubes and Proposed Plan for Testing and Disposing of Discrete Waste Items, Lawrence Livermore National Laboratory, Livermore, CA (UCRL-ID-110728).

Carlsen, T.M., Ecological Assessments During Site Investigation and Remediation, prepared for the Technical Information Exchange Workshop, Pleasanton, CA, November 17-19, 1992, Lawrence Livermore National Laboratory, Livermore, CA (UCRL-JC-110599 ABS). 
Carlsen, T.M., Ecological Risks at Superfund Sites: Research/Regulatory Drivers in the 1990s, prepared for the Spectrum '92 Nuclear and Hazardous Waste Management International Topical Meeting, Boise, ID, August 23-27, 1992, Lawrence Livermore National Laboratory, Livermore, CA (UCRL-JC109669).

Carlsen, T.M., Ecological Risks: Beyond Hazardous Chemicals. The Vulnerability and Persistence of Rare and Endangered Species, prepared for the Society for Risk Analysis 1992 Annual Meeting, San Diego, CA, December 6-9, 1992, Lawrence Livermore National Laboratory, Livermore, CA (UCRL-JC110542 ABS).

Carlsen, T.M., Integrated Ecological Risks: Multiple Risks at Larger Federal Superfund Sites, prepared for the Society for Risk Analysis 1992 Annual Meeting, San Diego, CA, December 6-9, 1992, Lawrence Livermore National Laboratory, Livermore, CA (UCRL-JC-110543 ABS).

Carlsen, T.M., S.D. Gregory, and L.L. Berg, LLNL Site 300 Environmental Restoration Project Standard Operating Procedures (SOPS), Lawrence Livermore National Laboratory, Livermore, CA (UCRL-MA-109115).

Carlsen, T.M., and M.N. Ridley, Draft Quality Assurance Project Plan, Lawrence Livermore National Laboratory Site 300 Environmental Restoration Project, Lawrence Livermore National Laboratory, Livermore, CA (UCRL-AR103160 Rev. 1 DR).

Carlsen, T.M., M.N. Ridley, and V.R. Kiszka, Quality Assurarce Project Plan, LLNL Site 300 Environmental Restoration Project, Lawrence Livermore National Laboratory, Livermore, CA (UCRL-AR-103160 Rev.1).

Cederwall, P.L., Critical Steps in the Flow of Environmental Data Through a Successful Data Manageinent System, prepared for the GIS Information Exchange Meeting, Santa Fe, NM, May 18-19, 1992, Lawrence Livermore National Laboratory, Livermore, CA (UCRL-JC-110441 ABS).

Cederwall, P.L., Providing Quality Data: Environmental Data Management, prepared for the U.S. EPA Region IX Workshop, San Francisco, CA, September 10-11, 1992, Lawrence Livermore National Laboratory, Livermore, CA (UCRL-JC-110587 ABS).

Crow, N.B., W.L. McConachie, L.L. Berg, T.A. Miller, J.L. Monroe, J.P. Pavletich, and J.P. Wilgard, Log of Wells and Boreholes Drilled During Hydrogeologic Studies at Lawrence Livermore National Laboratory Site 300, January 1, 1991September 1, 1992, Lawrence Livermore National Laboratory, Livermore, CA (UCID-21536 Addendum 2).

DeGrange, C.E., and H.L. Galles, Environmental Protection Handbook, Lawrence Livermore National Laboratory, Livermore, CA (UCRL-AR-110237).

Devany, R.O., M.D. Dresen, R.W. Bainer, and J.P. Ziagos, LLNL Ground Water Project Monthly Progress Report, July 1991, Lawrence Livermore National Laboratory, Livermore, CA (UCAR-10160-91-7). 
Devany, R.O., M.D. Dresen, and J.P. Ziagos, LLNL Ground Water Project Monthly Progress Report, September 1991, Lawrence Livermore National Laboratory, Livermore, CA (UCAR-10160-91-9).

Devany, R.O., M.D. Dresen, J.P. Ziagos, and R.W. Bainer, LLNL Ground Water Project Monthly Progress Report, November 1991, Lawrence Livermore National Laboratory, Livermore, CA (UCAR-10160-91-11).

Devany, R.O., M.D. Dresen, J.D. Hoffman, P.F. McKereghan, J.P. Ziagos, and R.W. Bainer, LLNL Ground Water Project Monthly Progress Report, January 1992, Lawrence Livermore National Laboratory, Livermore, CA (UCAR10160-92-1).

Devany, R.O., B.J. Qualheim, and S. Addison, Computer-Assisted Visualization and Volume Estimates of a Subsurface Gasoline Plume, prepared for the American Geophysical Union Fall Meeting, San Francisco, CA, December 7-11, 1992, Lawrence Livermore National Laboratory, Livermore, CA (UCRL-JC110610 ABS).

DeYoung, S.A., Closure Plan for the Building 169 Waste Accumulation Area (Temporary), Lawrence Livermore National Laboratory, Livermore, CA (UCRL-AR-110230).

DeYoung, S.A., Closure Plan for the Building 419 Size Reduction Unit, Lawrence Livermore National Laboratory, Livermore, CA (UCRL-AR-109411).

DeYoung, S.A., Closure Plan for the Building 419 Size Reduction Unit, Lawrence Livermore National Laboratory, Livermore, CA (UCRL-AR-109411 Rev. 1).

DeYoung, S.A., Closure Plan for the Building 419 Solidification Unit, Lawrence Livermore National Laboratory, Livermore, CA (UCRL-AR-109412).

DeYoung, S.A., Closure Plan for the Building 419 Solidification Unit, Lawrence Livermore National Laboratory, Livermore, CA (UCRL-AR-109412 Rev. 1).

Diaz, J.C., and D.J. Shoemaker, Process Waste Assessment for 1990-Main Site, Lawrence Livermore National Laboratory, Livermore, CA (UCRLAR-109107).

Diaz, J.C., and D.J. Shoemaker, Process Waste Assessment for 1990-Site 300, Lawrence Livermore National Laboratory, Livermore, CA (UCRLAR-109108).

Dresen, M.D., J.P. Ziagos, A.J. Boegel, and E.M. Nichols, Draft Remedial Action Implementation Plan for the LLNL Livermore Site, Livermore, California, Lawrence Livermore National Laboratory, Livermore, CA (UCRL-AR110532 DR).

Ferry, R.A., and A.L. Lamarre, Environmental Site Characterization and Remediation Activities at Lawrence Livermore National Laboratory Site 300, prepared for the American Institute of Hydrology's Second USA/USSR Joint Conference of Environmental Hydrology and Hydrogeology, Washington, DC, May 1620, 1993, Lawrence Livermore National Laboratory, Livermore, CA (UCRL-JC-109114 ABS). 
Ferry, R.A., A.L. Lamarre, and R.K. Landgraf, Draft Feasibility Study sf the General Services Area (GSA), Lawrence Livermore National Laboratory Site 300, Lawrence Livermore National Laboratory, Livermore, CA (UCRL-AR104517 DR).

Fischer, K.G., W.G. Hoppes, and D.H. MacQueen, LLNL Site 300 Groundwater Monitoring Program Quarterly Report, October-December 1991, Lawrence Livermore National Laboratory, Livermore, CA (UCAR-10191-91-4).

Fischer, K.G., W.G. Hoppes, and D.H. MacQueen, LLNL Site 300 Groundwater Monitoring Program Quarterly Report, January-March 1992, Lawrence Livermore National Laboratory, Livermore, CA (UCAR-10191-92-1).

Fischer, K.G., W.G. Hoppes, and D.H. MacQueen, LLNL Site 300 Groundwater Monitoring Program Quarterly Report, April-June 1992, Lawrence Livermore National Laboratory, Livermore, CA (UCAR-10191-92-2).

Fischer, K.G., W.G. Hoppes, and D.H. MacQueen, LLNL Site 300 Groundwater Monitoring Program Quarterly Report, July-September 1992, Lawrence Livermore National Laboratory, Livermore, CA (UCAR 10191-92-3).

Folsom, E.N., LLNL Experience with UV-Light Technologies Used for Ground Water Restoration, prepared for the Technology Information Exchange Workshop, Pleasanton, CA, November 17-19, 1992, Lawrence Livermore National Laboratory, Livermore, CA (UCRL-JC-110611 ABS).

Gallegos, G.M., B.K. Balke, K.A. Surano, W.G. Hoppes, P.J. Tate, J.C. Steenhoven, B.C. Fields, L.M. Garcia, and K.C. Lamson, Environmental Report for 1991, Lawrence Livermore National Laboratory, Livermore, CA (UCRL-50027-91).

Gallegos, G.M., K.A. Surano, K.C. Lamson, B.K. Balke, A.H. Biermann, W.G. Hoppes, B.C. Fields, F.J. Gouveia, R.L. Berger, F.S. Miller, D.W. Rueppel, and J.M. Sims, Environmental Monitoring Plan, Environmental Monitoring Section, Lawrence Livermore National Laboratory, Livermore, CA (UCRL-ID-106132).

Grandfield, C.H., Lawrence Livermore National Laboratory Environmental Protection Implementation Plan (November 9, 1991-November 9, 1992), Lawrence Livermore National Laboratory, Livermore, CA (UCAR-10307-92).

Grayson, A.R., Wastewater Discharge Permit Application 1992-1993, Lawrence Livermore National Laboratory, Livermore, CA (UCRL-AR-106905-92).

Grayson, A.R., Wastewater Point-Source Monitoring at Lawrence Livermore National Laboratory Livermore Site, Lawrence Livermore National Laboratory, Livermore, CA (UCAR-10204-92-1).

Grayson, A.R., and S.L. Brigdon, Wastewater Point-Source Monitoring at LLNL, Livermore Site Semiannual Report, June-November 1991, Lawrence Livermore National Laboratory, Livermore, CA (UCAR-10204-91-2).

Gregory, S.D., Seasonal Variability in Ground Water Tritium Activities Adjacent to a Closed Mixed Waste Landfill, prepared for the American Geophysical Union Fall Meeting, San Francisco, CA, December 7-11, 1992, Lawrence Livermore National Laboratory, Livermore, CA (UCRL-JC-110607 ABS). 
Hagen, A., Criteria and Procedures for the Certification of Nonradioactive Hazardous Waste, Lawrence Livermore National Laboratory, Livermore, CA (UCRL-AR-109662 Vols. 1-3).

Hallisey, M.L., Resource Conservation and Recovery Act Parts $A$ and B Permit Application, Hazardous Waste Treatment and Storage Facilities, Lawrence Livermore National Laboratory Livermore Site, Lawrence Livermore National Laboratory, Livermore, CA (UCAR-10275-91, Part A-Vols. 1-5, Part B-Vols. 1-38).

Hoffman, F., Characterization of Ground Water Contamination at the LLNL Main Site: A Case Study, prepared for Technologies for Environmental Cleanup: Soil and Ground Water, Ispra, Italy, September 21-25, 1992, Lawrence Livermore National Laboratory, Livermore, CA (UCRL-JC-110525).

Hoffman, F., Ground Water Remediation Using "Smart Pump and Treat," prepared for Ground Water, Lawrence Livermore National Laboratory, Livermore, CA (UCRL-JC-109663).

Hoffman, F., Innovative Characterization Techniques and Decision Support Systems for Ground Water Contamination Projects, prepared for Technologies for Environmental Cleanup: Soil and Ground Water, Ispra, Italy, September 21-25, 1992, Lawrence Livermore National Laboratory, Livermore, CA (UCRL-JC-110528).

Hoffman, F., A Strategy for Improving Pump and Treat Ground Water Remediation, prepared for Technologies for Environmental Cleanup: Soil and Ground Water, Ispra, Italy, September 21-25, 1992, Lawrence Livermore National Laboratory, Livermore, CA (UCRL-JC-110530).

Hoppes, W.G., D.H. MacQueen, and K.G. Fischer, Draft Report of Waste Discharge, LLNL Site 300, Lawrence Livermore National Laboratory, Livermore, CA (UCRL-AR-110071 DR).

Howe, R.E., and K.N. Williams, Waste Inventory System Manual, Lawrence Livermore National Laboratory, Livermore, CA (UCRL-MA-111775).

Iovenitti, J.L., J.J. Nitao, and D.J. Bishop, Vadose Zone Investigations at the Lawrence Livermore National Laboratory Superfund Site: An Overview, prepared for the Second USA/CIS Joint Conference on Environmental Hydrology and Hydrogeology, Washington, DC, May 1993, Lawrence Livermore National Laboratory, Livermore, CA (UCRL-JC-110606).

Isherwood, W.F., Are We Paying Too Much for Environmental Protection?, prepared for the AAAS-93 Annual Meeting, Boston, MA, February 11-16, 1993, Lawrence Livermore National Laboratory, Livermore, CA (UCRL-JC111757 ABS).

Isherwood, W.F., J.P. Ziagos, E.M. Nichols, P.W. Krauter, and D.W. Rice, Jr., Enhancing Aquifer Cleanup with Reinjection, prepared for the National Ground Water Association Conference, Houston, TX, November 4-6, 1992, Lawrence Livermore National Laboratory, Livermore, CA (UCRLJC-110235). 
Johnson, V.M., INTEGRATOR: Managing Data Flow Among GIS and Other Software Packages to Support Environmental Decisionmaking, prepared for the U.S. EPA Region IX Workshop, San Francisco, CA, September 10-11, 1992, Lawrence Livermore National Laboratory, Livermore, CA (UCRL-JC110584 ABS).

Johnson, V.M., and R.R. Johnson, INTEGRATOR: A Graphical Tool for Managing Data Flow Among Geographic Information System and Other Software Packages, prepared for the GIS Information Exchange Meeting, Santa Fe, NM, May 18-19, 1992, Lawrence Livermore National Laboratory, Livermore, CA (UCRL-JC-110227 ABS).

Johnson, V.M., and L.L. Rogers, Application of Neural Network Technology to the Optimization of Field-Scale Pump Configurations in Smart Pump-and-Treat Remediation, prepared for the Technology Information Exchange Workshop, Pleasanton, CA, November 17-19, 1992, Lawrence Livermore National Laboratory, Livermore, CA (UCRL-JC-110600 ABS).

Jovanovich, M.C., and G.L. McQueen, Evaluation of the Dynatech PTA-30 W/S for Extracting Trichloroethylene (TCE) and Benzene from Subsurface Sediments, prepared for the American Geophysical Union Fall Meeting, San Francisco, CA, December 7-11, 1992, Lawrence Livermore National Laboratory, Livermore, CA (UCRL-JC-110608 ABS).

Jovanovich, M.C., R.T. Taylor, W.B. Durham, A.G. Duba, K.J. Jackson, R.B. Knapp, A.M. Wijesinghe, and M.L. Hanna, Field Application of In Situ Microbial Filters for Remediation of the Contaminated Ground Water, prepared for the Technology Information Exchange Workshop, Pleasanton, CA, November 17-19, 1992, Lawrence Livermore National Laboratory, Livermore, CA (UCRL-JC-110603 ABS).

Kansa, E.J., Modern Optimization Procedures Applied to Ground Water Remediation, prepared for the IMACS India Symposium, Bangalore, India, December 711, 1992, Lawrence Livermore National Laboratory, Livermore, CA (UCRL-JC-110063 ADU).

Krauter, P.W., Micronucleus Incidence and Hematological Effects in Bullfrog Tadpoles (Rana catesbeiana) Exposed to 2-AAF and 2-AF, prepared for the Archives of Environmental Contamination and Toxicology, Lawrence Livermore National Laboratory, Livermore, CA (UCRL-JC-110447).

Krauter, P.W., M.H. Hanna, R.T. Taylor, and D.W. Rice, Jr., Bacterial Distribution and Metabolic Activity in Subsurface Sediments from a Gasoline Spill, prepared for the Spectrum '92 Nuclear and Hazardous Waste Management International Topical Meeting, Boise, ID, August 23-27, 1992, Lawrence Livermore National Laboratory, Livermore, CA (UCRL-JC-109677).

Krauter, P.W., R.E. Martinelli, and D.W. Rice Jr., BETX Degradation Rates of Indigenous Microorganisms from a Gasoline Spill Site, prepared for the Technology Information Exchange Workshop, Pleasanton, CA, November 17-19, 1992, Lawrence Livermore National Laboratory, Livermore, CA (UCRL-JC-110601 ABS). 
Kulshrestha, A., and A.L. Lamarre, Vadose Zone Modeling to Estimate the Impact of Contaminant Transport Through Fractured Media at LLNL Site 300, prepared for the American Geophysical Union Fall Meeting, San Francisco, CA, December 7-11, 1992, Lawrence Livermore National Laboratory, Livermore, CA (UCRL-JC-111727 ABS).

Lamarre, A.L., and E.M. Hill, Draft Engineering/Evaluation/Cost Analysis for the Central General Services Area (GSA) CERCLA Removal Action, Lawrence Livermore National Laboratory Site 300, Lawrence Livermore National Laboratory, Livermore, CA (UCRL-AR-110626 DR).

Lamarre, A.L., and R.A. Ferry, Environmental Site Characterization and Remediation at Lawrence Livermore National Laboratory Site 300, prepared for the Spectrum '92 Nuclear and Hazardous Waste Management International Topical Meeting, Boise, ID, August 23-27, 1992, Lawrence Livermore National Laboratory, Livermore, CA (UCRL-JC-109668).

Lamson, K.C., LLNL NESHAPS Project Quarterly Progress Report, March 31, 1992, Lawrence Livermore National Laboratory, Livermore, CA (UCRL-AR108419-92-1).

Lamson, K.C., Quarterly Progress Report, LLNL NESHAPs Project, December 31, 1991, Lawrence Livermore National Laboratory, Livermore, CA (UCRL-AR-108419-91-4).

Low, K.I., Monitoring Program for New Underground Storage Tanks 611-G1U1, 611-G2U1, 611-D1U1, and 611-O1U1, Lawrence Livermore National Laboratory, Livermore, CA (UCRL-AR-103195).

Landgraf, R.K., and V.M. Madrid, Application of Borehole Geophysics to Enhance Site Characterization and Reduce Remedial Investigation Costs at Lawrence Livermore National Laboratory Site 300, Southeast of Livermore, CA, prepared for the Technical Information Exchange Workshop, Albuquerque, NM, May 1921, 1992, Lawrence Livermore National Laboratory, Livermore, CA (UCRL-JC-11042.7 ABS).

Macdonald, J.K., M.D. Dresen, P.F. McKereghan, J.D. Hoffman, J.P. Ziagos, and R.W. Bainer, LLNL Ground Water Project Monthly Progress Report February 1992, Lawrence Livermore National Laboratory, Livermore, CA (UCAR-10160-92-2).

Macdonald, J.K., M.D. Dresen, and J.P. Ziagos, LLNL Ground Water Monthly Progress Report, June 1991, Lawrence Livermore National Laboratory, Livermore, CA (UCAR-10160-91-6).

Macdonald, J.K., M.D. Dresen, and J.P. Ziagos, LLNL Ground Water Project Monthly Progress Report, August 1991, Lawrence Livermore National Laboratory, Livermore, CA (UCAR-10160-91-8).

Macdonald, J.K., M.D. Dresen, and J.P. Ziagos, LLNL Ground Water Project Monthly Progress Report, October 1991, Lawrence Livermore National Laboratory, Livermore, CA (UCAR-10160-91-10). 
Macdonald, J.K., J.L. Iovenitti, and D.J. Bishop, Use of Soil Moisture Tritium Data for Delineation of Infiltration Patterns and the Implications for Remediation, prepared for the Technology Information Exchange Workshop, Pleasanton, CA, November 17-19, 1992, Lawrence Livermore National Laboratory, Livermore, CA (UCRL-JC-110614 ABS).

Mallon, B.J., S.A. Martins, J. Shinn, and J.J. Nitao, Detailed Study of a Small Area That Can Be Applied to the Water Movement Patterns of a Larger Site in the Vadose Zone, prepared for the Technology Information Exchange Workshop, Pleasanton, CA, November 17-19, 1992, Lawrence Livermore National Laboratory, Livermore, CA (UCRL-JC-110591 ABS).

Mallon, B.J., S.A. Martins, J.L. Houpis, B.W. Lowry, and C.D. Cremer, SEAMIST Soil Sampling for Tritiated Water: First Year's Results, prepared for the Sixth Annual Outdoor Action Conference on Aquifer Restoration, Ground Water Monitoring, and Geophysical Methods, Las Vegas, NV, May 9-13, 1992, Lawrence Livermore National Laboratory, Livermore, CA (UCRL-JC109015).

Martins, S.A., Collecting Soil Vapor from the Vadose Zone with an Instrumented Membrane System, Lawrence Livermore National Laboratory, Livermore, CA (UCRL-ID-109765).

Martins, S.A., G.L. McQueen, R.E. Martinelli, and M.C. Jovanovich, Factors Affecting Trichloroethylene (TCE) Recovery from SEAMIST Pads, prepared for the American Geophysical Union Fall Meeting, San Francisco, CA, December 7-11, 1992, Lawrence Livermore National Laboratory, Livermore, CA (UCRL-JC-110604 ABS).

Mayr, M., Spill Prevention Control and Countermeasure (SPCC) Plan, Site 300, Lawrence Livermore National Laboratory, Lawrence Livermore National Laboratory, Livermore, CA (UCRL-MA-105700).

McConachie, W.A., and J.P. Ziagos, Environmental Restoration and Optimized Remediation at Lawrence Livermore National Laboratory, prepared for the Technology Information Exchange Workshop, Pleasanton, CA, November 17-19, 1992, Lawrence Livermore National Laboratory, Livermore, CA (UCRL-JC-110619 ABS).

McPherrin, R.V., Using the SLICE Subterranean Geological Information System to Model a Monitor Well Matrix, prepared for the U.S. EPA Region IX Workshop, San Francisco, CA, September 10-11, 1992, Lawrence Livermore National Laboratory, Livermore, CA (UCRL-JC-110586 ABS).

Meltzer, M., and D.J. Shoemaker, Waste Minimization Activity Report for 1991, Lawrence Livermore National Laboratory, Livermore, CA (UCRLID-107268).

Nelson-Lee, J.C., P.W. Krauter, D.W. Rice, Jr., and D.J. Bishop, Relationship of Soil Physical Parameters and Contaminant Concentrations to Subsurface Biological Populations, prepared for the American Geophysical Union Fall Meeting, San Francisco, CA, December 7-11, 1992, Lawrence Livermore National Laboratory, Livermore, CA (UCRL-JC-110617 ABS). 
Nisbet, B.A., Waste Matters: A Hazardous Waste Management Division Bulletin, Lawrence Livermore National Laburatory, Livermore, CA (UCRLAR-110229).

Nisbet, B.A., Waste Matters (July 1992), Lawrence Livermore National Laboratory, Livermore, CA (UCRL-AR-110229-92-2).

Noyes, C.M., J.L. Iovenitti, J.C. Martin, and D.J. Bishop, Hydrogeochemical Characterization for Remedial Design Optimization, The Gasoline Spill Area, LLNL, A Case Study, prepared for the Technology Information Exchange Workshop, Pleasanton, CA, November 17-19, 1992, Lawrence Livermore National Laboratory, Livermore, CA (UCRL-JC-110613 ABS).

Otsuki, K.H., R.J. Harrach, and R.L. Berger, An Assessment of Potential Risk Resulting from a Maximum Credible Accident Scenario at the Proposed Explosive Waste Storage Facility (EWSF), Lawrence Livermore National Laboratory, Livermore, CA (UCRL-ID-111736).

Qualheim, B.J., Imaging of Geologic and Geochemical Data, prepared for the U.S. EPA Region IX Workshop, San Francisco, CA, September 10-11, 1992, Lawrence Livermore National Laboratory, Livermore, CA (UCRL-JC110585 ABS).

Qualheim, B.J., P.R. Daley, V.M. Johnson, R.V. McPherrin, and G.W. Laguna, Geologic Studies Critical to the Placement of Optimized Extraction Wells, prepared for the Technology Information Exchange Workshop, Pleasanton, CA, November 17-19, 1992, Lawrence Livermore National Laboratory, Livermore, CA (UCRL-JC-110605 ABS).

Qualheim, B.J., P.F. Daley, V.M. Johnson, R.V. McPherrin, and G.W. Laguna, Imaging Subsurface Geology and Volatile Organic Compound Plumes, prepared for Image VI Conference, Scottsdale, AZ, July 14-17, 1992, Lawrence Livermore National Laboratory, Livermore, CA (UCRL-JC-108055).

Qualheim, B.J., and R.V. McPherrin, SLICE: A Subsurface Mapping Program, prepared for the GIS Information Exchange Meeting, Santa Fe, NM, May 18-19, 1992, Lawrence Livermore National Laboratory, Livermore, CA (UCRL-JC-110436 ABS).

Radian Corporation, Chemical Health Risk Assessment for Hazardous and Mixed Waste Management Units at Lawrence Livermore National Laboratory, Lawrence Livermore National Laboratory, Livermore, CA (UCRLCR-109772 Vols. I and II).

Ragaini, R.C., Technologies for Environmental Cleanup of Soil and Groundwater, prepared for Technologies for Environmental Cleanup: Soil and Ground Water, Ispra, Italy, September 21-25, 1992, Lawrence Livermore National Laboratory, Livermore, CA (UCRL-JC-110959).

Rice, D.W., Jr., A.J. Boegel, V.M. Johnson, P.L. Cederwall, B.J. Qualheim, R.R. Johnson, R.D. Aines, T.W. Canales, M.N. Ridley, E.M. Nichols, and J.P. Ziagos, The Role of Data Management and Decision Support Systems in the Implementation of Smart Pump and Treat, prepared for the International School of Innovative Technology for Cleaning the Environment, Erice- 
Trapani, Sicily, April 22-29, 1992, Lawrence Livermore National Laboratory, Livermore, CA (UCRL-JC-110957).

Rice, D.W., Jr., V.M. Johnson, and P.L. Cederwall, Rapid Interpretation of Chemical and Geologic Data to Guide Borehole Sampling and Siting, prepared for the Technical Information Exchange Workshop, Albuquerque, NM, May 1921, 1992, Lawrence Livermore National Laboratory, Livermore, CA (UCRL-JC-110430 ABS).

Richardson, D., Community Relations Plan for the Lawrence Livermore National Laboratory Site 300 Superfund Site, San Joaquin and Alameda Counties, California, Lawrence Livermore National Laboratory, Livermore, CA (UCRL-AR-107435).

Richardson, D., and A.L. Lamarre, Site 300 Fact Sheet Number One, Lawrence Livermore National Laboratory, Livermore, CA (UCRL-TB-110590-92-1).

Ridley, M.N., and R.E. Martinelli, Chromium Characterization and Treatment at Lawrence Livermore National Laboratory, prepared for the American Geophysical Union Fall Meeting, San Francisco, CA, December 7-11, 1992, Lawrence Livermore National Laboratory, Livermore, CA (UCRLJC-110602 ABS).

Ridley, M.N., M.L. Hallisey, S.H. Terusaki, and M. Steverson, Closure of Building 624 Incinerator, prepared for the Incineration ' 92 Conference, Albuquerque, NM, May 14-15, 1992, Lawrence Livermore National Laboratory, Livermore, CA (UCRL-JC-110079).

Salazar, R.J., and S. Lane, Final Safety Analysis Document for Building a Chemical Waste Storage Building at the Lawrence Livermore National Laboratory, Lawrence Livermore National Laboratory, Livermore, CA (UCRLID-109144).

Schwartz, W.W., and B.W. Gracely, Engineering Evaluation of the Sewer Diversion Facility, Lawrence Livermore National Laboratory, Livermore, CA (UCRLID-107444).

Schwartz, W.W., and D.A. Graser, Engineering Assessment and Certification of Integrity of the 325-I1U1 Tank System, Lawrence Livermore National Laboratory, Livermore, CA (UCRL-ID-107633).

Schwartz, W.W., B.W. Gracely, and D.A. Graser, Engineering Assessment and Certification of Integrity of the 177-R1 Tank System, Lawrence Livermore National Laboratory, Livermore, CA (UCRL-ID-110440).

Science Applications International Corporation, Draft LLNL Livermure Site Groundwater Surveillance Plan, Lawrence Livermore National Laboratory, Livermore, CA (UCRL-CR-110539).

Sheets, B., and M.L. Hallisey, Environmental Legislation and Regulation Analysis Pamphlet, Lawrence Livermore National Laboratory, Livermore, CA (UCRL-TB-111726). 
Smith, D.K., D.W. Peifer, and C.K. Rood, Clay Mineralogy of Selected Borehole Sediments from the Lawrence Livermore National Laboratory and Sandia National Laboratories, Livermore, California, Lawrence Livermore National Laboratory, Livermore, CA (UCRL-ID-110534).

Taffet, M.J., Draft Remedial Investigation of Landfill Pit 6 at Lawrence Livermore National Laboratory Site 300, Lawrence Livermore National Laboratory, Livermore, CA (UCRL-ID-103961 DR).

Taffet, M.J., Status of Landfill Remediation at Lawrence Livermore National Laboratory Site 300, prepared for the Technical Information Exchange Workshop, Pleasanton, CA, November 17-19, 1992, Lawrence Livermore National Laboratory, Livermore, CA (UCRL-JC-110596 ABS).

Taffet, M.J., A.L. Lamarre, T.M. Carlsen, D.W. Carpenter, J.A. Oberdorfer, and W.R. Dugan, Tritium as an Indicator of Geologic Controls on Ground Water Migration at a DOE Site in the Northern California Coast Ranges, prepared for the GSA Conference, Eugene, OR, May 11-13, 1992, Lawrence Livermore National Laboratory, Livermore, CA (UCRL-JC-109380 ABS).

Taffet, M.J., J.A. Oberdorfer, T.M. Carlsen, W.R. Dugan, and R.S. Mateik, Draft Remedial Investigation of the Building 850 East Firing Area, Lawrence Livermore National Laboratory Site 300, Lawrence Livermore National Laboratory, Livermore, CA (UCRL-ID-104335 DR).

Terusaki, S.H., Closure Report for the Building 624 Incinerator at Lawrence Livermore National Laboratory-Review of Analytical Data and Waste Disposition Determination, Lawrence Livermore National Laboratory, Livermore, CA (UCRL-AR-109112 Vols. 1 and 2).

Thompson, R., Cost-Benefit Analysis for Waste Compaction Alternatives at Lawrence Livermore National Laboratory, Addendum $A$ to the Waste Minimization and Pollution Prevention Awareness Plan of May 31, 1991, Lawrence Livermore National Laboratory, Livermore, CA (UCRL-21215-91 ADD A).

Thompson, R., Cost-Benefit Analysis for Waste Segregation at Lawrence Livermore National Laboratory, Addendum B to the Waste Minimization and Pollution Prevention Awareness Plan of May 31, 1991, Lawrence Livermore National Laboratory, Livermore, CA (UCRL-21215-91 ADD B).

Van Wármerdam, C., Area 514 Facility Contingency Plan, Lawrence Livermore National Laboratory, Livermore, CA (UCRL-AR-109117).

Van Warmerdam, C., Area 612 Facility Contingency Plan, Lawrence Livermore National Laboratory, Livermore, CA (UCRL-AR-109118).

Van Warmerdam, C., Area 693 Facility Contingency Plan, Lawrence Livermore National Laboratory, Livermore, CA (UCRL-AR-109119).

Vattuone, G.M., S.M. Kluz, P. Genovese, and K. Fitzgerald, Connecting Isolated Workstations to Remote Computer Applications, prepared for the Technology Information Exchange Workshop, Pleasanton, CA, November 17-19, 1992, Lawrence Livermore National Laboratory, Livermore, CA (UCRL-JC111766 ABS). 
Wade, W.M., R.A. Ferry, S.P. Vonder Haar, V.M. Madrid, L.S. Rueth, J.K. Gardner, J.R. Copland, and R.K. Landgraf, Site Characterization of Buried Debris Trenches at Lawrence Livermore National Laboratory Site 300, prepared for the Technical Information Exchange Workshop, Pleasanton, CA, November 17-19, 1992, Lawrence Livermore National Laboratory, Livermore, CA (UCRL-JC-110597 ABS).

Wade, W.M., R.K. Landgraf, R.A. Ferry, S.P. Vonder Haar, V.M. Madrid, E.M. Hill, and J.R. Copland, Application of Multi-Technique Investigations for Site Characterization of a Buried Debris Pile at Lawrence Livermore National Laboratory Site 300, prepared for the Spectrum '92 Nuclear and Hazardous Waste Management International Topical Meeting, Boise, ID, August 2327, 1992, Lawrence Livermore National Laboratory, Livermore, CA (UCRL-JC-109667 ABS).

Willes, J.A., ORAD-New Employee Orientation Manual, Lawrence Livermore National Laboratory, Livermore, CA (UCRL-MA-111755).

William Self Associates, Documentation and Assessment of the History of the LLNL Livermore Facility, and Site CA-SJO-173H, the Carnegie Town Site at LLNL Site 300, Alameda and San Joaquin Counties, CA, Lawrence Livermore National Laboratory, Livermore, CA (UCRL-CR-110610).

Ziagos, J.P., Record of Decision for the Lawrence Livermore National Laboratory Livermore Site, Lawrence Livermore National Laboratory, Livermore, CA (UCRL-AR-109105).

Ziagos, J.P., R.W. Bainer, R.O. Devany, M.D. Dresen, and E.M. Nichols, LLNL Ground Water Project-Monthly Progress Report March 1992, Lawrence Livermore National Laboratory, Livermore, CA (UCAR-10160-92-3).

Ziagos, J.P., R.W. Bainer, M.D. Dresen, and J.D. Hoffman, Environmental Restoration at the Lawrence Livermore National Laboratory Livermore Site, prepared for the Spectrum '92 Nuclear and Hazardous Waste Management International Topical Meeting, Boise, ID, August 23-27, 1992, Lawrence Livermore National Laboratory, Livermore, CA (UCRL-JC-109493 EXT ABS).

Ziagos, J.P., R.W. Bainer, R.B. Weiss, and J.K. Macdonald, LLNL Ground Water Project 1991 Annual Report, Lawrence Livermore National Laboratory, Livermore, CA (UCAR-10160-92-12).

Ziagos, J.P., and M.D. Dresen, Case Study: Environmental Restoration at the Livermore Site Lawrence Livermore National Laboratory, prepared for the Technology Information Exchange Workshop, Pleasanton, CA, November 17-19, 1992, Lawrence Livermore National Laboratory, Livermore, CA (UCRL-JC-110594 ABS).

Ziagos, J.P., and E.M. Nichols, Ground Water Modeling for CERCLA Compliance at Lawrence Livermore National Laboratory, prepared for the Technology Information Exchange Workshop, Pleasanton, CA November 17-19, 1992, Lawrence Livermore National Laboratory, Livermore, CA (UCRL-JC110595 ABS). 


\section{Appendix B. Methods of Dose Calculations}

Robert J. Harrach

Kris A. Surano

\section{Introduction}

The effective dose equivalents shown in this report have been calculated using EPA-approved computer models, as described in Chapter 11. In particular, the technique used to calculate environmental transport and to estimate dose and risk in the CAP88-PC model are detailed in Chapter 8 of the EPA's Users Guide for CAP88-PC, Version 1.0 (Parks 1992).

Insight into these code results is provided by the explicit dose calculation methods described here. The methods are based on the NRC Regulatory Guide 1.109, Calculation of Annual Doses to Man from Routine Releases of Reactor Effluent (U.S. Nuclear Regulatory Commission 1977). The dose and dose-rate conversion factors used in these calculations were obtained from the committed dose equivalent tables for DOE dose calculations. These dose conversion factors are consistent with those specified in ICRP 30, Limits of Intakes of Radionuclides by Workers (ICRP 1980). Examples of these calculations and assumptions are presented below, following a brief description of the types of doses of major interest.

1. External perimeter dose. The dose rate at points along the perimeter of LLNL is a measure of the maximum external dose at locations of nearest uncontrolled public access. Dose rates from external penetrating radiation (primarily gamma rays and neutrons) are measured using environmental dosimeters (thermoluminescent dosimeters and neutron detectors). The environmental dosimeters measure dose rates from all external radiation sources, including cosmic radiations, naturally occurring radioactivity in the environment, fallout from nuclear weapons testing, and any contribution from LLNL operations. Hence, these doses are not solely attributable to LLNL, and in most instances they are a measure of regional background. This is determined by comparing the perimeter measurements with those made at off-site (Livermore Valley) locations. Moreover, these off-site measurements are made at points of maximum exposure and assume an occupancy of 24 hours per day, 365 days per year. In actuality, no member of the public resides at these locations for extended periods of time; therefore, these estimates are conservative.

2. Ingestion dose. Potential doses from ingestion of locally produced foodstuff and surface water are based on actual measurements of radionuclide concentrations in the various media (determined by sampling). Conservative exposure data and current ICRP dosimetric factors are used to estimate doses to 
the individual. The following radionuclide exposure pathways are assessed by means of field measurements: tritium in water, tritium in milk, tritium in wine, and tritium in vegetation (which includes the forage-cow-milk pathway).

3. Inhalation immersion dose. Air pathway doses are calculated for each airborne radioactive discharge to the atmosphere. Inhalation and immersion doses are calculated for the site-wide maximally exposed individual (SW-MEI), as defined and discussed in Chapter 11, and at other perimeter points of interest. The amount of radioactivity released by LLNL usually is too low to measure radionuclide concentrations accurately in ambient air and to determine dose. Therefore, environmental transport and exposure pathway models are used to calculate potential doses from effluent emissions.

Dose calculations were made using the AIRDOS and CAP88-PC computer codes, as discussed in Chapter 11. These codes utilize the the Gaussian plume model (Peterson et al. 1976) and provide concentrations through sixteen 22.5-degree compass sectors and distances up to 80 kilometers from potential release points. Local meteorological data is input to the codes. These data were obtained from sensors on the LLNL meteorological tower from heights of 10 and 40 meters. From records of these data, wind speed, wind direction, and atmospheric stability estimates were tabulated at one-quarter-hour intervals during the calendar year.

4. Maximally exposed individual dose. This dose represents the maximum credible dose to a "hypothetical" member of the public. Examples are the dose to the SW-MEI as described in Chapter 11, or the maximum dose at or beyond the site perimeter in any direction. Contributions from all potential exposure pathways are included and this dose is derived using the most conservative but realistic exposure data and assumptions. This hypothetical person is assumed to reside at the point of highest ground-level radionuclide air concentration continuously for 24 hours per day, 365 days per year. During this occupancy period, a portion of this person's diet consists of locally produced foodstuffs and drinking water containing the highest measured radionuclide concentration. Thus, this is not a dose actually received by any individual and should be viewed as a conservative estimate (i.e., overestimate) of the highest possible dose to any member of the public.

5. Collective population dose. The collective population dose is the dose for all individuals residing within an 80-kilometer radius of the site. It is calculated by summing for each sector the average individual dose multiplied by the number of individuals residing in the sector. The collective population dose is referred to as the "collective effective dose equivalent"; it is expressed in terms of person-sievert (person-rem).

The following dose calculation methods for water, vegetation, meat and milk ingestion pathways, and for air inhalation and immersion pathways are outlined 


\section{Appendix B. Methods of Dose Calculations}

in conventional activity units of picocuries (pCi) and dose units of millirem (mrem). Below are the conversion constants that apply when converting to Systéme International (SI) activity units of becquerels $(\mathrm{Bq})$ and dose units of sieverts (Sv):

$$
\begin{aligned}
1 \mathrm{pCi} & =\left(3.7 \times 10^{-2}\right) \mathrm{Bq} \\
1 \mathrm{mrem} & =\left(1 \times 10^{-5}\right) \mathrm{Sv}=10 \mu \mathrm{Sv}=1 \times 10^{-2} \mathrm{mSv}
\end{aligned}
$$

These conversions can be applied universally throughout any section of this appendix.

\section{Annual Dose from Potable Water}

Based on the assumption that all water sampled is available as drinking water, the annual whole-body dose for tritium in mrem/y can be calculated using the following equation:

$D_{\text {whole body }}(\mathrm{mrem} / \mathrm{y})=\mathrm{C}_{\mathrm{w}} \times \mathrm{U}_{\mathrm{w}} \times \mathrm{D}_{\mathrm{w}}$

where

$$
\begin{aligned}
C_{\mathrm{w}}= & \text { concentration of tritium in water }(\mathrm{pCi} / \mathrm{L}) \\
U_{\mathrm{w}}= & \begin{array}{l}
\text { intake rate }(\mathrm{L} / \mathrm{y})=730 \mathrm{~L} / \mathrm{y} \text { for maximally exposed } \\
\text { individual }
\end{array} \\
D_{\mathrm{w}}= & \text { dose factor }(\mathrm{mrem} / \mathrm{pCi}) \\
= & 6.3 \times 10^{-8} \mathrm{mrem} / \mathrm{pCi} \text { for tritium for the whole-body } \\
& \text { ingestion pathway for an adult } \\
D_{\text {whole body }=} & \text { effective dose equivalent }(\text { mrem } / \mathrm{y}) \text { from ingestion of } 730 \mathrm{~L} \\
& \text { of potable water with concentration } \mathrm{C}_{\mathrm{w}}
\end{aligned}
$$

\section{Annual Dose from Forage-Cow-Milk Pathway for Tritium in Vegetation}

Based on the assumption that all feed for the cattle was pasture grass, the effective dose equivalent per $\mu \mathrm{Ci} / \mathrm{mL}$ of tritiated water (HTO) for the maximum exposed individual can be calculated using the following equation:

$$
D_{\text {whole body }}(\mathrm{mrem} / \mathrm{y})=D_{\text {veg }}+D_{\text {meat }}+D_{\text {milk }}
$$

where

$$
D_{\text {veg }}=\text { dose from ingestion of vegetables }
$$




$$
\begin{aligned}
& D_{\text {meat }}=\text { dose from ingestion of meat } \\
& D_{\text {milk }}=\text { dose from ingestion of milk }
\end{aligned}
$$

\section{Vegetation}

$$
D_{\text {veg }}(\text { leafy })=\mathrm{U}_{\text {veg }} \times \mathrm{C}_{\text {veg }} \times \mathrm{D}_{\mathrm{HTO}}
$$

where

$$
\begin{aligned}
U_{\mathrm{veg}}= & \begin{array}{l}
\text { intake rate }(\mathrm{kg} / \mathrm{y}): 64 \mathrm{~kg} / \mathrm{y} \text { for maximally exposed } \\
\text { individual }
\end{array} \\
C_{\mathrm{veg}}= & \text { concentration }(\mathrm{pCi} / \mathrm{kg}): 10^{9} \frac{\mathrm{pCi} / \mathrm{kg}}{\mu \mathrm{Ci} / \mathrm{mL}} \times\left(\mathrm{C}_{\mathrm{veg}}[\mu \mathrm{Ci} / \mathrm{mL}\right. \\
& \text { measured }]) \\
D_{\mathrm{HTO}}= & \begin{array}{l}
\text { dose factor }(\mathrm{mrem} / \mathrm{pCi}): 6.3 \times 10^{-8} \mathrm{mrem} / \mathrm{pCi} \text { for }{ }^{3} \mathrm{H} \text { for } \\
\text { the adult whole-body ingestion pathway }
\end{array}
\end{aligned}
$$

thus,

$$
D_{\text {veg }}(\mathrm{mrem} / \mathrm{y})=\left(0.40 \times 10^{4}\right) \times\left(C_{\mathrm{veg}}[\mu \mathrm{Ci} / \mathrm{mL} \text { measured }]\right)
$$

\section{Meat}

$$
D_{\text {meat }}(\mathrm{mrem} / \mathrm{y})=U_{\text {meat }} \times C_{\text {meat }} \times D_{\text {HTO }}
$$

where

$$
\begin{aligned}
& U_{\text {meat }}=\text { intake rate }(\mathrm{kg} / \mathrm{y}): 110 \mathrm{~kg} / \mathrm{y} \text { for maximally exposed } \\
& \text { individual } \\
& C_{\text {meat }}=\left(F_{\mathrm{f}}\right) \times\left(Q_{\mathrm{f}}\right) \times\left(C_{\text {veg }}\right) \times\left(\mathrm{e}^{\left[-\lambda_{i} t_{s}\right]}\right) \\
& D_{\text {HTO }}=\text { dose factor }(\mathrm{mrem} / \mathrm{pCi}): 6.3 \times 10^{-8} \mathrm{mrem} / \mathrm{pCi} \text { for }{ }^{3} \mathrm{H} \text { for } \\
& \text { the adult whole-body ingestion pathway } \\
& F_{\mathrm{f}}=\text { fraction of daily intake of nuclide per } \mathrm{kg} \text { of animal } / \mathrm{fish} \\
& \text { ( } \mathrm{pCi} / \mathrm{kg} \text { in meat per } \mathrm{pCi} / \mathrm{d} \text { ingested by the animal) }(\mathrm{d} / \mathrm{kg}) \\
& Q_{\mathrm{f}}=\text { amount } \text { of feed consumed }(\mathrm{kg} / \mathrm{d})
\end{aligned}
$$




$$
\begin{aligned}
C_{\text {veg }}= & \text { concentration }(\mathrm{pCi} / \mathrm{kg}): 10^{9} \frac{\mathrm{pCi} / \mathrm{kg}}{\mu \mathrm{Ci} / \mathrm{mL}} \\
& \times\left(\mathrm{C}_{\mathrm{veg}}[\mu \mathrm{Ci} / \mathrm{mL} \text { measured }]\right) \\
\lambda_{\mathrm{i}}= & \text { radiological decay constant }\left(\mathrm{d}^{-1}\right) \\
t_{\mathrm{s}}= & \text { time between slaughter to consumption }(\mathrm{d}) \\
C_{\text {meat }}= & \left(1.2 \times 10^{-2} \mathrm{~d} / \mathrm{kg}\right) \times(50 \mathrm{~kg} / \mathrm{d}) \times\left(C_{\mathrm{veg}}[\mu \mathrm{Ci} / \mathrm{mL}]\right) \\
& \left.\times\left(10^{9} \frac{\mathrm{pCi} / \mathrm{kg}}{\mu \mathrm{Ci} / \mathrm{mL}}\right) \times\left(\mathrm{e}\left[1-1.5 \times 10^{-4}\right] \times[20]\right]\right) \\
= & \left(0.6 \times 10^{9} \frac{\mathrm{pCi} / \mathrm{kg}}{\mu \mathrm{Ci} / \mathrm{mL}}\right) \times\left(C_{\mathrm{veg}}[\mu \mathrm{Ci} / \mathrm{mL} \text { measured }]\right)
\end{aligned}
$$

thus,

$$
\begin{aligned}
D_{\text {meat }}(\mathrm{mrem} / \mathrm{y})= & (110 \mathrm{~kg}) \times\left(\left[0.6 \times 10^{9} \frac{\mathrm{pCi} / \mathrm{kg}}{\mu \mathrm{Ci} / \mathrm{mL}}\right] \times\left[\mathrm{C}_{\text {veg }}\{\mu \mathrm{Ci} / \mathrm{mL} \text { measured }\}\right]\right) \\
& \times\left(6.3 \times 10^{-8} \mathrm{mrem} / \mathrm{pCi}\right) \\
= & \left(0.41 \times 10^{4}\right) \times\left(C_{\text {veg }}[\mu \mathrm{Ci} / \mathrm{mL} \text { measured }]\right)
\end{aligned}
$$

Milk

$$
D_{\text {milk }}(\mathrm{mrem} / \mathrm{y})=U_{\text {milk }} \times C_{\text {milk }} \times D_{\text {HTO }}
$$

where

$$
\begin{aligned}
U_{\text {milk }}= & \text { intake rate }(\mathrm{L} / \mathrm{y}): 310 \mathrm{~L} / \mathrm{y} \text { for maximally exposed } \\
& \text { individual } \\
D_{\mathrm{HTO}}= & \begin{array}{l}
\text { dose factor }(\mathrm{mrem} / \mathrm{pCi}): 6.3 \times 10^{-8} \mathrm{mrem} / \mathrm{pCi} \text { for }{ }^{3} \mathrm{H} \text { for } \\
\text { the adult whole-body ingestion pathway }
\end{array} \\
C_{\text {milk }}= & \left(F_{\mathrm{m}}\right) \times\left(Q_{\mathrm{f}}\right) \times\left(C_{\mathrm{veg}}\right) \times\left(\mathrm{e}^{\left[-\lambda_{i} t \mathrm{f}\right)}\right) \\
F_{\mathrm{m}}= & \text { fraction of daily intake of nuclide per liter of milk }(\mathrm{pCi} / \mathrm{L} \\
& \text { in milk per pCi } / \mathrm{d} \text { ingested by the animal })(\mathrm{d} / \mathrm{L}) \\
Q_{\mathrm{f}}= & \text { amount of feed consumed }(\mathrm{kg} / \mathrm{d}) \\
C_{\mathrm{veg}}= & \text { concentration }(\mathrm{pCi} / \mathrm{kg}):\left(10^{9} \frac{\mathrm{pCi} / \mathrm{mL}}{\mu \mathrm{Ci} / \mathrm{mL}}\right) \times\left(C_{\mathrm{veg}}[\mu \mathrm{Ci} / \mathrm{mL}\right. \\
& \text { measured }]) \\
\lambda_{\mathrm{i}}= & \text { radiological decay constant }\left(\mathrm{d}^{-1}\right)
\end{aligned}
$$




$$
\begin{aligned}
t_{\mathrm{f}}= & \text { transport time from milking to milk consumption }(\mathrm{d}) \\
C_{\text {milk }}= & \left(1.0 \times 10^{-2} \mathrm{~d} / \mathrm{L}\right) \times(50 \mathrm{~kg} / \mathrm{d}) \times\left(\mathrm{C}_{\mathrm{veg}}[\mu \mathrm{Ci} / \mathrm{mL}]\right) \\
& \times\left(10^{9} \frac{\mathrm{pCi} / \mathrm{mL}}{\mu \mathrm{Ci} / \mathrm{mL}}\right) \\
& \times\left(\mathrm{e}^{\left.\left(1-1.5 \times 10^{-4} / \times[2]\right]\right)}\right. \\
= & \left(0.5 \times 10^{9} \frac{\mathrm{pCi} / \mathrm{mL}}{\mu \mathrm{Ci} / \mathrm{mL}}\right) \times\left(C_{\text {veg }}[\mu \mathrm{Ci} / \mathrm{mL} \text { measured }]\right)
\end{aligned}
$$

thus,

$$
\begin{aligned}
D_{\text {milk }}(\text { mrem } / \mathrm{y})= & (310 \mathrm{~L} / \mathrm{y}) \times\left(6.3 \times 10^{-8} \mathrm{mrem} / \mathrm{pCi}\right) \\
& \times\left(\left[0.5 \times 10^{9} \frac{\mathrm{pCi} / \mathrm{L}}{\mu \mathrm{Ci} / \mathrm{mL}}\right] \times\left[\mathrm{C}_{\text {veg }}\{\mu \mathrm{Ci} / \mathrm{mL} \text { measured }\}\right]\right) \\
= & \left(0.97 \times 10^{4}\right) \times\left(C_{\text {veg }}[\mu \mathrm{Ci} / \mathrm{mL} \text { measured }]\right)
\end{aligned}
$$

Whole Body

$$
\begin{aligned}
D_{\text {whole body }}(\mathrm{mrem} / \mathrm{y}) & =\left(\left[0.40 \times 10^{4}\right] \times\left[C_{\text {veg }}\{\mu \mathrm{Ci} / \mathrm{mL} \text { measured } \mid]\right)\right. \\
& +\left(\left[0.41 \times 10^{4}\right] \times\left[C_{\text {veg }}\{\mu \mathrm{Ci} / \mathrm{mL} \text { measured }\}\right]\right) \\
& +\left(\left[0.97 \times 10^{4}\right] \times\left[C_{\text {veg }}\{\mu \mathrm{Ci} / \mathrm{mL} \text { measured }\}\right]\right)
\end{aligned}
$$

thus,

$$
\left.D_{\text {whole body }}(\mathrm{mrem} / \mathrm{y})=\left[1.78 \times 10^{4}\right] \times\left[C_{\text {veg }}\{\mu \mathrm{Ci} / \mathrm{mL} \text { measured }\}\right]\right)
$$

\section{Inhalation/Immersion Dose}

Inhalation Dose $\left({ }^{3} \mathrm{H}\right)$

$D_{\text {whole body }}(\mathrm{mrem} / \mathrm{y})=C \times U \times D$

where

$$
\begin{aligned}
C= & \begin{array}{r}
\text { radionuclide concentration at the receptor } \\
([F] \times[\chi / Q] \times[Q])
\end{array} \\
F= & \frac{1 \times 10^{12} \mathrm{pCi} / \mathrm{Ci}}{3.15 \times 10^{7} \mathrm{~s} / \mathrm{y}}=3.17 \times 10^{4} \\
\chi / Q= & \text { diffusion parameter }\left(\mathrm{s} / \mathrm{m}^{3}\right) \text { (calculated) } \\
Q= & \text { release rate, } \mathrm{Ci} / \mathrm{y}
\end{aligned}
$$




\section{Appendix B. Methods of Dose Calculations}

$$
\begin{aligned}
& U= \text { intake rate (inhalation rate for adult is } 8,400 \mathrm{~m}^{3} / \mathrm{y} \text { ) } \\
& D= \text { dose conversion factor (for }{ }^{3} \mathrm{H}: 9.45 \times 10^{-8} \mathrm{mrem} / \mathrm{pCi} \text {; the } \\
& \text { tritium dose conversion factor was obtained by } \\
& \text { multiplying the inhalation dose factor, } 6.3 \times 10^{-8} \\
& \text { mrem } / \mathrm{pCi} \text {, by } 1.5 \text { to include absorption through the skin) }
\end{aligned}
$$

thus

$$
\begin{aligned}
D_{\text {wholebody }}(\text { mrem } / \mathrm{y})= & \left(3.17 \times 10^{4}\left[\frac{\mathrm{pCi} / \mathrm{Ci}}{\mathrm{s} / \mathrm{y}}\right]\right) \times\left(\chi / \mathrm{Q}\left[\mathrm{s} / \mathrm{m}^{3}\right]\right) \times(\mathrm{Q}[\mathrm{Ci} / \mathrm{y}]) \\
& \times\left(8,400\left[\mathrm{~m}^{3} / \mathrm{y}\right]\right) \times(D[\mathrm{mrem} / \mathrm{pCi}])
\end{aligned}
$$

\section{External Immersion Dose $\left({ }^{13} \mathrm{~N}\right.$ and $\left.{ }^{15} \mathrm{O}\right)$}

This dose calculation was not used for 1992 because we had no ${ }^{13} \mathrm{~N}$ and ${ }^{15} \mathrm{O}$ emissions.

$$
D_{\text {whole body }}(\mathrm{mrem} / \mathrm{y})=\mathrm{C} \times(D R F)
$$

where $D R F$, the dose-rate factor, is the external dose-equivalent rate per unit radionuclide concentration, $\mathrm{mrem} / \mathrm{y}$ $\mathrm{pCi} / \mathrm{m}^{3}$

$$
\begin{aligned}
& \text { For }{ }^{13} \mathrm{~N}, D R F=5.11 \times 10^{-3} \frac{\mathrm{mrem} / \mathrm{y}}{\mathrm{pCi} / \mathrm{m}^{3}} \\
& \text { For }{ }^{15} \mathrm{O}, D R F=5.12 \times 10^{-3} \frac{\mathrm{mrem} / \mathrm{y}}{\mathrm{pCi} / \mathrm{m}^{3}}
\end{aligned}
$$

and

$$
\begin{aligned}
C & =\text { radionuclide concentration at the receptor }([F] \times[\chi / Q] \times[Q]) \\
F & =\frac{1 \times 10^{12} \mathrm{pCi} / \mathrm{Ci}}{3.15 \times 10^{7} \mathrm{~s} / \mathrm{y}}=3.17 \times 10^{4} \\
\chi / Q & =\text { diffusion parameter }\left(\mathrm{s} / \mathrm{m}^{3}\right) \text { (calculated) } \\
Q & =\text { release rate, } \mathrm{Ci} / \mathrm{y}
\end{aligned}
$$


thus,

$$
\begin{aligned}
D_{\text {wholebody }}(\mathrm{mrem} / \mathrm{y})= & \left(3.17 \times 10^{4}\left[\frac{\mathrm{pCi} / \mathrm{Ci}}{\mathrm{s} / \mathrm{y}}\right]\right) \times\left(\chi / \mathrm{Q}\left[\mathrm{s} / \mathrm{m}^{3}\right]\right) \times(\mathrm{Q}[\mathrm{Ci} / \mathrm{y}]) \\
& \times\left(\mathrm{DRF}\left[\frac{\mathrm{mrem} / \mathrm{y}}{\mathrm{pCi} / \mathrm{m}^{3}}\right]\right)
\end{aligned}
$$

\section{Annual Population Dose}

For population dose, the diffusion parameters and population figures for a sector $(i)$ within 80 kilometers of LLNL are summed over all directions $(n)$. These values are determined in person-mrem.

Dose (person-mrem $/ y)=(F) \times\left(\sum_{i}^{n}[\chi / Q]_{i} P_{i}\right) \times Q \times U \times D$

where

$$
F=\frac{1 \times 10^{12} \mathrm{pCi} / \mathrm{Ci}}{3.15 \times 10^{7} \mathrm{~s} / \mathrm{y}}=3.17 \times 10^{4}
$$

$$
\begin{aligned}
\left(\sum_{i}^{n}[\chi / Q]_{\mathrm{i}} \mathrm{P}_{i}\right)= & \begin{array}{l}
\text { Summation of the calculated }(\chi / Q) \text { values for sector } i \\
\text { times the population in sector } i\left(\mathrm{P}_{\mathrm{i}}\right) \text { for all regions } n, \\
\text { person-s } / \mathrm{m}^{3}
\end{array} \\
\mathrm{Q}= & \text { release rate, } \mathrm{Ci} / \mathrm{y} \\
U & =\text { inhalation rate, average adult individual, is } 8400 \mathrm{~m}^{3} / \mathrm{y} \\
D & =\text { dose factor, } \mathrm{mrem} / \mathrm{pCi}
\end{aligned}
$$




\section{Appendix C. Ground Water Protection Tables}

Table C-1. Parameters sampled in Well NC2-07, Site 300, 1992.

\begin{tabular}{|c|c|c|c|}
\hline Parameters & NC2-07 & Parameters & NC2-07 \\
\hline \multicolumn{2}{|c|}{ Purgeable pollutants (EPA Method $601 \mu \mathrm{g} / \mathrm{L}$ ) } & \multicolumn{2}{|l|}{ Radiologicals (Bq/L) } \\
\hline 1,1,1-Trichloroethane & 4 n.d at $<0.5$ & Gross alpha & $0.12 \pm 0.13$ \\
\hline $1,1,2,2 \cdot$ Tetrachloroethane & $4 \mathrm{n} . \mathrm{d}$ at $<0.5$ & & $0.19 \pm 0.13$ \\
\hline 1,1,2-Trichloroethane & 4 n.d at $<0.5$ & Gross beta & $0.22 \pm 0.07$ \\
\hline 1,1-Dichloroethane & 4 n.d at $<0.5$ & & $0.23 \pm 0.05$ \\
\hline 1,1-Dichloroethene & 4 n.d at $<0.5$ & Tritium & $-1.9 \pm 3.4$ \\
\hline 1,2-Dichlorobenzene & $4 \mathrm{n} . \mathrm{d}$ at $<0.5$ & & $-0.7 \pm 3.9$ \\
\hline 1,2-Dichloroethane & 4 n.d at $<0.5$ & & \\
\hline 1,2-Dichloroethene (total) & $4 \mathrm{n} . \mathrm{d}$ at $<0.5$ & & \\
\hline 1,2-Dichloropropane & 4 n.d at $<0.5$ & & \\
\hline 1,3-Dichlorobenzene & 4 n.d at $<0.5$ & & \\
\hline 1,4-Dichlorobenzene & 4 n.d at $<0.5$ & & \\
\hline 2-Chloroethylvinylether & 4 n.d at $<0.5$ & & \\
\hline Bromodichloromethane & $4 \mathrm{n} . \mathrm{d}$ at $<0.5$ & & \\
\hline Bromoform & 4 n.d at $<0.5$ & & \\
\hline Bromomethane & $4 \mathrm{n} . \mathrm{d}$ at $<0.5$ & & \\
\hline Carbon tetrachloride & 4 n.d at $<0.5$ & & \\
\hline Chlorobenzene & 4 n.d at $<0.5$ & & \\
\hline Chloroethane & 4 n.d at $<0.5$ & & \\
\hline Chloroform & 4 n.d at $<0.5$ & & \\
\hline Chloromethane & 4 n.d at $<0.5$ & & \\
\hline cis-1,2-Dichloroethene & 4 n.d at $<0.5$ & & \\
\hline cis-1,3-Dichloropropene & $4 \mathrm{n} . \mathrm{d}$ at $<0.5$ & & \\
\hline Dibromochloromethane & $4 \mathrm{n} . \mathrm{d}$ at $<0.5$ & & \\
\hline Dichlorodifluoromethane & $4 \mathrm{n} . \mathrm{d}$ at $<0.5$ & & \\
\hline Freon-113 & 4 n.d at $<0.5$ & & \\
\hline Methylene chloride & 4 n.d at $<0.5$ & & \\
\hline Tetrachloroethene & $4 \mathrm{n} . \mathrm{d}$ at $<0.5$ & & \\
\hline trans-1,2-Dichloroethene & $4 \mathrm{n} . \mathrm{d}$ at $<0.5$ & & \\
\hline trans-1,3-Dichloropropene & 4 n.d at $<0.5$ & & \\
\hline Trichloroethene & $4 \mathrm{n} . \mathrm{d}$ at $<0.5$ & & \\
\hline Trichlorofluoromethane & $4 \mathrm{n} . \mathrm{d}$ at $<0.5$ & & \\
\hline Vinyl chloride & $4 \mathrm{n} . \mathrm{d}$ at $<0.5$ & & \\
\hline
\end{tabular}

Note: The abbreviation "n.d." stands for nondetection. 


\section{Appendix C. Ground Water Protection Tables}

Table C-2. Parameters in the Pit 1 Area monitoring wells, Site 300, 1992.

\begin{tabular}{|c|c|c|c|c|}
\hline \multirow[b]{2}{*}{ Parameters } & \multicolumn{4}{|c|}{ Well } \\
\hline & K1-01C & K1-02B & K1-03 & K1-04 \\
\hline \multicolumn{5}{|l|}{ Physical and chemical (mg/L) } \\
\hline \multirow[t]{4}{*}{ Ammonia nitrogen (as $\mathrm{N}$ ) } & 0.02 & 0.02 & 0.02 & \\
\hline & 0.04 & 0.04 & 0.06 & \\
\hline & 0.04 & 0.03 & 0.03 & $\therefore$ \\
\hline & 0.033 & $<0.03$ & $<0.03$ & ذن. \\
\hline \multirow[t]{4}{*}{ Arsenic } & 0.011 & 0.01 & 0.012 & 0.01 \\
\hline & 0.01 & 0.009 & 0.015 & 0.011 \\
\hline & 0.011 & 0.01 & 0.013 & 0.01 \\
\hline & 0.01 & 0.01 & 0.013 & 0.011 \\
\hline Barium & 4 n.d. at $<0.05$ & 4 n.d. at $<0.05$ & 4 n.d. at $<0.05$ & 4 n.d. at $<0.05$ \\
\hline Beryllium & 4 n.d. at $<0.0002$ & 4 n.d. at $<0.0002$ & 4 n.d. at $<0.0002$ & 4 n.d. at $<0.0002$ \\
\hline \multirow[t]{4}{*}{ Bicarbonate alk (as $\mathrm{CaCO}_{3}$ ) } & 150 & 140 & 150 & 150 \\
\hline & 150 & 140 & 150 & 150 \\
\hline & 160 & 150 & 150 & 300 \\
\hline & 160 & 150 & 150 & 150 \\
\hline Cadmium & 4 n.d. at $<0.0005$ & 4 n.d. at $<0.0005$ & 4 n.d. at $<0.0005$ & 4 n.d. at $<0.0005$ \\
\hline \multirow[t]{4}{*}{ Calcium } & 51 & 47 & 48 & 52 \\
\hline & 47 & 54 & 51 & 45 \\
\hline & 47 & 50 & 44 & 46 \\
\hline & 51 & 51 & 42 & 45 \\
\hline \multicolumn{2}{|c|}{ Carbonate alk (as $\mathrm{CaCO}_{3}$ ) 4 n.d. at } & 4 n.d. at $<1$ & 4 n.d. at $<1$ & $\begin{array}{l}3 \text { n.d. at }<1 \\
1 \text { n.d. at }<2\end{array}$ \\
\hline \multirow[t]{4}{*}{ Chloride } & & 53 & 34 & 36 \\
\hline & 38 & 52 & 35 & 39 \\
\hline & 40 & 53 & 37 & 76 \\
\hline & 38 & 55 & 34 & 38 \\
\hline Chromium & 4 n.d. at $<0.005$ & 4 n.d. at $<0.005$ & 4 n.d. at $<0.005$ & 4 n.d. at $<0.005$ \\
\hline \multirow[t]{4}{*}{ Conductivity ( $\mu \mathrm{mhos} / \mathrm{cm})$} & 540 & 650 & 530 & 560 \\
\hline & 480 & 530 & 490 & 490 \\
\hline & 490 & 540 & 440 & 490 \\
\hline & 470 & 590 & 450 & 510 \\
\hline \multirow[t]{2}{*}{ Copper } & 4 n.d. at $<0.05$ & 4 n.d. at $<0.05$ & 4 n.d. at $<0.05$ & 3 n.d. at $<0.05$ \\
\hline & & & & 1 n.d. at $<0.1$ \\
\hline \multirow[t]{4}{*}{ Fluoride } & 0.4 & 0.4 & 0.3 & 0.4 \\
\hline & 0.4 & 0.4 & 0.3 & 0.4 \\
\hline & 0.4 & 0.3 & 0.3 & 0.8 \\
\hline & 0.32 & 0.3 & 0.31 & 0.38 \\
\hline Hydroxide alk (as $\mathrm{CaCO}_{3}$ ) & 4 n.d. at $<1$ & 4 n.d. at $<1$ & 4 n.d. at $<1$ & $\begin{array}{l}3 \text { n.d. at }<1 \\
1 \text { n.d. at }<2\end{array}$ \\
\hline \multirow[t]{2}{*}{ Iron } & 4 n.d. at $<0.1$ & 4 n.d. at $<0.1$ & 4 n.d. at $<0.1$ & 3 n.d. at $<0.1$ \\
\hline & & & & 1 n.d. at $<0.2$ \\
\hline
\end{tabular}

...continued 
Table C-2. Parameters in the Pit 1 Area monitoring wells, Site 300, 1992 (continued).

\begin{tabular}{|c|c|c|c|c|}
\hline \multirow[b]{2}{*}{ Parameters } & \multicolumn{4}{|c|}{ Well } \\
\hline & K1-05 & K1-07 & K1-08 & K1-09 \\
\hline \multicolumn{5}{|l|}{ Physical and chemical (mg/L) } \\
\hline \multirow[t]{4}{*}{ Ammonia nitrogen (as $N$ ) } & 0.02 & $<0.02$ & 0.02 & 0.02 \\
\hline & $<0.02$ & $<0.02$ & $<0.02$ & $<0.02$ \\
\hline & 0.08 & 0.04 & 0.04 & 0.04 \\
\hline & $<0.03$ & $<0.06$ & $<0.03$ & $<0.03$ \\
\hline \multirow[t]{4}{*}{ Arsenic } & 0.014 & 0.014 & 0.014 & 0.013 \\
\hline & 0.012 & 0.013 & 0.013 & 0.015 \\
\hline & 0.015 & 0.013 & 0.014 & 0.014 \\
\hline & 0.013 & 0.013 & 0.013 & 0.013 \\
\hline Barium & 4 n.d. at $<0.05$ & 4 n.d. at $<0.05$ & 4 n.d. at $<0.05$ & 4 n.d. at $<0.05$ \\
\hline Beryllium & 4 n.d. at $<0.0005$ & 4 n.d. at $<0.0005$ & 4 n.d. at $<0.0005$ & 4 n.d. at $<0.0005$ \\
\hline \multirow[t]{4}{*}{ Bicarbonate alk (as $\mathrm{CaCO}$} & 3) 130 & 140 & 130 & 250 \\
\hline & 130 & 280 & 90 & 130 \\
\hline & 130 & 140 & 130 & 130 \\
\hline & 130 & 280 & 130 & 130 \\
\hline Cadmium & 4 n.d. at $<0.0005$ & 4 n.d. at $<0.0005$ & 4 n.d. at $<0.0005$ & 4 n.d. at $<0.0005$ \\
\hline \multirow[t]{4}{*}{ Calcium } & 42 & 44 & 43 & 82 \\
\hline & 37 & 39 & 40 & 40 \\
\hline & 36 & 38 & 39 & 42 \\
\hline & 41 & 76 & 40 & 38 \\
\hline \multirow{2}{*}{\multicolumn{2}{|c|}{ Carbonate alk (as $\mathrm{CaCO}_{3}$ ) 4 n.d. at $<1$}} & 2 n.d. at $<1$ & 4 n.d. at $<1$ & 3 n.d. at $<1$ \\
\hline & & 2 n.d. at $<2$ & & 1 n.d. at $<2$ \\
\hline \multirow[t]{4}{*}{ Chloride } & 36 & 36 & 36 & 70 \\
\hline & 36 & 72 & 38 & 35 \\
\hline & 36 & 37 & 37 & 37 \\
\hline & 38 & 75 & 37 & 38 \\
\hline Chromium & 4 n.d. at $<0.005$ & 4 n.d. at $<0.005$ & 4 n.d. at $<0.005$ & 4 n.d. at $<0.005$ \\
\hline \multirow[t]{4}{*}{ Conductivity $(\mu \mathrm{mhos} / \mathrm{cm})$} & 520 & 550 & 570 & 540 \\
\hline & 480 & 460 & 480 & 490 \\
\hline & 460 & 440 & 450 & 470 \\
\hline & 480 & 460 & 520 & 440 \\
\hline \multirow[t]{2}{*}{ Copper } & 4 n.d. at $<0.05$ & 2 n.d. at $<0.05$ & 4 n.d. at $<0.05$ & $<0.1$ \\
\hline & & 2 n.d. at $<0.1$ & & $<0.05$ \\
\hline \multirow[t]{4}{*}{ Fluoride } & 0.5 & 0.5 & 0.5 & 1 \\
\hline & 0.5 & 1 & 0.5 & 0.5 \\
\hline & 0.4 & 0.4 & 0.4 & 0.4 \\
\hline & 0.48 & 0.84 & 0.48 & 0.5 \\
\hline \multirow{2}{*}{\multicolumn{2}{|c|}{ Hydroxide alk (as $\mathrm{CaCO}_{3}$ ) 4 n.d. at $<1$}} & 2 n.d. at < 1 & 4 n.d. at $<1$ & 3 n.d. at $<1$ \\
\hline & & 2 n.d. at $<2$ & & 1 n.d. at $<2$ \\
\hline \multirow[t]{2}{*}{ Iron } & 4 n.d. at $<0.1$ & 2 n.d. at $<0.1$ & 4 n.d. at $<0.1$ & 3 n.d. at $<0.1$ \\
\hline & & 2 n.d. at $<0.2$ & & 1 n.d. at $<0.2$ \\
\hline
\end{tabular}

...continued 


\section{Appendix C. Ground Water Protection Tables}

Table C-2. Parameters in the Pit 1 Area monitoring wells, Site 300, 1992 (continued).

\begin{tabular}{|c|c|c|c|c|}
\hline \multirow[b]{2}{*}{ Parameters } & \multicolumn{4}{|c|}{ Well } \\
\hline & K1-01C & K1-02B & $\mathrm{K} 1-03$ & K1-04 \\
\hline \multirow[t]{4}{*}{ Lead } & 0.006 & 0.007 & 0.002 & 0.002 \\
\hline & 0.006 & 0.002 & 0.02 & \\
\hline & 0.006 & & 0.002 & \\
\hline & 0.002 & & & \\
\hline \multirow[t]{4}{*}{ Magnesium } & 16 & 18 & 15 & 16 \\
\hline & 18 & 23 & 19 & 16 \\
\hline & 19 & 22 & 17 & 34 \\
\hline & 18 & 23 & 16 & 16 \\
\hline \multirow[t]{4}{*}{ Manganese } & 0.06 & $<0.05$ & $<0.05$ & $<0.05$ \\
\hline & $<0.05$ & $<0.05$ & $<0.05$ & $<0.05$ \\
\hline & $<0.05$ & $<0.05$ & $<0.05$ & $<0.1$ \\
\hline & $<0.05$ & $<0.05$ & $<0.05$ & $<0.1$ \\
\hline \multirow[t]{2}{*}{ Mercury } & & & 0.0002 & \\
\hline & 4 n.d. at $<0.0002$ & 4 n.d. at $<0.0002$ & 3 n.d. at $<0.0002$ & 4 n.d. at $<0.0002$ \\
\hline \multirow[t]{4}{*}{ Nickel } & $<0.005$ & $<0.005$ & $<0.005$ & $<0.005$ \\
\hline & $<0.1$ & $<0.1$ & $<0.1$ & $<0.1$ \\
\hline & $<0.005$ & $<0.005$ & $<0.005$ & $<0.005$ \\
\hline & 0.046 & 0.006 & 0.006 & $<0.005$ \\
\hline \multirow[t]{4}{*}{ Nitrate plus nitrite (as N) } & 7.1 & 7 & 6.4 & 5.5 \\
\hline & 6 & 5.8 & 5.2 & 4.6 \\
\hline & 7.5 & 7 & 6.2 & 10.9 \\
\hline & 7.3 & 7.1 & 6.7 & 5.6 \\
\hline \multirow[t]{4}{*}{ Nitrate $\left(\right.$ as $\left.\mathrm{NO}_{3}\right)$} & 31 & 31 & 28 & 24 \\
\hline & 25 & 25 & 23 & 20 \\
\hline & 33 & 31 & 28 & 24 \\
\hline & 32 & 31 & 30 & 25 \\
\hline \multirow[t]{2}{*}{ Nitrite (as N) } & $<0.01$ & $<0.01$ & $<0.01$ & $<0.02$ \\
\hline & 0.09 & $<0.01$ & $<0.01$ & $<0.01$ \\
\hline \multirow[t]{2}{*}{ Nitrite (as $\left.\mathrm{NO}_{2}\right)$} & $<0.03$ & $<0.03$ & $<0.03$ & $<0.06$ \\
\hline & 0.3 & $<0.03$ & $<0.03$ & $<0.03$ \\
\hline \multirow[t]{2}{*}{ Total Kjeldahl nitrogen } & 4 n.d. at $<0.5$ & 4 n.d. at $<0.5$ & 4 n.d. at $<0.5$ & 3 n.d. at $<0.5$ \\
\hline & & & & 1 n.d. at $<1$ \\
\hline \multirow[t]{2}{*}{ Low level phenolics } & 4 n.d. at $<0.005$ & 4 n.d. at $<0.005$ & 4 n.d. at $<0 . C \supset 5$ & 3 n.d. at $<0.005$ \\
\hline & & & & 1 n.d. at $<0.01$ \\
\hline \multirow[t]{4}{*}{$\mathrm{pH}$} & 7.5 & 7.5 & 7.6 & 7.6 \\
\hline & 7.6 & 7.6 & 7.6 & 7.6 \\
\hline & 7.6 & 7.5 & 7.4 & 7.5 \\
\hline & 7.1 & 7.1 & 7.2 & 7.1 \\
\hline
\end{tabular}


Table C-2. Parameters in the Pit 1 Area monitoring wells, Site 300, 1992 (continued).

\begin{tabular}{|c|c|c|c|c|}
\hline \multirow[b]{2}{*}{ Parameters } & \multicolumn{4}{|c|}{ Well } \\
\hline & K1-05 & K1-07 & K1-08 & K1-09 \\
\hline \multirow[t]{4}{*}{ Lead } & 0.003 & 0.005 & $<0.002$ & 0.002 \\
\hline & $<0.002$ & $<0.002$ & $<0.002$ & $<0.002$ \\
\hline & & $<0.002$ & $<0.002$ & $<0.002$ \\
\hline & & $<0.002$ & $<0.002$ & $<0.002$ \\
\hline \multirow[t]{4}{*}{ Magnesium } & 17 & 17 & 16 & 32 \\
\hline & 18 & 35 & 17 & 18 \\
\hline & 16 & 18 & 17 & 16 \\
\hline & 18 & 36 & 17 & 17 \\
\hline \multirow[t]{4}{*}{ Manganese } & $<0.05$ & $<0.05$ & $<0.05$ & $<0.1$ \\
\hline & $<0.05$ & $<0.1$ & $<0.05$ & $<0.05$ \\
\hline & $<0.05$ & $<0.05$ & $<0.05$ & $<0.05$ \\
\hline & $<0.05$ & $<0.1$ & $<0.05$ & $<0.05$ \\
\hline Mercury & 4 n.d. at $<0.0002$ & 4 n.d. at $<0.0002$ & 4 n.d. at $<0.0002$ & 4 n.d. at $<0.0002$ \\
\hline \multirow[t]{4}{*}{ Nickel } & $<0.005$ & $<0.005$ & 0.008 & $<0.005$ \\
\hline & $<0.1$ & $<0.1$ & 0.2 & $<0.1$ \\
\hline & $<0.005$ & $<0.005$ & 0.006 & $<0.005$ \\
\hline & $<0.005$ & $<0.005$ & $<0.005$ & $<0.005$ \\
\hline \multirow[t]{4}{*}{ Nitrate plus nitrite (as $\mathrm{N}$ ) } & 8.5 & 7.7 & 8.3 & 16.8 \\
\hline & 8.4 & 15.4 & 8.4 & 8.3 \\
\hline & 8.2 & 7.5 & 8 & 8 \\
\hline & 8.6 & 15.2 & 8.7 & 8.3 \\
\hline \multirow[t]{4}{*}{ Nitrate (as $\mathrm{NO}_{3}$ ) } & 37 & 34 & 37 & 37 \\
\hline & 37 & 34 & 37 & 36 \\
\hline & 36 & 33 & 35 & 35 \\
\hline & 38 & 32 & 38 & 37 \\
\hline \multirow[t]{2}{*}{ Nitrite (as N) } & $<0.01$ & $<0.01$ & $<0.01$ & $<0.01$ \\
\hline & $<0.01$ & $<0.02$ & $<0.01$ & $<0.01$ \\
\hline \multirow[t]{2}{*}{ Nitrite (as $\mathrm{NO}_{2}$ ) } & $<0.03$ & $<0.03$ & $<0.03$ & $<0.03$ \\
\hline & $<0.03$ & $<0.66$ & $<0.03$ & $<0.03$ \\
\hline \multirow[t]{2}{*}{ Total Kjeldahl nitrogen } & 4 n.d. at $<0.5$ & 2 n.d. at $<0.5$ & 4 n.d. at $<0.5$ & 3 n.d. at $<0.5$ \\
\hline & & 2 n.d. at $<1$ & & 1 n.d. at $<1$ \\
\hline \multirow[t]{2}{*}{ Low level phenolics } & 4 n.d. at $<0.005$ & 2 n.d. at $<0.005$ & 4 n.d. at $<0.005$ & 3 n.d. at $<0.005$ \\
\hline & & 2 n.d. at $<0.01$ & & 1 n.d. at $<0.01$ \\
\hline \multirow[t]{4}{*}{$\mathrm{pH}$} & 7.6 & 7.6 & 7.6 & 7.7 \\
\hline & 7.6 & 7.6 & 7.6 & 7.6 \\
\hline & 7.5 & 7.5 & 7.6 & 7.5 \\
\hline & 7.4 & 7.3 & 7.4 & 7.4 \\
\hline
\end{tabular}


Table C-2. Parameters in the Pit 1 Area monitoring wells, Site 300, 1992 (continued).

\begin{tabular}{|c|c|c|c|c|}
\hline \multirow[b]{2}{*}{ Parameters } & \multicolumn{4}{|c|}{ Well } \\
\hline & K1-01C & K1-02B & K1-03 & K1-04 \\
\hline \multirow[t]{4}{*}{ Potassium } & 3 & 2 & 2 & 2 \\
\hline & 6 & 3 & 3 & 3 \\
\hline & 6 & 3 & 2 & 3 \\
\hline & $<5$ & $<5$ & $<5$ & $<5$ \\
\hline \multirow[t]{4}{*}{ Selenium } & 0.003 & 0.002 & 0.003 & $<0.002$ \\
\hline & $<0.002$ & 0.003 & $<0.002$ & 0.003 \\
\hline & 0.004 & $<0.002$ & 0.004 & 0.005 \\
\hline & $<0.002$ & $<0.002$ & $<0.002$ & $<0.002$ \\
\hline Silver & n.d. at $<0.0005$ & 4 n.d. at $<0.0005$ & 4 n.d. at $<0.0005$ & 4 n.d. at $<0.0005$ \\
\hline \multirow[t]{4}{*}{ Sodium } & 35 & 32 & 31 & 37 \\
\hline & 34 & 38 & 38 & 35 \\
\hline & 36 & 39 & 34 & 41 \\
\hline & 35 & 38 & 29 & 36 \\
\hline \multirow[t]{4}{*}{ Sulfate } & 42 & 64 & 39 & 54 \\
\hline & 39 & 65 & 39 & 53 \\
\hline & 40 & 60 & 40 & 107 \\
\hline & 42 & 68 & 41 & 54 \\
\hline \multirow[t]{4}{*}{ Total alkalinity $\left(\mathrm{as} \mathrm{CaCO}_{3}\right)$} & 150 & 140 & 150 & 150 \\
\hline & 150 & 140 & 150 & 150 \\
\hline & 160 & 150 & 150 & 300 \\
\hline & 160 & 150 & 150 & 150 \\
\hline \multirow[t]{4}{*}{ Total organic carbon (TOC) } & 1 & 1.2 & 0.7 & 0.7 \\
\hline & 1 & 0.8 & 0.6 & 0.6 \\
\hline & 0.9 & 1 & 0.7 & 0.8 \\
\hline & 0.7 & 0.8 & 0.7 & 0.6 \\
\hline Total organic haiides (TOX) & \multicolumn{2}{|c|}{8 n.d. at $<0.028$ n.d. at $<0.02$} & 8 n.d. at $<0.02$ & 8 n.d. at $<0.02$ \\
\hline \multirow[t]{4}{*}{ Total dissolved solids (TDS) } & 340 & 400 & 340 & 360 \\
\hline & 360 & 380 & 350 & 370 \\
\hline & 360 & 400 & 350 & 750 \\
\hline & 390 & 440 & 330 & 340 \\
\hline \multirow[t]{3}{*}{ Total suspended solids (TSS) } & 2 & $<1$ & $<1$ & $<1$ \\
\hline & 4 & $<1$ & 3 & $<1$ \\
\hline & 14 & 2 & $<1$ & $<1$ \\
\hline \multirow[t]{4}{*}{ Vanadium } & 0.06 & $<0.05$ & $<0.05$ & $<0.05$ \\
\hline & 0.05 & 0.07 & $<0.05$ & $<0.05$ \\
\hline & $<0.05$ & $<0.05$ & $<0.05$ & $<0.05$ \\
\hline & 0.054 & 0.062 & $<0.05$ & 0.054 \\
\hline \multirow[t]{2}{*}{ Zinc } & 4 n.d. at $<0.05$ & 4 n.d. at $<0.05$ & 4 n.d. at $<0.05$ & 3 n.d. at $<0.05$ \\
\hline & & & & 1 n.d. at $<0.1$ \\
\hline
\end{tabular}




\section{Appendix C. Ground Water Protection Tables}

Table C-2. Parameters in the Pit 1 Area monitoring wells, Site 300, 1992 (continued).

\begin{tabular}{|c|c|c|c|c|}
\hline \multirow[b]{2}{*}{ Parameters } & \multicolumn{4}{|c|}{ Well } \\
\hline & K1-05 & K1-07 & K1-08 & K1-09 \\
\hline \multirow[t]{4}{*}{ Potassium } & 2 & 2 & 2 & 5 \\
\hline & 3 & 6 & 3 & 3 \\
\hline & 2 & 3 & 3 & 3 \\
\hline & $<5$ & $<10$ & $<5$ & $<5$ \\
\hline \multirow[t]{4}{*}{ Selenium } & $<0.002$ & $<0.002$ & $<0.002$ & $<0.002$ \\
\hline & $<0.002$ & 0.002 & $<0.002$ & $<0.002$ \\
\hline & 0.002 & 0.004 & 0.003 & 0.003 \\
\hline & $<0.002$ & $<0.002$ & $<0.002$ & $<0.002$ \\
\hline Silver & n.d. at $<0.0005$ & 4 n.d. at $<0.0005$ & 4 n.d. at $<0.0005$ & 4 n.d. at $<0.0005$ \\
\hline \multirow[t]{4}{*}{ Sodium } & 38 & 40 & 38 & 75 \\
\hline & 44 & 45 & 40 & 43 \\
\hline & 37 & 41 & 43 & 38 \\
\hline & 42 & 79 & 39 & 40 \\
\hline \multirow[t]{4}{*}{ Sulfate } & 42 & 44 & 45 & 96 \\
\hline & 41 & 82 & 44 & 48 \\
\hline & 45 & 45 & 46 & 49 \\
\hline & 45 & 92 & 46 & 48 \\
\hline \multirow[t]{4}{*}{ Total alkalinity (as $\mathrm{CaCO}_{3}$ ) } & 130 & 140 & 130 & 250 \\
\hline & 130 & 280 & 90 & 130 \\
\hline & 130 & 140 & 130 & 130 \\
\hline & 130 & 280 & 130 & 130 \\
\hline \multirow[t]{4}{*}{ Total organic carbon (TOC) } & 0.9 & 0.8 & 0.7 & 0.7 \\
\hline & 0.8 & 0.8 & 0.8 & 0.7 \\
\hline & 0.7 & 0.7 & 0.7 & 0.7 \\
\hline & 0.7 & 0.7 & 0.6 & 0.7 \\
\hline Total organic halides (TOX) & 8 n.d. at $<0.02$ & 8 n.d. at $<0.02$ & 8 n.d. at $<0.02$ & 8 n.d. at $<0.02$ \\
\hline \multirow[t]{4}{*}{ Total dissolved solids (TDS) } & 330 & 340 & 340 & 670 \\
\hline & 310 & 760 & 360 & 360 \\
\hline & 360 & 360 & 360 & 350 \\
\hline & 310 & 750 & 320 & 320 \\
\hline \multirow[t]{3}{*}{ Total suspended solids (TSS) } & $<1$ & $<1$ & $<1$ & 10 \\
\hline & 1 & 1 & $<1$ & 1 \\
\hline & $<1$ & 10 & $<1$ & 3 \\
\hline \multirow[t]{4}{*}{ Vanadium } & 0.07 & 0.07 & 0.07 & $<0.05$ \\
\hline & 0.07 & 0.06 & 0.07 & 0.07 \\
\hline & $<0.05$ & $<0.05$ & $<0.05$ & $<0.05$ \\
\hline & 0.063 & 0.093 & 0.066 & 0.072 \\
\hline \multirow[t]{2}{*}{ Zinc } & 4 n.d. at $<0.05$ & 2 n.d. at $<0.05$ & 4 n.d. at $<0.05$ & 3 n.d. at $<0.05$ \\
\hline & & 2 n.d. at $<0.1$ & & 1 n.d. at $<0.1$ \\
\hline
\end{tabular}


Table C-2. Parameters in the Pit 1 Area monitoring wells, Site 300, 1992 (continued).

\begin{tabular}{|c|c|c|c|c|}
\hline \multirow[b]{2}{*}{ Parameters } & \multicolumn{4}{|c|}{ Well } \\
\hline & K1-01C & $\mathrm{K} 1-02 \mathrm{~B}$ & K1-03 & K1-04 \\
\hline \multicolumn{5}{|c|}{ Purgeable pollutants (EPA Method $601, \mu \mathrm{g} / \mathrm{L}$ ) } \\
\hline $1,1,1-$ Trichloroethane & 4 n.d. at $<0.5$ & 4 n.d. at $<0.5$ & 4 n.d. at $<0.5$ & $\begin{array}{l}3 \text { n.d. at }<0.5 \\
1 \text { n.d. at }<1\end{array}$ \\
\hline 1,1,2,2-Tetrachloroethane & 4 n.d. at $<0.5$ & 4 n.d. at $<0.5$ & 4 n.d. at $<0.5$ & $\begin{array}{l}3 \text { n.d. at }<0.5 \\
1 \text { n.d. at }<1\end{array}$ \\
\hline 1,1,2-Trichloroethane & 4 n.d. at $<0.5$ & 4 n.d. at $<0.5$ & 4 n.d. at $<0.5$ & $\begin{array}{l}3 \text { n.d. at }<0.5 \\
1 \text { n.d. at }<1\end{array}$ \\
\hline 1,1-Dichloroethane & 4 n.d. at $<0.5$ & 4 n.d. at $<0.5$ & 4 n.d. at $<0.5$ & $\begin{array}{l}3 \text { n.d. at }<0.5 \\
1 \text { n.d. at }<1\end{array}$ \\
\hline 1,1-Dichloroethene & 4 n.d. at $<0.5$ & 4 n.d. at $<0.5$ & 4 n.d. at $<0.5$ & $\begin{array}{l}3 \text { n.d. at }<0.5 \\
1 \text { n.d. at }<1\end{array}$ \\
\hline 1,2-Dichlorobenzene & 4 n.d. at $<0.5$ & 4 n.d. at $<0.5$ & 4 n.d. at $<0.5$ & $\begin{array}{l}3 \text { n.d. at }<0.5 \\
1 \text { n.d. at }<1\end{array}$ \\
\hline 1,2-Dichloroethane & 4 n.d. at $<0.5$ & 4 n.d. at $<0.5$ & 4 n.d. at $<0.5$ & $\begin{array}{l}3 \text { n.d. at }<0.5 \\
1 \text { n.d. at }<1\end{array}$ \\
\hline 1,2-Dichloroethene (total) & 4 n.d. at $<0.5$ & 4 n.d. at $<0.5$ & 4 n.d. at $<0.5$ & $\begin{array}{l}3 \text { n.d. at }<0.5 \\
1 \text { n.d. at }<1\end{array}$ \\
\hline 1,2-Dichloropropane & 4 n.d. at $<0.5$ & 4 n.d. at $<0.5$ & 4 n.d. at $<0.5$ & $\begin{array}{l}3 \text { n.d. at }<0.5 \\
1 \text { n.d. at }<1\end{array}$ \\
\hline 1,3-Dichlorobenzene & 4 n.d. at $<0.5$ & 4 n.d. at $<0.5$ & 4 n.d. at $<0.5$ & $\begin{array}{l}3 \text { n.d. at }<0.5 \\
1 \text { n.d. at }<1\end{array}$ \\
\hline 1,4-Dichlorobenzene & 4 n.d. at $<0.5$ & 4 n.d. at $<0.5$ & 4 n.d. at $<0.5$ & $\begin{array}{l}3 \text { n.d. at }<0.5 \\
1 \text { n.d. at }<1\end{array}$ \\
\hline 2-Cnloroethylvinylether & 4 n.d. at $<0.5$ & 4 n.d. at $<0.5$ & 4 n.d. at $<0.5$ & $\begin{array}{l}3 \text { n.d. at }<0.5 \\
1 \text { n.d. at }<1\end{array}$ \\
\hline Bromodichloromethane & 4 n.d. at $<0.5$ & 4 n.d. at $<0.5$ & 4 n.d. at $<0.5$ & $\begin{array}{l}3 \text { n.d. at }<0.5 \\
1 \text { n.d. at }<1\end{array}$ \\
\hline Bromoform & 4 n.d. at $<0.5$ & 4 n.d. at $<0.5$ & 4 n.d. at $<0.5$ & $\begin{array}{l}3 \text { n.d. at }<0.5 \\
1 \text { n.d. at }<1\end{array}$ \\
\hline Bromomethane & 4 n.d. at $<0.5$ & 4 n.d. at $<0.5$ & 4 n.d. at $<0.5$ & $\begin{array}{l}3 \text { n.d. at }<0.5 \\
1 \text { n.d. at }<1\end{array}$ \\
\hline Carbon tetrachloride & 4 n.d. at $<0.5$ & 4 n.d. at $<0.5$ & 4 n.d. at $<0.5$ & $\begin{array}{l}3 \text { n.d. at }<0.5 \\
1 \text { n.d. at }<1\end{array}$ \\
\hline Chlorobenzene & 4 n.d. at $<0.5$ & 4 n.d. at $<0.5$ & 4 n.d. at $<0.5$ & $\begin{array}{l}3 \text { n.d. at }<0.5 \\
1 \text { n.d. at }<1\end{array}$ \\
\hline Chloroethane & 4 n.d. at $<0.5$ & 4 n.d. at $<0.5$ & 4 n.d. at $<0.5$ & $\begin{array}{l}3 \text { n.d. at }<0.5 \\
1 \text { n.d. at }<1\end{array}$ \\
\hline Chloroform & 4 n.d. at $<0.5$ & 4 n.d. at $<0.5$ & 4 n.d. at $<0.5$ & $\begin{array}{l}3 \text { n.d. at }<0.5 \\
1 \text { n.d. at }<1\end{array}$ \\
\hline Chloromethane & 4 n.d. at $<0.5$ & 4 n.d. at $<0.5$ & 4 n.d. at $<0.5$ & $\begin{array}{l}3 \text { n.d. at }<0.5 \\
1 \text { n.d. at }<1\end{array}$ \\
\hline cis-1,2-Dichloroethene & 4 n.d. at $<0.5$ & 4 n.d. at $<0.5$ & 4 n.d. at $<0.5$ & $\begin{array}{l}3 \text { n.d. at }<0.5 \\
1 \text { n.d. at }<1\end{array}$ \\
\hline
\end{tabular}

c..continued 
Table C-2. Parameters in the Pit 1 Area monitoring wells, Site 300, 1992 (continued).

\begin{tabular}{|c|c|c|c|c|}
\hline \multirow[b]{2}{*}{ Parameters } & \multicolumn{4}{|c|}{ Well } \\
\hline & K1-05 & K1-07 & $\mathrm{K} 1-08$ & K1.09 \\
\hline \multicolumn{5}{|c|}{ Purgeable pollutants (EPA Method 601, $\mu \mathrm{g} / \mathrm{L}$ ) } \\
\hline \multirow[t]{2}{*}{ 1,1,1-Trichloroethane } & 4 n.d. at $<0.5$ & 2 n.d. at $<0.5$ & 4 n.d. at $<0.5$ & 3 n.d. at $<0.5$ \\
\hline & & 2 n.d. at $<1$ & & 1 n.d. at $<1$ \\
\hline \multirow[t]{2}{*}{$1,1,2,2$-Tetrachloroethane } & 4 n.d. at $<0.5$ & 2 n.d. at $<0.5$ & 4 n.d. at $<0.5$ & 3 n.d. at $<0.5$ \\
\hline & & 2 n.d. at $<1$ & & 1 n.d. at $<1$ \\
\hline \multirow[t]{2}{*}{ 1,1,2-Trichloroethane } & 4 n.d. at $<0.5$ & 2 n.d. at $<0.5$ & 4 n.d. at $<0.5$ & 3 n.d. at $<0.5$ \\
\hline & & 2 n.d. at $<1$ & & 1 n.d. at $<1$ \\
\hline \multirow[t]{2}{*}{ 1,1-Dichloroethane } & 4 n.d. at $<0.5$ & 2 n.d. at $<0.5$ & 4 n.d. at $<0.5$ & 3 n.d. at $<0.5$ \\
\hline & & 2 n.d. at $<1$ & & 1 n.d. at $<1$ \\
\hline \multirow[t]{2}{*}{ 1,1-Dichloroethene } & 4 n.d. at $<0.5$ & 2 n.d. at $<0.5$ & 4 n.d. at $<0.5$ & 3 n.d. at $<0.5$ \\
\hline & & 2 n.d. at $<1$ & & 1 n.d. at $<1$ \\
\hline \multirow[t]{2}{*}{ 1,2-Dichlorobenzene } & 4 n.d. at $<0.5$ & 2 n.d. at $<0.5$ & 4 n.d. at $<0.5$ & 3 n.d. at $<0.5$ \\
\hline & & 2 n.d. at $<1$ & & 1 n.d. at $<1$ \\
\hline 1,2-Dichloroethane & 4 n.d. at $<0.5$ & 2 n.d. at $<0.5$ & 4 n.d. at $<0.5$ & 3 n.d. at $<0.5$ \\
\hline 1,2-Dichloroethene (total) & 4 n.d. at $<0.5$ & $\begin{array}{l}2 \text { n.d. at }<1 \\
2 \text { n.d. at }<0.5\end{array}$ & 4 n.d. at $<0.5$ & $\begin{array}{l}1 \text { n.d. at }<1 \\
3 \text { n.d. at }<0.5\end{array}$ \\
\hline 1,2-Dichloropropane & 4 n.d. at $<0.5$ & $\begin{array}{l}2 \text { n.d. at }<1 \\
2 \text { n.d. at }<0.5\end{array}$ & 4 n.d. at $<0.5$ & $\begin{array}{l}1 \mathrm{n} . \text { d. at }<1 \\
3 \text { n.d. at }<0.5\end{array}$ \\
\hline 1,3-Dichlorobenzene & 4 n.d. at $<0.5$ & $\begin{array}{l}2 \text { n.d. at }<1 \\
2 \text { n.d. at }<0.5\end{array}$ & 4 n.d. at $<0.5$ & $\begin{array}{l}1 \text { n.d. at }<1 \\
3 \text { n.d. at }<0.5\end{array}$ \\
\hline 1,4-Dichlorobenzene & 4 n.d. at $<0.5$ & $\begin{array}{l}2 \text { n.d. at }<1 \\
2 \text { n.d. at }<0.5\end{array}$ & 4 n.d. at $<0.5$ & $\begin{array}{l}1 \text { n.d. at }<1 \\
3 \text { n.d. at }<0.5\end{array}$ \\
\hline \multirow[t]{2}{*}{ 2-Chloroethylvinylether } & 4 n.d. at $<0.5$ & $\begin{array}{l}2 \text { n.d. at }<1 \\
2 \text { n.d. at }<0.5\end{array}$ & 4 n.d. at $<0.5$ & $\begin{array}{l}1 \text { n.d. at }<1 \\
3 \text { n.d. at }<0.5\end{array}$ \\
\hline & & 2 n.d. at $<1$ & & 1 n.d. at $<1$ \\
\hline \multirow[t]{2}{*}{ Bromodichloromethane } & 4 n.d. at $<0.5$ & 2 n.d. at $<0.5$ & 4 n.d. at $<0.5$ & 3 n.d. at $<0.5$ \\
\hline & & 2 n.d. at $<1$ & & 1 n.d. at $<1$ \\
\hline \multirow[t]{2}{*}{ Bromoform } & 4 n.d. at $<0.5$ & 2 n.d. at $<0.5$ & 4 n.d. at $<0.5$ & 3 n.d. at $<0.5$ \\
\hline & & 2 n.d. at $<1$ & & 1 n.d. at $<1$ \\
\hline \multirow[t]{2}{*}{ Bromomethane } & 4 n.d. at $<0.5$ & 2 n.d. at $<0.5$ & 4 n.d. at $<0.5$ & 3 n.d. at $<0.5$ \\
\hline & & 2 n.d. at $<1$ & & 1 n.d. at $<1$ \\
\hline \multirow[t]{2}{*}{ Carbon tetrachloride } & 4 n.d. at $<0.5$ & 2 n.d. at $<0.5$ & 4 n.d. at $<0.5$ & 3 n.d. at $<0.5$ \\
\hline & & 2 n.d. at $<1$ & & 1 n.d. at $<1$ \\
\hline \multirow[t]{2}{*}{ Chlorobenzene } & 4 n.d. at $<0.5$ & 2 n.d. at $<0.5$ & 4 n.d. at $<0.5$ & 3 n.d. at $<0.5$ \\
\hline & & 2 n.d. at $<1$ & & 1 n.d. at $<1$ \\
\hline \multirow[t]{2}{*}{ Chloroethane } & 4 n.d. at $<0.5$ & 2 n.d. at $<0.5$ & 4 n.d. at $<0.5$ & 3 n.d. at $<0.5$ \\
\hline & & 2 n.d. at $<1$ & & 1 n.d. at $<1$ \\
\hline \multirow[t]{2}{*}{ Chloroform } & 4 n.d. at $<0.5$ & 2 n.d. at $<0.5$ & 4 n.d. at $<0.5$ & 3 n.d. at $<0.5$ \\
\hline & & 2 n.d. at $<1$ & & 1 n.d. at $<1$ \\
\hline \multirow[t]{2}{*}{ Chloromethane } & 4 n.d. at $<0.5$ & 2 n.d. at $<0.5$ & 4 n.d. at $<0.5$ & 3 n.d. at $<0.5$ \\
\hline & & 2 n.d. at $<1$ & & 1 n.d. at $<1$ \\
\hline \multirow[t]{2}{*}{ cis-1,2-Dichloroethene } & 4 n.d. at $<0.5$ & 2 n.d. at $<0.5$ & 4 n.d. at $<0.5$ & 3 n.d. at $<0.5$ \\
\hline & & 2 n.d. at $<1$ & & 1 n.d. at $<1$ \\
\hline
\end{tabular}

...continued 
Table C-2. Parameters in the Pit 1 Area monitoring wells, Site 300, 1992 (continued).

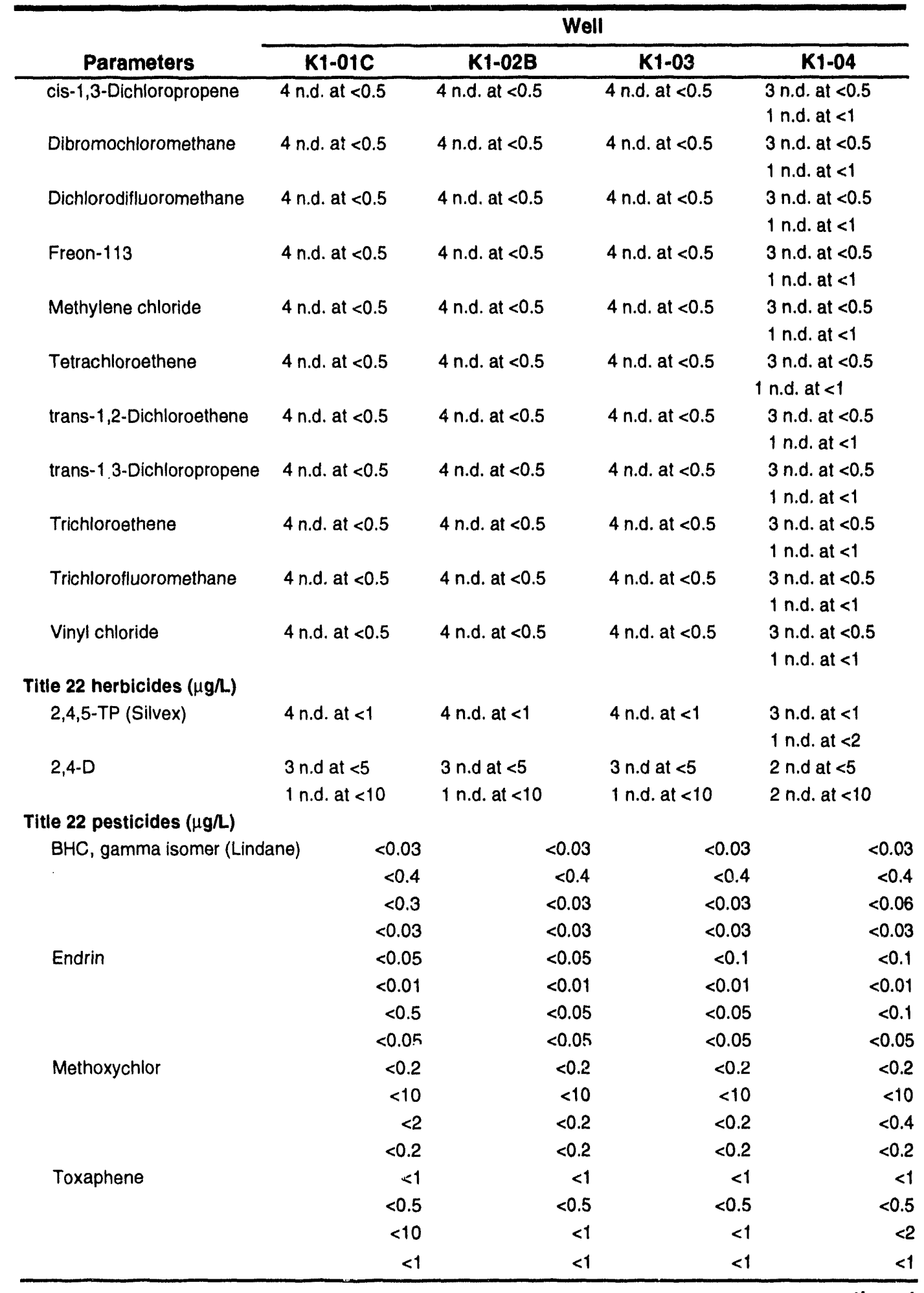


Table C-2. Parameters in the Pit 1 Area monitoring wells, Site 300, 1992 (continued).

\begin{tabular}{|c|c|c|c|c|}
\hline \multirow[b]{2}{*}{ Parameters } & \multicolumn{4}{|c|}{ Well } \\
\hline & K1-05 & K1-07 & K1-08 & K1-09 \\
\hline cis-1,3-Dichloropropene & 4 n.d. at $<0.5$ & $\begin{array}{l}2 \text { n.d. at }<0.5 \\
2 \text { n.d. at }<1\end{array}$ & 4 n.d. at $<0.5$ & $\begin{array}{l}3 \text { n.d. at }<0.5 \\
1 \text { n.d. at }<1\end{array}$ \\
\hline Dibromochloromethane & 4 n.d. at $<0.5$ & $\begin{array}{l}2 \text { n.d. at }<0.5 \\
2 \text { n.d. at }<1\end{array}$ & 4 n.d. at $<0.5$ & $\begin{array}{l}3 \text { n.d. at }<0.5 \\
1 \text { n.d. at }<1\end{array}$ \\
\hline Dichlorodifluoromethane & 4 n.d. at $<0.5$ & $\begin{array}{l}2 \text { n.d. at }<0.5 \\
2 \text { n.d. at }<1\end{array}$ & 4 n.d. at $<0.5$ & $\begin{array}{l}3 \text { n.d. at }<0.5 \\
1 \text { n.d. at }<1\end{array}$ \\
\hline \multirow[t]{4}{*}{ Freon-113 } & 2 & 2 n.d. at $<0.5$ & 20 & 59 \\
\hline & $<0.5$ & 2 n.d. at $<1$ & 13 & 41 \\
\hline & 3 & & 26 & 49 \\
\hline & 3 & & 14 & 37 \\
\hline Methylene chloride & 4 n.d. at $<0.5$ & $\begin{array}{l}2 \text { n.d. at }<0.5 \\
2 \text { n.d. at }<1\end{array}$ & 4 n.d. at $<0.5$ & $\begin{array}{l}3 \text { n.d. at }<0.5 \\
1 \text { n.d. at }<1\end{array}$ \\
\hline Tetrachloroethene & 4 n.d. at $<0.5$ & $\begin{array}{l}2 \text { n.d. at }<0.5 \\
2 \text { n.d. at }<1\end{array}$ & 4 n.d. at $<0.5$ & $\begin{array}{l}3 \text { n.d. at }<0.5 \\
1 \text { n.d. at }<1\end{array}$ \\
\hline trans-1,2-Dichloroethene & 4 n.d. at $<0.5$ & $\begin{array}{l}2 \text { n.d. at }<0.5 \\
2 \text { n.d. at }<1\end{array}$ & 4 n.d. at $<0.5$ & $\begin{array}{l}3 \text { n.d. at }<0.5 \\
1 \text { n.d. at }<1\end{array}$ \\
\hline trans-1,3-Dichloropropene & 4 n.d. at $<0.5$ & $\begin{array}{l}2 \text { n.d. at }<0.5 \\
2 \text { n.d. at }<1\end{array}$ & 4 n.d. at $<0.5$ & $\begin{array}{l}3 \text { n.d. at }<0.5 \\
1 \text { n.d. at }<1\end{array}$ \\
\hline Trichloroethene & 4 n.d. at $<0.5$ & $\begin{array}{l}2 \text { n.d. at }<0.5 \\
2 \text { n.d. at }<1\end{array}$ & 4 n.d. at $<0.5$ & $\begin{array}{l}3 \text { n.d. at }<0.5 \\
1 \text { n.d. at }<1\end{array}$ \\
\hline Trichlorofluoromethane & 4 n.d. at $<0.5$ & $\begin{array}{l}2 \text { n.d. at }<0.5 \\
2 \text { n.d. at }<1\end{array}$ & 4 n.d. at $<0.5$ & $\begin{array}{l}3 \text { n.d. at }<0.5 \\
1 \text { n.d. at }<1\end{array}$ \\
\hline Vinyl chloride & 4 n.d. at $<0.5$ & $\begin{array}{l}2 \text { n.d. at }<0.5 \\
2 \text { n.d. at }<1\end{array}$ & 4 n.d. at $<0.5$ & $\begin{array}{l}3 \text { n.d. at }<0.5 \\
1 \text { n.d. at }<1\end{array}$ \\
\hline \multicolumn{5}{|l|}{ Title 22 herbicides ( $\mu \mathrm{g} / \mathrm{L}$ ) } \\
\hline 2,4,5-TP (Silvex) & 4 n.d. at $<1$ & $\begin{array}{l}2 \text { n.d. at }<1 \\
2 \text { n.d. at }<2\end{array}$ & 4 n.d. at $<1$ & $\begin{array}{l}3 \text { n.d. at }<1 \\
1 \text { n.d. at }<2\end{array}$ \\
\hline $2,4-D$ & $\begin{array}{l}3 \text { n.d at }<5 \\
1 \text { n.d. at }<10\end{array}$ & $\begin{array}{l}1 \text { n.d at }<5 \\
3 \text { n.d. at }<10\end{array}$ & $\begin{array}{l}3 \text { n.d at }<5 \\
1 \text { n.d. at }<10\end{array}$ & $\begin{array}{l}3 \text { n.d at }<5 \\
1 \text { n.d. at }<20\end{array}$ \\
\hline \multicolumn{5}{|l|}{ Title 22 pesticides $(\mu \mathrm{g} / \mathrm{L})$} \\
\hline \multirow[t]{4}{*}{ BHC, gamma isomer (Lindan } & $<0.03$ & $<0.03$ & $<0.03$ & $<0.06$ \\
\hline & $<0.4$ & $<0.8$ & $<0.4$ & $<0.4$ \\
\hline & $<0.03$ & $<0.03$ & $<0.03$ & $<0.03$ \\
\hline & $<0.03$ & $<0.06$ & $<0.03$ & $<0.03$ \\
\hline \multirow[t]{4}{*}{ Endrin } & $<0.1$ & $<0.1$ & $<0.1$ & $<0.2$ \\
\hline & $<0.01$ & $<0.02$ & $<0.01$ & $<0.01$ \\
\hline & $<0.05$ & $<0.05$ & $<0.05$ & $<0.05$ \\
\hline & $<0.05$ & $<0.1$ & $<0.05$ & $<0.05$ \\
\hline \multirow[t]{4}{*}{ Methoxychlor } & $<0.2$ & $<0.2$ & $<0.2$ & $<0.4$ \\
\hline & $<10$ & $<20$ & $<10$ & $<10$ \\
\hline & $<0.2$ & $<0.2$ & $<0.2$ & $<0.2$ \\
\hline & $<0.2$ & $<0.4$ & $<0.2$ & $<0.2$ \\
\hline \multirow[t]{4}{*}{ Toxaphene } & $<1$ & $<1$ & $<1$ & $<2$ \\
\hline & $<0.5$ & $<1$ & $<0.5$ & $<0.5$ \\
\hline & $<1$ & $<1$ & $<1$ & $<1$ \\
\hline & $<1$ & $<2$ & $<1$ & $<1$ \\
\hline
\end{tabular}


Table C-2. Parameters in the Pit 1 Area monitoring wells, Site 300, 1992 (continued).

\begin{tabular}{|c|c|c|c|c|}
\hline \multirow[b]{2}{*}{ Parameters } & \multicolumn{4}{|c|}{ Well } \\
\hline & K1-01C & $\mathrm{K} 1-02 \mathrm{~B}$ & K1-03 & K1-04 \\
\hline \multicolumn{5}{|c|}{ Explosive compounds ( $\mu \mathrm{g} / \mathrm{L})$} \\
\hline \multirow[t]{2}{*}{$\mathrm{HMX}$} & 3 n.d. at $<20$ & 4 n.d. at $<20$ & 4 n.d. at $<20$ & 3 n.d. at $<20$ \\
\hline & & & & 1 n.d. at $<40$ \\
\hline \multirow[t]{2}{*}{ RDX } & 3 n.d. at $<30$ & 4 n.d. at $<30$ & 4 n.d. at $<30$ & 3 n.d. at $<30$ \\
\hline & & & & 1 n.d. at $<60$ \\
\hline TNT & 3 n.d. at $<30$ & 4 n.d. at $<30$ & 4 n.d. at $<30$ & 3 n.d. at $<30$ \\
\hline \multicolumn{5}{|c|}{ Radiologicals (Bq/L) } \\
\hline \multirow[t]{4}{*}{ Gross alpha } & $0.22 \pm 0.14$ & $0.09 \pm 0.10$ & $0.029 \pm 0.087$ & $0.039 \pm 0.093$ \\
\hline & $0.18 \pm 0.12$ & $0.052 \pm 0.081$ & $0.043 \pm 0.068$ & $0.010 \pm 0.087$ \\
\hline & $0.13 \pm 0.10$ & $-0.020 \pm 0.078$ & $0.023 \pm 0.081$ & $-0.016 \pm 0.088$ \\
\hline & $0.18 \pm 0.13$ & $0.05 \pm 0.08$ & $0.04 \pm 0.14$ & $0.03 \pm 0.09$ \\
\hline \multirow[t]{4}{*}{ Gross beta } & $0.113 \pm 0.068$ & $0.014 \pm 0.078$ & $0.067 \pm 0.076$ & $0.144 \pm 0.083$ \\
\hline & $0.238 \pm 0.080$ & $0.042 \pm 0.061$ & $0.180 \pm 0.067$ & $0.225 \pm 0.069$ \\
\hline & $0.157 \pm 0.068$ & $0.081 \pm 0.073$ & $0.045 \pm 0.074$ & $0.112 \pm 0.069$ \\
\hline & $0.18 \pm 0.08$ & $0.18 \pm 0.08$ & $0.08 \pm 0.07$ & $0.13 \pm 0.05$ \\
\hline \multirow[t]{4}{*}{ Tritium } & $4.0 \pm 3.4$ & $74.6 \pm 6.0$ & $1.5 \pm 3.4$ & $-1.4 \pm 3.0$ \\
\hline & $1.3 \pm 3.5$ & $68.5 \pm 5.7$ & $0.8 \pm 3.5$ & $-0.8 \pm 3.3$ \\
\hline & $3.6 \pm 2.6$ & $65.5 \pm 4.9$ & $3.6 \pm 2.6$ & $0.5 \pm 2.3$ \\
\hline & $5.4 \pm 3.6$ & $84 \pm 6$ & $2.5 \pm 3.5$ & $-1.3 \pm 3.2$ \\
\hline \multirow[t]{4}{*}{ Uranium-234 } & $0.140 \pm 0.017$ & $0.049 \pm 0.009$ & $0.033 \pm 0.008$ & $0.041 \pm 0.008$ \\
\hline & $0.162 \pm 0.021$ & $0.050 \pm 0.009$ & $0.033 \pm 0.009$ & $0.048 \pm 0.010$ \\
\hline & $0.699 \pm 0.053$ & $0.078 \pm 0.011$ & $0.188 \pm 0.019$ & $0.045 \pm 0.009$ \\
\hline & $0.176 \pm 0.020$ & $0.049 \pm 0.010$ & $0.036 \pm 0.007$ & $0.045 \pm 0.007$ \\
\hline \multirow[t]{4}{*}{ Uranium-235 } & $0.002 \pm 0.003$ & $0.000 \pm 0.001$ & $0.002 \pm 0.002$ & $0.001 \pm 0.002$ \\
\hline & $0.003 \pm 0.003$ & $0.001 \pm 0.002$ & $0.001 \pm 0.003$ & $0.001 \pm 0.002$ \\
\hline & $0.006 \pm 0.003$ & $0.006 \pm 0.003$ & $0.001 \pm 0.002$ & $0.001 \pm 0.001$ \\
\hline & $0.005 \pm 0.004$ & $0.001 \pm 0.002$ & $0.0013 \pm 0.0017$ & $-0.0007 \pm 0.0007$ \\
\hline \multirow[t]{4}{*}{ Uranium-238 } & $0.080 \pm 0.012$ & $0.025 \pm 0.006$ & $0.017 \pm 0.005$ & $0.025 \pm 0.006$ \\
\hline & $0.085 \pm 0.014$ & $0.034 \pm 0.008$ & $0.018 \pm 0.007$ & $0.022 \pm 0.007$ \\
\hline & $0.090 \pm 0.012$ & $0.026 \pm 0.006$ & $0.015 \pm 0.004$ & $0.020 \pm 0.006$ \\
\hline & $0.099 \pm 0.014$ & $0.030 \pm 0.007$ & $0.014 \pm 0.004$ & $0.026 \pm 0.006$ \\
\hline \multirow[t]{4}{*}{ Radium-226 } & $-0.022 \pm 0.021$ & $-0.002 \pm 0.021$ & $-0.005 \pm 0.028$ & $0.001 \pm 0.020$ \\
\hline & $-0.016 \pm 0.015$ & $0.031 \pm 0.141$ & $-0.005 \pm 0.019$ & $0.038 \pm 0.325$ \\
\hline & $-0.015 \pm 0.018$ & $0.005 \pm 0.000$ & $-0.005 \pm 0.016$ & $-0.008 \pm 0.022$ \\
\hline & $0.026 \pm 0.027$ & $-0.006 \pm 0.019$ & $0.019 \pm 0.014$ & $0.024 \pm 0.026$ \\
\hline
\end{tabular}




\section{Appendix C. Ground Water Protection Tables}

Table C-2. Parameters in the Pit 1 Area monitoring wells, Site 300, 1992 (concluded).

\begin{tabular}{|c|c|c|c|c|}
\hline \multirow[b]{2}{*}{ Parameters } & \multicolumn{4}{|c|}{ Well } \\
\hline & K1-05 & K1-07 & K1-08 & K1-09 \\
\hline \multicolumn{5}{|c|}{ Explosive compounds $(\mu \mathrm{g} \Omega)$} \\
\hline \multirow[t]{2}{*}{ HMX } & 4 n.d. at $<20$ & 2 n.d. at $<20$ & 4 n.d. at $<20$ & 3 n.d. at $<20$ \\
\hline & & 2 n.d. at $<40$ & & 1 n.d. at $<40$ \\
\hline \multirow[t]{2}{*}{ RDX } & 4 n.d. at $<30$ & 2 n.d. at $<30$ & 4 n.d. at $<30$ & 3 n.d. at $<30$ \\
\hline & & 2 n.d. at $<60$ & & 1 n.d. at $<60$ \\
\hline TNT & 4 n.d. at $<30$ & 2 n.d. at $<30$ & 4 n.d. at $<30$ & 3 n.d. at $<30$ \\
\hline \multicolumn{5}{|c|}{ Radiologicals (Bq/L) } \\
\hline \multirow[t]{4}{*}{ Gross alpha } & $-0.031 \pm 0.085$ & $0.057 \pm 0.089$ & $0.04 \pm 0.11$ & $-0.077 \pm 0.081$ \\
\hline & $0.039 \pm 0.072$ & $0.041 \pm 0.070$ & $0.002 \pm 0.069$ & $0.007 \pm 0.091$ \\
\hline & $-0.047 \pm 0.068$ & $0.045 \pm 0.081$ & $-0.010 \pm 0.088$ & $0.049 \pm 0.068$ \\
\hline & $0.02 \pm 0.13$ & $0.05 \pm 0.09$ & $0.05 \pm 0.15$ & $-0.01 \pm 0.14$ \\
\hline \multirow[t]{4}{*}{ Gross beta } & $0.083 \pm 0.068$ & $0.148 \pm 0.095$ & $0.121 \pm 0.067$ & $0.105 \pm 0.080$ \\
\hline & $0.159 \pm 0.069$ & $0.089 \pm 0.071$ & $0.145 \pm 0.070$ & $0.092 \pm 0.065$ \\
\hline & $-0.10 \pm 0.11$ & $0.077 \pm 0.063$ & $0.123 \pm 0.067$ & $0.019 \pm 0.064$ \\
\hline & $0.11 \pm 0.07$ & $0.12 \pm 0.07$ & $0.09 \pm 0.08$ & $0.10 \pm 0.07$ \\
\hline \multirow[t]{4}{*}{ Tritium } & $-3.0 \pm 3.2$ & $-1.2 \pm 3.1$ & $-1.2 \pm 3.2$ & $-2.8 \pm 3.1$ \\
\hline & $-1.3 \pm 3.3$ & $-0.4 \pm 3.2$ & $1.8 \pm 3.4$ & $0.7 \pm 3.4$ \\
\hline & $1.4 \pm 3.3$ & $-0.4 \pm 3.2$ & $-1.1 \pm 3.1$ & $0.4 \pm 3.1$ \\
\hline & $-0.1 \pm 3.3$ & $1.7 \pm 3.4$ & $1.0 \pm 3.4$ & $0.7 \pm 3.4$ \\
\hline \multirow[t]{4}{*}{ Uranium-234 } & $0.043 \pm 0.010$ & $0.056 \pm 0.011$ & $0.036 \pm 0.008$ & $0.035 \pm 0.008$ \\
\hline & $0.039 \pm 0.008$ & $0.057 \pm 0.010$ & $0.032 \pm 0.007$ & $0.035 \pm 0.008$ \\
\hline & $0.259 \pm 0.023$ & $0.067 \pm 0.008$ & $0.049 \pm 0.010$ & $0.043 \pm 0.009$ \\
\hline & $0.036 \pm 0.007$ & $0.050 \pm 0.011$ & $0.044 \pm 0.007$ & $0.044 \pm 0.008$ \\
\hline \multirow[t]{4}{*}{ Uranium-235 } & $0.001 \pm 0.002$ & $0.001 \pm 0.002$ & $0.001 \pm 0.002$ & $0.000 \pm 0.002$ \\
\hline & $0.001 \pm 0.002$ & $0.000 \pm 0.001$ & $0.001 \pm 0.002$ & $0.000 \pm 0.002$ \\
\hline & $0.001 \pm 0.001$ & $0.005 \pm 0.003$ & $0.001 \pm 0.002$ & $-0.001 \pm 1001$ \\
\hline & $0.0003 \pm 0.0013$ & $0.0022 \pm 0.0022$ & $0.0010 \pm 0.0014$ & $0.0012 \pm 0.0024$ \\
\hline \multirow[t]{4}{*}{ Uranium-238 } & $0.016 \pm 0.006$ & $0.028 \pm 0.007$ & $0.014 \pm 0.005$ & $0.013 \pm 0.005$ \\
\hline & $0.020 \pm 0.005$ & $0.027 \pm 0.007$ & $0.015 \pm 0.005$ & $0.016 \pm 0.005$ \\
\hline & $0.023 \pm 0.005$ & $0.025 \pm 0.005$ & $0.019 \pm 0.006$ & $0.020 \pm 0.006$ \\
\hline & $0.019 \pm 0.005$ & $0.026 \pm 0.007$ & $0.017 \pm 0.005$ & $0.020 \pm 0.005$ \\
\hline \multirow[t]{4}{*}{ Padium-226 } & $-0.012 \pm 0.024$ & $-0.015 \pm 0.016$ & $0.011 \pm 0.018$ & $-0.003 \pm 0.021$ \\
\hline & $0.006 \pm 0.016$ & $0.014 \pm 0.018$ & $0.019 \pm 0.019$ & $0.006 \pm 0.016$ \\
\hline & $-0.006 \pm 0.019$ & $-0.012 \pm 0.021$ & $0.028 \pm 0.016$ & $0.002 \pm 0.020$ \\
\hline & $0.038 \pm 0.021$ & $0.019 \pm 0.023$ & $0.020 \pm 0.023$ & $-0.002 \pm 0.021$ \\
\hline
\end{tabular}

Note: The abbreviation "n.d." stands for nondetection. 
Table C-3. Parameters sampled in Pit 2 Area monitoring wells, Site 300, 1992.

\begin{tabular}{|c|c|c|c|}
\hline \multirow[b]{2}{*}{ Parameters } & \multicolumn{3}{|c|}{ Well } \\
\hline & $\mathrm{K} 2-01 \mathrm{C}$ & K2-04D & K2-04S \\
\hline \multicolumn{4}{|l|}{ Physical and chemical (mg/L) } \\
\hline \multirow[t]{3}{*}{ Ammonia nitrogen (as $\mathrm{N}$ ) } & 0.06 & $<0.02$ & 0.12 \\
\hline & 0.06 & 0.13 & 0.06 \\
\hline & 0.05 & $<0.03$ & 0.03 \\
\hline \multirow[t]{2}{*}{ Beryllium } & 3 n.d. at $<0.0005$ & 3 n.d. at $<0.0005$ & 3 n.d. at $<0.0005$ \\
\hline & 1 n.d. at $<0.0002$ & 1 n.d. at $<0.0002$ & 1 n.d. at $<0.0002$ \\
\hline \multirow[t]{2}{*}{ Lead } & 0.011 & & \\
\hline & 3 n.d. at $<0.002$ & 4 n.d. at $<0.002$ & 4 n.d. at $<0.002$ \\
\hline \multirow[t]{4}{*}{ Nitrate plus nitrite (as $\mathrm{NO}_{3}$ ) } & 24 & 32 & 33 \\
\hline & 29 & 35 & 36 \\
\hline & 23 & 29 & 32 \\
\hline & 21 & 32 & 36 \\
\hline Total Kjeldahi nitrogen & 3 n.d. at $<0.5$ & 3 n.d. at $<0.5$ & 3 n.d. at $<0.5$ \\
\hline \multicolumn{4}{|c|}{ Purgeable pollutants (EPA Method $601, \mu \mathrm{g} / \mathrm{L}$ ) } \\
\hline 1,1,1-Trichloroethane & 4 n.d. at $<0.5$ & 4 n.d. at $<0.5$ & 4 n.d. at $<0.5$ \\
\hline 1,1,2,2-Tetrachloroethane & 4 n.d. at $<0.5$ & 4 n.d. at $<0.5$ & 4 n.d. at $<n . j$ \\
\hline 1,1,2-Trichloroethane & 4 n.d. at $<0.5$ & 4 n.d. at $<0.5$ & 4 n.d. at $<0.5$ \\
\hline 1,1-Dichloroethane & 4 n.d. at $<0.5$ & 4 n.d. at $<0.5$ & 4 n.d. at $<0.5$ \\
\hline 1,1-Dichloroethene & 4 n.d. at $<0.5$ & 4 n.d. at $<0.5$ & 4 n.d. at $<0.5$ \\
\hline 1,2-Dichlorobenzene & 4 n.d. at $<0.5$ & 4 n.d. at $<0.5$ & 4 n.d. at $<0.5$ \\
\hline 1,2-Dichloroethane & 4 n.d. at $<0.5$ & 4 n.d. at $<0.5$ & 4 n.d. at $<0.5$ \\
\hline 1,2-Dichloroethene (total) & 4 n.d. at $<0.5$ & 4 n.d. at $<0.5$ & 4 n.d. at $<0.5$ \\
\hline 1,2-Dichloropropane & 4 n.d. at $<0.5$ & 4 n.d. at $<0.5$ & 4 n.d. at $<0.5$ \\
\hline 1,3-Dichlorobenzene & 4 n.d. at $<0.5$ & 4 n.d. at $<0.5$ & 4 n.d. at $<0.5$ \\
\hline 1,4-Dichlorobenzene & 4 n.d. at $<0.5$ & 4 n.d. at $<0.5$ & 4 n.d. at $<0.5$ \\
\hline 2-Chloroethylvinylether & 4 n.d. at $<0.5$ & 4 n.d. at $<0.5$ & 4 n.d. at $<0.5$ \\
\hline Bromodichloromethane & 4 n.d. at $<0.5$ & 4 n.d. at $<0.5$ & 4 n.d. at $<0.5$ \\
\hline Bromoform & 4 n.d. at $<0.5$ & 4 n.d. at $<0.5$ & 4 n.d. at $<0.5$ \\
\hline Bromomethane & 4 n.d. at $<0.5$ & 4 n.d. at $<0.5$ & 4 n.d. at $<0.5$ \\
\hline Carbon tetrachloride & 4 n.d. at $<0.5$ & 4 n.d. at $<0.5$ & 4 n.d. at $<0.5$ \\
\hline Chlorobenzene & 4 n.d. at $<0.5$ & 4 n.d. at $<0.5$ & 4 n.d. at $<0.5$ \\
\hline Chloroethane & 4 n.d. at $<0.5$ & 4 n.d. at $<0.5$ & 4 n.d. at $<0.5$ \\
\hline Chloroform & 4 n.d. at $<0.5$ & 4 n.d. at $<0.5$ & 4 n.d. at $<0.5$ \\
\hline Chloromethane & 4 n.d. at $<0.5$ & 4 n.d. at $<0.5$ & 4 n.d. at $<0.5$ \\
\hline cis-1,2-Dichloroethene & 4 n.d. at $<0.5$ & 4 n.d. at $<0.5$ & 4 n.d. at $<0.5$ \\
\hline cis-1,3-Dichloropropene & 4 n.d. at $<0.5$ & 4 n.d. at $<0.5$ & 4 n.d. at $<0.5$ \\
\hline
\end{tabular}


Table C-3. Parameters sampled in Pit 2 Area monitoring wells, Site 300, 1992 (concluded).

\begin{tabular}{|c|c|c|c|}
\hline \multirow[b]{2}{*}{ Parameters } & \multicolumn{3}{|c|}{ Well } \\
\hline & $\mathrm{K} 2-01 \mathrm{C}$ & K2-04D & K2-04S \\
\hline Dibromochloromethane & 4 n.d. at $<0.5$ & 4 n.d. at $<0.5$ & 4 n.d. at $<0.5$ \\
\hline \multirow[t]{2}{*}{ Dichlorodifluoromethane } & 0.6 & 4 n.d. at $<0.5$ & 4 n.d. at $<0.5$ \\
\hline & 3 n.d. at $<0.5$ & & \\
\hline Freon-113 & $4 \mathrm{n} . \mathrm{d}$. at $<0.5$ & 4 n.d. at $<0.5$ & 4 n.d. at $<0.5$ \\
\hline Methylene chloride & 4 n.d. at $<0.5$ & 4 n.d. at $<0.5$ & 4 n.d. at $<0.5$ \\
\hline Tetrachloroethene & 4 n.d. at $<0.5$ & 4 n.d. $a^{\prime} !<0.5$ & 4 n.d. at $<0.5$ \\
\hline trans-1,2-Dichloroethene & 4 n.d. at $<0.5$ & 4 n.d. at $<0.5$ & 4 n.d. at $<0.5$ \\
\hline trans-1,3-Dichloropropene & 4 n.d. at $<0.5$ & 4 n.d. at $<0.5$ & 4 n.d. at $<0.5$ \\
\hline Trichloroethene & 4 n.d. at $<0.5$ & 4 n.d. at $<0.5$ & 4 n.d. at $<0.5$ \\
\hline Trichlorofluoroniethane & 4 n.d. at $<0.5$ & 4 n.d. at $<0.5$ & 4 n.d. at $<0.5$ \\
\hline Vinyl chloride & 4 n.d. at $<0.5$ & 4 n.d. at $<0.5$ & 4 n.d. at $<0.5$ \\
\hline Radiologicals (Bq/L) & & & \\
\hline Gross alpha & $0.22 \pm 0.17$ & $-0.024 \pm 0.093$ & $0.16 \pm 0.14$ \\
\hline & $0.28 \pm 0.15$ & $0.037 \pm 0.095$ & $0.10 \pm 0.13$ \\
\hline & $0.05 \pm 0.15$ & $0.057 \pm 0.079$ & $0.10 \pm 0.12$ \\
\hline & $0.08 \pm 0.09$ & $0.08 \pm 0.13$ & $0.67 \pm 0.20$ \\
\hline Gross beta & $0.174 \pm 0.074$ & $0.148 \pm 0.069$ & $0.111 \pm 0.087$ \\
\hline & $0.163 \pm 0.078$ & $0.157 \pm 0.072$ & $0.215 \pm 0.071$ \\
\hline & $0.141 \pm 0.071$ & $0.035 \pm 0.075$ & $0.048 \pm 0.073$ \\
\hline & $0.071 \pm 0.048$ & $0.113 \pm 0.048$ & $0.340 \pm 0.075$ \\
\hline Tritium & $345 \pm 18$ & $300 \pm 16$ & $327 \pm 17$ \\
\hline & $490 \pm 25$ & $286 \pm 15$ & $330 \pm 17$ \\
\hline & $340 \pm 18$ & $322 \pm 17$ & $340 \pm 18$ \\
\hline & $433 \pm 23$ & $403 \pm 22$ & $422 \pm 22$ \\
\hline
\end{tabular}

Note: The abbreviation "n.d." stands for nondetection. 
Table C-4. Parameters sampled in the Pit 6 Area monitoring wells, Site 300, 1992.

\begin{tabular}{|c|c|c|c|}
\hline \multirow[b]{2}{*}{ Parameters } & \multicolumn{3}{|c|}{ Well } \\
\hline & K6-01 & K6-03 & K6-04 \\
\hline \multicolumn{4}{|l|}{ Physical and chemical (mg/L) } \\
\hline \multirow[t]{4}{*}{ Ammonia nitrogen (as $N$ ) } & 0.1 & 0.24 & 0.03 \\
\hline & 0.2 & 0.43 & 0.1 \\
\hline & 0.17 & 0.34 & 0.04 \\
\hline & 0.1 & 0.28 & $<0.03$ \\
\hline \multirow[t]{4}{*}{ Arsenic } & 0.013 & 0.02 & 0.016 \\
\hline & 0.13 & 0.02 & 0.15 \\
\hline & 0.013 & 0.02 & 0.016 \\
\hline & 0.0086 & 0.016 & 0.013 \\
\hline Barium & 4 n.d. at $<0.05$ & 4 n.d. at $<0.05$ & 4 n.d. at $<0.05$ \\
\hline \multirow[t]{2}{*}{ Beryllium } & 1 n.d. at $<0.0005$ & 1 n.d. at $<0.0002$ & 1 n.d. at $<0.0002$ \\
\hline & 3 n.d. at $<0.0005$ & 3 n.d. at $<0.0005$ & 3 n.d. at $<0.0005$ \\
\hline \multirow[t]{4}{*}{ Bicarbonate alk (as $\mathrm{CaCO}_{3}$ ) } & 190 & 190 & 170 \\
\hline & 200 & 190 & 170 \\
\hline & 190 & 180 & 170 \\
\hline & 200 & 180 & 170 \\
\hline Cadmium & 4 n.d. at $<0.0005$ & 4 n.d. at $<0.0005$ & 4 n.d. at $<0.0005$ \\
\hline Carbonate alk (as $\mathrm{CaCO}_{3}$ ) & 4 n.d. at $<1$ & 4 n.d. at $<1$ & 4 n.d. at $<1$ \\
\hline \multirow{4}{*}{ Chloride } & 87 & 88 & 84 \\
\hline & 88 & 88 & 85 \\
\hline & 90 & 93 & 86 \\
\hline & 85 & 91 & 79 \\
\hline Chromium & 4 n.d. at $<0.005$ & 4 n.d. at $<0.005$ & 4 n.d. at $<0.005$ \\
\hline \multirow[t]{4}{*}{ Fluoride } & 0.4 & 0.5 & 0.5 \\
\hline & 0.4 & 0.5 & 0.5 \\
\hline & 0.5 & 0.5 & 0.5 \\
\hline & 0.4 & 0.49 & 0.45 \\
\hline Hydroxide alk (as $\mathrm{CaCO}_{3}$ ) & 4 n.d. at $<1$ & 4 n.d. at $<1$ & 4 n.d. at $<1$ \\
\hline \multirow[t]{4}{*}{ Iron } & 0.4 & 0.2 & 0.1 \\
\hline & 0.3 & 0.1 & 0.2 \\
\hline & 0.33 & 2 n.d. at $<0.1$ & 2 n.d. at $<0.1$ \\
\hline & 1 n.d. at $<0.1$ & & \\
\hline \multirow[t]{2}{*}{ Lead } & 4 n.d. at $<0.002$ & 0.0041 & 4 n.d. at $<0.002$ \\
\hline & 3 n.d. at $<0.002$ & 3 n.d. at $<0.002$ & \\
\hline Low level phenolics & 4 n.d. at $<0.005$ & 4 n.d. at $<0.005$ & 4 n.d. at $<0.005$ \\
\hline \multirow[t]{4}{*}{ Manganese } & $<0.05$ & $<0.05$ & 4 n.d. at $<0.05$ \\
\hline & 0.07 & 0.08 & \\
\hline & 0.08 & 0.09 & \\
\hline & 0.062 & 0.074 & \\
\hline Mercury & 4 n.d. at $<0.0002$ & 4 n.d. at $<0.0002$ & 4 n.d. at $<0.0002$ \\
\hline
\end{tabular}


Table C-4. Parameters sampled in the Pit 6 Area monitoring wells, Site 300, 1992 (continued).

\begin{tabular}{|c|c|c|c|}
\hline \multirow[b]{2}{*}{ Parameters } & \multicolumn{3}{|c|}{ Well } \\
\hline & EP6-07 & EP6-08 & EP6-09 \\
\hline \multicolumn{4}{|l|}{ Physical and chemical (mg/L) } \\
\hline \multirow[t]{4}{*}{ Ammonia nitrogen (as N) } & 0.23 & 0.03 & 0.02 \\
\hline & 0.25 & 0.06 & 0.04 \\
\hline & 0.35 & 0.05 & 0.03 \\
\hline & 0.26 & 0.031 & 0.093 \\
\hline \multirow[t]{4}{*}{ Arsenic } & 0.02 & 0.018 & 0.016 \\
\hline & 0.02 & 0.019 & 0.016 \\
\hline & 0.02 & 0.016 & 0.014 \\
\hline & 0.016 & 0.014 & 0.011 \\
\hline Barium & 4 n.d. at $<0.05$ & 4 n.d. at $<0.05$ & 4 n.d. at $<0.05$ \\
\hline \multirow[t]{2}{*}{ Beryllium } & 1 n.d. at $<0.0002$ & 1 n.d. at $<0.0002$ & 1 n.d. at $<0.0002$ \\
\hline & 3 n.d. at $<0.0005$ & 3 n.d. at $<0.0005$ & 3 n.d. at $<0.0005$ \\
\hline \multirow[t]{4}{*}{ Bicarbonate alk (as $\mathrm{CaCO}_{3}$ ) } & 180 & 190 & 200 \\
\hline & 190 & 190 & 200 \\
\hline & 180 & 180 & 190 \\
\hline & 190 & 200 & 190 \\
\hline Cadmium & 4 n.d. at $<0.0005$ & 4 n.d. at $<0.0005$ & 4 n.d. at $<0.0005$ \\
\hline Crirbonate alk (as $\mathrm{CaCO}_{3}$ ) & 4 n.d. at $<1$ & 4 n.d. at $<1$ & 4 n.d. at $<1$ \\
\hline \multirow[t]{4}{*}{ Chloride } & 88 & 95 & 120 \\
\hline & 88 & 94 & 110 \\
\hline & 91 & 96 & 120 \\
\hline & 85 & 90 & 110 \\
\hline Chromium & 4 n.d. at $<0.005$ & 4 n.d. at $<0.005$ & 4 n.d. at $<0.005$ \\
\hline \multirow[t]{4}{*}{ Fluoride } & 0.5 & 0.4 & 0.4 \\
\hline & 0.5 & 0.4 & 0.4 \\
\hline & 0.5 & 0.4 & 0.4 \\
\hline & 0.47 & 0.44 & 0.42 \\
\hline Hydroxide alk (as $\mathrm{CaCO}_{3}$ ) & 4 n.d. at $<1$ & 4 n.d. at $<1$ & 4 n.d. at $<1$ \\
\hline \multirow[t]{2}{*}{ Iron } & 0.1 & 4 n.d. at $<0.1$ & 4 n.d. at $<0.1$ \\
\hline & 3 n.d. at $<0.1$ & & \\
\hline \multirow[t]{2}{*}{ Lead } & 4 n.d. at $<0.002$ & $<0.002$ & 4 n.d. at $<0.002$ \\
\hline & & 3 n.d. at $<0.002$ & \\
\hline Low level phenolics & 4 n.d. at $<0.005$ & 4 n.d. at $<0.005$ & 4 n.d. at $<0.005$ \\
\hline \multirow[t]{4}{*}{ Manganese } & 0.12 & 4 n.d. at $<0.05$ & 4 n.d. at $<0.05$ \\
\hline & 0.14 & & \\
\hline & 0.16 & & \\
\hline & 0.14 & & \\
\hline Mercury & 4 n.d. at $<0.0002$ & 4 n.d. at $<0.0002$ & 4 n.d. at $<0.0002$ \\
\hline
\end{tabular}




\section{Appendix C. Ground Water Protection Tables}

Table C-4. Parameters sampled in the Pit 6 Area monitoring wells, Site 300, 1992 (continued).

\begin{tabular}{|c|c|c|c|}
\hline \multirow[b]{2}{*}{ Parameters } & \multicolumn{3}{|c|}{ Well } \\
\hline & K6-01 & K6-03 & K6-04 \\
\hline \multirow[t]{3}{*}{ Nickel } & 3 n.d. at $<0.005$ & 0.0054 & 3 n.d. at $<0.005$ \\
\hline & 1 n.d. at $<0.1$ & 2 n.d. at $<0.005$ & 1 n.d. at $<0.1$ \\
\hline & & 1 n.d. at $<0.1$ & \\
\hline \multirow[t]{2}{*}{ Nitrate plus nitrite (as N) } & 2 n.d. at $<0.1$ & 2 n.d. at $<0.1$ & 1.6 \\
\hline & & & 1.6 \\
\hline \multirow[t]{4}{*}{ Nitrate (as $\mathrm{NO}_{3}$ ) } & 4 n.d. at $<0.4$ & 4 n.d. at $<0.4$ & 7.1 \\
\hline & & & 6.9 \\
\hline & & & 6.3 \\
\hline & & & 7.3 \\
\hline Nitrite (as N) & 2 n.d. at $<0.01$ & 2 n.d. at $<0.01$ & 2 n.d. at $<0.01$ \\
\hline Nitrite (as $\left.\mathrm{NO}_{2}\right)$ & 2 n.d. at $<0.03$ & 2 n.d. at $<0.03$ & 2 n.d. at $<0.03$ \\
\hline \multirow[t]{3}{*}{ Selenium } & 4 n.d. at $<0.002$ & 4 n.d. at $<0.002$ & 0.002 \\
\hline & & & 0.0024 \\
\hline & & & 2 n.d. at $<0.002$ \\
\hline Silver & 4 n.d. at $<0.0005$ & 4 n.d. at $<0.0005$ & 4 n.d. at $<0.0005$ \\
\hline \multirow[t]{4}{*}{ Sodium } & 130 & 130 & 110 \\
\hline & 130 & 120 & 110 \\
\hline & 160 & 140 & 130 \\
\hline & 140 & 110 & 94 \\
\hline \multirow[t]{4}{*}{ Sulfate } & 290 & 240 & 200 \\
\hline & 320 & 250 & 190 \\
\hline & 280 & 240 & 200 \\
\hline & 280 & 250 & 200 \\
\hline \multirow[t]{4}{*}{ Total alkalinity (as $\mathrm{CaCO}_{3}$ ) } & 190 & 190 & 170 \\
\hline & 200 & 190 & 170 \\
\hline & 190 & 180 & 170 \\
\hline & 200 & 180 & 170 \\
\hline \multirow[t]{4}{*}{ Total dissolved solids (TDS) } & 780 & 700 & 620 \\
\hline & 790 & 710 & 640 \\
\hline & 740 & 700 & 620 \\
\hline & 760 & 700 & 620 \\
\hline Total Kjeldahl nitrogen & 4 n.d. at $<0.5$ & 4 n.d. at $<0.5$ & 4 n.d. at $<0.5$ \\
\hline \multirow[t]{4}{*}{ Total organic carbon (TOC) } & $<0.5$ & 0.7 & 0.5 \\
\hline & 1.8 & 0.8 & 0.7 \\
\hline & $<0.5$ & 0.5 & 0.7 \\
\hline & 0.57 & $<0.5$ & $<0.5$ \\
\hline
\end{tabular}


Table C-4. Parameters sampled in the Pit 6 Area monitoring wells, Site 300, 1992 (continued).

\begin{tabular}{|c|c|c|c|}
\hline \multirow[b]{2}{*}{ Parameters } & \multicolumn{3}{|c|}{ Well } \\
\hline & EP6-07 & EP6-08 & EP6-09 \\
\hline \multirow[t]{2}{*}{ Nickel } & 3 n.d. at $<0.005$ & 3 n.d. at $<0.005$ & 3 n.d. at $<0.005$ \\
\hline & 1 n.d. at $<0.1$ & $1 \mathrm{n} . \mathrm{d}$. at $<0.1$ & 1 n.d. at $<0.1$ \\
\hline \multirow[t]{2}{*}{ Nitrate plus nitrite (as N) } & 2 n.d. at $<0.1$ & 0.5 & 0.9 \\
\hline & & 0.6 & 1.1 \\
\hline \multirow[t]{4}{*}{ Nitrate (as $\mathrm{NO}_{3}$ ) } & 4 n.d. at $<0.4$ & 2.9 & 4.7 \\
\hline & & 2.2 & 4.1 \\
\hline & & 2.4 & 4.5 \\
\hline & & 2.6 & 4.7 \\
\hline \multirow[t]{2}{*}{ Nitrite (as N) } & $<0.01$ & 2 n.d. at $<0.01$ & $<0.01$ \\
\hline & 0.02 & & 0.013 \\
\hline \multirow[t]{2}{*}{ Nitrite (as $\mathrm{NO}_{2}$ ) } & $<0.03$ & 2 n.d. at $<0.03$ & $<0.03$ \\
\hline & 0.07 & & 0.043 \\
\hline \multirow[t]{4}{*}{ Selenium } & 4 n.d. at $<0.002$ & 0.003 & 0.002 \\
\hline & & 0.0031 & 0.002 \\
\hline & & 2 n.d. at $<0.002$ & 0.0034 \\
\hline & & & $<0.002$ \\
\hline \multirow[t]{2}{*}{ Silver } & 4 n.d. at $<0.0005$ & 0.0007 & 4 n.d. at $<0.0005$ \\
\hline & & 3 n.d. at $<0.0005$ & \\
\hline \multirow[t]{4}{*}{ Sodium } & 120 & 130 & 130 \\
\hline & 110 & 130 & 130 \\
\hline & 140 & 150 & 150 \\
\hline & 120 & 130 & 120 \\
\hline \multirow[t]{4}{*}{ Sulfate } & 250 & 230 & 230 \\
\hline & 250 & 240 & 240 \\
\hline & 240 & 210 & 230 \\
\hline & 240 & 240 & 240 \\
\hline \multirow[t]{4}{*}{ Total alkalinity (as $\left.\mathrm{CaCO}_{3}\right)$} & 180 & 190 & 200 \\
\hline & 190 & 190 & 200 \\
\hline & 180 & 180 & 190 \\
\hline & 190 & 200 & 190 \\
\hline \multirow[t]{4}{*}{ Total dissolved solids (TDS) } & 690 & 680 & 730 \\
\hline & 700 & 690 & 750 \\
\hline & 720 & 680 & 760 \\
\hline & 680 & 670 & 720 \\
\hline Total Kjeldahl nitrogen & 4 n.d. at $<0.5$ & 4 n.d. at $<0.5$ & 4 n.d. at $<0.5$ \\
\hline \multirow[t]{4}{*}{ Total organic carbon (TOC) } & 0.5 & 0.8 & $<0.5$ \\
\hline & 0.6 & 0.9 & 0.6 \\
\hline & 0.7 & 0.6 & 0.6 \\
\hline & $<0.5$ & $<0.5$ & $<0.5$ \\
\hline
\end{tabular}


Table C-4. Parameters sampled in the Pit 6 Area monitoring wells, Site 300, 1992 (continued).

\begin{tabular}{|c|c|c|c|}
\hline \multirow[b]{2}{*}{ Parameters } & \multicolumn{3}{|c|}{ Well } \\
\hline & K6-01 & K6-03 & K6-04 \\
\hline \multirow[t]{4}{*}{ Total suspended solids (TSS } & $<1$ & 5 & 2 \\
\hline & $<1$ & $<1$ & $<1$ \\
\hline & 1 & 1 & 1 \\
\hline & $<1$ & $<1$ & $<1$ \\
\hline TOX, 1st replicate & 4 n.d. at $<0.02$ & 4 n.d. at $<0.02$ & 4 n.d. at $<0.02$ \\
\hline TOX, 2nd replicate & 4 n.d. at $<0.02$ & 4 n.d. at $<0.02$ & 4 n.d. at $<0.02$ \\
\hline Vanadium & 4 n.d. at $<0.05$ & 4 n.d. at $<0.05$ & 4 n.d. at $<0.05$ \\
\hline \multirow{4}{*}{$\begin{array}{l}\text { Specific conductance } \\
(\mu \mathrm{mhos} / \mathrm{cm})\end{array}$} & 1000 & 950 & 900 \\
\hline & 1130 & 950 & 900 \\
\hline & 1000 & 990 & 920 \\
\hline & 1100 & 1050 & 910 \\
\hline \multirow[t]{4}{*}{$\mathrm{pH}$} & 7.7 & 7.7 & 7.7 \\
\hline & 8 & 7.7 & 7.7 \\
\hline & 7.7 & 7.7 & 7.7 \\
\hline & 7.6 & 7.6 & 7.7 \\
\hline \multicolumn{4}{|c|}{ Purgeable pollutants (EPA Method 601, $\mu \mathrm{g} / \mathrm{L}$ ) } \\
\hline 1,1,1-Trichloroethane & 4 n.d. at $<0.5$ & 4 n.d. at $<0.5$ & 4 n.d. at $<0.5$ \\
\hline 1,1,2,2-Tetrachloroethane & 4 n.d. at $<0.5$ & 4 n.d. at $<0.5$ & 4 n.d. at $<0.5$ \\
\hline 1,1,2-Trichloroethane & 4 n.d. at $<0.5$ & 4 n.d. at $<0.5$ & 4 n.d. at $<0.5$ \\
\hline 1,1-Dichloroethane & 4 n.d. at $<0.5$ & 4 n.d. at $<0.5$ & 4 n.d. at $<0.5$ \\
\hline 1,1-Dichloroethene & 4 n.d. at $<0.5$ & $4 \mathrm{n}$. d. at $<0.5$ & 4 n.d. at $<0.5$ \\
\hline 1,2-Dichlorobenzene & 4 n.d. at $<0.5$ & 4 n.d. at $<0.5$ & 4 n.d. at $<0.5$ \\
\hline 1,2-Dichloroethane & 4 n.d. at $<0.5$ & 4 n.d. at $<0.5$ & 4 n.d. at $<0.5$ \\
\hline 1,2-Dichloroethene (total) & 4 n.d. at $<0.5$ & 4 n.d. at $<0.5$ & 4 n.d. at $<0.5$ \\
\hline 1,2-Dichloropropane & 4 n.d. at $<0.5$ & 4 n.d. at $<0.5$ & 4 n.d. at $<0.5$ \\
\hline 1,3-Dichlorobenzene & $4 \mathrm{n} . \mathrm{d}$ at $<0.5$ & 4 n.d. at $<0.5$ & 4 n.d. at $<0.5$ \\
\hline 1,4-Dichlorobenzene & 4 n.d. at $<0.5$ & 4 n.d. at $<0.5$ & 4 n.d. at $<0.5$ \\
\hline 2-Chloroethylvinylether & 4 n.d. at $<0.5$ & $4 \mathrm{n}$.d. at $<0.5$ & 4 n.d. at $<0.5$ \\
\hline Bromodichloromethane & 4 n.d. at $<0.5$ & 4 n.d. at $<0.5$ & 4 n.d. at $<0.5$ \\
\hline Bromoform & 4 n.d. at $<0.5$ & 4 n.d. at $<0.5$ & 4 n.d. at $<0.5$ \\
\hline Bromomethane & 4 n.d. at $<0.5$ & $4 \mathrm{n} . \mathrm{d}$. at $<0.5$ & 4 n.d. at $<0.5$ \\
\hline Carbon tetrachloride & 4 n.d. at $<0.5$ & $4 \mathrm{n}$.d. at $<0.5$ & 4 n.d. at $<0.5$ \\
\hline Chlorobenzene & 4 n.d. at $<0.5$ & 4 n.d. at $<0.5$ & 4 n.d. at $<0.5$ \\
\hline Chloroethane & 4 n.d. at $<0.5$ & 4 n.d. at $<0.5$ & 4 n.d. at $<0.5$ \\
\hline Chloroform & 4 n.d. at $<0.5$ & 4 n.d. at $<0.5$ & 4 n.d. at $<0.5$ \\
\hline Chloromethane & 4 n.d. at $<0.5$ & $4 \mathrm{n}$.d. at $<0.5$ & 4 n.d. at $<0.5$ \\
\hline cis-1,2-Dichloroethene & 4 n.d. at $<0.5$ & 4 n.d. at $<0.5$ & 4 n.d. at $<0.5$ \\
\hline cis-1,3-Dichloropropene & 4 n.d. at $<0.5$ & 4 n.d. at $<0.5$ & 4 n.d. at $<0.5$ \\
\hline Dibromochloromethane & 4 n.d. at $<0.5$ & 4 n.d. at $<0.5$ & 4 n.d. at $<0.5$ \\
\hline Dichlorodifluoromethane & 4 n.d. at $<0.5$ & 4 n.d. at $<0.5$ & 4 n.d. at $<0.5$ \\
\hline Freon-113 & 4 n.d. at $<0.5$ & 4 n.d. at $<0.5$ & 4 n.d. at $<0.5$ \\
\hline
\end{tabular}


Table C-4. Parameters sampled in the Pit 6 Area monitoring wells, Site 300, 1992 (continued).

\begin{tabular}{|c|c|c|c|}
\hline \multirow[b]{2}{*}{ Parameters } & \multicolumn{3}{|c|}{ Well } \\
\hline & EP6-07 & EP6-08 & EP6-09 \\
\hline \multirow{4}{*}{ Total suspended solids (TSS) } & 2 & $<1$ & 1 \\
\hline & 1 & $<1$ & $<1$ \\
\hline & 2 & 1 & 1 \\
\hline & $<1$ & $<1$ & $<1$ \\
\hline TOX, 1st replicate & 4 n.d. at $<0.02$ & 4 n.d. at $<0.02$ & 4 n.d. at $<0.02$ \\
\hline TOX, 2nd replicate & 4 n.d. at $<0.02$ & 4 n.d. at $<0.02$ & 4 n.d. at $<0.02$ \\
\hline Vanadium & 4 n.d. at $<0.05$ & 4 n.d. at $<0.05$ & 4 n.d. at $<0.05$ \\
\hline Specific conductance & 970 & 1010 & 990 \\
\hline \multirow[t]{3}{*}{$(\mu \mathrm{mhos} / \mathrm{cm})$} & 1040 & 860 & 1130 \\
\hline & 1010 & 1030 & 1060 \\
\hline & 1080 & 1050 & 1080 \\
\hline \multirow[t]{4}{*}{$\mathrm{pH}$} & 7.8 & 7.6 & 7.8 \\
\hline & 7.6 & 7.6 & 7.9 \\
\hline & 7.7 & 7.6 & 7.7 \\
\hline & 7.7 & 7.6 & 7.6 \\
\hline \multicolumn{4}{|c|}{ Purgeable pollutants (EPA Method 601, $\mu \mathrm{g} / \mathrm{L}$ ) } \\
\hline 1,1,1-Trichloroethane & 4 n.d. at $<0.5$ & 4 n.d. at $<0.5$ & 4 n.d. at $<0.5$ \\
\hline 1,1,2,2-Tetrachloroethane & 4 n.d. at $<0.5$ & 4 n.d. at $<0.5$ & 4 n.d. at $<0.5$ \\
\hline 1,1,2-Trichloroethane & 4 n.d. at $<0.5$ & 4 n.d. at $<0.5$ & 4 n.d. at $<0.5$ \\
\hline 1,1-Dichloroethane & 4 n.d. at $<0.5$ & 4 n.d. at $<0.5$ & 4 n.d. at $<0.5$ \\
\hline 1,1-Dichloroethene & 4 n.d. at $<0.5$ & 4 n.d. at $<0.5$ & 4 n.d. at $<0.5$ \\
\hline 1,2-Dichlorobenzene & 4 n.d. at $<0.5$ & 4 n.d. at $<0.5$ & 4 n.d. at $<0.5$ \\
\hline \multirow[t]{2}{*}{ 1,2-Dichloroethane } & 4 n.d. at $<0.5$ & 4 n.d. at $<0.5$ & 0.6 \\
\hline & & & 3 n.d. at $<0.5$ \\
\hline 1,2-Dichloroethene (total) & 4 n.d. at $<0.5$ & 4 n.d. at $<0.5$ & 4 n.d. at $<0.5$ \\
\hline 1,2-Dichloropropane & 4 n.d. at $<0.5$ & 4 n.d. at $<: 0.5$ & 4 n.d. at $<0.5$ \\
\hline 1,3-Dichlorobenzene & 4 n.d. at $<0.5$ & 4 n.d. at $<0.5$ & 4 n.d. at $<0.5$ \\
\hline 1,4-Dichlorobenzene & 4 n.d. at $<0.5$ & 4 n.d. at $<0.5$ & 4 n.d. at $<0.5$ \\
\hline 2-Chloroethylvinylether & 4 n.d. at $<0.5$ & 4 n.d. at $<0.5$ & 4 n.d. at $<0.5$ \\
\hline Bromodichloromethane & 4 n.d. at $<0.5$ & 4 n.d. at $<0.5$ & 4 n.d. at $<0.5$ \\
\hline Bromoform & 4 n.d. at $<0.5$ & 4 n.d. at $<0.5$ & 4 n.d. at $<0.5$ \\
\hline Bromomethane & 4 n.d. at $<0.5$ & 4 n.d. at $<0.5$ & 4 n.d. at $<0.5$ \\
\hline Carbon tetrachloride & 4 n.d. at $<0.5$ & 4 n.d. at $<0.5$ & 4 n.d. at $<0.5$ \\
\hline Chlorobenzene & 4 n.d. at $<0.5$ & 4 n.d. at $<0.5$ & 4 n.d. at $<0.5$ \\
\hline Chloroethane & 4 n.d. at $<0.5$ & 4 n.d. at $<0.5$ & 4 n.d. at $<0.5$ \\
\hline Chloroform & 4 n.d. at $<0.5$ & 4 n.d. at $<0.5$ & 4 n.d. at $<0.5$ \\
\hline Chloromethane & 4 n.d. at $<0.5$ & 4 n.d. at $<0.5$ & 4 n.d. at $<0.5$ \\
\hline cis-1,2-Dichloroethene & 4 n.d. at $<0.5$ & 4 n.d. at $<0.5$ & 4 n.d. at $<0.5$ \\
\hline cis-1,3-Dichloropropene & 4 n.d. at $<0.5$ & 4 n.d. at $<0.5$ & 4 n.d. at $<0.5$ \\
\hline Dibromochloromethane & 4 n.d. at $<0.5$ & 4 n.d. at $<0.5$ & 4 n.d. at $<0.5$ \\
\hline Dichlorodifluoromethane & 4 n.d. at $<0.5$ & 4 n.d. at $<0.5$ & 4 n.d. at $<0.5$ \\
\hline Freon-113 & 4 n.d. at $<0.5$ & 4 n.d. at $<0.5$ & 4 n.d. at $<0.5$ \\
\hline
\end{tabular}

...continued 
Table C-4. Parameters sampled in the Pit 6 Area monitoring wells, Site 300, 1992 (continued).

\begin{tabular}{|c|c|c|c|}
\hline \multirow[b]{2}{*}{ Parameters } & \multicolumn{3}{|c|}{ Well } \\
\hline & K6-01 & K6-03 & K6-04 \\
\hline Methylene chloride & 4 n.d. at $<0.5$ & 4 n.d. at $<0.5$ & 4 n.d. at $<0.5$ \\
\hline Tetrachloroethene & 4 n.d. at $<0.5$ & 4 n.d. at $<0.5$ & 4 n.d. at $<0.5$ \\
\hline trans-1,2-Dichloroethene & 4 n.d. at $<0.5$ & 4 n.d. at $<0.5$ & 4 n.d. at $<0.5$ \\
\hline trans-1,3-Dichloropropene & 4 n.d. at $<0.5$ & $4 \mathrm{n}$.d. at $<0.5$ & 4 n.d. at $<0.5$ \\
\hline Trichloroethene & 4 n.d. at $<0.5$ & 4 n.d. at $<0.5$ & 4 n.d. at $<0.5$ \\
\hline Trichlorofluoromethane & 4 n.d. at $<0.5$ & 4 n.d. at $<0.5$ & 4 n.d. at $<0.5$ \\
\hline Vinyl chloride & 4 n.d. at $<0.5$ & 4 n.d. at $<0.5$ & 4 n.d. at $<0.5$ \\
\hline \multicolumn{4}{|l|}{ Title 22 herbicides $(\mu \mathrm{g} / \mathrm{L})$} \\
\hline 2,4,5-TP (Silvex) & 4 n.d. at $<1$ & 4 n.d. at $<1$ & 4 n.d. at $<1$ \\
\hline \multirow[t]{2}{*}{$2,4-D$} & 3 n.d. at $<5$ & 3 n.d. at $<5$ & 3 n.d. at $<5$ \\
\hline & 1 n.d. at $<10$ & 1 n.d. at $<10$ & 1 n.d. at $<10$ \\
\hline \multicolumn{4}{|l|}{ Title 22 pesticides $(\mu \mathrm{g} / \mathrm{L})$} \\
\hline \multirow{2}{*}{$\begin{array}{l}\text { BHC, gamma isomer } \\
\text { (Lindane) }\end{array}$} & 3 n.d. at $<0.03$ & 3 n.d. at $<0.03$ & 3 n.d. at $<0.03$ \\
\hline & 1 n.d. at $<0.4$ & 1 n.d. at $<0.4$ & 1 n.d. at $<0.4$ \\
\hline \multirow{2}{*}{ Endrin } & 2 n.d. at $<0.05$ & 2 n.d. at $<0.05$ & 2 n.d. at $<0.05$ \\
\hline & 2 n.d. at $<0.1$ & 2 n.d. at $<0.1$ & 2 n.d. at $<0.1$ \\
\hline \multirow[t]{2}{*}{ Methoxychlor } & 3 n.d. at $<0.2$ & 3 n.d. at $<0.2$ & 3 n.d. at $<0.2$ \\
\hline & 1 n.d. at $<10$ & 1 n.d. at $<10$ & 1 n.d. at $<10$ \\
\hline \multirow[t]{2}{*}{ Toxaphene } & 1 n.d. at $<0.5$ & 1 n.d. at $<0.5$ & 1 n.d. at $<0.5$ \\
\hline & 2 n.d. at $<1$ & 2 n.d. at $<1$ & 2 n.d. at $<1$ \\
\hline \multicolumn{4}{|c|}{ Explosive compounds $(\mu \mathrm{g} / \mathrm{L})$} \\
\hline HMX & 4 n.d. at $<20$ & 4 n.d. at $<20$ & 4 n.d. at $<20$ \\
\hline RDX & 4 n.d. at $<30$ & 4 n.d. at $<30$ & 4 n.d. at $<30$ \\
\hline TNT & 4 n.d. at $<30$ & 4 n.d. at $<30$ & 4 n.d. at $<30$ \\
\hline \multicolumn{4}{|l|}{ Radiologicals (Bq/L) } \\
\hline \multirow[t]{4}{*}{ Gross alpha } & $0.03 \pm 0.10$ & $-0.072 \pm 0.087$ & $-0.01 \pm 0.11$ \\
\hline & $-0.02 \pm 0.10$ & $-0.05 \pm 0.12$ & $-0.018 \pm 0.089$ \\
\hline & $0.00 \pm 0.14$ & $-0.07 \pm 0.12$ & $-0.04 \pm 0.11$ \\
\hline & $-0.09 \pm 0.16$ & $0.04 \pm 0.13$ & $0.01 \pm 0.10$ \\
\hline \multirow[t]{4}{*}{ Gross beta } & $0.223 \pm 0.074$ & $0.272 \pm 0.077$ & $0.264 \pm 0.075$ \\
\hline & $0.119 \pm 0.086$ & $0.294 \pm 0.089$ & $0.207 \pm 0.090$ \\
\hline & $0.250 \pm 0.086$ & $0.336 \pm 0.082$ & $0.14 \pm 0.12$ \\
\hline & $0.19 \pm 0.08$ & $0.28 \pm 0.11$ & $0.21 \pm 0.06$ \\
\hline
\end{tabular}

...continued 
Table C-4. Parameters sampled in the Pit 6 Area monitoring wells, Site 300, 1992 (continued).

\begin{tabular}{|c|c|c|c|}
\hline \multirow[b]{2}{*}{ Parameters } & \multicolumn{3}{|c|}{ Well } \\
\hline & EP6-07 & EP6-08 & EP6-09 \\
\hline Methylene chloride & 4 n.d. at $<0.5$ & 4 n.d. at $<0.5$ & 4 n.d. at $<0.5$ \\
\hline \multirow[t]{2}{*}{ Tetrachloroethene } & 4 n.d. at $<0.5$ & 0.92 & 4 n.d. at $<0.5$ \\
\hline & & 3 n.d. at $<0.5$ & \\
\hline trans-1,2-Dichloroethene & 4 n.d. at $<0.5$ & 4 n.d. at $<0.5$ & 4 n.d. at $<0.5$ \\
\hline trans-1,3-Dichloropropene & 4 n.d. at $<0.5$ & 4 n.d. at $<0.5$ & 4 n.d. at $<0.5$ \\
\hline \multirow[t]{4}{*}{ Trichloroethene } & 4 n.d. at $<0.5$ & 4 n.d. at $<0.5$ & 21 \\
\hline & & & 25 \\
\hline & & & 20 \\
\hline & & & 15 \\
\hline Trichlorofluoromethane & 4 n.d. at $<0.5$ & 4 n.d. at $<0.5$ & 4 n.d. at $<0.5$ \\
\hline Vinyl chloride & 4 n.d. at $<0.5$ & 4 n.d. at $<0.5$ & 4 n.d. at $<0.5$ \\
\hline \multicolumn{4}{|l|}{ Title 22 herbicides ( $\mu \mathrm{g} / \mathrm{L}$ ) } \\
\hline 2,4,5-TP (Silvex) & 4 n.d. at $<1$ & 4 n.d. at $<1$ & 4 n.d. at $<1$ \\
\hline \multirow[t]{2}{*}{$2,4-D$} & 3 n.d. at $<5$ & 3 n.d. at $<5$ & 3 n.d. at $<5$ \\
\hline & 1 n.d. at $<10$ & 1 n.d. at $<10$ & 1 n.d. at $<10$ \\
\hline \multicolumn{4}{|l|}{ Title 22 pesticides $(\mu \mathrm{g} / \mathrm{L})$} \\
\hline $\mathrm{BHC}$, gamma isomer & 3 n.d. at $<0.03$ & 3 n.d. at $<0.03$ & 3 n.d. at $<0.03$ \\
\hline (Lindane) & 1 n.d. at $<0.4$ & 1 n.d. at $<0.4$ & 1 n.d. at $<0.4$ \\
\hline \multirow[t]{2}{*}{ Endrin } & 2 n.d. at $<0.05$ & 2 n.d. at $<0.05$ & 2 n.d. at $<0.05$ \\
\hline & 2 n.d. at $<0.1$ & 2 n.d. at $<0.1$ & 2 n.d. at $<0.1$ \\
\hline \multirow[t]{2}{*}{ Methoxychlor } & 3 n.d. at $<0.2$ & 3 n.d. at $<0.2$ & 3 n.d. at $<0.2$ \\
\hline & 1 n.d. at $<10$ & 1 n.d. at $<10$ & 1 n.d. at $<10$ \\
\hline \multirow[t]{2}{*}{ Toxaphene } & 1 n.d. at $<0.5$ & 1 n.d. at $<0.5$ & 1 n.d. at $<0.5$ \\
\hline & 2 n.d. at $<1$ & 2 n.d. at $<1$ & 2 n.d. at $<1$ \\
\hline \multicolumn{4}{|c|}{ Explosive compounds $(\mu g / L)$} \\
\hline $\mathrm{HMX}$ & 4 n.d. at $<20$ & 4 n.d. at $<20$ & 4 n.d. at $<20$ \\
\hline RDX & 4 n.d. at $<30$ & 4 n.d. at $<30$ & 4 n.d. at $<30$ \\
\hline TNT & 4 n.d. at $<30$ & 4 n.d. at $<30$ & 4 n.d. at $<30$ \\
\hline \multicolumn{4}{|l|}{ Kadiologicals (Bq/L) } \\
\hline \multirow[t]{4}{*}{ Gross alpha } & $-0.09 \pm 0.09$ & $0.06 \pm 0.12$ & $0.00 \pm 0.13$ \\
\hline & $-0.07 \pm 0.13$ & $0.02 \pm 0.12$ & $0.08 \pm 0.14$ \\
\hline & $0.02 \pm 0.11$ & $0.01 \pm 0.14$ & $-0.02 \pm 0.13$ \\
\hline & $0.00 \pm 0.10$ & $-0.02 \pm 0.07$ & $0.04 \pm 0.11$ \\
\hline \multirow[t]{4}{*}{ Gross beta } & $0.251 \pm 0.075$ & $0.224 \pm 0.074$ & $0.263 \pm 0.074$ \\
\hline & $0.258 \pm 0.079$ & $0.298 \pm 0.109$ & $0.293 \pm 0.093$ \\
\hline & $0.293 \pm 0.081$ & $0.245 \pm 0.076$ & $0.313 \pm 0.090$ \\
\hline & $0.26 \pm 0.06$ & $0.27 \pm 0.05$ & $0.28 \pm 0.06$ \\
\hline
\end{tabular}

...continued 
Table C-4. Parameters sampled in the Pit 6 Area monitoring wells, Site 300, 1992 (continued).

\begin{tabular}{cccc}
\hline & \multicolumn{3}{c}{ Well } \\
\cline { 2 - 4 } Parameters & K6-01 & K6-03 & K6-04 \\
\hline Tritium & $1.0 \pm 3.3$ & $-0.4 \pm 3.4$ & $-0.7 \pm 3.2$ \\
& $0.0 \pm 3.1$ & $3.3 \pm 4.3$ & $-0.7 \pm 3.3$ \\
Uranium-234 & $-1.7 \pm 3.6$ & $-2.5 \pm 3.6$ & $-5.2 \pm 3.5$ \\
& $3 \pm 4$ & $3 \pm 4$ & $1 \pm 4$ \\
& $0.015 \pm 0.006$ & $0.011 \pm 0.005$ & $0.022 \pm 0.006$ \\
& $0.009 \pm 0.008$ & $0.011 \pm 0.003$ & $0.026 \pm 0.010$ \\
Uranium-235 & $0.015 \pm 0.005$ & $0.011 \pm 0.004$ & $0.025 \pm 0.006$ \\
& $0.027 \pm 0.006$ & $0.014 \pm 0.005$ & $0.023 \pm 0.007$ \\
& $0.000 \pm 0.001$ & $0.002 \pm 0.003$ & $0.001 \pm 0.002$ \\
& $0.000 \pm 0.003$ & $0.001 \pm 0.001$ & $0.003 \pm 0.003$ \\
Uranium-238 & $0.000 \pm 0.001$ & $0.000 \pm 0.001$ & $0.001 \pm 0.002$ \\
& $0.0003004 \pm 0.0012$ & $0.0004 \pm 0.0017$ & $0.0021 \pm 0.0025$ \\
& $0.013 \pm 0.005$ & $0.009 \pm 0.004$ & $0.021 \pm 0.006$ \\
& $0.011 \pm 0.009$ & $0.009 \pm 0.002$ & $0.020 \pm 0.007$ \\
& $0.011 \pm 0.004$ & $0.004 \pm 0.003$ & $0.015 \pm 0.004$ \\
Radium-226 & $0.0109 \pm 0.0035$ & $0.0040 \pm 0.0022$ & $0.0149 \pm 0.0051$ \\
& $0.009 \pm 0.028$ & $0.008 \pm 0.023$ & $0.006 \pm 0.008$ \\
& $-0.009 \pm 0.016$ & $-0.008 \pm 0.017$ & $0.018 \pm 0.019$ \\
& $-0.012 \pm 0.016$ & $0.004 \pm 0.013$ & $-0.016 \pm 0.019$ \\
& $0.022 \pm 0.017$ & $0.011 \pm 0.014$ & $0.016 \pm 0.021$ \\
\hline
\end{tabular}




\section{Appendix C. Ground Water Protection Tables}

Table C-4. Parameters sampled in the Pit 6 Area monitoring wells, Site 300, 1992 (concluded).

\begin{tabular}{cccc}
\hline & \multicolumn{3}{c}{ Well } \\
\cline { 2 - 4 } Paranieters & EP6-07 & EP6-08 & EP6-09 \\
\hline Tritium & $1.9 \pm 3.5$ & $1.3 \pm 3.3$ & $-2.0 \pm 3.3$ \\
& $0.3 \pm 3.6$ & $-0.1 \pm 3.3$ & $1.7 \pm 4.4$ \\
Uranium-234 & $-3.2 \pm 3.4$ & $1.0 \pm 3.7$ & $-5.3 \pm 3.5$ \\
& $2 \pm 4$ & $-4 . \pm 4$ & $3 \pm 4$ \\
& $0.011 \pm 0.005$ & $0.024 \pm 0.006$ & $0.040 \pm 0.008$ \\
& $0.011 \pm 0.002$ & $0.024 \pm 0.004$ & $0.040 \pm 0.005$ \\
Uranium-235 & $0.010 \pm 0.004$ & $0.024 \pm 0.006$ & $0.051 \pm 0.008$ \\
& $0.013 \pm 0.004$ & $0.031 \pm 0.006$ & $0.045 \pm 0.008$ \\
& $0.000 \pm 0.001$ & $0.002 \pm 0.002$ & $0.001 \pm 0.002$ \\
& $0.001 \pm 0.001$ & $0.000 \pm 0.001$ & $0.002 \pm 0.001$ \\
Uranium-238 & $0.000 \pm 0.001$ & $0.001 \pm 0.002$ & $0.001 \pm 0.001$ \\
& $-0.0006 \pm 0.0011$ & $0.0003 \pm 0.0012$ & $0.0017 \pm 0.0013$ \\
& $0.007 \pm 0.004$ & $0.016 \pm 0.005$ & $0.033 \pm 0.007$ \\
& $0.007 \pm 0.002$ & $0.017 \pm 0.003$ & $0.034 \pm 0.004$ \\
& $0.007 \pm 0.003$ & $0.016 \pm 0.005$ & $0.037 \pm 0.007$ \\
Radium-226 & $0.0038 \pm 0.0024$ & $0.0134 \pm 0.0036$ & $0.0347 \pm 0.0065$ \\
& $-0.010 \pm 0.018$ & $-0.007 \pm 0.024$ & $0.003 \pm 0.021$ \\
& $0.004 \pm 0.019$ & $0.024 \pm 0.020$ & $0.014 \pm 0.015$ \\
& $0.029 \pm 0.022$ & $0.029 \pm 0.000$ & $0.019 \pm 0.013$ \\
& $0.002 \pm 0.011$ & $-0.002 \pm 0.013$ & $-0.005 \pm 0.019$ \\
\hline
\end{tabular}

Note: The abbreviation "n.d." stands for nondetection. 
Table C-5. Parameters sampled in the Pit 7 Complex Area monitoring wells, Site 300, 1992.

\begin{tabular}{|c|c|c|c|c|}
\hline \multirow[b]{2}{*}{ Parameters } & \multicolumn{4}{|c|}{ Well } \\
\hline & K7-01 & K7-03 & K7-06 & K7-10 \\
\hline \multicolumn{5}{|l|}{ Physical and chemical (mg/L) } \\
\hline \multirow[t]{4}{*}{ Barium } & 0.17 & 0.07 & 0.07 & 4 n.d. at $<0.05$ \\
\hline & 0.17 & 0.09 & 0.07 & \\
\hline & 0.19 & 0.07 & 0.07 & \\
\hline & 0.17 & 0.06 & 0.08 & \\
\hline \multirow[t]{2}{*}{ Beryllium } & 1 n.d. at $<0.0002$ & 1 n.d. at $<0.0002$ & 1 n.d. at $<0.0002$ & 1 n.d. at $<0.0002$ \\
\hline & 3 n.d. at $<0.0005$ & 3 n.d. at $<0.0005$ & 3 n.d. at $<0.0005$ & 3 n.d. at $<0.0005$ \\
\hline \multirow[t]{2}{*}{ Lead } & 0.004 & 0.004 & 0.003 & 0.003 \\
\hline & 3 n.d. at $<0.002$ & 3 n.d. at $<0.002$ & 3 n.d. at $<0.002$ & 3 n.d. at $<0.002$ \\
\hline \multirow[t]{2}{*}{ Vanadium } & 4 n.d. at $<0.05$ & 4 n.d. at $<0.05$ & 0.05 & 4 n.d. at $<0.05$ \\
\hline & & & 3 n.d. at $<0.05$ & \\
\hline \multicolumn{5}{|c|}{ Purgeable pollutants (EPA Method 601/624, $\mu \mathrm{g} / \mathrm{L}$ ) } \\
\hline 1,1,1-Trichloroethane & 4 n.d. at $<0.5$ & 4 n.d. at $<0.5$ & 4 n.d. at $<0.5$ & 4 n.d. at $<0.5$ \\
\hline 1,1,2,2-Tetrachloroethane & 4 n.d. at $<0.5$ & 4 n.d. at $<0.5$ & 4 n.d. at $<0.5$ & 4 n.d. at $<0.5$ \\
\hline 1,1,2-Trichloroethane & 4 n.d. at $<0.5$ & 4 n.d. at $<0.5$ & 4 n.d. at $<0.5$ & 4 n.d. at $<0.5$ \\
\hline 1,1-Dichloroethane & 4 n.d. at $<0.5$ & 4 n.d. at $<0.5$ & 4 n.d. at $<0.5$ & $4 \mathrm{n} . \mathrm{d}$. at $<0.5$ \\
\hline \multirow[t]{4}{*}{ 1,1-Dichloroethene } & 1.3 & & 4 n.d. at $<0.5$ & 4 n.d. at $<0.5$ \\
\hline & 1 & 0.8 & & \\
\hline & $<0.05$ & 2 n.d. at $<0.05$ & & \\
\hline & 2 & 0.78 & & \\
\hline 1,2-Dichlorobenzene & 4 n.d. at $<0.5$ & 4 n.d. at $<0.5$ & 4 n.d. at $<0.5$ & 4 n.d. at $<0.5$ \\
\hline 1,2-Dichloroethane & 4 n.d. at $<0.5$ & 4 n.d. at $<0.5$ & 4 n.d. at $<0.5$ & 4 n.d. at $<0.5$ \\
\hline 1,2-Dichloroethene (total) & 4 n.d. at $<0.5$ & 4 n.d. at $<0.5$ & 4 n.d. at $<0.5$ & 4 n.d. at $<0.5$ \\
\hline 1,2-Dichloropropane & 4 n.d. at $<0.5$ & 4 n.d. at $<0.5$ & 4 n.d. at $<0.5$ & 4 n.d. at $<0.5$ \\
\hline 1,3-Dichlorobenzene & 4 n.d. at $<0.5$ & 4 n.d. at $<0.5$ & 4 n.d. at $<0.5$ & 4 n.d. at $<0.5$ \\
\hline 1,4-Dichlorobenzene & 4 n.d. at $<0.5$ & 4 n.d. at $<0.5$ & 4 n.d. at $<0.5$ & 4 n.d. at $<0.5$ \\
\hline 2-Chloroethylvinylether & 4 n.d. at $<0.5$ & 4 n.d. at $<0.5$ & 4 n.d. at $<0.5$ & 4 n.d. at $<0.5$ \\
\hline Bromodichloromethane & 4 n.d. at $<0.5$ & 4 n.d. at $<0.5$ & 4 n.d. at $<0.5$ & 4 n.d. at $<0.5$ \\
\hline Bromoform & 4 n.d. at $<0.5$ & 4 n.d. at $<0.5$ & 4 n.d. at $<0.5$ & 4 n.d. at $<0.5$ \\
\hline Bromomethane & 4 n.d. at $<0.5$ & 4 n.d. at $<0.5$ & 4 n.d. at $<0.5$ & 4 n.d. at $<0.5$ \\
\hline Carbon tetrachloride & 4 n.d. at $<0.5$ & 4 n.d. at $<0.5$ & 4 n.d. at $<0.5$ & 4 n.d. at $<0.5$ \\
\hline Chlorobenzene & 4 n.d. at $<0.5$ & 4 n.d. at $<0.5$ & 4 n.d. at $<0.5$ & 4 n.d. at $<0.5$ \\
\hline
\end{tabular}

...continued 


\section{Appendix C. Ground Water Protection Tables}

Table C-5. Parameters sampled in the Pit 7 Complex Area monitoring wells, Site 300, 1992 (continued).

\begin{tabular}{|c|c|c|c|c|}
\hline \multirow[b]{2}{*}{ Parameters } & \multicolumn{4}{|c|}{ Well } \\
\hline & NC7-25 & NC7-26 & NC7-47 & NC7-48 \\
\hline \multicolumn{5}{|l|}{ Physical and chemical (mg/L) } \\
\hline \multirow[t]{4}{*}{ Barium } & 0.05 & 4 n.d. at $<0.05$ & 4 n.d. at $<0.05$ & 0.12 \\
\hline & 0.06 & & & 0.19 \\
\hline & 0.06 & & & \\
\hline & 0.06 & & & \\
\hline \multirow[t]{2}{*}{ Beryllium } & 1 n.d. at $<0.0002$ & 1 n.d. at $<0.0002$ & 1 n.d. at $<0.0002$ & $<0.0005$ \\
\hline & 3 n.d. at $<0.0005$ & 3 n.d. at $<0.0005$ & 3 n.d. at $<0.0005$ & $<0.0002$ \\
\hline \multirow[t]{3}{*}{ Lead } & 0.002 & 0.003 & 0.003 & 2 n.d. at $<0.002$ \\
\hline & 0.002 & 0.003 & 3 n.d. at $<0.002$ & \\
\hline & 2 n.d. at $<0.002$ & 2 n.d. at $<0.002$ & & \\
\hline \multirow[t]{3}{*}{ Vanadium } & 4 n.d. at $<0.05$ & 3 n.d. at $<0.05$ & 0.051 & 2 n.d. at $<0.05$ \\
\hline & & 1 n.d. at $<0.06$ & 0.08 & \\
\hline & & & 2 n.d. at $<0.05$ & \\
\hline \multicolumn{5}{|c|}{ Purgeable pollutants (EPA Method 601/624, $\mu \mathrm{g} / \mathrm{L}$ ) } \\
\hline \multirow[t]{2}{*}{ 1,1,1-Trichloroethane } & 4 n.d. at $<0.5$ & 1.2 & 4 n.d. at $<0.5$ & 2 n.d. at $<0.5$ \\
\hline & & 3 n.d. at $<0.5$ & & \\
\hline 1,1,2,2-Tetrachloroethane & 4 n.d. at $<0.5$ & 4 n.d. at $<0.5$ & 4 n.d. at $<0.5$ & 2 n.d. at $<0.5$ \\
\hline 1,1,2-Trichloroethane & 4 n.d. at $<0.5$ & 4 n.d. at $<0.5$ & 4 n.d. at $<0.5$ & 2 n.d. at $<0.5$ \\
\hline 1,1-Dichloroethane & 4 n.d. at $<0.5$ & 4 n.d. at $<0.5$ & 4 n.d. at $<0.5$ & 2 n.d. at $<0.5$ \\
\hline 1,1-Dichloroethene & 4 n.d. at $<0.5$ & 4 n.d. at $<0.5$ & 4 n.d. at $<0.5$ & 2 n.d. at $<0.5$ \\
\hline 1,2-Dichlorobenzene & 4 n.d. at $<0.5$ & 4 n.d. at $<0.5$ & 4 n.d. at $<0.5$ & 2 n.d. at $<0.5$ \\
\hline 1,2-Dichloroethane & 4 n.d. at $<0.5$ & 4 n.d. at $<0.5$ & 4 n.d. at $<0.5$ & 2 n.d. at $<0.5$ \\
\hline 1,2-Dichloroethene (total) & 4 n.d. at $<0.5$ & 4 n.d. at $<0.5$ & 4 n.d. at $<0.5$ & 2 n.d. at $<0.5$ \\
\hline 1,2-Dichloropropane & 4 n.d. at $<0.5$ & 4 n.d. at $<0.5$ & 4 n.d. at $<0.5$ & 2 n.d. at $<0.5$ \\
\hline 1,3-Dichlorobenzene & 4 n.d. at $<0.5$ & 4 n.d. at $<0.5$ & 4 n.d. at $<0.5$ & 2 n.d. at $<0.5$ \\
\hline 1,4-Dichlorobenzene & 4 n.d. at $<0.5$ & 4 n.d. at $<0.5$ & 4 n.d. at $<0.5$ & 2 n.d. at $<0.5$ \\
\hline 2-Chloroethylvinylether & 4 n.d. at $<0.5$ & 4 n.d. at $<0.5$ & 4 n.d. at $<0.5$ & 2 n.d. at $<0.5$ \\
\hline Bromodichloromethane & 4 n.d. at $<0.5$ & 4 n.d. at $<0.5$ & 4 n.d. at $<0.5$ & 2 n.d. at $<0.5$ \\
\hline Bromoform & 4 n.d. at $<0.5$ & 4 n.d. at $<0.5$ & 4 n.d. at $<0.5$ & 2 n.d. at $<0.5$ \\
\hline Bromomethane & 4 n.d. at $<0.5$ & 4 n.d. at $<0.5$ & 4 n.d. at $<0.5$ & 2 n.d. at $<0.5$ \\
\hline Carbon tetrachloride & 4 n.d. at $<0.5$ & 4 n.d. at $<0.5$ & 4 n.d. at $<0.5$ & 2 n.d. at $<0.5$ \\
\hline Chlorobenzene & 4 n.d. at $<0.5$ & 4 n.d. at $<0.5$ & 4 n.d. at $<0.5$ & 2 n.d. at $<0.5$ \\
\hline
\end{tabular}


Table C-5. Parameters sampled in the Pit 7 Complex Area monitoring wells, Site 300, 1992 (continued).

\begin{tabular}{|c|c|c|c|c|}
\hline \multirow[b]{2}{*}{ Parameters } & \multicolumn{4}{|c|}{ Well } \\
\hline & K7-01 & K7-03 & K7-06 & K7-10 \\
\hline Chloroethane & 4 n.d. at $<0.5$ & 4 n.d. at $<0.5$ & 4 n.d. at $<0.5$ & 4 n.d. at $<0.5$ \\
\hline Chloroform & 4 n.d. at $<0.5$ & 4 n.d. at $<0.5$ & 4 n.d. at $<0.5$ & 4 n.d. at $<0.5$ \\
\hline Chloromethane & 4 n.d. at $<0.5$ & 4 n.d. at $<0.5$ & 4 n.d. at $<0.5$ & 4 n.d. at $<0.5$ \\
\hline cis-1,2-Dichloroethene & 4 n.d. at $<0.5$ & 4 n.d. at $<0.5$ & 4 n.d. at $<0.5$ & 4 n.d. at $<0.5$ \\
\hline cis-1,3-Dichloropropene & 4 n.d. at $<0.5$ & 4 n.d. at $<0.5$ & 4 n.d. at $<0.5$ & 4 n.d. at $<0.5$ \\
\hline Dibromochloromethane & 4 n.d. at $<0.5$ & 4 n.d. at $<0.5$ & 4 n.d. at $<0.5$ & 4 n.d. at $<0.5$ \\
\hline Dichlorodifluoromethane & 4 n.d. at $<0.5$ & 4 n.d. at $<0.5$ & 4 n.d. at $<0.5$ & 4 n.d. at $<0.5$ \\
\hline Freon-113 & 4 n.d. at $<0.5$ & 4 n.d. at $<0.5$ & 4 n.d. at $<0.5$ & 4 n.d. at $<0.5$ \\
\hline Methylene chloride & 4 n.d. at $<0.5$ & 4 n.d. at $<0.5$ & 4 n.d. at $<0.5$ & 4 n.d. at $<0.5$ \\
\hline Tetrachloroethene & 4 n.d. at $<0.5$ & 4 n.d. at $<0.5$ & 4 n.d. at $<0.5$ & 4 n.d. at $<0.5$ \\
\hline trans-1,2-Dichloroethene & 4 n.d. at $<0.5$ & 4 n.d. at $<0.5$ & 4 n.d. at $<0.5$ & 4 n.d. at $<0.5$ \\
\hline trans-1,3-Dichloropropene & 4 n.d. at $<0.5$ & 4 n.d. at $<0.5$ & 4 n.d. at $<0.5$ & 4 n.d. at $<0.5$ \\
\hline \multirow[t]{4}{*}{ Trichloroethene } & 12 & 3.5 & 4 n.d. at $<0.5$ & 4 n.d. at $<0.5$ \\
\hline & 10 & 7.6 & & \\
\hline & 11 & 6.7 & & \\
\hline & 11 & 3.1 & & \\
\hline Trichlorofluoromethane & 4 n.d. at $<0.5$ & 4 n.d. at $<0.5$ & 4 n.d. at $<0.5$ & 4 n.d. at $<0.5$ \\
\hline Vinyl chloride & $4 \mathrm{n} . \mathrm{d}$. at $<0.5$ & 4 n.d. at $<0.5$ & 4 n.d. at $<0.5$ & 4 n.d. at $<0.5$ \\
\hline \multicolumn{5}{|l|}{ 2-Hexanone } \\
\hline \multicolumn{5}{|l|}{ 4-Methyl-2-pentanone } \\
\hline \multicolumn{5}{|l|}{ Acetone } \\
\hline \multicolumn{5}{|l|}{ Acrolein } \\
\hline \multicolumn{5}{|l|}{ Acrylonitrile } \\
\hline \multicolumn{5}{|l|}{ Benzene } \\
\hline \multicolumn{5}{|l|}{ Carbon disulfide } \\
\hline \multicolumn{5}{|l|}{ Ethylbenzene } \\
\hline \multicolumn{5}{|l|}{ Methyl ethyl ketone } \\
\hline \multicolumn{5}{|l|}{ Styrene } \\
\hline \multicolumn{5}{|l|}{ Toluene } \\
\hline \multicolumn{5}{|l|}{ Total xylene isomers } \\
\hline Vinyl acetate & & & & \\
\hline
\end{tabular}




\section{Appendix C. Ground Water Protection Tables}

Table C-5. Parameters sampled in the Pit 7 Complex Area monitoring wells, Site 300, 1992 (continued).

\begin{tabular}{|c|c|c|c|c|}
\hline \multirow[b]{2}{*}{ Parameters } & \multicolumn{4}{|c|}{ Well } \\
\hline & NC7-25 & NC7-26 & NC7-47 & NC7-48 \\
\hline Chloroethane & 4 n.d. at $<0.5$ & 4 n.d. at $<0.5$ & 4 n.d. at $<0.5$ & 2 n.d. at $<0.5$ \\
\hline \multirow[t]{2}{*}{ Chloroform } & 4 n.d. at $<0.5$ & 0.6 & 4 n.d. at $<0.5$ & 2 n.d. at $<0.5$ \\
\hline & & 3 n.d. at $<0.5$ & & \\
\hline Chloromethane & 4 n.d. at $<0.5$ & 4 n.d. at $<0.5$ & 4 n.d. at $<0.5$ & 2 n.d. at $<0.5$ \\
\hline cis-1,2-Dichloroethene & 4 n.d. at $<0.5$ & 4 n.d. at $<0.5$ & 4 n.d. at $<0.5$ & 2 n.d. at $<0.5$ \\
\hline cis-1,3-Dichloropropene & 4 n.d. at $<0.5$ & 4 n.d. at $<0.5$ & 4 n.d. at $<0.5$ & 2 n.d. at $<0.5$ \\
\hline Dibromochloromethane & 4 n.d. at $<0.5$ & 4 n.d. at $<0.5$ & 4 n.d. at $<0.5$ & 2 n.d. at $<0.5$ \\
\hline Dichlorodifluoromethane & 4 n.d. at $<0.5$ & 4 n.d. at $<0 . \dot{E}$ & 4 n.d. at $<0.5$ & 2 n.d. at $<0.5$ \\
\hline Freon-113 & 4 n.d. at $<0.5$ & 4 n.d. at $<0.5$ & 4 n.d. at $<0.5$ & 2 n.d. at $<0.5$ \\
\hline \multirow[t]{2}{*}{ Methylene chloride } & 4 n.d. at $<0.5$ & 4 n.d. at $<0.5$ & 4 n.d. at $<0.5$ & 2 n.d. at $<0.5$ \\
\hline & & & & 1 n.d. at $<2$ \\
\hline Tetrachloroethene & 4 n.d. at $<0.5$ & 4 n.d. at $<0.5$ & 4 n.d. at $<0.5$ & 2 n.d. at $<0.5$ \\
\hline trans-1,2-Dichloroethene & 4 n.d. at $<0.5$ & 4 n.d. at $<0.5$ & 4 n.d. at $<0.5$ & 2 n.d. at $<0.5$ \\
\hline trans-1,3-Dichloropropene & 4 n.d. at $<0.5$ & 4 n.d. at $<0.5$ & 4 n.d. at $<0.5$ & 2 n.d. at $<0.5$ \\
\hline Trichloroethene & 4 n.d. at $<0.5$ & 4 n.d. at $<0.5$ & 4 n.d. at $<0.5$ & 2 n.d. at $<0.5$ \\
\hline Trichlorofluoromethane & 4 n.d. at $<0.5$ & 4 n.d. at $<0.5$ & 4 n.d. at $<0.5$ & 0.9 \\
\hline Vinyl chloride & 4 n.d. at $<0.5$ & 4 n.d. at $<0.5$ & 4 n.d. at $<0.5$ & 4 n.d. at $<0.5$ \\
\hline 2-Hexanone & & & & $<0.5$ \\
\hline 4-Methyl-2-pentanone & & & & $<5$ \\
\hline Acetone & & & & $<5$ \\
\hline Acrolein & & & & $<5$ \\
\hline Acrylonitrile & & & & $<5$ \\
\hline Benzene & & & & $<0.5$ \\
\hline Carbon disulfide & & & & $<0.5$ \\
\hline Ethylbenzene & & & & $<0.5$ \\
\hline Methyl ethyl ketone & & & & $<10$ \\
\hline Styrene & & & & $<0.5$ \\
\hline Toluene & & & & $<0.5$ \\
\hline Total xylene isomers & & & & $<0.5$ \\
\hline Vinyl acetałe & & & & $<0.5$ \\
\hline
\end{tabular}


Table C-5. Parameters sampled in the Pit 7 Complex Area monitoring wells, Site 300, 1992 (continued).

\begin{tabular}{|c|c|c|c|c|}
\hline \multirow[b]{2}{*}{ Parameters } & \multicolumn{4}{|c|}{ Well } \\
\hline & K7-01 & K7-03 & K7.06 & K7-10 \\
\hline \multicolumn{5}{|l|}{ Radioiogicals (Bq/L) } \\
\hline \multirow[t]{4}{*}{ Gross alpha } & $0.22 \pm 0.13$ & $0.15 \pm 0.11$ & $0.125 \pm 0.094$ & $0.01 \pm 0.13$ \\
\hline & $0.44 \pm 0.17$ & $0.10 \pm 0.13$ & $0.032 \pm 0.097$ & $-0.06 \pm 0.11$ \\
\hline & $0.15 \pm 0.13$ & $0.19 \pm 0.12$ & $0.046 \pm 0.082$ & $0.02 \pm 0.12$ \\
\hline & $0.27 \pm 0.15$ & $0.10 \pm 0.10$ & $0.038 \pm 0.042$ & $0.15 \pm 0.19$ \\
\hline \multirow[t]{4}{*}{ Gross beta } & $0.190 \pm 0.076$ & $0.088 \pm 0.072$ & $0.137 \pm 0.072$ & $0.534 \pm 0.087$ \\
\hline & $0.300 \pm 0.082$ & $0.162 \pm 0.079$ & $0.111 \pm 0.083$ & $0.219 \pm 0.077$ \\
\hline & $-0.02 \pm 0.11$ & $0.225 \pm 0.073$ & $0.087 \pm 0.064$ & $0.234 \pm 0.075$ \\
\hline & $0.40 \pm 0.08$ & $0.19 \pm 0.07$ & $-0.023 \pm 0.035$ & $0.322 \pm 0.081$ \\
\hline \multirow[t]{4}{*}{ Tritium } & $1882 \pm 94$ & $2540 \pm 130$ & $-4.7 \pm 2.9$ & $-4.0 \pm 4.6$ \\
\hline & $1596 \pm 79$ & $6420 \pm 320$ & $0.9 \pm 2.4$ & $1.4 \pm 3.4$ \\
\hline & $1438 \pm 71$ & $1600 \pm 79$ & $0.5 \pm 3.4$ & $1.5 \pm 2.5$ \\
\hline & $1795 \pm 89$ & $1436 \pm 72$ & & $-3.1 \pm 3.9$ \\
\hline \multirow[t]{4}{*}{ Uranium-233, -234} & $0.239 \pm 0.025$ & $0.036 \pm 0.008$ & $0.027 \pm 0.006$ & $0.047 \pm 0.009$ \\
\hline & $0.232 \pm 0.014$ & $0.075 \pm 0.010$ & $0.021 \pm 0.003$ & $0.027 \pm 0.006$ \\
\hline & $0.255 \pm 0.031$ & $0.051 \pm 0.009$ & $0.192 \pm 0.020$ & $0.035 \pm 0.006$ \\
\hline & $0.232 \pm 0.029$ & $0.031 \pm 0.006$ & $0.025 \pm 0.006$ & $0.018 \pm 0.005$ \\
\hline \multirow[t]{4}{*}{ Uranium-235 } & $0.016 \pm 0.005$ & $0.001 \pm 0.002$ & $0.000 \pm 0.001$ & $0.001 \pm 0.002$ \\
\hline & $0.011 \pm 0.003$ & $0.003 \pm 0.002$ & $0.001 \pm 0.001$ & $0.002 \pm 0.001$ \\
\hline & $0.010 \pm 0.004$ & $0.003 \pm 0.002$ & $0.002 \pm 0.002$ & $0.003 \pm 0.002$ \\
\hline & $0.009 \pm 0.006$ & $0.0006 \pm 0.0012$ & $0.0003 \pm 0.0019$ & $0.0012 \pm 0.0015$ \\
\hline \multirow[t]{4}{*}{ Uranium-238 } & $0.224 \pm 0.023$ & $0.037 \pm 0.008$ & $0.017 \pm 0.005$ & $0.024 \pm 0.006$ \\
\hline & $0.215 \pm 0.014$ & $0.078 \pm 0.010$ & $0.017 \pm 0.003$ & $0.019 \pm 0.005$ \\
\hline & $0.233 \pm 0.028$ & $0.041 \pm 0.008$ & $0.021 \pm 0.005$ & $0.014 \pm 0.004$ \\
\hline & $0.220 \pm 0.028$ & $0.029 \pm 0.006$ & $0.024 \pm 0.005$ & $0.017 \pm 0.005$ \\
\hline \multirow[t]{4}{*}{ Radium-226 } & $0.032 \pm 0.024$ & $0.006 \pm 0.012$ & & \\
\hline & $0.081 \pm 0.031$ & $0.000 \pm 0.012$ & & $0.007 \pm 0.018$ \\
\hline & $0.044 \pm 0.019$ & $0.005 \pm 0.017$ & & $0.021 \pm 0.015$ \\
\hline & $0.057 \pm 0.025$ & $0.023 \pm 0.017$ & & $0.024 \pm 0.024$ \\
\hline
\end{tabular}

...continued 


\section{Appendix C. Ground Water Protection Tables}

Table C-5. Parameters sampled in the Pit 7 Complex Area monitoring wells, Site 300, 1992 (concluded).

\begin{tabular}{|c|c|c|c|c|}
\hline \multirow[b]{2}{*}{ Parameters } & \multicolumn{4}{|c|}{ Well } \\
\hline & NC7-25 & NC7-26 & NC7-47 & NC7-48 \\
\hline \multicolumn{5}{|l|}{ Radiologicals (Bq/L) } \\
\hline \multirow[t]{4}{*}{ Gross alpha } & $0.29 \pm 0.18$ & $-0.008 \pm 0.092$ & $0.03 \pm 0.10$ & $0.57 \pm 0.26$ \\
\hline & $0.63 \pm 0.23$ & $-0.047 \pm 0.066$ & $0.01 \pm 0.11$ & $0.05 \pm 0.14$ \\
\hline & $0.32 \pm 0.16$ & $0.034 \pm 0.088$ & $0.01 \pm 0.08$ & \\
\hline & $0.98 \pm 0.26$ & $0.00 \pm 0.10$ & $0.10 \pm 0.10$ & \\
\hline \multirow[t]{4}{*}{ Gross beta } & $0.70 \pm 0.10$ & $0.104 \pm 0.081$ & $0.233 \pm 0.075$ & $1.16 \pm 0.12$ \\
\hline & $0.593 \pm 0.097$ & $0.125 \pm 0.076$ & $0.272 \pm 0.090$ & $0.114 \pm 0.072$ \\
\hline & $0.80 \pm 0.11$ & $0.080 \pm 0.076$ & $0.08 \pm 0.11$ & \\
\hline & $0.80 \pm 0.11$ & $0.11 \pm 0.05$ & $0.18 \pm 0.07$ & \\
\hline \multirow[t]{4}{*}{ Tritium } & $11060 \pm 540$ & $36.8 \pm 5.4$ & $-6.1 \pm 3.9$ & $4.1 \pm 3.6$ \\
\hline & $11690 \pm 574$ & $40.1 \pm 4.8$ & $1.4 \pm 3.6$ & $2 \pm 3$ \\
\hline & $8532 \pm 417$ & $32.1 \pm 3.8$ & $0.2 \pm 2.3$ & $1.5 \pm 3.4$ \\
\hline & $10360 \pm 511$ & $29 \pm 5$ & $-0.63 \pm 4.18$ & \\
\hline \multirow[t]{4}{*}{ Uranium-233, -234 } & $0.423 \pm 0.104$ & $0.007 \pm 0.003$ & $0.048 \pm 0.008$ & $0.130 \pm 0.017$ \\
\hline & $0.407 \pm 0.033$ & $0.005 \pm 0.002$ & $0.042 \pm 0.005$ & $0.69 \pm 0.05$ \\
\hline & $0.443 \pm 0.020$ & $0.017 \pm 0.003$ & $0.060 \pm 0.005$ & \\
\hline & $0.45 \pm 0.04$ & $0.012 \pm 0.008$ & $0.041 \pm 0.008$ & \\
\hline \multirow[t]{4}{*}{ Uranium-235 } & $0.018 \pm 0.005$ & $0.0004 \pm 0.001$ & $0.001 \pm 0.001$ & $0.008 \pm 0.004$ \\
\hline & $0.019 \pm 0.006$ & $0.001 \pm 0.001$ & $0.001 \pm 0.004$ & $0.048 \pm 0.010$ \\
\hline & $0.019 \pm 0.003$ & $0.001 \pm 0.001$ & $0.001 \pm 0.001$ & \\
\hline & $0.018 \pm 0.005$ & $-0.0012 \pm 0.0023$ & $0.0021 \pm 0.0016$ & \\
\hline \multirow[t]{4}{*}{ Uranium-238 } & $0.338 \pm 0.030$ & $0.002 \pm 0.002$ & $0.026 \pm 0.005$ & $0.326 \pm 0.031$ \\
\hline & $0.347 \pm 0.030$ & $0.003 \pm 0.002$ & $0.027 \pm 0.004$ & $1.38 \pm 0.09$ \\
\hline & $0.383 \pm 0.018$ & $0.005 \pm 0.002$ & $0.023 \pm 0.003$ & \\
\hline & $0.392 \pm 0.035$ & $0.007 \pm 0.006$ & $0.029 \pm 0.007$ & \\
\hline \multirow[t]{4}{*}{ Radium-226 } & $0.036 \pm 0.031$ & & & $0.169 \pm 0.040$ \\
\hline & $0.017 \pm 0.022$ & & & $1.310 \pm 0.093$ \\
\hline & $0.016 \pm 0.022$ & & & \\
\hline & $0.039 \pm 0.028$ & & & \\
\hline
\end{tabular}

Note: The abbreviation "n.d." stands for nondetection. 
Table C-6. Parameters sampled in Well K8-01, Site 300, 1992.

\begin{tabular}{|c|c|c|c|}
\hline Parameters & K8-01 & Parameters & K8-01 \\
\hline Physical and chemical (mg/L) & & Sulfate & 78 \\
\hline Aluminum & $<0.2$ & & 76 \\
\hline \multirow[t]{2}{*}{ Ammonia nitrogen (as $\mathrm{N}$ ) } & 0.02 & Total alkalinity (as $\mathrm{CaCO}_{3}$ ) & 200 \\
\hline & 0.049 & & 210 \\
\hline \multirow[t]{2}{*}{ Arsenic } & 0.017 & Total dissolved solids (TDS) & 500 \\
\hline & 0.014 & & 550 \\
\hline Barium & 2 n.d. $<0.05$ & Total Kjeldahl nitrogen & 2 n.d. $<0.5$ \\
\hline \multirow[t]{2}{*}{ Beryllium } & $<0.0005$ & Total organic carbon (TOC) & 4.4 \\
\hline & $<0.0002$ & & 3.79 \\
\hline \multirow[t]{2}{*}{ Bicarbonate alk (as $\mathrm{CaCO}_{3}$ ) } & 200 & Total organic halides (TOX) & 0.021 \\
\hline & 210 & & 8 n.d. $<0.02$ \\
\hline \multirow{4}{*}{$\begin{array}{l}\text { Cadmium } \\
\text { Carbonate alk (as } \mathrm{CaCO}_{3} \text { ) } \\
\text { Chloride }\end{array}$} & 2 n.d. $<0.0005$ & Specific conductance & 670 \\
\hline & 2 n.d. $<1$ & $(\mu \mathrm{mhos} / \mathrm{cm})$ & \\
\hline & 64 & & 740 \\
\hline & 68 & $\mathrm{pH}$ & 7.6 \\
\hline \multirow[t]{2}{*}{ Chromium } & 0.022 & & 7.6 \\
\hline & 0.026 & $\begin{array}{l}\text { Purgeable pollutants } \\
\text { (EPA Methods 601/624, } \mu g / \text { ) }\end{array}$ & \\
\hline \multirow[t]{2}{*}{ Fluoride } & 0.5 & 1,1,1-Trichloroethane & 3 n.d. $<0.5$ \\
\hline & 0.44 & 1,1,2,2-Tetrachloroethane & 3 n.d. $<0.5$ \\
\hline \multirow[t]{2}{*}{ Lead } & $<0.002$ & 1,1,2-Trichloroethane & 2 n.d. $<0.5$ \\
\hline & 0.0025 & 1,1-Dichloroethane & 1 n.d. $<0.4$ \\
\hline Low level phenolics & 2 n.d. $<0.005$ & & 2 n.d. $<0.5$ \\
\hline Mercury & 2 n.d. $<0.0002$ & 1,1-Dichloroethene & 1 n.d. $<0.2$ \\
\hline Nitrate plus nitrite (as N) & 9.2 & & 2 n.d. $<0.5$ \\
\hline \multirow[t]{2}{*}{ Nitrate plus nitrite (as $\mathrm{NO}_{3}$ ) } & 41 & 1,2-Dichlorobenzene & $\begin{array}{r}2 \text { n.d. }<0.5 \\
1 \text { n.d. }<4\end{array}$ \\
\hline & 2 n.d. $<0.01$ & 1,2-Dichloroethane & 2.7 \\
\hline Nitrite (as $\mathrm{NO}_{2}$ ) & 2 n.d. $<0.03$ & & 2.6 \\
\hline \multirow[t]{2}{*}{ Selenium } & 0.003 & 1 2 Dighlarnathang (tall & 2.1 \\
\hline & 0.0029 & 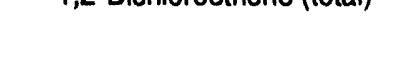 & 2 n.d. $<0.5$ \\
\hline Silica (as $\mathrm{SiO}_{2}$ ) & 66 & 1,2-Dichloropropane & 3 n.d. $<0.5$ \\
\hline \multirow[t]{2}{*}{ Silver } & $<0.0005$ & 1,3-Dichlorobenzene & 2 n.d. $<0.5$ \\
\hline & $<0.05$ & 14-Dichlarobenzene & $2 n d<05$ \\
\hline \multirow[t]{2}{*}{ Sodium } & 62 & & 1 n.d. $<4$ \\
\hline & 60 & & \\
\hline
\end{tabular}


Table C-6. Parameters sampled in Well K8-01, Site 300, 1992 (concluded).

\begin{tabular}{|c|c|c|c|}
\hline Parameters & K8-01 & Parameters & K8-01 \\
\hline \multirow[t]{3}{*}{ 2-Chloroethylvinylether } & $<0.5$ & Methyl ethyl ketone & $<10$ \\
\hline & $<2$ & & $<5$ \\
\hline & $<1$ & Methylene chloride & 2 n.d. $<2$ \\
\hline 2-Hexanone & 2 n.d. $<0.5$ & & 1 n.d. $<0.5$ \\
\hline \multirow[t]{2}{*}{ 4-Methyl-2-pentanone } & $<0.5$ & Styrene & 2 n.d. $<0.5$ \\
\hline & $<5$ & Tetrachloroethene & 3 n.d. $<0.5$ \\
\hline Acetone & 2 n.d. $<5$ & Toluene & 2 n.d. $<0.5$ \\
\hline \multirow[t]{2}{*}{ Acrolein } & $<5$ & Total xylene isomers & 2 n.d. $<0.5$ \\
\hline & $<10$ & trans-1,2-Dichloroethene & 1 n.d. $<0.4$ \\
\hline Acrylonitrile & 2 n.d. $<5$ & & 2 n.d. $<0.5$ \\
\hline Benzene & 2 n.d. $<0.5$ & trans-1,3-Dichloropropene & 2 n.d. $<0.5$ \\
\hline \multirow[t]{2}{*}{ Bromodichloromethane } & 2 n.d. $<0.5$ & & 1 n.d. $<0.6$ \\
\hline & 1 n.d. $<0.7$ & Trichloroethene & 6 \\
\hline Bromoform & 1 n.d. $<0.7$ & & 4.4 \\
\hline Bromomethane & 1 n.d. $<0.7$ & & 3.2 \\
\hline Carbon disulfide & 2 n.d. $<0.5$ & Trichlorofluoromethane & 1 n.d. $<0.4$ \\
\hline \multirow[t]{2}{*}{ Carbon tetrachloride } & 2 n.d. $<0.5$ & & 2 n.d. $<0.5$ \\
\hline & 1 n.d. $<0.6$ & Vinyl acetate & 2 n.d. $<0.5$ \\
\hline \multirow[t]{2}{*}{ Chlorobenzene } & 2 n.d. $<0.5$ & Vinyl chloride & 3 n.d. $<0.5$ \\
\hline & 1 n.d. $<0.7$ & & \\
\hline \multirow[t]{2}{*}{ Chloroethane } & 2 n.d. $<0.5$ & Explosive compounds $(\mu g / \Omega)$ & \\
\hline & 1 n.d. $<1$ & $H M X$ & 2 n.d. at $<20$ \\
\hline \multirow[t]{2}{*}{ Chloroform } & 0.9 & RDX & 2 n.d. at $<30$ \\
\hline & 2 n.d. $<0.5$ & TNT & 2 n.d. at $<30$ \\
\hline \multirow[t]{3}{*}{ Chloromethane } & 0.5 & & \\
\hline & $<0.5$ & Radiologicals (Bq/L) & \\
\hline & $<0.6$ & Gross alpha & $0.11 \pm 0.11$ \\
\hline \multirow[t]{2}{*}{ cis-1,2-Dichloroethene } & 1 n.d. $<0.4$ & Gross beta & $0.19 \pm 0.07$ \\
\hline & 2 n.d. $<0.5$ & Tritium & $0.0 \pm 4.8$ \\
\hline cis-1,3-Dichloropropene & 3 n.d. $<0.5$ & & $-1.6 \pm 3.4$ \\
\hline \multirow[t]{2}{*}{ Dibromochloromethane } & 2 n.d. $<0.5$ & & $2.7 \pm 3.4$ \\
\hline & 1 n.d. $<0.6$ & Uranium-233,-234 & $0.16 \pm 0.01$ \\
\hline Dichlorodifluoromethane & $<1$ & Uranium-235 & $0.00 \pm 0.01$ \\
\hline Ethylbenzene & 2 n.d. $<0.5$ & Uranium-238 & $0.10 \pm 0.01$ \\
\hline \multirow[t]{3}{*}{ Freon-113 } & $<0.5$ & Radium-226 & $0.00 \pm 0.00$ \\
\hline & $<1$ & Radium-228 & $0.00 \pm 0.15$ \\
\hline & $<0.6$ & & \\
\hline
\end{tabular}

Note: The abbreviation "n.d." stands for nondetection. 
Table C-7. Parameters analyzed in the explosives rinse water surface impoundments area monitoring wells, Site 300, 1992.

\begin{tabular}{|c|c|c|c|c|c|}
\hline \multirow[b]{2}{*}{ Parameters } & \multicolumn{5}{|c|}{ Well } \\
\hline & W-817-01 & W-817-02 & W-817-03 & $W-817-13 A$ & W-817-04 \\
\hline \multicolumn{6}{|l|}{ Physical and chemical (mg/L) } \\
\hline Antimony & 4 n.d. at $<0.2$ & 4 n.d. at $<0.2$ & 4 n.d. at $<0.2$ & & 4 n.d. at $<0.2$ \\
\hline \multirow[t]{16}{*}{ Arsenic } & 0.041 & 0.043 & 0.044 & & 0.052 \\
\hline & 0.04 & 0.051 & 0.025 & & 0.031 \\
\hline & 0.044 & 0.047 & 0.045 & & 0.046 \\
\hline & 0.045 & 0.046 & 0.044 & & 0.053 \\
\hline & 0.044 & 0.05 & 0.047 & & 0.055 \\
\hline & 0.027 & 0.038 & 0.038 & & 0.031 \\
\hline & 0.046 & 0.046 & 0.045 & & 0.046 \\
\hline & 0.05 & 0.053 & 0.044 & & 0.055 \\
\hline & 0.039 & 0.046 & 0.045 & & 0.047 \\
\hline & 0.041 & 0.045 & 0.039 & & 0.048 \\
\hline & 0.037 & 0.043 & 0.043 & & 0.043 \\
\hline & 0.04 & 0.043 & 0.039 & & 0.043 \\
\hline & 0.041 & 0.044 & 0.042 & & 0.038 \\
\hline & 0.043 & 0.048 & 0.042 & & 0.048 \\
\hline & 0.045 & 0.047 & 0.044 & & 0.053 \\
\hline & 0.025 & 0.018 & 0.051 & & 0.054 \\
\hline Barium & 4 n.d. at $<0.05$ & 4 n.d. at $<0.05$ & 4 n.d. at $<0.05$ & & 4 n.d. at $<0.05$ \\
\hline \multirow[t]{2}{*}{ Beryllium } & 0.0005 & 4 n.d. at $<0.0002$ & 4 n.d. at $<0.0002$ & & 4 n.d. at $<0.0002$ \\
\hline & $\begin{array}{l}4 \text { n.d. at }<0.0002 \\
11 \text { n.d. at }<0.0005\end{array}$ & 12 n.d. at $<0.0005$ & 12 n.d. at $<0.0005$ & & 12 n.d. at $<0.0005$ \\
\hline \multirow[t]{4}{*}{ Bicarbonate alk (as $\mathrm{CaCO}_{3}$ ) } & 260 & 240 & 250 & & 250 \\
\hline & 260 & 240 & 250 & & 250 \\
\hline & 260 & 240 & 250 & & 250 \\
\hline & 250 & 240 & 250 & & 230 \\
\hline Cadmium & 4 n.d. at $<0.0005$ & 4 n.d. at $<0.0005$ & 4 n.d. at $<0.0005$ & & 4 n.d. at $<0.0005$ \\
\hline \multirow[t]{4}{*}{ Calcium } & 14 & 38 & 22 & & 22 \\
\hline & 13 & 41 & 23 & & 23 \\
\hline & 13 & 41 & 21 & & 22 \\
\hline & 15 & 37 & 19 & & 19 \\
\hline \multirow[t]{2}{*}{ Carbonate alk (as $\mathrm{CaCO}_{3}$ ) } & 4 n.d. at $<1$ & 4 n.d. at $<1$ & 4 n.d. at $<1$ & & 20 \\
\hline & & & & & 3 n.d. at $<1$ \\
\hline \multirow[t]{4}{*}{ Chloride } & 140 & 280 & 220 & & 220 \\
\hline & 150 & 290 & 220 & & 230 \\
\hline & 150 & 290 & 230 & & 240 \\
\hline & 140 & 300 & 230 & & 230 \\
\hline \multirow[t]{4}{*}{ Chromium } & 0.008 & 16 n.d. at $<0.005$ & 0.01 & & 0.0052 \\
\hline & 0.007 & & 0.008 & & 0.006 \\
\hline & 14 n.d. at $<0.005$ & & 0.005 & & 14 n.d. at $<0.005$ \\
\hline & & & 13 n.d. at $<0.005$ & & \\
\hline Cobalt & 4 n.d. at $<0.05$ & 4 n.d. at $<0.05$ & 4 n.d. at $<0.05$ & & 4 n.d. at $<0.05$ \\
\hline Copper & 4 n.d. at $<0.05$ & 4 n.d. at $<0.05$ & 4 n.d. at $<0.05$ & & 4 n.d. at $<0.05$ \\
\hline Hydroxide alk (as $\mathrm{CaCO}_{3}$ ) & 4 n.d. at $<1$ & 4 n.d. at $<1$ & 4 n.d. at $<1$ & & 4 n.d. at $<1$ \\
\hline
\end{tabular}




\section{Appendix C. Ground Water Protection Tables}

Table C-7. Parameters analyzed in the explosives rinse water surface impoundments area monitoring wells, Site 300, 1992 (continued).

\begin{tabular}{|c|c|c|c|c|c|}
\hline \multirow[b]{2}{*}{ Parameters } & \multicolumn{5}{|c|}{ Well } \\
\hline & $W-817-01$ & $W-817-02$ & $W-817-03$ & $W-817-03 A$ & $W-817-04$ \\
\hline \multirow[t]{2}{*}{ Iron } & 0.2 & 4 n.d. at $<0.1$ & 4 n.d. at $<0.1$ & & 4 n.d. at $<0.1$ \\
\hline & 3 n.d. at $<0.1$ & & & & \\
\hline \multirow[t]{5}{*}{ Lead } & 0.002 & 0.002 & 16 n.d. at $<0.002$ & & 0.003 \\
\hline & 0.004 & 15 n.d. at $<0.002$ & & & 14 n.d. at $<0.002$ \\
\hline & 0.002 & & & & 1 n.d. at $<0.004$ \\
\hline & 0.005 & & & & \\
\hline & 12 n.d. at $<0.002$ & & & & \\
\hline \multirow[t]{4}{*}{ Magnesium } & 5 & 17 & 10 & & 9.8 \\
\hline & 5.7 & 20 & 11 & & 10 \\
\hline & 5 & 18 & 9.5 & & 9.7 \\
\hline & 5.3 & 15 & 9.4 & & 85 \\
\hline Manganese & 4 n.d. at $<0.05$ & 3 n.d. at $<0.05$ & 3 n.d. at $<0.05$ & & 3 n.d. at $<0.05$ \\
\hline \multirow[t]{2}{*}{ Mercury } & 4 n.d. at $<0.0002$ & 4 n.d. at $<0.0002$ & 0.0002 & & 4 n.d. at $<0.0002$ \\
\hline & & & 3 n.d. at $<0.0002$ & & \\
\hline \multirow[t]{2}{*}{ Molybdenum } & 3 n.d. at $<0.2$ & 3 n.d. at $<0.2$ & 3 n.d. at $<0.2$ & & 3 n.d. at $<0.2$ \\
\hline & 1 n.d at $<0.1$ & $1 \mathrm{n} . \mathrm{d}$ at $<0.1$ & $1 \mathrm{n} . \mathrm{d}$ at $<0.1$ & & $1 \mathrm{n} . \mathrm{d}$ at $<0.1$ \\
\hline \multirow[t]{2}{*}{ Nickel } & 2 n.d. at $<0.1$ & 1 n.d. at $<0.1$ & 1 n.d. at $<0.1$ & & 1 n.d. at $<0.1$ \\
\hline & 2. n.d at $<0.003$ & $3 \mathrm{n} . \mathrm{d}$ at $<0.005$ & $3 \mathrm{n} . \mathrm{d}$ at $<0.005$ & & 3 n.d at $<0.005$ \\
\hline \multirow[t]{4}{*}{ Nitrate plus nitrite (as N) } & 17 & 20 & 17 & & 21 \\
\hline & 20 & 23 & 23 & & 24 \\
\hline & 17 & 20 & 21 & & 22 \\
\hline & 40 & 21 & 21 & & 22 \\
\hline \multirow[t]{4}{*}{ Nitrate (as $\mathrm{NO}_{3}$ ) } & 75 & 90 & 75 & & 94 \\
\hline & 90 & 100 & 100 & & 110 \\
\hline & 76 & 87 & 92 & & 96 \\
\hline & 79 & 91 & 93 & & 95 \\
\hline \multirow[t]{2}{*}{ Nitrite (as N) } & 0.015 & $<0.01$ & $<0.01$ & & $<0.01$ \\
\hline & 0.01 & $<0.01$ & $<0.01$ & & $<0.01$ \\
\hline \multirow[t]{2}{*}{ Nitrite (as $\mathrm{NO}_{2}$ ) } & 0.05 & $<0.03$ & $<0.03$ & & $<0.03$ \\
\hline & 0.04 & $<0.03$ & $<0.03$ & & $<0.03$ \\
\hline \multirow[t]{4}{*}{ Potassium } & 9 & 14 & 10 & & 11 \\
\hline & 9.4 & 15 & 11 & & 12 \\
\hline & 8.4 & 14 & 10 & & 11 \\
\hline & 9 & 12 & 11 & & 10 \\
\hline \multirow[t]{10}{*}{ Selenium } & 0.038 & 0.098 & 0.039 & & 0.031 \\
\hline & 0.039 & 0.07 & 0.021 & & 0.018 \\
\hline & $<0.002$ & 0.09 & 0.038 & & 0.033 \\
\hline & 0.033 & 0.091 & 0.032 & & 0.029 \\
\hline & 0.033 & 0.08 & 0.032 & & 0.028 \\
\hline & 0.026 & 0.069 & 0.028 & & 0.021 \\
\hline & 0.035 & 0.081 & 0.034 & & 0.028 \\
\hline & 0.023 & 0.067 & 0.031 & & 0.021 \\
\hline & 0.038 & 0.091 & 0.037 & & 0.029 \\
\hline & 0.034 & 0.088 & 0.034 & & 0.029 \\
\hline
\end{tabular}




\section{Appendix C. Ground Water Protection Tables}

Table C-7. Parameters analyzed in the explosives rinse water surface impoundments area monitoring wells, Site 300, 1992 (continued).

\begin{tabular}{|c|c|c|c|c|c|}
\hline \multirow[b]{2}{*}{ Parameters } & \multicolumn{5}{|c|}{ Well } \\
\hline & $W-817-01$ & $W-817-02$ & $W-817-03$ & $W-817-03 A$ & $W-817-04$ \\
\hline \multirow[t]{6}{*}{ Selenium (continued) } & 0.035 & 0.03 & 0.011 & & 0.009 \\
\hline & 0.024 & 0.071 & 0.027 & & 0.022 \\
\hline & 0.015 & 0.073 & 0.012 & & 0.015 \\
\hline & 0.019 & 0.062 & 0.029 & & 0.02 \\
\hline & 0.006 & 0.049 & 0.016 & & 0.014 \\
\hline & 0.019 & 0.075 & 0.025 & & 0.022 \\
\hline \multirow[t]{2}{*}{ Silver } & 0.0005 & 0.0007 & 0.0005 & & 0.0017 \\
\hline & 15 n.d. at $<0.0005$ & 15 n.d. at $<0.0005$ & 15 n.d. at $<0.0005$ & & 15 n.d. at $<0.0005$ \\
\hline \multirow[t]{4}{*}{ Sodium } & 250 & 420 & 310 & & 360 \\
\hline & 270 & 450 & 330 & & 350 \\
\hline & 240 & 420 & 300 & & 330 \\
\hline & 280 & 380 & 310 & & 330 \\
\hline \multirow[t]{4}{*}{ Sulfate } & 110 & 390 & 220 & & 260 \\
\hline & 120 & 420 & 260 & & 300 \\
\hline & 120 & 410 & 250 & & 290 \\
\hline & 120 & 370 & 220 & & 230 \\
\hline \multirow[t]{2}{*}{ Thallium } & 3 n.d. at $<0.2$ & 3 n.d. at $<0.2$ & 3 n.d. at $<0.2$ & & 3 n.d. at $<0.2$ \\
\hline & 1 n.d. at $<0.4$ & 1 n.d. at $<0.4$ & 1 n.d. at $<0.4$ & & 1 n.d. at $<0.4$ \\
\hline \multirow[t]{4}{*}{ Total alkalinity (as $\mathrm{CaCO}_{3}$ ) } & 260 & 240 & 250 & & 250 \\
\hline & 260 & 240 & 250 & & 250 \\
\hline & 260 & 240 & 250 & & 250 \\
\hline & 250 & 240 & 250 & & 250 \\
\hline \multirow[t]{4}{*}{ Total dissolved solids (TDS) } & 810 & 1400 & 1100 & & 1200 \\
\hline & 830 & 1400 & 1100 & & 1200 \\
\hline & 860 & 1400 & 1100 & & 1200 \\
\hline & 830 & 1400 & 1100 & & 1100 \\
\hline \multirow[t]{16}{*}{ Total organic carbon (TOC) } & 1.1 & 0.94 & 1 & & 1 \\
\hline & 0.81 & 0.74 & 0.88 & & 0.92 \\
\hline & 1.2 & 0.7 & 1 & & 1.1 \\
\hline & 1.1 & 1 & 0.9 & & 1.1 \\
\hline & 0.92 & 1 & 0.78 & & 0.81 \\
\hline & 0.8 & 0.57 & 0.69 & & 0.82 \\
\hline & 1 & 0.7 & 0.9 & & 0.9 \\
\hline & 1.1 & 0.9 & 1.4 & & 1.2 \\
\hline & 0.86 & 0.81 & 0.79 & & 0.84 \\
\hline & 1.6 & 1.1 & 0.7 & & 1.3 \\
\hline & 4.2 & 0.8 & 0.9 & & 1 \\
\hline & 1.2 & 0.9 & 1.1 & & 1 \\
\hline & 1 & 0.73 & 0.89 & & 0.97 \\
\hline & 1.1 & 1.1 & 1 & & 1.5 \\
\hline & 1.4 & 1 & 1.4 & & 1.2 \\
\hline & 0.88 & 0.69 & 0.78 & & 0.93 \\
\hline \multirow[t]{2}{*}{ TOX, 1st replicate } & 0.03 & 0.03 & 0.12 & & 0.03 \\
\hline & 0.03 & 0.02 & 0.02 & & 0.05 \\
\hline
\end{tabular}


Table C-7. Parameters analyzed in the explosives rinse water surface impoundments area monitoring wells, Site 300, 1992 (continued).

\begin{tabular}{|c|c|c|c|c|c|}
\hline \multirow[b]{2}{*}{ Parameters } & \multicolumn{5}{|c|}{ Wells } \\
\hline & $W-817-01$ & W-817-02 & $W-817-03$ & W-817-03A & $W-817-04$ \\
\hline TOX, 1st replicate & 0.03 & 0.02 & 0.02 & & 0.02 \\
\hline \multirow[t]{10}{*}{ (continued) } & 0.025 & 13 n.d. at $<0.02$ & 0.04 & & 0.021 \\
\hline & 0.025 & & 0.02 & & 0.02 \\
\hline & 0.03 & & 0.02 & & 0.02 \\
\hline & 0.03 & & 10 n.d. at $<0.02$ & & 10 n.d. at $<0.02$ \\
\hline & 0.03 & & & & \\
\hline & 0.03 & & & & \\
\hline & 0.02 & & & & \\
\hline & 0.03 & & & & \\
\hline & 0.02 & & & & \\
\hline & 4 n.d. at $<0.02$ & & & & \\
\hline \multirow[t]{12}{*}{ TOX, 2nd replicate } & 0.03 & 0.03 & 0.08 & & 0.02 \\
\hline & 0.03 & 0.02 & 0.02 & & 0.03 \\
\hline & 0.04 & 0.02 & 0.02 & & 0.02 \\
\hline & 0.023 & 13 n.d. at $<0.02$ & 0.03 & & 0.02 \\
\hline & 0.024 & & 0.02 & & 0.02 \\
\hline & 0.02 & & 0.02 & & 11 n.d. at $<0.02$ \\
\hline & 0.03 & & 0.02 & & \\
\hline & 0.03 & & 9 n.d. at $<0.02$ & & \\
\hline & 0.03 & & & & \\
\hline & 0.02 & & & & \\
\hline & 0.03 & & & & \\
\hline & 5 n.d. at $<0.02$ & & & & \\
\hline \multirow[t]{4}{*}{ Vanadium } & 0.15 & 0.15 & 0.14 & & 0.13 \\
\hline & 0.16 & 0.14 & 0.13 & & 0.12 \\
\hline & 0.14 & 0.12 & 0.12 & & $<0.05$ \\
\hline & 0.16 & 0.16 & 0.13 & & 0.13 \\
\hline Zinc & 4 n.d. at $<0.05$ & 4 n.d. at $<0.05$ & 4 n.d. at $<0.05$ & & 4 n.d. at $<0.05$ \\
\hline Specific conductance & 1120 & 2010 & 1540 & & 1660 \\
\hline \multirow[t]{15}{*}{$(\mu \mathrm{mhos} / \mathrm{cm})$} & 1100 & 2080 & 1780 & & 1720 \\
\hline & 1270 & 2140 & 1630 & & 1840 \\
\hline & 1470 & 2350 & 2080 & & 1880 \\
\hline & 1200 & 2240 & 1580 & & 1760 \\
\hline & 1120 & 2010 & 1680 & & 1730 \\
\hline & 1050 & 1790 & 1560 & & 1730 \\
\hline & 1180 & 2110 & 1880 & & 1870 \\
\hline & 1080 & 2060 & 1740 & & 1640 \\
\hline & 1370 & 2060 & 1700 & & 1700 \\
\hline & 1230 & 1820 & 1430 & & 1760 \\
\hline & 1160 & 2030 & 1700 & & 1860 \\
\hline & 1080 & 2010 & 1740 & & 1560 \\
\hline & 1180 & 2020 & 1880 & & 1680 \\
\hline & 1150 & 2150 & 1610 & & 1730 \\
\hline & 1220 & 2310 & 1720 & & 1870 \\
\hline
\end{tabular}


Table C-7. Parameters analyzed in the explosives rinse water surface impoundments area monitoring wells, Site 300, 1992 (continued).

\begin{tabular}{|c|c|c|c|c|c|}
\hline \multirow[b]{2}{*}{ Parameters } & \multicolumn{5}{|c|}{ Well } \\
\hline & $W-817-01$ & $W-817-02$ & $W-817-03$ & $W-817-03 A$ & $W-817-04$ \\
\hline \multirow[t]{16}{*}{$\mathrm{pH}$} & 7.9 & 7.7 & 7.9 & & 8 \\
\hline & 7.9 & 8.1 & 8 & & 8 \\
\hline & 8.3 & 7.7 & 7.8 & & 8.2 \\
\hline & 8.1 & 8 & 8.1 & & 8.2 \\
\hline & 7.8 & 7.6 & 7.7 & & 7.8 \\
\hline & 8 & 7.8 & 7.9 & & 7.9 \\
\hline & 8.2 & 7.9 & 8 & & 8.1 \\
\hline & 8 & 7.8 & 7.9 & & 8.1 \\
\hline & 7.7 & 7.5 & 7.7 & & 7.8 \\
\hline & 8.1 & 7.7 & 7.9 & & 9 \\
\hline & 8 & 7.8 & 7.8 & & 8 \\
\hline & 7.9 & 8.4 & 7.8 & & 8.4 \\
\hline & 7.7 & 7.9 & 8 & & 8 \\
\hline & 8.1 & 7.9 & 8.1 & & 7.8 \\
\hline & 7.8 & 7.8 & 7.7 & & 8.6 \\
\hline & 8 & 7.8 & 7.9 & & 8 \\
\hline
\end{tabular}

\section{Purgeable pollutants (EPA Method 601, $\mu \mathrm{g} /$ )}

1,1,1-Trichloroethane

1,1,2,2-Tetrachloroethane

1,1,2-Trichloroethane

1,1-Dichloroethane

1,1-Dichloroethene

1,2-Dichlorobenzene

1,2-Dichloroethane

1,2-Dichloroethene (total)

1,2-Dichloropropane

1,3-Dichlorobenzene

1,4-Dichlorobenzene

2-Chloroethylvinylether

Bromodichloromethane

Bromoform

Bromomethane

Carbon tetrachloride

Chlorobenzene

Chloroethane

Chloroform

Chloromethane
4 n.d. at $<0.5$

4 n.d. at $<0.5$

4 n.d. at $<0.5$

3 n.d at $<0.5$

4 n.d. at $<0.5$

4 n.d. at $<0.5$

4 n.d. at $<0.5$

4 n.d. at $<0.5$

4 n.d. at $<0.5$

4 n.d. at $<0.5$

4 n.d. at $<0.5$

4 n.d. at $<0.5$

4 n.d. at $<0.5$

4 n.d. at $<0.5$

4 n.d. at $<0.5$

4 n.d. at $<0.5$

4 n.d. at $<0.5$

4 n.d. at $<0.5$

4 n.d. at $<0.5$

4 n.d. at $<0.5$

4 n.d. at $<0.5$
4 n.d. at $<0.5$

4 n.d. at $<0.5$

4 n.d. at $<0.5$

4 n.d. at $<0.5$

4 n.d. at $<0.5$

4 n.d. at $<0.5$

4 n.d. at $<0.5$

4 n.d. at $<0.5$

4 n.d. at $<0.5$

4 n.d. at $<0.5$

4 n.d. at $<0.5$

4 n.d. at $<0.5$

4 n.d. at $<0.5$

4 n.d. at $<0.5$

4 n.d. at $<0.5$

4 n.d. at $<0.5$

4 n.d. at $<0.5$

4 n.d. at $<0.5$

4 n.d. at $<0.5$

4 n.d. at $<0.5$

\begin{abstract}
4 n.d. at $<0.5$
4 n.d. at $<0.5$

4 n.d. at $<0.5$

4 n.d. at $<0.5$

4 n.d. at $<0.5$

4 n.d. at $<0.5$

4 n.d. at $<0.5$

4 n.d. at $<0.5$
\end{abstract}

$\begin{aligned} & 4 \mathrm{n} . \mathrm{d} \text { at }<0.5 \\ & 4 \mathrm{n} . \mathrm{d} \text { at }<0.5 \\ & 0.65 \\ & 4 \mathrm{n} . \mathrm{d} \text { at }<0.5 \\ & 4 \mathrm{n} . \mathrm{d} \text { at }<0.5 \\ & 4 \mathrm{n} . \mathrm{d} \text { at }<0.5 \\ & 4 \mathrm{n} . \mathrm{d} \text { at }<0.5 \\ & 1.2 \\ & 2.8 \\ & 2.6 \\ & \quad 0.9 \\ & 4 \mathrm{n} . \mathrm{d} \text { at }<0.5 \\ & 4 \mathrm{n} . \mathrm{d} \text { at }<0.5 \\ & 4 \mathrm{n} . \mathrm{d} \text { at }<0.5 \\ & 4 \mathrm{n} . \mathrm{d} \text { at }<0.5 \\ & 4 \mathrm{n} . \mathrm{d} \text { at }<0.5 \\ & 4 \mathrm{n} . \mathrm{d} \text { at }<0.5 \\ & 4 \mathrm{n} . \mathrm{d} \text { at }<0.5 \\ & 4 \mathrm{n} . \mathrm{d} \text { at }<0.5 \\ & 4 \mathrm{n} . \mathrm{d} \text { at }<0.5 \\ & 4 \mathrm{n} . \mathrm{d} \text { at }<0.5 \\ & 4 \mathrm{n} . \mathrm{d} \text { at }<0.5 \\ & 4 \mathrm{n} . \mathrm{d} \text { at }<0.5\end{aligned}$

5 n.d. at $<0.5$

5 n.d. at $<0.5$

5 n.d. at $<0.5$

5 n.d. at $<0.5$

5 n.d. at $<0.5$

5 n.d. at $<0.5$

5 n.d. at $<0.5$

5 n.d. at $<0.5$

4 n.d. at $<0.5$

4 n.d. at $<0.5$

4 n.d. at $<0.5$

4 n.d. at $<0.5$

4 n.d. at $<0.5$

4 n.d. at $<0.5$

4 n.d. at $<0.5$

4 n.d. at $<0.5$

4 n.d. at $<0.5$

4 n.d. at $<0.5$

4 n.d. at $<0.5$

4 n.d. at $<0.5$
4 n.d at $<0.5$

5 n.d. at $<0.5$

5 n.d. at $<0.5$

$5 \mathrm{n}$.d. at $<0.5$

5 n.d. at $<0.5$

5 n.d. at $<0.5$

5 n.d. at $<0.5$

5 n.d. at $<0.5$

5 n.d. at $<0.5$

5 n.d. at $<0.5$

5 n.d. at $<0.5$

5 n.d. at $<0.5$

5 n.d. at $<0.5$ 


\section{Appendix C. Ground Water Protection Tables}

Table C-7. Parameters analyzed in the explosives rinse water surface impoundments area monitoring wells, Site 300, 1992 (concluded).

\begin{tabular}{|c|c|c|c|c|c|}
\hline \multirow[b]{2}{*}{ Parameters } & \multicolumn{5}{|c|}{ Well } \\
\hline & W-817-01 & $W-817-02$ & $W-817.03$ & $W-817-03 A$ & $W-817-04$ \\
\hline \multirow[t]{4}{*}{ cis-1,2-Dichloroethene } & 4 n.d. at $<0.5$ & 4 n.d. at $<0.5$ & 4 n.d. at $<0.5$ & 1.2 & 5 n.d. at $<0.5$ \\
\hline & & & & 2.8 & \\
\hline & & & & 2.6 & \\
\hline & & & & 0.9 & \\
\hline cis-1,3-Dichloropropene & 4 n.d. at $<0.5$ & 4 n.d. at $<0.5$ & 4 n.d. at $<0.5$ & 4 n.d at $<0.5$ & 5 n.d. at $<0.5$ \\
\hline Dibromochloromethane & 4 n.d. at $<0.5$ & 4 n.d. at $<0.5$ & 4 n.d. at $<0.5$ & 4 n.d at $<0.5$ & 5 n.d. at $<0.5$ \\
\hline Dichlorodifluoromethane & 4 n.d. at $<0.5$ & 4 n.d. at $<0.5$ & 4 n.d. at $<0.5$ & $4 \mathrm{n} . \mathrm{d}$ at $<0.5$ & 5 n.d. at $<0.5$ \\
\hline Freon-113 & 4 n.d. at $<0.5$ & 4 n.d. at $<0.5$ & 4 n.d. at $<0.5$ & $4 \mathrm{n} . \mathrm{d}$ at $<0.5$ & 5 n.d. at $<0.5$ \\
\hline Methylene chloride & 4 n.d. at $<0.5$ & 4 n.d. at $<0.5$ & 4 n.d. at $<0.5$ & $4 \mathrm{n} . \mathrm{d}$ at $<0.5$ & 5 n.d. at $<0.5$ \\
\hline Tetrachloroethene & 4 n.d. at $<0.5$ & 4 n.d. at $<0.5$ & 4 n.d. at $<0.5$ & $4 \mathrm{n} . \mathrm{d}$ at $<0.5$ & 5 n.d. at $<0.5$ \\
\hline trans-1,2-Dichloroethene & 4 n.d. at $<0.5$ & 4 n.d. at $<0.5$ & 4 n.d. at $<0.5$ & 4 n.d at $<0.5$ & 5 n.d. at $<0.5$ \\
\hline trans-1,3-Dichloropropene & 4 n.d. at $<0.5$ & 4 n.d. at $<0.5$ & 4 n.d. at $<0.5$ & 4 n.d at $<0.5$ & 5 n.d. at $<0.5$ \\
\hline \multirow[t]{5}{*}{ Trichloroethene } & 4 n.d. at $<0.5$ & 4 n.d. at $<0.5$ & 22 & 47 & 9.6 \\
\hline & & & 19 & 64 & 17 \\
\hline & & & & 71 & 14 \\
\hline & & & 21 & 66 & 15 \\
\hline & & & 21 & & 7.7 \\
\hline Trichlorofluoromethane & 4 n.d. at $<0.5$ & 4 n.d. at $<0.5$ & 4 n.d. at $<0.5$ & $4 \mathrm{n} . \mathrm{d}$ at $<0.5$ & 5 n.d. at $<0.5$ \\
\hline Vinyl chloride & 4 n.d. at $<0.5$ & 4 n.d. at $<0.5$ & 4 n.d. at $<0.5$ & 4 n.d at $<0.5$ & 5 n.d. at $<0.5$ \\
\hline \multicolumn{6}{|l|}{ Explosive compounds $(\mu g / L)$} \\
\hline $\mathrm{HMX}$ & 16 n.d. at $<20$ & 16 n.d. at $<20$ & 17 n.d. at $<20$ & 3 n.d at $<20$ & 17 n.d. at $<20$ \\
\hline \multirow[t]{13}{*}{ RDX } & 107 & 16 n.d. at $<30$ & 17 n.d. at $<30$ & 3 n.d at $<30$ & 17 n.d. at $<30$ \\
\hline & 200 & & & & \\
\hline & 72 & & & & \\
\hline & 109 & & & & \\
\hline & 116 & & & & \\
\hline & 74 & & & & \\
\hline & 94 & & & & \\
\hline & 92 & : & & & \\
\hline & 76 & & & & \\
\hline & 163 & & & & \\
\hline & 74 & & & & \\
\hline & 189 & & & & \\
\hline & 4 n.d. at $<30$ & & & & \\
\hline TNT & 16 n.d. at $<30$ & 16 n.d. at $<30$ & 17 n.d. at $<30$ & 3 n.d at $<30$ & 17 n.d. at $<30$ \\
\hline \multicolumn{6}{|l|}{ Radiologicals (Bq/L) } \\
\hline \multirow[t]{4}{*}{ Tritium } & $-4.1 \pm 3.1$ & $-3.0 \pm 3.2$ & $1.4 \pm 3.4$ & $2.7 \pm 3.5$ & $-4.4 \pm 3.2$ \\
\hline & $3.2 \pm 4.6$ & $-2.4 \pm 4.4$ & $1.5 \pm 4.6$ & $-0.1 \pm 4.5$ & $-3.9 \pm 4.4$ \\
\hline & $1.9 \pm 2.5$ & $0.1 \pm 3.2$ & $1.6 \pm 3.3$ & $-0.7 \pm 3.2$ & $-2.6 \pm 3.2$ \\
\hline & $-3.6 \pm 3.0$ & $-3.0 \pm 3.0$ & $1.5 \pm 3.4$ & $-1.1 \pm 3.2$ & $-1.5 \pm 3.2$ \\
\hline
\end{tabular}

Note: The abbreviation "n.d." starids for nondetection. 
Table C-8. Parameters sampled in the Pit 9 Area monitoring wells, Site 300 , 1992.

\begin{tabular}{|c|c|c|c|c|}
\hline \multirow[b]{2}{*}{ Parameters } & \multicolumn{4}{|c|}{ Well } \\
\hline & K9-01 & K9-02 & K9-03 & K9-04 \\
\hline \multicolumn{5}{|l|}{ Physical and chemical (mg/L) } \\
\hline Ammonia nitrogen (as N) & 1.3 & 0.89 & 1.3 & \\
\hline Arsenic & 0.003 & 0.036 & 0.012 & $<0.002$ \\
\hline Barium & $<0.05$ & $<0.05$ & $<0.05$ & $<0.05$ \\
\hline Beryllium & $<0.0005$ & $<0.0005$ & $<0.0005$ & $<0.0005$ \\
\hline Bicarbonate alk (as $\mathrm{CaCO}_{3}$ ) & 160 & 200 & 170 & \\
\hline Cadmium & $<0.0005$ & $<0.0005$ & $<0.0005$ & $<0.0005$ \\
\hline Calcium & 80 & 64 & 83 & \\
\hline Carbonate alk (as $\mathrm{CaCO}_{3}$ ) & $<1$ & $<1$ & $<1$ & \\
\hline Chloride & 150 & 170 & 140 & \\
\hline Chromium & $<0.005$ & $<0.005$ & $<0.005$ & $<0.005$ \\
\hline Copper & $<0.05$ & $<0.05$ & $<0.05$ & \\
\hline Fluoride & 0.24 & 0.38 & 0.28 & \\
\hline Hydroxide alk (as $\mathrm{CaCO}_{3}$ ) & $<1$ & $<1$ & $<1$ & \\
\hline Iron & $<0.1$ & $<0.1$ & $<0.1$ & 0.54 \\
\hline Lead & $<0.002$ & $<0.002$ & 0.003 & 0.003 \\
\hline Low level phenolics & $<0.005$ & 0.0084 & $<0.005$ & \\
\hline Magnesium & 37 & 35 & 39 & \\
\hline Manganese & 0.1 & 0.061 & 0.069 & 0.15 \\
\hline Mercury & $<0.0002$ & $<0.0002$ & $<0.0002$ & $<0.0002$ \\
\hline Nickel & $<0.005$ & $<0.005$ & $<0.005$ & $<0.005$ \\
\hline \multicolumn{5}{|l|}{ Nitrate nitrogen } \\
\hline Nitrate (as N) & $<0.1$ & $<0.1$ & $<0.1$ & \\
\hline Nitrate (as $\mathrm{NO}_{3}$ ) & $<0.4$ & $<0.4$ & $<0.4$ & \\
\hline Nitrite (as N) & $<0.01$ & $<0.01$ & $<0.01$ & \\
\hline Nitrite (as $\mathrm{NO}_{2}$ ) & $<0.03$ & $<0.03$ & $<0.03$ & \\
\hline $\mathrm{pH}$ & 7.6 & 7.8 & 7.8 & \\
\hline Potassium & 8.4 & 10 & 9.2 & \\
\hline Selenium & $<0.002$ & $<0.002$ & $<0.002$ & $<0.002$ \\
\hline Silver & 0.0021 & $<0.0005$ & $<0.0005$ & 0.0005 \\
\hline Sodium & 350 & 310 & 321 & 280 \\
\hline Specific conductance ( $\mu \mathrm{mhos} / \mathrm{cm})$ & 1820 & 1760 & 1880 & \\
\hline Sulfate & 620 & 520 & 680 & \\
\hline Total alkalinity (as $\mathrm{CaCO}_{3}$ ) & 160 & 200 & 170 & \\
\hline Total dissolved solids (TDS) & 1300 & 1200 & 1300 & \\
\hline Total Kjeldahl nitrogen & $<0.5$ & $<0.5$ & $<0.5$ & \\
\hline Total organic carbon (TOC) & 0.58 & 0.95 & 0.54 & \\
\hline Total suspended solids (TSS) & $<1$ & $<1$ & 4 & \\
\hline TOX, 1st replicate & $<0.02$ & $<0.02$ & $<0.02$ & \\
\hline TOX, 2nd replicate & $<0.02$ & $<0.02$ & $<0.02$ & \\
\hline Vanadium & $<0.05$ & $<0.05$ & $<0.05$ & $<0.05$ \\
\hline Zinc & $<0.05$ & $<0.05$ & $<0.05$ & \\
\hline
\end{tabular}




\section{Appendix C. Ground Water Protection Tables}

Table C-8. Parameters sampled in the Pit $\$$ Area monitoring wells, Site 300 , 1992 (continued).

\begin{tabular}{|c|c|c|c|c|}
\hline \multirow[b]{2}{*}{ Parameters } & \multicolumn{4}{|c|}{ Well } \\
\hline & K9-01 & K9-02 & $\mathbf{K} 9 \overline{03}$ & K9-04 \\
\hline \multicolumn{5}{|c|}{ Purgeable pollutants (EPA Method 624, $\mu \mathrm{g} /$ L) } \\
\hline 1,1,1-Trichloroethane & $<0.5$ & $<0.5$ & $<0.5$ & \\
\hline 1,1,2,2-Tetrachloroethane & $<0.5$ & $<0.5$ & $<0.5$ & \\
\hline 1,1,2-Trichloroethane & $<0.5$ & $<0.5$ & $<0.5$ & \\
\hline 1,1-Dichloroethane & $<0.5$ & $<0.5$ & $<0.5$ & \\
\hline 1,1-Dichloroethene & $<0.5$ & $<0.5$ & $<0.5$ & \\
\hline 1,2-Dichlorobenzene & $<0.5$ & $<0.5$ & $<0.5$ & \\
\hline 1,2-Dichloroethane & $<0.5$ & $<0.5$ & $<0.5$ & \\
\hline 1,2-Dichloroethene (total) & $<0.5$ & $<0.5$ & $<0.5$ & \\
\hline 1,2-Dichloropropane & $<0.5$ & $<0.5$ & $<0.5$ & \\
\hline 1,3-Dichlorobenzene & $<0.5$ & $<0.5$ & $<0.5$ & \\
\hline 1,4-Dichlorobenzene & $<0.5$ & $<0.5$ & $<0.5$ & \\
\hline 2-Chloroethylvinylether & $<0.5$ & $<0.5$ & $<0.5$ & \\
\hline 2-Hexanone & $<0.5$ & $<0.5$ & $<0.5$ & \\
\hline 4-Methyl-2-pentanone & $<0.5$ & $<0.5$ & $<0.5$ & \\
\hline Acetone & $<5$ & $<5$ & $<5$ & \\
\hline Acrolein & $<5$ & $<5$ & $<5$ & \\
\hline Acrylonitrile & $<5$ & $<5$ & $<5$ & \\
\hline Benzene & $<0.5$ & $<0.5$ & $<0.5$ & \\
\hline Bromodichloromethane & $<0.5$ & $<0.5$ & $<0.5$ & \\
\hline Bromoform & $<0.5$ & $<0.5$ & $<0.5$ & \\
\hline Bromomethane & $<0.5$ & $<0.5$ & $<0.5$ & \\
\hline Carbon disulfide & $<0.5$ & $<0.5$ & $<0.5$ & \\
\hline Carbon tetrachloride & $<0.5$ & $<0.5$ & $<0.5$ & \\
\hline Chlorobenzene & $<0.5$ & $<0.5$ & $<0.5$ & \\
\hline Chloroethane & $<0.5$ & $<0.5$ & $<0.5$ & \\
\hline Chloroform & $<0.5$ & $<0.5$ & $<0.5$ & \\
\hline Chloromethane & $<0.5$ & $<0.5$ & $<0.5$ & \\
\hline cis-1,2-Dichloroethene & $<0.5$ & $<0.5$ & $<0.5$ & \\
\hline cis-1,3-Dichloropropene & $<0.5$ & $<0.5$ & $<0.5$ & \\
\hline Dibromochloromethane & $<0.5$ & $<0.5$ & $<0.5$ & \\
\hline Ethylbenzene & $<0.5$ & $<0.5$ & $<0.5$ & \\
\hline Freon-113 & $<0.5$ & $<0.5$ & $<0.5$ & \\
\hline Methyl ethyl ketone & $<10$ & $<10$ & $<10$ & \\
\hline Methylene chloride & $<2$ & $<2$ & $<2$ & \\
\hline Styrene & $<0.5$ & $<0.5$ & $<0.5$ & \\
\hline Tetrachloroethene & $<0.5$ & $<0.5$ & $<0.5$ & \\
\hline Toluene & $<0.5$ & $<0.5$ & $<0.5$ & \\
\hline Total xylene isomers & $<0.5$ & $<0.5$ & $<0.5$ & \\
\hline trans-1,2-Dichloroethene & $<0.5$ & $<0.5$ & $<0.5$ & \\
\hline trans-1,3-Dichloropropene & $<0.5$ & $<0.5$ & $<0.5$ & \\
\hline
\end{tabular}


Table C-8. Parameters sampled in the Pit 9 Area monitoring wells, Site 300 , 1992 (concluded).

\begin{tabular}{|c|c|c|c|c|c|}
\hline \multirow{2}{*}{\multicolumn{2}{|c|}{ Parameters }} & \multicolumn{4}{|c|}{ Well } \\
\hline & & K9-01 & K9-02 & K9-03 & K9-04 \\
\hline \multicolumn{2}{|l|}{ Trichloroethene } & $<0.5$ & $<0.5$ & $<0.5$ & \\
\hline \multicolumn{2}{|c|}{ Trichlorofluoromethane } & $<0.5$ & $<0.5$ & $<0.5$ & $一^{a}$ \\
\hline \multicolumn{2}{|c|}{ Vinyl acetate } & $<0.5$ & $<0.5$ & $<0.5$ & $-a$ \\
\hline \multicolumn{2}{|l|}{ Vinyl chloride } & $<0.5$ & $<0.5$ & $<0.5$ & $-^{a}$ \\
\hline \multicolumn{6}{|c|}{ Title 22 herbicides ( $\mu g /$ ) } \\
\hline \multicolumn{2}{|c|}{ 2,4,5-TP (Silvex) } & $<1$ & $<1$ & $<1$ & $-a^{a}$ \\
\hline \multicolumn{2}{|c|}{$2,4-D$} & $<5$ & $<5$ & $<5$ & $-^{a}$ \\
\hline \multicolumn{6}{|c|}{ Tite 22 pesticides ( $\mu \mathrm{g} / \mathrm{L})$} \\
\hline \multicolumn{2}{|c|}{ BHC, gamma isomer (Lindane) } & $<0.03$ & $<0.03$ & $<0.03$ & -a \\
\hline \multicolumn{2}{|l|}{ Endrin } & $<0.05$ & $<0.05$ & $<0.05$ & $-\mathbf{a}$ \\
\hline \multicolumn{2}{|l|}{ Methoxychlor } & $<0.2$ & $<0.2$ & $<0.2$ & $-\mathbf{a}^{a}$ \\
\hline \multicolumn{2}{|l|}{ Toxaphene } & $<1$ & $<1$ & $<1$ & $-a$ \\
\hline \multicolumn{6}{|c|}{ Explosive compounds $(\mu g \Omega)$} \\
\hline \multicolumn{2}{|l|}{$H M X$} & $<20$ & $<20$ & $<20$ & $-{ }^{a}$ \\
\hline \multicolumn{2}{|l|}{ RDX } & $<30$ & $<30$ & $<30$ & $-a$ \\
\hline \multicolumn{2}{|l|}{ TNT } & $<30$ & $<30$ & $<30$ & - \\
\hline Parameters & K9-01 & K9-02 & $\mathrm{Kg}-03$ & \multicolumn{2}{|c|}{ K9-04 } \\
\hline \multicolumn{6}{|c|}{ Radiological Data (BqL) ${ }^{b}$} \\
\hline Gross alpha & $-0.13 \pm 0.16$ & $0.03 \pm 0.20$ & $0.01 \pm 0.15$ & \multicolumn{2}{|c|}{$-{ }^{a}$} \\
\hline Gross beta & $0.29 \pm 0.15$ & $0.26 \pm 0.13$ & $0.24 \pm 0.13$ & \multicolumn{2}{|c|}{-a } \\
\hline Tritium & $0.9 \pm 3.5$ & $-2.6 \pm 3.4$ & $-2.8 \pm 3.0$ & \multicolumn{2}{|c|}{$-a$} \\
\hline Uranium-234 & $0.012 \pm 0.004$ & $0.040 \pm 0.008$ & $0.044 \pm 0.007$ & \multicolumn{2}{|c|}{$0.029 \pm 0.007$} \\
\hline Uranium-235 & $-0.000 \pm 0.001$ & $-0.000 \pm 0.001$ & $0.001 \pm 0.001$ & \multicolumn{2}{|c|}{$0.001 \pm 0.002$} \\
\hline Uranium-238 & $0.000 \pm 0.000$ & $0.006 \pm 0.003$ & $0.007 \pm 0.003$ & \multicolumn{2}{|c|}{$0.003 \pm 0.003$} \\
\hline Radium-226 & $0.005 \pm 0.021$ & $0.003 \pm 0.011$ & $0.014 \pm 0.015$ & \multicolumn{2}{|c|}{$-{ }^{a}$} \\
\hline
\end{tabular}

a No sainple; see Quality Assurance chapter.

b Radionuclide results are reported $\pm 2 \sigma$; see Quality Assurance chapter. 
Air Resources Board

J. Morgester

Compliance Division

1102 " $Q$ " Street

Sacramento, CA 95814

Alameda County Department of Environmental Health

Jeff Shapiro

Hazardous Materials Division

800 Swan Way, Room 350

Oakland, CA 94621

Alameda County Flood

Control District

J. Killingstad

Zone 7

5997 Parkside Drive

Pleasanton, CA 94566

Alameda County Health Care

Services Agency

T. Shirasawa

Environmental Health Planning

470 27th Street

Oakland, CA 94612

Alameda County Water District

E. L. Lenahan

3805 Fremont Blvd.

Fremont, CA 94537

Argonne National Laboratory

Norbert Golchert

9700 S. Cass Avenue

Building 200, Room B-117

Argonne, IL 60439

Assistant Administrator for Air

Radiation (ANR-443)

U.S. Environmental Protection Agency

Washington, DC 20460

Association of Bay Area Governments

T. Bursztynsky

P.O. Box 2050

Oakland, CA 94604
Battelle NW

Richard E. Jaquish

P.O. Box 999

Richland, WA 99352

Bay Area Air Quality

Management District

Milton Feldstein

939 Ellis Street

San Francisco, CA 94109

Biomedical \& Environmental

Sciences Lab

Dr. O. R. Lunt, Director

University of California

900 Veteran Avenue

Los Angeles, CA 90024

Brookhaven National Laboratory

Robert Miltenberger

Bldg. 535A

Upton, NY 11973

California Department of Energy

Barbara J. Byron

Executive Office

1515 - 9th Street/MS-36

Sacramento, CA 95814

California Department of

Health Services

J. S. McGurk

K. Jackson

Radiologic Health Branch

714 "P" Street, Box 942732

Sacramento, CA 95814

California Environmental

Protection Agency

C. Williams

Department of Toxic

Substances Control

700 Heinz Avenue, Bldg. F

Berkeley, CA 94710 
California Regional Water Quality

Control Board

W.H. Crooks

S. Timm

Central Valley Region

3443 Routier Road

Sacramento, CA 95827-3098

California Regional Water Quality

Control Board

S. Ritchie

E. Adams

San Francisco Bay Region

2101 Webster Street \#500

Oakland, CA 94612

California State Water Resources

Control Board

W. Pettit

J. Diaz, Chief

Division of Water Quality

901 "P" Street

Sacramento, CA 95814

EG \& G Mound Applied Technologies

Daniel G. Carfagno

P.O. Box 3000

Miamisburg, $\mathrm{OH} 45343$

Environmental Measurements Lab

Edward P. Hardy, Jr., Director

Environmental Studies Division

U.S. Department of Energy

376 Hudson Street

New York, NY 10014-3621

Fermilab

Sam Baker

P.O. Box 500, MS-119

Batavia, IL 60510

Hanford Environmental Health

Foundation

L. J. Maas, Manager

Environmental Health Services

P.O. Box 100

Richland, WA 99352
Lawrence Berkeley Laboratory

Gary E. Schleimer

University of California

One Cyclotron Road

Berkeley, CA 94720

Livermore Water Reclamation Plant

$W$. Adams

1250 Kitty Hawk Road

Livermore, CA 94550

Los Alamos National Laboratory

John M. Puckett, Division Leader

Health Safety \& Environmental Div.

University of California

P.O. Box 1663

Los Alamos, NM 87545

Los Alamos National Laboratory

Lars Soholt

Environmental Surveillance Group

MS-K490

P.O. Box 1663

Los Alamos, NM 87545

Los Alamos National Laboratory

Doris Garvey

Environmental Assessments and

Resource Evaluation

Section HSE-8, MS-K490

Los Alamos, NM 87545

Los Alamos National Laboratory

Tom Buhl

Group HSE-8

MS-K490

Los Alamos, NM 87545

Nevada Operations Office

Bruce W. Church

Asst. Manager for Environment,

Safety and Health

P.O. Box 98518

Las Vegas, NV 89193-8518 
Oak Ridge National Laboratory

Karen Daniels

Building $4500 \mathrm{~S}$.

MS-6102

Oak Ridge, TN 37831-6102

Oak Ridge National Laboratory

John B. Murphy

Head, Environmental Surveillance and Protection Section

P.O. Box 2008

Oak Ridge, TN 37831-6102

Pacific Northwest Laboratory

W. W. Laity, General Manager

Environmental Mgmt. Operations

Battelle Blvd.

P.O. Box 999

Richland, WA 993252

Radiobiology \& Environmental

Health Laboratory

Dr. Sheldon Wolff, Director

University of California

Medical Center

San Francisco, CA 94143

REECO

Daniel A. Gonzalez

Health Physics Department

P.O. Box 98521, MS-708

Las Vegas, NV 89193-8521

Rockwell International

George H. Setlock

Rocky Flats Plant

P.O. Box 464

Golden, CO 80402-0464

Rocky Flats Area Office Chief, Candice C. Jierree

Safety, Environmental Program Branch

U.S. Department of Energy

P.O. Box 928

Golden, CO 80402-0928
San Joaquin Valley Unified Air

Pollution Control District

Anthony Mendes

Engineering Manager

4230 Kiernan Avenue, Suite 130

Modesto, CA 95356

Sandia National Laboratories,

Albuquerque

N. R. Ortiz, Director

Environment, Safety and Health

P.O. Box 5800

Albuquerque, NM 87185

Sandia National Laboratories, California

A. West

P.O. Box 969

Livermore, CA 94551-0969

Sandia National Laboratories,

California

D. Brekke

P.O. Box 969

Livermore, CA 94551

San Joaquin County Public

Health Services

Mr. Doug Wilson

Environmental Health Division

P. O. Box 2009

Stockton, CA 95201

San Joaquin Local Health District

V. V. Williams

P.O. Box 2009

Stockton, CA 95201

Savannah River Plant

Dennis Stevenson, Chief Supervisor,

Environmental Monitoring

Health Protection Department

Building 735A

Aiken, SC 29808 
Stanford University

H. Gusterson

Anthropology Department

Stanford, CA 94305

U.S. Department of Energy

Eddie Chew

785 Department of Energy Place

Idaho Falls, ID 83401-4149

U.S. Environmental Protection Agency

S. Rosenblum

D. McGovern

Region IX

75 Hawthorne Street

San Francisco, CA 94105

\&U.S. GOVERavaNT PRTNITNG OFFICE: 1993-583-562/19020 
$r$
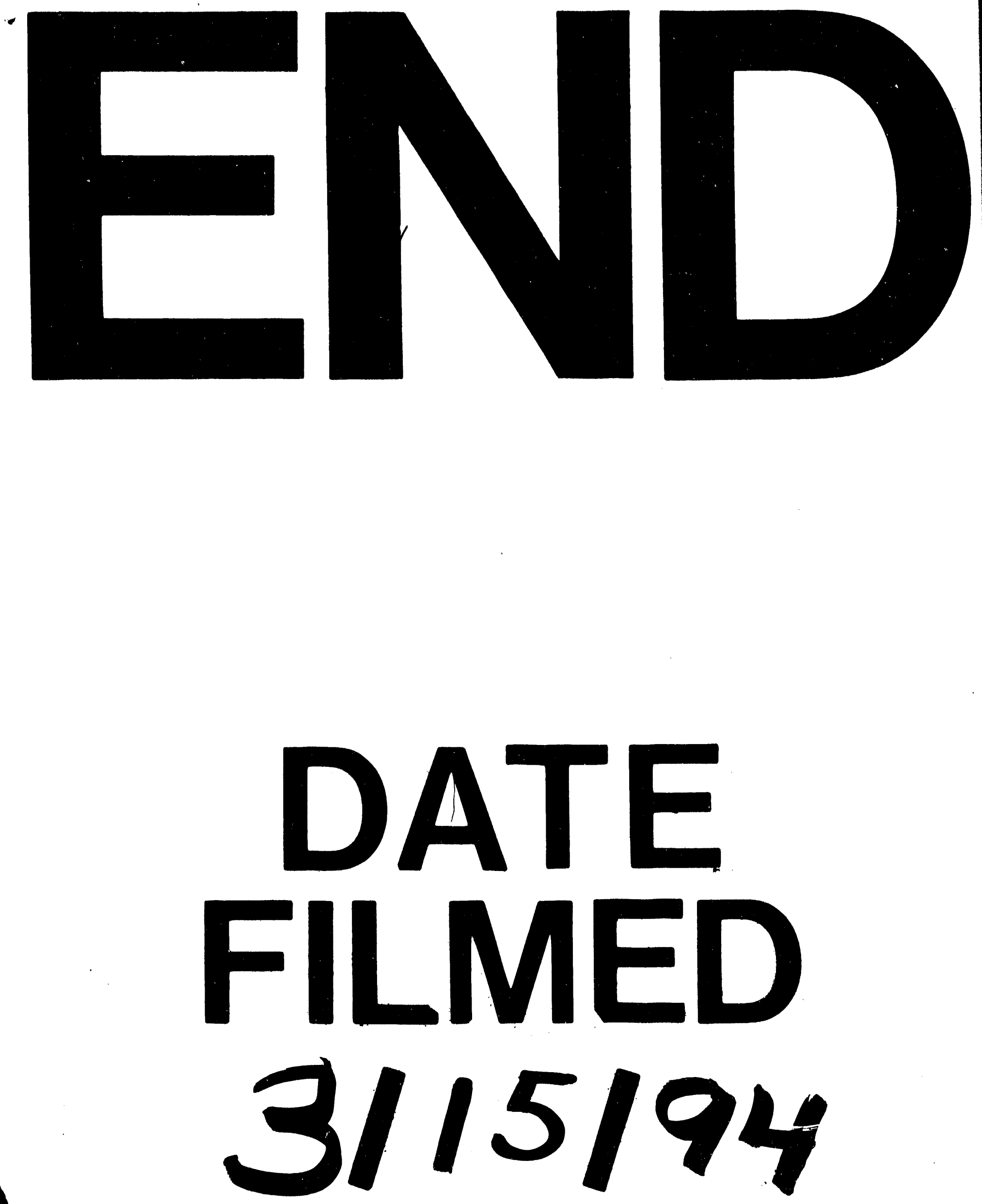
$\longrightarrow$

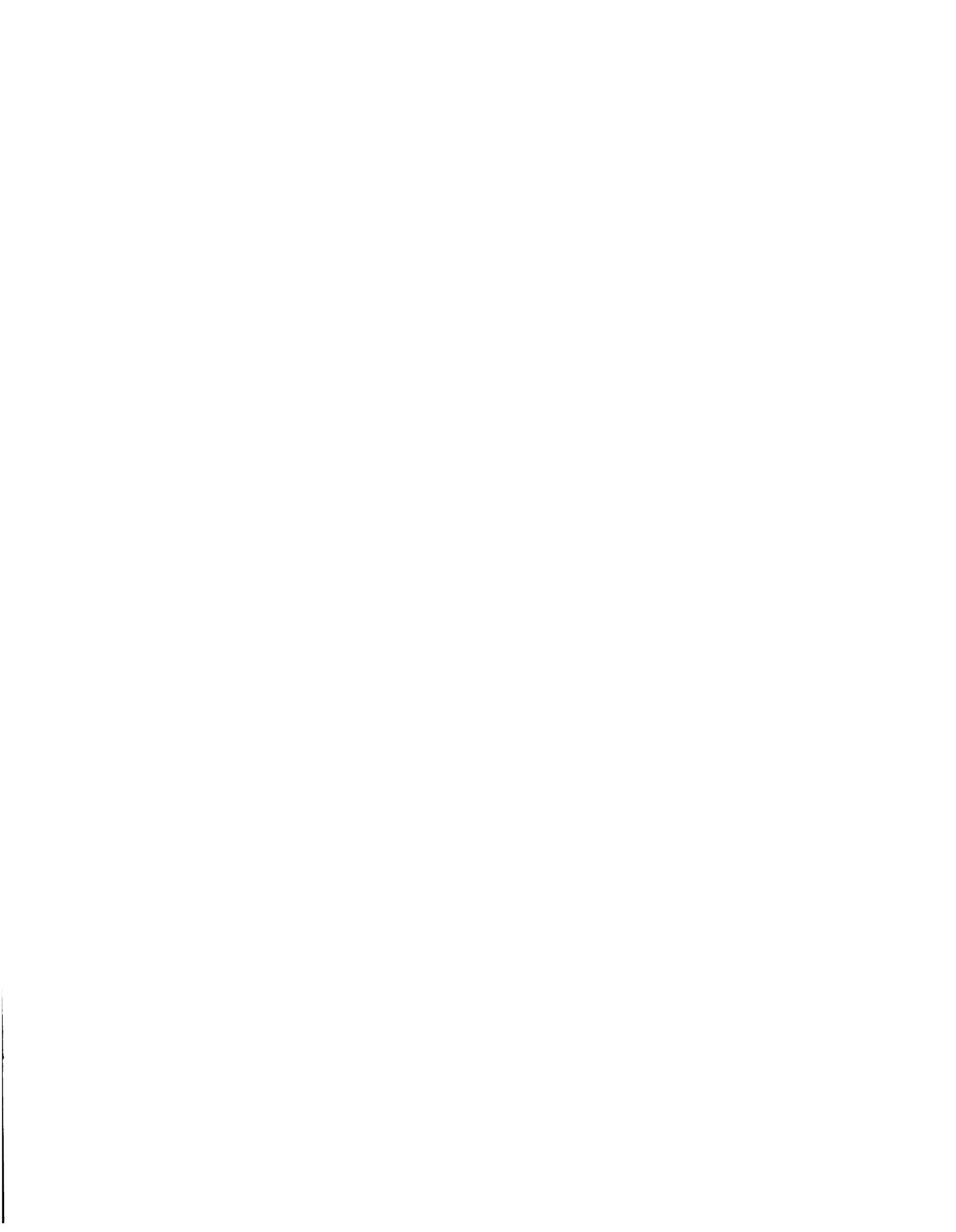

